$$
\begin{gathered}
\text { SAND 79-0369 } \\
\text { NUREG/CR-0743 } \\
\text { Unlimited Release } \\
\text { RT }
\end{gathered}
$$

NUREG/CR--0743

TI86 000909

\title{
TRANSPORTATION OF RADIONUCLIDES IN URBAN ENVIRONS: \\ DRAFT ENVIRONMENTAL ASSESSSMENT
}

\author{
Nancy C. Finley (Project Coordinator) \\ David C. Aldrich \\ Sharon L. Daniel \\ David M. Ericson \\ Corinne Henning-Sachs \\ Peter C. Kaestner \\ Nestor R. Ortiz \\ Danie1 D. Sheldon \\ John M. Taylor \\ Scott F. Herreid \\ University of New Mexìco \\ Printed July 1980
}

Sandia National Laboratories

Albuquerque, NM 87185

operated by

Sandia Corporation

for the

U.S. Department of Energy

Prepared for

Transportation and Product Standards Branch

office of Standards Development

U.S. Nuclear Regulatory Commission

Washington, D.C. 20555

This document is

NRC FIN No.' A1077
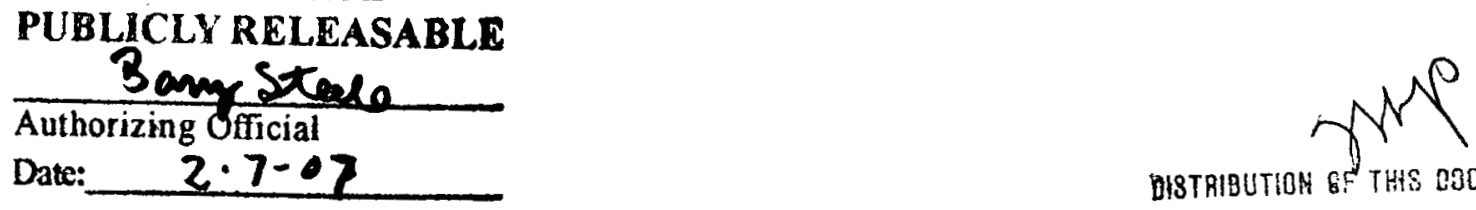

DHSTRISUTION GE THS DSCUMENT IS URUMAREB 


\section{DISCLAIMER}

This report was prepared as an account of work sponsored by an agency of the United States Government. Neither the United States Government nor any agency Thereof, nor any of their employees, makes any warranty, express or implied, or assumes any legal liability or responsibility for the accuracy, completeness, or usefulness of any information, apparatus, product, or process disclosed, or represents that its use would not infringe privately owned rights. Reference herein to any specific commercial product, process, or service by trade name, trademark, manufacturer, or otherwise does not necessarily constitute or imply its endorsement, recommendation, or favoring by the United States Government or any agency thereof. The views and opinions of authors expressed herein do not necessarily state or reflect those of the United States Government or any agency thereof. 


\section{DISCLAIMER}

Portions of this document may be illegible in electronic image products. Images are produced from the best available original document. 
ABSTRACT

This report assesses the environmental consequences of the transportation of radioactive materials in densely populated urban areas, including estimates of the radiological, nonradiological, and social impacts arising from this process. The chapters of the report and the appendices which follow detail the methodology and results for each of four causative event categories: incident free transport, vehicular accidents, human errors or deviations from accepted quality assurance practices, and sabotage or malevolent acts. The numerical results are expressed in terms of the expected radiological and economic impacts from each. Following these discussions, alternatives to the current transport practice are considered. Then, the detalled analysis is extended from a limited area of New York City to other urban areas. The appendices contain the data bases and specific models used to evaluate these impacts, as well as discussions of chemical toxicity and the social Impacts of radioactive material transport in urban areas. The latter are evaluated for each causative event category in terms of psychological, sociological, political, legal, and organizational impacts. The report is followed by an extensive bibliography covering the many fields of study which were required in performing the analysis.

\section{DISCLAIMER}

This report was prepared as an account of work sponsored by an agency of the United States Government. Neither the United States Government nor any agency thereof, nor any of their employees, makes any warranty, express or implied, or assumes any legal liability or responsibility for the accuracy, completeness, or usefulness of any information, apparatus, product, or process disclosed, or represents that its use would not infringe privately owned rights. Reference herein to any specific commercial product, process, or service by trade name, trademark, manufacturer, or otherwise does not necessarily constitute or imply its endorsement, recommendation, or favoring by the United States Government or any agency thereof. The views and opinions of authors expressed herein do not necessarily state or reflect those of the United States Government or any agency thereof. 
ACKNOWLEDGEMENT

The authors acknowledge the following for their contributions to the overall effort: Norman Alan Eisenberg of the U.S. Nuclear Regulatory Commission; Brian H. Finley, Arthur R. DuCharme, Jr., Lynn T. Ritchie, and Martin S. Tierney of Sandia National Laboratories; staff members of the Transportation Technology Center, DOE; and Christopher Cluett and the project team at Battelle Human Affairs Research Centers, Seattle. The authors also wish to acknowledge the contributions of Gene Runkle of the University of New Mexico in the area of health-effects modeling. 


\section{CONTENTS}

Chapter

Executive Summary

1 Introduction

2 Incident-Free Transport

3 Impacts of Transportation Accidents

4 Environmental Impacts from Human Errors and Deviations from Accepted Quality Assurance Practices

5 Sabotage, Security, and Safeguards in Urban Transport

6 Alternatives

7 Generic Extension

179

Appendix A -- Urban Area Data Bases

A-1

Appendix B -- Dose Model for Incident-Free Transport of Radioactive Materials

$\mathrm{B}-1$

Appendix C -- Analysis of the Radiological Consequences of Accidents Involving Radioactive Materials

$\mathrm{C}-1$

Appendix D -- Sensitivity and Error Analyses

D-I

Appendix E -- Meteorological Models -- MICMET and PICMET

$\mathrm{E}-1$

Appendix F - Meteorological Data and Approach

F-1

Appendix G -- Radiological Health Effects

G-1

Appendix H - DOT Records for Radioactive Material

Transportation Incidents, 1971-1977

$\mathrm{H}-1$

Appendix I -- Radioactive Material Transportation Incidents in 1975 in NRC Agreement States

$\mathrm{I}-1$

Appendix J -- Impacts of Chemical Toxicity

$\mathrm{J}-1$

Appendix K -- Direct Economic Impact

$\mathrm{K}-1$

Appendix L -- Social Impacts

$\mathrm{L}-1$

Appendix M -- Task Group

M-1

Bibliography

By -1 


\section{ILLUSTRATIONS}

Figure

$\underline{\text { Page }}$

1-1 Basic Grid for Portions of Manhattan, Queens, and Brooklyn

$1-2$

Four-Cell Grid

$1-3$

Radiological Consequences Method

20

2-1 Geometry for Incident-Free Dose to Pedestrians

2-2 Geometry for Incident-Free Dose to People in Buildings and Vehicles $\quad 32$

2-3 Geometry for Calculating Dose to Warehouse Personnel

2-4 Geometry for Calculating Dose to People along Rail Right-of-Way (Integration along a Strip) 35

3-1 Possible Accidents and Their Consequences 43

3-2 Economic Impact versus Amount of Material Aerosolized $\quad 57$

3-3 Cost Components for Urban Releases of Long-Lived Materia1s 58

3-4 Cost Components for Urban Release of Short-Lived Materials 59

3-5 Relationship of Economic Impacts to Amount of Long-Lived Material Released for Different Cleanup Levels $\quad 61$

3-6 Relationship of Economic Impacts to Amount of Short-Lived Material Released for Different Cleanup Levels

5-1 Blast Overpressure versus Distance for High-Explosive Charges

5-2 Cumulative Latent Cancer Fatalities as Function of Distance from Release Point (Baseline Estimate Release from Spent Fue1)

5-3 Projected 1-Year Lung Dose as a Function of Distance from Release Point (Baseline Estimate Release from Spent Fuel)

5-4 Pattern of Grid Sectors Affected by a Release in Cell B

5-5 Mean Area Affected by Release in Cel1 B (CRAC Estimate) Superimposed on METRAN Cells Affected by Rush Hour Release in Cell B

5-6 CRAC Estimate of Cumulative Area Traversed by Radioactive Cloud as a Function of Downwind Distance from Release Point ( 10 to $10^{3}$ metres)

5-7 CRAC Estimate of Cumulative Area Traversed by Radioactive Cloud as a Function of Downwind Distance from Release Point ( 1 to $1000 \mathrm{~km}$ )

6-1 Routes through and around New York City 153

6-2 Release at Point A, Northwest Winds 162

6-3 Release at Point $A$, Southeast Winds 


\section{ILLUSTRATIONS (Continued)}

Figure

Page

6-4 Release at Point $B$, West Winds

164

6-5 Release at Point $C$, Northwest Winds

165

6-6 Projected Bone Marrow Dose versus Downwind Distance

169

6-7 Projected Lung Dose versus Downwind Distance

7-1 Major Freeway Patterns for New York City

7-2 Major Freeway Patterns for Houston

190

A-1 Basic Grid for Portions of Manhattan, Queens, and Brooklyn

A-3

A-2 Differing Criteria for Shipment Routes into, out of, through, and in the Vicinity of a Given Latitude and Longitude

A-3 Vector Directions of Travel within a Cell

A-33

B-1 Basic Geometry for Integrated Dose Calculations

$\mathrm{B}-2$

B-2 Geometry for Incident-Free Dose to Pedestrians

B-3

B-3 Albedo Dose Contributions

B-6

B-4 Gamma-Ray Scattering from a Concrete Surface

B-7

B-5 Total Dose Albedos for Gama Rays Normally Incident on Concrete

$\mathrm{B}-8$

B-6 Geometry for Incident-Free Dose to Pedestrians on Sidewalks

B-10

B-7 Geometry for Incident-Free Dose to Pedestrians in Crosswalks ahead of Vehicle

B -11

B-8 Geometry for Incident-Free Dose to People in Buildings

B-12

B-9 Linear Attenuation Coefficients as a Function of Energy for Some Materials

B-14

B-10 Dose Buildup Factor for Concrete

B-15

B-11 Multiple Slab Geometry

B-16

B-12 Peebles Buildup Factor (Iron Buildup Used)

B-17

$B-13$ Obliqueness Factor

B-17

B-14 Obliqueness Geometry

$\mathrm{B}-18$

B-15 Raypaths to People in Buildings

B-20

B-16 Incident-Free Dose to People in Vehicles B-21

B-17 Model of Dose to People in Vehicles Including the Phenomenon of Platooning

B-24

C-1 Possible Accidents and Their Consequences $\mathrm{C}-2$

C-2 Release Fraction C-3

C-3 Radiological Health-Effect Hazard Categories C-5 


\section{ILLUSTRATIONS (Continued)}

$\underline{\text { Figure }}$

Page

C-4 Dose Due to Nondispersible Sources

C-7

C-5 Nondispersible Accident Geometry (Intersection Shown)

$\mathrm{C}-8$

C-6 Exposure Geometry for Direct Exposure to People in Vehicles Resulting from an Intersection Accident

C-13

C-7 Nondispersible Source Dose to People in Vehicles for Freeway Accidents

C-16

E-1 Comparison of Different Treatments of Horizontal Diffusion

E-9

F-1 Kennedy Airport Surface Wind Data, 1948-1965 (All Months, All Hours): Probability versus Wind Direction

F-5

G-1 Estimated Dose-Response Curves

G-2

G-2 Dose-Response Curves for Mortality Due to Acute Pulmonary Effects of Radiation

G-3

G-3 Biological Pathways for Inhaled Material

G-4 Plot of Radioactive Half Life versus Resuspension Dose Factor

G-11

G-20

K-1 Calculationa1 Flow Diagram for Economic Impact Assessment

$\mathrm{K}-12$ 


\section{EXECUTIVE SUMMARY}

\section{Purpose of This Assessment}

The Final Environmental Statement on the Transportation of Radioactive Material by Air and Other Modes (NUREG 0170) concluded that the risks associated with such transportation are low, although severe accidents in urban areas have the potential for large radiological and economic consequences. The present study investigates radioactive material transport in urban areas and addresses the specific urban features which influence the resulting environmental impacts. These features include the geographic and demographic makeup, and vehicular, population, and transportation patterns in the city.

Previous efforts have not identified a most important population exposure pathway or group. This assessment examines several pathways and a number of urban-specific population groups to evaluate their relative significance. In addition, because different causative events contribute to the overall environmental impacts, this assessment addresses four of these: incident-free transport, vehicular accidents, human errors, and sabotage or malevolent act.

Not only does radioactive material transport produce radiological and economic consequences, but also it can have social impacts. The objective of this study is to examine both the quantitative environmental impacts of radioactive material transport in urban areas and the more subjective effects of this process.

Method of Approach

Using previous studies on radioactive material transport in the United States as a general foundation, we examined the urban area to determine which population groups were most likely to be exposed to materials in transit. After identifying these groups, we constructed computer simulation models to assess the expected population exposure, from either incident-free transport or vehicular accidents, to each group. The impacts from human errors or sabotage were examined using adaptations of the accident analyses.

A section of New York City encompassing portions of the boroughs of Manhattan, Brooklyn, and Queens was selected for the initial application of the computer models. Using the results of sensitivity analyses and insights gained from detailed examination of several classes of radioactive materials, the initial results were extended to a selected set of the top Standard Metropolitan Statistical Areas (SMSAs) in the United States. Twenty sites were selected, ranging from several of the densely populated areas on the eastern seaboard to the more spread out, but still heavily populated, areas on the west coast. In addition, several alternatives to current transport practice were examined. These include transport mode changes, increased damage resistance for shipping packages, rerouting of materials 
around the urban area, and a series of operational alternatives which would affect the population groups within the urban area most closely associated with the transport process (warehouse personnel, handlers, etc.).

This assessment contains detailed descriptions of the urban area studied, the simulation and health-effects models used for evaluating radiological and economic impacts, and an evaluation of the social impacts of the transport of radioactive materials in urban areas. The material included in this assessment comes from a number of different sources, including the Task Group on Radioactive Material Transport in Urban Environs, established to assist the Sandia staff in developing the scope and details of the study. The results of all of the analyses are presented in terms of expected numbers of health effects per shipment year.

\section{Results}

Incident-Free Transport

For the selected New York City study area, the overall population dose from incident-free transport of radioactive materials were found to be less than 10 person rem per shipment year. This is equivalent to an number of expected latent cancer fatalities (LCF) for the total population at risk of the order of $10^{-4}$ per shipment year. The population groups receiving the majority of this dose were handlers, warehouse personnel, transport vehicle crew, pedestrians, and people in vehicles.

When the analysis was extended to other urban areas, the values for population dose ranged from 4 person rem $\left(\sim 10^{-4}\right.$ latent cancer fatality) per shipment year, for the San Diego area, to a high of $\sim 200$ person rem $\left(\sim 10^{-3}\right.$ latent cancer fatality) for Newark. This range is not unexpected since there are widely different population and shipment patterns across the nation.

\section{Vehicular Accidents}

Radioactive materials are shipped in either nondispersible (or special) form (metals or doubly encapsulated sources) or in dispersible (or normal) form. Both types of materials were included in the shipment model developed for this analysis and have required different methods for assessing the expected consequences of vehicular accidents involving them. When the results of the separate analyses were combined, the expected numbers of health effects per shipment year were calculated. For the baseline accident analysis, the expected results are approximately $10^{-5}$ early morbidity, $10^{-3}$ latent cancer fatality, and $10^{-3}$ genetic effect per shipment year (1imited New York City area). The results calculated using METRAN show no vast differences from those found in NUREG 0170. Economic risks from a year's shipment activity were calculated to be $\sim \$ 1$ million.

For other urban areas, the expected number of latent cancer fatalities per shipment year (shipment level for each urban area) ranges from $\sim 10^{-5}$ for San Diego to a high of $\sim 0.2$ for Chicago. These variations arise from several different sources including the population and shipment differences referred to earlier, but here an additional factor is the spectfic character of the shipments in each urban area. 
Human Errors or Deviations from Accepted Quality Assurance Practices

Human errors can occur at any stage during the transport sequence, and pre-transport errors can cause impacts during transit. To evaluate environmental impacts, the accident analysis model was altered, with the accident rates replaced by incident or occurrence rates for known incidents involving human errors. From this point, the analysis paralleled that of vehicular accidents insofar as expected numbers of health effects per shipment year were calculated. In this case, no early effects have been observed, but approximately $10^{-3}$ latent cancer fatality is expected per shipment year for the limited New York City area. Economic risk from human errors would total approximately $\$ 10^{4}$.

Extension to other urban areas resulted in a range of expected values from $\sim 10^{-6}$ latent cancer fatality per shipment year for San Diego to 0.4 latent cancer fatality per shipment year for Chicago. This overall range is consistent with that for vehicular accidents.

\section{Sabotage or Malevolent Acts}

There are no data available with which to estimate the probability of occurrence of sabotage or malevolent acts. This precludes calculating risk values. Although a successful attack is considered unlikely, we assumed that one occurred and calculated the consequences in terms of expected numbers of health effects. Several different materials were examined in terms of the numbers of health effects to be expected given that the event occurred. Results of this analysis indicate that for a range of materials, expected numbers of early fatalities would be small, expected numbers of early morbidities would range from ones to hundreds, and expected numbers of latent cancer fatalities would range from ones to thousands in the limited area considered. Economic effects are strongly dependent upon the amount of radioactive material released. Thus, there is unquestionably a wide range of environmental impacts potentially exceeding $\$ 1$ billion for the largest radioactive material release.

Generic extension of the sabotage calculations is restricted by several factors. In general, it can be stated that the results for a particular event can be scaled, approximately linearly, with population densities. However, the scaling must be done with caution since several assumptions are inherent in the calculations which affect the extrapolation.

\section{Alternatives to Current Transport Practice}

A number of alternatives to current transport practice have been considered, and the expected values for radiological consequences or risk have been determined, assuming the operation of the alternative for an entire year of shipment activity. In general, the effects of implementing these alternatives ranged from no change in the calculated risk to reduction in the expected numbers of early morbidities resulting from vehicular accidents. Decreases in the expected number of latent cancer fatalities by factors of two to five were also observed. In some cases, where implementation of the alternative involved changes of shipment time, a range of values for expected radiological health effects was calculated, with some shipment times resulting in a decrease in the expected number of health effects. In the sabotage case, a range of expected number of health effects was also observed, depending upon the particular alternative. For the alternative involving changes in transport mode, no change in the expected number of health effects was observed for the incident-free and human error situations, although consequences from a sabotage event increased significantly and the accident risk doubled. Rerouting 
materials outside the urban area resulted in decreased numbers of expected health effects from vehicular accidents, sabotage, and human errors because of the lower populations and vehicular traffic patterns in these areas. The treatment of rerouting has involved comparisons of expected consequences of severe accidents for a limited number of shipment types; thus, no risk comparisons are made.

Social Impacts of the Transport of Radioactive Materials in Urban Areas

The social impacts study concludes that transportation issues are a particularly visible component of the nuclear energy controversy. Although the number of transportation incidents which have occurred has been small and the consequences slight, the political and legal attention given transportation is likely to increase. These impacts may ultimately prove more significant in decisions regarding the transportation of radioactive materials than will strictly technical concerns. 
INTRODUCTION

Radioactive material transport is increasing as additional uses of these substances are found whether for diagnostic procedures, industrial purposes, production of electrical power, or disposition of waste. Studies have been performed that address the pattern of radioactive material shipments and the environmental impacts of the transport of these materials across the United States. 12 The results reported in Reference 2 raised specific questions about the transport of materials through densely populated urban areas. The current effort is directed at answering some of these questions.

For the purposes of this study, radiological impacts are assumed to be produced by four causative events: incident-free transport (also called normal or accidentfree transport), vehicular accidents, human errors or deviations from accepted quality assurance practices, and sabotage or malevolent act. The calculated impacts for the first three of these are evaluated both from consequence and risk perspectives. Only radiological consequences of sabotage or malevolent act are addressed. All types and modes of shipment are examined with the exceptions of materials shipped in limited quantities (the radiological impact of shipments of these materials has been found to be insignificant ${ }^{2}$ ) and defense shipments. Materials have been categorized on the basis of their ultimate use, with four separate end uses considered: medical, industrial, fuel cycle, and waste.

The remaining chapters of this report describe the results of the analysis for each causative event, in the order given above, and also address the questions of a1ternatives to current transport practice. The final chapter contains the details of an extension of the specific analysis for a single urban area to a variety of other urban areas. These other areas, the 20 largest Standard Metropolitan Statistical Areas (SMSAs) as defined for $1978,{ }^{3}$ have various radioactive material shipping patterns which are quite different from the limited New York City area chosen for the initial analysis. They also have different population characteristics and personal travel patterns. An extensive set of detailed appendices is provided which includes the data collection methods, analytical methodology, and sensitivity analysis techniques used for. the determination of the reported impacts. Also included as appendices are a brief examination of the chemical toxicity of the transported materials and a summary of the social impacts of radioactive material shipment in urban areas.

\subsection{Background of the Study}

In March 1977, the U.S. Nuclear Regulatory Commission (NRC) announced in the Federal Register its intention to prepare a generic Environmental Impact Statement (EIS) on the transportation of radioactive materials in urban areas and that Sandia 
National Laboratories had been selected and had begun work to provide NRC with additional technical information on the expected environmental impacts. ${ }^{4}$ The present draft environmental assessment is a part of the technical basis for NRC consideration of possible rule changes in Parts 71 and 73 to Title 10 of the Code of Federal Regulations (10CFR71 and 10CFR73) -- those pertaining to transport of radioactive materials. The environmental impacts addressed include radiological, nonradiological, and economic impacts. This investigation uses a risk methodology approach similar to that in Reference 2 but includes detailed consideration of the special characteristics of urban areas as they affect the transport of radioactive materials.

In response to the diversity of opinions, and technical and nontechnical concerns, Sandia National Laboratories, with the approval of NRC, formed a task group of individuals, from industry environmental groups, and other government agencies, with some knowledge in this area. The function of this group was to assist in obtaining the most comprehensive and useful assessment possible. Membership of the Task Group on Transportation of Radioactive Materials in Urban Environs is given in Appendix M.

\subsection{Purpose and Scope}

The purpose of this assessment is to examine the overall environmental impacts from radioactive material transport in densely populated urban areas. Impacts addressed in this study include radiological health effects, economic costs, nonradiological health effects arising from the chemical nature of the materials, and social impacts of the transportation of these materials. The radiological health effects included are expected numbers of latent cancer fatalities, genetic effects, early fatalities, and early morbidities.* These would result from exposure to external penetrating radiation from or inhalation of the materials. Economic impacts can arise from any spillage or delay in transit of materials and include those from emergency response to an accident, survey and cleanup costs, evacuation costs, and possible land-use denial expenses for large accidents, which include relocation costs for those affected. Several nonradiological impacts are addressed in the report. First, there are the additional accidental injuries and deaths attributable to the movement of radioactive materials by exclusive-use vehicles. Second, materials may present health hazards which are attributable to the chemical forms of materials. Finally, there are important social impacts resulting from urban radioactive material transport.

To address the radiological impacts described above, four causative events are used to describe various aspects of the transport process. First, incident-free transport includes all portions of the process during which nothing out of the ordinary occurs during shipment. Reference 2 uses the term "normal" transport for this situation, but the present report views "normal" transport as divided into two parts: (1) incident-free transport, defined above, and (2) human errors or deviations from accepted quality assurance practices, where something abnormal occurs, affecting the shipment, which is not the result of a vehicular accident. This subdivision allows the separate evaluation of the risks to the public from these types of occurrences. For comparisons with earlier work, the two sets of impacts must be summed to obtain the previously defined "normal" transport case. In

*The expected radiological health effects are calculated using the computer models developed for this study. 
incident-free transport, then, there is no release of radioactivity and no loss of shielding. Resultant impacts come from exposure of nearby people to levels of external penetrating radiation which normally exist outside the package shielding.

The second causative event, vehicular accidents, can result in damage to the package with either a reduction in the effective amount of shielding around the material or a release of material from the package, or perhaps both. If the transported material is nondispersible, the major effects observed would be decrease or destruction of the package shielding and/or delay in transporting the material, resulting in increased external exposure or longer exposure times to normal levels. On the other hand, for dispersible materials, several pathways for exposure exist. As in the nondispersible case, increased external exposure is a possibility, but the dispersible material can also become aerosolized and present other hazards. The aerosol might be inhaled as the cloud passes, or it might expose individuals to external radiation during cloud passage (cloudshine). Also, material in the cloud can be redeposited on the ground, away from the accident site, and this material would be an additional external exposure source (groundshine).

For human errors, the third causative event, the analysis considers problems resulting from labeling or packaging faults or deviations from accepted quality assurance practices. The resulting environmental impacts are similar to those from vehicular accidents but are dependent upon the frequency and magnitude of the human errors. Most human errors do not produce compromises of package integrity; thus the risk contributions most probably result from the shipments acting only as exposure sources. Substantial economic losses can result either from vehicular accidents or from incidents involving human errors.

The final causative event, sabotage or malevolent act, differs from the first three because it is deliberate. Because of this aspect, there is the potential for large releases of material, resulting in radiological and economic consequences on the same scale as an extremely severe accident. Theft or diversion of radioactive materials can potentially result in significant environmental consequences if material is deliberately moved to locations where large consequences may be expected.

Beyond considering the four causative event categories, this analysis considers all radioactive materials shipped in urban areas, except limited and defense shipments. As mentioned earlier, materials are considered to have four end uses, and through analysis of data collected for the summary reports included in Reference 1 , several transport modes are included. These modes are truck, air passenger, air freight, rail, and barge (or ship). Detailed analysis of the urban area selected for the initial assessment, a limited area in the center of New York City, indicated that only the first three transport modes were found in the data base. Thus, to address the effects of other transport modes and urban area characteristics, this study extends the initial analysis to other densely populated urban areas. The study also considers certain alternatives to current transport practice as well as the social impacts of radioactive material transport in urban areas.

\subsection{General Approach}

The general approach to this environmental assessment involved the development of computer models to quantify the radiological impacts using dynamic simulation techniques and the use of more qualitative techniques for the evaluation of nonradiological impacts. The task group previously described provided comments and suggestions, and advice on the scope of work, data bases, important subprograms, and key tasks developed in the assessment. 
Initially, an approximate representation of the urban environment was developed. The urban area was divided into geographical units (cells) which were characterized by demographic, traffic, and shipment patterns as required by the analysts. A limited section of New York City, comprising portions of the boroughs of Brooklyn, Manhattan, and Queens, was chosen for application of the computer models and to provide an urban basis for interpretation of the results.

More qualitative techniques have been employed to assess the nonradiological impacts such as chemical toxicity of the materials and observed or presently perceived social impacts resulting from the transport of radioactive materials in urban areas. Anticipated social impacts were assessed by a review of the recent literature and application of pertinent information to develop the important social consequences of transport of radioactive materials in urban areas. These social impacts were evaluated for each of the causative event categories.

Results of sensitivity analysis and other insights gained from the limited New York City case were used to extend the initial study to other urban areas. Also, these techniques were used, in part, to evaluate the effects of certain alternatives to current transport practice.

\subsection{Radiologica1 Impact Assessment}

The models developed to calculate the radiological impacts incorporate those features of urban areas believed to affect the radiation doses received by the surrounding population. Included are such features as shielding afforded by buildings, large and variable traffic flow and population patterns (time and location dependent), and urban pedestrian patterns. Location-dependent variables have been specified using a grid composed of cells of equal and specified geographical extent. Each cell was characterized by a set of parameters, some of which varied with time of day. The resulting data bases served as input to a radiological consequence mode1, METRAN, which allows quantification of the impacts from incidentfree transport and from vehicular accidents. Adaptation of the vehicular accident sections of METRAN allowed calculation of the impacts from human errors. Using METRAN in a consequence-only mode, the expected health effects from sabotage or malevolent act could also be assessed. Where the radioactive material could be released and aerosolized, separate meteorological dispersion models were developed to describe the street channeling and other urban-specific effects on the aerosol cloud movement. These calculations were performed independently and served as additional input to the METRAN code. Radiological consequences were described in terms of expected numbers of health effects and expected economic impacts.

\subsubsection{Description of the Urban Area}

A 100-square-kilometre region in the boroughs of Manhattan, Brooklyn, and Queens was used in the detailed analysis. This area was subdivided into one hundred $1-\mathrm{km}^{2}$ cells to facilitate data collection. The area was chosen to cover the maximum amount of land area with as much variation of land use as was possible within the calculational constraints. Figure 1-1 illustrates the selected area. To investigate the meteorological dispersion of aerosolized material, it is assumed that there are four 30-metre-high vertical cells, so that the meteorological grid is $10 \times 10 \times 4$ and Cartesian in three directions. 


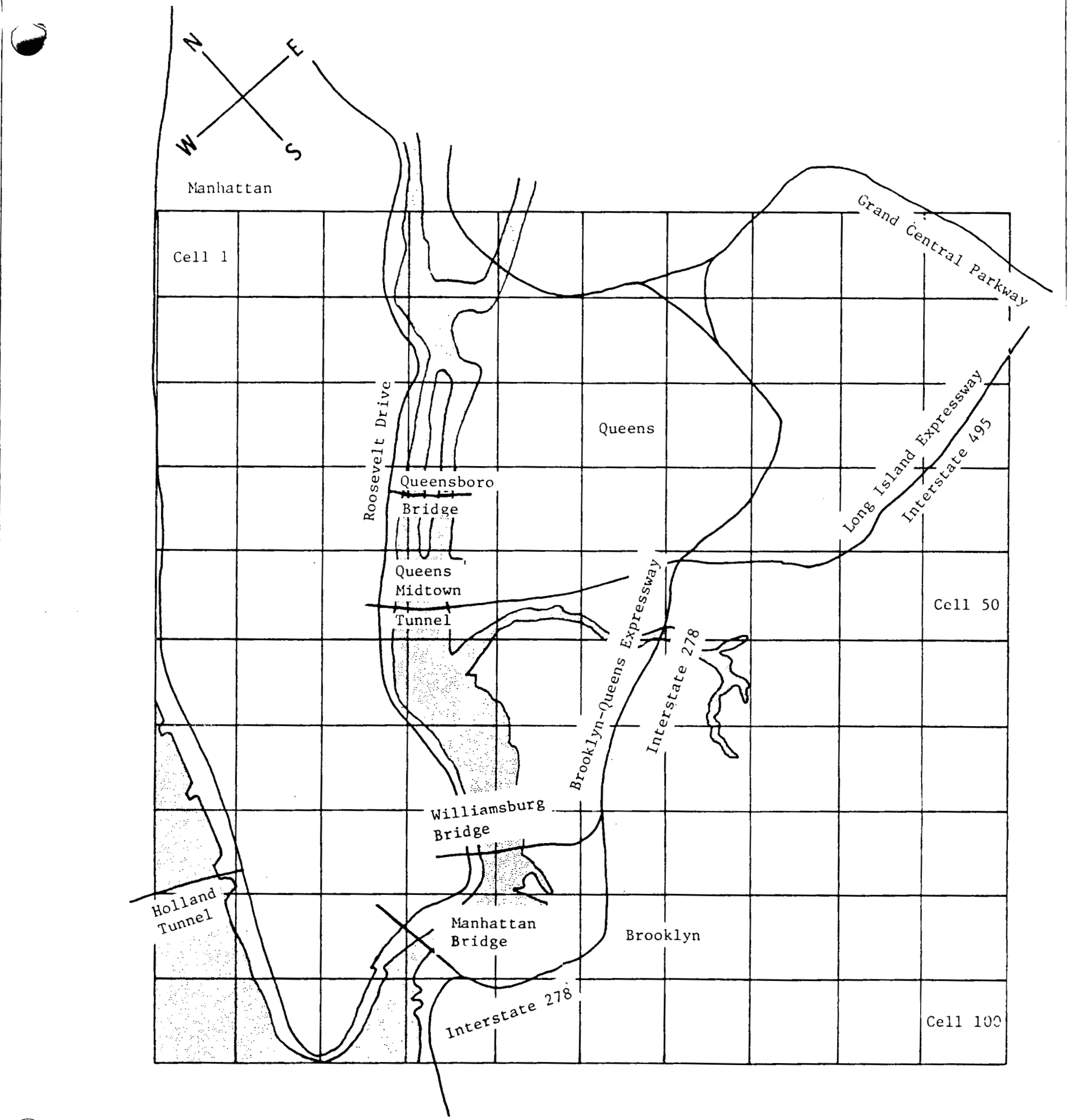

Figure 1-1. Basic Grid for Portions of Manhattan, Queens, and Brooklyn 
The parameters required for the analysis have several different kinds of dependencies: some are assumed to be independent, and thus individual values of these suffice; others are dependent upon geographical location, and 100 values for each must be determined. Several parameters were required that possessed a time dependence. To facilitate the collection of these data, the urban day was divided into time spans or intervals to reflect the fluxes of people and vehicles which result from work patterns in the city. Certain of these time-dependent parameters are independent of geography; thus a single value, assumed valid for the length of a specific time span, has been obtained for each span. Others, both geographical and time dependent, required much more specific data collection. Table $1-1$ lists the urban day/time span breakdown and gives examples of the parameters which fall into each of the classes described above. A complete description of the data base can be found in Appendix A.

Once the geographical and demographic characteristics had been determined, information on the radioactive material shipment patterns was collected. The grid representation allowed for the determination of specific shipment routes. Constraints in the computer modeling allowed only eight directions for movement from one cell center to another. Figure 1-2 illustrates idealized routes through a four-cell grid. Exact routing information for shipments in New York City were approximated using the constraints mentioned earlier. End-use classification of the materials in the standard shipments model allowed assumptions to be made about the times at which different substances would be shipped into, out of, or through the area (Appendix A).

\subsubsection{Computational Method for Radiological Consequences}

The general program structure used to calculate radiological consequences and risk is illustrated in Figure 1-3. The executive program, METRAN, quantifies radiological and economic impacts as a function of the input data described above. METRAN receives additional input information for dispersible materials, as predicted by the meteorological dispersion codes, MICMET and PICMET in terms of downwind volumetric and surface concentrations of the dispersed material. MICMET is a micrometeorological dispersion model developed to treat some of the features of airflows likely to be encountered in urban street channeling and at street intersections. The model is used to provide both estimates of dispersion to the environment shortly after the release and initial conditions for the urban/regional transport model, PICMET, which follows the concentrations of radioactive material for larger distances and times. In PICMET, the mean wind field is constructed from available measurements of the horizontal mean wind field, the mean building height, and the fraction of open area in the surface layer of cells. For incident-free transport, the results are expressed in terms of total integrated population dose which can then be converted into expected numbers of health effects. For vehicular accidents, human errors and sabotage, METRAN estimates the expected numbers of latent cancer fatalities, genetic effects, early fatalities, and early morbidities.

\subsubsection{Estimated Radiological Impacts}

The approach to an assessment of the radiological impacts involved the development of models for several urban dose groups. Table 1-2 lists the dose groups for incident-free transport, vehicular accidents, and human errors. Only totalpopulation radiological consequences are reported for sabotage. 
Table 1-1

Time Span Description and Parameter List

Time Span

Number

1

2

3

4

5

6

$$
\begin{aligned}
& \text { Interval } \\
& \text { (hours) } \\
& 1800-0700 \\
& 0700-0830 \\
& 0830-1130 \\
& 1130-1300 \\
& 1300-1630 \\
& 1630-1800
\end{aligned}
$$

\section{Independent Parameters}

Population density/warehouse

Crew separation distance

Vehicle length

Vehicle stop time at intersections

Stopped vehicle separation distance

Minimum and maximum exposure distances for warehouse personnel, people in air terminals, etc.

Time-Dependent

Parameters

Vehicle velocities

Fraction of intersections stopped at

Air terminal population densities

People per vehicle (average)

Pedestrian density
Ce11-Dependent Parameters

Fraction of cell area occupied by streets, buildings

Street width

Sidewalk width

Construction material for buildings

Number of floors per building (average)

Height of building story

Number of lanes per side of street

Ce11- and TimeDependent Parameters

Population density

Vehicle separation distance

Pedestrian velocity

Traffic counts

Transient population density 


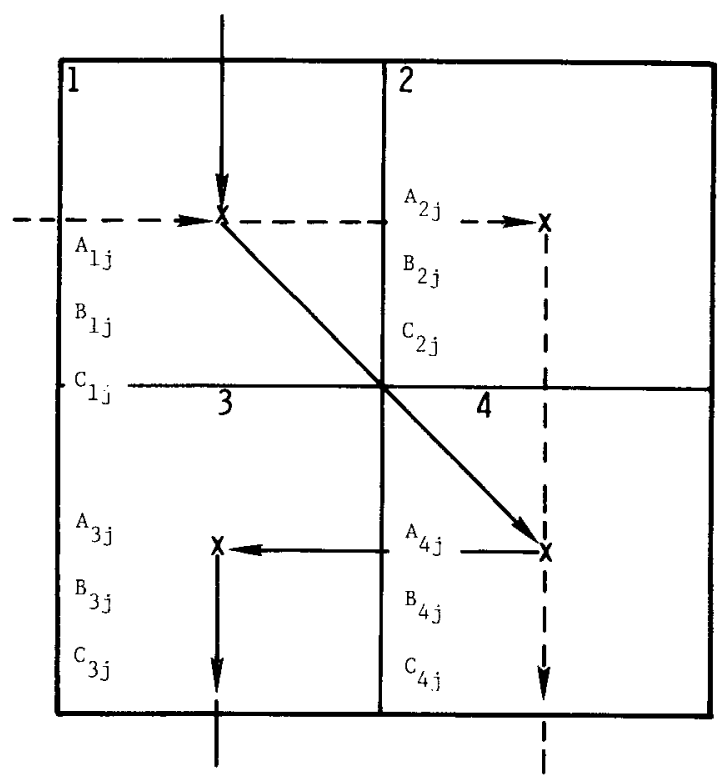

Figure 1-2. Four-Cel1 Grid

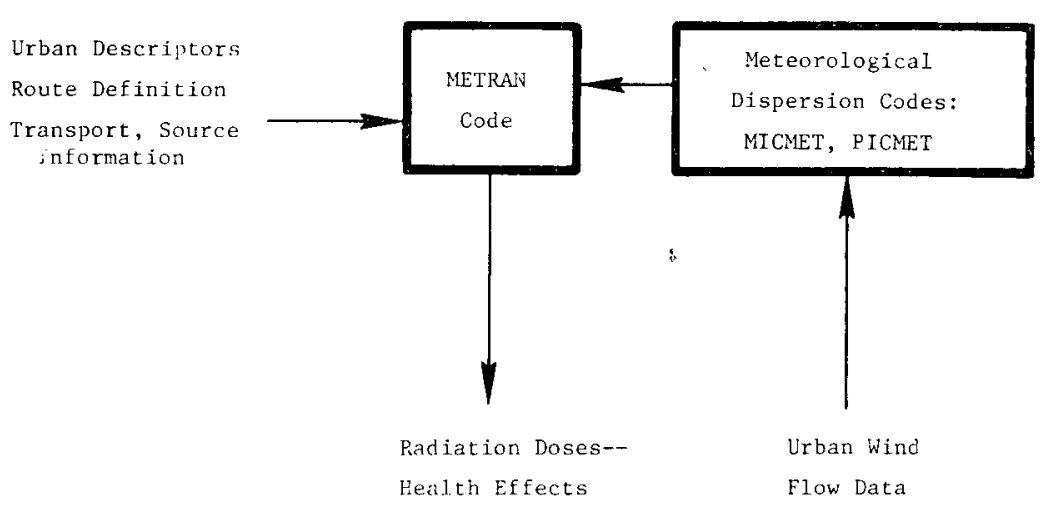

Figure 1-3. Radiological Consequences Method 
Table 1-2

Urban Dose Groups

Incident-Free Transport

Truck Mode:

Pedestrians

People in, Vehicles

People in Buildings

Transport Vehicle Crew

Warehouse Personnel ${ }^{b}$

Handlers ${ }^{b}$

Air Mode :

Persons in Air Terminals

(Cargo/Passenger)

Water Mode:

Dock Personne1

Rail Mode:

Persons in Rail Terminals

Persons Sharing Transport Link

Persons along Rail Right of Way

$a_{\text {Both }}$ consequences from individual accidents and accident risk are calculated.

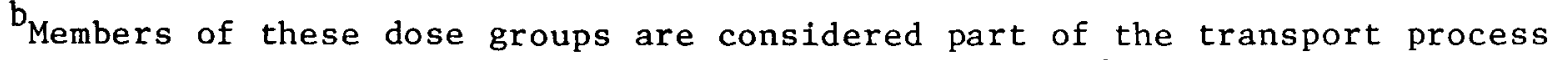
although the shipment is not moving when they are exposed.

In the incident-free case, the dose rate from a point source of ionizing radiation was integrated over distance and time to deduce exposure to several surrounding population groups, including crewmen, pedestrians, people in vehicles, people in buildings, handlers, and warehouse personnel. Subdividing the population at risk in this manner allowed the inclusion of unique radiation exposure geometries for each subgroup.

Although the environmental impact of accidents may not be severe from an annual risk perspective, individual vehicular accidents during the transport of radioactive materials in urban areas have the potential for causing significant 
health and economic consequences. Radioactive material in transit was characterized as dispersible or nondispersible under accident conditions. Impacts from nondispersible material accidents were treated like impacts from incidentfree transport accidents, with the added factor of accident delay time. As already mentioned, inhalation of aerosolized materia1, cloudshine, and groundshine are all possible causes of impacts from dispersible materials. Impacts can also come from nonaerosolized material remaining at the scene of an accident. Risk (probability of an event multiplied by the consequences of the event) was computed using vehicular accident rates and a corresponding release fraction adapted in part from Reference 2. Economic consequences of a given accident or human error were also calculated using techniques described in Appendix K.

The contribution to the risk from human errors was assessed using an approach similar to that for vehicular accidents. The probability term, however, has been computed using estimated urban incident rates evaluated by package type.

The radiological impacts from sabotage were computed using the urban accident consequences model with appropriate modification in the source terms considered. In this case, comparisons were also made with another model used to determine the consequences of such events.

\subsubsection{Nonradiological Impacts}

Analysis of nonradiological impacts, such as social impacts or chemical toxicity, usually involved less quantitative techniques than those described previously. This more qualitative approach was needed for social impacts since the responses of individuals and groups to occurrences within any of the causative event categories could not be rigorously quantified. Personnel at Battelle Human Affairs Research Centers, Seattle, performed an assessment of the social impacts resulting from the transport of radioactive materials in densely populated urban areas. The executive summary of their report is included in this document as Appendix L.

Additional nonradiological impacts from chemical toxicity of the materials, and those resulting from transport by exclusive-use vehicles were also assessed. The analysis of chemical toxicity yielded a rank ordering of those materials presenting acute toxir hazards from their chemical nature. Details of the approach to this problem can be found in Appendix $J$ of this report. The results of the analysis performed in Reference 1 for the estimated number of nonradiological deaths and injuries attributable to use of exclusive-use vehicles were extrapolated for the limited New York City analysis. Nonradiological impacts resulting from explosive use during sabotage events are discussed in Chapter 5.

\subsection{Sensitivity and Error Analyses}

The objectives of the sensitivity analyses were to determine the order of importance of the input parameters in characterizing the output variables and, where possible, to provide simplified, closed-form equations for calculating doses and for performing error analyses. Generally, the input parameters were varied over plausible ranges, and the resulting output from METRAN was fitted by linear least-squares methods to obtain regression equations. The values for input variables were selected according to appropriate response surface designs. The fitting was done in a stepwise fashion until only the most important input 
variables appeared in the regression equations. In the case of incident-free transport, this process has produced sufficiently adequate results so that the equations could be rescaled, using multiplicative factors not treated in the regression analysis. This has resulted in a set of simplified equations that can be used in lieu of the more complicated METRAN program. For vehicular accidents and, by analogy, for human errors, the regression analysis had to stop short of producing simplifying equations because of the greater complexity of the models and the interactions of the METRAN code with the independent meteorological models.

Using the fitted regression equations for each population group, the change in each input variable producing a doubling ( $100 \%$ change) was calculated. The magnitudes of these variations measure the sensitivity of the response variables to changes in the input and permit the latter to be ranked in order of their influence.

The error analyses were performed on the regression equations obtained above. Error propagation formulas were derived from Taylor expansions of the regression equations (Appendix D). Since the model equations consist mostly of series of product terms, and magnitudes or distribution of errors in the input variables are not known, these are expressed as relative errors.

\subsection{Alternatives to Current Transport Practices}

Several alternatives to current transport practice were examined. These include time of. shipment restriction, package type changes/overpack, rerouting of materials, and operational alternatives (e.g., decrease in storage time). In each case, the authors have attempted to quantify the effects that a particular alternative would have on the impacts resulting from current transport practice. For incident-free transport, the simplifying equations derived in the sensitivity analysis have been used, where possible, to evaluate the effects of a particular alternative. For vehicular accidents and human errors, the METRAN code has been used to evaluate the alternative's impacts as needed. Rerouting certain types of shipments has been treated explicitly by routing the materials outside the New York City SMSA. Different demographic and traffic parameters are used. Accidents are postulated to occur on sections of highway outside New York City, and the consequences of these accidents are evaluated. The two sets of accident consequences are then compared (within and outside the city). A similar assessment is made for incident-free transport by considering the changes in travel distances, pedestrian populations, etc. and calculating the resulting integrated doses. The consequences from a sabotage event occurring during rerouting are also discussed. In the case of some alternatives, where quantification of the expected change has not been feasible, the reasons for not giving numerical results are discussed.

\subsection{Generic Extension}

Using insights gained from detafled consideration of the mechanics of the calculational tools (METRAN, sensitivity analysis, etc.) and the analysis results, it was possible to develop techniques for extending the analysis for the limited New York City case to other urban areas. As mentioned earlier, the 20 top SMSAs for $1978^{3}$ were chosen for examination. Shipment patterns for each SMSA were obtained from data forming the basis for Reference 1. The techniques used to acquire this information are the same as those described in Appendix $A$ for the limited New York City case. 
For incident-free transport, the analysis has consisted of applying the simplified equations for the major dose groups (handlers, warehouse personnel, pedestrians, people in vehicles, and crew) to the shipment patterns described above. Several assumptions are made to allow the application of the equations. These include constant values for number of vehicles in any urban area, distance travelled across the area, and city-specific pedestrian densities. The important shipment parameter for this portion of the analysis is total transport index (TI) shipped in the area.

For vehicular accidents and human errors, materials were categorized on the bases of dispersibility, shipment size, average photon energy per disintegration, and radiological toxicity. The radiological toxicity is related to the average dose received per curie of material deposited in the body organs. The calculated risk values for the limited New York City case were subdivided into the same categories and further broken down by major contributing dose groups. These data were then coupled with demographic and traffic data and the total curies shipped by category to produce a first-order approximation to the risks from transport of materials in each SMSA.

In the case of sabotage or malevolent act, some statements can be made about scaling of the reported consequences with population density in other urban areas. This is discussed in more detail in Chapter 7.

\subsection{Social Impacts of the Transportation of Radioactive Materials}

An extensive search of available literature was made to assess the social impacts from radioactive material transport in urban areas. 5 Sociological, psychological, organizational, legal, and political impacts have been examined. These areas were addressed for each causative event category. The results of the analysis are included, in summary form, as Appendix L of this report.

\subsection{Comments on 1985 Shipment Estimation for New York City}

Reference 2, the analysis of radioactive material transport for the United States, includes an extension of the 1975 results to shipping estimations and expected results for 1985. Several problems were encountered when examining this process for New York City, including tremendous uncertainties about demographic, traffic, and radioactive material use for the city. Resident population is expected to decrease gradually, although commercial (and perhaps residential) expansion is being attempted. Changes in population will affect traffic flow patterns and could alter radioactive material use in the city (more or fewer hospitals, greater industrial use, etc.). Because of these and other problems associated with meaningful data collection, the authors feel that extension of the 1975 analysis to 1985 is not possible with any degree of accuracy.

\subsection{Related Studies}

Several studies performed in recent years act as foundations for or relate closely to this investigation.

WASH-1238 and its two supplements address the shipment of fresh fuel, spent fuel, and waste associated with the operation of light-water reactors. 678 The reports treat shipment by truck, rail, and barge and estimate the effects along the transport routes to and from reactor sites. 
NUREG-0073 and BNWL-1972 report the results of a survey conducted on quantities and types of shipments of radioactive materials between March 1974 and February 1975.19 In this investigation, questionnaires were sent to about 2300 of about 18000 licensees. Detailed questionnaires were mailed to Special Nuclear Material licensees who shipped 1 gram or more of material and also to 150 "major shippers." This information serves as the foundation for a data base on the U.S. radioactive material shipment pattern. This data base has provided important information for Sandia studies on radioactive material transport.

NUREG-0170, the predecessor to the current effort, assesses the environmental impacts resulting from transport of radioactive materials throughout the U.S. ${ }^{2}$ This study estimates that "normal" transport produces no short-term deaths, but in a statistical basis induces 1.2 latent cancer fatalities per shipment year. The comparable value is an existing rate of $3 \times 10^{5}$ cancer fatalities per year from all causes. Transportation accidents were estimated to produce only 1 latent cancer fatality in 200 years of shipping activity at the 1975 level. In spite of this risk level, specific accidents, occurring in high population zones, were found to have the potential for significant consequences -- as many as 150 latent cancer fatalities and more than $\$ 100$ million in decontamination costs. The details of the effects of the urban environment on these estimates were provided an impetus for another study to specifically analyze transportation impacts in urban areas.

\subsection{Summary}

The other chapters of this report detail the methodology and results for each of the four causative events in terms of the expected radiological and economic impacts from each. Following these discussions, alternatives to the current transport practice are considered and the detailed analysis is extended to other urban areas. The appendices contain the data bases and specific models used to evaluate these impacts, as well as discussions of chemical toxicity and social impacts. Collectively, the report assesses the environmental consequences of the transportation of radioactive materials in urban areas, including estimates of radiological, nonradiological, and social impacts arising from this process.

\section{NOTES}

1J. L. Simmons et al, Survey of Radioactive Material Shipments in the United States, BNWL-1972, Richland, WA: Battelle Pacific Northwest Laboratories, April 1976. Hereafter cited as BNWL-1972.

${ }^{2}$ U.S. Nuclear Regulatory Commission, Office of Standards Development, Final Environmental Statement on the Transportation of Radioactive Materials by Air or Other Modes, NUREG-0170, Washington: USNRC, December 1977. Hereafter cited as NUREG -0170 . 1978.

${ }^{3}$ U.S. Department of Commerce, Statistical Abstract of the United States,

442FR 12271 (Federal Register). 
${ }^{5}$ C. Cluett et a1, Identification and Assessment of the Social Impacts of Transportation of Radioactive Materials in Urban Environment, SAND79-7032, NUREG/CR-0744, Seattle, WA: Battelle Human Affairs Research Centers, May 1980.

${ }^{6}$ U.S. Atomic Energy Commission, Directorate of Regulatory Standards, Environmental Survey of Transportation of Radioactive Material to and from Nuclear Power Plants, WASH-1238, Washington: USAEC, 1972. Hereafter cited as WASH-1238.

${ }^{7}$ U.S. Nuclear Regulatory Commission, Office of Standards Development, Environmental Survey of Transportation of Radioactive Material to and from Nuclear Power Plants, Supplement 1, NUREG-75/038, Washington: USNRC, Apri1 1975.

8U.S. Nuclear Regulatory Commission, Office of Standards Development, Potential Releases of Cesium from Irradiated Fuel in a Transportation Accident, Supplement 2 to WASH-1238, NUREG-0069, Washington: USNRC, July 1976. Hereafter cited as NUREG-0069.

${ }^{9}$ U.S. Nuclear Regulatory Commission, Office of Standards Development, A Detailed Summary of "Survey of Radioactive Material Shipments in the United States," -1-BNWL-1972, NUREG-0073, Washington: USNRC, May 1976. Hereafter cited as NUREG-0073. 
INCIDENT-FREE TRANSPORT

\subsection{General Discussion of the Incident-Free Transport Model}

Transport of radioactive materials can cause environmental impacts even when no acccidents or other abnormalities compromise package integrity. These impacts result from exposure of surrounding populations to external penetrating radiation. Reference 1 indicates that the radiological impacts from incident-free or "normal" transport could be significantly greater on an annual risk basis than those resulting from vehicular accidents.

Although the consequences to individuals may be small, overall population impacts from low-dose, low-dose-rate radiation exposure can occur and may be significant because of the large number of annual shipments, many of which occur in urban areas. 1-3 The model used to evaluate the radiological impacts and the results of the analysis are described in this chapter.

\subsubsection{Standard Shipments Model}

A standard shipments model is used to describe the movement of radioactive material through a $100-\mathrm{km}^{2}$ section of New York City and to account for shipments which originate in or are destined for that area. (Information from Reference 3 was accepted as the best available data, although errors may be contained therein.) The specific $100 \mathrm{~km}^{2}$ chosen for study -- comprising parts of Manhattan, Brook1yn, and Queens -- is shown in Figure 1-1 and described in detail in Appendix A.

Isotopes have been assigned an end use on the basis of general information about the material, including half life and accepted use.* The end uses considered are medical (including research materials), industrial, fuel cycle (including fuel cycle wastes), and waste materials. Tables 4.6 and 4.7 on pp 4-19 and 4-20 of Reference 3 supply specific information on transport mode and package type.

The standard shipments model lists the isotopes by shipment mode and further categorizes them by end use and package type (see Tables 2-1 through 2-3). Specific routes are established for each shipment mode (Table 2-4): truck, train, barge, air freight and air passenger. The air shipments include overflights across the grid and overflights with return to the grid by truck as the secondary mode. Detalls of these routes are in Appendix A.

*Examination of supplier catalogues frequently supplied information on material end use. 
Table 2-1

Standard Shipments Information by End Use ${ }^{a}$

\begin{tabular}{|c|c|c|c|c|}
\hline End Use & $\begin{array}{l}\text { Packages } \\
\text { Per Year } \\
\end{array}$ & $\begin{array}{c}\text { Percent } \\
\text { of } \\
\text { Total } \\
\end{array}$ & TI Per Year & $\begin{array}{c}\text { Percent } \\
\text { of } \\
\text { Total } \\
\end{array}$ \\
\hline Medical & $2.23 \times 10^{4}$ & 80.1 & $8.12 \times 10^{3}$ & 76.5 \\
\hline Industrial & $3.10 \times 10^{3}$ & 11.1 & $1.85 \times 10^{3}$ & 17.5 \\
\hline Fuel Cycle & $2.34 \times 10^{3}$ & 8.4 & $5.89 \times 10^{2}$ & 5.6 \\
\hline Waste & $9.60 \times 10^{1}$ & 0.4 & $4.58 \times 10^{1}$ & 0.4 \\
\hline Totals & $2.78 \times 10^{4}$ & & $1.06 \times 10^{4}$ & \\
\hline \multicolumn{5}{|c|}{$\begin{array}{l}\text { a Materials shipped as limited or exempt quantities are not in- } \\
\text { cluded. A limited shipment does not have to conform to the same } \\
\text { packaging regulations as other shipments because even a } 100 \% \\
\text { release would contribute only negligibly to overall impact. } \\
\qquad \mathrm{b}_{\mathrm{TI}}=\text { Transport index, a measure of the dose rate at } \sim 1 \text { metre } \\
\text { from the package surface. }\end{array}$} \\
\hline
\end{tabular}

Table 2-2

Standard Shipments Information by Primary Mode

\begin{tabular}{|c|c|c|c|c|}
\hline $\begin{array}{l}\text { Shipment } \\
\text { Mode }\end{array}$ & $\begin{array}{l}\text { Packages } \\
\text { Per Year } \\
\end{array}$ & $\begin{array}{c}\text { Percent } \\
\text { of } \\
\text { Total } \\
\end{array}$ & TI Per Year & $\begin{array}{c}\text { Percent } \\
\text { of } \\
\text { Tota } 1 \\
\end{array}$ \\
\hline Truck & $1.48 \times 10^{4}$ & 53.3 & $5.58 \times 10^{3}$ & 52.7 \\
\hline Air Freight* & $4.75 \times 10^{3}$ & 17.0 & $2.94 \times 10^{3}$ & 27.7 \\
\hline Air Passenger* & $5.95 \times 10^{3}$ & 21.4 & $1.51 \times 10^{3}$ & 14.2 \\
\hline Barge & $2.31 \times 10^{3}$ & 8.3 & $5.75 \times 10^{2}$ & 5.4 \\
\hline Totals & $2.78 \times 10^{4}$ & & $1.06 \times 10^{4}$ & \\
\hline
\end{tabular}

*The total packages per year given for air freight and air passenger include those which are eventually rerouted into the area by truck. 
Table 2-3

Standard Shipments Information by Package Type

$\begin{array}{crrrr}\begin{array}{c}\text { Package } \\ \text { Type }\end{array} & \begin{array}{c}\text { Packages } \\ \text { Per Year }\end{array} & \begin{array}{c}\text { Percent } \\ \text { of } \\ \text { Total }\end{array} & \text { TI Per Year } & \begin{array}{c}\text { Percent } \\ \text { of } \\ \text { Total }\end{array} \\ \text { A } & 2.50 \times 10^{4} & 90.0 & 9.34 \times 10^{3} & 88.1 \\ \text { B } & 2.69 \times 10^{3} & 9.7 & 1.23 \times 10^{3} & 11.6 \\ \text { Drum } & 7.0 \times 10^{1} & 0.3 & 1.66 \times 10 & 0.2 \\ \text { Cask } & \underline{1.2 \times 10^{1}} & 0.1 & \frac{1.2 \times 10}{1.06 \times 10^{4}} & 0.1 \\ \text { Totals } & 2.78 \times 10^{4} & & & \end{array}$

Table 2-4

Standard Shipments Information by Route ${ }^{a}$

\begin{tabular}{|c|c|c|}
\hline $\begin{array}{l}\text { Route } \\
\text { Number }\end{array}$ & $\begin{array}{l}\text { Material } \\
\text { End Use }\end{array}$ & Mode ${ }^{b}$ \\
\hline 1 & Medical & $\begin{array}{l}1^{c}-\text { Air freight } \\
2^{\circ}-\text { Truck }\end{array}$ \\
\hline 2 & Medical & Truck \\
\hline 3 & Medical & Air freight overflight \\
\hline 4 & Medical & Truck \\
\hline 5 & Industrial & Truck \\
\hline 6 & Industrial & Air passenger overflight \\
\hline 7 & Industrial & $\begin{array}{l}1^{\circ} \text { - Air freight } \\
2^{\circ} \text { - Truck }\end{array}$ \\
\hline 8 & Industrial & Truck \\
\hline 9 & Fuel cycle & Ship \\
\hline 10 & Industrial & $\begin{array}{l}1^{\circ} \text { - Air passenger } \\
2^{\circ}-\text { Truck }\end{array}$ \\
\hline 11 . & Waste & Truck \\
\hline 12 & Fuel cycle & Truck \\
\hline 13 & Medical & Air passenger overflight \\
\hline 14 & Industrial & Air freight overflight \\
\hline 15 & Medical & $\begin{array}{l}1^{\circ} \text { - Air passenger } \\
2^{\circ}-\text { Truck }\end{array}$ \\
\hline 16 & Waste & Truck \\
\hline 17 & $\begin{array}{l}\text { Fuel cycle } \\
\text { (spent fuel } \\
\text { shipments) }\end{array}$ & Truck \\
\hline
\end{tabular}


Comparing the shipment model for the limited New York City area with the nationwide shipping pattern reported in Reference 1, the New York data represent $1.3 \%$ of the total number of packages shipped per year, $0.5 \%$ of the total curies per year, and only $0.2 \%$ of the total transport index (TI). Unlike the Reference 1 case, medical-use shipments represent $80 \%$ of the total number of packages compared with approximately $4.2 \%$ for the nationwide data, and they represent $\sim 77 \%$ of the total TI compared with the nationwide value of $14 \%$. When the total curies shipped are compared, the limited New York City and Reference 1 data are remarkab1y consistent: both values are between $1 \%$ and $2 \%$. The observed differences are due at least in part to the specific uses of radioactive materials in the urban situation.

\subsubsection{Radiological Consequence Model}

The model to evaluate the radiological consequences from incident-free transport is based on the formula for dose rate from a point source of ionizing radiation. This formula contains an exponential factor accounting for the attenuation of photons in the absorbing medium which surrounds the receptor, a second term which accounts for the inverse-square dose-rate reduction with distance from the source, and a third term which accounts for dose-rate buildup caused by inelastic photon scattering in the attenuating medium. This dose-rate buildup factor (DBF) can be for a single medium (e.g., air, in the case of pedestrians) or for a series (e.g., air/building material/air, for people in buildings). To adapt this equation for materials in transport, terms specifically related to the transport process are introduced. These terms include the $\mathrm{TI}^{3}{ }^{3}$ packages per. shipment, and a package shape factor to account for actual source geometry.

In addition, the movement of the shipment must be considered; i.e., the vehicle carrying the radioactive material is moving at some velocity $V$. The resulting formulation, Equation 6 of Appendix B, forms the basis for many of the integrated dose expressions used in this analysis.

At this point, the analysis diverges from previous work in that the exposure to groups peculiar to urban areas is calculated. The dose groups considered are

- Pedestrians

- People in vehicles

- People in buildings

- Crew of the transport vehicle

- Warehouse personnel exposed while the package is in storage

- Those who handle the package during transfers from one vehicle to another or at freight depots

- People at rail terminals

- People sharing the rail transport link

- People along the rail right-of-way

- People in dock areas (considered to be freight docks, not passenger docks)

- People at air passenger terminals

- People at air freight terminals 
Specific dose calculations for each of these groups can be found in Appendix $B$. The following sections will briefly describe the techniques used to evaluate the doses to the various groups.

\section{Pedestrians}

The most important characteristic of this dose group is the number of pedestrians present in any grid cell (given by PedD: persons $/ \mathrm{km}^{2}$ of sidewalk). Other important parameters include street and sidewalk widths, average vehicle velocity, pedestrian velocity, and the distance the transport vehicle travels in the cell. The direct dose to pedestrians is calculated both for those moving in the same direction as the transport vehicle and those moving in the opposite direction. In addition, albedo dose is calculated to account for the dose received by pedestrians due to scattering from the ground and adjacent structures. Figure $2-1$ is a diagram of the geometry used for the incident-free dose to pedestrians.

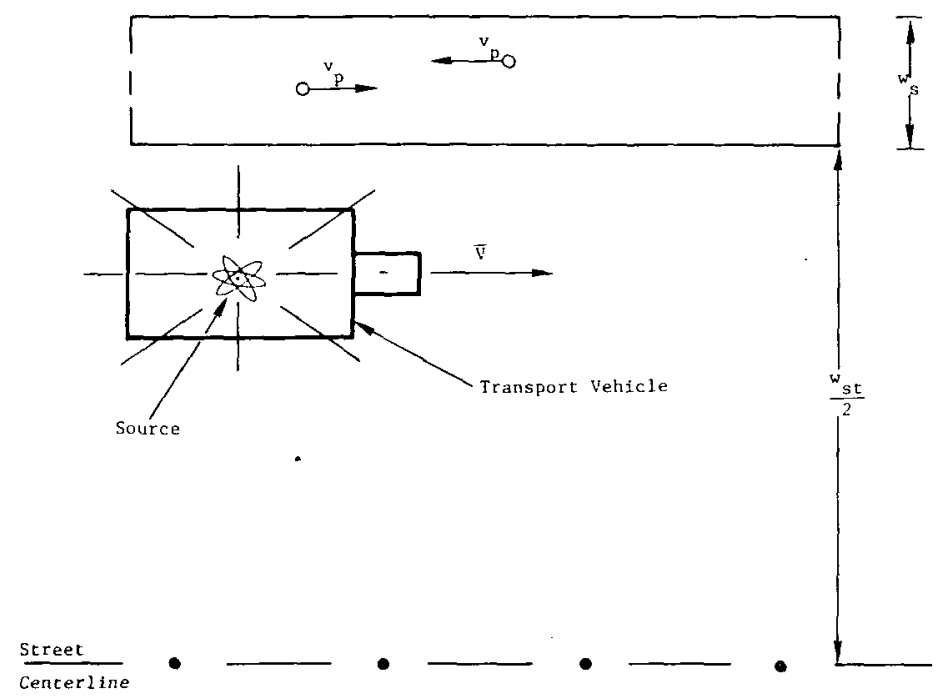

Figure 2-1. Geometry for Incident-Free Dose to Pedestrians

The overall expression used to calculate dose to pedestrians from truck travel on two-way streets is given in Equation 9, Appendix B. An adaptation of this expression (Equation 41, Appendix B) is made for truck travel on one-way streets. For truck travel on freeways, dose to pedestrians is assumed to be zero.

\section{People in Buildings}

Dose to people in buildings is computed in a way similar to that for pedestrians. The basic differences are that structural shielding is considered and that people are assumed to be stationary inside the building (see Figure 2-2). The model can account for attenuation coefficients for the various building construction materials, buildup factor for air, and building materials, etc. 


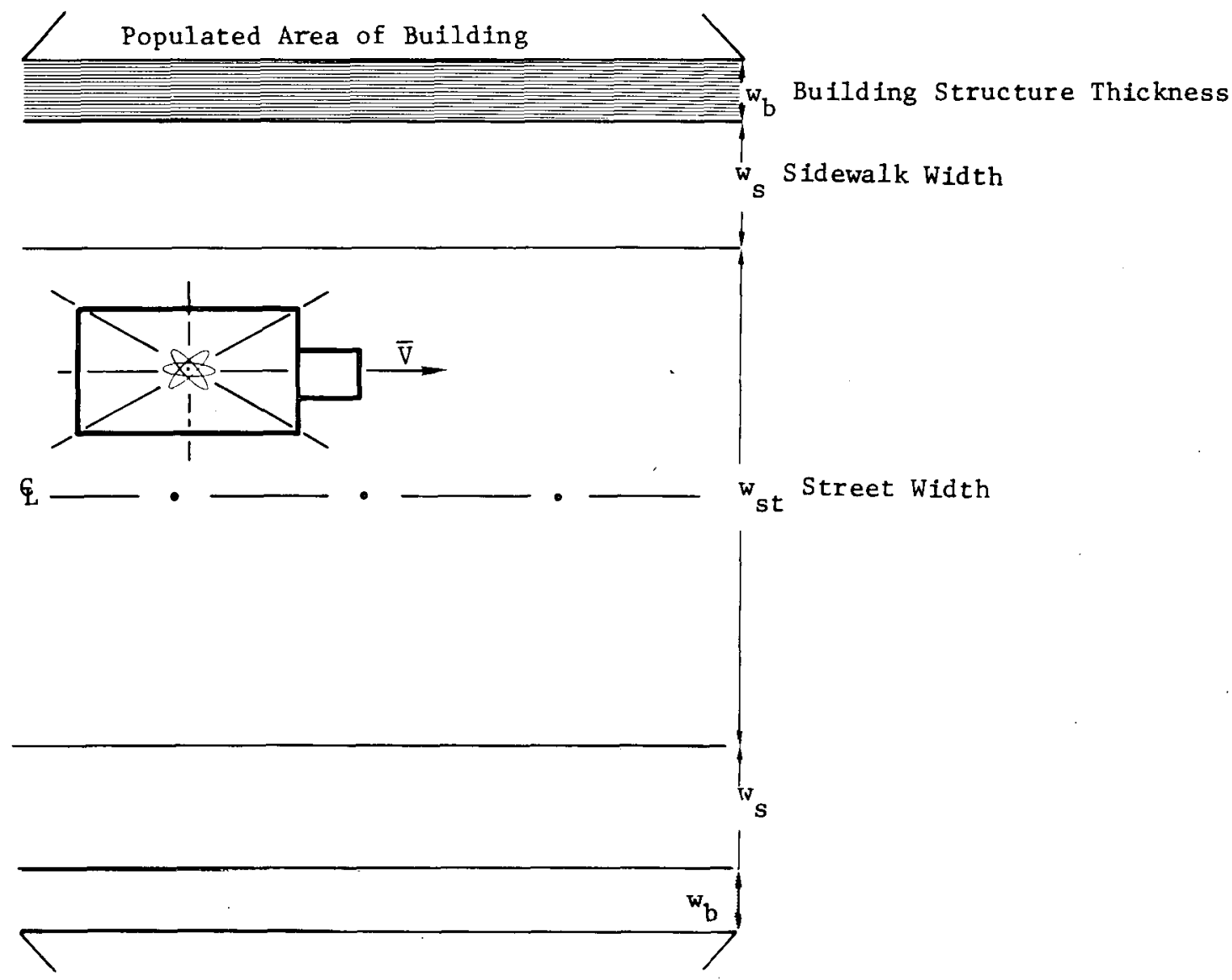

Figure 2-2. Geometry for Incident-Free Dose to People in Buildings and Vehicles

In the transport process, the vehicle is moving past the people it exposes. To account for this, the model calculates the dose attenuation due to oblique impingement of the radiation on the building surface. When these factors are combined with the necessary population density information, dose to people in buildings can be calculated. The expression for integrated dose to people in buildings for one-way and two-way street travel is given in Equation 28, Appendix B; for freeway travel, Equation 44, Appendix B.

People in Vehicles

The urban-specific parameters necessary for the analysis of dose to people in vehicles include average traveling speed (including intersection delays), people per vehicle, and number of vehicles in any grid cell at any time. Vehicles traveling in urban areas tend to platoon (form groups due to traffic signals, freeway entrances, etc.), and this is also factored into the analysis.

The expression for same-direction dose is given in Equation 36, Appendix B. For vehicles traveling in the opposite direction, the model treats people in vehicles as stationary with the shipment passing by at twice the cruising speed (Equation 38, Appendix B). The total integrated dose for people in vehicles is expressed in Equation 39 of Appendix B. 
Crew

The integrated dose to the crew is calculated by using an expression with three multiplicative terms: one accounting for attenuation of photons in the absorbing medium, one for inverse-square geometric dose reduction, and the third for dose-rate buildup caused by inelastic scattering in the attenuating medium. The exact expression for integrated dose to crew is given in Equation 40, Appendix B. For travel on freeways, travel velocity is adjusted, resulting in the expression in Equation 40 with freeway velocity substituted.

\section{Warehouse Personne1}

For this dose group, it is assumed that personnel maintain a minimum distance from the shipment when stored. (Because of building geometry, a maximum achievable distance from the shipment is used.) The computation of integrated dose for warehouse personnel is performed over an annulus of integration with a constant warehouse population density assumed over the entire annulus (see Figure 2-3). When the length of time the shipment remains in storage is included, the expression for integrated dose to warehouse personnel is given in Equation 53, Appendix B.

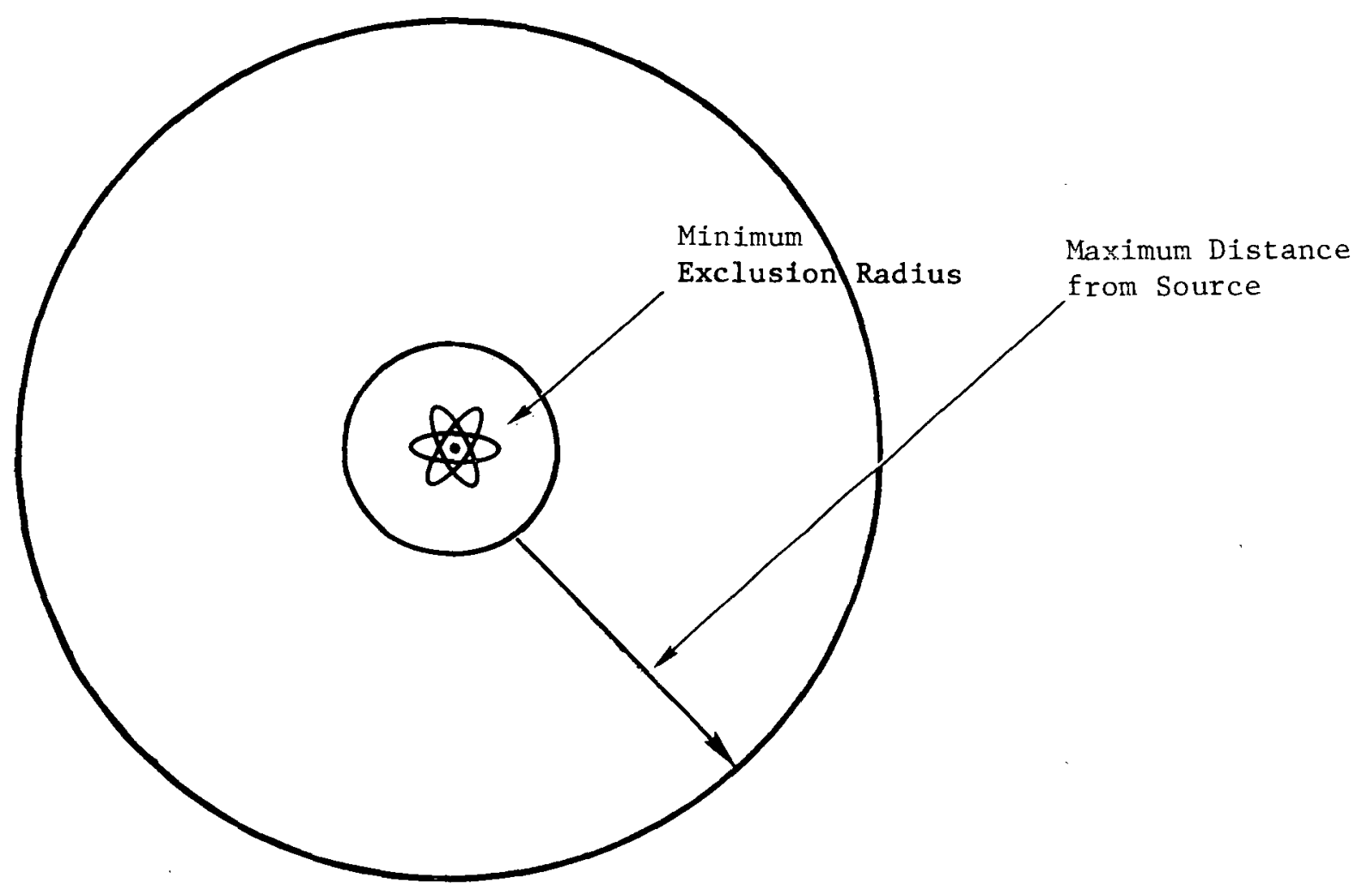

Figure 2-3. Geometry for Calculating Dose to Warehouse Personnel. This geometry is also applicable to people at rail terminals, in dock areas, or in air passenger terminals. 
Handlers

Handlers are exposed to materials in transit only during transfers. The expression for integrated dose to handlers is simply a function of the number of handlings, packages per shipment, and TI per package. The expression for integrated dose is given in Equation 51, Appendix B. A different expression is used for dose to handlers of casks. This is given in Equation 52, Appendix B.

People at Rail Terminals

People in terminal areas are exposed only during the time the train remains in the terminal area. The integrated dose to people in rail terminals is a function of the population density in the terminal, TI per package, and package shape factor, which accounts for actual package dimensions. An annulus within which the exposable population is located is assumed, giving the expression found in Equation 45, Appendix B. The analysts have used an annular integration technique in this instance to account for the geometry of the population around the source.

People Sharing the Transport Link

For people aboard trains, the development of integrated dose expressions is similar to that for people in vehicles during truck transport (see Equation 40 , Appendix B). The differences are basically those specifically related to trains: velocity and people per train. The resulting expression is Equation 46, Appendix B.

People Along Rail Right-of-Way

An overall population density along rail right-of-way was calculated (Equation 47, Appendix B) because it was not possible to perform the calculation for discrete dose groups. Instead of an annulus of integration, the limits of integration have been taken from the minimum rail right-of-way distance to 400 metres* (Figure 2-4). Equation 48 in Appendix $B$ was derived by combining the calculated population density with the other train-specific parameters described earlier.

People in Dock Areas

The technique described earlier for warehouse personnel is used again here, i.e., the exposable population (here the dock population) is assumed to be within an annulus of integration. The expression is dependent upon the length of time the water transport vehicle remains in the dock area. Docks are assumed to occupy only one-half of the area, the other half being water. A factor of $1 / 2$ is introduced to account for this assumption (Equation 50, Appendix B).

People in Air Terminals (Passenger or Cargo)

The derivations for air passenger and air freight terminals are the same except for population densities and maximum exposure radii. The basic expression is the same as that used for people in rail terminals or dock areas. It is dependent upon the length of time the plane remains in the terminal area, which may vary for passenger and cargo planes (Equation 49, Appendix B). 


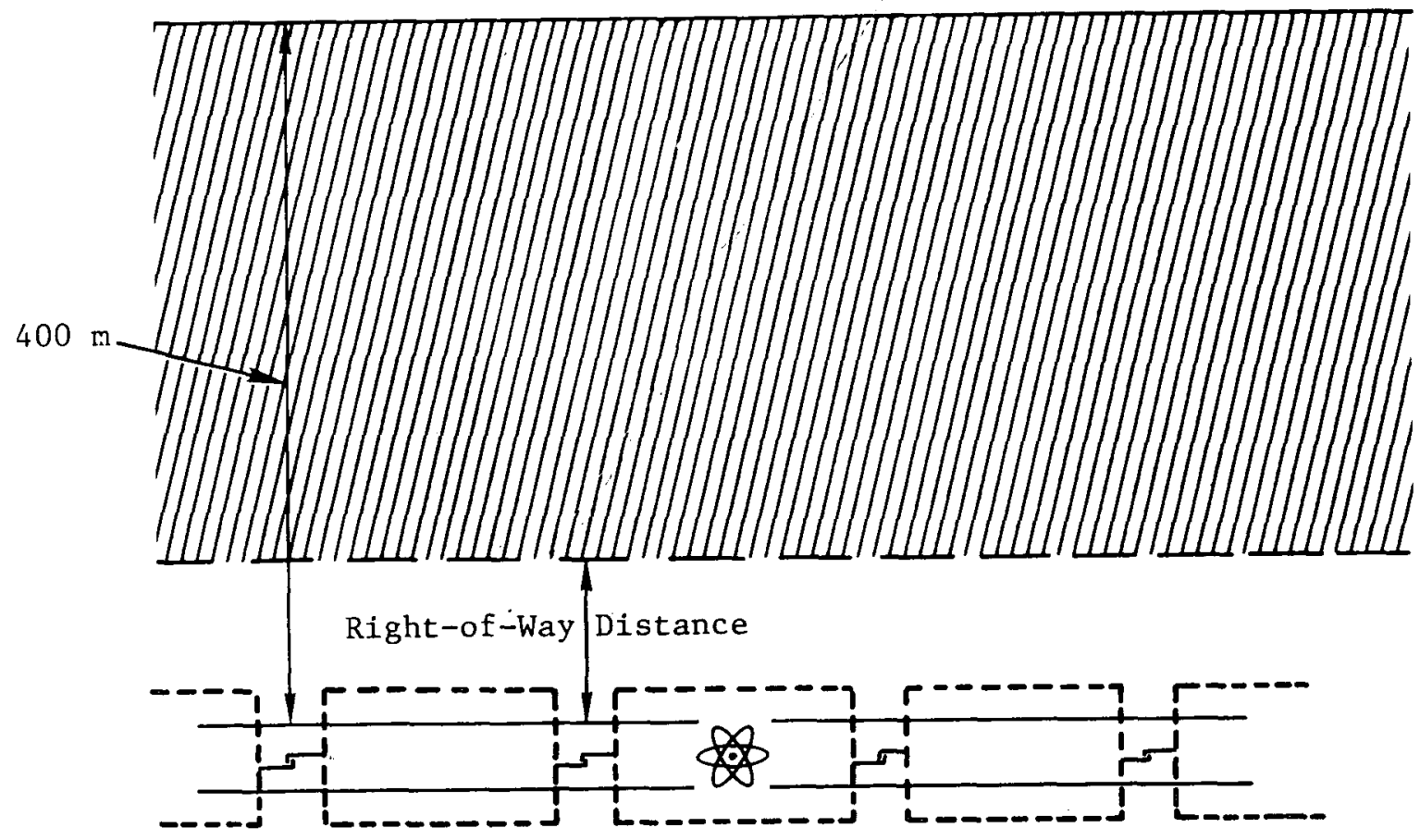

Figure 2-4. Geometry for Calculating Dose to People along Rail Right-of-Way (integration along a strip)

\subsection{Radiological Impact}

The radiological consequence code, METRAN, quantifies the incident-free environmental impacts resulting from the transport of standard shipments of radioactive materials through the New York City grid in terms of annual person rem for the shipment year considered (1974-1975). The total radiological impact for the limited New York City grid is about 10 person rem per year.

\subsubsection{Incident-Free Radiological Impacts by End Use (for NYC shipment model compressed to $100-\mathrm{km}$ study area)}

Medical-use radioisotopes constitute the largest component (78\%) of the incident-free impacts. This result is to be expected since Table 2-1 shows that these radioisotopes constitute more than $80 \%$ of the yearly shipments and about $77 \%$ of the annual $\mathrm{TI}$.

Industrial-use isotopes rank second (17\%). This is also predictable since $11 \%$ of the shipments in the urban area involve these radioisotopes with $18 \%$ of the total TI.

Nuclear fuel cycle shipments and shipments of radioactive waste account for the remaining $5 \%$. The small contribution from the waste category is mainly due to the small number of waste shipments in the urban region during the survey year. 
The impacts received by specific urban population groups are summarized by material end use in Table 2-5.

\subsubsection{Incident-Free Radiological Impacts by Transport Mode}

Essentially all of the incident-free impacts result from truck transport of the radioactive materials and from the storage and handling of the materials shipped by air. Only $4 \%$ of the total impacts result from exposure to persons in air terminals while the materials are being on-loaded or off-loaded from aircraft. Approximately $70 \%$ of the incident-free impacts result from truck transport and $26 \%$ from exposure of handlers and warehouse personnel during storage of air freight and air passenger shipments. This exposure may well be considered occupational in nature and thus would further reduce the incident-free impacts. A summary of radiological impacts by transport mode is given in Table 2-6.

\subsubsection{Incident-Free Radiological Impacts by Package Type}

A summary of incident-free impacts by package type is presented in Table 2-7. Eighty-six percent of annual person rem is attributable to lightly shielded Type A packages. Type B packages, drums, and casks account for the remaining $15 \%$ of the radiological impact. Some of these packages are transported by barge through the grid. These shipments do not expose the large number of people adjoining routes followed by trucks carrying Type A packages.

\subsubsection{Summary of Impacts from Incident-Free Transport}

The impacts from incident-free transport in the urban area are summarized in Table 2-8 by person rem per shipment year for the exposed population groups.

These groups, in descending order of impact, are handlers, people in vehicles, warehouse personnel, pedestrians, crew, people in air passenger terminals, and people in buildings. More than $95 \%$ of the impact is distributed among the first five of these dose groups.

About 10 person rem have been estimated for the limited New York City analysis of incident-free transport. The use of 25 expected latent cancer fatalities per million person rem (see Appendix G) for the low-dose, low-dose-rate exposures typical of incident-free transport yields a prediction of less than 1 latent cancer fatality per shipment year.

Through rough scaling, the approximately 7 person rem for the $1.06 \times 10^{4} \mathrm{TI}$ per year for the limited New York City example can be extended to the $4.54 \times 10^{6} \mathrm{TI}$ per year included in the analysis of Reference 1 for the entire nation. The comparison is on the basis of total TI shipped per year since it is external exposure resulting from radiation levels outside the package which is significant. The extrapolation gives a value of 3000 person rem compared with the 9790 person rem estimated in Reference 1 .

\subsection{Sensitivity and Error Analysis}

The analysis for the incident-free model evaluates the sensitivity of the response variable (integrated dose, in person rem) for each dose group when the shipment proceeds on a one-cell route by a given transport mode/road type. 
Table 2-5

Incident-Free Radiological Impacts (person rem/year of shipping activity) on Population Groups by End Use

\begin{tabular}{|c|c|c|c|c|c|c|c|c|c|c|c|c|c|c|}
\hline End Use & $\begin{array}{l}\text { Percent } \\
\text { of Total }\end{array}$ & Handlers & $\begin{array}{l}\text { Warehouse } \\
\text { Personnel } \\
\text { (Storage) }\end{array}$ & $\begin{array}{c}\text { Crew } \\
\text { (Truck) } \\
\end{array}$ & Pedestrians & $\begin{array}{c}\text { People } \\
\text { in } \\
\text { Vehicles }\end{array}$ & $\begin{array}{l}\text { People in } \\
\text { Buildings }\end{array}$ & $\begin{array}{l}\text { People } \\
\text { in Rail } \\
\text { Depots }\end{array}$ & $\begin{array}{l}\text { People } \\
\text { Sharing } \\
\text { Rail } \\
\text { Link } \\
\end{array}$ & $\begin{array}{l}\text { People } \\
\text { on Rail } \\
\text { Right- } \\
\text { of-Way }\end{array}$ & \begin{tabular}{l}
\multicolumn{1}{c}{ Air } \\
Passenger \\
Terminal \\
\end{tabular} & $\begin{array}{c}\text { Air } \\
\text { Cargo } \\
\text { Terminal } \\
\end{array}$ & Ship & Totals \\
\hline Medical & 74 & 1.6 & 0.73 & 0.52 & 0.95 & 1.3 & 0.012 & - & - & - & 0.19 & - & - & 5.3 \\
\hline Industrial & 18 & 0.46 & 0.46 & 0.07 & 0.07 & 0.16 & $<0.01$ & - & - & - & 0.03 & - & - & 1.3 \\
\hline Fuel Cycle & 8 & $0.48 *$ & - & 0.003 & 0.009 & 0.07 & $<0.01$ & - & - & - & - & - & - & 0.6 \\
\hline Waste & $<1$ & - & - & 0.006 & 0.01 & 0.02 & $<0.001$ & - & - & - & - & - & - & $<0.1$ \\
\hline Totals & & 2.5 & 1.2 & 0.6 & 1.0 & 1.6 & $\sim 0.015$ & - & - & - & 0.22 & - & - & $\sim 7.2$ \\
\hline
\end{tabular}

*This hypothetical value assumes one handling of a cask during transport.

Table 2-6

Incident-Free Radiological Impacts (person rem/year of shipping activity) on Population Groups by Mode

\begin{tabular}{|c|c|c|c|c|c|c|c|c|c|c|c|c|c|c|}
\hline Mode & $\begin{array}{l}\text { Percent } \\
\text { of Total } \\
\end{array}$ & Hand lers & $\begin{array}{l}\text { Warehouse } \\
\text { Per sonneI } \\
\text { (Storage) }\end{array}$ & $\begin{array}{c}\text { Crew } \\
\text { (Truck) } \\
\end{array}$ & Pedestrians & $\begin{array}{c}\text { People } \\
\text { in } \\
\text { Vehicles } \\
\end{array}$ & $\begin{array}{l}\text { People in } \\
\text { Buildings }\end{array}$ & $\begin{array}{l}\text { People } \\
\text { in Rail } \\
\text { Depots }\end{array}$ & $\begin{array}{c}\text { People } \\
\text { Sharing } \\
\text { Rail } \\
\text { Link } \\
\end{array}$ & $\begin{array}{l}\text { People } \\
\text { on Rail } \\
\text { Right- } \\
\text { of-Way }\end{array}$ & \begin{tabular}{l}
\multicolumn{1}{c}{ Air } \\
Passenger \\
Terminal \\
\end{tabular} & $\begin{array}{c}\text { Air } \\
\text { Cargo } \\
\text { Terminal } \\
\end{array}$ & $\underline{\text { Ship }}$ & Totals \\
\hline $\begin{array}{l}\text { Air pass. } \\
\text { over- } \\
\text { flights }\end{array}$ & 14 & 0.4 & 0.4 & - & - & - & - & - & - & - & 0.22 & - & - & $\sim 1.0$ \\
\hline $\begin{array}{l}\text { Air freigh } \\
\text { over- } \\
\text { flights }\end{array}$ & 21 & 0.7 & 0.8 & - & - & - & - & - & - & - & - & - & - & 1.5 \\
\hline Truck $^{a}$ & 65 & $1.4^{b}$ & - & 0.6 & 1.0 & 1.6 & 0.015 & - & - & - & - & - & - & $\sim 4.7$ \\
\hline Barge & - & - & - & - & - & - & - & - & - & - & - & - & - & - \\
\hline Totals & & 2.5 & 1.2 & 0.6 & 1.0 & 1.6 & $\sim 0.015$ & - & - & - & 0.22 & - & - & $\sim 7.2$ \\
\hline
\end{tabular}

\footnotetext{
ancludes contributions from alr freight and air passenger shipments which are rerouted into NYC grid by truck.

${ }^{b}$ Includes 0.48 person rem per shipment year from a hypothetical handing of a cask during transport.
}

Table 2-7

Incident-Free Radiological Impacts (person rem/year af shipping activity) on Population Groups by Package Type

\begin{tabular}{|c|c|c|c|c|c|c|c|c|c|c|c|c|c|c|}
\hline $\begin{array}{c}\text { Package } \\
\text { Type }\end{array}$ & $\begin{array}{l}\text { Percent } \\
\text { of Total } \\
\end{array}$ & Handlers & $\begin{array}{l}\text { Warehouse } \\
\text { Personne1 } \\
\text { (Storage) }\end{array}$ & $\begin{array}{c}\text { Crew } \\
\text { (Truck) }\end{array}$ & Pedestrians & $\begin{array}{c}\text { People } \\
\text { in } \\
\text { Vehicles } \\
\end{array}$ & $\begin{array}{l}\text { People in } \\
\text { Buildings }\end{array}$ & $\begin{array}{l}\text { People } \\
\text { in Rail } \\
\text { Depots }\end{array}$ & $\begin{array}{l}\text { People } \\
\text { Sharing } \\
\text { Rail } \\
\text { Link } \\
\end{array}$ & $\begin{array}{l}\text { People } \\
\text { on Rail } \\
\text { Right- } \\
\text { of-Way } \\
\end{array}$ & $\begin{array}{c}\text { Air } \\
\text { Passenger } \\
\text { Terminal } \\
\end{array}$ & $\begin{array}{c}\text { Air } \\
\text { Cargo } \\
\text { Terminal } \\
\end{array}$ & Ship & Totals \\
\hline A & 86 & 1.9 & 1.0 & 0.6 & 1.0 & 1.5 & 0.012 & - & - & - & 0.17 & - & - & 6.2 \\
\hline B & 7 & 0.16 & 0.2 & 0.04 & 0.02 & 0.06 & $<0.01$ & - & - & - & 0.05 & - & - & 0.5 \\
\hline Drum & $<0.1$ & $<0.001$ & $<0.001$ & $<0.01$ & 0.005 & 0.006 & $<0.001$ & - & $=$ & - & - & - & - & 0.01 \\
\hline Cask & 8 & $0.48^{*}$ & - & $<0.01$ & $<0.007$ & 0.07 & $<0.01$ & - & - & - & - & - & - & 0.56 \\
\hline Totals & & 2.5 & 1.2 & 0.6 & 1.0 & 1.6 & 0.015 & - & - & - & 0.22 & - & - & 7.2 \\
\hline
\end{tabular}

*This hypothetical value assumes one handling of a cask durtng transport. 
Table 2-8

Summary of Radiological Impacts from

Incident-Free Transport by

Dose Group

Dose Group

Handlers

People in Vehicles

Warehouse Personnel

Pedestrians

Crew

People in Air

Passenger Terminals

People in Buildings

People in Rail Depots*

People Sharing Rail

Transport Link*

People in Rail

Right-of-way*

People in Air Cargo

Terminals*

People in Dock Areas*
Total Person Rem/Yr Percent of Total

2.5

$\sim 35$

1.6

22

1.2

17

1.0

$>14$

0.6

$\sim 8.4$

0.2

$\sim 3$

0.015

$\sim 0 . ?$

.
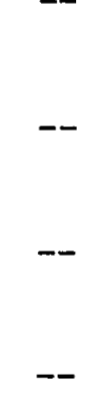

$-$

7.2

Tota1

* No impact has been calculated for people in dock areas since the reported shipments in the limited New York City shipment model are all through shipments. No shipments by rail are found in the shipment model. Air freight shipments have been assumed to arrive at facilities outside the terminal area, hence no impact has been calculated for people in air cargo terminals. 
For multiplicative factors, the sensitivity is easily determined; i.e., doubling a factor doubles the dose. Therefore multiplicative factors are fixed at nominal values and the remaining variables are examined, resulting in scaled regression equations.

The process of producing scaled regression equations allows for the development of specific information on a desired route. If each of these equations is applied to one cell at a time, it is only necessary to determine values of the appropriate parameters for individual cells and sum the integrated doses for each to determine the overall integrated dose for a given route. Using this technique, the incident-free model is no longer specifically tied to $100 \mathrm{~km}^{2}$ in New York City but can be transposed to any urban area, given standard shipment information and data on the new urban area.

The error analysis of the incident-free transport model indicates the following:

- With the exceptions listed below, most of the coefficients of the error equations are less than unity. The variables for which relative errors are magnified are

$\begin{array}{ll}\frac{\text { Dose Groups }}{\text { Crew }} & \text { Source to crew distance }\left(d_{2}\right) \\ \begin{array}{l}\text { People in vehicles } \\ \text { (two-way streets) }\end{array} & \text { Street width }\left(w_{s t}\right) \\ \text { People in buildings } & \text { Building wall thickness }\left(w_{b}\right) \\ \text { People in air terminals } & \text { Minimum exposure distance }\left(r_{4}\right) \\ \text { A complete list of the variables appearing in the error } \\ \text { equations can be found in Appendix D. }\end{array}$

- For a predominant dose group, pedestrians, a $10 \%$ error in each of the six variables appearing in the error equation will result in a maximum relative error of $60 \%$. Thus the integrated dose to pedestrians could be underestimated by 0.6 or overestimated by the same amount. Note that these results do not lend themselves to a probabilistic interpretation.

\subsection{Conclusions}

The overall population dose received as a result of the incident-free shipment of radioactive materials in $100 \mathrm{~km}^{2}$ of New York City is about 10 person rem per shipment year. The predominant contributors to this dose are medical/research shipments in Type A packages transported by truck. A majority of the incidentfree population dose is received by two specific dose groups (people in vehicles and handlers) and three other groups, two of which are associated with the mechanics of the transport process (pedestrians, warehouse personnel, and crew). The urban-specific dose groups receive $36 \%$ of the incident-free dose, while the other three groups receive $60 \%$ of the dose. If the contribution to the dose from crew, handlers, and warehouse personnel exposures are considered occupational exposure, the overall incident-free population dose would decrease by almost a factor of 2 .

Use of scaled regression equations resulting from the sensitivity analysis of the incident-free model allows for simple application of the urban-specific 
transport model to other urban areas given special features such as standard shipment data characteristic of the site- and city-specific demographic information.

\section{NOTES}

${ }^{1}$ NUREG-0170.

${ }^{2}$ NUREG-0073.

${ }^{3}$ Analysis of raw data from BNWL-1972.

${ }^{4}$ WASH-1 238.

${ }^{5} \mathrm{~J}$. Shapiro, Exposure of Airport Workers to Radiation from Shipment of Radioactive Materials, NUREG-0154, Washington: U.S. Nuclear Regulatory Commission, January 1977.

${ }^{6} \mathrm{U} . \mathrm{S}$. Department of Commerce, Statistical Abstract of the United States, 1978. 
IMPACTS OF TRANSPORTATION ACCIDENTS

The analysis of the impacts of transportation accidents involving radioactive materials in urban areas is subdivided initially into two categories based on the dispersibility of the material involved in the accident. Impacts from accidents involving nondispersible or special-form materials result from increased exposure either because of the delay in transit of the material or because of reduction in the available shielding due to accident deformation forces. Dispersible materials can be aerosolized, producing an inhalation hazard to people near the accident. In addition, because the aerosol cloud disperses as a result of the prevailing meteorological conditions, dispersible materials can expose people in downwind areas. Part of a dispersible shipment can remain at the accident site, resulting in increased exposure to the surrounding population. Material in the aerosol cloud causes increased cloudshine and groundshine doses to the population along the route of cloud passage. If some of the deposited material is resuspended by wind, traffic, or other means, it can become a long-term inhalation hazard as well.

Two factors are involved in evaluating the impact of an accident from a risk (or expected value) perspective: probability and consequence. The probability that an accident occurs is presented in terms of the expected number of accidents of a given severity per year as a function of transport mode. The amount of dispersal or increased exposure is related to package condition after the accident by the release fraction, which describes the severity dependence of package response for various package types. These release fractions, together with mode-specific accident rates and severity-dependent fractional occurrences for accidents, form the basis for the calculations of probability of package response.

The consequence of an accident is defined as the effect of the release or exposure which results from an accident of a specific severity involving a particular shipment and package type. The product of consequence and overall package response is defined as the "expected value of radiological risk" and is expressed in terms of expected radiological consequences for a given type of shipment. For each shipment type, four potential health effects are considered: (1) early fatalities, (2) early morbidities, (3) latent cancer fatalities, and (4) genetic effects.*

Expected radiological risk values are calculated for each shipment type and sumed over all shipments to obtain the annual radiological risk resulting from a year of shipment activity at the given level. The value of expected annual

*Values for each of the health effects are calculated using the METRAN computer models. 
radiological accident risk represents a statistical measure of the annual public hazard posed by urban transport of radioactive materials. This type of analysis does not distinguish the contributions to overall risk of high-probability/lowconsequence accidents from those of low-probability/high-consequence accidents. Because of the heightened public and regulatory concern over low-probability/ high-consequence events, shipments which could cause potentially severe consequences if involved in an accident are considered separately to complete the analysis. In some cases, these shipments occur within the limited shipment data base for New York City and so are included in the expected value risk calculation. For those shipments not included in the data base, the calculated values do not contribute to the expected value of risk. Although the passage of these shipments through urban areas may not be documented, that possibility exists and, as a result, must be considered.

The remainder of this chapter is divided into five sections. Section 3.1 discusses the accident model in general.* This section touches on the use of data discussed in detail in Appendices $A$ and $C$ and on the meteorological model used in the dispersal accident case. The next section describes the method used for obtaining the expected values of radiological risk for the limited New York City standard shipment model. Following this discussion is a section on the potential health effects and indirect economic impact of an accident. The next section, 3.4, analyzes low-probability/high-consequence accidents. Finally, there is a discussion of a sensitivity and error analysis performed on the METRAN accident models and a short summary of the overall accident results.

\subsection{METRAN Accident Mode1}

As shown in Figure 3-1, the METRAN accident analysis involves the melding of the outputs of several submodels to provide either individual accident consequences or expected annual radiological risk. The model, described in detail in Appendix C, considers three types of accidents: (1) those involving release of a dispersible material, (2) those involving reduction or elimination of shielding from a nondispersible material, and (3) those which simply delay a package without any release of contents or shielding reduction. Two specific accident geometries -- midblock and intersection; four population groups -- crew, pedestrians, people in vehicles, and people in buildings; and three street types -freeways, one-way streets, and two-way streets are analyzed. Various other modes, rail, water, and air, result in other exposure situations, as discussed in Appendix C. Meteorological input based on the MICMET and PICMET codes (Appendices $E$ and $F$ ) provide the atmospheric dispersal input. The extensive land-use and population distribution data base (Appendix A) provides an estimate of the time and location dependencies of the population at risk. Accident rates, accident severity data, and release fraction information are also extracted for the data base to help determine the consequence and/or risk.

In the case of both nonrelease accidents and accidents involving exposure to nondispersible sources, the exposure magnitude depends directly on the length of exposure. This so-called delay time is a severity-dependent parameter specified in Appendix A. and 2 .

*Details can be found in the appendices to this document and in References 1 


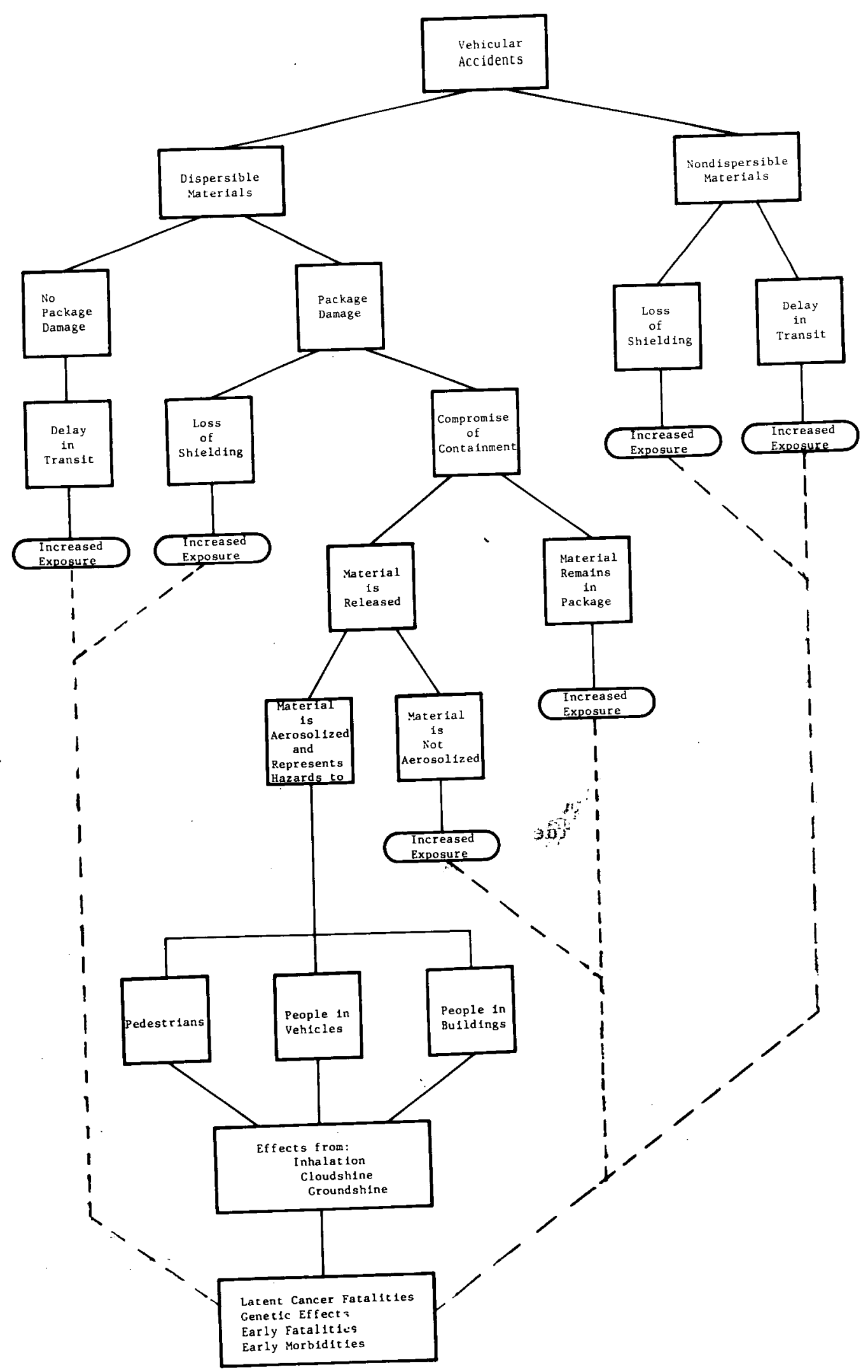

Figure 3-1. Potential Accidents and Their Consequences as Analyzed Using METRAN Code 


\subsubsection{Accidents Involving Nondispersible Materials}

The estimation of nonrelease accident consequences are dependent on the transport index of the shipment, the accident delay time, the number of people in the vicinity in each of the four dose groups, and the actual exposure geometry. Nonrelease accidents can involve either dispersible or nondispersible materials and fall in the category of high-probability/low-consequence accidents since they include "fender-bender" accidents which may significantly delay a transporting vehicle without any substantial cargo damage.

The consequences of accidents above the package damage threshold involving nondispersible materials are a strong function of the number of curies exposed, the number of exposable people, the particular exposure geometry for each dose group, and the accident delay time. Consequences of nondispersible source accidents are generally confined to the immediate area of the accident, because of the $1 / \mathrm{r}^{2}$ reduction in dose rate with distance coupled with the shielding afforded by the buildings present in urban areas.

\section{Nondispersal Source Accidents on Two-Way Streets}

Dose to Pedestrians -- The expression for dose to pedestrians depends upon the location of the individual along the linear path which the vehicle was following when the accident occurred, and his perpendicular location on the sidewalk. The expression (Equation 6, Appendix C) includes consideration of pedestrian density, accident delay time, the source strength, and intersection and midblock accident occurrence factors.

Dose to People in Buildings -- The dose to people in buildings will depend upon the location of the individuals in buildings and on the distance "down the block" from the accident site. The model considers oblique geometry as in Appendix $B$ (dose to people in buildings). Since people are assumed to be 10cated only adjacent to the outside walls of the buildings, a single integral is used to account for distance down the street. The expression includes a summation over all floors to account for the vertical distribution of people in buildings. Equation 10, Appendix $\mathrm{C}$, is the expression for integrated dose to people in buildings; it includes consideration of the number of people involved (population density plus transient population), building wall thickness, doserate buildup factor for the building material, fraction of the cell area occupied by buildings, and accident delay time. Terms to account for unshielded as well as shielded material and for accident location appear in the expression for people in buildings.

Dose to People in Vehicles -- Vehicles are assumed to provide no shielding, so the individual dose to people in vehicles is calculated in the same manner as for pedestrians (Equation 2, Appendix C). For accidents occurring in intersections, vehicles are assumed to be stopped at the outer edge of the intersection. In the midblock case, the vehicles traveling in the direction opposite to that in which the accident occurs are allowed to move at a reduced speed (one-half the cruising velocity). Vehicles moving in the same direction as that in which the accident occurs are assumed to be stopped. Two terms in the midblock expression account for same and opposite direction doses. Equation 18, Appendix $C$, gives the overall expression for dose to people in vehicles and includes consideration of source strength, accident delay time, and number of people per vehicle. 
Nondispersal Source Accidents on Freeways

Since it is assumed that there are no pedestrians on freeways, the pedestrian dose is set to zero. Geometry for dose to people in buildings is similar to the earlier expression for a midblock accident occurring on a very wide street. The basic changes include the minimum exposure distance and the number of exposed building faces. The method of calculating oblique impingement also changes, resulting in Equation 20, Appendix C, for dose to people in buildings from freeway accidents.

Since accidents on freeways seldom disable traffic in both directions, the model assumes, as in the two-way street midblock analysis, that only the side on which the accident occurs is stopped. Traffic in the opposite direction continues to move normally. The phenomenon of platooning, developed in Appendix B, is applied here, and the dose from the expression in Appendix $B$ for people traveling opposite to the shipment is modified to account for the fact that the source is stopped instead of moving. The net result is given in Equation 22, Appendix $C$.

Nondispersal Source Accidents on One-Way Streets

Since there is no opposite direction traffic stream on one-way streets, the major change is in dose to people in vehicles. A midblock accident is surrounded by streams of traffic moving past it in the same direction. The adjusted expression for dose to people in vehicles is given in Equation 23, Appendix C.

Nondispersal Source Air Transport Accidents

Aircraft accidents in urban areas can occur anywhere. The dose expressions used are assumed to be those for truck transport accidents on two-way streets (see Appendix C).

Nondispersal Source Rail Transport Accidents

Although rail shipments do not appear in the New York City shipment data base, the model is capable of handling this type of accident. Although accidents could occur anywhere in the urban area, high-speed collisions causing significant damage to packages would most likely occur outside of terminal areas. Thus, a development analogous to incident-free dose to people along rail rightof-ways is used, resulting in Equation 24, Appendix C.

Nondispersal Source Water Transport Accidents

Only water transport accidents occurring in the dock area could cause significant exposure in an urban area. Equation 25, Appendix $C$, an equation analogous to Equation 50, Appendix B, is used.

\subsubsection{Accidents Involving Dispersible Material}

Accidents severe enough to disperse radioactive material can result in up to five different doses: from inhalation of airborne material, from cloudshine exposure, from groundshine exposure, from material remaining at the scene of the 
accident, and from inhalation and cloudshine from material resuspended after being initially deposited on the ground. Ingestion of radioactive contamination from a vehicular accident is not considered in this analysis.

Because of the additional complexity afforded by consideration of meteorological conditions, the calculations of these consequences are more involved and the discussion of this source of exposure is expanded somewhat.

Direct Inhalation Dose

The consequences of radiation exposure from the direct inhalation pathway are strongly dependent on both the radioactive source term, the prevailing atmospheric conditions, and the location of the population. The source-term parameters of interest include the number of curies of material released from the package and aerosolized, the physiological pathway followed by material once inhaled, and the dose to a specific organ which results from inhalation. The relationships between these factors and their use are discussed in greater detail in Appendices $B$ and $H$. The atmospheric dispersion processes, described in Appendices $E$ and $F$, control the downwind cloud dimensions and material concentrations as a function of mean wind velocity, local surface roughness (including building height and fraction of area occupied by buildings), and atmospheric turbulence parameters.

\section{Cloudshine Dose}

Those persons who are immersed in the passing cloud of radioactive material may consequently be exposed to external penetrating radiation. The amount of dose received by this pathway is dependent on the energy of the emitted photons, the length of exposure, and the concentration of the cloud.

\section{Remnant Dose}

In many cases, a significant amount of material may remain undispersed at the scene of an accident. This material will act as an exposure source, giving some additional dosage to persons in the immediate area depending on their exposure geometry, length of exposure, and the amount of material present.

Groundshine Dose

Some of the material which is dispersed in an accident will be deposited downwind of the accident site on building floors, walls, streets, etc. This material can act as a direct radiation source, causing substantial exposure to people traveling in the contaminated area. The model computes this exposure by assuming an infinite plane source of monoenergetic photons. If the area is contaminated above cleanup level and/or land-use denial (interdiction) levels, the mitigating effect of these actions on exposure is considered.

\section{Resuspension Dose}

Deposited material can be resuspended by various mechanical forces such as wind, vehicular traffic, street cleaning, etc. The resuspended material then becomes a direct inhalation source. This exposure pathway is evaluated using a resuspension dose factor which operates directly on the direct inhalation dose to give the total dose due both to direct inhalation and inhalation of resuspended material. 
Additional discussion of inhalation dose follows to illustrate the various aspects of the process used to evaluate a particular type of dispersal accident dose.

\subsubsection{Inhalation Dose from Accidents Involving Dispersible Materials}

A complicating factor with vehicular accident analysis is the dependence of health-effect consequences of an individual inhalation dose received on the particular body organ or set of organs exposed. The inhalation dose to a particular organ is dependent upon radionuclide characteristics as well as upon the concentration of the material in the cloud. This analysis considers individual organ doses from inhalation to lungs (1-year and 50-year doses), bone marrow (1-year and 50-year doses), lower large intestine, bone, thyroid, and gonads. For the last four, only 50-year total doses are considered.

\section{Pedestrians}

As a cloud of radioactive material leaves the accident site, the meteorological models (Appendices $\mathrm{E}$ and $\mathrm{F}$ ) calculate an atmospheric dilution factor at street level which describes the concentration of particles to which different dose groups are exposed during cloud passage and the amount of material deposited from the cloud as it passes. Since these factors change as the cloud disperses, it is assumed that a pedestrian remains within a specific cell for the duration of cloud passage. The dose received by a particular pedestrian is the sum over all time steps of the product of the dilution factors, the length of each time step, and the radiological source term (Equation 27, Appendix C). As indicated in Appendix E, there are short time steps (ce11 of release) and long time steps (other affected cells); thus the summation must be across both sets of time steps. The integrated dose across all time steps and for the midblock and intersection cases is given in Equation 29, Appendix C. Pedestrian density is still allowed to vary to simulate the diurnal variation, but it is no longer a simple multiplicative factor becoming part of the summation process. Details of the development of this expression are given in Appendix C.

People in Vehicles

The development of this expression is analogous to that for dose to pedestrians. No allowance is made for filtration of the cloud of debris as it enters the vehicle. The total individual dose to people in vehicles is the same as for pedestrians (Equation 27, Appendix C). The total number of people in vehicles is required in this case (people per vehicle times number of vehicles). When this factor is incorporated, the general expression for integrated dose to people in vehicles is given by Equation 30, Appendix $C$.

\section{People in Buildings}

The concentration of airborne radioactive material inside a building depends on the rate of exchange of outside air with the air inside the building. Equation 31, Appendix $\mathrm{C}$, gives an expression for the building dose factor (ratio of dose inside to dose. outside). The model takes account of two ventilation types (continuous intake and air-conditioned systems). For details of these systems and the remainder of the building dose development, the reader is referred to Appendix $C$. The expression for integrated dose to people in buildings is given in Equation 32, Appendix C. 
Exact development of expressions for other accident doses can be found also in Appendix C.

\subsection{Summary of Direct Radiological Effects from Accidents}

Once the integrated and individual doses from a specific accident have been calculated as described above, it is necessary to combine the results, determine the expected number of health effects, and express the consequences in terms of expected annual values. The output parameters are early fatalities, early morbidities, latent cancer fatalities, and genetic effects, and result from inhalation and/or external exposure. Details of the health effects models and conversion techniques are described in Appendices $\mathrm{C}$ and $\mathrm{G}$.

Expected numbers of early fatalities are obtained by multiplying doses received by individuals by the probability of a fatality given a specific dose and then summing over the various computed individual doses (Equation 43, Appendix C). Early morbidities are calculated analogously except that in this case there is assumed to be a 100\% occurrence of morbidity above a specified dose threshold (Equation 44, Appendix $C$ ). In order to predict the number of latent cancer fatalities and/or genetic effects, the integrated exposure associated with early fatalities is subtracted from the total integrated exposure. The remainder is then converted to expected number of long-term effects by multiplying the adjusted dose to a particular organ received in an accident by the appropriate health-effects coefficient for that organ and the dose-rate effectiveness factor and then summing over all organs (Equation 45, Appendix $C$ ). The dose-rate effectiveness factor is a variable in the analysis and can be chosen by the analyst. For this study, a dose-rate effectiveness factor of 0.2 is used for all dose rates less than $1 \mathrm{rem} /$ day $(\sim 400 \mathrm{rem} /$ year $)$ and a dose-rate effectiveness factor of 1.0 is used for doses greater than $1 \mathrm{rem} /$ day. In addition a dose-rate effectiveness factor of 1.0 is used for all high linear energy transfer (LET) radiation sources, regardless of dose rate.

To calculate the total expected health effects, it is necessary to evaluate the expected number of accidents of a given severity in a particular cell. The results described above are for accidents of a given severity occurring in a specific cell along the route. The expected number of accidents (Equation 46, Appendix $C$ ) is the product of the distance traveled in a cell, the accident rate per unit distance of travel, and the fraction of accidents of a given severity. It should be noted that the expected number of accidents for a given cell and severity is dependent on both transport mode and time span. Combining the above with the calculated number of consequences for a given cell and severity, the expected numbers of health effects are obtained (Equation 47, Appendix C). Finally, the expected numbers of health effects are summed over all modes and routes to obtain the total expected values for a specific set of standard shipments.

\subsection{Results of Analysis}

The results of this portion of the analysis are presented from several different perspectives for easy comparison of the impacts of vehicular accidents with those from the other causative events. The impacts from the entire shipments model are presented, and then impacts from particular shipments are analyzed. In evaluating these results, the reader should keep four concepts in mind. First, the expected value of annual radiological risk, as defined here, is a 
statistical measurement of the hazard to the public over all time from 1 year of shipment activity, not the number of health effects expected to occur annually. Second, the population at risk is the entire population of the specified urban area since, for dispersible materials, the aerosol cloud could pass over any portion of the city given the appropriate meteorological conditions. Similarly, for nondispersible materials, the accident could occur anywhere in the city, not just along the routes specified in this study. Finally, the exposed population is that portion of the overall population directly affected by increased external exposure and/or by radioactive debris from a specific release.

Before summarizing the results of the analysis, a few comments on data changes since the working draft of this report (Reference 10) was published are in order. The major change has been in the consideration of material which can be released from a spent fuel shipping cask. In the previous analysis, a fraction of the entire cask contents was assumed to be released and aerosolized. After consideration of test data on cask response to severe accidents, the authors decided that this assumption was not reasonable. ${ }^{3}$ The analysis of cask accidents in the present study is based upon the more realistic assumption that the major source of radioactive material which would be released in a severe accident is the material deposited on the cask interior and exterior surfaces of the fuel elements during their lifespan in the reactor (known as reactor "crud" and containing predominantly primary system corrosion products). References 4 and 5 indicate that for 150-day cooled fuel, the major contaminant in the crud is cobalt-60. The other materials present after the fuel has been removed from the reactor have relatively short half lives and, for practical purposes, are gone at the end of the first 150 days. For a generic 3-element, water-cooled cask (similar to a NFS-4 or NAC-1 cask) containing $16 \times 16$ arrays of fuel pins per element, the available inventory of Co-60 can be calculated using the conservative data in References 4 and 5: namely, 180 microcuries per $\mathrm{cm}^{2}\left(\mu \mathrm{Ci} / \mathrm{cm}^{2}\right)$ of pin surface. The result of the computation is that about 154 curies of Co-60 are present on the surface of the fuel pins. Recent information indicates that approximately $20 \%$ of the adhered crud could be easily removed by physical shock. other sources of "crud" are also present in the cask; however, these have not been quantified, and the assumption is made that the 154 curies represents the fraction of the total available inventory which could be released. It is recognized that other materials (not corrosion products) are available for release. In particular, noble gases and cesium-137 are present as a result of normal levels of perforation of the fuel pins in a reactor. There may be significant quantities of krypton-85 available for release. Since the levels of noble gases in general would contribute negligibly to the overall population dose in comparison to the levels of $\mathrm{Co}-60, \mathrm{Kr}-85$ is not considered further. Cs-137 is recognized to produce health effects at a level comparable to those of Co-60. However, Reference 6 indicates that in contaminated cask coolant water, $90 \%$ of the contaminant is Co-60 while only $1 \%$ is Cs-137. Thus, the assumption is made here that the available Co-60 produces the total health effects which will be observed. In conclusion, the source term in Reference 10 for spent fuel dispersal has been replaced by a more realistic value, consisting solely of Co-60, which would become available for release if the transport vehicle were shocked severely enough in collision to jar loose and release the deposited "crud." The authors believe this is an improved approach to the spent fuel 
vehicular accident analysis.* The quantity of "crud" available for release would be a strong function of the cask type. That is, the use of air or inert gas coolants, instead of water will strongly influence the "crud" source terms.

To examine the influence of meteorology on the accident risk, the analysis was performed for one time of day ( 1630 hours), the four cardinal wind directions, and three separate wind speeds $(2,4$, and $8 \mathrm{~m} / \mathrm{s})$. These calculations indicate that as wind speed increases, the calculated number of expected early morbidities disappears since the materials are dispersed more rapidly and the initial exposure levels to individuals close to the accident site are accordingly lowered. Values for latent effects do not change markedly with different wind speeds and directions. Thus, the tables which follow are for a single set of meteorological conditions ( $2 \mathrm{~m} / \mathrm{s}$, south wind) with accident occurrence time set arbitrarily to 1630 hours.

Table 3-1 summarizes the contributions to the expected value of radiological risk by material end-use categories. The major contributors to the expected risk are fuel cycle and medical shipments contributing together more than $80 \%$ of the expected effects.

Table 3-1

End-Use Contributions to Expected Radiological Risk Values from Vehicular Accidents per Year of Shipping Activity

\begin{tabular}{|c|c|c|c|c|c|c|c|c|c|c|}
\hline End Use & $\begin{array}{l}\text { Amount } \\
\text { Shipped } \\
\text { (Ci/yr) } \\
\end{array}$ & $\mathrm{f}$ & $\begin{array}{c}\text { No. of } \\
\text { Shipments per year }\end{array}$ & $f$ & $\begin{array}{l}\text { Expected } \\
\text { Number of } \\
\text { Latent } \\
\text { Cancer } \\
\text { Fatalities } \\
\end{array}$ & $E^{a}$ & $\begin{array}{l}\text { Expected } \\
\text { Number of } \\
\text { Genetic } \\
\text { Effects } \\
\end{array}$ & $\mathbf{F}^{\mathrm{a}}$ & $\begin{array}{l}\text { Expected } \\
\text { Number of } \\
\text { Early } \\
\text { Morbidities } \\
\end{array}$ & $F^{a}$ \\
\hline Medical & $4.6 \times 10^{4}$ & 0.017 & $2.2 \times 10^{4}$ & 0.80 & $5.3 \times 10^{-4}$ & $\sim 0.39$ & $7.4 \times 10^{-4}$ & 0.39 & - & - \\
\hline Industrial & $7.1 \times 10^{4}$ & 0.026 & $3.1 \times 10^{3}$ & $\sim 0.11$ & $2.3 \times 10^{-4}$ & $\sim 0.17$ & $3.4 \times 10^{-4}$ & 0.18 & - & - \\
\hline $\begin{array}{l}\text { Fuel cycle } \\
\quad \text { (including fuel } \\
\text { cycle wastes) }\end{array}$ & $2.6 \times 10^{6}$ & 0.96 & $2.3 \times 10^{3}$ & $\sim 0.08$ & $6.1 \times 10^{-4}$ & $\sim 0.44$ & $8.1 \times 10^{-4}$ & 0.43 & $2.7 \times 10^{-5}$ & 1.0 \\
\hline Waste & $5.7 \times 10^{-3}$ & -- & $9.6 \times 10^{1}$ & $\sim 0.004$ & $8.2 \times 10^{-7}$ & $<0.001$ & $1.1 \times 10^{-6}$ & $<0.001$ & - & - \\
\hline Total & $\sim 2.7 \times 10^{6}$ & & $2.8 \times 10^{4}$ & & $1.4 \times 10^{-3}$ & & $1.9 \times 10^{-3}$ & & $2.7 \times 10^{-5}$ & - \\
\hline
\end{tabular}

*Inclusion of a Cs-137 release in addition to the assumed Co-60 value was determined to alter the radiological consequences from a spent fuel transport accident by less than $10 \%$. This was confirmed by using METRAN and CRAC analyses. The Cs-137 release (25 curies) was calculated from the assumptions in Reference 12. 
Table 3-2 displays the contributions to expected risk by transport mode. Only aircraft overflights are allowed in the limited New York City area, with secondary mode rerouting by truck, since no air terminals are located there. Examination of these air/truck shipments shows that the truck contribution predominates. Therefore, when the rows labeled "Truck," "Air," and "Air and Truck" are combined to reflect this assumption, the contribution of truck transport to latent cancer fatalities and to genetic effects is $\sim 100 \%$.

Table 3-2

Contributions of Transport Modes to Expected Radiological Risk Values from Vehicular Accidents per Year of Shipping Activity

\begin{tabular}{|c|c|c|c|c|c|c|c|c|c|c|}
\hline $\begin{array}{l}\text { Transport } \\
\text { Mode } \\
\end{array}$ & $\begin{array}{r}\text { Amount } \\
\text { Shipped } \\
\text { (ci/yr) } \\
\end{array}$ & $f$ & $\begin{array}{c}\text { No. of } \\
\text { Shipments per year }\end{array}$ & $f$ & $\begin{array}{l}\text { Expected } \\
\text { Number } \\
\text { of Latent } \\
\text { Cancer } \\
\text { Fatalities }\end{array}$ & $\boldsymbol{F}$ & $\begin{array}{l}\text { Expected } \\
\text { Number of } \\
\text { Genetic } \\
\text { Effects } \\
\end{array}$ & $\mathbf{F}$ & $\begin{array}{l}\text { Expected } \\
\text { Number } \\
\text { of Early } \\
\text { Morbidities }\end{array}$ & $F$ \\
\hline Truck & $2.7 \times 10^{6}$ & 0.98 & $1.5 \times 10^{4}$ & 0.53 & $7.2 \times 10^{-4}$ & 0.53 & $9.7 \times 10^{-4}$ & 0.51 & $2.7 \times 10^{-5}$ & 1.0 \\
\hline Ai $r^{a}$ & $5.5 \times 10^{4^{b}}$ & 0.02 & $1.1 \times 10^{4} \mathrm{~b}$ & $\sim 0.39$ & $9.7 \times 10^{-9}$ & $<0.001$ & $1.4 \times 10^{-8}$ & $<0.001$ & - & - \\
\hline $\begin{array}{l}\text { Air and } \\
\text { Truck }\end{array}$ & - & - & - & - & $6.5 \times 10^{-4}$ & 0.47 & $9.2 \times 10^{-4}$ & 0.49 & - & - \\
\hline Barge & 4.9 & $<0.001$ & $2.3 \times 10^{3}$ & $\sim 0.08$ & $3.2 \times 10^{-\theta}$ & $<0.001$ & $4.1 \times 10^{-8}$ & $<0.001$ & $2.7 \times 10^{-5}$ & - \\
\hline Total & $-2.7 \times 10^{6}$ & & $2.8 \times 10^{4}$ & & $\sim 1.4 \times 10^{-3}$ & & $1.9 \times 10^{-3}$ & & & \\
\hline
\end{tabular}

Table 3-3 displays contributions by package type. Type A and Type B packages account for $57 \%$ of the expected risk values while cask shipments produce $43 \%$ of the calculated values.

Table 3-4 summarizes the risk contributions from materials as a function of the dispersibility of the shipment. Dispersible shipments cause slightly more than two-thirds of the total health effects. As might be expected, the entire early morbidity risk results from dispersible shipments, specifically the recorded spent fuel shipments, as seen in Tables 3-1 through 3-3.

Table 3-5 lists the most significant standard shipments in terms of contribution to the various health effect categories. These 10 shipments (out of a total shipment population of 130 ) result in over $90 \%$ of the expected value of radiological risk from accidents. Shipments of spent fuel and magnesium-28 contribute $1.7 \%$ of latent cancers and genetic effects. Spent fuel produces all of the early effects (early morbidities). The remainder of the shipments listed in the table are various industrial and medical materials. 
Table 3-3

Contributions of Package Type to Expected Radiological Risk Values from Vehicular Accidents per Year of Shipping Activity

\begin{tabular}{|c|c|c|c|c|c|c|c|c|c|c|}
\hline $\begin{array}{c}\text { Package } \\
\text { Type }\end{array}$ & $\begin{array}{l}\text { Amount } \\
\text { Shipped } \\
\text { (Ci/yr) } \\
\end{array}$ & $\mathrm{f}$ & $\begin{array}{l}\text { No. of } \\
\text { Shipments per year }\end{array}$ & $\underline{f}$ & $\begin{array}{l}\text { Expected } \\
\text { Number } \\
\text { of Latent } \\
\text { Cancer } \\
\text { Fatalities }\end{array}$ & $\mathbf{F}$ & $\begin{array}{l}\text { Expected } \\
\text { Number of } \\
\text { Genetic } \\
\text { Effects } \\
\end{array}$ & $\mathrm{F}$ & $\begin{array}{l}\text { Expected } \\
\text { Number } \\
\text { of Early } \\
\text { Morbidities } \\
\end{array}$ & $E$ \\
\hline A & $2.4 \times 10^{4}$ & 0.01 & $2.5 \times 10^{4}$ & 0.90 & $5.8 \times 10^{-4}$ & 0.41 & $7.9 \times 10^{-4}$ & 0.41 & - & - \\
\hline B & $9.2 \times 10^{4}$ & 0.03 & $2.7 \times 10^{3}$ & $\sim 0.10$ & $2.2 \times 10^{-4}$ & 0.16 & $3.0 \times 10^{-4}$ & 0.16 & - & - \\
\hline Cask & $2.6 \times 10^{6}$ & 0.96 & $1.2 \times 10^{1}$ & $<0.001$ & $6.1 \times 10^{-4}$ & 0.43 & $8.1 \times 10^{-4}$ & 0.43 & $2.7 \times 10^{-5}$ & 1.0 \\
\hline Drum & $9.7 \times 10^{-3}$ & $<0.001$ & $7.0 \times 10^{1}$ & $<0.01$ & $8.9 \times 10^{-7}$ & $<0.001$ & $1.2 \times 10^{-6}$ & $<0.001$ & - & - \\
\hline Total & $2.7 \times 10^{6}$ & & $2.8 \times 10^{4}$ & & $1.4 \times 10^{-3}$ & & $1.9 \times 10^{-3}$ & & $2.7 \times 10^{-5}$ & \\
\hline
\end{tabular}

Table 3-4

Contributions to Expected Radiological Risk Values from Vehicular Accidents on the Basis of Dispersible Nature of Shipments

\begin{tabular}{|c|c|c|c|c|c|c|c|c|c|c|}
\hline $\begin{array}{l}\text { Nature of } \\
\text { Shipment }\end{array}$ & $\begin{array}{l}\text { Amount } \\
\text { Shipped } \\
\text { (Ci/yr) } \\
\end{array}$ & $\mathrm{f}$ & $\begin{array}{l}\text { Number of } \\
\text { Shipments } \\
\text { per Year }\end{array}$ & $f$ & $\begin{array}{l}\text { Expected } \\
\text { Number of } \\
\text { Latent Cancer } \\
\text { Fatalities } \\
\end{array}$ & $\mathbf{F}$ & $\begin{array}{l}\text { Expected } \\
\text { Number of } \\
\text { Genetic } \\
\text { Effects } \\
\end{array}$ & $\mathbf{F}$ & $\begin{array}{l}\text { Expected } \\
\text { Number of } \\
\text { Early } \\
\text { Morbidities }\end{array}$ & $\underline{F}$ \\
\hline Dispersible & $2.4 \times 10^{4}$ & 0.01 & $2.6 \times 10^{4}$ & 0.94 & $1.1 \times 10^{-3}$ & 0.82 & $1.5 \times 10^{-3}$ & 0.81 & $2.7 \times 10^{-5}$ & 1.0 \\
\hline $\begin{array}{l}\text { Nondispersible } \\
\text { (Special Form) }\end{array}$ & $2.7 \times 10^{6}$ & 0.99 & $1.7 \times 10^{3}$ & 0.06 & $2.5 \times 10^{-4}$ & 0.18 & $3.5 \times 10^{-4}$ & 0.19 & - & - \\
\hline Totals & $\sim 2.7 \times 10^{6}$ & & $\sim 2.8 \times 10^{4}$ & & $\sim 1.4 \times 10^{-3}$ & & $\sim 1.9 \times 10^{-3}$ & & $2.7 \times 10^{-5}$ & \\
\hline
\end{tabular}

Changes in consequence modeling have resulted in enhanced sensitivity of the results to the level of contamination caused by an accident. Table 3-6 illustrates the population group contributions to health effects for the materials given in Table 3-5. The breakdown is by curies per package and dispersibility. For dispersible materials, the dose groups receiving the majority of the effects are pedestrians and people in buildings, although the fractional contributions to these groups depend upon the nature of the materials. For nondispersible materials, the major recipients of the health effects are pedestrians and people in vehicles. For exposure sources the contributions to health effects arise from (1) nonrelease accidents (release fraction is zero), and (2) direct exposure and remnant material for nonzero release fractions. Several dose pathways are possible for dispersible materials. 
Table 3-5

\section{Contributions of Specific Standard Shipments to Expected Radiological Risk Values \\ from Vehicular Accidents}

Material

Fractional Contribution to Expected Number of Latent Cancer Fatalities

Spent Fuel ${ }^{\mathrm{a}}$

Mg-28 (52 Ci/Type A pkg)

Co-60 (4700 Ci/Type B pkg) ${ }^{b}$

Ir-192 (100 Ci/Type B pkg)

Mo-99 ( 1.3 Ci/Type A pkg)

Mo-99 (1.2 Ci/Type A pkg)

Ir-192 (80 Ci/Type A pkg)

Au-198 (13 Ci/Type A pkg)

Co-60 (4700 Ci/Type B pkg $)^{b}$

Mo-99 (91 Ci/Type B pkg)

Subtotal

All Others ${ }^{c}$

$$
0.44
$$

0.23

0.07

0.06

0.04

0.03

$\sim 0.03$

0.02

0.01

0.01

0.94

0.06

Fractional Contribution

to Expected Number of

Early Morbidities
Fractional Contribution to Expected Number of of Genetic Effects
Material

Spent Fue1
1.0

\subsection{3}

0.24

0.07

0.07

0.04

0.04

$\sim 0.03$

0.02

0.01

0.01

0.96

0.04

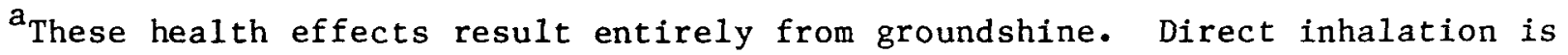
not a significant contributor except in high-severity accidents which do not contribute greatly to the overall risk.

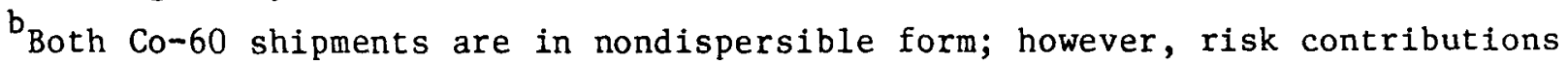
vary because of different routes through the study area.

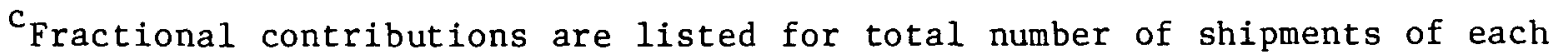
material type, e.g., for $\mathrm{Mg}-28$ the number of shipments is 46 , for spent fuel the number is 12 , etc. 
Table 3-6

Population and Dose Pathway Contributions to Health Effects

Latent Cancer Fatalities*

Total
Contribution to

\section{Material}

Mo-99

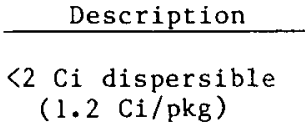

Mo-99

$<2$ Ci dispersible

( $1.3 \mathrm{Ci} / \mathrm{pkg}$ )

Au-198

$>2 \mathrm{Ci}$ dispersible

(13 $\mathrm{Ci} / \mathrm{pkg}$ )

$\mathrm{Mg}-28>2 \mathrm{Ci}$ dispersible

(52 Ci/pkg)

$\mathrm{S} p \mathrm{~F}-\mathrm{INH} \quad>2 \mathrm{Ci}$ dispersible

Mo-99 >2 Ci dispersible

(9l $\mathrm{Ci} / \mathrm{pkg}$ )

Co-60. $>2 \mathrm{Ci}$ nondispersible ( $4700 \mathrm{Ci} / \mathrm{pkg}$ )

Ir-192 >2 Ci nondispersible ( $80 \mathrm{Ci} / \mathrm{pkg}$ )

Ir-192 >2 Ci nondispersible $\left(100^{\circ} \mathrm{Ci} / \mathrm{pkg}\right)$

Co-60 Route 5

Type A Type B
Fractional Contribution

Buildings Vehicles Pedestrians

0.6

$$
6 \times 10^{-5}
$$

$3 \times 10^{-5}$

$3 \times 10^{-4}$

$6 \times 10^{-4}$

$2 \times 10^{-5}$

$1 \times 10^{-4}$

$4 \times 10^{-5}$

$9 \times 10^{-5}$

$2 \times 10^{-5}$
0.4

0.4

0.3

0.4

0.98

0.3

$-$

$-$

-

$-$

0.6

0.7

0.6

0.02

0.7

0.3

0.3

0.3

0.3

0.7 (4700 Ci/pkg) Route 2

* Only latent cancer fatalities are expressed here since the pattern of fractional contributions is the same for genetic effects, although the

total contributions are not the same.

Fractional Contribution by Dose Pathway

$\begin{aligned} & \text { Expo- } \\ & \text { sure }\end{aligned}$ Inhala- $\begin{gathered}\text { Ground- } \\ \text { tion }\end{gathered}$ shine
shine

$-$

$-$

1.0

$-\quad-\quad 1.0$

$-\quad-\quad 1.0$

- $\quad-\quad 1.0$

$-$

1.0

1.0

1.0

1.0 
For low-severity accidents, the entire effect comes from the nonrelease accident. When the release fraction is not zero and the material is aerosolized, three exposure pathways are possible: remnant, cloudshine, and groundshine. There is also the inhalation dose pathway. Analysis of these pathways has revealed that cloudshine is not significant. Groundshine is the most significant contributor to both latent cancer fatalities and genetic effects (see Table 3-6).

Further analysis of the data reveals that certain accident severities contribute most significantly to the overall risk. For dispersible materials shipped in Type A packages, accidents of severities II through $\mathrm{V}$ produce more than $95 \%$ of the observed health effects. For dispersible materials shipped in Type B packages (non-fuel-cycle materials), accidents of severities III through VI result in more than $98 \%$ of the calculated health effects. If dispersible fuel cycle materials shipped in casks are examined, severity III and severity IV accidents produce $98 \%$ of the observed health effects. Nondispersible material accidents of intermediate severities contribute the major portion of the health effects. Given the above information, we conclude that accidents of intermediate severity are the most significant in the calculation of risk values.

In summary, the expected values of radiological risk are dominated by the few spent fuel shipments and by medical-use shipments, with all of the expected early morbidities coming from the spent fuel shipments. Essentially $100 \%$ of the expected health effects come from truck transport of the materials, although this may well be an artifact of the computer models. Shipments in Type A packages and casks dominate the expected health effects, contributing equally to the calculated values. Dispersible materials dominate the expected number of health effects, although much of the total results from exposure to material deposited on the ground during cloud passage. Several routes used in the analysis pass through cells adjacent to the northern boundary of the study area. Since accidents occurring in these cells during prevailing southerly winds (assumed for Tables 3-1 through 3-6) could affect areas of the city outside the grid, the analysis was repeated using the four cardinal wind directions and three distinct wind speeds to examine the variation of the overall results for these different conditions. Table 3-7 presents the results of this analysis. The greatest variation is observed in the calculated numbers of expected early morbidities. As wind speed increases independent of direction, the number of early effects decreases. As the wind speed increases above $8 \mathrm{~m} / \mathrm{s}$, the calculated number of early morbidities becomes zero. The analysis indicates that, for the most part, a single choice of wind direction and speed is sufficient to gauge the level of estimated radiological effects.

\subsection{Direct Economic Impacts}

The extensive radioactive contamination of an area from a major accident involving radioactive material can result in large economic costs to homeowners, businesses, and governmental agencies. These costs consist of immediate emergency response costs; cleanup and recovery costs, radiological survey costs, street cleanup costs, building cleanup costs, evacuation costs, security costs, and land-use denial costs as determined by the particular situation. Each of these costs, detailed in Appendix $\mathrm{K}$, is a function of accident severity.

The general methodology in economic impact assessment involves five principal steps : 
Table 3-7

Expected Radiological Risk Values from Vehicular Accidents by Wind Direction and Speed

\begin{tabular}{|c|c|c|c|c|}
\hline $\begin{array}{c}\text { Wind } \\
\text { Direction }\end{array}$ & $\begin{array}{c}\text { Wind } \\
\text { Speed }(\mathrm{m} / \mathrm{s})\end{array}$ & $\begin{array}{c}\text { Expected Number of } \\
\text { Latent Cancer } \\
\text { Fatalities } \\
\end{array}$ & $\begin{array}{c}\text { Expected Number } \\
\text { of Genetic } \\
\text { Effects } \\
\end{array}$ & $\begin{array}{l}\text { Expected } \\
\text { Number of } \\
\text { Early } \\
\text { Morbidities } \\
\end{array}$ \\
\hline$S$ & 2 & $1.4 \times 10^{-3}$ & $1.9 \times 10^{-3}$ & $2.7 \times 10^{-5}$ \\
\hline $\mathrm{s}$ & 4 & $1.4 \times 10^{-3}$ & $1.9 \times 10^{-3}$ & $4.2 \times 10^{-6}$ \\
\hline $\mathrm{s}$ & 8 & $1.4 \times 10^{-3}$ & $2.0 \times 10^{-3}$ & 0 \\
\hline w & 2 & $1.5 \times 10^{-3}$ & $2.0 \times 10^{-3}$ & $3.3 \times 10^{-5}$ \\
\hline w & 4 & $1.5 \times 10^{-3}$ & $2.1 \times 10^{-3}$ & $8.4 \times 10^{-6}$ \\
\hline W & 8 & $1.4 \times 10^{-3}$ & $1.9 \times 10^{-3}$ & 0 \\
\hline $\mathrm{N}$ & 2 & $1.5 \times 10^{-3}$ & $2.1 \times 10^{-3}$ & $2.7 \times 10^{-5}$ \\
\hline $\mathbf{N}$ & 4 & $1.6 \times 10^{-3}$ & $2.2 \times 10^{-3}$ & $4.2 \times 10^{-5}$ \\
\hline $\mathrm{N}$ & 8 & $1.6 \times 10^{-3}$ & $2.3 \times 10^{-3}$ & 0 \\
\hline E & 2 & $1.3 \times 10^{-3}$ & $1.7 \times 10^{-3}$ & $3.3 \times 10^{-5}$ \\
\hline E & 4 & $1.3 \times 10^{-3}$ & $1.8 \times 10^{-3}$ & $8.4 \times 10^{-6}$ \\
\hline $\mathbf{E}$ & 8 & $1.2 \times 10^{-3}$ & $1.7 \times 10^{-3}$ & 0 \\
\hline
\end{tabular}

Average Values

$1.4 \times 10^{-3}$

$2.0 \times 10^{-3}$

$1.2 \times 10^{-5}$

1. Calculation of the actual downwind contamination levels

2. Comparison of actual levels with desired cleanup levels

3. Selection of cleanup technique required

4. Assessment of costs based on cleanup technique selected

5. Calculation of economic risk in a parallel fashion to radiological risk.

The costs are a strong function of both the amount of material released and the desired cleanup level. Figure 3-2 shows the relationship between the direct economic impact and the amount of material released and aerosolized for the cleanup level of $0.2 \mu \mathrm{Ci} / \mathrm{m}^{2}$ currently recommended by the Environmental Protection Agency (EPA) for both long- and short-lived materials. 11 Figures $3-3$ and 3-4 show the contribution of each of the components of economic impact to the total amount for long- and short-lived materials. For smaller releases of long-lived materials, the costs of surveying the area dominate the overall costs. As the released amount increases, costs associated with street cleanup, building cleanup, and evacuation become significant. At high levels $(>100$ curies), costs associated with permanent land-use denial account for virtually all of the economic impact. For releases of short-lived material, survey, street cleanup, and building cleanup costs dominate the smaller releases. In the case of intermediate and larger releases, however, costs associated with evacuation and building cleanup account for virtually all of the cost impact. The figures display the costs for the limited New York City study area and thus are truncated at the edges of the grid. This truncation levels off some of the cost curves and is an artifact of the modeling. 


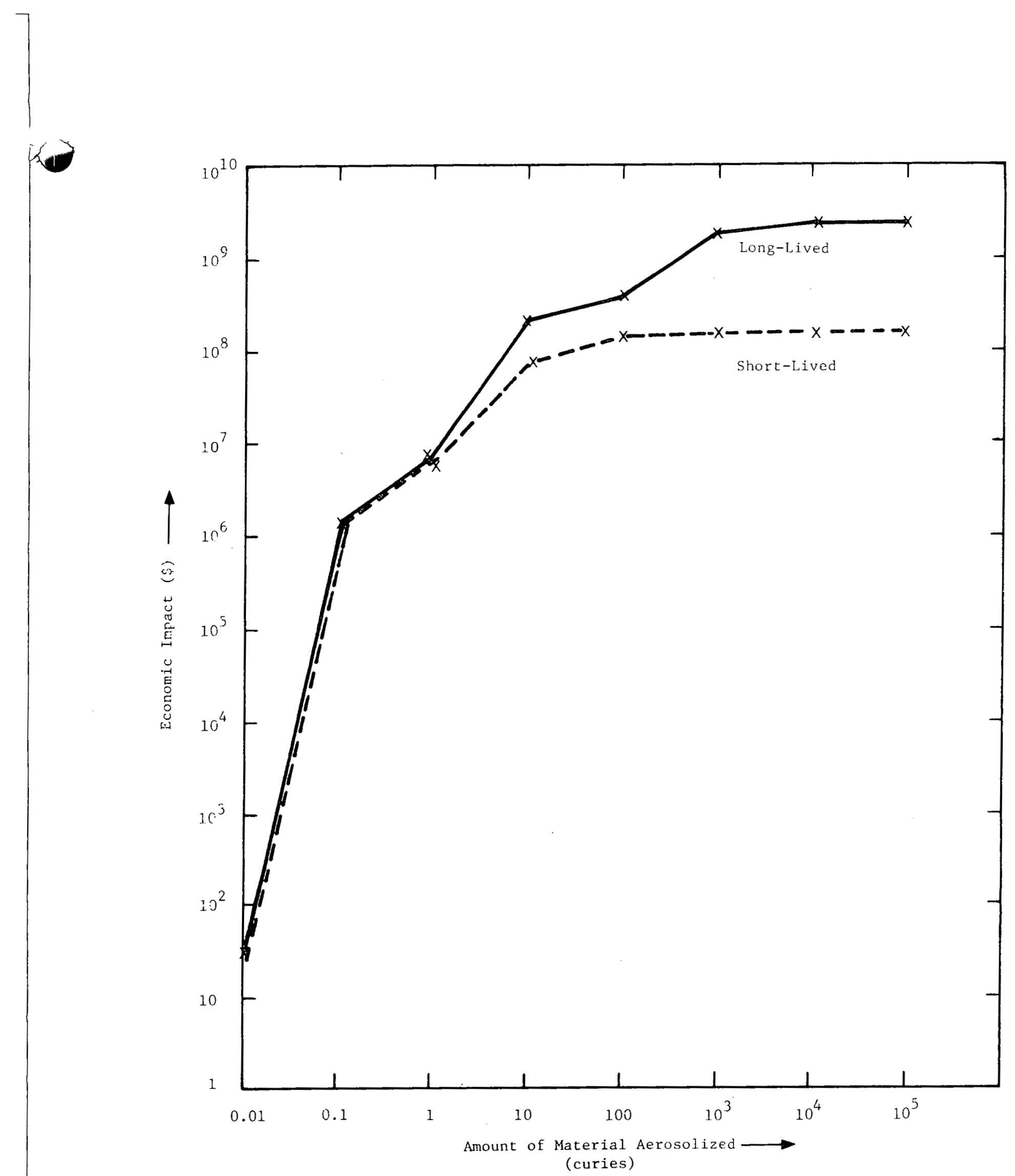

Figure 3-2. Economic Impact versus Amount of Material Aerosolized. The cleanup level is $0.2 \mathrm{\mu Ci} / \mathrm{m}^{2}$ for both long- and short-lived materials. 


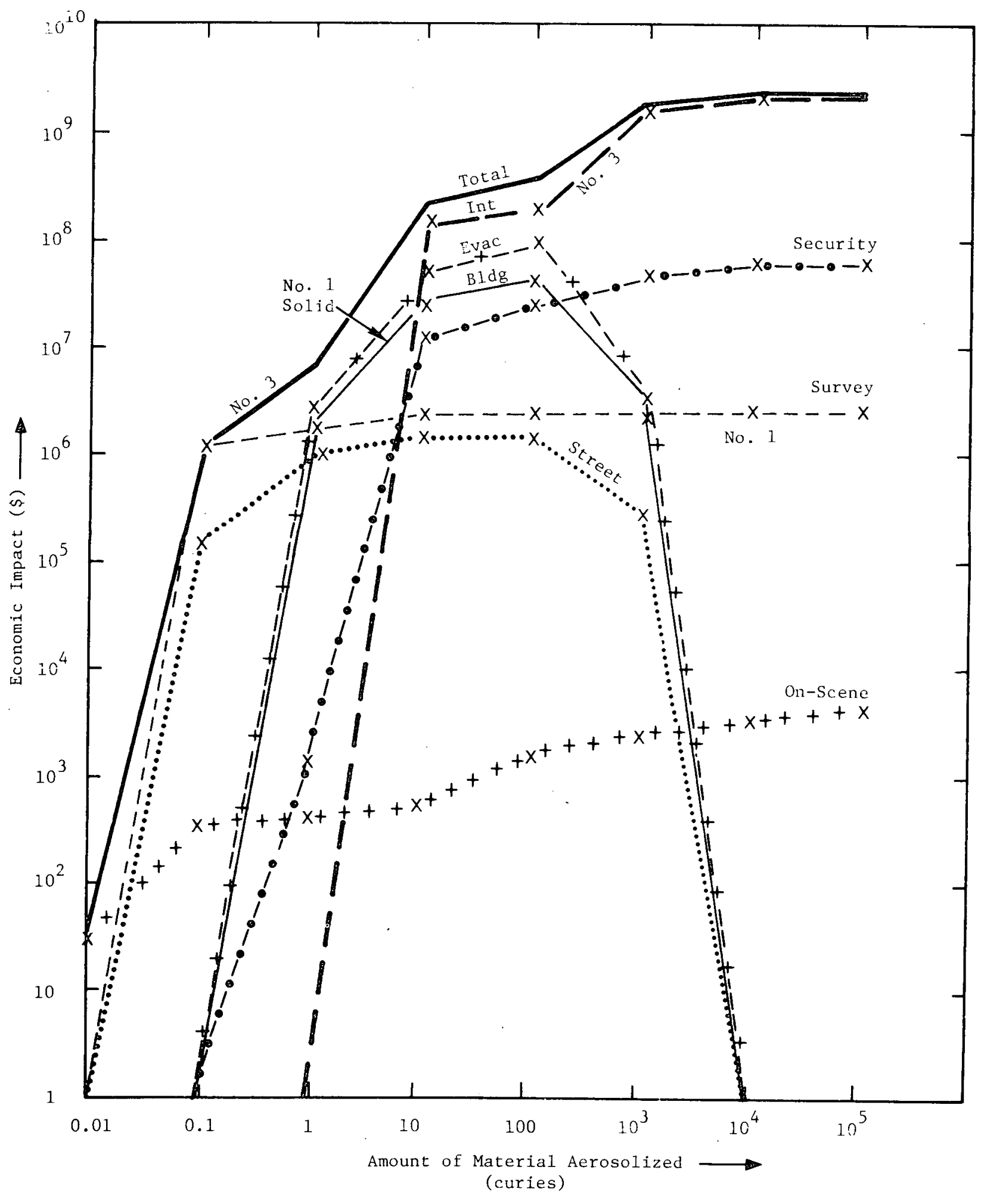

Figure 3-3. Cost Components for Urban Releases of Long-Lived Materials. The cleanup level is $0.2 \mu \mathrm{Ci} / \mathrm{m}^{2}$. 


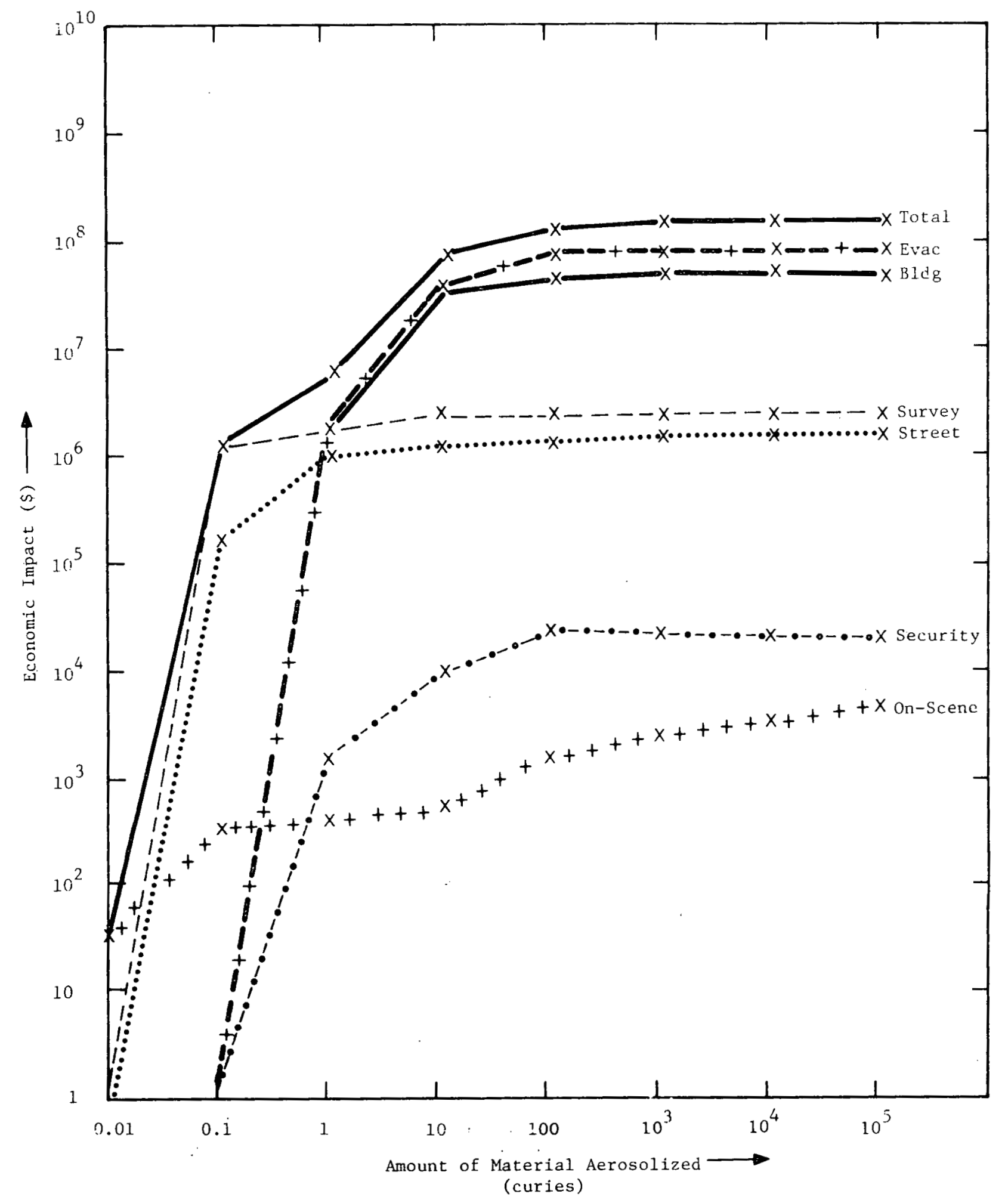

Figure 3-4. Cost Components for Urban Release of Short-Lived Materials. The cleanup level is $0.2 \mu \mathrm{Ci} / \mathrm{m}^{2}$. 
Figure 3-5 shows the relationship of cleanup level to total cost for releases of long-lived materials. For small releases, there is a very large spread in costs, reflecting the large difference between on-scene emergency response costs, which require a relatively small amount of time, and costs for survey which take a relatively large amount of time and technical expertise. The curves are roughly parallel for intermediate-level releases where costs are dominated by cleanup and evacuation. For very large releases, where land-use denial occurs and where the decontamination factors are extremely large, the costs are relatively insensitive to cleanup level because permanent land-use denial is required for all cases. The overlap between $0.2 \mu \mathrm{Ci} / \mathrm{m}^{2}$ and $0.1 \mu \mathrm{Ci} / \mathrm{m}^{2}$ is a result of being near the threshold for permanent land-use denial. In the case of the $0.2 \mu \mathrm{Ci} / \mathrm{m}^{2}$ release, the total of evacuation and building cleanup costs is slightly greater in the neighborhood of 10 curies than in the interdiction cost for the same geographical area for a cleanup level of $0.1 \mu \mathrm{Ci} / \mathrm{m}^{2}$.

As discussed in Appendix $\mathrm{K}$, the half life of a radionuclide determines, in part, the type of approach taken to the overall cleanup effort. Figure 3-6 shows the effect of cleanup level on total cost for various releases of short-1ived material. In the low and intermediate ranges, the costs are comparable to those for long-lived materials, although they are somewhat smaller. At levels greater than 10 curies, however, the costs associated with releases of short-lived materials are significantly less, principally due to the permanent land-use denial in the case of long-lived materials. This factor is omitted from the costs for short-lived materials because the approach assumed is one of "evacuate and wait for decay."

Because of the overwhelming contribution of permanent land-use denial to costs of large releases of long-lived materials, these costs are relatively insensitive to the particular urban area being studied (assuming, of course, that urban land value is relatively constant from city to city). Similarly, the dominance of survey costs for small releases means that these costs, too, will not vary much from city to city. Evacuation and building cleanup costs dominate intermediate releases, and these costs scale roughly with population density. Therefore, costs for releases of long-lived materials can be approximated for other cities knowing only population density variations.

Intermediate and large releases of short-1ived materials are dominated by evacuation and building cleanup costs so these can be approximated from city to city knowing the ratio of population densities for the various cities. Low-level release costs are dominated by survey costs, which are essentially independent of the city involved.

In evaluating these economic impacts, two things must be kept in mind. First, these are, at best, order-of-magnitude estimates. More accurate predictions would require detailed descriptions of the actual accident site, prevailing meteorology, and downwind land-use patterns rather than the more generic descriptions used in METRAN. Second, METRAN cannot quantify all economic costs since there are several indirect costs which are more a function of public response than a function of the actual contamination "footprint." Examples of these costs include costs of litigation, indirect business losses (due, for example, to fear of possible danger, even after cleanup), and actual costs to government agencies dealing with the incident. 


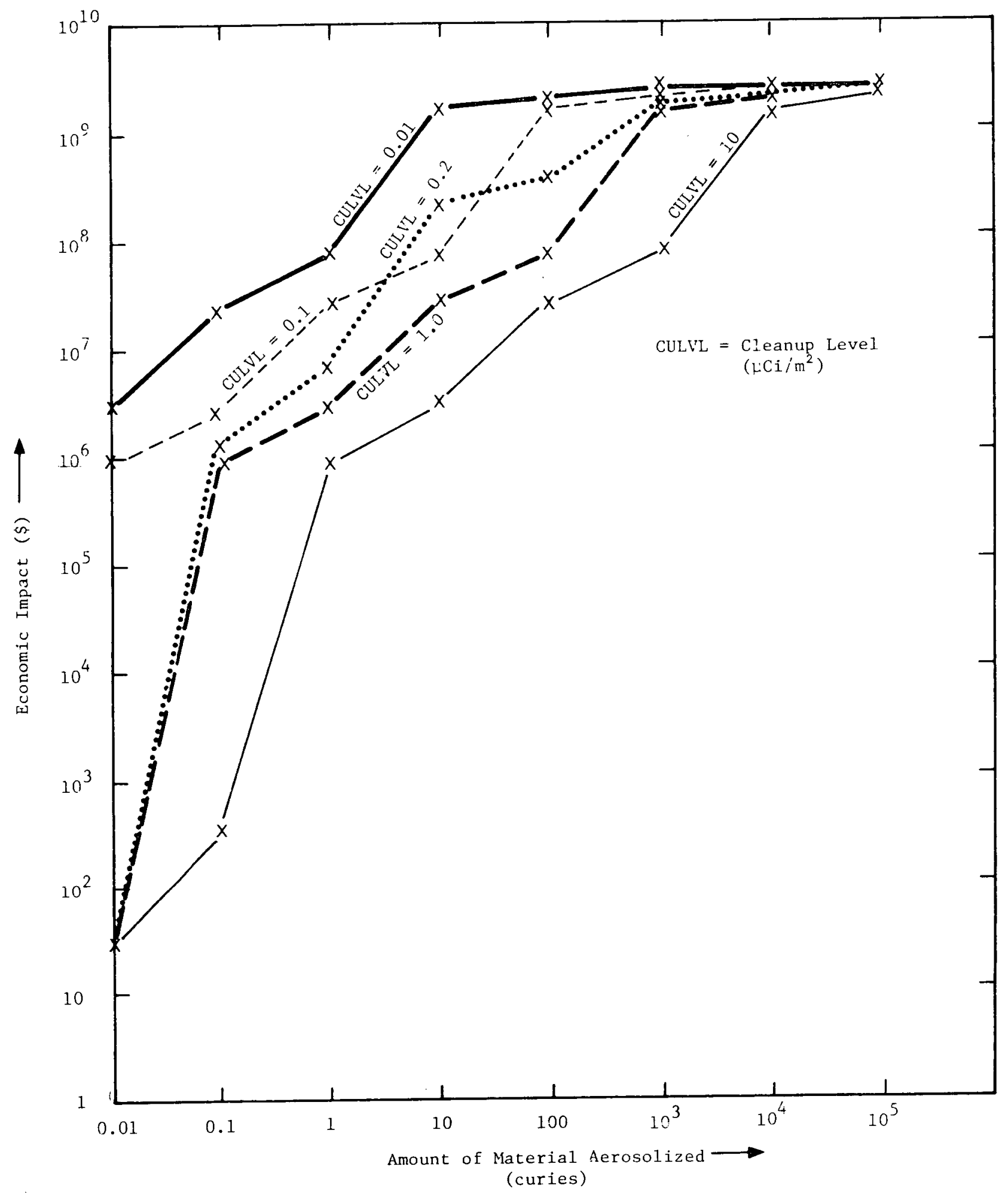

Figure 3-5. Relationship of Economic Impacts to Amount of Long-Lived Material Released for Different Cleanup Levels 


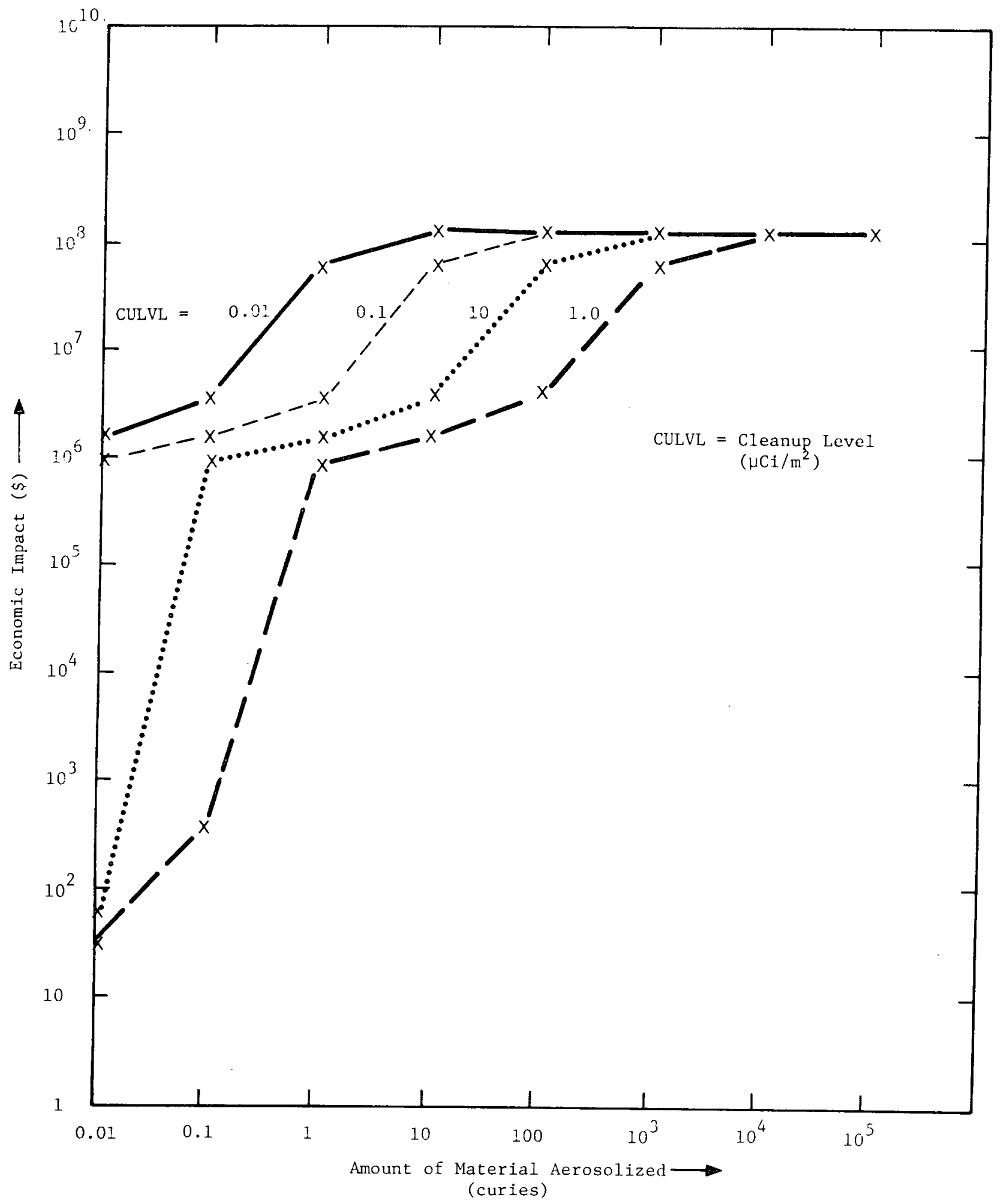

Figure 3-6. Relationship of Economic Impacts to Amount of Short-Lived Material Released for Different Cleanup Levels 
Estimation of the expected economic risks from the transport of radioactive materials for the limited New York City study area is accomplished in a parallel fashion to that for expected radiological risks, i.e., the probability that an accident of a given severity will occur is multiplied by the expected economic consequences of that accident. The severity dependent "economic risks" are then summed across all severities and shipment types to obtain an overall value for expected economic risk. Table 3-8 summarizes the economic risk calculations for the same wind conditions used elsewhere $(2 \mathrm{~m} / \mathrm{s}$, south wind). The estimated economic risk is summarized by end use, mode, and package type. The expected numbers of latent cancer fatalities are included for ease of comparison with the earlier tables in this chapter. Examination of the contributions to economic risk resulting from the dispersible nature of the shipments indicates that essentially all of the risk arises from dispersible shipments. This is reasonable since in the case of nondispersible materials, there is no requirement for survey, decontamination, etc.

Table 3-8

Expected Economic Risk Values from Vehicular Accidents

\begin{tabular}{|c|c|c|c|c|}
\hline End Use & $\begin{array}{c}\text { Expected Value } \\
\text { of Economic Risk } \\
\text { per Year of Shipping } \\
\text { Activity }(\$) \\
\end{array}$ & F & $\begin{array}{l}\text { Expected Number } \\
\text { of Latent Cancer Fatalities } \\
\text { per Year of Shipping Activity }\end{array}$ & $\underline{F}$ \\
\hline Medical & $1.4 \times 10^{6}$ & 0.97 & $5.3 \times 10^{-4}$ & 0.39 \\
\hline Industrial & $1.9 \times 10^{4}$ & 0.01 & $2.3 \times 10^{-4}$ & 0.17 \\
\hline Fuel Cycle & $3.1 \times 10^{4}$ & 0.02 & $6.1 \times 10^{-4}$ & 0.44 \\
\hline Waste & $5.1 \times 10^{0}$ & $<0.001$ & $8.2 \times 10^{-7}$ & $<0.001$ \\
\hline \multicolumn{5}{|l|}{ Mode } \\
\hline Truck & $6.4 \times 10^{4}$ & 0.04 & $7.2 \times 10^{-4}$ & 0.53 \\
\hline Air & $1.0 \times 10^{1}$ & $<0.001$ & $9.7 \times 10^{-9}$ & $<0.001$ \\
\hline Air and Truck & $1.4 \times 10^{6}$ & 0.96 & $6.5 \times 10^{-4}$ & 0.47 \\
\hline Barge & $1.3 \times 10^{0}$ & $<0.001$ & $3.2 \times 10^{-8}$ & $<0.001$ \\
\hline \multicolumn{5}{|l|}{ Package Type } \\
\hline A & $1.4 \times 10^{6}$ & 0.94 & $5.8 \times 10^{-4}$ & 0.41 \\
\hline B & $6.2 \times 10^{4}$ & 0.04 & $2.2 \times 10^{-4}$ & 0.16 \\
\hline Cask & $3.1 \times 10^{4}$ & 0.02 & $6.1 \times 10^{-4}$ & 0.43 \\
\hline Drum & $4.1 \times 10^{0}$ & $<0.001$ & $8.9 \times 10^{-7}$ & $<0.001$ \\
\hline Totals & $\sim 1.5 \times 10^{6}$ & & $1.4 \times 10^{-3}$ & \\
\hline
\end{tabular}


Table 3-9 examines the effect of wind speed on estimated economic risks and latent cancer fatalities for three wind speeds and four directions. The small variation observed in estimates of the economic risk are consistent with those observed for expected numbers of latent cancer fatalities, indicating that wind speed and direction are not significant factors in the determination of economic risk.

Table 3-9

Estimates of Economic Risk Values as a Function of Wind Speed for Vehicular Accidents

\begin{tabular}{cccc}
$\begin{array}{c}\text { Wind Speed } \\
(\mathrm{m} / \mathrm{s})\end{array}$ & $\begin{array}{c}\text { Wind } \\
\text { Direction }\end{array}$ & $\begin{array}{c}\text { Estimated } \\
\text { Economic } \\
\text { Risk }(\$)\end{array}$ & $\begin{array}{c}\text { Expected Number } \\
\text { of Latent Cancer } \\
\text { Fatalities }\end{array}$ \\
\hline 2 & $\mathrm{~S}$ & $1.5 \times 10^{6}$ & $1.4 \times 10^{-3}$ \\
4 & $\mathrm{~S}$ & $1.5 \times 10^{6}$ & $1.4 \times 10^{-3}$ \\
8 & $\mathrm{~S}$ & $1.2 \times 10^{6}$ & $1.4 \times 10^{-3}$ \\
2 & $\mathrm{~W}$ & $\sim 1.5 \times 10^{6}$ & $1.5 \times 10^{-3}$ \\
4 & $\mathrm{~W}$ & $1.4 \times 10^{6}$ & $1.5 \times 10^{-3}$ \\
8 & $\mathrm{~W}$ & $9.9 \times 10^{5}$ & $1.4 \times 10^{-3}$ \\
2 & $\mathrm{~N}$ & $1.4 \times 10^{6}$ & $1.5 \times 10^{-3}$ \\
4 & $\mathrm{~N}$ & $1.5 \times 10^{6}$ & $1.6 \times 10^{-3}$ \\
8 & $\mathrm{~N}$ & $1.1 \times 10^{6}$ & $1.6 \times 10^{-3}$ \\
2 & $\mathrm{E}$ & $1.6 \times 10^{6}$ & $1.3 \times 10^{-3}$ \\
4 & $\mathrm{E}$ & $1.8 \times 10^{6}$ & $1.3 \times 10^{-3}$ \\
8 & $\mathrm{E}$ & $9.5 \times 10^{5}$ & $1.2 \times 10^{-3}$
\end{tabular}

\subsection{Low-Probability/High-Consequence Accidents}

Quantification of risk using the product of probability and consequence is only one form of risk analysis used in decision making. In dealing with potentially high-consequence but low-probability events, an approach called "mini-max" is also useful.7 This technique involves the calculation of the consequences of certain events separate from their probability, keeping in mind that at some point the consequences will be intolerably severe, even at an extremely low probability. This section considers the consequences of high-level releases that might occur. Several shipments were selected from the actual New York City shipment model specified in Appendix A. However, since these shipments were averaged to some degree, other potentially high-consequence shipments were 
added. These additional shipments were selected from the Battelle survey ${ }^{8}$ and various other sources, and represent shipments that could conceivably have an urban destination or origin, or that could pass through an urban area. The shipments selected for this mini-max analysis are specified in Table 3-10. The first three entries are standard shipments evaluated in the risk analysis; the remainder are additions from the Battelle survey.

Table 3-10

Shipments Used for Mini-Max Risk Analysis

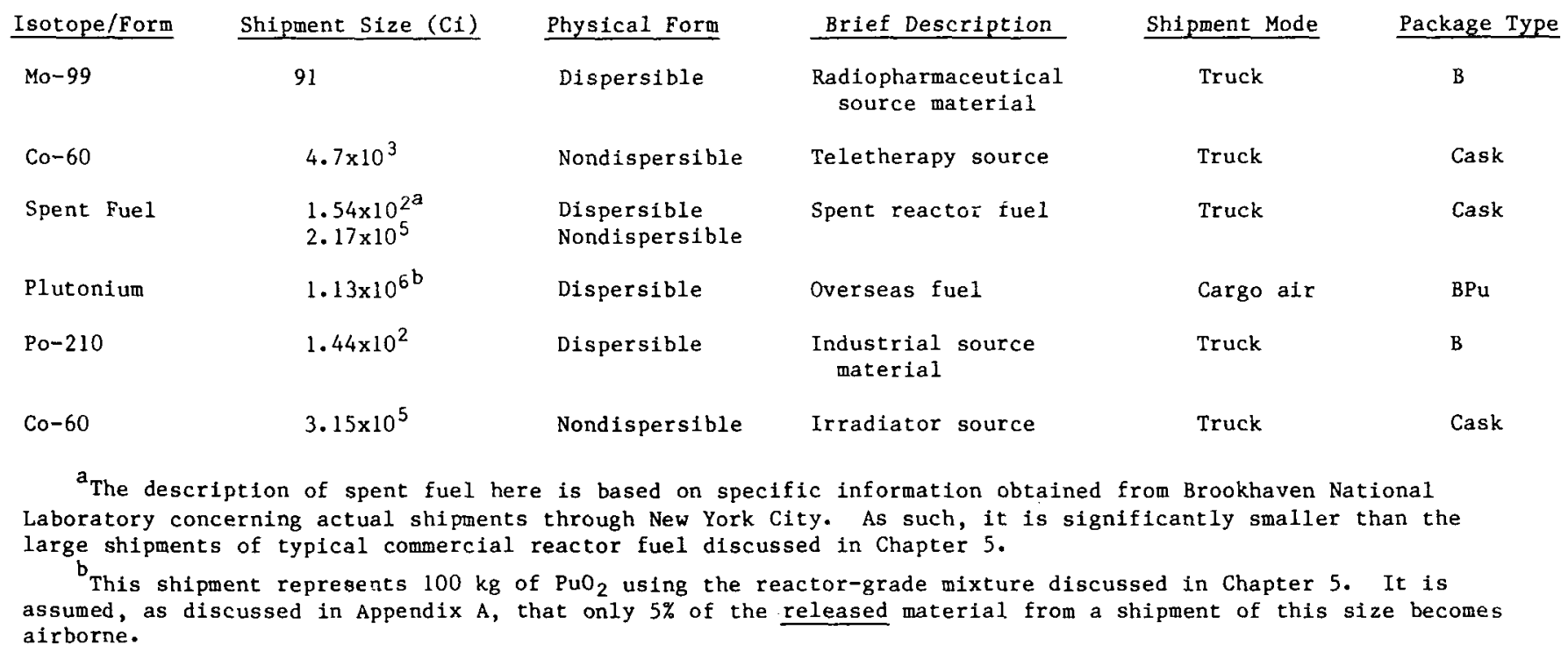

The consequence analysis has been performed using the METRAN code to evaluate the effects of maximum severity accidents. Results for latent cancer fatalities, early morbidities, early fatalities, and economic costs are presented in Table 3-11.

\subsection{Sensitivity and Error Analysis}

Techniques similar to those described in Chapter 2 for incident-free transport were applied to the nondispersal accident case since the two problems are similarly structured. In incident-free transport the source is moving, whereas in the nondispersal accident case, the shipment is stationary for an accident delay time, $\Delta \mathrm{T}_{\mathrm{a}} \cdot$ Because of the similarity, it was possible to generate simpler equations than those in METRAN to "mimic" the model. These equations and their derivation are discussed in detail in Appendix D. Briefly, the equations for dose to pedestrians involve street width, pedestrian density, and accident delay time. For people in vehicles, there are three equations for different road types which consider street (or freeway) width, traffic count, people per vehicle, photon energy (only for freeways) and accident delay time. For people in buildings, there are equations for different road types involving population density, transient population, street width (or freeway width), fraction of the cell which is buildings, sidewalk width, and accident delay time. Error equations were also developed and are presented in detail in Appendix D. 
Table 3-11

Results of Low-Probability/High-Consequence Accident Analysis ${ }^{a}$ b c

\begin{tabular}{|c|c|c|c|c|c|}
\hline Isotope & $\begin{array}{l}\text { Latent Cancer } \\
\text { Fatalities } \\
\end{array}$ & $\begin{array}{c}\text { Early } \\
\text { Morbidities } \\
\end{array}$ & $\begin{array}{c}\text { Early } \\
\text { Fatalities }\end{array}$ & $\begin{array}{l}\text { Direct Economic } \\
\text { Impact }(\$) \\
\end{array}$ & Comments \\
\hline $\begin{array}{l}\text { Mo-99 } \\
\quad(91 \mathrm{Ci})\end{array}$ & 0.05 & 0 & 0 & $2 \times 10^{8}$ & $\begin{array}{ll}\text { On-scene costs: } \quad<0.1 \% \\
\text { Survey costs: } \sim 4 \% \\
\text { Street cleanup: } \quad 0.6 \% \\
\text { Building cleanup: } \sim 0.18 \% \\
\text { Evacuation costs: } \sim 77 \% \\
\text { Security costs: } \sim 18 \%\end{array}$ \\
\hline $\begin{array}{l}\mathrm{Co}-60 \\
\left(4.7 \times 10^{3} \mathrm{Ci}\right)^{\mathrm{d}}\end{array}$ & 0.01 & 0 & 0 & $4 \times 10^{4}$ & $\begin{array}{l}\text { Emergency response: } \quad 20 \% \\
\text { On-scene recovery: }\end{array}$ \\
\hline $\begin{array}{l}\text { Spent Fue } 1 \\
\qquad\left(154 / 2.17 \times 10^{5} \mathrm{Ci}\right)^{\mathrm{e}}\end{array}$ & 1 & 0 & 0 & $2 \times 10^{9}$ & $\begin{array}{l}\text { On-scene costs: }<0.1 \% \\
\text { Survey costs: } 0.7 \% \\
\text { Street cleanup: }<0.1 \% \\
\text { Building cleanup: } 0.7 \% \\
\text { Evacuation costs: } \quad \sim 1 \% \\
\text { Security costs: } \quad 2 \% \\
\text { Land-use denial: } \quad 95 \%\end{array}$ \\
\hline $\begin{array}{l}\text { P1utonium } \\
\qquad\left(1.13 \times 10^{6} \mathrm{Ci}\right)^{\mathrm{f}}\end{array}$ & 1800 & 290 & 5 & $2 \times 10^{9}$ & $\begin{array}{l}\text { On-scene costs: }<0.1 \% \\
\text { Survey costs: } 0.5 \% \\
\text { Street cleanup: } 0 \\
\text { Building cleanup: } 0 \\
\text { Evacuation: } 0 \\
\text { Security costs: } \quad 4 \% \\
\text { Land-use denial: } \quad 95 \%\end{array}$ \\
\hline $\begin{array}{l}\text { Po-210 } \\
\quad(144 \mathrm{C} 1)\end{array}$ & 10 & 60 & 3 & $9 \times 10^{9}$ & $\begin{array}{l}\text { On-scene costs: }<0.1 \% \\
\text { Survey costs: } 0.1 \% \\
\text { Street cleanup: }<0.1 \% \\
\text { Building cleanup: } 0.5 \% \\
\text { Evacuation: } \sim 100 \% \\
\text { Security costs: }<0.1 \%\end{array}$ \\
\hline $\begin{array}{l}\mathrm{Co}-60 \\
\left(3.15 \times 10^{5} \mathrm{C} 1\right)^{\mathrm{d}}\end{array}$ & 3 & 0 & 0 & $4 \times 10^{4}$ & $\begin{array}{l}\text { Emergency response: } \\
\text { On-scene recovery: }\end{array}$ \\
\hline
\end{tabular}

aelease fractions for maximum credible accidents are assumed to be the maximum value of release fraction for the accident analysis, i.e., package for Types A, B, drum, and cask release; release fraction = 1 ; for $B P$, release fraction $=0.1$, for exposure source casks, release fraction $=3.1 \times 10^{-3}$.

brobability of occurrence of these accidents can be estimated by multiplying the accident rate (No. of accidents $/ \mathrm{km}$ of travel) times the distance traveled in the cell $(\mathrm{km})$ times the fraction of all accidents occurring in a densely populated urban area that result in the maximum release. ${ }^{1}$ The distance traveled in the cell is taken to be $1 \mathrm{~km}$. For the materials in this table, the probabilities are as follows:

Probability of Occurrence per Shipment

for a Single cell of Travel

$\begin{array}{lll}\text { Mo-99 } & \text { Type B Package } & 2 \times 10^{-11} \\ \text { Co-60 (both shipments) } & \text { Cask -- exposure } & 8 \times 10^{-14} \\ \text { Spent Fuel } & \text { Cask -- release } & 2 \times 10^{-11} \\ \text { Spent Fue1 } & \text { Cask -- exposure } & 8 \times 10^{-14} \\ \text { Po-210 } & \text { Type B Package } & 2 \times 10^{-11} \\ \text { Plutonium } & \text { Type BPu Package } & 2 \times 10^{-12}\end{array}$

Using this technfque, the probability of occurrence of the most severe accident for material transported in Type A or LSA packages is $3 \times 10^{-9}$.

\footnotetext{
${ }^{c}$ Values in table represent effects weighted for midblock and intersection locations for accidents.

dspecial form (nondispersible).

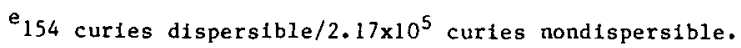

$\mathrm{f}_{10 \%}$ released; $5 \%$ of released material is aerosolized.
} 
For the dispersal accident case, the situation is quite different. Because of the complicating meteorological factors, it has not been possible to produce simplifying equations as in the other cases. Treatment of the dispersal accident involved parameters common to the meteorological models MICMET and PICMET (see Appendix E) and to METRAN, as well as those unique to the consequence calculations. Output variables examined have been latent cancer fatalities (from groundshine and inhalation pathways -- the others, e.g., cloudshine, were found to be several orders of magnitude less significant), genetic effects (as in latent cancer fatalities), early fatalities, and early morbidities. The variables examined are not expected values of radiological risk but simply the consequences resulting from the most severe accident postulated. Simplifying assumptions were used to decrease the total number of input variables to be considered. The nature of the assumptions are detailed in Appendix $D$. When the reduction process was complete, the input variables remaining to be considered are street width, sidewalk width, fraction of cell area occupied by buildings, total curies per package, cleanup level, as well as the meteorological data, which included street and sidewalk widths, wind speed, and fraction of material striking the lowest boundary which is reflected (or absorbed). An added complication in this portion of the analysis is that the building intake system can also be varied, i.e., continuous building intake or an air-conditioning system can be assumed.

Taking all of these factors into account and varying the input parameters over reasonable ranges, it is possible to rank order the importance of these to each of the output variables. The details of this rank ordering are in Appendix $D$ of this report.

\subsection{Nonradiological Impacts from Transport in Exclusive-Use Vehicles}

Because radioactive materials are a negligible fraction of the total shipments of all commercial cargo, the only nonradiological impacts which can be attributed to radioactive material shipments are those which result from shipments in exclusive-use vehicles. To consider the nonradiological risks in transportation accidents for exclusive-use vehicles, previously developed methods are used. Data from Reference 1 provide accident information for fuel cycle shipments. Shipments from only one reactor passed through the grid during the 1975 survey period used elsewhere in this study. Values of 0.03 injury and 0.003 fatality are obtained for exclusive-use shipments of fuel cycle material. 1

The other significant use of exclusive-use trucks is in the shipment of molybdenum-99/technetium-99m generators. Reference 1 assumes that $10 \%$ of the generators are transported by exclusive-use trucks. For the New York City study area, this would imply an average TI quantity of 1.2 TI per shipment and a total of $2.83 \times 10^{2}$ shipments per year (see Appendix A -- Routing Information) carried by exclusive-use vehicles. The maximum distance traveled in the grid by one of these shipments is $12 \mathrm{~km}$ (Appendix A). As a conservative estimate, the upper bound of the total exclusive-use vehicle travel in the grid would be $3.48 \times 10^{3}$ $\mathrm{km} / \mathrm{yr}$. Comparing this distance to data in Reference 1 leads to values of 0.002 injury and about 0.0001 fatality per year.

Similarly for cargo airlines, the assumption is made that routine flights are made primarily for Mo-99/Tc-99m generators. For the New York City study area, a total of $1.16 \times 10^{3}$ air freight shipments of Mo-99/Tc-99m are made. Assuming, again, that $10 \%$ of all air cargo shipments are exclusive-use shipments gives a 
total of $1.16 \times 10^{2}$ shipments/yr by dedicated carrier. The average air freight shipment distance traveled in the study area is $10 \mathrm{~km}$; thus the total distance traveled is $1.16 \times 10^{3} \mathrm{~km} / \mathrm{yr}$. Using the average accident rate of $1.44 \times 10^{-8}$ accident $/ \mathrm{km}$, these flights would be expected to result in about $1.7 \times 10^{-7}$ accident/ year. 1 Assuming a crew of two, a value of $3.4 \times 10^{-7}$ fatality per year would be expected.

Summarizing, nonradiological impacts resulting from exclusive-use transport vehicles for radioactive materials shipments would be approximately 0.03 injury and 0.003 fatality per shipment year for the limited New York City area.

\subsection{Conclusions}

The overall expected annual radiological risks due to vehicular accidents in the limited New York City study area are quite small $\left(1.4 \times 10^{-3}\right.$ latent cancer fatality, $1.9 \times 10^{-3}$ genetic effect, $2.7 \times 10^{-5}$ early morbidity). The risk is dominated by Type A packages of medical-use isotopes and spent fuel shipments by truck. The largest contributor to the overall risk is the groundshine dose received by the population exposed to dispersed material which is deposited during cloud passage and remains on the ground. Dispersible materials contribute approximately $80 \%$ of the overall risk, while large nondispersible shipments contribute most of the remainder. Economic risk from the shipments is dominated by medical-use shipments in Type A packages transported at least in part by truck. For very severe accidents, the economic impacts could be up to several billion dollars, although most accidents would be less costly, especially those involving short-lived radioisotopes used for medical purposes. Extremely severe accidents have the potential for causing significant numbers (tens to thousands) of latent effects and lesser numbers (ones to hundreds) of early effects. Since these accidents have very low probabilities of occurrence, they do not contribute significantly to the total expected values of risk. Sensitivity analysis results provide rank ordering of important variables for dispersal accidents and simplifying equations for nondispersal accidents. Thus, the parameters important for the use of the METRAN methodology in other cities can be identified. 
${ }^{1}$ NUREG-0170.

2J. M. Taylor and S. L. Daniel, RADTRAN: A Computer Code to Analyze Transportation of Radioactive Material, SAND76-0243, Albuquerque: Sandia Laboratories, April 1977.

${ }^{3}$ R. Yoshimura and M. Huerta, A Crash Test of a Nuclear Spent Fuel Cask and Truck Transport System, SAND77-0419, Albuquerque: Sandia Laboratories, January 1978.

${ }^{4}$ W. E. Berry and R. B. Diegle, Survey of Corrosion Product Generation, Transport, and Deposition in Light Water Nuclear Reactors, Final Report, EPRI NP-522, TPS 76-663, Palo Alto, CA: Electric Power Research Institute, March 1979 .

${ }^{5}$ G. E. Zima, Comments on Fuel Crud as a Safety and Operational Factor of Independent Spent Fuel Storage Installations (IS-FSI), NUREG/CR-0163, PNL-2657, Richland, WA: Battelle Pacific Northwest Laboratories, November 1978.

${ }^{6}$ K. J. Eger and G. E. Zima, Commentary on Spent Fuel Storage at Morris Operation, NUREG/CR-0956, PNL-3065, Richland, WA: Battelle Pacific Northwest Laboratories, July 1979; and personal communication with K. Eger, 25 June 1979.

7J. R. Benjamin, M. Shinozuka, and H. C. Shah, "Acceptable Risk in Reactor Safety Studies," in Proceedings of the 3rd International Conference on Structural Mechanics in Reactor Technology, September 1975 .

$8_{\mathrm{BNWL}}-1972$.

${ }^{9}$ I. B. Wall et al, Overview of Reactor Safety Study Consequence Model, NUREG-0340, Washington: U.S. Nuclear Regulatory Commission, 1977; Reactor Safety Study Consequence Model CRAC, Computer Code Users Manual, available from Argonne Code Center, Argonne National Laboratory, IL.

${ }^{10}$ A. R. DuCharme et al, Transport of Radionuclides in Urban Environs: Working Draft Assessment, SAND77-1927, Albuquerque: Sandia Laboratories, May 1978.

${ }^{11}$ U.S. Environmental Protection Agency, Proposed Guidance on Dose Limits for Persons Exposed to Transuranium Elements in the General Environment, EPA 520/4-77-016, Washington: USEPA, September 1977.

$$
12 \text { NUREG-0069. }
$$




\author{
ENVIRONMENTAL IMPACTS FROM HUMAN ERRORS AND \\ DEVIATIONS FROM ACCEPTED QUALITY ASSURANCE PRACTICES
}

\begin{abstract}
Human errors and deviations from accepted quality assurance (QA) practices can produce environmental impacts similar to those produced by vehicular accidents, i.e., through loss of shielding, loss of containment, or through delay of the shipment. The detailed incidents selected for analysis in this chapter are those specifically related to the transport process and include problems in packaging, labeling, handling and stowage of the radioactive material. Human errors which result in vehicular accidents are treated as such (Chapter 3). Deviations from accepted QA practices include both failure to adhere to normal procedure and lack of quality control.
\end{abstract}

Records of actual incidents involving radioactive material transport in urban areas reported to governmental agencies were analyzed to estimate the probability of occurrence of an incident on a per-shipment basis. Since quality assurance practices vary depending on the package type (e.g., Type A packages are controlled differently from spent fuel casks), the probability of occurrence of an incident is estimated as a function of package type. A separate analysis estimates the probability of occurrence of an incident involving a spent fuel cask since there have been no reports filed with the appropriate agencies for this shipment type.

Package-dependent incident probabilities are used in the radiological consequence code METRAN, operating in a special mode, to estimate the contribution of human errox or QA deviations to the risk of transporting radioactive materials in urban areas. The definition of estimated value of radiological risk, given in the introduction of Chapter 3, applies here also.

From a systems point of view, human error occurs when there is a reduction or potential reduction in system reliability or safety, e.g., failure to perform the necessary task, performance of a required task out of sequence, or inaccurate marking of the transport index on the package.

\title{
4.1 Transportation
}

Radioactive materials are not unique in the complexity of the transport process. Operations specifically related to transportation in which human errors could occur include packaging and labeling of the shipment; temporary stowage of packages, handling, securing, stowing, and routing operations prior to initial movement of the material; in-transit transfers; and movements of the shipment by the receiver to its final destination. Incident reports, examined in this analysis, describe several of the previously listed error types and form the basis for determination of occurrence rates for human errors and deviations from accepted QA practices as a function of package type. 


\subsection{Methodology for Risk Assessment}

Risk from human errors and deviations from accepted QA practices are expressed as expected health effects as a function of the type of package in which the material is shipped. In general terms, the risk may be formulated as follows:

$$
R=\sum_{i=1}^{a} \sum_{j=1}^{b} \sum_{k=1}^{c} N_{j, k} \cdot \operatorname{PPS}_{j, k} \cdot R F_{i, j} \cdot k_{k} \cdot S P Y_{j, k} \cdot C_{i, j}
$$

where

$$
\begin{aligned}
& R=\text { total annual risk from human errors and deviations from } \\
& \text { QA practices (expected number of human health effects) } \\
& i=\text { index over severity categories } \\
& a=\text { number of severity categories }(=3 \text { for casks; }=8 \text { for } \\
& \text { all other package types) } \\
& \mathrm{j}=\text { index over package types } \\
& b=\text { number of package types } \\
& \mathrm{k}=\text { index over materials } \\
& c=\text { number of materials } \\
& \begin{aligned}
& N_{j, k}= \text { curies per package for } k t h \text { material shipped in } j \text { th } \\
& \text { package type }
\end{aligned} \\
& \text { PPS }_{j, k}=\begin{array}{l}
\text { packages per shipment for } k \text { th material shipped in } j \text { th } \\
\text { package type }
\end{array} \\
& \mathrm{RF}_{i, j}=\begin{array}{l}
\text { release fraction for } j \text { th package in accident of } i t h \\
\text { severity }
\end{array} \\
& K_{k}=\text { health effects conversion factor for } k \text { th material } \\
& \text { (expected health effects per curie released or exposed) } \\
& \text { SPY }_{j, k}=\text { shipments per year of } k t h \text { material in } j \text { th package type } \\
& C_{i, j}=\text { incident rate for ith severity incident involving } j \text { th }
\end{aligned}
$$

Severity-dependent fractional occurrences for human errors were developed from the data provided in the DOT and NRC incident reports and are reflected in Table 4-5. Release fractions consistent with the accident analysis are used for package Types A, B, LSA, and drum. A separate section of the chapter is devoted to fractional occurrences, release fractions, and incident rates for cask transport. 
Sources of information on the number and type of incidents involving radioactive material shipments have been developed with the assistance of the Task Group on Transportation of Radioactive Material in Urban Environs. Several members supplied contacts within their own organizations or have suggested individuals, agencies, or groups that led to relevant information.

Unfortunately, most potential data sources have not maintained records that could readily be applied to this study. Frequently, applicable data could only be extracted from the actual reports of investigations made by the regulatory agencies. Regulations require that a detailed incident report be submitted to the Department of Transportation (DOT) within 15 days if death, injury, fire, breakage, spillage, or suspected radioactive contamination occurs as a result of transportation of radioactive materials. ${ }^{1}$ Similar reports must be filed with the NRC for any instance in which there is substantial reduction in the effectiveness of any authorized packaging during use. ${ }^{2}$ If a local (city, county, state) surveillance agency exists, that agency will usually make and file a report of an incident investigation. False alarms or insignificant events are rarely reported to the federal level but do remain a matter of record at the local level for short periods of time. Reports of incidents thought to be newsworthy are also generally filed and thus made a part of the record.

\subsubsection{DOT Incident Reports}

DOT reports on incidents involving transportation of radioactive materials in urban areas are available for the period 1 January $197 \mathrm{I}$ through 3 August 1977. These investigative reports, which describe the events as reported at the time of the incident, are summarized in Appendix $\mathrm{H}$. Of the 251 incidents for that period, only the 153 occurring in urban areas are included. Other information derived from the detailed reports, such as the probable cause of the incidents and transport mode affected, are summarized in Table 4-1. Human errors or deviations from accepted QA practices were found to affect 141 of the total 153 incidents. Incidents were about equally divided among air and surface modes of transport.*

The probable causes of the incidents studied include the following:

- Stowage -- Shipments are blown off vehicles, crushed by following vehicles, run over by forklifts, damaged by other freight, fall from vehicles, or suffer water damage as a result of insecure or ineffective placement on a vehicle or within a terminal area.

- Handling -- When dropped or punctured, shipments lose package integrity through damage to internal containers or external packaging material.

- Packaging -- Shipments lose integrity by failure of external containers, omission of internal padding, defective valve closures, corrosion, improper packaging, welding failures, or drum rupture.

- Theft/Loss -- Radioactive materials are stolen or misdirected in shipment.

* The events charged to air shipments usually occur as a result of actions performed during ground operations before or after flight (a package falls off a loading dock, faulty tiedowns, etc.). 
- Disposal -- A damaged radioactive material container is discarded in an unauthorized fashion.

- Labeling -- Improper label or radiation level is given on package.

As shown by Table 4-1, stowage, handling, and packaging account for the bulk of the human error incidents. Traffic accidents are not considered, and theft is considered a purposeful act rather than a human error. This set was further reduced to include only those for 1975 before the incident rates were calculated.

Table 4-1

Department of Transportation Investigative Reports on Radioactive Material Incidents in Urban Areas -- 1971-1977

\begin{tabular}{|c|c|c|c|c|c|}
\hline Incident Cause & $\begin{array}{l}\text { No. of } \\
\text { Reports }\end{array}$ & $\begin{array}{l}\text { Percent } \\
\text { of Total }\end{array}$ & Human & $\begin{array}{l}\text { Error/Deviations } \\
\text { from QA }\end{array}$ & $\begin{array}{l}\text { Percent } \\
\text { of Total }\end{array}$ \\
\hline Stowage & 51 & 33.3 & & 51 & 36.2 \\
\hline Handling & 39 & 25.5 & & 39 & 27.6 \\
\hline Packaging & 50 & 32.7 & & 50 & 35.5 \\
\hline Theft/Loss & 4 & 2.6 & & & \\
\hline Unknown & 9 & 5.9 & & 1.0 & 0.7 \\
\hline TOTAL & 153 & & & 141 & \\
\hline Transport Mode & $\begin{array}{l}\text { No. of } \\
\text { Reports } \\
\end{array}$ & $\begin{array}{l}\text { Percent } \\
\text { of Total }\end{array}$ & & & \\
\hline Air & 78 & 51.0 & & & \\
\hline Road & 72 & 47.1 & & & \\
\hline Train & 2 & 1.3 & & & \\
\hline Water & 1 & 0.6 & & & \\
\hline TOTAL & 153 & & & & \\
\hline
\end{tabular}

\subsubsection{NRC Incident Reports}

Transportation incident reports for 1975 were provided by the NRC from its five regional offices. Reports pertinent to urban areas are synopsized in Appendix $I$. As summarized in Table 4-2, 8 of the 19 incidents contained in the NRC files which occurred in urban areas (excluding those also reported by DOT) can be attributed to human errors. As in the case of the incidents reported to the DOT, packaging, handling, and stowage account for the majority of human errors or deviations from accepted QA practices. 
Additional information was obtained from the NRC for incidents reported by its agreement states for the period July 1976-July 1977. These reports are summarized in Table 4-3. Of the 23 incidents related to transportation, 7 involved human errors of the types in the other incident reports.

Table 4-2

Nuclear Regulatory Commission Regional Office Reports of Transportation-Related Radioactive Material Incidents in Urban Areas, 1975

\begin{tabular}{|c|c|c|c|c|c|}
\hline Incident & Cause & $\begin{array}{r}\text { No. of } \\
\text { Reports } \\
\end{array}$ & $\begin{array}{l}\text { Percent } \\
\text { of Total }\end{array}$ & $\begin{array}{c}\text { Human Error/ } \\
\text { Deviations } \\
\text { from QA } \\
\end{array}$ & $\begin{array}{l}\text { Percent } \\
\text { of Total }\end{array}$ \\
\hline Stowage & & 2 & 10.5 & 2 & 25.0 \\
\hline Handling & & 2 & 10.5 & 2 & 25.0 \\
\hline Packaging & & 3 & 15.8 & 3 & 37.5 \\
\hline Procedure & & 1 & 5.3 & 1 & 12.5 \\
\hline Theft/Loss & & 4 & 21.1 & - & - \\
\hline Unknown* & & $\underline{7}$ & 36.8 & $=$ & - \\
\hline TOTAL & & 19 & & 8 & \\
\hline
\end{tabular}

Table 4-3

Nuclear Regulatory Commission Agreement States Reports on Incidents Related to Urban Transportation of Radioactive Materials 1976-1977

\begin{tabular}{|c|c|c|c|c|}
\hline Incident Cause & $\begin{array}{l}\text { No. of } \\
\text { Reports }\end{array}$ & $\begin{array}{l}\text { Percent } \\
\text { of Total }\end{array}$ & $\begin{array}{c}\text { HumanError/ } \\
\text { Deviations } \\
\text { from QA } \\
\end{array}$ & $\begin{array}{l}\text { Percent } \\
\text { of Total }\end{array}$ \\
\hline Stowage & 2 & 8.7 & 2 & 28.6 \\
\hline Handling & 4 & 17.4 & 4 & 57.1 \\
\hline Procedure & 1 & 4.4 & 1 & $14 \cdot 3$ \\
\hline Theft/Loss & 9 & 39.1 & - & - \\
\hline $\begin{array}{l}\text { Equipment } \\
\text { Failure }\end{array}$ & 1 & 4.4 & - & - \\
\hline Unknown & 6 & 26.0 & $=$ & - \\
\hline TOTAL & 23 & & 7 & \\
\hline
\end{tabular}




\subsubsection{Other Data Sources}

Other data sources have been investigated in order to obtain a better perspective on the types of human error and general error rates in shipping to be expected.

Studies performed in nine states plus New York City, and collated by Los Alamos Scientific Laboratories, indicate that the same procedures are usually followed at terminals for all types of shipments, including radioactive materials. ${ }^{3}$ No special procedures, special stowage, or special loading are consistently applied to radioactive material shipments.

Additional information necessary to this analysis is actual shipment frequency by package type. The 1975 shipment data base is coupled with the incident reports for 1975 to estimate the incident rates by package type.

\subsection{Estimation of Urban Incident Rates}

Equation 1 requires an incident rate as a function of radioactive material shipment type. Data described in the previous sections indicate that few incidents have occurred which involved a small fraction of the hundreds of different isotopes shipped annually. Therefore, a reliable incident rate by isotope cannot be calculated directly from the data. The package type employed may be a more significant parameter affecting the occurrences of errors, since only a few package types are typically employed. Thus, the available data can be used to estimate incident rates as a function of packaging.

The incident rate per package for package Type $\mathrm{k}$ may be expressed as

$$
E_{k}=\frac{\text { Total No. of incidents involving package Type } k}{\text { Total No. of packages of Type } k \text { shipped }}
$$

Since the incident reports do not normally indicate package type, the total number of incidents for a particular material in a given package type is estimated as follows:

$$
\left[\begin{array}{l}
\text { Incidents involving } \\
\text { isotope X in Type A } \\
\text { packages }
\end{array}\right]=\left[\begin{array}{c}
\text { Total incidents } \\
\text { involving X }
\end{array}\right] \cdot\left[\frac{\text { Total Type A packages for X }}{\text { Total packages of } X}\right]
$$

Thus the expression for $\mathrm{E}_{\mathrm{k}}$ in Equation $2 \mathrm{a}$ can be replaced by

where

$$
E_{k}=\frac{\sum_{j=1}^{N} B_{j} F_{j k}}{\sum_{j=1}^{N} n_{j k}}
$$

$$
\begin{aligned}
B_{j} & =\text { total urban incidents per year for isotope } j \\
F_{j k} & =\text { fraction of isotope } j \text { shipments made in Type } k \text { packages }
\end{aligned}
$$




$$
\begin{aligned}
n_{j k} & =\text { number of Type } k \text { packages of isotope } j \text { shipped per year } \\
N & =\text { total number of isotopes in the shipment model }
\end{aligned}
$$

Note that for those materials with no reported urban incidents, the value of $\mathrm{B}=$ 0 . However, the sum expressed in the denominator of Equation 3 equals the total number of shipments of Type $k$ packages per year. Nonzero values for $B$ have been obtained from the summarized urban incident reports in Appendices $H$ and $I$ and combined with the data from Reference 4.

Values obtained for the terms in Equation 3 are given in Table 4-4. The calculated urban incident rates are per package shipped on a nationwide annual basis. other incident rates can be calculated using all 1975 incidents (DOT and NRC) if an evaluation of a national average and not of an urban-specific set is desired. The estimated urban incident rates by package type are as shown in Table 4-5.

The release fractions by severity and package type are summarized in Table 4-6. DOT and NRC incident reports indicated that in $71 \%$ of the cases, no measurable release to the environment occurred. For a Category 1 accident, the probability of occurrence was set at 0.71 . The remaining seven probabilities of occurrence were scaled in comparison with the fractional occurrences for vehicular accidents discussed in Appendix A. The resulting set of occurrence probabilities are given in Table 4-6. Hypothetical descriptions are also provided for the severity categories used in the analysis.

\subsection{Analysis of Cask Incident Rate}

Data from Reference 5, Table 7.2 indicate the following information:

- Total number of cask shipments (rail and truck) -- 3939

- Number of incidents which could be traced directly to a human error or deviation from accepted QA practices -- 16

A tabulation (by cause) of these human error or quality assurance incidents is given in Table 4-7.

The 16 occurrences in 3939 shipments result in an overall rate of $4.1 \times 10^{-3}$ incidents per shipment. It is assumed that the maximum result of a human error is the release of all contaminated coolant water in the cask. Reference 6 provides information on the quantity of material that could be released. This information is summarized in Table 4-8.

It is recognized that inert gases and traces of tritium and iodine would also be released from the perforated rods. Using the assumptions in Reference 7 , the quantity of these materials in the cask during "normal" transport is approximately 12 curies. As in the accident analysis, these materials would contribute negligibly to the overall population dose, hence they are not considered further (see Section 3.3 in Chapter 3 ). Additionally, the conservative assumption is made that all of the material in Table 4-8 is cobalt 60 . Reference 6 indicates that for the "normal" transport situation, $90 \%$ of the activity in the coolant is cobalt (dissolved "crud" material) and $1 \%$ is cesium (leached from fuel rods). Thus, the assumption that all contamination is $\mathrm{Co}-60$ is not unreasonable. The health effects coefficients (rem per $\mu \mathrm{Ci}$ values - see Appendix $\mathrm{H}$ ) used for the "human error" cask incident are those for Co-60. 
Table 4-4

Determination of Urban Incident Rates for 1975 DOT and NRC Data

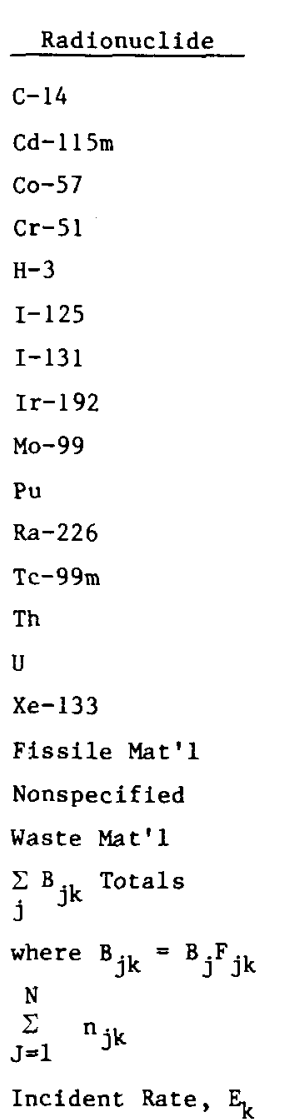

\begin{tabular}{|c|c|c|c|c|c|c|c|c|c|c|c|c|}
\hline$B_{j}$ & $\mathrm{~F}_{\mathrm{A}}$ & $\mathrm{B}_{\mathrm{jA}}$ & $\mathrm{F}_{\mathrm{L}}$ & $\mathrm{B}_{j \mathrm{~B}}$ & $\mathrm{~F}_{\mathrm{L}}$ & ${ }^{B}{ }_{j L}$ & $\mathrm{~F}_{\mathrm{LSA}}$ & ${ }^{B}{ }_{j L S A}$ & $F_{\mathrm{LQ}}$ & ${ }^{B}{ }_{j} L Q$ & $\mathrm{~F}_{\mathrm{NS}}$ & ${ }^{8}{ }_{j N S}$ \\
\hline 5 & $1.0 \times 10^{0}$ & $5.0 \times 10^{0}$ & - & - & $3.8 \times 10^{-3}$ & $1.9 \times 10^{-2}$ & - & - & - & - & $2.8 \times 10^{-4}$ & $1.4 \times 10^{-3}$ \\
\hline 1 & $1.1 \times 10^{-1}$ & $1.1 \times 10^{-1}$ & $8.9 \times 10^{-1}$ & $8.9 \times 10^{-1}$ & - & - & - & - & - & - & - & - \\
\hline I & 9. $1 \times 10^{-1}$ & $9.1 \times 10^{-1}$ & - & - & $8.5 \times 10^{-2}$ & $8.5 \times 10^{-2}$ & $3.5 \times 10^{-3}$ & $3.5 \times 10^{-3}$ & - & - & - & - \\
\hline 1 & $1.0 \times 10^{0}$ & $1.0 \times 10^{0}$ & - & - & - & - & $1.0 \times 10^{-3}$ & $1.0 \times 10^{-3}$ & - & - & $1.8 \times 10^{-3}$ & $1.8 \times 10^{-3}$ \\
\hline 3 & $9.8 \times 10^{-1}$ & $2.9 \times 10^{0}$ & $1.4 \times 10^{-2}$ & $4.1 \times 10^{-2}$ & $5.9 \times 10^{-3}$ & $1.8 \times 10^{-2}$ & $1.7 \times 10^{-3}$ & $5.0 \times 10^{-3}$ & - & - & $1.9 \times 10^{-4}$ & $5.7 \times 10^{-4}$ \\
\hline 2 & $8.5 \times 10^{-1}$ & $1.7 \times 10^{0}$ & - & - & $1.4 \times 10^{-1}$ & $2.9 \times 10^{-1}$ & $9.1 \times 10^{-4}$ & $1.8 \times 10^{-3}$ & - & - & $3.5 \times 10^{-3}$ & $7.0 \times 10^{-3}$ \\
\hline 6 & $9.8 \times 10^{-1}$ & $5.9 \times 10^{0}$ & $1.4 \times 10^{-3}$ & $8.1 \times 10^{-3}$ & $1.2 \times 10^{-3}$ & $7.3 \times 10^{-3}$ & $2.0 \times 10^{-5}$ & $1.2 \times 10^{-4}$ & - & - & $1.9 \times 10^{-2}$ & $1.1 \times 10^{-1}$ \\
\hline 2 & $1.8 \times 10^{-1}$ & $3.5 \times 10^{-1}$ & $8.1 \times 10^{-1}$ & $1.6 \times 10^{0}$ & $9.8 \times 10^{-3}$ & $2.0 \times 10^{-2}$ & - & - & - & - & $1.9 \times 10^{-3}$ & $3.7 \times 10^{-3}$ \\
\hline 3 & $9.7 \times 10^{-1}$ & $2.9 \times 10^{0}$ & $2.5 \times 10^{-2}$ & $7.5 \times 10^{-2}$ & $6.0 \times 10^{-4}$ & $1.8 \times 10^{-3}$ & - & - & - & - & - & - \\
\hline 2 & $5.1 \times 10^{-1}$ & $1.0 \times 10^{0}$ & $3.9 \times 10^{-1}$ & $7.8 \times 10^{-1}$ & $6.5 \times 10^{-2}$ & $1.3 \times 10^{-1}$ & $7.1 \times 10^{-3}$ & $1.4 \times 10^{-2}$ & $6.1 \times 10^{-3}$ & $1.2 \times 10^{-2}$ & $2.4 \times 10^{-2}$ & $4.7 \times 10^{-2}$ \\
\hline 1 & $1.5 \times 10^{-1}$ & $1.5 \times 10^{-1}$ & $3.9 \times 10^{-1}$ & $3.9 \times 10^{-1}$ & $2.8 \times 10^{-2}$ & $2.8 \times 10^{-2}$ & - & - & - & - & $4.3 \times 10^{-1}$ & $4.3 \times 10^{-1}$ \\
\hline 2 & $9.9 \times 10^{-1}$ & $2.0 \times 10^{0}$ & - & - & - & - & $7.2 \times 10^{-5}$ & $1.4 \times 10^{-4}$ & - & - & $1.2 \times 10^{-2}$ & $2.4 \times 10^{-2}$ \\
\hline 2 & $6.5 \times 10^{-1}$ & $1.3 \times 10^{0}$ & $5.3 \times 10^{-3}$ & $1.1 \times 10^{-2}$ & $9.0 \times 10^{-3}$ & $1.8 \times 10^{-2}$ & $3.4 \times 10^{-1}$ & $6.7 \times 10^{-1}$ & - & - & $1.7 \times 10^{-3}$ & $3.4 \times 10^{-3}$ \\
\hline 2 & $4.1 \times 10^{-2}$ & 8. $2 \times 10^{-2}$ & $1.1 \times 10^{-1}$ & $2.3 \times 10^{-1}$ & $1.2 \times 10^{-2}$ & $2.5 \times 10^{-2}$ & $8.3 \times 10^{-1}$ & $1.7 \times 10^{0}$ & $4.8 \times 10^{-3}$ & $9.6 \times 10^{-3}$ & $1.2 \times 10^{-3}$ & $2.5 \times 10^{-3}$ \\
\hline 1 & $9.9 \times 10^{-1}$ & $9.9 \times 10^{-1}$ & - & - & - & - & - & - & - & - & $5.7 \times 10^{-3}$ & $5.7 \times 10^{-3}$ \\
\hline 1 & $7.3 \times 10^{-2}$ & $7.3 \times 10^{-2}$ & $9.3 \times 10^{-1}$ & $9.3 \times 10^{-1}$ & - & - & - & - & - & - & - & - \\
\hline 2 & $9.8 \times 10^{-1}$ & $2.0 \times 10^{0}$ & - & - & - & - & $1.7 \times 10^{-2}$ & $3.5 \times 10^{-2}$ & - & - & - & - \\
\hline 3 & $3.8 \times 10^{-1}$ & $1.1 \times 10^{0}$ & $4.9 \times 10^{-3}$ & $1.5 \times 10^{-2}$ & $3.7 \times 10^{-3}$ & $1.1 \times 10^{-2}$ & $5.9 \times 10^{-1}$ & $1.8 \times 10^{0}$ & $5.0 \times 10^{-4}$ & $1.5 \times 10^{-3}$ & $2.1 \times 10^{-2}$ & $6.4 \times 10^{-2}$ \\
\hline & & $2.9 \times 10^{1}$ & & $5.0 \times 10^{0}$ & & $6.5 \times 10^{-1}$ & & $4.2 \times 10^{0}$ & & $2.3 \times 10^{-2}$ & & $7.0 \times 10^{-1}$ \\
\hline & & $1.71 \times 10^{6}$ & & $1.04 \times 10^{5}$ & & $4.35 \times 10^{4}$ & & $6.24 \times 10^{5}$ & & $3.45 \times 10^{3}$ & & $2.21 \times 10^{4}$ \\
\hline & & $1.7 \times 10^{-5}$ & & $4.8 \times 10^{-5}$ & & $1.5 \times 10^{-5}$ & & $6.7 \times 10^{-6}$ & & $6.7 \times 10^{-6}$ & & $3.2 \times 10^{-5}$ \\
\hline
\end{tabular}


Table 4-5

Urban Area Incident Rates by Package Type

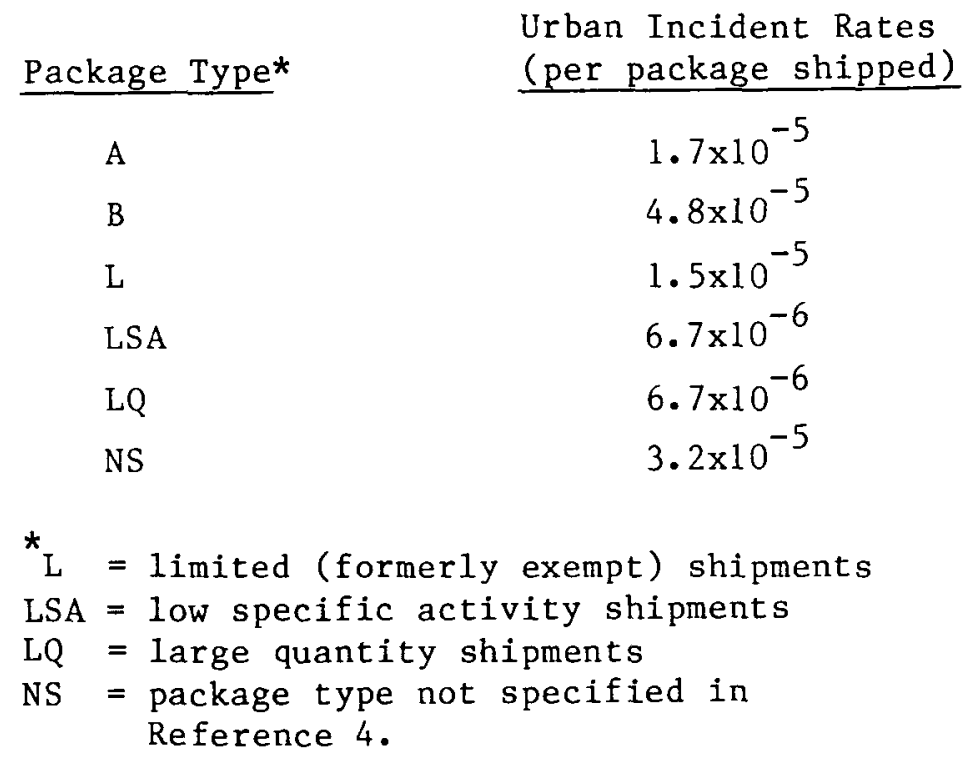

Table 4-6

Probability of Occurrence

Category

1

2

3

4

5

6

7

8
Description

No measurable release

No significant release

For fragile packaging--

partial release of contents

For fragile packaging--

total release of contents

For sturdy packaging (e.g., Type B) total release of contents

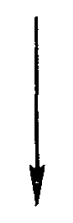

Release Fractions by Package Type $\left(R F_{i j}\right)$

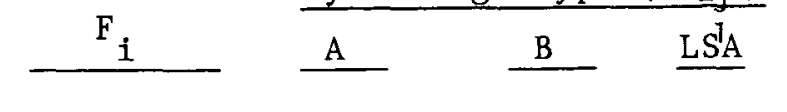

$\begin{array}{cccc}0.710 & 0 & 0 & 0 \\ 0.232 & 0.01 & 0 & 0.01\end{array}$

0.045

0.1

0.01

0.1

0.010

1.0

0.1

1.0

0.0018

1.0

1.0

1.0

0.00071

1.0

1.0

1.0

$5.5 \times 10^{-5} \quad 1.0$

1.0

1.0

$9.7 \times 10^{-6}$

1.0

1.0

1.0 
Tab1e 4-7

Summary of Causes of Cask Incidents

Cause

No. of Incidents

Impact limiters not properly installed

1

Higher external radiation readings than permitted

Closure bolts not properly torqued

Missing closure bolts

Closure seal leaking*

Vent valve not closed

TOTAL

2

16

*This was the only recorded case where a release to the environment was documented.

Table 4-8

Levels of Contamination in Cask Coolant

Level of

Transport

Situation

"Normal"

"Maximum Con-"

3.0
Source of Contamination

Dissolved "crud" material and cesium leached from perforated fuel rods

Dissolved "crud" material and abnormal levels of cesium from additional perforated fuel rods
Tota1 Quantity ${ }^{a}$

of Material (Ci)

0.042

1.25

a This calculation assumes $4.18 \times 10^{5} \mathrm{~cm}^{3}$ of coolant, characteristic of the NFS-4 or NAC-1 truck cask.

$\mathrm{b}_{\text {This }}$ level occurred in 1 shipment out of 800 . 
In order to apply the METRAN model to human errors involving the special case of spent fuel casks, release fractions and fractional occurrences must be determined. Information from Table 4-7 reveals that in $94 \%$ of the incidents, no release to the environment occurred. The information from Reference 5 is used to subdivide the remaining $6.25 \%$ ( 1 incident out of 16 ) between categories of nominal release to the environment and a maximum release to the environment. This information is also summarized in Table 4-9.

Table 4-9

Fractional Occurrences and Release Fractions for Human Errors/Cask Incidents

Occurrence

Categories

A

B

C

\section{Description}

No release to environment

Minimum release

Maximum release

\section{Fractional}

Occurrences

0.94

0.06

$7.8 \times 10^{-5}$
Release

Fractions

of $\mathrm{Co}-60$.

0

0.034

1

For the cask exposure case, it is assumed that a human error or deviation from QA practices would not create the kinds of forces necessary to cause a circumferential crack in the cask wall (this is the assumption used in the accident release fraction determination). Thus the release fractions for all severities for cask exposure are set to zero.

\subsection{Environmental Impacts of Human Errors or Deviations from Accepted QA Practices}

Equation 1 contains a term $C_{i, j}$ which represents the probability per year of a human error of severity $i$ for package Type $j$. Since the incident rates are only a function of package type, $C_{i j}$ may be expressed as follows:

$$
C_{i j}=F_{i} \cdot E_{j}
$$

where

$$
\begin{aligned}
F_{i}= & \text { probability of occurrence of a human error or } \\
& \text { deviation from accepted quality assurance practices } \\
& \text { of severity } i \\
E_{j}= & \text { package type } j \text { incident rate }
\end{aligned}
$$

Table 4-10 summarizes the results of the application of the METRAN consequence code with the human error incident rates and occurrence probabilities replacing the similar accident-related parameters (accident rates, etc.). 
Package-Type Contributions to Expected Risk Values from Human Errors or Deviations from Accepted QA Practices

\begin{tabular}{|c|c|c|c|c|}
\hline \multirow[b]{2}{*}{ Package Type } & \multirow{3}{*}{ Time of Day: } & \\
\hline & & 1200 & 1630 & 2400 \\
\hline$A^{b}$ & & $4.1 \times 10^{-5}$ & $3.6 \times 10^{-5}$ & $5.1 \times 10^{-6}$ \\
\hline$B^{b}$ & & $2.4 \times 10^{-5}$ & $1.2 \times 10^{-5}$ & $3.9 \times 10^{-6}$ \\
\hline Drum ${ }^{b}$ & & $1.0 \times 10^{-8}$ & $1.1 \times 10^{-8}$ & $1.1 \times 10^{-8}$ \\
\hline Cask $^{\mathrm{C}}$ & & $1.8 \times 10^{-3}$ & $1.4 \times 10^{-3}$ & $1.4 \times 10^{-3}$ \\
\hline
\end{tabular}

${ }^{a}$ Values are presented only for expected number of latent cancer fatalities since the breakdown for expected numbers of genetic effects is quite similar with totals of $2.0 \times 10^{-3}$ at 1630 hours, $2.5 \times 10^{-3}$ at 1200 hours, and $2 \times 10^{-3}$ at 2400 hours.

${ }^{b}$ Calculated using DOT HMIR data for incident rates (see Section 4.3).

${ }^{c}$ Calculated using data from Reference 5 (see Section 4.4).

As in the accident case, there is only a small variation between the time of day runs; thus a single set of values to estimate the radiological risk is used. Specifically, the time 1630 is chosen, with a $4-\mathrm{m} / \mathrm{s}$ south wind. For this set of data, Tables 4-11 and 4-12 present the breakdown on the basis of end use and transport mode.

Results of the analysis are expressed as total expected health effects as in the vehicular accident case. Again, the expected risk values are per shipment year; however, in this instance the most meaningful breakdown is on the basis of package type.

Major contributions to the total expected risk are from casks, Type A, and Type B packages. Examination of the economic risks from human errors or deviations from accepted QA practices reveals that the major contributors to the total of $\$ 2.9 \times 10^{4}$ are medical-use shipments (92\%) and shipments in Type A packages (75\%), with Type B packages contributing an additional $18 \%$. Shipments having at least part of their transport by truck constitute $99 \%$ of the economic risk. 
Table 4-11

End-Use Category Contributions to Expected Number of Latent Cancer Fatalities from Human Errors

\author{
Expected Number of \\ $\frac{\text { End Use }}{\text { Medical }^{a}}$ \\ $\frac{\text { Latent Cancer Fatalities }}{1.3 \times 10^{-5}}$ \\ Industrial ${ }^{a}$ \\ $6.4 \times 10^{-6}$ \\ Fuel Cycle $e^{b}$ \\ $1.4 \times 10^{-3}$ \\ Waste ${ }^{a}$ \\ $1.2 \times 10^{-8}$ \\ TOTAL \\ $\sim 1.4 \times 10^{-3}$ \\ ${ }^{a}$ Calculated using DOT HMIR data for incident rates (see \\ Section 4.3). \\ ${ }^{b}$ Calculated using DOT HMIR data from Reference 5 (see \\ Section 4.4).
}

\author{
Table 4-12 \\ Transport-Mode Contributions to Expected Number of \\ Latent Cancer Fatalities from Human Errors \\ Transport Mode \\ Truck ${ }^{\mathrm{a}}$ \\ Air ${ }^{b}$ \\ Air and truck ${ }^{b}$ \\ Barge ${ }^{b}$ \\ Expected Number of \\ Latent Cancer Fatalities

$$
1.4 \times 10^{-3}
$$$$
3.5 \times 10^{-8}
$$$$
7.7 \times 10^{-6}
$$$$
2.7 \times 10^{-7}
$$ \\ ${ }^{a}$ Calculated using DOT HMIR data for incident rates \\ (see Section 4.3). \\ ${ }^{\mathrm{b}}$ Calculated using data from Reference 5 (see \\ Section 4.4).
}

\title{
4.6 Summary
}

Contributions to total expected radiological risk from human errors has been evaluated using urban incident rates by package type. Expected health effects are $\sim 1.4 \times 10^{-3}$ latent cancer fatality and $\sim 2 \times 10^{-3}$ genetic effect. These results 
are obtained using the accident consequence portion of METRAN and represent a conservative estimate of the effects of human errors, since it is assumed that the error results in release and dispersal of materials in a manner similar to a vehicular accident, i.e., release fractions and aerosol fractions are assumed in most cases to be the same as in the accident analysis. In reality, the aerosol fractions (and possibly release fractions) would probably be smaller than estimated, but in the absence of better data, the conservative assumptions have been utilized. As mentioned earlier, human errors resulting in accidents are not included in this analysis. Although there are possible synergisms that would connect the human error with a vehicular accident, the two were considered separable for this treatment. The results should be interpreted carefully since the source of the initial data for the determination of incident rates were vastly different.

\section{NOTES}

149 CFR 171.15

${ }^{2} 10 \mathrm{CFR} 71.61$.

${ }^{3}$ U.S. Nuclear Regulatory Commission, Summary Report of the State Surveillance Program on the Transportation of Radioactive Materials, NUREG-0393, Washington: USNRC, March 1978.

${ }^{4}$ BNWL-1972.

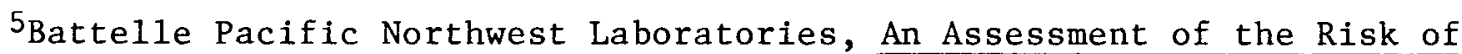
Transporting Spent Nuclear Fue1 By Truck, PNL-2588, Richland, WA: PNL, November 1978.

${ }^{6}$ Personal communication from K. Eger, General Electric, Morris, Illinois, 25 June 1979.

${ }^{7}$ WASH-1238. 
SABOTAGE, SECURITY, AND SAFEGUARDS IN URBAN TRANSPORT

\subsection{Introduction}

As a result of the continued growth of the nuclear industry, the level of public concern about the safety and physical security of nuclear materials in transit has increased. A significant portion of this concern is engendered by the world-wide increase in terrorist activity, ${ }^{-4}$ together with the possibility that nuclear materials and facilities may become the targets of such attacks. 56 Such public concern has led to studies on possible threats to the nuclear industry ${ }^{7}$ and evaluations of the environmental impacts of the transportation of nuclear materials. ${ }^{8} 9$ Therefore, although there appear to be no documented incidents of sabotage to nuclear materials in transit, the question must be addressed. Sabotage involves human motivations and the probability of human actions which are unquantifiable with our present knowledge. Thus, in order to examine the impact of sabotage, this study assumes that a sabotage attempt is made and that it is successful. In addition, it is assumed that the intent of the adversary is to inflict public harm (both radiologic and economic) either by dispersal of radioactive material or direct radiation exposure. Other actions, e.g., theft for ransom, are certainly possible, but they would not have the immediate effects of a dispersal. Various shipments are analyzed from this viewpoint as possible targets. Assuming an attack which leads to a release of radioactive materials, estimates of the consequences of postulated radioactive releases from these shipments are made using the consequence model developed for the safety portion of this study.

At the time this study was initiated, spent fuel, low enriched fresh fuel, radiography sources, and radiopharmaceuticals were not subject to safeguards requirements during transit. Subsequently, the NRC published interim rules requiring certain. safeguards for spent fuel. The effect of these interim requirements has not been addressed in this study, primarily because treating such a situation from a risk viewpoint would require knowledge (or an estimate) of the probability of an adversary attack against spent fuel and knowledge of adversary capabilities. This information is not available and any such estimates would have considerable uncertainty. Therefore, for purposes of this study, spent fuel is considered without safeguards. The study considers the nature of the shipments, the quantity of radioactivity per shipment, and material dispersibility and toxicity, as well as the access to the shipment that a potential adversary might have in the urban environment (Sections 5.2 and 5.3). The public consequences of a successful direct sabotage or theft (with ultimate dispersal) are estimated in Section 5.4. Special characteristics of the urban area are included in the analysis, namely (1) the high population densities with attendant heavy vehicular and pedestrian traffic densities, (2) large diurnal variation in population, numbers of vehicles, and pedestrians, and (3) the effect of high-rise buildings on the dispersal of radioactive material and the radiation shielding of occupants afforded by such buildings. 
For radioactive material shipments subject to safeguards (special nuclear materials (SNM), such as plutonium, uranium-233 (U-233), or uranium enriched to greater than $20 \%$ in U-235 and, now, spent fuel), the impact of the urban environment on the function of the safeguards system is discussed. In particular, attention is directed toward the response times, capabilities, and tactics of law enforcement agencies, and how these may be affected by the increased population and traffic densities associated with an urban area. The potential consequences of theft of such materials with the intent of manufacturing nuclear explosive devices has been treated elsewhere and is not included in this study.

\subsection{Potential Modes of Sabotage}

It is possible to divide the various types of shipments and related packaging in use in the nuclear industry into two broad classes based upon the degree of resistance they offer to unauthorized penetration. One group includes the large packages (usually casks) that are used for material such as spent fuel and large teletherapy sources. Special tools and heavy equipment are normally required to handle and open these packages; therefore, unauthorized penetration will require energy-intensive techniques such as explosives. The second group includes packages which contain low-level sources. Many of these packages can be opened with simple hand tools or even, in some instances, without tools. Because the contained material has low levels of radioactivity, there is litle hazard to the public. This is discussed further in a later section.

The packages (casks) which require energy-intensive methods for unauthorized penetration also contain the largest amounts of radioactive material and thus provide the greatest potential for public harm. Therefore, it is appropriate to consider further some of the potential methods of sabotage that might be employed against these packages.

\subsubsection{Explosives}

To avoid providing potential adversaries with a "cookbook" of methods, much of the ensuing discussion is qualitative and may appear subjective. However, the discussion is based on more quantitative analyses. High explosives are available commercially in a variety of chemical and physical forms. However, for this study, the exact form of the explosive is not of as much interest as the manner in which the explosives might be used. For purposes of this study, high-explosive attacks sufficient to cause a release of material are categorized as (1) airblast, (2) contact or breaching charges, (3) shaped charges, and (4) platter charges. Each of these methods is discussed in the following paragraphs. A variety of hand-held, antitank-type weapons might puncture a package but not cause a rupture sufficient to release a large amount of the contents.

\section{Airblast}

In a sabotage attack involving airblast, a high-explosive charge would be positioned close to a package and detonated, employing the resulting air shock wave to disrupt package integrity. The inherent strength and massive nature of large packages, such as shipping casks, suggest that the amount of high explosive would have to be large. There are some precedents for terrorist use of relatively large amounts of high explosive, such as the attack on the University of Wisconsin in 1970 , which involved approximately $770 \mathrm{~kg}$ of a fertilizer-fuel oil mixture. ${ }^{1}$ Generally speaking, terrorist activities have not involved such large amounts of high explosives. Nevertheless, such a mixture may be attractive to an adversary because 
the components can be obtained and the explosive prepared without revealing to suppliers the end purpose.11

There are some constraints on the use of airblast that reduce its effectiveness and attractiveness from an adversary's point of view. First, the readily available explosives are bulky. For example, several tonnes of fertilizer-fuel oil mixture occupy several cubic metres and would require truck transport. Second, the quantity of high explosive involved would necessitate that the adversary place the charge and then move to a safe distance before detonation. The airblast overpressures can cause personnel and property damage at considerable distances (hundreds of metres for tonnes of high explosive) so moving to a safe distance would reduce the adversary's control over the situation. Third, use of airblast would require that the target (truck or railcar) be detained within range of the blast, or that the charge be prepositioned with assurance that the target would pass close by. This would also require that the firing system be sufficiently sophisticated to insure charge detonation at the appropriate time.

In summary, although airblast is straightforward for an adversary to employ, the practical constraints and target characteristics discussed above decrease the likelihood of success if employed against large radioactive material packages.

\section{Breaching Charges}

In a sabotage attempt involving breaching charges, high explosives would be placed in direct contact with a package and detonated. This is analogous to the use of high explosives to breach large concrete structures. 12 The energy of the charge would be coupled directly into the package, possibly leading to fracture, spallation, and rupture. Here, as with airblast, the strength and massive nature of the large packages would necessitate the use of significant quantities of explosives. It is generally conceded that explosives useful in this type of at tack are available "on the street" in quantities such that an adversary could acquire the necessary explosives without contacting government agencies controlling the sale of such material.

With this method of attack it would be necessary for the adversary to gain access to the shipment so that the charge could be placed. With sufficient preparation (knowledge of routes, type of package to be attacked, materials of construction, etc.), a small group could presumably complete such an attack in a short time. Access could be achieved while the truck is parked in a terminal or rest stop. Alternatively, the truck could be hijacked and then driven to some point where the sabotage would cause the desired public harm. Although the amount of high explosives required is large enough so that the adversary would have to leave the immediate area before detonation, the use of simple time-delay fuses would be sufficient to permit the adversary to accomplish the attack because the high explosive is in contact with the surface.

As with airblast attack, there are some inherent constraints which will affect the adversary's success. The weight of high explosives required makes it unlikely that "hit and run" tactics would be successful if employed. A potential alternative is the theft or hijacking of the truck, the installation of explosives at some hidden or remote location, followed by detonation and release at some predetermined point. Such a scheme also has inherent constraints. For instance, although mobile, a truck with a large radioactive material cask is quite distinct and would be quite obvious unless it were hidden or camouflaged in some manner. Also, movement of 
hazardous cargo of any type, although it may not be guarded per se, is frequent1y coordinated with law enforcement agencies. It is unlikely that a theft or hijacking would go undetected for a significant period of time. Furthermore, a number of the truck systems are over legal roadway weight limits, and, therefore, their routes are carefully planned and coordinated, including in many instances requirements for some type of escort vehicle or movement only during specified hours. All of these serve to complicate the hijacking of a truck shipment.

Because casks designed for rail shipment are the most massive, the weight of explosive required to breach them is quite large. This negates the possibility of an attack on foot (i.e., while the shipment is in a rail yard). Similarly, an attempt to place explosives while the train was halted by an obstruction or similar means would appear to require a reasonably sized, well trained group. Because the location of the release would be difficult for an adversary to control (dictated more by access than availability of public to intimidate or harm), and the effort to accomplish sabotage extensive, the use of breaching charges against rail packages would seem unattractive for an adversary intent upon maximizing public harm.

Therefore, attacks with breaching charges against truck-mounted packages appear possible, although logistical and other constraints may reduce their attractiveness to an adversary. Successful attacks against the very massive rail packages are considered beyond the capabilities of small groups.

\section{Shaped Charges}

In this type of attack, specially shaped high explosives are placed on the package and detonated. A high-temperature, high-pressure jet from the shaped charge punches a hole into and through the material. In contrast to breaching charges, which must be placed in direct contact with the target, shaped charges have standoff requirements which are a function of target thickness and the charge weight and geometry. 121314 Shaped charges have been fabricated for the military and could presumably be stolen by an adversary. Also, with the information available in the open literature it would be possible for a moderately skilled explosives handler to fabricate a rudimentary shaped charge.

As with breaching charges, it would be necessary for the adversary to gain access to the target to place the charge. Again, simple access could be obtained while a truck is parked in a terminal or rest stop. However, use of shaped charges would require that the attacker know the design features of the package in some detail to properly place the charge. Thus, although shaped charges can be handled by one or two men, the "hit and run" tactic is not considered a realistic way to initiate a release from a large radioactive material cask. As with the breaching charge, hijacking of the truck followed by explosive installation would perhaps be easier for the adversary. Therefore, the constraints (truck visibility, prearranged routes, hazardous cargo escort, etc.) discussed under breaching charges also apply here.

For railcar casks, an attack with shaped charges is possible since the requisite materials can be carried by men on foot. However, the requirement to modify the target to insure effective charge placement is more significant with these larger packages than those that are truck mounted. Therefore, the adversary would have to have some unobserved and uninterrupted time before the target was moved (assuming it is on a siding or in the yard) or a way to control the movement. Here again, the release location is not readily controllable by the adversary without an effort involving the takeover of some rail facilities. 
Although it appears that an attack using shaped charges might conceivably be attempted against either truck- or rail-mounted shipping containers, the uncertainties in cask design and charge placement facing the adversary decrease his likelihood of success.

\section{Platter Charges}

In this attack, a flat steel plate becomes a blunt projectile under the action of a driving explosive. In such an attack, it is not necessary that the adversary have direct access to the target, although he does have to get reasonably close. Because the plate is simply driven by high explosive, it is not precisely aimable in the sense that an artillery weapon is aimed. However, it has been stated ${ }^{12}$ that with practice, a demolitionist can hit a target the size of a 55-gallon drum about $20 \%$ of the time at a range of 23 metres using a 1 to $3 \mathrm{~kg}$ projectile. The massiveness of the large packages discussed earlier suggests that very heavy platters would be required.

Considering the characteristics of platter charges, if an adversary were to attempt an "ambush" type of attack, he would have to know the physical dimensions of the target and have a means to insure that it passed within range of the charge. Because the platter charge is uncontained, any support system for the explosive (truck, for example) would be destroyed on detonation. Therefore, the adversary would have to have a firing system that enables him to operate from a safe distance. Uncertainties in target position and platter performance lead to the conclusion that such attacks would not be attractive to a potential adversary.

\section{2 .2 Mechanical}

Some energy-intensive techniques that might be employed in an attempt to penetrate massive casks may be described as mechanical instead of explosive. In most instances, the employment of a mechanical technique requires that an operator be close by: for example, gas cutting torches, power saws, burn bars, etc.* Although an adversary might attempt to use such devices, it is clear that for those packages which contain sufficient gamma-emitting radioactive material to pose a threat to the public, doing so would put him in considerable danger should he successfully penetrate the package. The levels of external penetrating radiation in close proximity to unshielded spent fuel, for example, can lead to fatal doses in seconds. Thus, it appears that the "hands-on" mechanical techniques would be unattractive to any adversary.

There have also been suggestions that a release from a stolen cask could be initiated by deliberate accidents: for example, running it from a highway overpass to drop on the roadway below, crashing it into a bridge or overpass abutment, or initiating similar accident-like events. Recent tests on the survivability of spent fuel packages in accident. situations have demonstrated that such an approach is unlikely to result in releasing radioactive material to the environment. $15-18 * *$ Other large packages are designed to resist the same accident environments.

*The study assumes that an adversary intent upon dispersal does not have sophisticated, radiation-shielded, remote handling equipment.

**Initial reports including photographs of test casks are also included in Nuclear News, Vo1. 20, Nos. 3, 4, 9, and 14, 1977. 
Another accident-like sabotage event that has been suggested is to run a package off a bridge into a river or lake. Because of the impact resistance demonstrated in the referenced crash tests, simply dropping a package into water is unlikely to cause release. Furthermore, packages are designed to withstand at least 170 kilopascals external pressure, so submergence into water 15 to 18 metres deep will have no effect. Other analyses suggest that seals will maintain their integrity to even greater depths. 9

\subsubsection{Summary of Possible Attacks}

Possible attacks against large radioactive material casks have been discussed, and some factors affecting the likelihood of success of such attacks have been outlined in a qualitative way, but there has been no attempt here to quantify that likelihood. It is assumed in later sections that an adversary successfully sabotages a radioactive material package. Based on that assumption, consideration is given to the amount and form of the radioactive material that might be released. Impacts of such releases are then estimated using consequence models. This underlying assumption must be kept in mind when interpreting the results.

\subsection{Nonsafeguarded Shipments, Potential Adversary Actions, and Release Fractions}

The shipments of radioactive materials that are currently unprotected may be conveniently grouped into seven categories for purposes of this analysis. These are

1. Irradiated or spent fuel from reactors using low enrichment uranium*

2. Nonfissile isotopes (1arge sources)

3. Nonfissile isotopes (small sources)

4. Less than strategic quantities of SNM

5. Radiopharmaceuticals

6. Low-level wastes

7. Low enriched uranium

These sources are listed in order of decreasing curie level per package, with the last three being nearly comparable. High-level waste is not considered here for several reasons. One, such waste is not currently being shipped. Two, because commercial spent fuel reprocessing has been deferred, the physical form and radionuclide content of any resultant high-level waste have not been completely defined. Three, shipping containers for high-level waste are only in the conceptual design stage. All of these facts combine to make any attempt at a sabotage analysis of this material speculative at best. Each of the seven groups is considered in further detail below, first in terms of potential adversary actions, next in terms of potential releases, and finally in terms of estimated consequences (Section 5.4).

*As noted earlier, this was the situation at the initiation of this study, and no attempt is made here to examine the effectiveness of the interim NRC rules regarding spent fuel. 


\subsubsection{Potential Adversary Actions}

For each category of shipment, potential adversary actions are discussed considering package contents and structure.

Irradiated (Spent) Fue1

Shipments of spent fuel from light-water reactors using low enriched uranium represent the largest single source of radioactivity routinely shipped. A single spent fuel element may contain in excess of $10^{6}$ curies of radioactivity even after 120-150 days cooling time at the reactor site. Fuel this age was selected as the source because under existing conditions, it is unlikely that fuel with less cooling time would be shipped. In fact, most shipments will be of much older fuel. To an adversary intent upon public harm by dispersal of radioactive material or direct radiation exposure, this level of radioactivity may represent an attractive target for sabotage or theft for later dispersal.*

However, the very radioactivity that makes spent fuel an attractive target also serves to enhance shipment resistance to adversary attack. Because of its high radiation level, spent fuel requires considerable shielding for safe handling, which leads to very massive, and therefore durable, shipping casks. These casks weigh from 23 to 91 tonnes depending upon the number of elements to be carried and the transport mode (truck or rail). Wall construction of these casks may include stainless steel along with lead and/or depleted uranium. Many of the newer designs also include a borated water jacket for shielding purposes.

Access to shipments of spent fuel would be possible for an adversary intent upon sabotage or theft. Truck shipments along the normal road system could be reached at rest and/or refueling stops by following the truck or by collusion with the driver. Traffic tie-ups could be caused which might stop the shipment and permit access. Of course, in the latter instance the adversary runs the risk of having his own progress impeded by traffic. If the shipment travels on urban thoroughfares, normal traffic control could cause stops and give an adversary an opportunity to approach the truck. This could be a possible alternative, especially if theft of the spent fuel were the intent.** Rail shipments could be reached enroute if the adversary had knowledge of the route and used vehicles or sabotage to block rail crossings. Railcars could be reached in the yards during train makeup; however, this would reduce the options available to the adversary since it generally would require him to move on foot. Therefore, although truck shipments are considered accessible, rail shipments are only accessible with difficulty.

The massiveness of the spent fuel cask and the type of access available to an adversary limit to a considerable degree the sabotage schemes that could be employed. The casks are quite invulnerable to small-arms fire or small explosive charges. Therefore, even though such items are possessed by dissident groups, a successful attack appears unlikely. If the intent is dispersal, the potential saboteur is forced to consider other alternatives. Any attempt to open the cask and mechanically disperse the fuel poses significant problems. The casks

*Theft with the intent to process spent fuel to recover nuclear weapon material is beyond the scope of this study and is not examined here. In addition, there is disagreement as to the technical capabilities required to accomplish such reprocessing. 519

**Again, the interim rules regarding spent fuel are not considered here. 
require heavy-duty handling equipment (overhead cranes, etc.) and, in some instances, special tools to open the closure system. If the cask were successfully opened, the adversary would have to contend with an intense radiation field while attempting to remove fuel from the cask. Thus, successful dispersion by simple mechanical means is unlikely.

With these considerations in mind, it is concluded that an attempt to cause a release with significant public impact will necessarily involve an attack with explosive charges, as described in Section 5.2.1.

\section{Nonfissile Isotopes (Large Sources)}

Some shipments ( $10^{2}$ to $10^{6}$ curies) of nonfissile isotopes present a source of radioactive material that, on a curie-per-shipment basis, may be only slightly less than that for spent fuel shipments. Therefore, like spent fuel, such shipments may appear to be an attractive target to an adversary intent upon creating public harm by dispersal or direct radiation exposure. Fortunately, the very level of radioactivity that may make it an attractive target for causing public harm serves to enhance resistance to attack. Large shipments of gamma-emitting sources require considerable shielding for safe handling. This in turn leads to massive, and therefore durable, shipping containers. Such containers or casks weigh from hundreds of kilograms to tens of tonnes depending upon the amount of material to be shipped. These containers are designed to meet DOT and NRC requirements for Type B or large-quantity packages. Typically, these containers will have a lead or depleted uranium shield material between an inner. and outer layer of stainless steel.

The sabotage potential of these containers is quite similar to that for spent fuel casks. Because these containers move in interstate commerce, an adversary intent upon sabotage or theft can conceivably gain access. Truck shipments can be reached in rest/refueling stops or truck terminals, or through driver collusion. Traffic tie-ups could be caused which would stop the shipment and permit access. If the container were being moved to an industrial or medical center, normal traffic control could cause stops that would permit an adversary to approach the vehicle. Rail shipments could also be reached if the adversary had knowledge of the shipment routing, although access to rail yards, transfer points, etc. could be more difficult than access to motor freight facilities. For example, a container in a rail yard may well be accessible only on foot.

The massiveness of these containers, which increases as the quantity of material increases, and the limited access available to a potential adversary limit his choice of attack schemes. If the intent is dispersal, the adversary has some alternatives, although they all present an associated hazard. Any container with shielding thicknesses sufficient for more than a few hundred curies of a gamma emitter will be invulnerable to small arms fire and attacks involving small amounts of high explosive. If the adversary considers opening the container he faces additional problems. To move the containers or open their closures will require some type of mechanical assistance (fork lifts, cranes, etc.) simply because of the weight of the shielding. If the container is opened, the adversary has to contend with an intense radiation field while attempting to remove the contents. Therefore, dispersion by mechanical means will be very difficult to achieve. A deliberate accident (crashing into an abutment, running off an overpass, etc.) is not likely to produce any significant release of material because the containers are designed to retain integrity under just such conditions. Given these factors, the saboteur is forced to consider the use of explosive charges to cause dispersal. 
The alternatives available to the adversary here are the high-explosive attacks presented in Section 5.2.1.

\section{Nonfissile Isotopes (Small Sources)}

Sources for radiography and well-logging are the principal concern in this grouping. Radiography sources are usually gamma emitters (Co-60, Cs-137, Ir-192) doubly encapsulated in stainless steel. When new, radiography source strengths are typically 100 curies or less. ${ }^{20}$ A well-logging source typically consists of a neutron source $(\mathrm{Am}-241 / \mathrm{Be})$ of a few tens of curies, combined with a gamma source (Cs-137) of several curies. 20 These sources are also double-canned in stainless steel. Because of the double encapsulation, both types of sources are considered special form (essentially nondispersible). Sources of these types do not offer a saboteur a very attractive target. In addition to the low source strength compared to spent fuel or the large nonfissile sources, the design of these sources is such that they will withstand considerable abuse without releasing their contents. Although the shipping containers could be stolen (in some instances they are small enough to be hand carried and even opened), the radiation field in close proximity to the unshielded source is sufficiently intense that adversaries could not handle the actual sources without some type of shielding and remote handling capability. If an adversary were to steal such sources with the intent to cause public harm by secreting the unshielded source in a public place, the potential effects would be extremely limited.

\section{Radiopharmaceuticals}

Radiopharmaceuticals are used in the diagnosis and treatment of disease. These products have two principal characteristics that make them unattractive targets for an adversary. First, these isotopes generally have relatively short half lives (a few days or less). Second, they are shipped with a few curies at most in a single package.

Although theft of (or from) such a shipment is a possibility, it would be nearly impossible for an adversary to accumulate sufficient material to create any widespread hazard because the activity in some packages would be decaying while other packages were collected. Any dispersal of a single package would be sufficiently diluted that no significant hazard would be presented to the public.

\section{Less-than-Strategic Quantities of SNM}

Under existing regulations, limited quantities of SNM may be shipped without safeguards. This quantity is 5000 grams, determined from this formula: grams = (grams contained U-235) $+2.5 \times$ (grams U-233 + grams plutonium). 21 If the shipment were a single isotope, then it could be as much as 5 kilograms of U-235, or 2 kilograms of U-233 or plutonium.** None of these materials presents a significant

\footnotetext{
*Source material for radiopharmaceuticals (such as Mo-99) is shipped in significant curie quantities. Multicurie shipments are considered in the earlier subsection, entitled "Nonfissile Isotopes (Large Sources)."

**As stated earlier, theft of material for purposes of producing or fabricating a nuclear explosive is not addressed in this study.
} 
direct radiation hazard because they are primarily alpha emitters and have low specific activity compared to other isotopes. 22

On the other hand, because these materials are alpha emitters they can pose a significant hazard if inhaled or absorbed through open wounds. For purposes of this investigation, absorption through open wounds is considered unlikely and is not addressed further. In addition, because plutonium has a much longer effective half 1 ife in the lung (between 200 and 500 days for Pu versus 100 days for $U$ ) and a much larger specific activity than uranium, only the misuse of plutonium is considered here.

At the present time, plutonium is shipped primarily as plutonium dioxide (powder or pellets) in Type B packaging. A recent survey indicates that some $88 \%$ of the shipments that involve less than $2 \mathrm{~kg}$ of plutonium are actually quantities less than 100 grams. 23 About $37 \%$ of the shipments ( $65 \%$ of the packages) involve amounts between 1 and 100 grams. The bulk of these shipments is by contract or common carrier. Because these materials move on interstate carriers, it must be presumed that a determined adversary could gain access at some point in the transportation cycle, for example, truck terminals, rest stops, etc. Because of the Type B packaging, it is unlikely that deliberate accidents (crashes into bridge abutments, etc.) would successfully release any significant quantities of these materials. Likewise, because it may be only part of a shipment inventory, direct explosive attack would not guarantee the adversary a successful dispersal. For an adversary intent upon public harm, the most attractive scheme appears to be theft of one or more packages followed by dispersal at some later time and location.

Low-Leve1 Wastes

Low-level wastes include the byproducts of various operations with radioactive materials. Such wastes include soft materials such as contaminated paper, clothing, rags, etc. These soft materials are usually compacted and placed in 55-gallon drums for shipment to disposal sites. An individual drum may weigh over $100 \mathrm{~kg}$ and contain up to a curie of activation and fission products. Liquid wastes--for example, contaminated resins and sludges--are dewatered, mixed with solidifying agents (frequently concrete), and placed in 55-gallon drums. These drums usually contain less than 20 curies total activity, although some small fraction may contain as much as 100 curies. The former are shipped in Type A packages, while the latter are shipped in Type B.

In all cases above, when the material is in the transportation sector, it is a solid inside at least a 55-gallon drum. The total activity available in a full shipment of soft waste (approximately 50 drums) is typically less than 50 curies. To disperse this activity effectively, an adversary would have to ensure that every drum was opened and the contents volatilized because the activity is bound to the cloth and paper surfaces, either mechanically or chemically. The only realistic path to such volatilization is fire. Although it is conceivable that a truck loaded with such containers could be set ablaze, it appears unlikely that the adversary could successfully release any significant amount of material for several reasons. First, in any populated area there would be a fire department response to extinguish the blaze. Second, not only would the fire have to be set, but the drums would have to be opened to ensure that their contents were exposed to the flames. 
For those wastes that have been solidified, an adversary would be forced to consider the use of high explosives to rupture the drums and break up the contents. Certainly in the case of Type A shipments, attack with explosives could rupture the drums and cause some breakup of the contents. For Type B shipments, this would be more difficult because of the extra packaging, but it could be done. This would necessitate reasonably long access to the vehicle, so that hijacking of a shipment would be required if an adversary selected such material as a target. Considering that a full load of waste represents a source of only 1000 to 5000 curies tctal activity--all solidified--such shipments do not appear to be attractive targets for an adversary intent upon creating public harm.

Low Enriched Uranium

Low enriched uranium (less than $5 \% \mathrm{U}-235$ ) is the fuel used in light-water power reactors. Typical shipments of fresh fuel may consist of 6 to 12 assemblies in specially designed containers ( 6 to 12 containers to a semitrailer). The total activity in such a fresh fuel shipment is 0.5 to perhaps 2 curies per container. The active material (uranium oxide) is encapsulated in the fuel rods and assemblies, which are then packaged. Dispersal by mechanical means would not produce airborne material. Dispersal by explosives could produce airborne particles containing only small amounts of radioactive material, primarily an alpha emitter. Simply scattering the fuel on the ground would not produce any significant radiological hazard because the available activity is so small.

\subsubsection{Estimation of Radioactive Material Releases for Various Shipping Categories}

In the preceding section, nonsafeguarded shipments were categorized and some possible adversary actions against them identified and discussed. These postulated attacks have been quantified to a limited extent. That is, a particular type of sabotage attack was assumed to be attempted. Based upon that assumption, the resources required by the attacker were considered, e.g., the amount of high explosive required to disrupt large package integrity. After the resources were examined, the amount of radioactive material that could be released was estimated based upon the damage to the package and contents that could reasonably be expected from the attack.

It must be emphasized that the material releases suggested and summarized here have not been verified experimentally. Although programs have been initiated to investigate the nature of the releases resulting from explosive attacks against spent fuel casks, it will be sometime yet before data are available. Therefore, the considerations presented here are based on engineering judgment and the extrapolation of available data to the present study. With this caveat, the release estimates will be used as the source term to estimate the public consequences of the postulated attack.

\section{Irradiated (Spent) Fuel}

As indicated earlier, spent fuel shipments represent the largest single radioactive material source in the transportation sector; this may make them a target for sabotage. Based upon the massive nature and other design characteristics of these casks, it has been concluded that the nnly realistic way to attack such shipments in order to cause dispersal is with high explosives. Analysis indicates that if airblast were used as the mechanism to transmit energy to the cask, large amounts of high explosive would have to be detonated in very close proximity to have any 
chance of disrupting the cask integrity. In such an attack, the most likely result is failure of the cask closure mechanism, with some fuel elements being exposed and perhaps even ejected. There is probably no mechanism to create airborne respirable material except for noble gases that might be released from cracked or ruptured fuel pins. In a breaching attack, the analysis indicates that a significant amount of high explosive, precisely employed, is necessary to disrupt package integrity. Because the explosives are in direct contact with the cask in this attack and because of the energy densities involved, coupled with the brittle nature of the spent fuel pellets, it is believed that some radioactive material of respirable size might become airborne. For such an attack, it is reasonable to assume that all the fuel elements are at least fractured and that some noble gases will be released.

In the analysis, mechanisms for creating respirable particles were also postulated. For example, with shaped charges, the jet energy may be intensely coupled with the cask and contents, creating respirable material. The release estimated for a platter charge attack is very similar to that for the breaching attack, since the interactions are primarily mechanical and may be intensely coupled. The release fractions are summarized later in the subsection entitled "Summary of Estimated Release Fractions."

\section{Nonfissile Isotopes (Large Sources)}

The situation with large quantities of nonfissile isotopes is very similar to that for spent fuel. That is, the shipping casks are inherently massive, so the only credible way to cause significant dispersion is with high explosives. Again the analysis indicates that significant amounts of high explosives are required. However, because the volume occupied by the radioactive material is much smaller in these shipments than for spent fuel $\left(0.03\right.$ to $0.06 \mathrm{~m}^{3}$ compared to 0.3 to 0.34 $\left.\mathrm{m}^{3}\right), 2425$ there is a potential for a larger fraction of the material to be released. Perhaps as much as several tenths of a percent may appear in respirable form, although in this instance it will be a single nuclide and not a mixture of fission products and actinides. The release fractions assumed are summarized in "Summary of Estimated Release Fractions."

\section{Nonfissile Isotopes (Small Sources)}

As indicated earlier, theft of such sources may be possible. If stolen, it is unlikely that an adversary could successfully disperse anything other than the encapsulated source. Any attempt to cut into or grind the capsules would expose the adversary to a hazardous radiation field. Furthermore, because these sources are solids, metals, or inorganic salts, it would be extremely difficult to create particles small enough for airborne dispersal. Attempts to disperse the material explosively could lead to the individual capsules being scattered around if there were more than one present. However, their small sizes, coupled with their generally metallic nature and double canning, make it quite improbable that there would be any release. The scattered capsules could be a source of direct exposure, although the affected area would be quite small.

Radiopharmaceuticals

Radiopharmaceuticals are shipped with so little activity in a single package that they are not considered to be a target for adversary activity. Therefore, no release is estimated for these packages. 
As indicated earlier, the majority of the plutonium shipments in this category involves packages containing 100 grams or less. Furthermore, it was suggested that the attack scheme most likely to produce public harm is theft followed by dispersal. If an adversary steals plutonium with the intent to disperse it, it is assumed that he is sufficiently aware of its properties and toxicity so as to protect himself while handling it. In this analysis, only dispersal which leads to inhalation of the material is considered as the threat. The fraction of any shipment that is respirable (particles less than approximately $10 \mathrm{~mm}$ aerodynamic diameter) is a strong function of the method of preparation. Particle size distributions have been reported that have a respirable content from 4 to $40 \%$, and to be consistent with earlier studies, ${ }^{9}$ this analysis assumes that approximately $20 \%$ of the material is respirable. Because the quantities of material involved are easily handled by one or two persons, a number of dispersal mechanisms are conceivable. One approach is for the adversary to simply scatter the stolen material in an area of heavy foot traffic (transportation terminals for instance) and rely on the movement of people to disperse it. However, even assuming $20 \%$ respirable material, the degree of public hazard from this approach may vary significantly because the amount of material that will become airborne and inhaled is uncertain. Of course, significant surface contamination would result. A second alternative is to introduce the material into the ventilation system of a major public facility (theater, sports arena, etc.). This would certainly cause major contamination and cleanup problems and would expose hundreds to tens of thousands of people to the respirable component. A third alternative would be explosive dispersal of the stolen material in a heavily populated area (e.g., business district during rush hour or an outdoor sports event). In the latter instance, a small explosion to scatter material could expose up to 100000 people to the respirable component as well as to surface contamination from scattered material. Based upon these considerations and recognizing the variability in shipments, this analysis will examine the effects of theft and dispersal of 100 to 1000 grams of plutonium assumed to be $20 \%$ respirable.

Low-Level Wastes

As indicated earlier, a typical shipment of soft waste represents a source of some 50 curies and requires a very unique set of conditions, probably involving extensive and intensive fire to initiate release. Therefore, these shipments are considered such unlikely targets for adversary action that no release fraction is estimated.

Shipments of solidified waste have a potential for 1000 to 5000 curies total activity in the shipment. Furthermore, such shipments can conceivably be attacked with high explosives. However, to cause an inhalation hazard, the contents must be reduced to particles with aerodynamic diameters less than $10 \mu \mathrm{m}$. Explosive attack against solid targets like these drums could certainly rupture them and fracture the contents, but it would not pulverize the contents enough to cause a significant airborne release. Such an attack would create a direct radiation hazard and a cleanup problem. For purposes of analysis and comparison with other events, it will be assumed that $1 \%$ of the contents is released in respirable form and that $50 \%$ of the contained activity becomes a direct radiation source. This accounts for self-shielding, etc. within large pieces that would probably result from the explosive attack. 
Low Enriched Uranium

Because the total available activity in fresh fuel is so low, it is not considered an attractive target to an adversary intent on public harm. Therefore no release fraction is estimated.

\section{Summary of Estimated Release Fractions}

Estimates, based upon possible sabotage attacks, of the radioactive materials released from the various shipping containers are summarized in Table 5-1. These estimates differ from those published previously because of changes in the quantitative analyses. Because the spent fuel elements contain gases as well as solids, the nature of the potential release will be different from other shipments where only solids are involved. For the spent fuel, it has been conservatively assumed that any fracturing of the pins will permit a significant fraction of gases that have migrated into the plenum to escape. This assumption is conservative because a fracture some distance away from the plenum may or may not permit the escape of these gases. Based upon these estimates and considering the absence of experimental data specifically addressing this question, three release-fraction combinations are examined for spent fuel to establish the sensitivity of the consequence estimates to the assumptions made regarding release fractions. These three combinations are

1. Baseline estimate: $10 \%$ of noble gases, $0.07 \%$ of solids as aerosolized respirable material

2. Upper estimate: $25 \%$ of noble gases, $0.2 \%$ of solids as aerosolized respirable material

3. Lower estimate: $10 \%$ of noble gases, $0.02 \%$ of solids as aerosolized respirable material

The release fraction estimates for the other types of shipments are also summarized in Table 5-1. In these cases, the radioactive material is presumed to be shipped as a single isotope in solid form. For the estimation of dispersal consequences for the nonfissile isotopes (large sources), three cases have been examined: $0.28 \%, 0.8 \%$, and $0.08 \%$ of the contents in respirable form. The direct radiation cases will be the same as for the spent fuel. For nonfissile isotopes (small sources), only a direct radiation source will be considered in the consequence estimation. No release is postulated for the radiopharmaceuticals or the low enriched uranium. For the remaining two groups, the consequences will be estimated for the release fractions indicated in Table 5-1.

\subsection{Estimation of the Consequences of Sabotage Attack}

The public consequence of sabotage directed toward radioactive material has several unique aspects. As an example, for fixed facilities such as power plants, an actual sabotage act would probably not involve any of the general public; only the ultimate potential result of such sabotage--the release of radioactive material-could have far-reaching effects. In contrast, for an act of sabotage directed against radioactive material in transit, the dispersal may be deliberately initiated in a public location, for example, a city street or a truck terminal, using significant quantities of high explosives. Therefore, the immediate consequences of the explosive attack itself must be considered along with the immediate and long-term radiological consequences related to the release of radioactive material. 
Table 5-1

Summary of Potential Release Fractions ${ }^{a}$

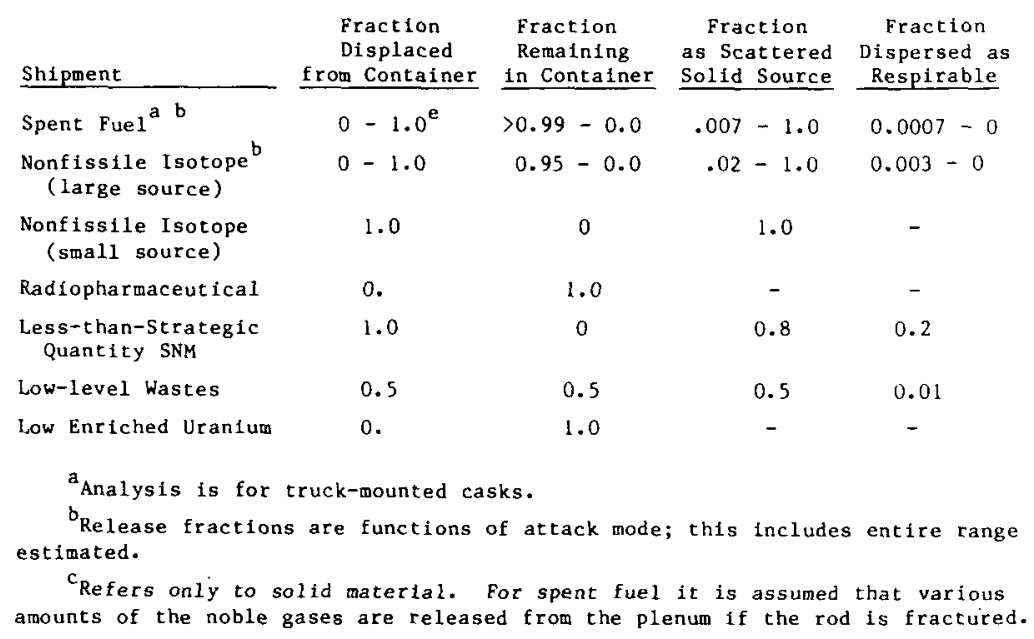

Such considerations are taken into account in this analysis, at least in a qualitative manner.

\subsubsection{The Consequence Mode1s}

The consequences of a release of radioactive material were estimated using the consequence model METRAN, which is described in Appendices B and C. Several specific features should be mentioned here. METRAN has provisions for considering the details of cloud dispersion on a small geographic scale. This is done using a combination of a three-dimensional layered Gaussian dispersion model and a particle-in-cell dispersion model (Appendices $E$ and F). Such a treatment is appropriate when small airborne releases in urban areas are considered. METRAN also has provisions for following airborne material concentrations vertically from ground level to a height of 120 metres. The basic calculational elements are cells 1 kilometre square and 30 metres high. The total calculational grid is a volume 10 kilometres by 10 kilometres by 120 metres high. As employed here, METRAN is used to estimate the effects of radiation from cloudshine, groundshine, and inhaled radionuclides. The model explicitly accounts for people in buildings, in vehicles, and pedestrians. The actual population densities are a function of time and location. The release point (any one of the surface cells) and the release time are selected by the analyst. No special protective action is assumed, and people are exposed to the cloud wherever they happen to be at time of cloud passage (e.g., inside buildings or on the street).

In addition to the estimates made with METRAN, some parallel estimates have been made using CRAC, the consequence model developed for the Reactor Safety Study. 2627 Although CRAC was not developed with the intent to apply it to releases that may be created in the transportation sector, consequence estimates made with CRAC can be useful if interpreted with appropriate regard for the modeling assumptions. CRAC uses time-averaged shielding factors to account for population location and does not require the type of detailed population information used in METRAN. Also, CRAC uses only a Gaussian diffusion approach to the cloud dispersion problem and, therefore, may not have sufficient detail close to the release point to examine street 
canyon effects on cloud progression. On the other hand, CRAC is not restricted to considering a $100-\mathrm{km}^{2}$ grid, but can be used to follow cloud progression out to any selected distance. CRAC also can be used to accumulate results for many different accident or sabotage times throughout a calendar year and thereby obtain mean values and distributions of the estimated consequences over a variety of meteorological conditions. In this particular study, the results using METRAN are consistent with those obtained using CRAC within the constraints of the two models.

With the preceding considerations concerning the range of consequences and the computational tools in mind, the consequence estimates for each of the various classes of shipments for which dispersal is considered realistic are presented in Sections 5.4 .2 through 5.4 .5 .

\subsubsection{Irradiated (Spent) Fuel}

As established in the earlier discussions, any realistic attack against spent fuel shipments can be expected to involve significant quantities of high explosives. Likewise, if an adversary is intent upon public harm, any such attack is likely to take place in densely populated areas. It therefore becomes appropriate to consider the nonradiological effects of such an attack along with the radiological effects.

Consequences of the Use of High Explosives

There are two principal mechanisms for creating damage and causing public consequences using large amounts of high explosive. One is the air shock or blast wave which propagates radially outward from the blast center. The second is the high-velocity debris created by the interaction of the explosive or the shock wave with surrounding structures. Assuming, for this discussion, that an attack on a spent fuel cask may involve as much as several tonnes of explosive, two types of consequences must be considered. These are blast damage to surrounding structures and direct blast effects on people. Blast damage to surrounding structures is considered first.

The blast overpressures associated with the detonation of tens, hundreds, and thousands of kilograms of high explosive are shown as a function of distance in Figure 5-1.* Depending upon the amount of explosive, overpressures greater than $6.9 \mathrm{kPa}$ can be expected to distances of approximately 40 to 200 metres. Based upon observed blast effects, ${ }^{30}$ windows will be broken to even greater distances. In addition, cinderblock buildings can be damaged at distances of 15 to 80 metres ( 20 $\mathrm{kPa}$ level), and reinforced concrete structures can be damaged at distances of 8 to 40 metres $(\sim 100 \mathrm{kPa}$ level). It is nearly impossible to estimate the casualties attributable to collapsing structures, flying debris, etc., but at the population densities typical of an urban environment, the number of casualties will be significant. For instance, at the evening rush hour $(4: 30 \mathrm{pm})$ in one calculational cell of the grid, as many as 15000 people could be within a 200-metre radius of any given point: some in buildings, some in vehicles, some pedestrians. Other locations will have comparable numbers, although the exact number is location and time dependent. All of these people will be exposed in one way or another to the direct and collateral effects of the detonation of high explosive.

*Adapted from data in References 28 and 29. 


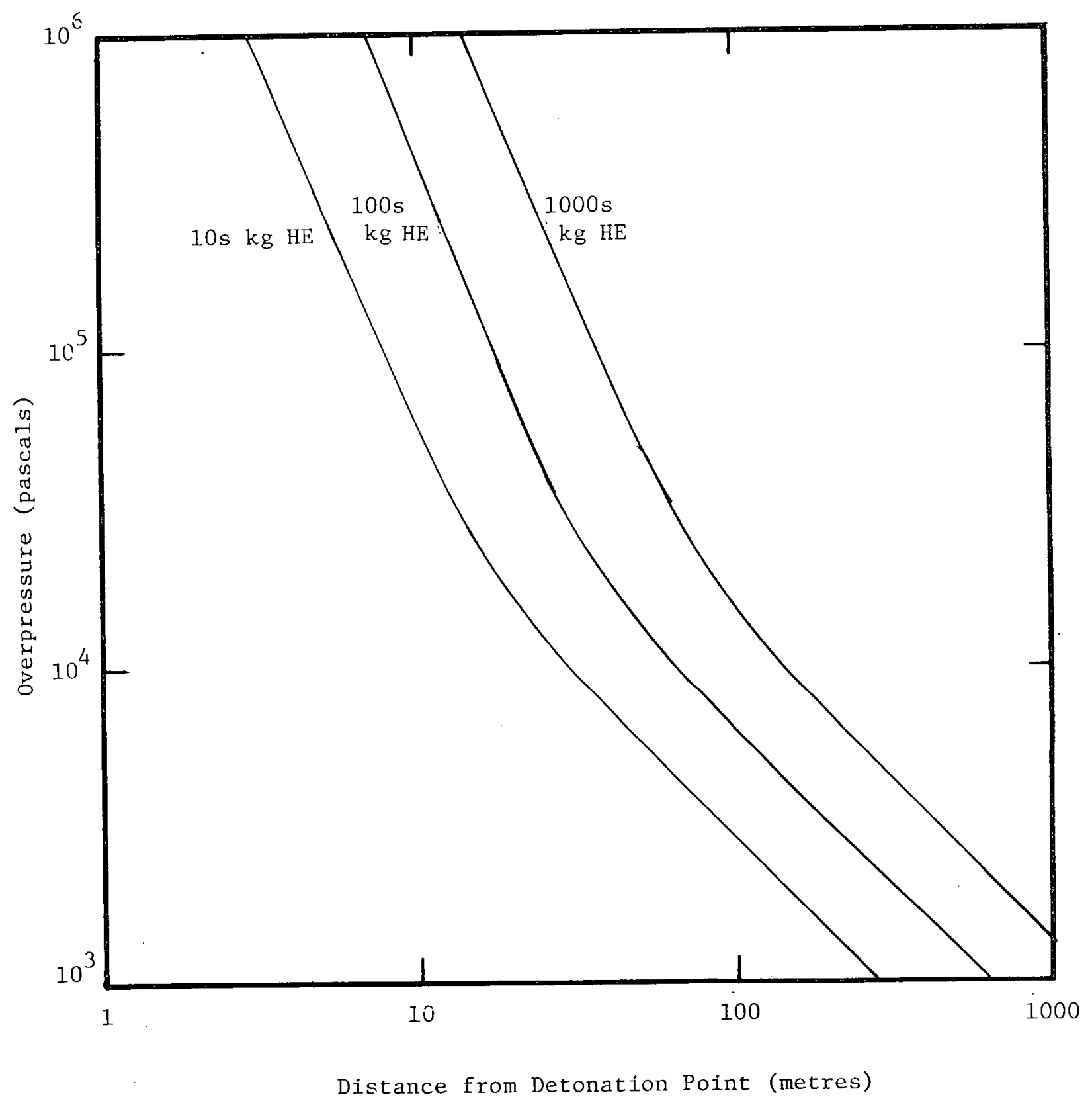

Figure 5-1. Blast Overpressure versus Distance for High-Explosive Charges 
People may be affected by the direct overpressure of a blast wave by being struck with flying debris from surrounding structures, or by striking the ground or other surfaces after being thrown by the gust loading from the blast wave. If just the direct effects of the overpressure are considered, Glasstone ${ }^{30}$ reports that there is a 0.99 probability of fatality for people exposed to overpressures of 380 to 450 $\mathrm{kPa}$. Assuming that an attack with tens to hundreds of kilograms of explosives occurs at a midblock location and in stalled traffic, and considering only pedestrians and those in vehicles, it is estimated that for the cited cell at rush hour, some 10 to 50 fatalities could result from overpressure alone. The total number of fatalities due to the explosion would be even greater due to the collateral damage mechanisms discussed above. Other cells would be comparable.

It will be noted later that these immediate, nonradiological (direct and collateral) effects of a sabotage attack in a densely populated area may be as significant or more significant than the radiological effects, even with attacks employing only tens of kilograms of high explosives.

\section{Consequences of the Radioactive Material Release--METRAN Estimates}

The METRAN model has been used to examine the three releases from a spent fuel cask summarized in "Summary of Estimated Release Fractions." The spent fuel radionuclide inventory has been generated using the latest version of the fuel burnup code ORIGEN, ${ }^{42}$ assuming light-water reactor fuel with $33000 \mathrm{MWd} /$ MTHM$^{*}$ burnup at $40 \mathrm{~kW} / \mathrm{kg}$ power density and 150 days cooling. The truck-mounted cask is assumed to contain radionuclides equivalent to 1.4 MTHM charged to the reactor. The resultant cask inventory is shown in Table 5-2. Consequence estimates have been generated for releases occurring in four different cells of the grid, at three times, and for midblock and intersection street locations. The calculation conditions are shown in Table 5-3. This calculational approach provides 24 separate consequence estimates for each assumed release magnitude. An example of one set is given in Table 5-4. Because of the limited area encompassed by the METRAN grid, the total population at risk is a strong function of the location of the release point, the weather (especially wind direction), and the time of day. Therefore, it is not appropriate to simply average all the estimates together to generate mean values for the city. For purposes of comparison, however, it is reasonable to average the consequence estimates for a release in a given cell at different times and wind speeds to obtain an average for each release location. Such averages are presented in Table 5-5 for the postulated releases from spent fuel casks.

The estimated consequences (Table 5-4) do show substantial sensitivity to the time of day for midblock releases and less so for intersection releases. However, varying the time at which a release occurs introduces two variables, population location and wind speed, the effects of which may be either offsetting or cumulative. The noontime and rush hour releases would have more people, both in buildings and outdoors, thus potentially increasing the number of exposures. But these times also have higher average wind speeds, so cloud dispersal (dilution) is enhanced. Nevertheless, for midblock releases in cell C, the influence of the diurnal variation in the population density in a predominantly business district is evident in that the estimated latent cancer fatalities for a midnight release are many times lower than those at noon or rush hour. Similar conditions exist for cell A. At the same time, the low wind speed at midnight permits sufficient exposure close-in to cause early fatalities, even with the smaller nighttime population. The reasons for the reduced sensitivity to time of day for intersection releases are less clear. It is presumed to be due in part to the manner in which the cloud splitting is handled in the model.

$\star_{\text {MTHM }}=$ Metric Tons of Heavy Meta1. 
Table 5-2

Spent Fuel Cask Radionuclide Inventory

1.4 MTHM Charged to Reactor (3 Elements) 33000 MWd/MTHM Burnup at $40 \mathrm{~kW} / \mathrm{kg}$ 150 Days Cooling

Radionuclide ${ }^{*}$

Co-58

Co- 60

$\mathrm{Kr}-85$

$\mathrm{Sr}-89$

$\mathrm{Sr}-90$

$\mathrm{Y}-90$

$\mathrm{Y}-91$

$\mathrm{Zr}-95$

Nb-95

Rn-103

$\mathrm{Ru}-106$

$\mathrm{Te}-127$

$\mathrm{Te}-127 \mathrm{~m}$

$\mathrm{Te}-129$

$\mathrm{Te}-129 \mathrm{~m}$

Cs- 134

Cs-136

Cs -137

$\mathrm{Ba}-140$

La- 140

$\mathrm{Ce}-141$

$\mathrm{Ce}-144$

Pr-143

Nd-147

$\mathrm{Np}-239$

Pu-238

Pu-239

Pu-240

$\mathrm{Pu}-241$

Am-241

Cm-242

Cm-244 $\frac{\text { Curies }}{3.277 \times 10^{3}}$

1. $55 \times 10^{2}$

1. $576 \times 10^{4}$

$2.243 \times 10^{5}$

$1.202 \times 10^{5}$

$1.202 \times 10^{5}$

$3.421 \times 10^{5}$

$5.434 \times 10^{5}$

$1.012 \times 10^{6}$

$1.738 \times 10^{5}$

$-5.848 \times 10^{5}$

$8.807 \times 10^{3}$

$8.991 \times 10^{3}$

$3.678 \times 10^{3}$

$5.793 \times 10^{3}$

$3.604 \times 10^{5}$

$3.295 \times 10^{1}$

$1.596 \times 10^{5}$

$8.371 \times 10^{2}$

$9.633 \times 10^{2}$

$1.113 \times 10^{5}$

$1.371 \times 10^{6}$

1. $268 \times 10^{3}$

$8.968 \times 10^{1}$

$3.046 \times 10^{1}$

$4.44 \times 10^{3}$

$4.648 \times 10^{2}$

$6.883 \times 10^{2}$

1. $555 \times 10^{5}$

$2.035 \times 10^{2}$

$2.620 \times 10^{4}$

4. $374 \times 10^{3}$

* Radionuclides with significant health effects based upon Reactor Safety Study (see Reference 26). 
Table 5-3

Summary of METRAN Case Conditions

1. Cell of Release

2. Time of Release

3. Location

4. Population Density

5. Wind Speed and Direction
A--Expressway

B--Industrial area

C--High population density

D--High population density

Noon

Evening rush hour

Midnight

Midblock

Intersection

Typical of hyperurban environs (varies with time and location)

Cel1 A--26 000 to $52000 / \mathrm{km}^{2}$

Ce11 B--24000 to $46000 / \mathrm{km}^{2}$

Ce11 C--50 000 to $77000 / \mathrm{km}^{2}$

Cell D--35 000 to $38000 / \mathrm{km}^{2}$

Noon--8 m/s, south

Rush hour-- $4 \mathrm{~m} / \mathrm{s}$, south

Midnight-- $2 \mathrm{~m} / \mathrm{s}$, south 
Table 5-4

METRAN Consequence Estimates -- Baseline Estimate: Spent Fuel ${ }^{a}$

\begin{tabular}{|c|c|c|c|c|c|}
\hline $\begin{array}{l}\text { Cell of } \\
\text { Release }\end{array}$ & Time & Location $^{c}$ & $\begin{array}{c}\text { Early d e } \\
\text { Fatalities }\end{array}$ & $\begin{array}{c}\text { Early } \\
\text { Morbidities }\end{array}$ & $\begin{array}{c}\text { Latent Cancer } \\
\text { Fatalities } \\
\end{array}$ \\
\hline \multirow[t]{6}{*}{ A } & 1200 & MB & 0 & 281 & 152 \\
\hline & 1630 & MB & 0 & 638 & 199 \\
\hline & 2400 & MB & $<1$ & 27 & 37 \\
\hline & 1200 & INT & 0 & 64 & 46 \\
\hline & 1630 & INT & $<1$ & 80 & 62 \\
\hline & 2400 & INT & 2 & 71 & 70 \\
\hline \multirow[t]{6}{*}{ B } & 1200 & MB & 0 & 20 & 42 \\
\hline & 1630 & $M B$ & 0 & 15 & 50 \\
\hline & 2400 & $M B$ & 2 & 22 & 30 \\
\hline & 1200 & INT & 0 & 71 & 67 \\
\hline & 1630 & INT & $<1$ & 90 & 75 \\
\hline & 2400 & INT & 1 & 59 & 47 \\
\hline \multirow[t]{6}{*}{ C } & 1200 & $M B$ & 0 & 697 & 269 \\
\hline & 1630 & MB & 0 & 880 & 318 \\
\hline & 2400 & $M B$ & 1 & 32 & 33 \\
\hline & 1200 & INT & 0 & 96 & 71 \\
\hline & 1630 & INT & $<1$ & 117 & 84 \\
\hline & 2400 & INT & $<2$ & 75 & 66 \\
\hline \multirow[t]{6}{*}{$\mathrm{D}$} & 1200 & $M B$ & 0 & 31 & 26 \\
\hline & 1630 & $\mathrm{MB}$ & 0 & 32 & 32 \\
\hline & 2400 & $M B$ & $<1$ & 21 & 34 \\
\hline & 1200 & INT & 0 & 31 & 26 \\
\hline & 1630 & INT & 0 & 32 & 32 \\
\hline & 2400 & INT & $<1$ & 21 & 34 \\
\hline
\end{tabular}

${ }^{a}$ Assumed release from a three-element cask: $10 \%$ of the noble gas and $0.07 \%$ of the solids as respirable material.

${ }^{b}$ See Table 5-3.

$\mathrm{C}_{\mathrm{MB}}=$ Midblock, INT = Intersection.

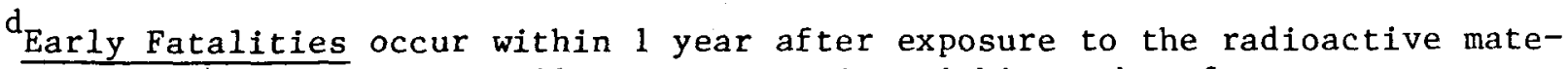
rial. Early Morbidities are illnesses appearing within weeks after exposure. Latent Cancer Fatalities occur over any time subsequent to the exposure as a result of the initial exposure and of any long-term exposure to low levels of contamination. I.e., 40 latent cancers would represent an average of less than 2 cancers per year over a 30-year period for the population group exposed.

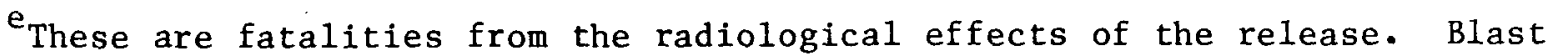
effects from explosives used to attack a cask could cause tens of fatalities (see the subsection, "Consequences of the Use of High Explosives"). In fact, if explosives are used, early radiological fatalities are highly unlikely because those close enough to receive lethal radiation doses would be killed by the explosion. That is, the lethal radius for blast effects exceeds the lethal radius for early deaths from radiation in this particular instance. 
Table 5-5

Consequence Estimates for Releases from Spent Fuel: Average for Three Release Times

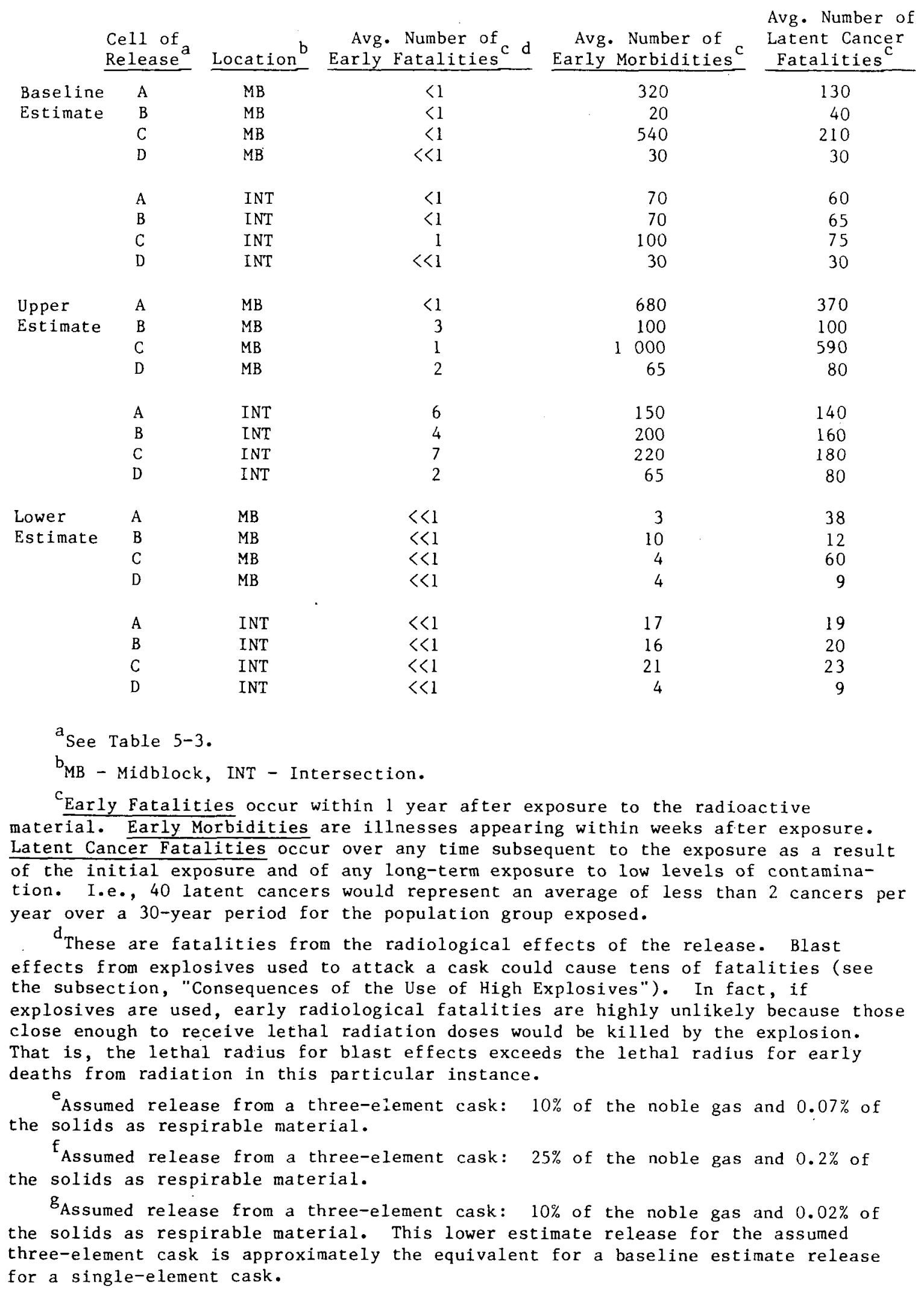


It should be noted that relatively small changes in population location can have a strong influence upon METRAN estimates of early effects. METRAN employs a threshold model for early effects; therefore, changes in where people are located can put significant numbers of people above or below a particular dose threshold.* At present, the pedestrian population density is a function of the time of day but not of the cell. The total number of pedestrians is cell dependent, however, because of the cell-to-cell variation in total street area. Building population and vehicular population are both time-of-day and cell dependent.

The magnitude of the averaged consequence estimates (Table 5-5) exhibits some correlation with the grid location of the release. Cell $\mathrm{C}$, which has the largest population density of the four cells considered, also exhibits the greatest number of estimated latent cancer fatalities for all release magnitudes. Similar trends hold for the early morbidities. Because there are so few early fatalities predicted for the smaller releases (baseline and lower estimates) and because they would necessarily occur very close to the release point, these results are not very sensitive to the cell of release.

In attempting to understand the variations in results shown in Tables $5-4$ and 5-5, it must be realized that the large number of variables in the METRAN model, many with rather subtle interactions, may make it impossible to isolate specific causes for the variations. As noted earlier, simply changing the time of day introduces both population and wind variations. Also, the finite size of the METRAN grid influences the results since for releases in cells near the boundary (such as cell A) and depending upon wind direction, the cloud may not traverse much of the grid. The doses to people in buildings depend on the total number of people in buildings and the average number of floors. Also, building heights or "surface roughness" will affect the atmospheric dispersion and consequently the projected dose. Finally, in the computation of cloud splitting, the interaction between street directions and wind direction is complex. For example, if a street is perpendicular to wind direction in a midblock release, there will be fewer early effects because the vertical dispersion reduces concentrations. But if the street is parallel to the wind vector, the cloud can move along the street and expose pedestrians. Given these and similar considerations, no attempt will be made to explain all the differences in the estimates. It should also be noted that evolutions in the data base and the modeling approach have led to results which differ from earlier drafts of this report. No attempt is made here to catalog all such changes. Rather, these predictions are the best estimates based on the current data and model.

The average consequence estimates for the three releases are shown in Table 5-6, when the release occurs in cell $\mathrm{B}$, near the grid center. The data simply indicate the relationship of consequences to release magnitude. It might be expected that the lower estimates would be an order of magnitude lower than the upper estimates since the principal change is reduction of the solids portion of the source term by an order of magnitude, and this is essentially the case.

*It is emphasized that a threshold model is used only for early effects. Long-term effects or latent cancer fatalities are estimated using population dose criteria in accord with accepted practice. 
Table 5-6

Average-Consequence Estimate Comparison, Midblock Releases from Spent Fuel in Cell B

\begin{tabular}{|c|c|c|c|}
\hline Estimate ${ }^{a}$ & $\begin{array}{c}\text { Early } b c \\
\text { Fatalities }\end{array}$ & $\begin{array}{c}\text { Early } \\
\text { Morbidities }\end{array}$ & $\begin{array}{l}\text { Latent Cancer } \\
\text { Fatalities } \\
\end{array}$ \\
\hline Baseline & $<1$ & 19 & 40 \\
\hline Upper & 2.8 & 99 & 104 \\
\hline Lower & $<<1$ & 10 & 12 \\
\hline
\end{tabular}

${ }^{a}$ See "Summary of Estimated Release Fractions."

${ }^{b}$ Early Fatalities occur within 1 year after exposure to the radioactive material. Early Morbidities are illnesses appearing within weeks after exposure. Latent Cancer Fatalities occur over any time subsequent to the exposure as a result of the initial exposure and of any long-term exposure to low levels of contamination; i.e., 40 latent cancers would represent an average of less than 2 cancers per year over a 30-year period for the population group exposed.

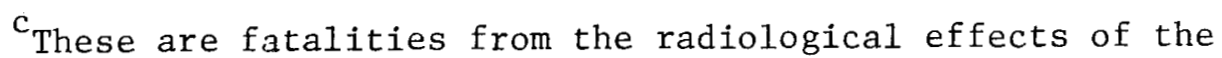
release. Blast effects from explosives used to attack a cask could cause tens of fatalities (see the subsection "Consequences of the Use of High Explosives.") In fact, if explosives are used, early radiological fatalities are highly unlikely because those close enough to receive lethal radiation doses would be killed by the explosion. That is, the lethal radius for blast effects exceeds the lethal radius for early deaths from radiation in this particular instance.

The estimates of early consequences also decline by a factor of 10 but this may be coincidental because the dose response is nonlinear and because of the threshold effects just discussed, which would not be expected to scale linearly with dose. That is, a change in source-term magnitude may or may not have a major impact upon the estimates, depending upon the number of persons receiving doses at or near a threshold. Also, in the computational scheme, the population dose to those who become early fatalities reduces the population dose available to induce latent cancers. This also contributes to the deviation of the latent cancer predictions from direct scaling.

In Table 5-7, the METRAN estimates for a release in cell $\mathrm{B}$ are compared with the estimates from a CRAC calculation for a release at the grid center and for the same area. 2627 (The CRAC estimates made in support of this work are described in the following subsection.) Generally speaking, there is agreement between the two calculational techniques. The larger estimates of early morbidities by METRAN are expected for four reasons:

1. METRAN is designed to handle the close-in meteorology with finer resolution than CRAC. 
2. Because METRAN was specifically designed for transportation accident conditions, it assumes diffusion of a "cold cloud;" that is, the release cloud has no thermal buoyancy, and therefore a large portion of the radioactive material stays near the ground where the pedestrians can be affected. This assumption also permits material to be deposited on the ground close-in, thus reducing the low-level population dose which leads to latent cancers.

3. The lower boundary of the METRAN grid is assumed to absorb $50 \%$ of the material that interacts with it, which tends to keep the centroid of the release cloud low.

4. METRAN assumes that all persons receiving doses above the early morbidity threshold become early morbidities, whereas CRAC uses dose response curves to estimate early morbidities.

The thermal source in CRAC was used to account for the effects of high explosives lofting the material and thus reducing the close-in ground level concentrations. The CRAC estimates were made with buoyancy in the cloud, the magnitude of which was chosen after consultation with personnel experienced with explosives. If CRAC is run without a thermal source in the cloud, the estimates of early effects rise. The authors suggest that thermal buoyancy be used for the sabotage cases. This can probably be treated in METRAN by the way the material is distributed in the four layers (see Appendix E).

Given the differences in the two models, the agreement of the latent cancer fatality estimates is considered good. The CRAC estimate of total latent cancer fatalities is greater than the METRAN estimate, but this is attributable in part to the differences in population and in part to the way the two models treat long-term exposure to contaminated ground. In METRAN, a permissible ground concentration level is specified by the analyst. As the release is followed, the resulting ground contamination is compared to the permissible levels. If contamination is greater than 40 times the permissible level, decontamination is assumed impossible, and the area is subject to permanent land-use denial, so there is no long-term exposure. In contrast, CRAC computes the dose commitment due to exposure to the ground contamination: if it is less than 25 rem lifetime (for urban populations), the population is exposed to that ground contamination. If the projected dose commitment in any spatial interval is greater than $25 \mathrm{rem}$, CRAC assumes that decontamination occurs (decontamination factor $(D F) \approx 20$ ) then again examines the dose commitment. If the projected dose still exceeds $25 \mathrm{rem}$ in any spatial interval, land-use denial is started. Because METRAN uses the fixed-level model with a low permissible level, there is more such area and thus less population exposure.

All of the consequence estimates have been made with the population "in-place." No attempt was made to model or account for evacuation to avoid early exposure because evacuation may not be possible in all instances. Effective evacuation could serve to reduce the estimated consequences in some cases. 
In summary, for the postulated baseline release from a truck-mounted spent fuel cask of $10 \%$ of the noble gases and $0.07 \%$ of the remaining radionuclides, a few early fatalities, tens to several hundreds of early morbidities, and tens to hundreds of latent fatalities may be expected.* If explosives are used, early radiological fatalities are highly unlikely because those close enough to receive lethal radiation doses would be killed by the explosion. That is, the lethal radius for blast effects exceeds the lethal radius for early deaths from radiation in this particular instance.

\section{Consequences of the Radioactive Material Release--CRAC Estimates}

CRAC, the consequence model of the Reactor Safety Study, was used to examine the postulated release from spent fuel casks for several reasons: (1) there is considerable experience available in the use of this model, (2) it allows consequences to be estimated out to considerable distances from the release point, and (3) parameters may be varied in the model to explore the effects of radiation exposure pathways.

In this particular analysis, the release is centered on the METRAN grid, using a single population distribution based on METRAN data for afternoon building occupancy. This single population distribution is just an approximation to the time varying METRAN data: CRAC does not have provisions for a time-varying distribution. For radial distances beyond the METRAN grid (approximately $5.25 \mathrm{~km}$ ), the detailed population distribution employed is equivalent to approximately 16000 people per $\mathrm{km}^{2}$ out to $16 \mathrm{~km}, 3800$ people per $\mathrm{km}^{2}$ between 16 and $48 \mathrm{~km}, 380$ people per $\mathrm{km}^{2}$ between 48 and $88 \mathrm{~km}$, and 38 people per $\mathrm{km}^{2}$ beyond $88 \mathrm{~km}$. The detailed distribution accounts for the fact that there is no population in the seaward area by setting the population equal to zero in certain segments. The total population thus included closely approximates the actual population within $800 \mathrm{~km}$ of the assumed release point.

CRAC operates basically on a radial computational mesh. The circular area is centered at the release point and divided into 16 segments of $22.5^{\circ}$. Each segment has 34 radial intervals. For this study, the interval spacing is approximately 60 metres out to 360 metres from the release point (to approximate METRAN); then the interval width expands. The outer radius of each interval is shown in Table 5-8. In this analysis, a release cloud is permitted to traverse each segment ( 16 segments for 91 sequences of weather conditions), representative of weather near the release point. The mean value of reported consequences represents the mean from 1456 separate trials. Computing the mean in this manner essentially accounts for any weather in any direction.

Each of the three releases mentioned earlier was examined under the following conditions. First, the population was limited to the area $\left(100 \mathrm{~km}^{2}\right)$ covered by the METRAN grid. Second, the population base was expanded to include people out to 800 $\mathrm{km}$, as described earlier. The results are shown in Table 5-9.

*Other analyses indicate that consequence estimates (LCFs) for spent fuel cooled as long as 5 years will be comparable to those for fuel cooled only 150 days. For example, using CRAC and the population to $800 \mathrm{~km}$ (see Table 5-9) the consequences of a release from 5-year-old fuel are $70 \%$ of those from 150-day-old fuel. 
Several observations are in order concerning these results:

1. Approximately $60 \%$ of the latent cancer fatalities predicted occur close to the release point (less than $5 \mathrm{~km}$ ). This is observed by comparing the results for only the METRAN grid with the results where there are people present to $800 \mathrm{~km}$.

2. Inhalation of radionuclides is the dominant exposure mode for latent effects (99\%). This is attributable to the presence of significant quantities of actinides (alpha emitters) which must be taken into the body to cause significant exposure.

3. The radius for early fatalities and early morbidities (see Table 5-9) are so close to the release point that the effects of any explosive used would dominate.

An alternate view of the spatial distribution of latent cancer fatalities is shown in Figure 5-2, where cumulative latent fatalities are plotted against downwind distance from the release point. The change in slope in the vicinity of $10 \mathrm{~km}$ indicates that, as the population distribution has been modeled here and using the CRAC model, the majority of the latent fatalities will occur in the population near the release point. This is further illustrated by Figure 5-3, in which the projected 1-year lung dose is shown as a function of distance from the release point. For these estimates, lung cancers account for more than $80 \%$ of the predicted fatalities due to initial exposure and nearly $50 \%$ of the total latent cancer fatalities.

Although the emphasis has been on releases from truck-mounted spent fuel casks (based upon analysis and arguments in Sections 5.2 and 5.3), there is a good possibility that shipments will also be made by rail. Therefore, the baseline estimate release has been examined assuming a rail cask as the source. Although it is certainly not clear that rail traffic would necessarily move through urban centers, this limited analysis with CRAC provides some comparison with truckmounted cask results. An attack against a rail cask would probably involve larger quantities of high explosive than an attack against a truck-mounted cask. The results are shown in Table 5-10. Comparison of these results with the earlier CRAC estimates for the release from truck-mounted casks indicates that the estimates of early fatalities and morbidities do not scale linearly with source strength; again, this is because of the response models discussed earlier. Using CRAC, the predicted latent cancer fatalities do scale approximately as the total source strength for the same population distribution. This was not the case using METRAN. Again, it should be noted that the majority of the fatalities occur close to the release point with the population distribution employed here. The population densities are so high that chronic exposure pathways other than inhalation and groundshine are insignificant. This would not be true for a uniform population distribution of hundreds per square kilometre.

One final comment on the CRAC analyses: In all of the consequence estimates reported here, the "central estimate" dose model was used based upon the previous usage in the Reactor Safety Study. 2627 Several calculations were made using the "linear response" dose model in which no thresholds or response curves are assumed. The net result is that latent cancer fatalities are approximately 2 to $2-1 / 4$ times greater if linear response is assumed. 
Table 5-8

CRAC Estimate -- Radial Intervals

\begin{tabular}{|c|c|c|}
\hline Interval No. & $\begin{array}{c}\text { Radius } \\
(\mathrm{km}) \\
\end{array}$ & $\begin{array}{l}\text { Radius } \\
\text { (mi) }\end{array}$ \\
\hline $\begin{array}{l}1 \\
2\end{array}$ & $\begin{array}{l}0.06 \\
0.12\end{array}$ & $\begin{array}{l}0.04 \\
0.08\end{array}$ \\
\hline 3 & 0.19 & 0.12 \\
\hline 4 & 0.26 & 0.16 \\
\hline 5 & 0.32 & 0.2 \\
\hline 6 & 0.37 & 0.23 \\
\hline 7 & 0.5 & 0.31 \\
\hline 8 & 0.8 & 0.5 \\
\hline 9 & 1.2 & 0.75 \\
\hline 10 & 1.6 & 1.0 \\
\hline 11 & 2.4 & 1.5 \\
\hline 12 & 3.2 & 2. \\
\hline 13 & 3.6 & 2.27 \\
\hline 14 & 7.2 & 4.5 \\
\hline 15 & 11.2 & 7.0 \\
\hline 16 & 16.0 . & 10.0 \\
\hline 17 & 28.0 & 17.5 \\
\hline 18 & 32.0 & 20 \\
\hline 19 & 40.0 & 25 . \\
\hline 20 & 48.0 & 30 . \\
\hline 21 & 56.0 & 35. \\
\hline 22 & 64.0 & 40 \\
\hline 23 & 72.0 & 45. \\
\hline 24 & 80.0 & 50. \\
\hline 25 & 88.0 & 55. \\
\hline 26 & 96.0 & 60. \\
\hline 27 & 104. & 65. \\
\hline 28 & 112 . & 70 \\
\hline 29 & 136 & 85. \\
\hline 30 & 160 & 100 \\
\hline 31 & 240 . & 150. \\
\hline 32 & 320 . & 200 \\
\hline 33 & 500. & 350. \\
\hline 34 & 800 & 500 \\
\hline
\end{tabular}


Table 5-9

CRAC Consequence Estimates for Releases from Spent Fuel Casks

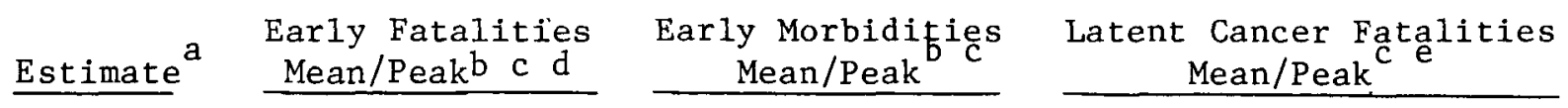

Initial Tota1

1. Population Limited to METRAN Grid

$\begin{array}{lcccr}\text { Baseline } & 0.2 / 19 & 1 / 76 & 17 / 89 & 77 / 310 \\ \text { Upper } & 1 / 86 & 11 / 220 & 51 / 220 & 190 / 790 \\ \text { Lower } & 0 & 0.3 / 32 & 5 / 23 & 24 / 100 \\ \text { Baseline } & 0.2 / 19 & 1 / 76 & 17 / 86 & 77 / 310\end{array}$

2. Population Present to $800 \mathrm{~km}$

$\begin{array}{lcccc}\text { Baseline } & 0.2 / 19 & 1 / 76 & 27 / 90 & 130 / 490 \\ \text { Upper } & 1 / 86 & 11 / 220 & 80 / 280 & 360 / 1350 \\ \text { Lower } & 0 & 0.3 / 32 & 7 / 26 & 40 / 140 \\ \text { Baseline } & 0.2 / 19 & 1 / 76 & 26 / 88 & 130 / 490\end{array}$

$\mathrm{a}_{\text {See }}$ "Summary of Estimated Release Fractions."

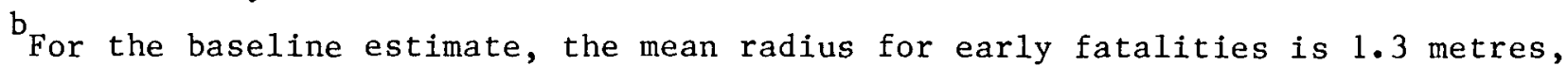
peak 60 metres. Mean radius for morbidities is 2.6 metres, peak 60 metres.

Early Fatalities occur within 1 year after exposure to the radioactive material. Early Morbidities are illnesses appearing within weeks after exposure. Latent Cancer Fatalities occur over any time subsequent to the exposure as a result of the initial exposure and of any long-term exposure to low levels of contamination. I.e., 40 latent cancers would represent an average of less than 2 cancers per year over a 30-year period for the population group exposed.

$d$ These are fatalities from the radiological effects of the release. Blast effects from explosives used to attack a cask could cause tens of fatalities (see the subsection, "Consequences of the Use of High Explosives"). In fact, if explosives are used, early radiological fatalities are highly unlikely because those close enough to receive lethal radiation doses would be killed by the explosion. That is, the lethal radius for blast effects exceeds lethal radius for early deaths from radiation in this particular instance.

Initial: These are latent cancer fatalities predicted to occur as result of initial exposure. In CRAC, this is direct cloudshine and 1-day exposure to ground contamination plus dose in 1 year due to radionuclides inhaled during cloud passage. Total: These are total latent cancer fatalities predicted as result of initial exposure, resuspension, and long-term exposure to contaminated ground.

${ }^{\mathrm{f}}$ Only exposure pathway is from inhaled radionuclides. 


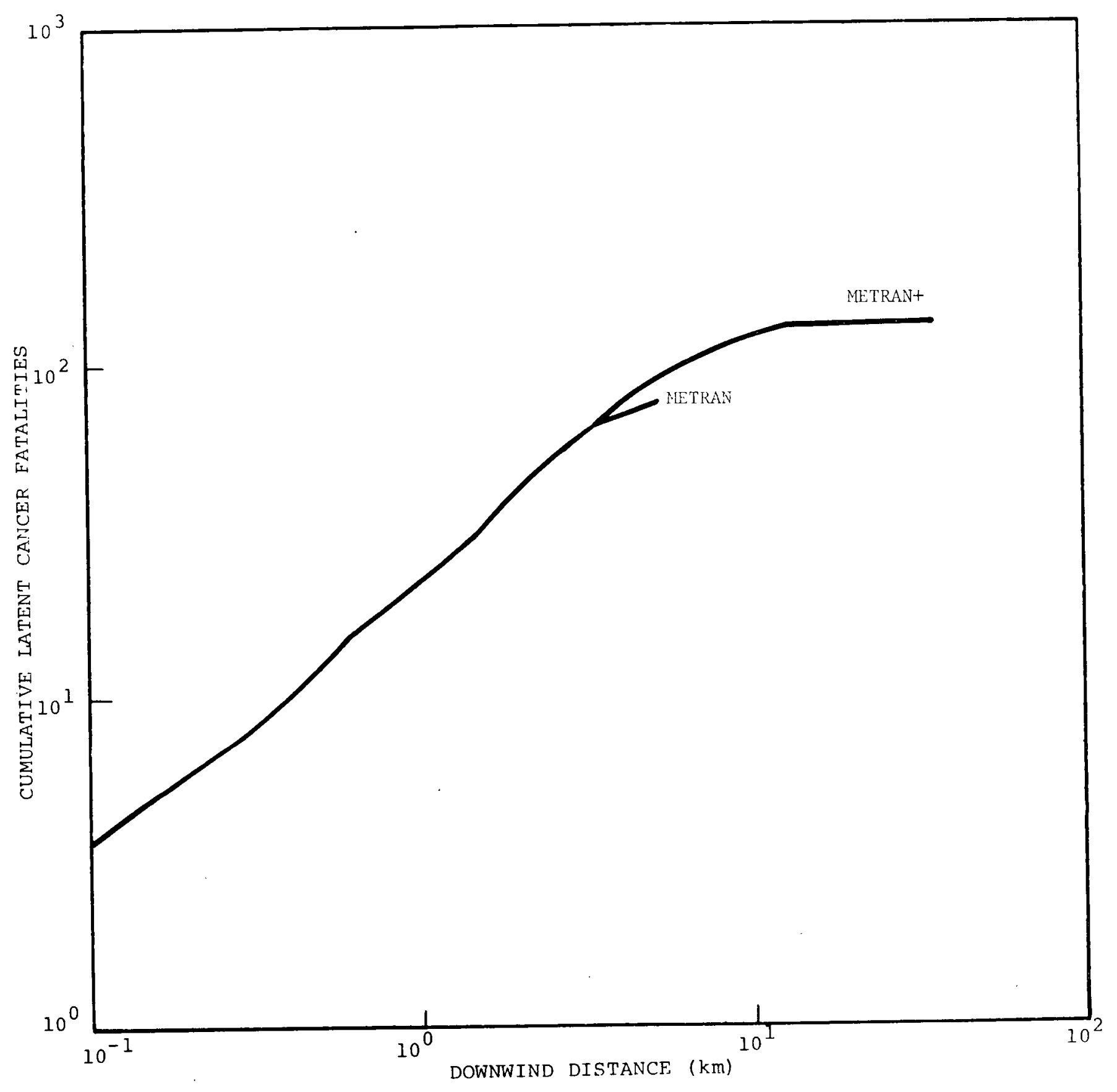

Figure 5-2. Cumulative Latent Cancer Fatalities as Function of Distance from Release Point (Baseline Estimate Release from Spent Fue1) 


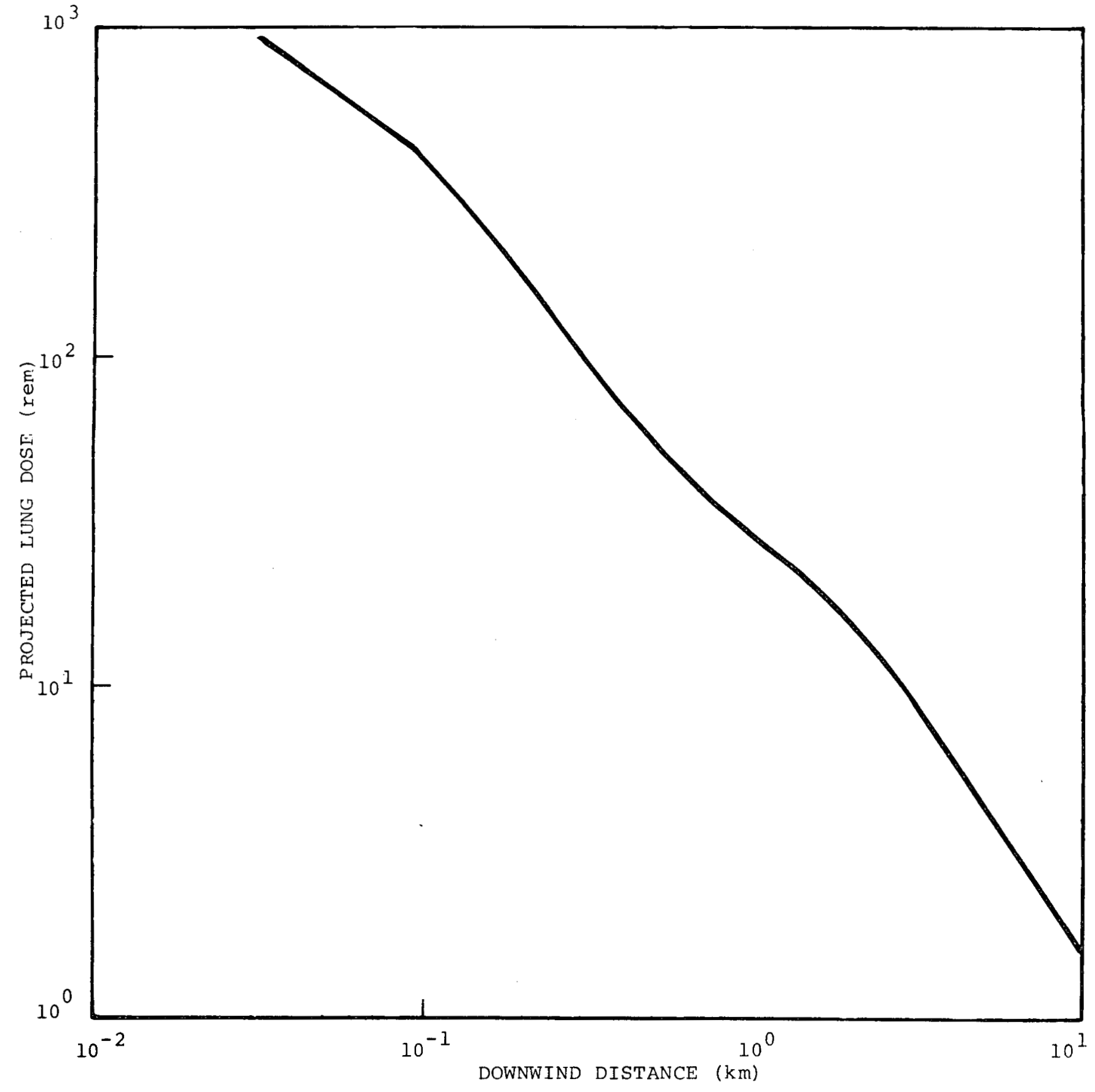

Figure 5-3. Projected 1-Year Lung Dose as a Function of Distance from Release Point (Baseline Estimate Release from Spent Fuel) 
Table 5-10

CRAC Consequence Estimates for Release from Railcar-Mounted Spent Fuel Cask

Estimate $\begin{gathered}\text { Early Fatalities } \\ \text { Mean/Peak }\end{gathered}$

1. Population Limited to METRAN Grid

Baseline

$2 / 92$

2. Population Present to $800 \mathrm{~km}$

Baseline

$2 / 92$

$15 / 240$

$95 / 360$

Early Morbidities Mean/Peak
Latent Cancer Fatalities Mean/Peak

Initial Total

$62 / 290$

$220 / 930$

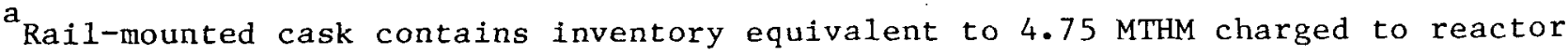
compared to the 1.4 MTHM for truck-mounted cask.

b Assumed release: $10 \%$ of the noble gases and $0.07 \%$ of the solids as respirable material.

In summary, estimates for latent cancer fatalities obtained from CRAC essentially agree with estimates from METRAN. The analysis indicates that tens to hundreds of latent fatalities may occur as a result of the baseline estimate release in a densely populated area. The analysis also indicates that for such releases, given these population densities, early fatalities and morbidities are restricted to areas very close to the release point, and the latent fatalities will be manifest mainly in the population located within 10 to $15 \mathrm{~km}$ of the release point.

\subsubsection{Nonfissile Isotopes (Large Sources)}

It was established earlier that any realistic attempt at dispersal of material shippec in large casks can be expected to involve significant quantities of high explosive. The discussion in "Consequences of the Use of High Explosives" of those consequences directly associated with the employment of high explosives also applies here. Although shipments of nonfissile isotopes can involve large quantities, as much as $10^{6}$ curies, there is usually only a single radionuclide involved, as contrasted to the tens of nuclides involved in a spent fuel shipment.

Both the METRAN and CRAC models were used to estimate the consequences for three levels of respirable release, based upon the data in "Summary of Estimated Release Fractions." The releases considered are $0.28 \%, 0.8 \%$, and $0.08 \%$ of the tota 1 
shipment. Based upon available data, ${ }^{23}$ two isotope shipments were examined: 200 kilocuries ( $\mathrm{kCi}$ ) of Co-60 in metal form and $15 \mathrm{kCi}$ of $\mathrm{Cs}-137$ as cesium chloride. The results from METRAN and CRAC are summarized in Table 5-11. The results from METRAN are for a cel1-B midblock release averaged over the three release times (noon, rush hour, and midnight). As before, cell $B$ is used because the CRAC estimates assume a release at the grid center and cell $\mathrm{B}$ is the adjacent cell.

METRAN consistently predicts early effects for the cobalt releases while CRAC does not. As discussed earlier, this is not unexpected because of the different techniques by which the two codes handle atmospheric dispersion and cloud depletion, and because of the use of thresholds in METRAN versus dose-response curves in CRAC. METRAN has more detail close to the release point, and the lower boundary is assumed to absorb $50 \%$ of the material which interacts with it. As discussed earlier under the results for spent fuel, the differences in total latent cancer predictions may be attributable to the differing techniques used to handle long-term exposure to contaminated ground and to population differences. In the event of a release from a Co-60 shipment, there could be several early morbidities and tens to hundreds of latent cancer fatalities. If high explosives are the means of dispersal, then the immediate effects of the blast might be expected to overshadow the radiological effects. Similar results are presented for a release of cesium, albeit the predicted consequences are much smaller than those for the cobalt shipment. The larger estimate of latent cancer fatalities for the smallest cesium release, as compared to the baseline release, is a result of the way METRAN handles cleanup versus interdiction. That is, if the contamination is above a specified level, public use is denied and chronic exposure is reduced. For the smaller release, this would not occur, and thus additional latent cancers are predicted. In this case, especially, it is observed that the effects of using high explosives to initiate the release from a shielded cask can dominate the public consequences of the event.

In summary, the atmospheric dispersal of material from shipments of nonfissile isotopes may lead to tens to hundreds of latent fatalities. The immediate consequences of the use of high explosives in the attack will likely be comparable.

\subsubsection{Less-than-Strategic Quantities of SNM}

Most shipments of less-than-strategic quantities of special nuclear materials (SNM) involve less than 100 grams of plutonium. ${ }^{23}$ Therefore, a scenario involving theft or multiple thefts followed by a dispersal on a city street is postulated. Although plutonium in oxide form is a powder that could be "dumped" or thrown from a high building, such an act would result in limited dispersal. Explosive dispersal is therefore assumed in this analysis. Because it is difficult to even get plutonium dioxide powder airborne in significant quantities, it was assumed for purposes of the consequence estimates that an adversary would not attempt a dispersal with less than 100 grams.

In this analysis, the plutonium mixture employed is characteristic of that from reprocessing of reactor fuel 1 year after removal from the reactor. The isotopic composition of this plutonium is given in Table 5-12. Based upon the earlier discussion, $20 \%$ of the dispersed material is assumed to be of respirable size. The consequences of an outdoor release were estimated with METRAN and CRAC. The averaged results from the METRAN predictions are presented in Table 5-13, and the METRAN results from a release in cell $\mathrm{B}$ are compared with CRAC estimates in Table 5-14. 
Table 5-11

\section{METRAN and CRAC Consequence Estimates -- Releases of Nonfissile Isotopes (Large Sources)}

$$
\begin{array}{ccc}
\text { Early a b } & \text { Early a } & \text { Latent Cancer } \\
\text { Fatalities } & \text { Fatalitics } \\
\hline
\end{array}
$$

1. Cobalt (200 liCi Shipment, $0.28 \%$ Respirable Release)

$\begin{array}{lrcr}\text { METRAN }^{2} & \ll 1 & 2.2 & 4.1 \\ \text { CRAC }^{\mathrm{C}} & 0 & 0 & 53 \\ \text { CRAC }^{2} & 0 & 0 & 106\end{array}$

2. Cobalt (200 kCi Shipment, $0.8 \%$ Respirable Release)

$\begin{array}{lccr}\text { METRAN } & & 11.2 & 9.9 \\ \text { CRAC }^{c} & 1 & 11.2 & 93 \\ \text { CRAC }^{e} & 0 & 0 & 240\end{array}$

3. Cobalt ( $200 \mathrm{kCi}$ Shipment, $0.08 \%$ Respirable Release)

$\begin{array}{lccc}\operatorname{METRAN}^{c} & 0 & 0.4 & 1.9 \\ \text { CRAC }^{\mathrm{C}} & 0 & 0 & 19 \\ \text { CRAC }^{\mathrm{N}} & 0 & 0 & 3.4\end{array}$

4. Cesium ( $15 \mathrm{kCi}$ Shipient, $0.28 \%$ Respirable Release)

$\begin{array}{llll}\text { METRAN }^{c} & 0 & 0 & 4.8 \\ \text { CRAC }^{\text {CRAC }} & 0 & 0 & 5.5 \\ & 0 & 0 & 9 .\end{array}$

5. Cesium ( $15 \mathrm{kCi}$ Shipment, $0.8 \%$ Respirable Release)

$\begin{array}{lccc}\text { METRAN }^{c} & 0 & 0.6 & 1.1 \\ \text { CRAC }^{\text {CRAC }} & 0 & 0 & 14 \\ & 0 & 0 & 25\end{array}$

6. Cesium ( $15 \mathrm{kCl}$ Shipment, $0.08 \%$ Respirable Release)

$\begin{array}{llll}\text { METRAN }^{c} & 0 & 0 & 4.5 \\ \text { CRAC }^{\text {CRAC }} & 0 & 0 & 1.7 \\ & 0 & 0 & 2.7\end{array}$

a Early fatalities occur within 1 year after exposure to the radioactive material. Early Morbidities are illresses appearing within weeks after exposure. Latent Cancer fatalities occur over any time subsequent to the exposure as a result of the initial exposure and of any Jong-term exposure to low levels of contamination. I.e., 40 latent cancers would represent an average of less than 2 cancers per year over a 30 -year period for the population group exposed.

b These are fatalities from the radiological effects of the release. Blast effects from explosives used to attack a cask could cause tens of fatalitifs (see the subsection, "Consequences of the Use of lligh Explosives"). In fact, if explosives are used, early radiological fatalities are highly unlikely because those close enough to receive lethal radiation doses would be killed by the explosion. That is, the lethal radius for blast effects cxcecds the lethal radius for early deaths from radiation in this particular instance.

CAverage of midblock release for threc release times in cell B.

diean from CRAC for grid population only.

CMean from CRAC for grid plus population to $800 \mathrm{~km}$. 
Table 5-12

Isotopic Composition of Plutonium Shipment*

\begin{tabular}{lr} 
Isotope & Weight Perc \\
\cline { 2 - 2 } & 1.79 \\
$\mathrm{Pu}-238$ & 60.65 \\
$\mathrm{Pu}-239$ & 22.64 \\
$\mathrm{Pu}-240$ & 10.95 \\
$\mathrm{Pu}-241$ & 3.63 \\
$\mathrm{Pu}-242$ & 0.43 \\
$\mathrm{Am}-241$ & 100.00
\end{tabular}

* Based upon LWR fuel having 33000

MWd/MTHM at $40 \mathrm{~kW} / \mathrm{kg}$ burnup, 1 year after

removal from the reactor.

In contrast to the other releases investigated, where early morbidities (especially with METRAN) often equal or exceed the latent cancer fatalities, the latent effects are by far the most dominant from the dispersal of SNM. This is not surprising because the plutonium is an alpha emitter; and cancers of the lung ( $42 \%)$ and bone (40\%), where the plutonium is deposited, dominate the latent fatalities. This also implies that under the overall assumptions of the study, the dispersal of a kilogram of plutonium has the potential, in the long term, to cause significant numbers of fatalities. The METRAN and CRAC estimates again show reasonable agreement in the latent predictions. In this instance, the METRAN early estimates are probably more reasonable because this dispersal, is essentially a cold cloud compared to the release from the spent fuel cask.

Neither METRAN nor CRAC was designed to analyze the effects of a release in an enclosed sports arena. In an attempt to explore the effects of such a release, CRAC was exercised for plutonium dispersal with small spatial zones, no heat in the release cloud, and a population density that approximates a large outdoor stadium holding 100000 spectators. These results suggest that tens to hundreds of early fatalities and hundreds to thousands of early morbidities and latent fatalities can be caused. The results also suggest that more than $90 \%$ of the effects would be manifest in those exposed at the stadium.

In summary, a public dispersal of approximately $1 \mathrm{~kg}$ of plutonium could produce a few early fatalities, tens to hundreds of early morbidities, and hundreds to thousands of latent fatalities. A similar release under the conditions of an outdoor sporting event could increase these consequences somewhat.

\subsubsection{Low-Level Waste}

As indicated earlier, because there is so little activity available in low-level waste shipments ( 1000 to 5000 curies), it is unlikely that any attempt at dispersal would be made with the intent to create public harm. Nevertheless, because an attack on low-level waste could have nuisance value, the effects of a release are considered. 
Table 5-13

Average METRAN Consequence Estimates -- Dispersal

of Less-than-Strategic Quantities of SNM

\begin{tabular}{cccc} 
Cel1 of Release & $\begin{array}{c}\text { Early } \\
\text { Fatalities* }\end{array}$ & $\begin{array}{c}\text { Early } \\
\text { Morbidities* }\end{array}$ & $\begin{array}{c}\text { Latent Cancer } \\
\text { Fatalities* }\end{array}$ \\
\hline 1000-g Dispersal & 0.2 & 486 & 1808 \\
A & 0.3 & 154 & 552 \\
B & 0.3 & 766 & 2897 \\
D & 0.1 & 127 & 411
\end{tabular}

$\underline{100-g \text { Dispersal }}$

$\begin{array}{rrrr}\text { A } & 0 & 93 & 192 \\ \text { B } & 0 & 8 & 59 \\ \text { C } & 0 & 237 & 296 \\ \text { D } & 0 & 13 & 45\end{array}$

*Early Fatalities occur within 1 year after exposure to the radioactive material. Early Morbidities are illnesses appearing within weeks after exposure. Latent Cancer Fatalities occur over any time subsequent to the exposure as a result of the initial exposure and of any long-term exposure to low leve1s of contamination. I.e., 40 latent cancers would represent an average of less than 2 cancers per year over a 30-year period for the population group exposed.

Table 5-14

Comparison of METRAN ${ }^{a}$ and CRAC Consequence Estimates -Dispersal of Less-than-Strategic Quantities of SNM

\begin{tabular}{|c|c|c|c|}
\hline & $\begin{array}{c}\text { Early } b \\
\text { Fatalities }\end{array}$ & $\begin{array}{c}\text { Early } \\
\text { Morbidities }\end{array}$ & $\begin{array}{c}\text { Latent Cancer } \\
\text { Fatalities } \\
\end{array}$ \\
\hline \multicolumn{4}{|l|}{ 1000-g Dispersal } \\
\hline $\begin{array}{l}\text { METRAN } \\
\text { CRAC }\end{array}$ & $\begin{array}{l}0.3 \\
0.08\end{array}$ & $\begin{array}{r}154 \\
5\end{array}$ & $\begin{array}{l}552 \\
660\end{array}$ \\
\hline 100-g Dispersal & . & & \\
\hline $\begin{array}{l}\text { METRAN } \\
\text { CRAC }\end{array}$ & $\begin{array}{l}0 \\
-\end{array}$ & $\begin{array}{l}8 \\
-\end{array}$ & $\begin{array}{l}59 \\
60\end{array}$ \\
\hline
\end{tabular}

average values for release in cell $\mathrm{B}$.

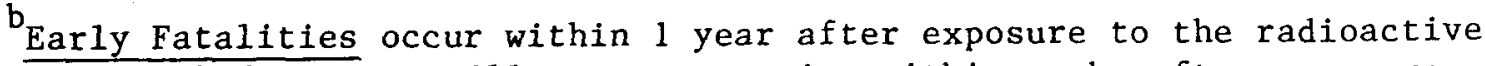
material. Early Morbidities are illnesses appearing within weeks after exposure. Latent Cancer Fatalities occur over any time subsequent to the exposure as a result of the initial exposure and of any long-term exposure to low levels of contamination. I.e., 40 latent cancers would represent an average of less than 2 cancers per year over a 30-year period for the population group exposed. 
Assuming that $1 \%$ of the shipment is released in respirable form, about 50 curies could be released. It is difficult to predict which radionuclides might be included in a specific shipment of such wastes. However, considering that the release of 560 curies of Co-60 only leads to tens of casualties and that the release of 42 curies of $\mathrm{Ce}-137$ leads to essentially no casualties in a densely populated area (see Section 5.4.3), it follows that the dispersal of 50 curies of low-level waste is unlikely to result in any illness or fatalities. To support this conclusion, CRAC has been exercised for a 50-curie release which contains approximately $30 \%$ cesium, $50 \%$ ruthenium and $18 \%$ strontium. For this situation, CRAC predicts no early effects and less than one latent cancer fatality (i.ẹ., mean and peak values are less than 1 ).

In summary, a release from low-level wastes would pose no significant hazard to the general public.

\subsubsection{Consequences of a Release of Radioactive Materia1--Areas Affected and Economic Impact}

In the preceding sections, the METRAN and CRAC models were employed to examine the public health consequences of a deliberate release of radioactive materials from various shipping containers. Another aspect of such releases that must be considered is the extent of. the area which may be contaminated by deposition from the passing cloud and the costs associated with cleanup, including those associated with temporary or permanent relocation of the affected population.

At present, the METRAN model does not include the capability to follow the cloud precisely and thereby estimate the actual affected area. However, METRAN results do indicate which cells are affected and the fractional area of each cell involved: that is, those cells within the computational grid in which some concentration of airborne material appears. Figure 5-4 indicates the cells affected by a specific release at midblock from a spent fuel cask in cell B for the three release times considered in the study. The prevailing wind flow is from the south. At noon, higher wind speeds cause some cells adjacent to the release to be skipped, while at midnight, low speed allows lateral diffusion to occur.

Estimated releases in other cells exhibit similar behavior. It is possible to extract an estimate of the surface area traversed by the cloud from the CRAC atmospheric dispersal model. Because the area estimate is a mean value from many computations, it is not directionally dependent; that is, it is a mean downwind area: For purposes of comparison, Figure 5-5 shows the CRAC estimate of cloud coverage superimposed on one of the METRAN calculations. This indicates that near the release, the affected area is much smaller than would be predicted by simply counting cells in the METRAN grid in which activity appears. Figure 5-6 shows the CRAC mean estimate of cloud area out to $2.5 \mathrm{~km}$ from the release point, and Figure 5-7 shows the mean cloud area out to $100 \mathrm{~km}$.

To estimate the economic impact of the releases due to sabotage, some idea of the potential surface contamination levels is required. For this analysis, it is assumed that those surface areas with more than $0.2 \mu \mathrm{Ci} / \mathrm{m}^{2}$ will require decontamination. 32 This is about a factor of 3 lower than cleanup levels reported and used elsewhere. $933 \quad 34$ It is further assumed that those building surfaces with contamination levels greater than $8 \mu \mathrm{Ci} / \mathrm{m}^{2}$ (DF factors greater than 40 ) could not be decontaminated and public use would have to be denied. The model employed here (described in detail in Appendix K) accounts for the costs of land-use denial as well as other costs such as emergency response, radiological surveys, evacuation, security, etc. 
TII Ncon Release

DA Rush-Hour Release

DV Midnight Release

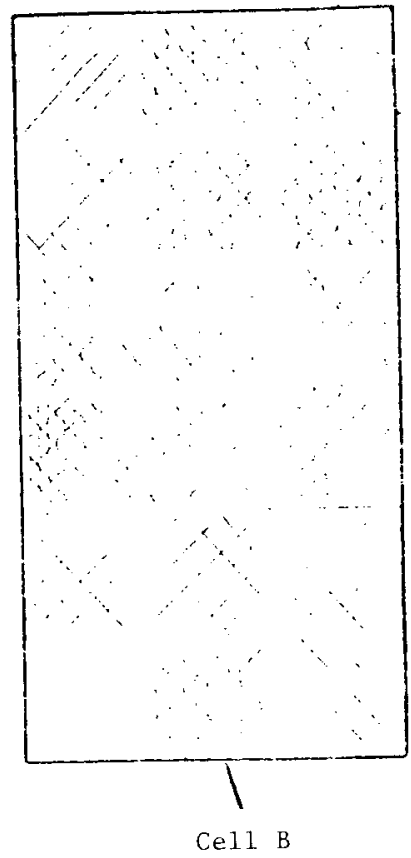

Figure 5-4. Pattern of Grid Sectors Affected by a Release in $\mathrm{Cell} \mathrm{B}$

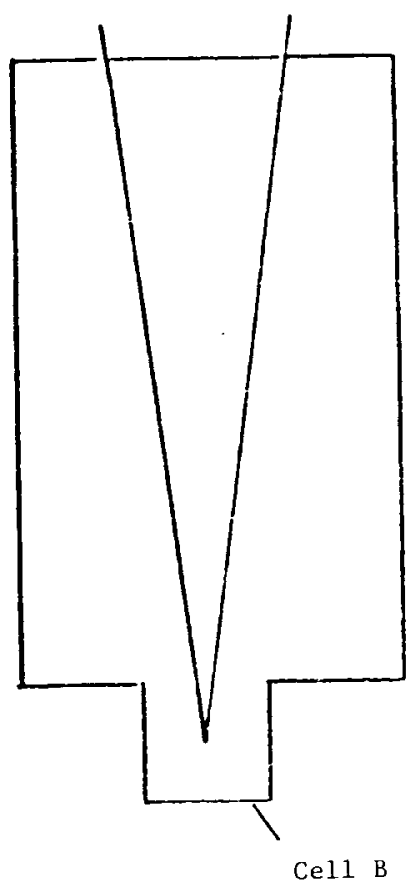

Figure 5-5. Mean Area Affected by Release in Cell B (CRAC Estimate) Superimposed on METRAN Cells Affected by Rush Hour Release in Cell B 


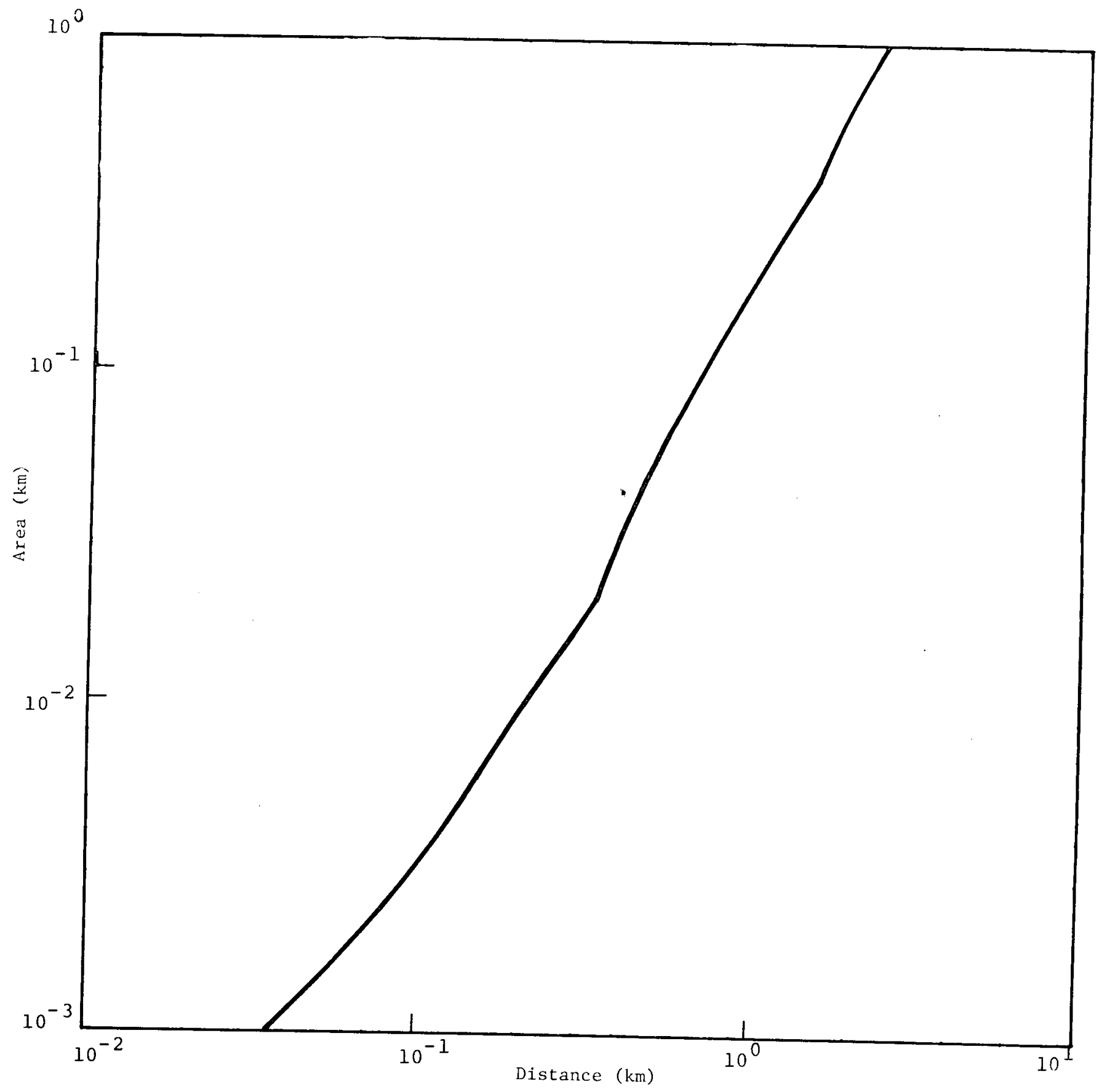

Figure 5-6. CRAC Estimate of Cumulative Area Traversed by Radioactive Cloud as Function of Downwind Distance from Release Point (10 metres to 10 kilometres) 


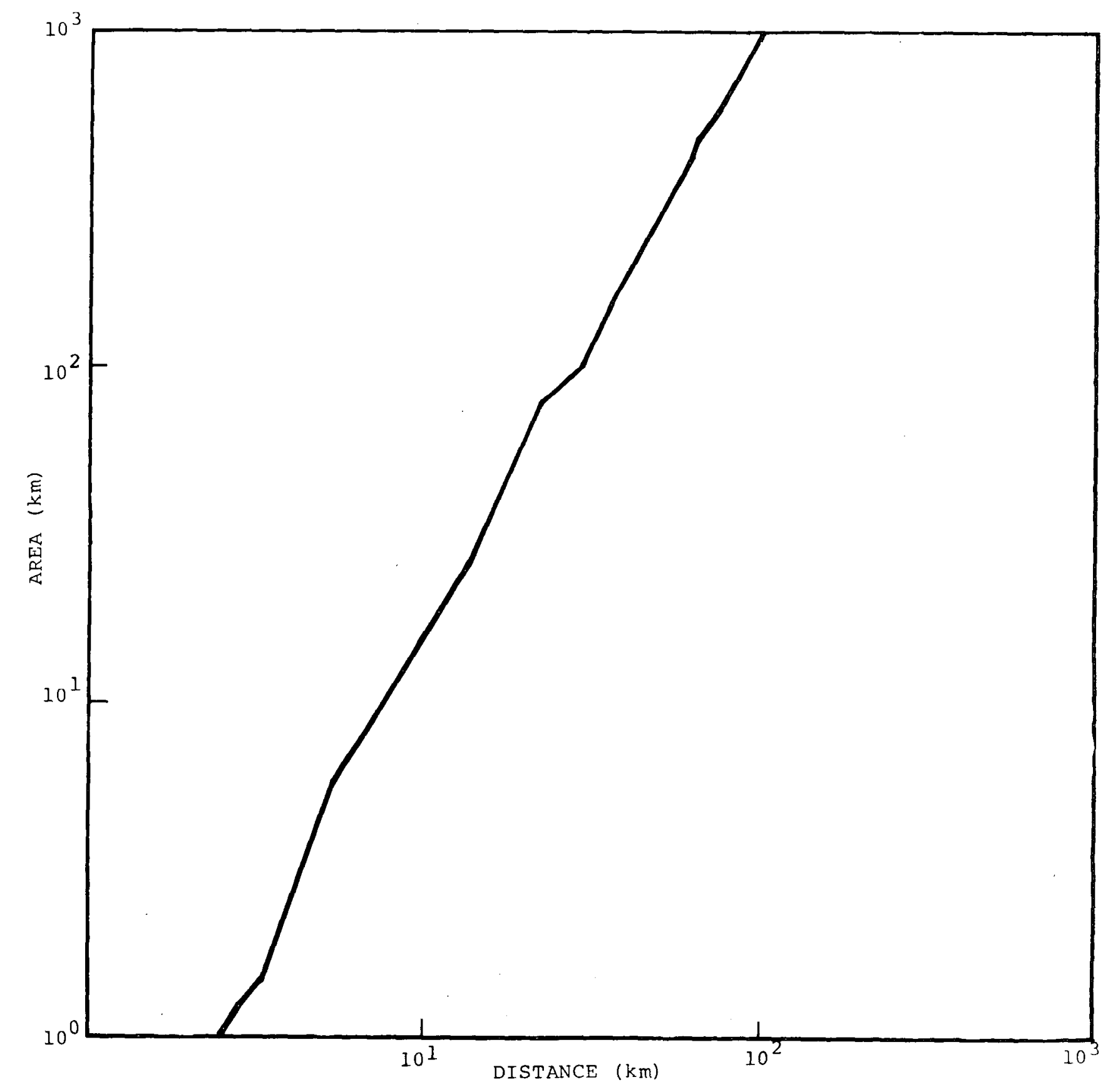

Figure 5-7. CRAC Estimate of Cumulative Area Traversed by Radioactive Cloud as a Function of Downwind Distance from Release Point (1 to 1000 kilometres) 
Any attempt to quantify economic impact involves many assumptions. Therefore, the following results are only order-of-magnitude estimates. More accurate analysis requires, among other things, details of land use near the actual point of release (and for larger releases, to considerable distances downwind), the nature of the release (magnitude, radionuclides involved), and the weather conditions at release time and for sometime thereafter. Nevertheless, the costs may be approximated as proportional to the area contaminated, the type of land use, and the population density. The Reactor Safety Study ${ }^{26}$ addresses such questions for the case of a reactor accident; similar methodology has been used in this study.

A brief summary of the parameters used in the economic analysis is presented in Table 5-15. These data are abstracted from a more complete tabulation in Appendix $\mathrm{K}$. The results of the analysis for the baseline estimate releases are shown on Table 5-16. The individual costs vary with time of release (affected by wind speed and population distribution) and location--midblock or intersection. In general, lower wind speeds mean higher contamination levels close to the release point. In this case, land-use denial is required and cleanup costs are not calculated. For the intersection release, the effect of the cloud "splitting" is observed in that even the midvelocity wind for the rush hour release produces lower levels of contamination thus allowing cleanup. Also, the total cost of a midblock release is relatively insensitive to time of release--the cost varies only about $10 \%--$ while the intersection release is more sensitive, as the midnight cost is only about $17 \%$ of the noontime cost. In Table 5-17, the cost data for the three spent fuel releases are presented, averaged for the three release times. The results are not very sensitive to the level of release over the range considered. This is attributable to land-use denial over significant areas, and the cost of this dominates. It is anticipated that there would be more sensitivity to the release magnitude if the entire affected area were considered rather than just the existing METRAN grid.

A number of features in this analysis warrant emphasis:

1. This analysis is based upon the release point being within an urban environment and is limited to the METRAN grid.

2. This analysis of necessity makes only an order-of-magnitude estimate.

3. The analysis does not take into account the repair and cleanup costs that would be associated with the damage resulting from the use of high explosives in the sabotage attack.

4. Although the results imply decreased costs as release magnitude decreases, in all likelihood there will be some minimum cost associated with any public release regardless of size. Likewise, the estimates here imply "leveling" of costs as release magnitude increases, due to the fact that once an area is above a contamination level which requires land-use denial, further contamination will not increase the cost.

5. The indirect sociopolitical, economic, and litigation costs of the loss (however temporary) of the business, finance, and government facets of an urban area have not been estimated or included.

6. Finally, the values here are in 1979 dollars. Projections should take appropriate account of inflation. 
Table 5-15

Summary of Economic Cost Parameters

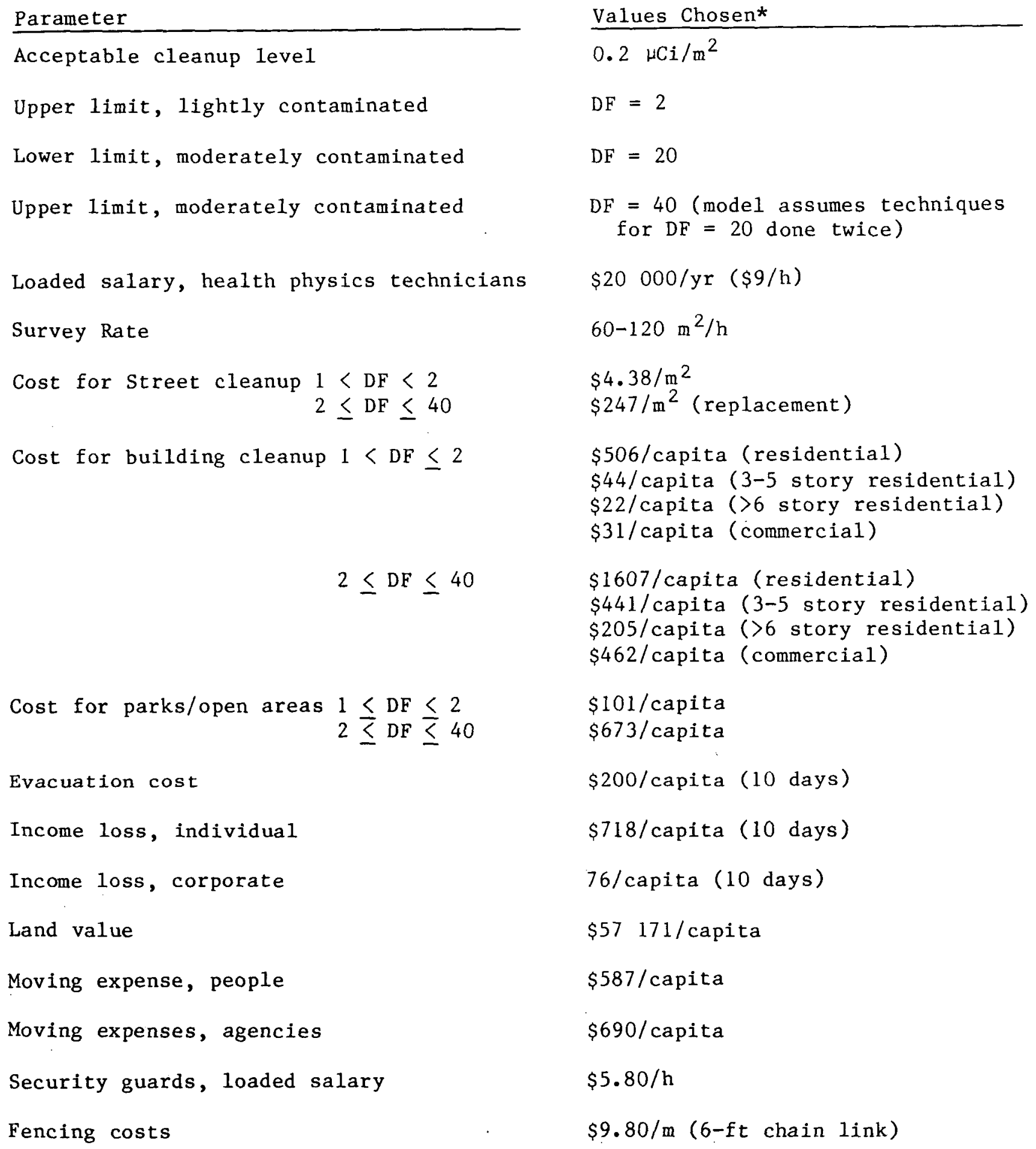

*All cost values in 1979 dollars. Cleanup costs cited are on a per-capita basis since such data are readily available (see, for example, Reference 26). A DF of 2 implies that contamination levels after cleanup are one-half those before cleanup; a DF of 20 implies post-cleanup levels that are $5 \%$ of original levels. 
Table 5-16

Summary of Economic Costs --

Cel1 B Baseline Estimate Release*

\begin{tabular}{|c|c|c|c|c|c|c|}
\hline \multirow{2}{*}{$\begin{array}{c}\text { Cost } \\
\text { Element }\end{array}$} & \multicolumn{3}{|c|}{ Midblock } & \multicolumn{3}{|c|}{ Intersection } \\
\hline & Noon & Rush hour & Midnight & Noon & Rush hour & Midnight \\
\hline $\begin{array}{l}\text { On-scene } \\
\text { emerg. resp. }\end{array}$ & $7.6 \times 10^{2}$ & $7.6 \times 10^{2}$ & $7.6 \times 10^{2}$ & $7.6 \times 10^{2}$ & $7.6 \times 10^{2}$ & $7.6 \times 10^{2}$ \\
\hline $\begin{array}{l}\text { On-scene } \\
\text { recovery }\end{array}$ & $3.7 \times 10^{3}$ & $3.7 \times 10^{3}$ & $3.7 \times 10^{3}$ & $3.7 \times 10^{3}$ & $3.7 \times 10^{3}$ & $3.7 \times 10^{3}$ \\
\hline Survey cost & $1.7 \times 10^{6}$ & $2.3 \times 10^{6}$ & $2.3 \times 10^{6}$ & $2.9 \times 10^{6}$ & $2.8 \times 10^{6}$ & $1.7 \times 10^{6}$ \\
\hline Street cost & $1.1 \times 10^{6}$ & 0 & 0 & $1.8 \times 10^{6}$ & $2.2 \times 10^{6}$ & 0 \\
\hline $\begin{array}{l}\text { Building } \\
\text { cost }\end{array}$ & $1.6 \times 10^{7}$ & 0 & 0 & $3.6 \times 10^{7}$ & $3.1 \times 10^{7}$ & 0 \\
\hline Evac. cost & $7.2 \times 10^{6}$ & 0 & 0 & $1.4 \times 10^{7}$ & $1.3 \times 10^{7}$ & 0 \\
\hline $\begin{array}{l}\text { Security } \\
\text { cost }\end{array}$ & $3.7 \times 10^{7}$ & $6.1 \times 10^{7}$ & $6.1 \times 10^{7}$ & $4.9 \times 10^{7}$ & $4.9 \times 10^{7}$ & $4.9 \times 10^{7}$ \\
\hline $\begin{array}{l}\text { Interdict. } \\
\text { cost }\end{array}$ & $2.0 \times 10^{9}$ & $2.1 \times 10^{9}$ & $2.2 \times 10^{9}$ & $2.9 \times 10^{9}$ & $1.8 \times 10^{9}$ & $4.5 \times 10^{8}$ \\
\hline Total & $2.1 \times 10^{9}$ & $2.2 \times 10^{9}$ & $2.3 \times 10^{9}$ & $3.0 \times 10^{9}$ & $1.9 \times 10^{9}$ & $5.0 \times 10^{8}$ \\
\hline
\end{tabular}

*A11 costs in 1979 dollars.

Table 5-17

Summary of Economic Costs -- Average for Three Release Times in Cell $\mathrm{B}^{*}$

\begin{tabular}{|c|c|c|c|c|c|c|}
\hline \multirow{2}{*}{$\begin{array}{c}\text { Cost } \\
\text { Element }\end{array}$} & \multicolumn{3}{|c|}{ Midblock } & \multicolumn{3}{|c|}{ Intersection } \\
\hline & Low & Baseline & $\mathrm{High}$ & Low & Baseline & High \\
\hline $\begin{array}{l}\text { On-scene } \\
\text { emerg. resp. }\end{array}$ & $7.6 \times 10^{2}$ & $7.6 \times 10^{2}$ & $7.6 \times 10^{2}$ & $7.6 \times 10^{2}$ & $7.6 \times 10^{2}$ & $7.6 \times 10^{2}$ \\
\hline $\begin{array}{l}\text { On-scene } \\
\text { recovery }\end{array}$ & $3.7 \times 10^{3}$ & $3.7 \times 10^{3}$ & $3.7 \times 10^{3}$ & $3.7 \times 10^{3}$ & $3.7 \times 10^{3}$ & $3.7 \times 10^{3}$ \\
\hline $\begin{array}{l}\text { Survey } \\
\text { cost }\end{array}$ & $2.4 \times 10^{6}$ & $2.1 \times 10^{6}$ & $2.4 \times 10^{6}$ & $2.5 \times 10^{6}$ & $2.5 \times 10^{6}$ & $2.5 \times 10^{6}$ \\
\hline Street cost & $4.4 \times 10^{5}$ & $3.7 \times 10^{5}$ & 0 & $1.3 \times 10^{6}$ & $1.3 \times 10^{6}$ & $1.3 \times 10^{6}$ \\
\hline Bldg cost & $6.7 \times 10^{6}$ & $5.3 \times 10^{6}$ & 0 & $1.1 \times 10^{7}$ & $2.2 \times 10^{7}$ & $2.5 \times 10^{7}$ \\
\hline Evac. cost & $3.4 \times 10^{6}$ & $2.4 \times 10^{6}$ & 0 & $5.7 \times 10^{6}$ & $9 \times 10^{6}$ & $8.0 \times 10^{6}$ \\
\hline $\begin{array}{l}\text { Security } \\
\text { cost }\end{array}$ & $4.9 \times 10^{7}$ & $5.3 \times 10^{7}$ & $5.7 \times 10^{7}$ & $4.9 \times 10^{7}$ & $4.9 \times 10^{7}$ & $4.9 \times 10^{7}$ \\
\hline $\begin{array}{l}\text { Interdict. } \\
\text { cost }\end{array}$ & $2.0 \times 10^{9}$ & $2.0 \times 10^{9}$ & $2.5 \times 10^{9}$ & $1.7 \times 10^{9}$ & $1.8 \times 10^{9}$ & $1.7 \times 10^{9}$ \\
\hline Tota1 & $2.0 \times 10^{9}$ & $2.7 \times 10^{9}$ & $2.6 \times 10^{9}$ & $1.8 \times 10^{9}$ & $1.8 \times 10^{9}$ & $1.8 \times 10^{9}$ \\
\hline
\end{tabular}

*A11 costs in 1979 dollars. 
In summary, the geographical coverage and the economic impact have been estimated for a range of deliberate releases within an urban area. For the largest release examined $(\sim \mathrm{ll} \mathrm{kCi})$, the impact is spread over numerous political subdivisions, and releases in the range 1 to $10 \mathrm{kCi}$ are estimated to have costs on the order of $\$ 10^{9}$.

\subsubsection{Consequences from Nondispersed Sources}

In several of the releases postulated, there may be a substantial amount of material ejected from the shipping container but not aerosolized. That is, it becomes a source of direct radiation and subsequently a cleanup problem. It was anticipated that this source would not contribute significantly to the public risk. To explore this question, the METRAN code has been exercised in the mode used for special form (i.e., nondispersible) material. In this mode, the source is treated as a point source in the middle of the street. Therefore, the pedestrian distance of closest approach is the sidewalk and the closest approach for vehicles is the crosswalk. The vehicles proceeding on one side of the street are presumed to be bumper-to-bumper. These conditions are presumed to exist at the time of sabotage and shortly thereafter.

The two shipment types of major concern insofar as direct radiation is concerned are the irradiated (spent) fuel and nonfissile isotope (large sources) shipments because of the source strengths involved. In these classes of shipments, release mechanisms can be postulated in which substantial amounts of the material are simply ejected from the cask and scattered on the ground. To establish an upper bound, it has been assumed that the entire contents are outside the shipping container-a conservative assumption in terms of the realistic release mechanisms. The results at three release times are shown in Table 5-18. As would be expected, the latent cancer fatalities as a result of direct radiation are minor compared to the early fatalities and morbidities.

Several other points should be noted. First, the early consequences estimated here are insensitive to the cell of release. This is due to the traffic "packing" assumption described above and to the fact that pedestrian density is time- but not cell-dependent. As modeled in METRAN, there are about three times more fatalities and morbidities among pedestrians than among people in vehicles during daylight hours and about six times more among people in vehicles than among pedestrians at midnight, which simply reflects the relative population densities.

Second, no early consequences are predicted for people in buildings because of the assumed distance from the source and the shielding afforded by the building materials. In contrast, the pedestrians and people in vehicles are not shielded. The dose to pedestrians includes an albedo term to account for radiation scattering from streets and adjacent building walls. Third, no early consequences are predicted for the case of a cesium release because of the constraints the model places on the distance of closest approach. For comparison purposes, the distances at which dose thresholds for early fatalities and morbidities (assuming 15-minute exposure) are equalled or exceeded are shown in Table 5-19.

It is clear from the data that direct radiation from the cesium source, even at 15 $\mathrm{kCi}$, does not pose a major threat to public health and safety. It is also apparent that for those releases involving dispersal and in which there is some residual material on the ground, the consequences resulting from inhaled radionuclides will outweigh those from direct radiation. 
Table 5-7

Comparison of Average METRAN Consequence Estimate to CRAC Mean Estimate for Midblock, Cell $\mathrm{B}^{\mathrm{a}}$

\begin{tabular}{|c|c|c|c|c|}
\hline Estimate $^{\mathrm{b}}$ & Mode1 & $\begin{array}{c}\text { Early } c d \\
\text { Fatalities }^{\text {Falitis }}\end{array}$ & $\begin{array}{c}\text { Early } \\
\text { Morbidities }\end{array}$ & $\begin{array}{c}\text { Latent } \\
\text { Cancer Fatalities } \\
\end{array}$ \\
\hline Baseline & $\begin{array}{l}\text { METRAN } \\
\text { CRAC }\end{array}$ & $\begin{array}{l}<1 \\
<1\end{array}$ & $\begin{array}{r}19 \\
1\end{array}$ & $\begin{array}{l}40 \\
77\end{array}$ \\
\hline Upper & $\begin{array}{l}\text { METRAN } \\
\text { CRAC }\end{array}$ & $\begin{array}{c}2.8 \\
1\end{array}$ & $\begin{array}{l}99 \\
11\end{array}$ & $\begin{array}{l}104 \\
195\end{array}$ \\
\hline Lower & $\begin{array}{l}\text { METRAN } \\
\text { CRAC }\end{array}$ & $\begin{array}{c}<<1 \\
0\end{array}$ & $\begin{array}{l}10 \\
<1\end{array}$ & $\begin{array}{l}12 \\
24\end{array}$ \\
\hline
\end{tabular}

${ }^{a}$ CRAC calculation limited to same 10 by $10 \mathrm{~km}$ area as METRAN, assuming a release at center (Cell B).

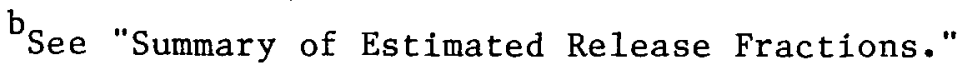

${ }^{c}$ Early Fatalities occur within 1 year after exposure to the radioactive material. Early Morbidities are illnesses appearing within weeks after exposure. Latent Cancer Fatalities occur over any time subsequent to the exposure as a result of the initial exposure and of any long-term exposure to low levels of contamination. I.e., 40 latent cancers would represent an average of less than 2 cancers per year over a 30-year period for the population group exposed.

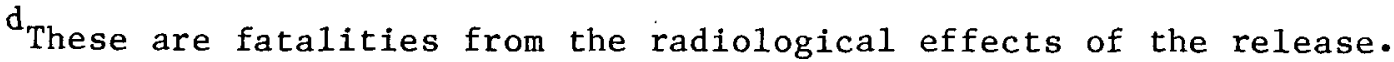
Blast effects from explosives used to attack a cask could cause tens of fatalities (see the subsection, "Consequences of the Use of High Explosives"). In fact, if explosives are used, early radiological fatalities are highly unlikely because those close enough to receive lethal radiation doses would be killed by the explosion. That is, the lethal radius for blast effects exceeds the lethal radius for early deaths from radiation in this particular instance.

These are total latent cancer fatalities predicted as result of initial exposure, resuspension, and long-term exposure to contaminated ground. The text discusses the differences in manner in which METRAN and CRAC treat the long-term exposure. 
Table 5-18

Consequences of Direct Radiation -- 15-Minute Exposure

Time of

Release

\begin{tabular}{c} 
Early \\
Fatalities \\
\hline \\
108 \\
65 \\
20 \\
66
\end{tabular}

Early
Morbidities $^{a}$

Latent Cancer

1. Spent Fuel (5.37 MCi)

Noon

Rush Hour

Midnight

Average

2. Cobalt $-60(200 \mathrm{kCi})$

Noon

Rush Hour

Midnight

Average

3. Cesium-137 (15. kCi)

Noon

Rush Hour

Midnight

Average

$\begin{array}{rr}24 & 392 \\ 15 & 227 \\ 6 & 85 \\ 15 & 235\end{array}$

509

295

109

304

Fatalities $^{\mathrm{a}}$

0
0
0
0

0
0
0
0

0.23

0.13

0.04

0.13

${ }^{a}$ See footnote d, Table 5-4.

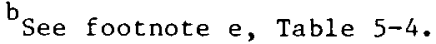

Table 5-19

Dose Threshold-Distance Relationship, 15-Minute Exposure

\section{Dose (rem)}

1. Spent Fuel (5.37 MCi)

$$
\begin{array}{r}
700^{a} \\
400^{b} \\
50^{c}
\end{array}
$$


One further point must be emphasized. An attack violent enough to overturn and disrupt the shipping container integrity sufficiently to "spill" the contents would have to employ thousands of kilograms of high explosives. In light of the possible effects of high explosives discussed earlier under "Consequences of the Use of High Explosives," it is highly likely that those persons close enough to be affected by direct radiation will have undergone severe physical trauma or died from the explosion, and therefore direct radiation becomes of secondary importance to direct blast consequences.

In summary, although direct radiation could cause hundreds of early fatalities if an entire spent fuel shipment were spilled, for the more realistic modes of potential sabotage, the dispersed material will dominate the public health consequences.

\subsubsection{Summary of Consequences}

The consequences in Table 5-20 have been estimated for the urban environment based upon the release fractions postulated in the subsection "Summary of Estimated Release Fractions" and the public health effects as defined in the blast effects analysis and as modeled by the METRAN and CRAC codes.

Economic effects are strongly dependent upon the amount of radioactive material released. Thus, there is unquestionably a wide range of environmental impacts potentially exceeding $\$ 1$ billion for the largest radioactive material release.

Table 5-20

Summary of Public Consequences ${ }^{a}$

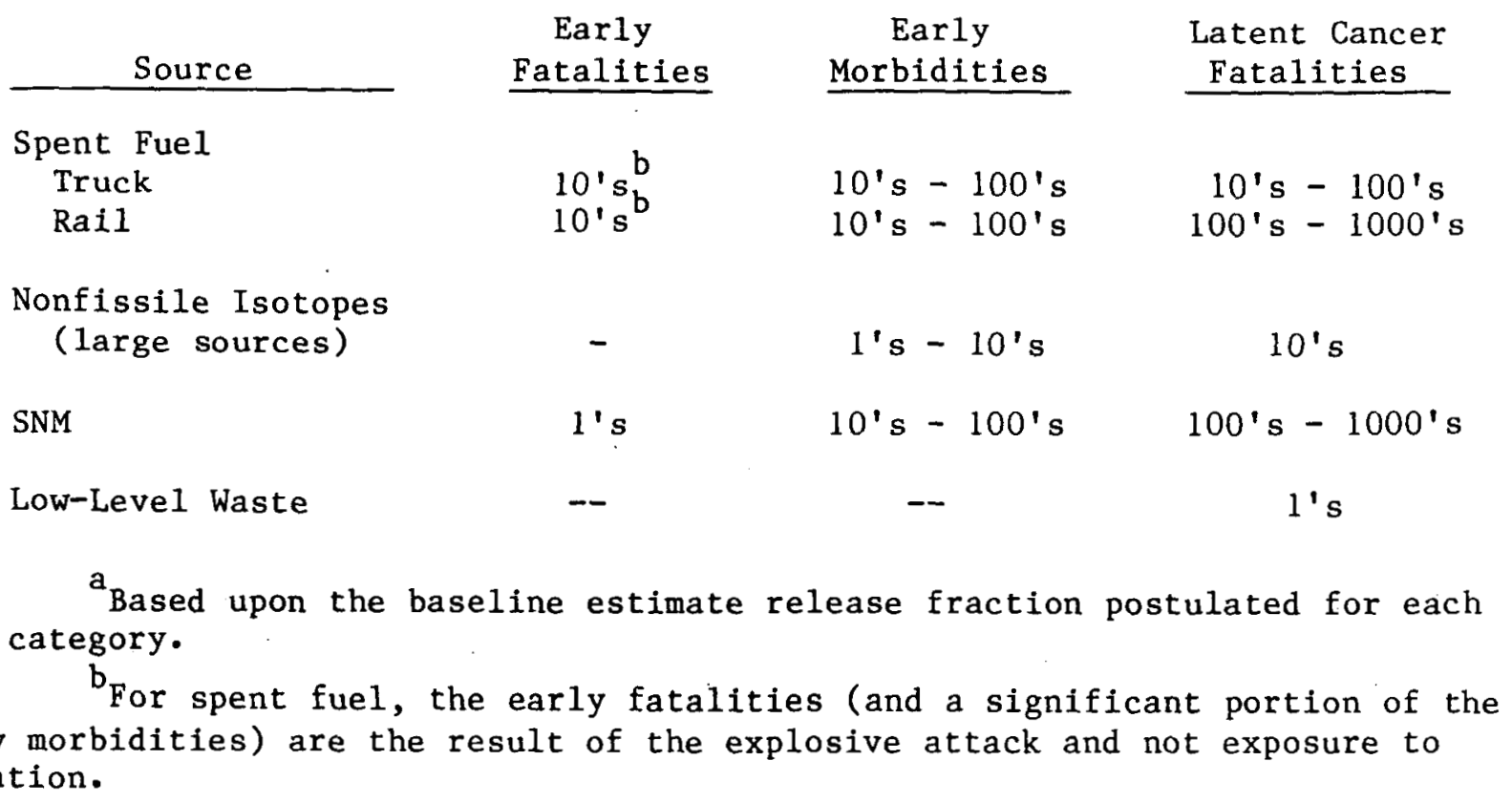




\subsection{Effects of Urban Areas on the Functions of Transportation Safeguards}

Unlike the majority of shipments discussed at length above, some shipments of SNM (such as plutonium and $\mathrm{U}-233$ ) and spent fuel are currently being safeguarded.* Additional safeguard measures have been proposed by the NRC. ${ }^{35}$ In this section, the impacts of the urban environment on the functioning of transportation safeguard systems are considered. Particular attention is paid to the response times, capabilities, and tactics of the supporting local law enforcement agencies (LEAs) and the effects of increased population and traffic densities in the urban areas as contrasted with suburban and semirural areas. There is an active program underway to systematically model transportation safeguard systems, including convoy configurations, conflict simulations, and law enforcement agency availability.

Because this detailed modeling and analysis is still in progress, the observations here are considered to be semiquantitative to qualitative based upon the available data. The analysis is being conducted assuming those systems outlined in the proposed rules to be the first line of response to any threat or attack.

The proposed rule 36 for transportation safeguards incorporates several concepts and features which are summarized here for convenient reference. The proposed rule has three principal objectives:

1. Restrict access and activity in the vicinity of transports.

2. Prevent the unauthorized access into, or removal of strategic special nuclear material from, transports.

3. Provide a response capability (force) to insure that the first two objectives can be accomplished.

For road shipments, whether by special truck or armored cars, there are some additional criteria established. These are

1. There will be seven armed escorts, possibly including drivers, with two in the cargo vehicle.

2. The cargo vehicle will have a bullet-resistant cab and a penetrationresistant cargo compartment.

3. There will be at least two bullet-resistant escort vehicles.

4. Shipments will use primary highways. (For most urban areas this tacitly implies the interstate highway system or comparable expressways.)

5. There will be continuous radio communication intraconvoy with backup, and there will be radio contact with a movement control center at least every 30 minutes.

6. There will be communications with local LEAs.

7. When stopped (refueling, rest, or emergency only) at least three escorts will have the transport under surveillance with nine available to respond. Two escorts will be sufficiently remote to retain contact with local LEAs in the event of a single attack.

*The interim rule for spent fuel was not considered explicitly in the discussion, although the comments appear generally applicable. 
With these conditions in mind, the effects of the urban environment may be explored. These observations and conclusions on local LEA response and capabilities are based upon a limited analysis of data provided by several thousand local LEAs.*

\subsubsection{Response Time and Numbers of Local LEAs}

The limited sample of available data suggests that in very large cities (those with population greater than 1000 000), 30 to 50 officers can respond to calls for assistance in 10 minutes or less. This response usually represents less than $10 \%$ of those on duty. Similar conditions appear to prevail in cities with populations in the 500000 to 1000000 category, although the response represents a larger fraction of those on duty, perhaps $25 \%$ or so. Even in cities with 100000 to 500000 population, the 10-minute response level is significant, in the range of 10 to 30 officers. However, in some areas this may be nearly one-half of the total number of officers on duty. In those cities of less than 100000 , the response is more likely 10 or so, which frequently represents nearly all the officers on duty. In small cities--those with less than 25000 but greater than $10000--$ the response is typically around 10 , but again. this represents nearly all those on duty. The actual numbers obviously vary from city to city and shift to shift; the numbers quoted here are "averaged" over three shifts for the cities examined. Nevertheless, the following conclusions may be drawn. In urban areas, the number of defenders (escorts plus local LEAs) can be quadrupled in 10 minutes or less, and even in smaller cities the number can be doubled. This simple difference in numbers appears to provide the urban LEA and the shipment escorts much more flexibility in responding to an attack than would be the case in small cities or a semirural environment. Also, because the responding force in large cities represents only a small fraction of the officers on duty, the urban LEA should be better able to cope with diversionary or multiple attacks.

If the impact of protracted engagements ( 1 hour or more) are considered, then the sample again suggests that the urban LEAs are able to respond with significantly greater numbers, five to seven times the initial response, without necessarily calling upon off-duty personnel or outside assistance. In the smaller cities, the total response in 1 hour may only be two times the initial response, and even at that will require personnel other than the duty shift.

\subsubsection{Capabilities of Responding LEA--Individuals}

It is interesting to note, based upon the sample, that the capabilities of individual officers, in terms of equipment they bring, are not strongly related to the

*The survey was conducted by the International Association of Chiefs of Police for Sandia Laboratories, Department 1710, under Department of Energy programs. The survey responses are being correlated and analyzed, and a detailed report will be published. It is emphasized that the observations made here are based upon a limited random sample of the survey. responses and are therefore subject to modification as the full analysis proceeds. 
size of city in which they serve. Patrol cars appear to routinely carry shotguns, and in the limited sample examined, about three-quarters indicated that at least some officers would respond with rifles, although the type (automatic or semiautomatic) was not specified. In larger urban areas, cities greater than 500000 , the responding officers would also have gas guns and personal body armor. In the smaller cities, the survey seems to indicate that about half the responding officers would be equipped with gas guns and armor. This would appear to provide the urban LEA more flexibility in dealing with a situation.

Individual officer training and experience is less amenable to quantification, but it is likely that the large city forces will have more formal and regular training than those in small cities and towns. It is anticipated that the urban officer is better prepared to handle an armed confrontation than an officer from a semirural area, simply because he meets such situations frequently in the course of his normal duties.

\subsubsection{Capabilities of Responding LEA--Special Teams}

The availability of specially trained teams to handle unique situations is very much a function of city size. The sample suggests that very large cities most likely have a special weapons team of some type (the so-called SWAT teams), teams of highly skilled marksmen (snipers), and specially equipped and trained riot squads. In addition, nearly all the large cities have personnel trained in hostage negotiations and qualified explosive ordnance disposal (EOD) experts. All of these special teams have come into existence in large urban areas over the past few decades in response to a variety of sociopolitical pressures and incidents. In cities of intermediate size (100 000 to 1000.000$)$, the sample indicates that more than half have SWAT, riot control, and explosive ordnance disposal teams. Less than about half of these cities have some sniper team capability, and even fewer have any trained negotiators for hostage situations. In cities smaller than 100 000 , less than half have any special teams, SWAT, sniper, riot control, or hostage negotiation teams. Above 25000 , about half will have some EOD capabilities, but such capability is nonexistent in smaller cities.

The obvious conclusion is that the larger the city, the more likely it is to have specially trained teams. Again, this provides the urban LEA with more flexibility in response to an attack on safeguarded shipments, as well as the ability to bring specially trained personnel into the action. The only drawback is that the response time of such teams is typically around 30 minutes, so that the escorts and the initially responding LEA would have to hold the situation until the special teams arrived.

\subsubsection{Capabilities of Responding LEA--Equipment}

As might reasonably be expected, the information in the survey again suggests a strong dependence upon city size. For example, more than half of the cities with greater than 1000000 population possess some type of armored vehicle. Cities under 1000000 typically do not but rely instead on agreements with the National Guard or State Police. Cities larger than 500000 nearly always have equipment vans and special communications vans (obviously correlating with the existence of special teams). Cities under 25000 seldom have either item, while about half of the cities between these two extremes will have one item or the other. In terns of airborne support, it appears that all of the larger cities have police helicopters and about half also have fixed-wing aircraft. In cities of 100000 to 1000000 , 
better than one-third have helicopters, and less than one-third have aircraft. Below 100000 population, city-owned helicopters and aircraft are nearly nonexistent, and below 25000 , they are nonexistent.

As with the special teams, other pressures--some routine, some extraordinary--have caused the urban LEA to be better equipped and prepared to respond to a variety of threats and events. Certainly, the availability of armored vehicles and air surveillance puts the urban LEA in a better position than the semirural LEA to cope with an armored attack.

\subsubsection{Influence of Higher Traffic Density}

There is perhaps a natural tendency to believe that the increased traffic density of the urban area as compared with the rural area will automatically inhibit the response of law enforcement agencies. This tendency is no doubt strengthened by the personal experience of bumper-to-bumper vehicles in rush hour city traffic creeping along at a few kilometres per hour. However, this situation must be examined from the perspective of the proposed rules.

If these shipments are required to use primary highways, this implies that they will move on the interstate highway system or similar controlled access expressways in most urban areas. It is also presumed, although not specified in the proposed rules, that because an escorted shipment is essentially a convoy operation, routing would be selected, or at least scheduled, to avoid the rush hour congestion in or near major urban areas.* With this consideration, it is then appropriate to compare the urban and rural situations based upon the average traffic densities.

The available data on highway use permit some comparison of urban and rural fourlane and six-lane two-way highways. Although eight-lane expressways exist in urban areas, they are seldom found in rural areas. For four-lane freeways (two lanes each direction) the average urban traffic flow is about 1.8 times the rural average on a vehicles-per-hour-per-lane basis. For six-lane freeways, this ratio increases to about 6.5 . This suggests that in urban areas it will be more difficult to operate a convoy of three vehicles. For example, the traffic density on the urban freeways (on the order of 1000 per hour per lane for the larger expressways) will make it difficult for the escorts to insure that they can reach the transport quickly because of the tendency of other drivers to fill in gaps which may exist between vehicles by "lane jumping" in an attempt to move faster than the general traffic flow. This situation is further aggravated by the frequent access points in the urban freeway system, with the attendant constant influx of vehicles from side roads. In some urban areas, freeway exits may be as close together as 0.8 to $1.2 \mathrm{~km}$, while in other areas there may well be tens of kilometres between exits. of course, this high frequency of access also works to assist the sàfeguards system simply because it provides more routes by which responding LEA can reach the scene and may permit more forces to respond.

This increased vehicle density in the urban area would also make it easier for vehicles carrying an adversary attack force to approach the transport (say in an adjacent lane) without arousing undue suspicion on the part of the escorts.

\footnotetext{
*This presumption is based in part on the requirement in the proposed rule to avoid areas of natural disaster and civil disturbances.
} 
Furthermore, it has been suggested that "staged" accidents could be employed by an adversary to halt the transport vehicle. 41 Again, such events are less likely to arouse suspicion in the urban area simply because traffic-delaying accidents are not uncommon on these freeways. Therefore, the escorts will have to be particularly alert for such events. The urban traffic density can also present the escorts and responding LEA with additional constraints on their response to an adversary. The presence of hundreds of "innocent bystanders" in the passing (or stopped) vehicles could generate considerable reluctance on the part of the escorts to engage in an armed confrontation. In fact, this readily available pool of potential hostages could be employed by an adversary to negate or inhibit escort response.

On the other hand, the very traffic density which inhibits the function of the safeguards team may also have a strong effect upon an adversary. For example, if the transport is effectively immobilized by the crew, the adversary must open the transport by forceful means and remove the contents in full view of hundreds of passing vehicles. In light of the current popularity of $C B$ radio usage, it is highly unlikely that such an undertaking would go unreported, even if the transport and escort vehicle radios had been disabled. In this regard, numerous postulated scenarios are set in isolated areas away from cities, apparently because of the presumed desire on the part of the adversary to operate clandestinely if possible.

In summary, the increased traffic density in the urban area will make it more difficult to maintain clear access to the transport, and the presence of other vehicles can inhibit escort responses. On the other hand, the same increased traffic density forces the adversary into the open and increases the likelihood of detection and intervention by LEAs.

\subsubsection{Influence of Pedestrian Density}

Upon cursory examination, it would appear that the pedestrian density associated with the urban area would have a detrimental effect upon safeguards. That is, the presence of many bystanders and potential hostages would seriously inhibit the escort response. However, when viewed in the light of the proposed rule requiring that routes be restricted to primary highways, the pedestrian population will have essentially no effect upon the functioning of the safeguards system: in most urban areas, primary highways are generally freeways and devoid of pedestrians.

\subsubsection{Influences of Other Urban Characteristics}

Other aspects of the urban environment can influence the functioning of the safeguards system, or at least influence the requirements of the system. For example, in most cities, especially those areas near primary highways, there are numerous shipping terminals, warehouses, factories, etc., many of which are abandoned or at least unoccupied at any given time. This means that if a safeguarded shipment is attacked with theft as the goal, there are many hiding places readily available if the adversary is successful. Therefore, there is increased need for the safeguards system to prevent access to and removal of material. This means that hardware systems, as well as escorts, can play a significant role in protecting material in transit.

This same condition exists with respect to possible handling and processing of stolen material. In most urban areas, especially semi-industrial areas, frequent movements of materials would hardly be noticed. Groups of individuals going in and 
out of a warehouse would simply be taken as part of the normal work force. Also, in these areas the necessary utilities are readily available to operate a clandestine laboratory. Again, this places a premium on maintaining control of the material and preventing an adversary from leaving the scene.

\section{NOTES}

lJ. B. Be11, Transnational Terror, Washington: American Enterprise Institute for Public Policy Research, and Palo Alto, CA: Hoover Institution on War, Revolution and Peace; 1975.

2Y. Alexander, International Terrorism - Nationa1, Regional and Global Perspectives, New York: Praeger, 1976.

3B. Jenkins, International Terrorism, A New Mode of Conflict, Research paper No. 48, California Seminar on Arms Control and Foreign Policy, Los Angeles: Cresent Publications, 1974.

${ }^{4}$ A. Deming et al, "War on Terrorism," Newsweek, 31 October 1977.

5M. Willrich and T. B. Taylor, Nuclear Theft: Risks and Safeguards, Cambridge, MA: Ballinger, 1974.

${ }^{6} \mathrm{~J}$. McPhee, The Curve of Binding Energy, New York: Farrar, Straus and Giroux, 1974.

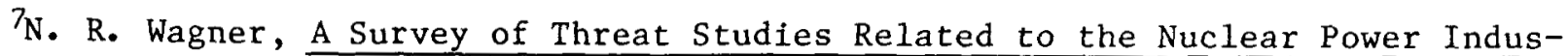
try, SAND77-8254, Albuquerque: Sandia Laboratories, August 1977.

${ }^{8}$ WASH-1238.

9NUREG-0170.

10 E. A. Pike, Protection Against Bombs and Incendaries for Business, Industrial and Educational Institutions, Springfield, IL: C. C. Thomas, 1972.

${ }^{11}$ E. I. DuPont de Nemours, Blasters Handbook, 15th Edition.

12 U.S. Department of Defense, Department of the Army, Explosives and Demolitions, FM5-25, 1967.

${ }^{13}$ Commercial Catalog, Jet Research Center, Arlington, TX.

14M. A. Cook, The Science of High Explosives, ACS Monograph 139, New York: Reinhold Publishers, 1958.

15R. M. Jefferson, "Cask Demolishes Train Superstructure - Sustains Minor Damage," Nuclear Engineering International, Vo1. 22, No. 260, July 1977.

16R. M. Jefferson and H. R. Yoshimura, Crash Testing of Nuclear Fuel Shipping Containers, SAND77-1462, Albuquerque: Sandia Laboratories, February 1978 . 
${ }^{17}$ R. Yoshimura and M. Huerta, A Crash Test of a Nuclear Spent Fuel Cask and Truck Transport System, SAND77-0419, Albuquerque: Sandia Laboratories, January 1978.

$18_{\mathrm{R}}$. Yoshimura, "Full Scale Tests of Spent-Fuel Shipping Systems," Transactions of the American Nuclear Society, Winter Mtg., 28 November to 2 December 1977.

19 . Meyer et al, "The Homemade Nuclear Bomb Syndrome," Nuclear Safety, 18(4): $427 \mathrm{ff}$, July-August 1977.

${ }^{2} \mathrm{C}$. W. Townley et a1, The Development and Evaluation of Safety Performance Criteria for Sealed Radiation Sources, BMI-1559, Columbus, OH: Battelle Memorial Institute, 5 December 1961.

21 10CFR73.

22U.S. Department of Health, Education, and Welfare, Radiological Health Handbook, January 1970.

$23_{\text {Battelle Pacific Northwest Laboratories, Transport of Radioactive Materials }}$ Data Base (This is an extension of material reported in Survey of Radioactive Material Shipments in the United States, BNWL-1972, Hanford, WA, April 1976).

24 U.S. Atomic Energy Commission, Directory of Shipping Containers for Radioactive Materials, Washington: USAEC, October 1969.

${ }^{25}$ S. H. Rust and L. E. Wesney, eds, Nuclear Power Safety, New York: Pergamon । Press, 1976.

26 NUREG75-014 (WASH 1400).

27I. B. Wall et al, Overview of the Reactor Safety Study Consequence Model, NUREG-0340, Washington: U.S. Nuclear Regulatory Commission, October 1977.

${ }^{28}$ G. G. Kennedy, Explosive Shocks in Air, New York: MacMillan and Co., 1962.

29 .S. Department of Defense, Department of the Army, Fundamentals of Protective Design (Non-Nuclear), TM5-855-1, July 1965.

${ }^{30}$ S. Glasstone, ed, The Effects of Nuclear Weapons, Washington: U.S. Atomic Energy Commission, April 1962; also issued as Air Force Pamphlet 136-1-3.

${ }^{3} \mathrm{I}_{\mathrm{M}}$. J. BelI, ORIGEN, The ORNL Isotope Generation and Depletion Code, ORNL4628, Oak Ridge, TN: Oak Ridge National Laboratory, May 1973.

${ }^{32}$ U.S. Environmental Protection Agency, Proposed Guidance on Dose Limits for Persons Exposed to Transuranium Elements in the General Environment, EPA520/ 4-77-016, Washington: USEPA, September 1977.

${ }^{33}$ F. Lewis, One of Our H-Bombs is Missing, New York: McGraw-Hill, 1967.

${ }^{34}$ SAND 77-1927. 
35.S. Nuclear Regulatory Commission "Physical Protection of Plants and Materials, Performance Oriented Safeguards Requirements," 10CFR, Parts 70,73; in the Federal Register, Vol. 42, No. 128, 5 July 1977 (FR Doc 77-19051).

$36_{R}$. L. Rinne, The Evaluation of Safeguards Systems for Nuclear Material in Transit - The Development of the Program Plan, SAND77-8249, Livermore, CA: Sandia Laboratories, July 1977.

37 R. J. Gallagher, K. G. Stemwell, and N. R. Wagner, The Configuration of Road Convoys: A Simulation Study, SAND77-8625, Livermore, CA: Sandia Laboratories, Ju1y 1977.

$38_{\mathrm{K}}$. P. Berkbigler, Estimating the Availability of LLEA Officers, SAND77-8626, Livermore CA: Sandia Laboratories, July 1977.

${ }^{39}$ S. C. Keaton and P. DeLaquell IV, Conflict Simulation for Surface Transport Systems, SAND77-8624, Livermore, CA: Sandia Laboratories, July 1977.

4 National Academy of Sciences, National Research Council, Highway Research Board, Highway Capacity Manual 1965, Special Report 87, Washington, DC: NAS, 1965.

410 . C. Baldonado et al, Safeguards Systems Concepts for Nuclear Material Transportation, NUREG-0335, Washington: U.S. Nuclear Regulatory Commission, Office of Nuclear Regulatory Research, September 1977.

42 D. E. Bennett, SANDIA-ORIGEN User's Manual, NUREG/CR-0987, SAND79-0299, Albuquerque: Sandia Laboratories, October 1979. 


\section{ALTERNATIVES}

\subsection{Introduction}

Chapters 2 through 5 described the radiological and economic impacts from the transportation of a specified set of radioactive materials in a densely populated urban area in the context of current transport practices. This chapter describes the effects of several alternatives to or regulatory changes from current practice. These alternatives include shifts in transport mode, changes in package types, and routing or time of day restrictions. In considering alternatives, the authors recognize that changes in radiological impacts may also result in economic and social impacts. Indeed, these latter impacts may be more significant, if not dominant, when considering alternatives to current transport practice. A change in impacts resulting from implementation of a particular alternative for one causative event may well result in no change or significant, but contradictory, changes for another.

As each alternative is addressed, a discussion of its effect on all four causative events (i.e., comparison with the baseline case) will be presented as well as a discussion of the feasibility of the alternative in view of the present urban environment, transport system and current regulatory controls.

Since it is not possible to detail the precise manner in which a particular alternative would be implemented, assumptions are made which are considered applicable to certain types or groups of materials. These assumptions are given in the discussion for each case. Because of these assumptions, the results should be interpreted carefuliy. The authors do not consider changes from baseline risk values of less than an order of magnitude to be significant, although such changes may well be important in regulatory decision making.

\subsection{Baseline or Reference Cases}

For incident-free transport, the reference case is that discussed in detail in Chapter 2. Materials with different end uses are allowed to enter the urban area at different times of day, consistent with actual shipping practices. Results of the incident-free analysis are expressed as total expected latent cancer fatalities (LCF) per year of shipment activity at the given level.

To obtain the baseline value for latent cancer fatalities, the whole-body integrated dose ( $~ 7$ person rem/shipment year) is converted to the health effects value using the appropriate coefficients described in Appendix G (25 latent cancer fatalities $/ 10^{6}$ person rem). Expected numbers of genetic effects (GE) could be similarly obtained using a conversion factor of 170 genetic effects/106 person rem. To simplify the presentation of results in this chapter, only expected numbers of latent cancer fatalities are reported since expected numbers of genetic effects would follow the same general pattern. The reader is referred to the earlier chapters of 
this document for more discussion on genetic effects. Application of the latent cancer fatality conversion factor to the incident-free population dose gives a value of $1.8 \times 10^{-4}$ expected $\mathrm{LCF} /$ shipment year at the specified leve1 $\left(\sim 1.2 \times 10^{-3}\right.$ $\mathrm{GE})$.

For the reference case for vehicular accidents, all shipments begin through the study area at 1630 hours, with a southerly wind speed of $4 \mathrm{~m} / \mathrm{s}$. These conditions are frequent at 1630 hours, and the appropriate population parameters are about midway between the high values observed at 1200 hours and the relatively low values at 2400 hours. Output variables examined are expected numbers of early fatalities (EF), early morbidities (EM) and LCF per shipment year. For the same reasons given above, genetic effects are not included in the tables. Changes corresponding to those for cancer fatalities would be expected.

For human errors or deviations from accepted quality assurance practices, the 1630-hour case is also used for reference. Output variables are as for vehicular accidents: namely, expected numbers of early fatalities, early morbidities, and latent cancer fatalities.

The precise meaning of a baseline or reference case for a sabotage event is unclear since the presumption in Chapter 5 is that the event has occurred. For consistency with the remainder of the analysis, a reference value from the sabotage calculations for 1630 hours will be used when appropriate and results will be expressed as expected numbers of health effects (consequences, not risk). The discussion of the alternatives and their effects on sabotage events will be more narrative and less quantitative than for the other causative events.

For each causative event, the effects of a particular alternative are expressed as the expected number of health effects resulting from a year's shipment activity with that alternative in operation. To examine the relative change resulting from the implementation of a particular alternative, the tabulated values can be divided by the given baseline results. If the ratio obtained is less than unity, the effect of implementing an alternative is to reduce the radiologial risk and vice versa. Where specific values are not available or, for some reason, not meaningful, the reasons for their absence will be explained.

\subsection{Transport Mode Shifts}

Although the majority (98\%) of the total curies transported in the limited New York City area are shipped at least in part by truck, other transport modes could be used for some portion of these shipments, thus having some effect on the radiological risk. These alternate transport modes could include aircraft, trains, and barges. Even with increased use of alternate modes, the major portion of traffic within the urban area will still be by truck since shipments received at terminals must still be delivered to urban destinations or picked up at their points of origin for transport away from the urban center. Data in Chapter 2 of this report indicate that the major portion of the incident-free dose to urban specific population groups arises from the truck link of the transport process. Overflights of the urban area do not contribute significantly to the urban-specific population group dose. Since the major contributors to the incident-free population dose are groups related to the storage and handling of the shipments received at terminals for eventual delivery in the urban area, requirements that shipments currently carried by truck be routed by an alternate mode, then stored for later delivery, could significantly affect the incident-free case. 
For the case of vehicular accidents, the effects of this alternative are less clear. Regardless of the transport mode chosen, there is still the possibility of an accident anywhere along the route within the urban area. The materials with different transport modes cannot be removed as contributors to the overall risk, although the probability and frequency of an accident and the mode-related accident rate would change. This argument also holds for the human error situation in that changes in transport mode could alter the overall risk, but estimation of the changes resulting from alternate transport modes would depend heavily on those materials chosen for alternate mode transport and the actual modes used. Another problem with alternate transport modes is the timely availability of sensitive materials, such as radiopharmaceuticals with short half lives, or viability for their intended purposes. If the alternate mode does not make these materials available as needed, the usefulness of the alternative is diminished.

To evaluate the effect of transport mode changes in the sabotage case, the use of rail as the alternate mode is considered. Because of the restrictions on rail cars (i.e., they cannot be maneuvered off the rail line), the materials could be intercepted anywhere along the route, much of which lies outside urban centers, but could not be as readily diverted to an optimal urban setting.

The effects of implementing this alternative are evaluated by assuming the following:

- All shipments routed through the grid are shifted to aircraft with flight paths of $10 \mathrm{~km}$ across the area (see Appendix A routing tables), with the exception of the observed spent fuel shipments.

- Origin/destination shipments by truck are altered to travel by air freight (arbitrary) across the grid and to be carried by truck into their destinations or the reverse.

- No mode shift is included for existing barge shipments.

The results obtained from this assessment are given in Table 6-1.

Table 6-1

Effects of Transport Mode Shifts

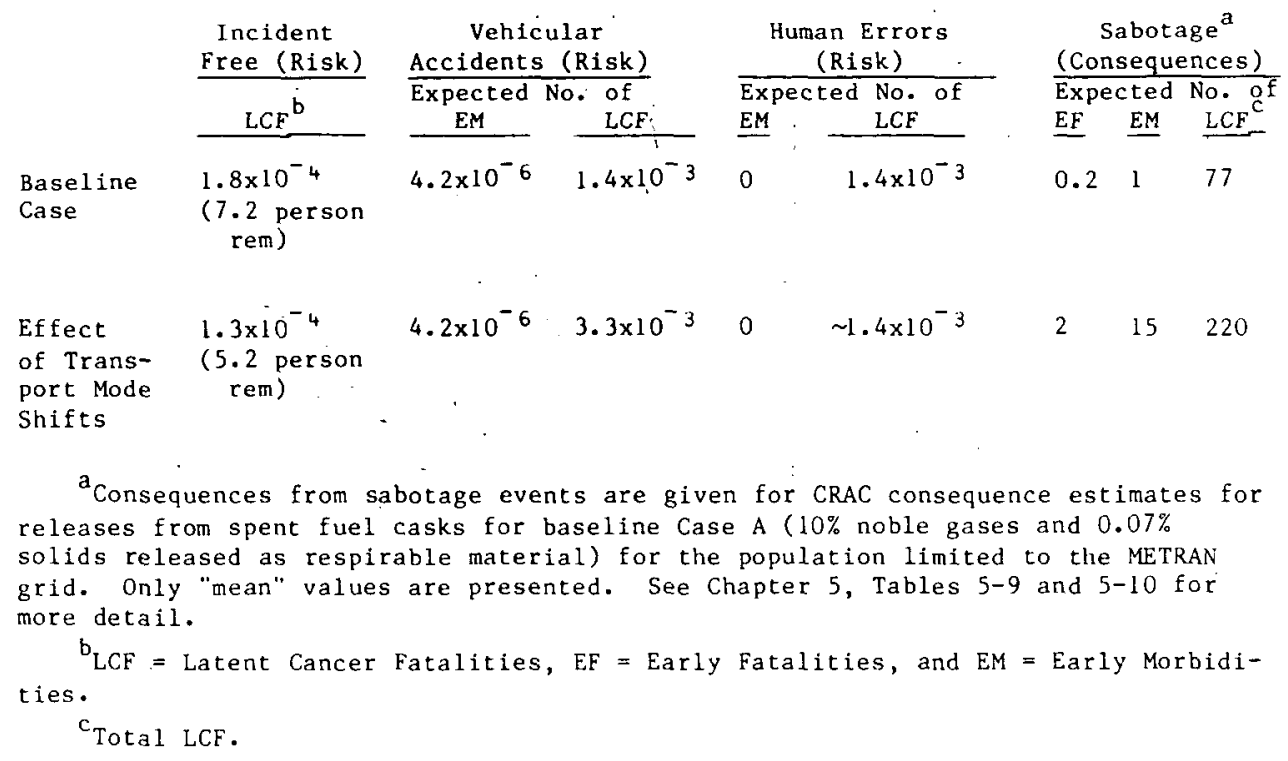


The change in radiological impacts from incident-free transport, vehicular accidents, and human errors is small when this alternative is implemented. In the case of the sabotage event, however, the consequences of sabotage of a shipment containing much larger quantities of material, such as a rail cask would have, could be significant. Earlier discussion alluded to the fact that it would be more difficult for a rail cask to be successfully diverted to an optimal location in the urban area. Since no consideration has been given to how the shipment arrived at a given point, the reported consequence estimates must be evaluated with some care. An important factor in considering transport mode shifts is the economic consequences of their use. To convert from trucks to rail or air transport would require a significant investment especially for large quantity shipments for which new-generation shipping containers might be required. The alternative is feasible, however, only with long-range planning and sizeable capital investment with only small benefit from the radiological risk perspective.

\section{4 . Package Type Changes/Overpack}

Changes in the accident resistance of current packages can effect the consequences or risk from transport of radioactive materials. Type B packages, having higher performance standards and used in place of current Type A packages, could be expected to resist accident damage to a greater degree. Shipments could also be required to have an overpack or additional layer of protection which would provide extra shielding as well as additional accident resistance. For the case of human errors, improved quality assurance practices generally associated with sturdier packages might serve to reduce the occurrence of some types of incidents discussed in Chapter 4. To examine the effects of package type changes the following adjustments are made:

- Al1 materials currently shipped in Type A packages are assumed to be shipped in Type B packages, with the exception of fuel cycle shipments in casks. No attempt has been made to assess the actual change in Transport Index (TI) that might occur with the package type shift. Instead, all TIs are assumed to be decreased by $50 \%$ for the affected shipments.

- For the accident and human error analyses, the increased accident resistance of the package due to overpack, etc. has been addressed by shifting the release fractions by one severity. Thus if the release fraction for a severity 3 accident were 0.1 , it is now 0.01 , and total release of package contents is expected to occur in a higher severity accident. (See Chapter 3, Chapter 4, and Appendix A for details.)

- For fuel cycle shipments in casks, it is assumed that the integrity of the cask provides more protection than a generic Type B package does, so no package type adjustment is made for these shipments. The release fraction change described above is also made for casks.

- Because the sabotage analysis assumes that an event and a release have occurred, this alternative is evaluated by examining the CRAC estimates for a Case $\mathrm{C}(10 \%$ of noble gases and $0.02 \%$ of the solids) release as described in Table 5-9. Again, only "mean" values are reported for this spent fuel release.

Results of implementing this alternative are given in Table 6-2. 
Table 6-2

Effects of Package Type Changes/Overpack

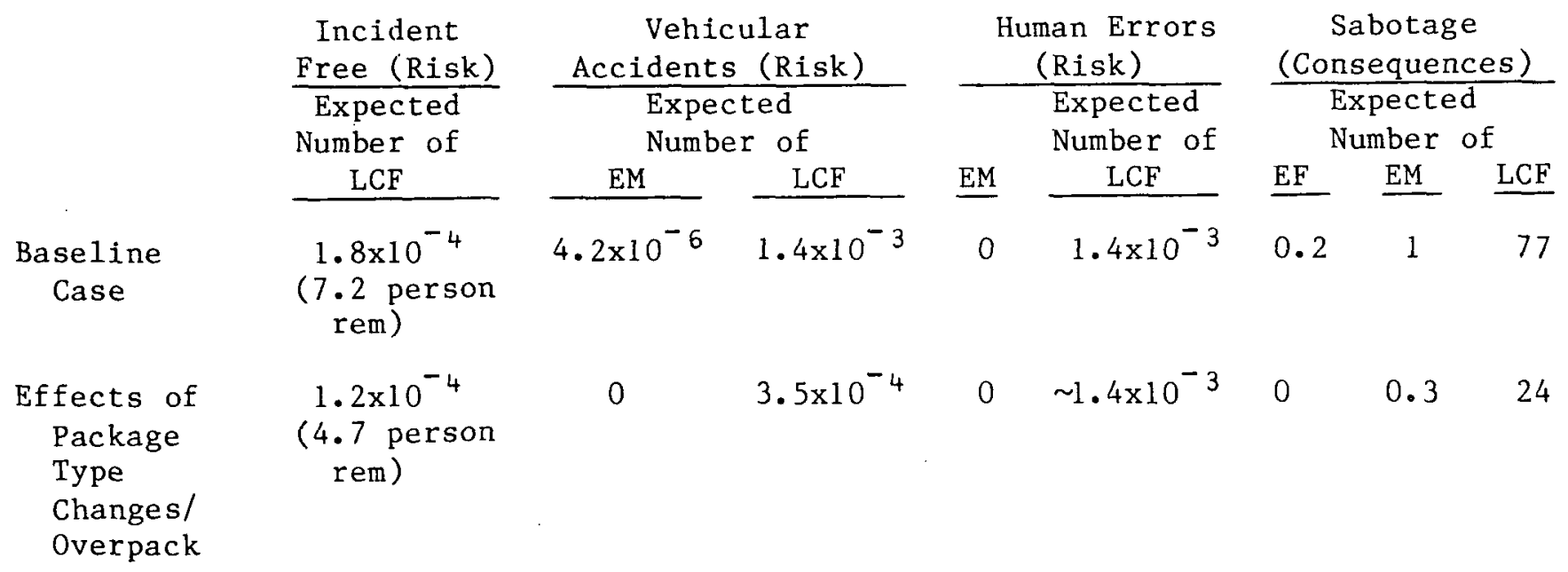

Economic factors are again a consideration, although the capital expenditures for application of this alternative are probably not as extensive as for transport mode shifts.

\subsection{Time of Shipment Restriction}

For some materials, particularly radiopharmaceuticals, a specific delivery time is important since the half life of the isotope may be short and use of the material during a small time window is critical. Also, the delivery or shipment of materials during normal working hours is an obvious convenience. Alternate shipment times can affect the dose to the population at risk since the exposable population shifts with the time of day. Total numbers of pedestrians drop dramatically from 1200 hours to 2400 hours, and there are fewer people in buildings also (see Appendix A for a discussion of time-of-day population variations). To examine the effect of time of shipment restriction on the risk or consequence values for each causative event, various assumptions are used. For incident-free transport, the route start times are adjusted so that the truck link of multimode shipments is set to enter the grid at the specified start time. That is, the air overflight portion of the air/truck shipments is set to occur far enough in advance so that the storage of the material (assumed to be 12 hours as in Chapter 2) would be completed before the truck shipment arrived at the boundary of the grid. For example, if the start time were 0700 hours, the air overflight was set to occur the preceeding evening. Table 6-3 tabulates the results of this analysis.

Similarly, route start time is varied across the maximum changes in population characteristics and meteorological conditions for vehicular accidents, human errors, and sabotage consequences. Meteorological conditions, coupled with time of day, are (1) 1200 hours -- $8 \mathrm{~m} / \mathrm{s}$, south wind; (2) 1630 hours -- $4 \mathrm{~m} / \mathrm{s}$, south wind; and (3) 2400 hours $-2 \mathrm{~m} / \mathrm{s}$, south wind. Results are summarized in Table 6-4. 
Table 6-3

Results of Incident-Free Analysis for Fixed Start Times

\begin{tabular}{|c|c|c|}
\hline $\begin{array}{l}\text { Start } \\
\text { Time }\end{array}$ & $\begin{array}{c}\text { Estimated } \\
\text { Person Rem/ } \\
\text { Shipment Year } \\
\end{array}$ & $\begin{array}{l}\text { Expected Number } \\
\text { of } \\
\text { LCF/Shipment Year }\end{array}$ \\
\hline 0700 & $\sim 12$ & $3.1 \times 10^{-4}$ \\
\hline 0830 & 9.6 & $2.4 \times 10^{-4}$ \\
\hline 1130 & $\sim 16$ & $3.9 \times 10^{-4}$ \\
\hline 1300 & 9.8 & $2.5 \times 10^{-4}$ \\
\hline 1630 & $\sim 13$ & $3.3 \times 10^{-4}$ \\
\hline 1800 & 5.2 & $1.3 \times 10^{-4}$ \\
\hline $\begin{array}{l}\text { Baseline } \\
\text { Case } \\
\text { (Variable } \\
\text { Time) }\end{array}$ & Start & $1.8 \times 10^{-4}$ \\
\hline
\end{tabular}

Table 6-4

Results of Shipment-Time Restriction Analysis:

Vehicular Accidents, Human Errors, Sabotage

\begin{tabular}{|c|c|c|c|}
\hline $\begin{array}{c}\text { Causative } \\
\text { Event }\end{array}$ & $\begin{array}{c}\text { Route Start } \\
\text { Time } \\
\end{array}$ & $\begin{array}{l}\text { Expected Number } \\
\text { of EM } \\
\end{array}$ & $\begin{array}{l}\text { Expected Number } \\
\text { of LCF }\end{array}$ \\
\hline $\begin{array}{l}\text { Vehicular } \\
\text { Accidents } \\
\text { (Risk) } \\
\text { Baseline Case }\end{array}$ & $\begin{array}{l}1200 \\
1630 \\
2400\end{array}$ & $\begin{array}{l}3.0 \times 10^{-6}-6 \\
4.2 \times 10^{-6} \\
2.5 \times 10^{-6} \\
4.2 \times 10^{-6}\end{array}$ & $\begin{array}{l}3.9 \times 10^{-4} \\
1.4 \times 10^{-3} \\
2.9 \times 10^{-3} \\
1.4 \times 10^{-3}\end{array}$ \\
\hline $\begin{array}{l}\text { Human Errors } \\
\text { (Risk) } \\
\text { Baseline Case }\end{array}$ & $\begin{array}{l}1200 \\
1630 \\
2400\end{array}$ & $\begin{array}{l}0 \\
0 \\
0 \\
0\end{array}$ & $\begin{array}{l}\sim 1.8 \times 10^{-3} 3 \\
\sim 1.4 \times 10^{-3} \\
\sim 1.4 \times 10^{-3} \\
\sim 1.4 \times 10^{-3}\end{array}$ \\
\hline $\begin{array}{l}\text { Sabotage } \\
\text { (Consequences) } \\
\text { of Spent Fuel } \\
\text { Sabotage } \\
\text { Case A, } \\
\text { Cell D, } \\
\text { METRAN Analysis }\end{array}$ & $\begin{array}{l}1200 \\
1630 \\
2400\end{array}$ & $\begin{array}{l}31 \\
32 \\
21\end{array}$ & $\begin{array}{l}26 \\
32 \\
34\end{array}$ \\
\hline
\end{tabular}


Economic factors are again a major consideration. Since staffing at receiving sites and test schedules would have to be altered if materials are delivered at odd hours, the economic impacts of this alternative might be significant. The economic factors must be weighed against possible changes in the small radiological risk observed using current transport practice in deciding on implementation of this alternative.

\subsection{Operational Alternatives}

Alternatives related to intermediate stops of materials during transit of the material as well as those related to changes in the transport vehicle or its total contents are referred to as operational alternatives. Five separate operational alternatives are considered:

- Reduction in storage time,

- Cordoning off of storage areas,

- Reduction in number of handlings,

- Extra shielding in the transport vehicle, and

- Containerized shipping.

\subsubsection{Reduction in Storage Time}

To examine the effect of storage-time reduction, the 12-hour (equivalent to overnight) storage of materials is arbitrarily reduced to 6 hours. The distance of closest approach to the shipment remains constant at 1.5 metres. The effect of storage time reduction is linear with time for warehouse personne1, thus the . baseline value from Chapter 2 is reduced from 1.2 person rem to 0.6 person rem per shipment year. The resultant $8 \%$ decrease in the total population dose is from 7.2 person rem to 6.6 person rem per shipment year. The $50 \%$ decrease in exposure to warehouse personnel could be considered important if the dose to these individuals is considered part of their occupational exposure. However, the very low value calculated for the given level of shipping activity is already quite small, and the achieved reduction is probably not significant.

With the possibility of start-time restrictions, the dose to warehouse personne 1 could be increased or decreased depending upon requirements that shipments remain at storage locations for shorter or longer times until the shipment can be transported to its destination.

Changes in storage time could have an effect on the consequences of vehicular accidents and/or human errors in the sense that accidents involving these materials could occur at times of day which would influence the exposable population. This question has been addressed already under time-of-shipment restrictions.

\subsubsection{Cordoning off Storage Areas}

If warehouse personnel are required to 'remain farther from a shipment while in storage, their dose could be reduced. To assess the effects of cordoning off storage areas, it was assumed that the minimum distance for exposure changes from 1.5 metres to 5 metres. The net effect of this change is to reduce the warehouse personne1 dose from 1.2 person rem per shipment year to $\sim 0.4$ person rem per shipment year. The net effect on the total population exposure is an $11 \%$ reduction, 
from 7.2 to 6.4 person rem per shipment year. If this alternative were coupled with a reduction in storage time, the already small warehouse personnel dose perhaps could be reduced even further. If the total warehouse personnel exposure is removed, the net population dose becomes 6 person rem per shipment year, a $17 \%$ reduction. No other causative event is affected by this alternative.

\subsubsection{Reduction in Number of Handlings}

The maximum number of handlings addressed in the analysis is one. If the number of handlings is set to zero (no handling in the urban area), the dose to handlers becomes zero, which results in a $35 \%$ reduction in the total incident-free population exposure. This alternative seems inapplicable since shipments must be loaded at their origins or off-loaded at their destinations.

6.6.4 Extra Shielding in the Transport Vehicle

The significant consequence of this alternative is its effect on exposure to crew members during transit. The dose rate allowable in the cab of an exclusive-use transport vehicle is set by regulation (see Appendix $B$ ). To assess the effect of extra shielding in the cab of the vehicle, all materials are assumed to be shipped in exclusive-use vehicles, in the absence of better information.

Reductions of the exclusive-use vehicle limit of $2 \mathrm{mrem} / \mathrm{h}$ to $1 \mathrm{mrem} / \mathrm{h}$ and to 0.5 $\mathrm{mrem} / \mathrm{h}$ results in no change in the calculated crew dose. This indicates that for this particular set of standard shipments, the calculated dose rates for crew exposure are below the $0.5 \mathrm{mrem} / \mathrm{h}$ level. The removal of all dose to crew from the incident-free population exposure would result in an $8 \%$ reduction in the baseline calculated value.

For the accident case, the effect of this alternative is less straightforward. In accidents where the material is not dispersed -- i.e., the accident results in increased external population exposure -- crew members may receive increased dose due to the accident delay time. Quantifying the reduction in exposure that would result from increased shielding, is assumed, to be similar to quantifying the reduction in the incident-free case. Because crew dose is much less significant in the accident case, the effect on total risk to the population is negligible. This same argument holds for human errors resulting in negligible reduction in risk. In the sabotage case, the entire dose results from dispersal of the materials; hence the effect of this alcernative would be zero.

\subsubsection{Containerized Shipping}

Containerized shipping means packaging together several (or many) shipments which are bound for a single destination. Examples of containerized shipments include packing of many materials into uniform-size containers for stowage in the cargo hold of a ship or aircraft and piggyback shipments of truck trailers aboard freight trains. One problem with generally applying this alternative is that except for large users of radioactive materials, (e.g., waste shipments) the destinations for shipments are too numerous to make containerization practical or profitable. To address this alternative, the assumption is made that only for waste shipments would containerized shipping be practical. Thus, all waste shipments are considered to be containerized, and the shipment-specific parameters (curies per package and TI per package) are scaled linearly. The now containerized shipments are allowed to proceed along their routes. 
Since the incident-free population dose is mostly dependent upon the total TI shipped, this alternative would not have any effect. In the case of vehicular accidents and human errors, however, the larger quantities of material could produce greater consequences. Table 6-5 presents the results of this analysis. In this instance, the baseline cases for vehicular accidents and human errors represent the expected numbers of latent cancer fatalities for waste shipments only and similarly for the containerized shipping case. Since the sabotage analysis considers that an event has occurred and calculates consequences from a given release, this alternative is not applicable.

\author{
Table 6-5 \\ Effects of Containerized Shipping \\ on Expected Number of Latent Cancer \\ Fatalities per Shipment Year \\ (Waste Shipments Only)
}

\begin{tabular}{lr} 
Causative Event & \\
$\begin{array}{c}\text { Vehicular Accidents } \\
\text { Baseline Case } \\
\text { Containerized Shipping }\end{array}$ & $\begin{array}{c}\text { Expected Number of } \\
\text { Latent Cancer } \\
\text { Fatalities }\end{array}$ \\
\hline & $8.3 \times 10^{-7}$ \\
& $8 \times 10^{-7}$
\end{tabular}

Human Error

Baseline Case

$1.2 \times 10^{-8}$

Containerized Shipping

$1.2 \times 10^{-8}$

The cost effectiveness of this alternative is questionable for most urban shipments because (1) much of the transport would still have to be on a per shipment destination basis, and (2) for the limited New York City case, no effect is observed in the expected numbers of latent cancer fatalities.

\title{
6.7 Reduction or Elimination of Some Shipments
}

Except for the sabotage case, where consequences are calculated for an event that is assumed to have already occurred, reducing or eliminating some shipments would remove from the total risk numbers the contributions of these shipments. The actual amount of the decrease would depend upon the particular shipments involved. To address this question, two separate calculations have been made. First, all shipments routed through the grid have been eliminated as nonessential to the urban area. Second, only large-quantity shipments have been eliminated in line with recent proposed restrictions on the shipment of these materials through densely populated urban areas. In the limited New York City case, the only shipments fal1ing within the regulatory definition of large quantity are the spent fuel shipments along Route 17 of the standard shipments mode1 (see Appendix A). The effects of 
these two options and the values for the baseline case are given in Table 6-6. Reducing or eliminating some shipments is equivalent to rerouting these materials around the urban area. The consequences of rerouting materials with the capacity to produce significant consequences in the urban setting (see Tables 3-10 and 3-11) are discussed in the next section of this chapter. Here, reducing or eliminating shipments as discussed above is a mechanism for examining the effectiveness of this alternative on a limited geographical area. The results of the rerouting discussion would, of course, have to be added to the values in Table 6-6, thus mitigating the effects of the shipment reductions. In the case of sabotage, this alternative would decrease the availability, of those vehicles within the urban setting for diversion. However, since that analysis presents only the consequences of an event assumed to have occurred, the effect of this alternative cannot be assessed.

\section{Table 6-6}

Effects of Reduction or Elimination of Some Shipments

Causative
Event

Incident-Free Transport (Risk)

LCF

Vehicular Accidents (Risk)

EM

LCF

Human Errors (Risk)

EM

LCF

Sabotage (Consequences)

$\begin{array}{lc}\text { EF } & 0.2 \\ \text { EM } & 1 \\ \text { LCF } & 77\end{array}$

Elimination of Elimination of Only A11 Through Large-Quantity Shipments Shipments

\begin{tabular}{cc}
$\begin{array}{c}\text { Baseline } \\
\text { Case }\end{array}$ & $\begin{array}{c}\text { A11 Through } \\
\text { Shipments }\end{array}$ \\
\hline
\end{tabular}

$1.8 \times 10^{-4}$

$4.2 \times 10^{-6}$

$1.4 \times 10^{-3}$

$7.7 \times 10^{-4}$

0

$7.7 \times 10^{-4}$

0

$1.4 \times 10^{-3}$

$$
4.8 \times 10^{-5}
$$

0 $4.8 \times 10^{-5}$

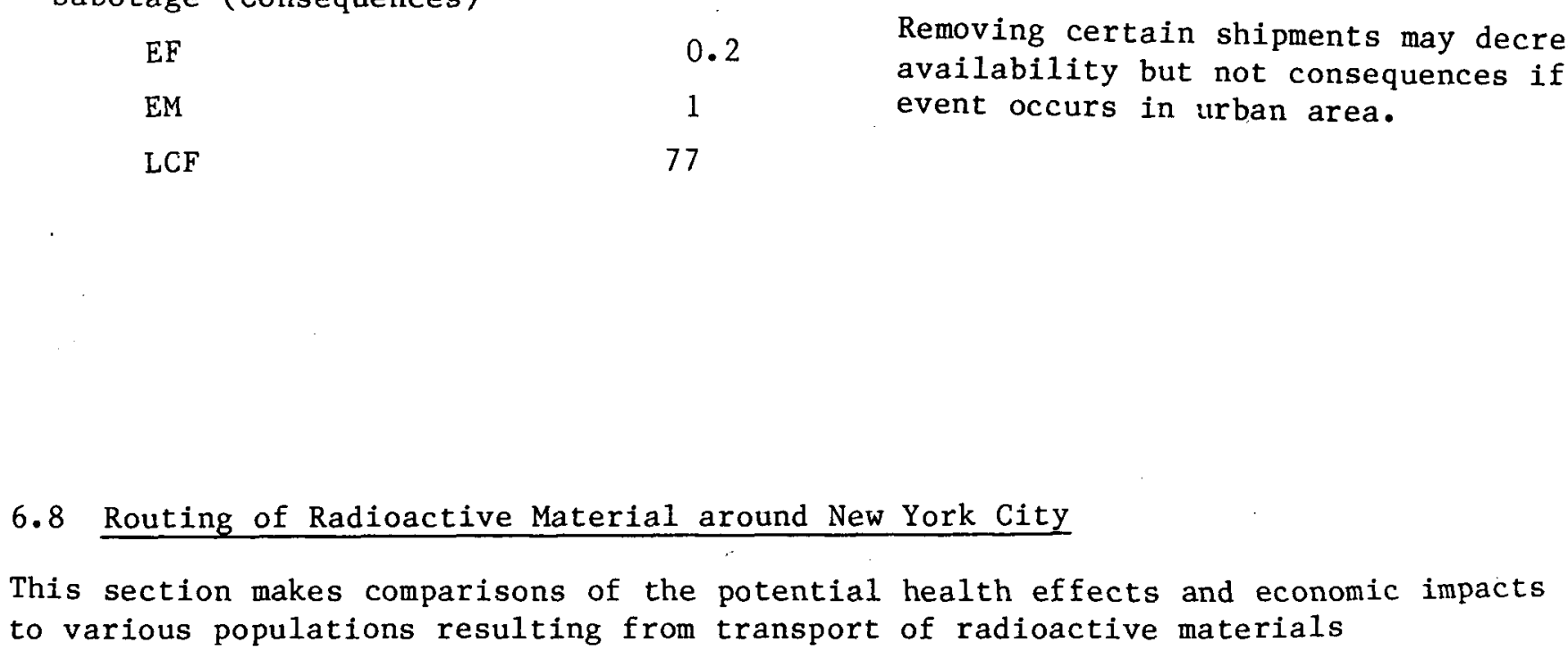


through and around New York City (NYC) for all causative event categories discussed in Chapters 2 through 5 of the report. Seven of the largest radioactive material shipments identified previously are investigated. The simplifying equations for incident-free transport, derived in Appendix D, are used to compare the impacts of routing changes in the NYC area. Calculations are performed using the METRAN code to compare the potential impacts from vehicular accidents and human errors. In these cases, the $100-\mathrm{km}^{2}$ grid is transposed to the geographical area of interest with appropriate adjustments of demographic, traffic, and meteorological data. The analysis is then performed assuming that the releases of radioactive material occur in a single cell. Comparisons of the potential impacts from sabotage of shipments of spent fuel and plutonium are made with the CRAC code (see Chapter 5). Four points along the two routes are used for comparison.

Emphasis in this section is on the relative changes in anticipated impacts from a given causative event rather than on the absolute values calculated. The analysis can be applied to other urban/rural areas, assuming that the necessary input data are available. Similar analyses can be performed on other specific routes as desired.

Routes chosen for the comparisons represent examples used to exercise the methodology and do not represent a recommendation for routing or not routing around the city. Since the results depend heavily on the selected path of travel, extrapolation to other routes should be made with extreme caution.

In order to demonstrate the methodology developed to compare the consequences from transporting radioactive material around versus through NYC, the following radioactive shipments are selected:*

1. Co-60; nondispersible

$4.7 \times 10^{3} \mathrm{Ci} / \mathrm{pkg}$

$\mathrm{TI}=1.3$

1 package/shipment, cask

6 shipments/year

2. Co-60; nondispersible

$3.15 \times 10^{5} \mathrm{Ci} / \mathrm{pkg}$

$\mathrm{TI}=1.3$

1 package/shipment, cask

6 shipments/year

3. Po-210; dispersible

$1.44 \times 10^{2} \mathrm{Ci} / \mathrm{pkg}$

$\mathrm{TI}=1.0$

1 Type B package/shipment

2 shipments/year

4. Mo-99; dispersible

$91 \mathrm{Ci} / \mathrm{pkg}$

$\mathrm{TI}=6.2$

1 Type B package/shipment

48 shipments/year

* Only truck transport is considered here since the majority of the impacts calculated in Chapters 2 through 5 result from this mode of transport. 
5. Pu; dispersible

$1.13 \times 10^{6} \mathrm{Ci} / \mathrm{pkg}$

$\mathrm{TI}=2$

1 Type B package/shipment

1 shipment/year

6. SPF-IH; dispersible (research reactor fuel)

$154 \mathrm{Ci} / \mathrm{pkg}$

$\mathrm{TI}=0$

1 package/shipment, cask

12 shipments/year

7. SPF-TH; dispersible (commercial reactor fuel)

$3.74 \times 10^{3} \mathrm{Ci} / \mathrm{pkg}$ (sabotage source)

$\mathrm{TI}=0$

1 package/shipment, cask

1 shipment/year

Shipments 1, 3, 4, and 6 were observed in the New York City shipment model for 1975 (see Appendix A). Shipments 2 and 5 are included for comparison since they have been observed in other urban areas. Shipment 7 is included because of the widespread interest in commercial spent fuel transport and because of the potential for shipments of this material through New York State in the future.

\subsubsection{Route Descriptions}

It is assumed that the route through the urban area follows Interstate 95 (I-95) (see Figure 6-1). The urban route starts at the northern intersection of U.S. Highway 287 (Hwy-287) and I-95, north of Yonkers, New York. It passes through New York City and Newark and ends at the southern intersection of Hwy-287 and I-95. The route is subdivided into five sections, $P_{1}$ to $P_{5}$. The section through the city is represented by $P_{2}$, through Newark by $P_{4}$, and near the urban areas by $P_{1}, P_{3}$, and $P_{5}$. The length of this route is about $85 \mathrm{~km}$.

The route around the urban area starts at the northern intersection of Hwy-287 and I-95. It follows Hwy-287 to the west and then south of New York City before ending at the southern intersection of $H w y-287$ and I-95. This route complies with the current requirement that spent fuel shipments should avoid, where practicable, heavily populated areas. ${ }^{2}$ The, closest route distance to an urban area is about 6.7 $\mathrm{km}$ from Paterson, New Jersey. The length of this route is $154 \mathrm{~km}$ and is represented by $\mathrm{P}_{6}$.

In the following subsections, the potential impacts of transporting the selected radioactive shipments along routes $\mathrm{P}_{1}-\mathrm{P}_{5}$ and $\mathrm{P}_{6}$ are compared. They are also compared with the calculated results for the urban grid (see Chapter 2).

\subsubsection{Radiological Impacts from Incident-Free Transport}

This subsection describes the potential health effects to the population due to incident-free transportation of radioactive material on freeways. The population groups at risk are mainly the crew and people in vehicles since it is assumed in the analysis that there are no pedestrians on freeways. The dose to people in buildings is negligible, as shown in Chapter 2. 


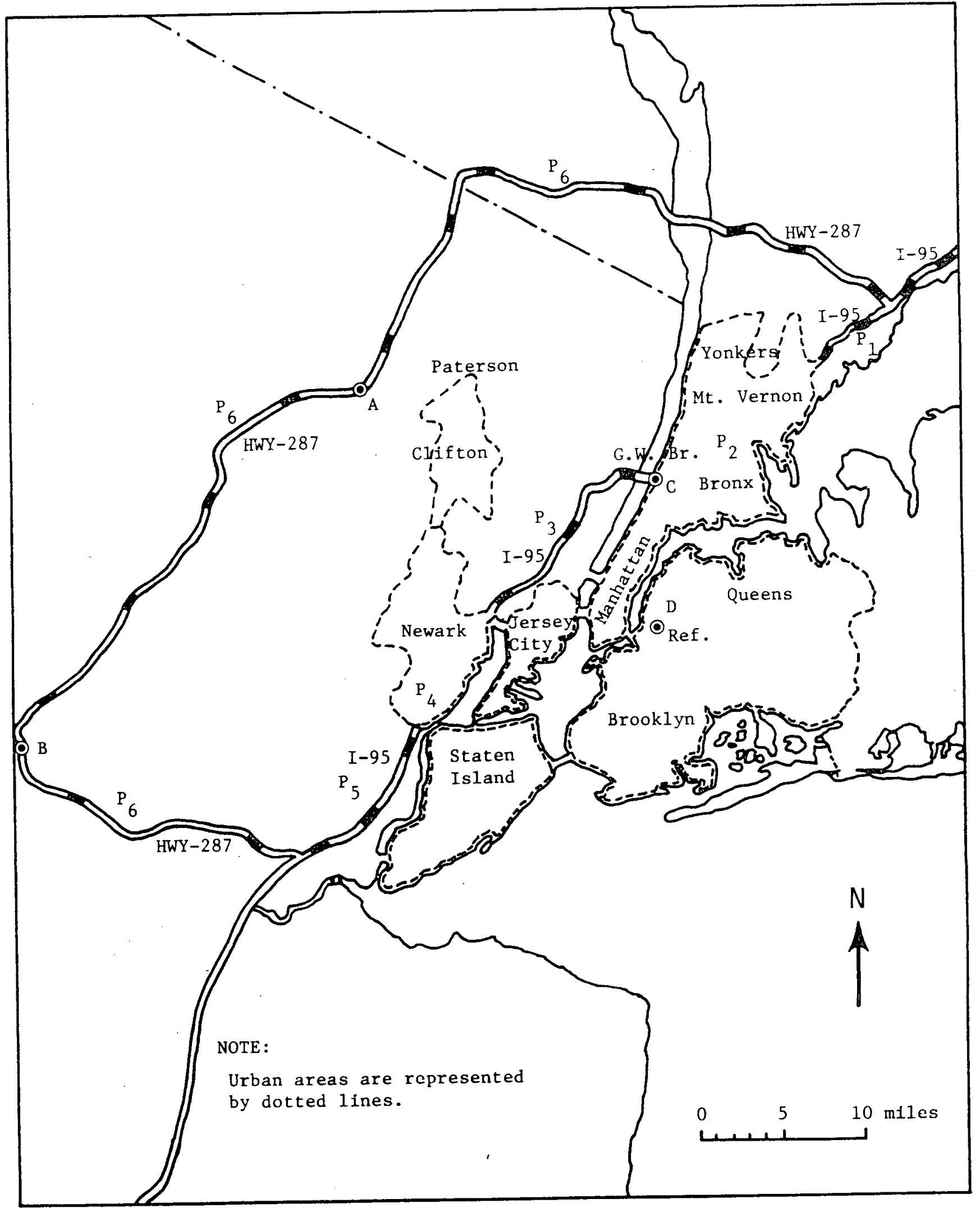

Figure 6-1. Routes through and around New York City 
Dose to Crew

The equations derived in Appendix $\mathrm{D}$ are used to estimate the integrated dose to crew for both routes.

The dose to crew for travel on freeways is given by*

$$
\mathrm{ID}_{\mathrm{c}} \approx \mathrm{Q}_{3} \cdot \mathrm{K}_{0} \cdot \frac{\mathrm{L}}{\mathrm{V}} \cdot \mathrm{N}_{\mathrm{c}} \cdot \mathrm{TI} \cdot \mathrm{PPS} \cdot \mathrm{SPY} \cdot \frac{\mathrm{e}^{-\mu_{\mathrm{air}} \mathrm{d}_{2}} \cdot \mathrm{B}_{\mathrm{air}}\left(\mathrm{d}_{2}\right)}{\mathrm{d}_{2}{ }^{2}}
$$

Since all parameters remain constant except for $L$ and $\bar{V}$, the ratio of the integrated dose for routing around versus through the urban area is given by

$$
\frac{\mathrm{ID}_{\mathrm{RR}}}{\mathrm{ID}_{\mathrm{UA}}}=\frac{\mathrm{L}_{\mathrm{RR}} / \mathrm{V}_{\mathrm{RR}}}{\mathrm{L}_{\mathrm{UA}} / \mathrm{V}_{\mathrm{UA}}}
$$

where

$$
\begin{aligned}
{ }^{I D} D_{R R} & =\text { integrated dose for rerouting (person rem) } \\
{ }^{I D} D_{U A} & =\text { integrated dose for urban area route (person rem) } \\
{ }^{{ }_{R R}} & =\text { rerouting length }\left(P_{6}\right. \text { route) (metres) } \\
V_{R R} & =\text { rerouting average speed }(\mathrm{m} / \mathrm{s}) \\
L_{U A} & =\text { route length through urban area }\left(P_{1}-P_{5}\right) \text { (metres) } \\
V_{U A} & =\text { average speed through urban area }\left(P_{1}-P_{5}\right)(m / s)
\end{aligned}
$$

The input data required for the comparison are listed in Table 6-7. The average speed will vary as a function of time of day; therefore the dose ratio will also vary as a function of time. For the afternoon rush period,

$$
\frac{\mathrm{ID}_{\mathrm{RR}}}{\mathrm{ID}_{\mathrm{UA}}} \approx 1.25
$$

Thus, the dose to crew is increased about $25 \%$ by routing around the urban area at this time.

* Regulations limit the maximum allowable dose rate in the exclusive-use vehicle cab to 2 milliroentgens per hour $(\mathrm{mR} / \mathrm{h})$. If this value is exceeded, the analysis proceeds as though sufficient shielding is in place to reduce the level to $2 \mathrm{mR} / \mathrm{h}$. Equation 1 is used for dose rates below the maximum allowed. 
Table 6-7

Input Data to Estimate Dose to Crew and People in Vehicles

\begin{tabular}{|c|c|c|c|c|}
\hline Population & Route & Average Vehicle & Number of Veh. & $\begin{array}{l}\text { Average } \\
\text { Persons }\end{array}$ \\
\hline Zones & Length $(\mathrm{km})$ & Speed $(\mathrm{km} / \mathrm{h})$ & per Cell (veh $\left./ \mathrm{km}^{2}\right)$ & per Vehicle \\
\hline $\mathrm{Pl}$ & 9.6 & 65 & 65 & 2.0 \\
\hline $\mathrm{P} 2$ & 24.0 & 32 & 170 & 2.7 \\
\hline P3 & 19.2 & 65 & 65 & 2.0 \\
\hline P4 & 14.4 & 58 & 120 & 2.4 \\
\hline P5 & 17.6 & 65 & 65 & 2.0 \\
\hline P6 & 154.0 & 72 & 50 & 1.5 \\
\hline
\end{tabular}

NOTES:

1. Data are for rush hour (1630 to 1800).

2. Values are estimated based on information from References 3 and 4. Data for highways in New Jersey and New York are used.

3. Number of vehicles per cell from Reference 3 is multiplied by 1.5 to account for increase in traffic volume from 1961 to 1975 , based on data from Reference 5 for New Jersey and New York.

If routing is compared at nighttime ( $\sim 100$ hours), the average speeds for urban and rural area freeways are similar and the dose ratio is approximately equal to the ratio of the route length. Thus, assuming $\mathrm{V}_{\mathrm{RR}}=\mathrm{V}_{\mathrm{UA}}$,

$$
\frac{\mathrm{ID}_{\mathrm{RR}}}{\mathrm{ID}_{\mathrm{UA}}} \approx \frac{\mathrm{L}_{\mathrm{RR}}}{\mathrm{L}_{\mathrm{UA}}} \approx 1.82
$$

The integrated dose to the crew $\left(\mathrm{ID}_{\mathrm{UA}}\right.$ ) from incident-free transport through New York City is about 0.6 person rem per shipment year (see Chapter 2). In general, it is expected that the dose to crew will increase by routing around New York City, due to the increase in travel time.

Dose to People in Vehicles

The dose to people in vehicles (PIV; see also Appendix D) for travel on freeways is given by

$\mathrm{ID}_{\mathrm{V}}=10^{-12} \cdot \mathrm{K}_{\mathrm{o}} ; \mathrm{TI} \cdot \mathrm{PPS} \cdot \mathrm{PPV} \cdot \mathrm{L} \cdot \mathrm{N} \cdot \mathrm{SPY}\left(17.38-1.244 \mathrm{~V}_{\mathrm{f}}+0.02665 \mathrm{~V}_{\mathrm{f}}^{2}\right)$

Since all parameters remain constant except for $P P V, L, N$, and $V_{f}$, the ratio of the integrated dose to people in vehicles for routing around versus through the urban area is given by 


$$
\frac{\mathrm{ID}_{\mathrm{VR}}}{\mathrm{ID}_{\mathrm{VU}}}=\frac{(\mathrm{PPV} \cdot \mathrm{L} \cdot \mathrm{N})_{\mathrm{RR}} \cdot\left(17.38-1.244 \mathrm{~V}_{\mathrm{RR}}+0.02665\left(\mathrm{~V}_{\mathrm{RR}}\right)^{2}\right.}{(\mathrm{PPV} \cdot \mathrm{L} \cdot \mathrm{N})_{\mathrm{UA}} \cdot\left(17.38-1.244 \mathrm{~V}_{\mathrm{UA}}+0.02665\left(\mathrm{~V}_{\mathrm{UA}}\right)^{2}\right.},
$$

where

$$
\begin{aligned}
\mathrm{ID}_{\mathrm{VR}}= & \begin{aligned}
\text { integrated dose to people in vehicles for rerouting } \\
\text { (person rem) }
\end{aligned} \\
\mathrm{ID}_{\mathrm{VU}}= & \begin{array}{l}
\text { integrated dose to people in vehicles for urban area route } \\
\text { (person rem) }
\end{array} \\
\mathrm{PPV}_{\mathrm{RR}}= & \text { people per vehicle for rerouting } \\
\mathrm{L}_{\mathrm{RR}}= & \text { rerouting length }(\mathrm{km}) \\
\mathrm{N}_{\mathrm{RR}}= & \text { number of vehicles in the cell for rerouting (veh/ } \left.\mathrm{km}^{2}\right) \\
\mathrm{V}_{\mathrm{RR}}= & \text { average speed rerouting (m/s) } \\
\mathrm{PPV}_{\mathrm{UA}}= & \text { people per vehicle on route through urban area } \\
\mathrm{L}_{\mathrm{UA}}= & \text { route length through urban area ( } \mathrm{km}) \\
\mathrm{N}_{\mathrm{UA}}= & \text { number of vehicles in the cell on route through urban area } \\
& \text { (veh/km } 2 \text { ) } \\
\mathrm{V}_{\mathrm{UA}}= & \text { average speed on route through urban area (m/s) }
\end{aligned}
$$

Using the input data in Table 6-7 for rush hour, the above ratio becomes

$$
\frac{I D_{V R}}{I D_{V U}}=0.28
$$

The dose to people in vehicles in the rural area is about $28 \%$ of the value for the urban area. However, if the urban shipments occurred late at night, the vehicle speeds would be similar to those in the rural route and also the differences in number of vehicles would be less. Under certain circumstances (e.g., similar speed and vehicle density but longer rural route), the integrated dose to people in vehicles could be higher for the rural route.

It should be observed that the ratio of the integrated dose to people in vehicles (and crew) from incident-free transport is independent of the radioactive source characteristics. For the specific routes considered in this section, the maximum value of the ratio $\mathrm{ID}_{\mathrm{RR}} / \mathrm{ID}_{\mathrm{UA}}$ is about 1.8 ( $80 \%$ increase). The integrated dose to people in vehicles from incident-free transport through New York City is about 1.6 person rem per shipment year (see Chapter 2).

In summary, the integrated dose to penple in vehicles and crew from incident-free transport may increase by routing around New York City. For the specific routes considered in this section, the maximum increase is about $80 \%$. However the total integrated dose to people in vehicles and crew in New York City is very small ( 2 person rem/shipment year). 


\subsubsection{Impacts from Vehicular Accidents}

This subsection compares the potential radiological and economic impacts from vehicular accidents involving the shipments described in the previous section. The impacts are estimated using the METRAN code with a 10-km by 10-km grid. The grid, located in the urban area (point D, Figure 6-1), is described in Appendix A. Similar grids with homogeneous characteristics (parameters shown in Table 6-8) are used for points A, B, and C (see Figure 6-1).

Table 6-8

Parameters at Points $\mathrm{A}, \mathrm{B}$, and $\mathrm{C}$

Population Distribution

\begin{tabular}{|c|c|c|c|c|}
\hline $\begin{array}{l}\text { Point of } \\
\text { Release }\end{array}$ & $\begin{array}{c}\text { Radius From } \\
\text { Release } \\
(\mathrm{km}) \\
\end{array}$ & $\begin{array}{l}\text { Population } \\
\text { Density } \\
\left.\text { (people } / \mathrm{km}^{2}\right)^{a}\end{array}$ & $\begin{array}{l}\text { Fraction of } \\
\text { Buildings } \\
\text { Per Cell } \\
\end{array}$ & $\begin{array}{c}\text { Number of } \\
\text { Vehicles Per } \\
\text { Cell } \\
\end{array}$ \\
\hline \multirow[t]{2}{*}{$A(N W)$} & $0-8$ & 390 & 0.02 & 65 \\
\hline & $8-10^{c}$ & 2070 & 0.12 & 65 \\
\hline$A(S E)$ & $0-10$ & 207 & 0.01 & 65 \\
\hline $\begin{array}{l}B(W) \\
B(E)\end{array}$ & $\begin{array}{l}0-10 \\
0-10\end{array}$ & $\begin{array}{l}90 \\
90\end{array}$ & $\begin{array}{l}0.005 \\
0.005\end{array}$ & $\begin{array}{l}65 \\
65\end{array}$ \\
\hline$C(N W)$ & $0-10$ & 24000 & 0.70 & 170 \\
\hline
\end{tabular}

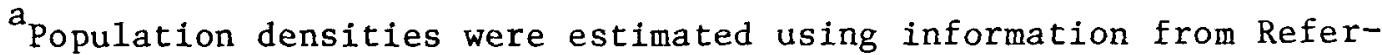
ence 5 (for 1976).

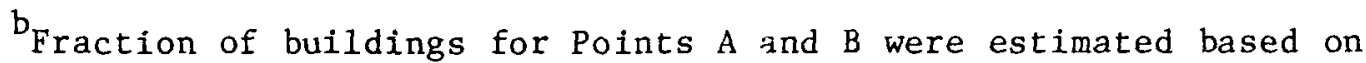
population densities.

${ }^{\mathrm{C}}$ At $>8 \mathrm{~km}$, a more densely populated area is observed.

Only vehicular accidents of severity VIII (see Chapter 3) are considered. The intent is to use these accidents as upper bounds to compare potential consequences on both routes.* The consequences are estimated in terms of expected numbers of latent cancer fatalities since specific data are not available to make reasonable estimates of early fatalities and early morbidities at points A, B, and C. Early fatalities and morbidities are very sensitive to wind speed, population location, and geometric arrangements of buildings, streets, and open areas. This information is not readily available for the above points. On the other hand, expected numbers of latent cancer fatalities are mostly dependent on the release source and population densities, for which there is more information. Therefore, all comparisons are made in terms of latent cancer fatalities.

The latent cancer fatalities expected from vehicular accidents involving nondispersible sources (e.g., Co-60) are similar for all points. This is due to the model assumption that vehicles (people in vehicles are the main exposed group) will

*It should be observed that the probability of occurrence of a severity VIII accident nationwide is $1.1 \times 10^{-5}$. 
stop in clusters near the accident area. The economic impacts are the same for all the points.

In summary, the radiological and economic impacts of shipping nondispersible sources are expected to be very small even if transported through urban areas. The economic impacts are small (a few thousand dollars) for both rural and urban areas. Table 6-9 summarizes the impacts from accidents.

Table 6-9

Comparsion of Impacts from Vehicular Accidents

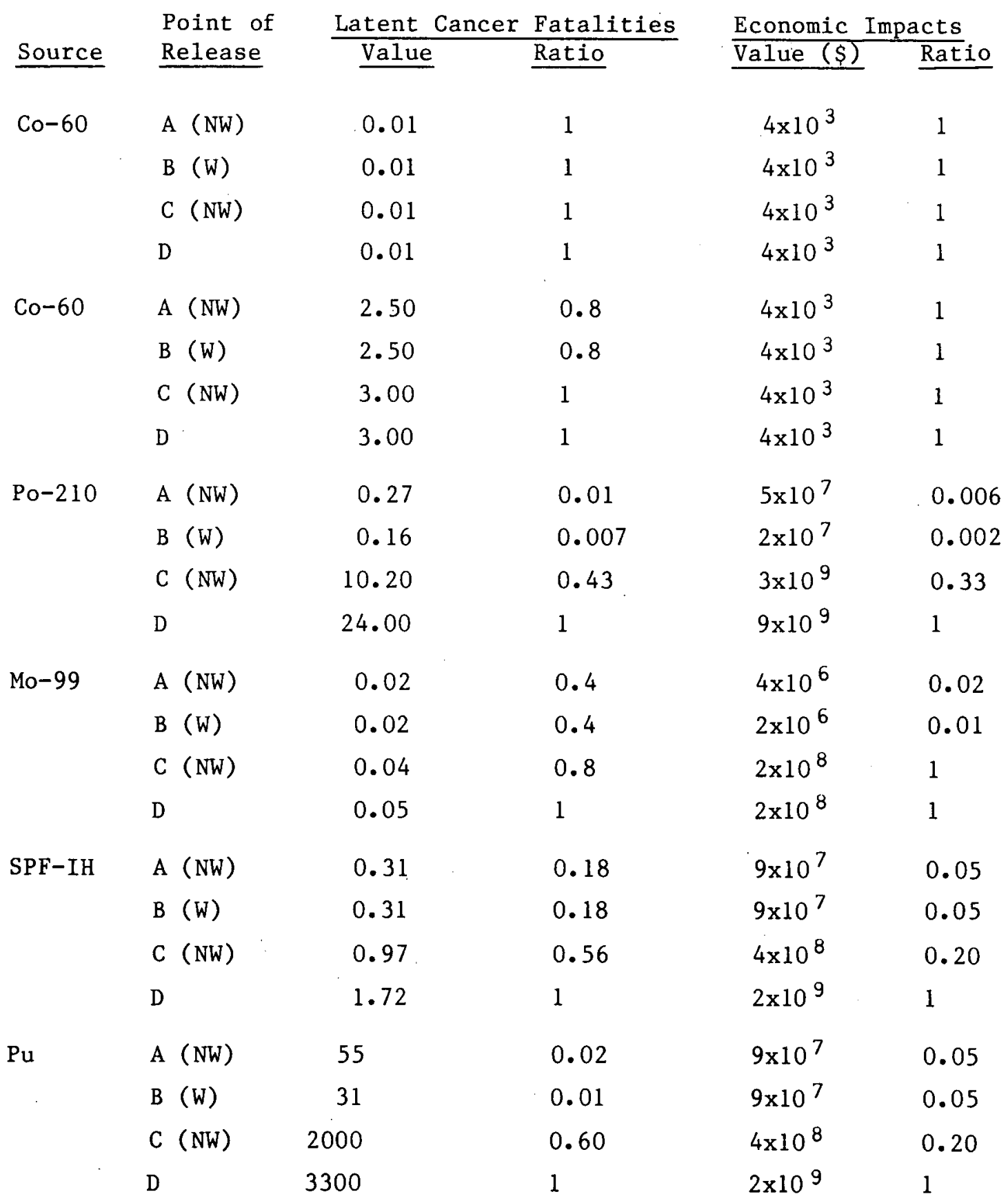


The potential latent cancer fatalities from Po-210 and Pu are reduced to a few percent $(1 \%-2 \%)$ of the values for the urban route. This is a significant reduction for the $\mathrm{Pu}$ source because of the relatively large number of expected latent cancer fatalities. The reduction in economic impacts is also significant $(95 \%-99 \%$ reduction), since the cost of severity VIII accidents could be of the order of a billion dollars in urban areas. The input data to estimate the economic impact for the rural area are shown in Table 6-10. The potential radiological consequences from Mo-99 and SPF-IH are relatively small on both routes, but the economic impacts are greatly reduced in the rural area.

Table 6-10

Economic Impact Parameters for Route $\mathrm{P}_{6}$

Parameter

1. Cleanup leve1

2. Upper limit of lightly contaminated regime

3. Lower limit of moderately contaminated regime

4. Loaded salary for health physics technician

5. Survey rate

6. Half-life check value

7. Filter density

8. Cost for street cleaning level

9. Cost for building cleanup $(N=1,2)$ level 1

10. Cost for building cleanup $(N=3,4,5)$ level 1

11. Cost for building cleanup (C/I) level 1

12. Cost for park/open area cleanup level 1

13. Evacuation costs

14. Individual income loss

15. Crops income loss

16. Cost for building cleanup $(N=1,2,3,4,5)$ level 2

17. Cost for parks/open area cleanup

18. Land value
Value

$0.2 \mu \mathrm{Ci} / \mathrm{m}^{2}$

$2 \mu \mathrm{Ci} / \mathrm{m}^{2}$

$20 \mu \mathrm{Ci} / \mathrm{m}^{2}$

$\$ 9 / \mathrm{h}$

$120 \mathrm{~m}^{2} / \mathrm{h}$

180 days

0.02 filters $/ \mathrm{m}^{2}$

$\$ 4.38 / \mathrm{m}^{2}$

$\$ 506 /$ capita

0

0

$\$ 101 /$ capita

$\$ 200 /$ capita

$\$ 575 /$ capita $^{5}$

$\$ 198 /$ capita $^{5}$

0

$\$ 673 /$ capita

$\$ 1314 /$ capita $^{5}$ 
The potential economic impacts from the dispersible sources considered in this section could be significantly reduced if an accident occurs in a rural area instead of the urban area. The percent reduction in radiological effects in the rural area would depend on the type of source, the source strength, and the curies per shipment. For example, rerouting of $\mathrm{Pu}$ and Po-210 shipments results in significant reductions, while Mo-99 shipments show small reductions.

Risk comparisons for the above shipments are not possible, due in part to the lack of accident rate information for the specific routes. Shipments of radioactive materials through routes where higher accident rates are possible could increase the risk to the population, even though the potential consequences from a specific accident are reduced. Therefore, the calculations in this section do not indicate whether the risk to the public from the above shipments is increased or decreased by routing around the urban areas.

\subsubsection{Impacts from Human Error Incidents}

Analysis of the potential consequences of a human error or deviation from accepted quality assurance practices is analogous to that for vehicular accidents. Since only consequences (and not risk) are evaluated, the results of the preceeding section are also valid for human errors. Changes in population risk are not evaluated because of lack of data on human error incident rates for the different routes.

\subsubsection{Impacts from Sabotage Acts}

The potential radiological and economic impacts from sabotage of spent fuel and plutonium shipments were sstimated for both routes. These shipments were selected for comparison because of their potential to cause significant radiological and economic impacts and are used as upper-bound cases for high consequences. Points A and $B$, having characteristics of the rural area, represent the closest and farthest points, respectively, from the urban area. Points $C$ and $D$ represent the urban area. The population distributions for points $A, B$, and $C$ are included in Table 6-11. CRAC, the model used in Chapter 5 for evaluating sabotage consequences, is also used here.

The spent fuel source consists of light water reactor fuel with $33000 \mathrm{MWd} / \mathrm{MTHM}$ (megawatts day/metric tonne of heavy metal) burnup at $40 \mathrm{~kW} / \mathrm{kg}$ power density and 150 days cooling. The truck-mounted cask contains three elements with radionuclides equivalent to $1.4 \mathrm{MTHM}$ charged to the reactor. The baseline estimate for the fractions of radioactive material released is $7 \times 10^{-4}$ of the solids and .1 of the noble gases. The basis for this release and a description of the potential sabotage acts are presented in Chapter 5.

In this analysis, the consequences were estimated assuming the same population density along 16 segments (each of $22.5^{\circ}$ spread) and 5 to 6 radial mesh points, depending on the population distributions (see Table 6-11 and Figures 6-1 to 6-5). This greatly reduces the needed input data and computational effort. It also provides reasonable lower and upper bound results.

Table 6-12 compares the potential radiological and economical impacts at points A, $B$, and $C$ with the reference case (point $D$ ) for the spent fuel release. It shows that for the rural area (points $A$ and $B$ ), the radiological impacts are reduced about one order of magnitude if the wind blows toward the high population areas, 
and about two orders of magnitude if it blows toward the low population areas. The economic impacts are also reduced to a few percent $(4 \%-5 \%)$ of the value for the urban area. The radiological impacts at point $C$ are slightly lower than the reference point. This was expected since $\mathrm{C}$ is also in an urban area but has a lower population density.

Table 6-11

Population Distributions at Points A, B and C

\begin{tabular}{|c|c|c|}
\hline $\begin{array}{l}\text { Point of } \\
\text { Release }\end{array}$ & $\begin{array}{l}\text { Population } \\
\text { Radius from Release } \\
(\mathrm{km})\end{array}$ & $\begin{array}{l}\text { Distribution } \\
\text { Population Density } \\
\left.\text { (people/ } / \mathrm{km}^{2}\right) \\
\end{array}$ \\
\hline \multirow[t]{5}{*}{$A(N W)$} & $0-7.5$ & 390 \\
\hline & $7.5-16$ & 2070 \\
\hline & $16-32$ & $54 \%$ \\
\hline & $32-48$ & 2230 \\
\hline & $>48$ & 0 \\
\hline$A(S E)$ & $0-800$ & 207 \\
\hline \multirow[t]{7}{*}{$B(W)$} & $0-16$ & 90 \\
\hline & $16-32$ & 1050 \\
\hline & $32-56$ & 5860 \\
\hline & $56-64$ & $24 \quad 220$ \\
\hline & $64-80$ & 7030 \\
\hline & $80-176$ & 390 \\
\hline & $>176$ & 0 \\
\hline$B(E)$ & $0-800$ & 90 \\
\hline \multirow[t]{3}{*}{$C(\mathrm{NW})$} & $0-16$ & $24 \quad 220$ \\
\hline & $16-28$ & 7030 \\
\hline & $>28$ & 0 \\
\hline
\end{tabular}

The comparisons for the sabotage of plutonium shipments are presented in Table 6-13. In this case, the potential latent cancer fatalities are reduced one to two orders of magnitude by routing around New York City. This is a significant reduction since several thousand latent cancer fatalities are predicted if a Pu shipment were successfully sabotaged in a city. The potential economic impact also shows a significant reduction (about $95 \%$ ) by rerouting. The analysis has as a basic assumption that no attempt is made to move a shipment to a more desirable location before the sabotage act is accomplished. 


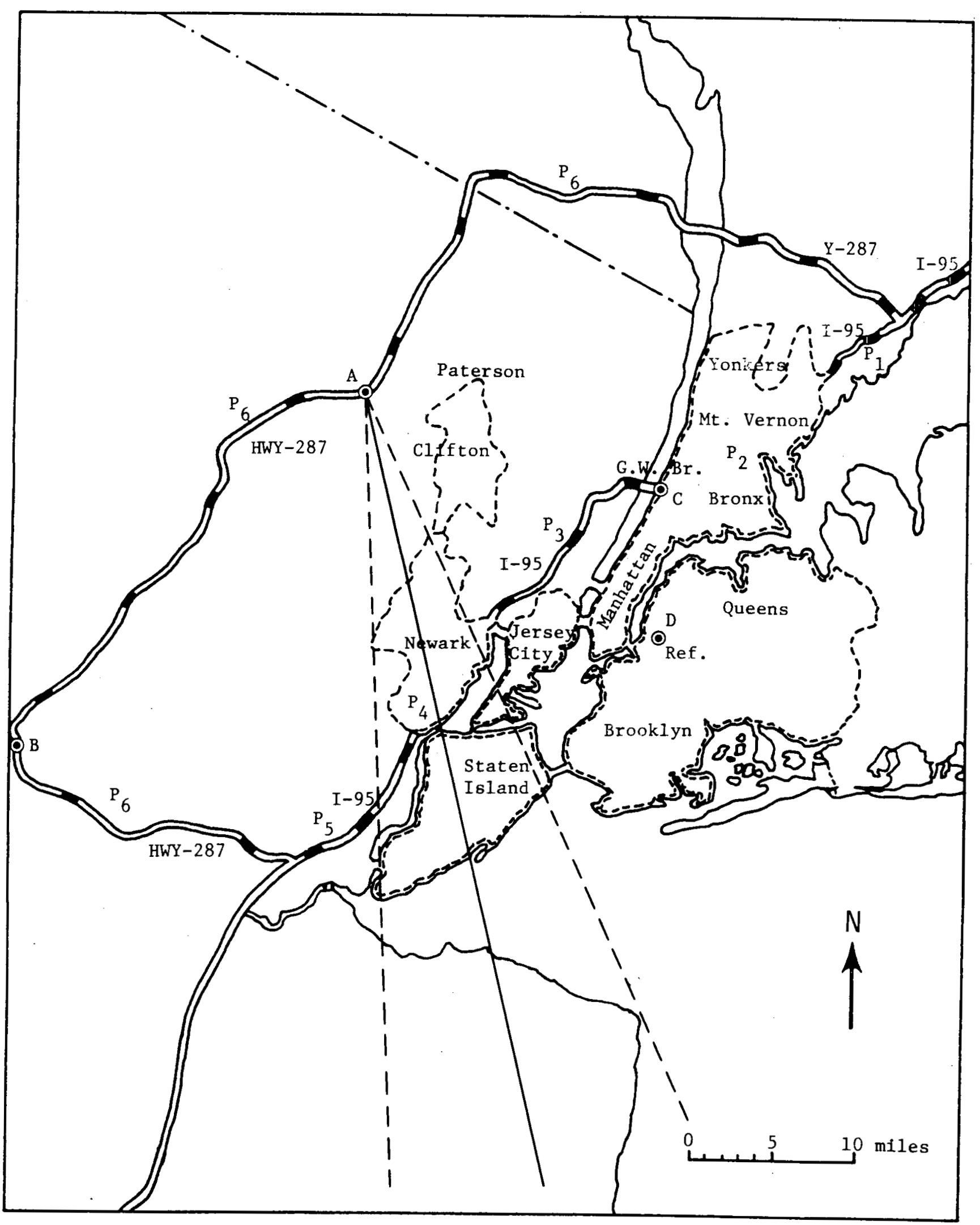

Figure 6-2. Release at Point A, Northwest Winds 


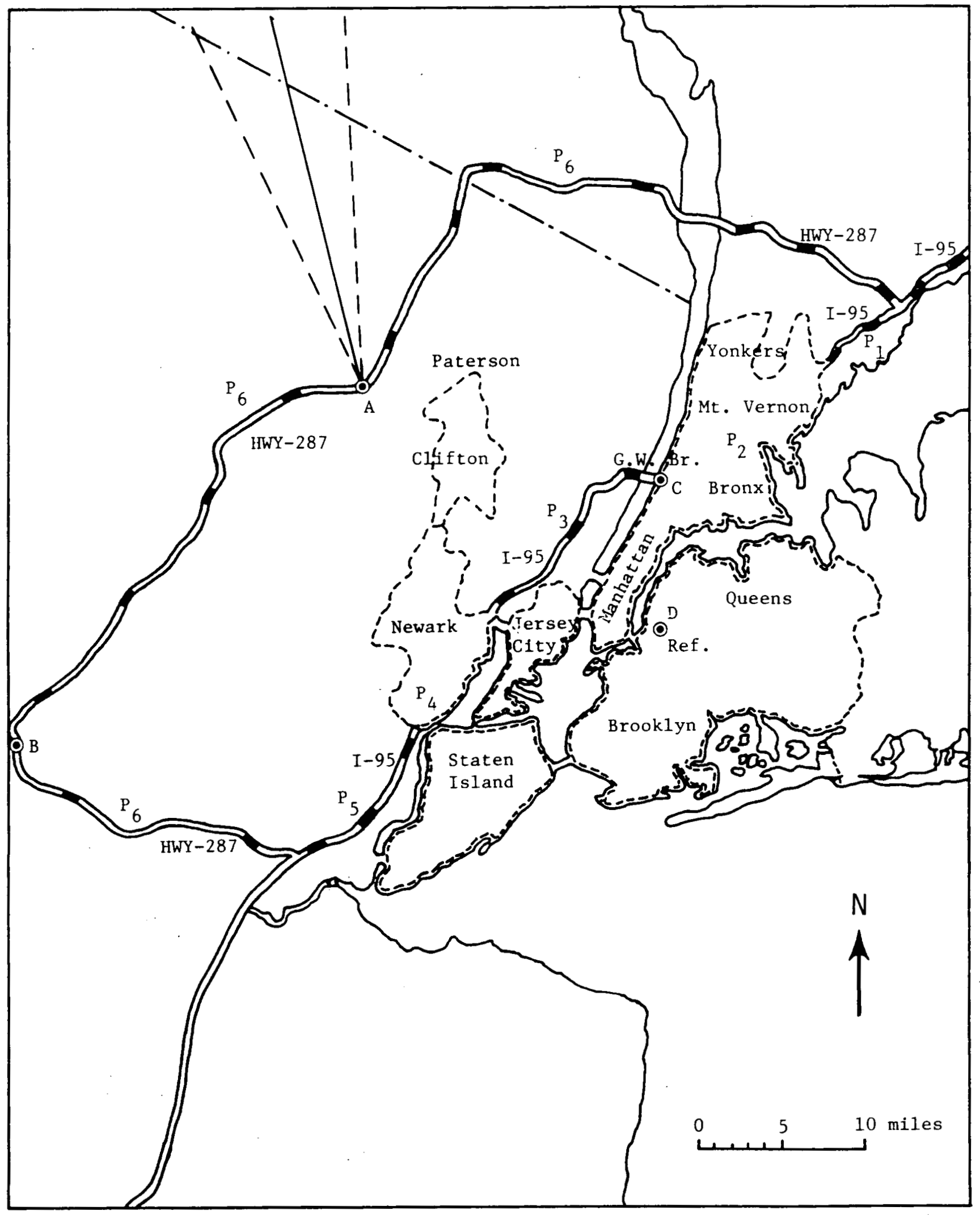

Figure 6-3. Release at Point A, Southeast Winds 


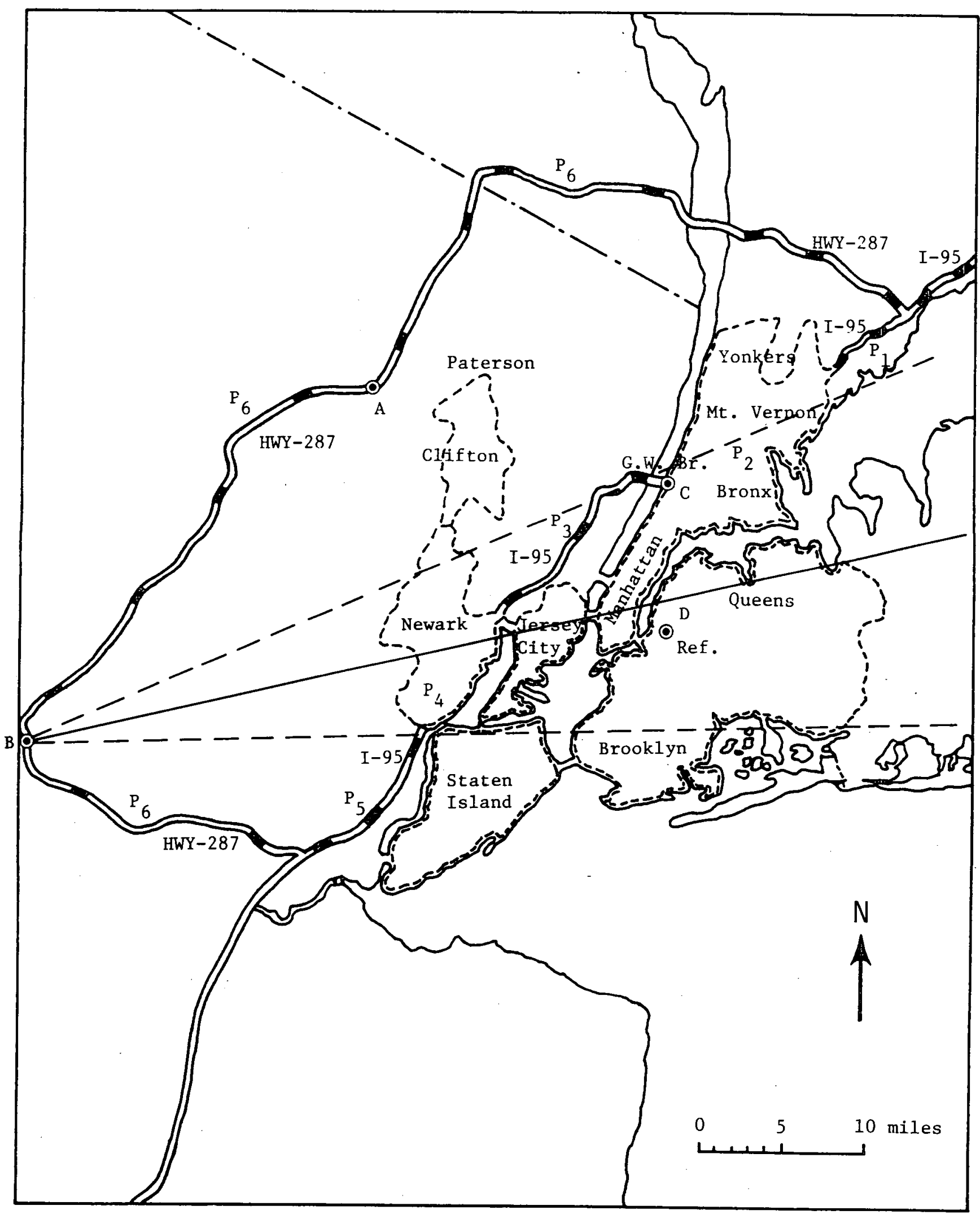

Figure 6-4. Release at Point B, West Winds 


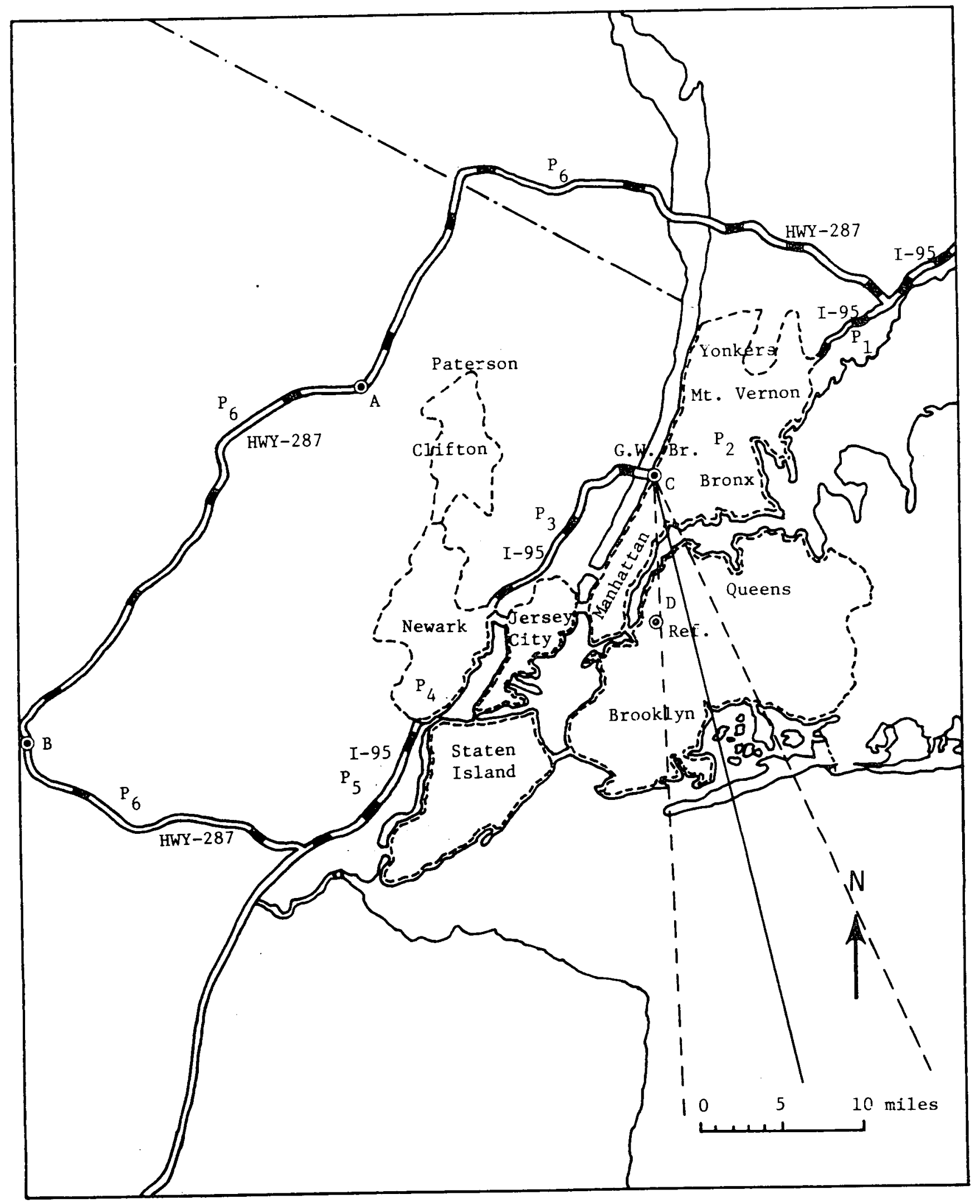

Figure 6-5. Release at Point C, Northwest Winds 
Table 6-12

Comparison of Impacts from Sabotage of Spent Fuel

Point of Release

A (SE)

A (NW)

$B(W)$

$B(E)$

C (NW)

D
Total Latent Cancer Fatalities Avg. (Max.) Ratio Avg. (Max.)

$2.6(3.0)$

13 (27)

16 (58)

$1.3(1.6)$

116 (252)

$140(532)$
$0.02(0.006)$

$0.09 \quad(0.05)$

$0.11(0.11)$

$0.009(0.003)$

$0.83(0.47)$

l (1)
Economic Impact Value (\$) Ratio

$8 \times 10^{7} \quad 0.04$

$9 \times 10^{7} \quad 0.05$

$9 \times 10^{7} \quad 0.05$

$8 \times 10^{7} \quad 0.04$

$4 \times 10^{8} \quad 0.20$

$2 \times 10^{9} \quad 1$

NOTES :

1. No early fatalities or early morbidities are predicted.

2. Source: 1ight_water reactor spent fuel Release: $7 \times 10^{-4}$ solids

0.1 noble gas

3. Point $D$ includes 91 weather sequences (see Chapter 5 ).

4. Economic impacts are calculated using the METRAN code.

Table 6-13

Comparison of Impacts from Sabotage of Pu Shipments

Point of Release

$$
\text { A (SE) }
$$

$A(N W)$

B (W)

$B(E)$

C (NW)

$\mathrm{D}$

NOTES :

1. Plutonium shipment: $1.13 \times 10^{6}$ curies

Release fraction: $0.1 \%$

Aerosolized fraction: $0.05 \%$

Particle size: 1.0 micron

2. Economic impacts calculated using the METRAN code.

$43(59.4) \quad 0.01 \quad(0.004)$

$0.04 \quad(0.03)$

$0.05(0.06)$

$0.005(0.002)$

$0.74(0.52)$

$1(1)$
Economic Impact Value (\$) Ratio

$\begin{array}{ll}8 \times 10^{7} & 0.04 \\ 9 \times 10^{7} & 0.05 \\ 9 \times 10^{7} & 0.05 \\ 8 \times 10^{7} & 0.04 \\ 4 \times 10^{8} & 0.20 \\ 2 \times 10^{9} & 1\end{array}$


The spatial distributions of projected marrow dose and lung dose to an individual due to initial ( 1 year) and chronic ( 30 to 40 years) exposures are shown in Figures 6-6 and 6-7, respectively. It is clear from these figures that most of the projected dose is received within $10 \mathrm{~km}$ of the release point.

In summary, the analysis indicates that the radiological consequences from a sabotage act could be reduced from one to two orders of magnitude by routing spent fuel and plutonium shipments around the urban area. The economic impacts could also be reduced to about $4 \%$ to $5 \%$ of the urban values. This is a significant reduction since the urban impacts could be several billion dollars. The results are heavily dependent on the assumptions and specific routes selected. Therefore, caution should be employed in generalizing to other cities or other routes. The analysis also indicates that the latent cancer fatalities would occur mainly in the population located within $10 \mathrm{~km}$ of the release point, given the assumed population densities.

\subsubsection{Summary and Conclusions}

The analysis shows that routing around New York City could affect the radiological consequences and economic impacts in the following ways.

Incident-Free Transport

1. Routing on freeways will eliminate the dose to pedestrians. However, shipments with origin and/or destination in urban areas can not be routed entirely by freeways.

2. Integrated dose to crew would increase due to longer driving time. The maximum increase is about $80 \%$ for the specific case discussed in this section.

3. Under optimal conditions, integrated dose to people in vehicles would decrease to about $30 \%$ of the value in the urban area (for the rush hour time period); however, depending on the traffic conditions, rerouting could increase the dose to people in vehicles up to about $80 \%$.

4. Dose to people in buildings would continue to be negligible in comparison to the other groups.

Vehicular Accidents (Similarly for Human Ėrror Incidents)

\section{Nondispersible Material Accidents}

1. Potential consequences (LCFs) to people in vehicles are relatively small for both routes.

2. Economic impacts are very small (less than a few thousand dollars) for both routes.

3. - Dose to people in buildings is negligible in comparison to other groups (at least six orders of magnitude smaller).

\section{Dispersible Material Accidents}

1. For $\mathrm{Pu}$ and $\mathrm{Po}-210$ shipments, the potential consequences to the population would decrease by one to two orders of magnitude by routing around New 
York City. This is a significant reduction for Pu shipments, since a few thousand latent cancer fatalities are expected from a severity-VIII accident in the urban area.

2. For Mo-99 and SpF-IH shipments, the potential consequences to the population would decrease less than one order of magnitude by routing around the urban area.

3. The potential economic impact in the rural area would decrease to a few percent $(1 \%-5 \%)$ of the values for the urban route. This is a significant reduction since the impact in the city would probably be greater than $\$ 1$ billion.

4. Even though routing certain radioactive materials around urban areas reduces the magnitude of potential consequences of a serious accident, it may also increase the probability that such accidents occur due to higher speeds, poorer road conditions, higher accident rates, etc. The probability of accident occurrence was not investigated in this section.

Sabotage Acts

1. For plutonium and spent fuel shipments, the potential consequences to the population would be decreased one to two orders of magnitude by rerouting (assuming the sabotage event occurs at the point of takeover of the shipment).

2. The potential economic impact for the above shipments would be decreased to a few percent ( $4 \%$ to $5 \%$ ) of the values for the urban area. This is a significant reduction since the impact in the city would probably be greater than $\$ 1$ billion.

\subsection{Changes in Form of Material Shipped}

Physical and chemical form of materials currently shipped in the New York City area vary from gases to solids and highly dispersible powders to nondispersible metals. Changes in either the physical or chemical form of material shipped can have an effect on the radiological impacts from the several causative events. For incident-free transport, the physical or chemical form of material is not an important parameter. In the accident case, the physical and chemical form determine whether the material is treated as an exposure source only or as an inhalation/ cloudshine/groundshine source. The same applies for the human errors situation. To evaluate the effects of material form changes for accidents and human errors, it is assumed that the materials currently shipped in dispersible form are converted to a nondispersible form. For the sabotage case, the assumption is made that energy-intensive devices are used, possibly vaporizing material otherwise considered nondispersible or special form. This alternative, then, is not really applicable to the sabotage event. Results for this alternative are given in Table 6-14.

It should be remembered that dispersible materials used in medical applications are responsible for a major portion of incident-free, vehicular accidents, and human error risk. To require that radiopharmaceuticals, for example, be transported in nondispersible form complicates if not denies their use. Since many materials are used in diagnostic testing, this alternative is at least impractical in these cases since each user facility would have to produce the chemical forms desired from the nondispersible shipping form, posing a significant economic burden on the users. 


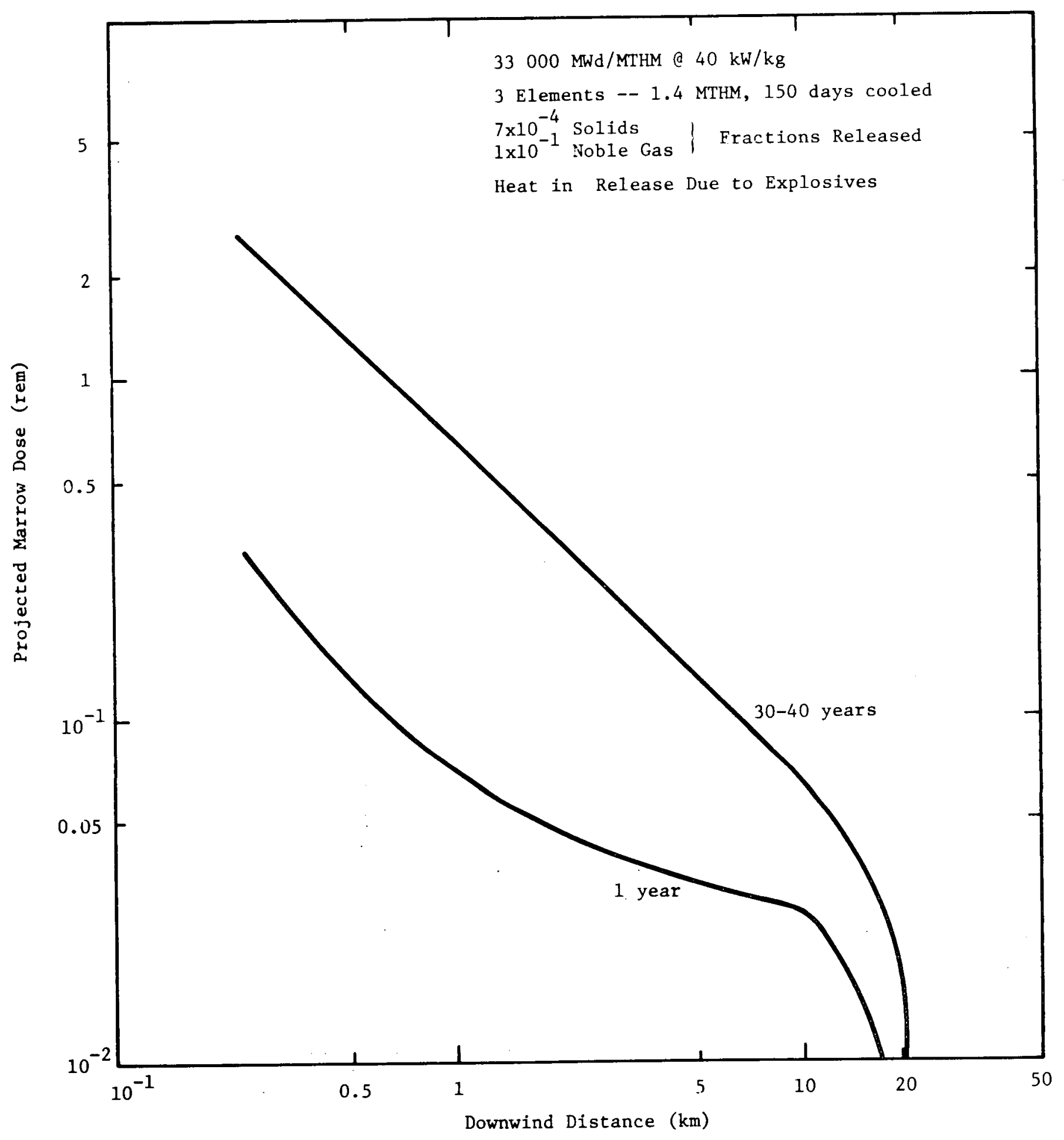

Figure 6-6. Projected Bone Marrow Dose versus Downwind Distance 


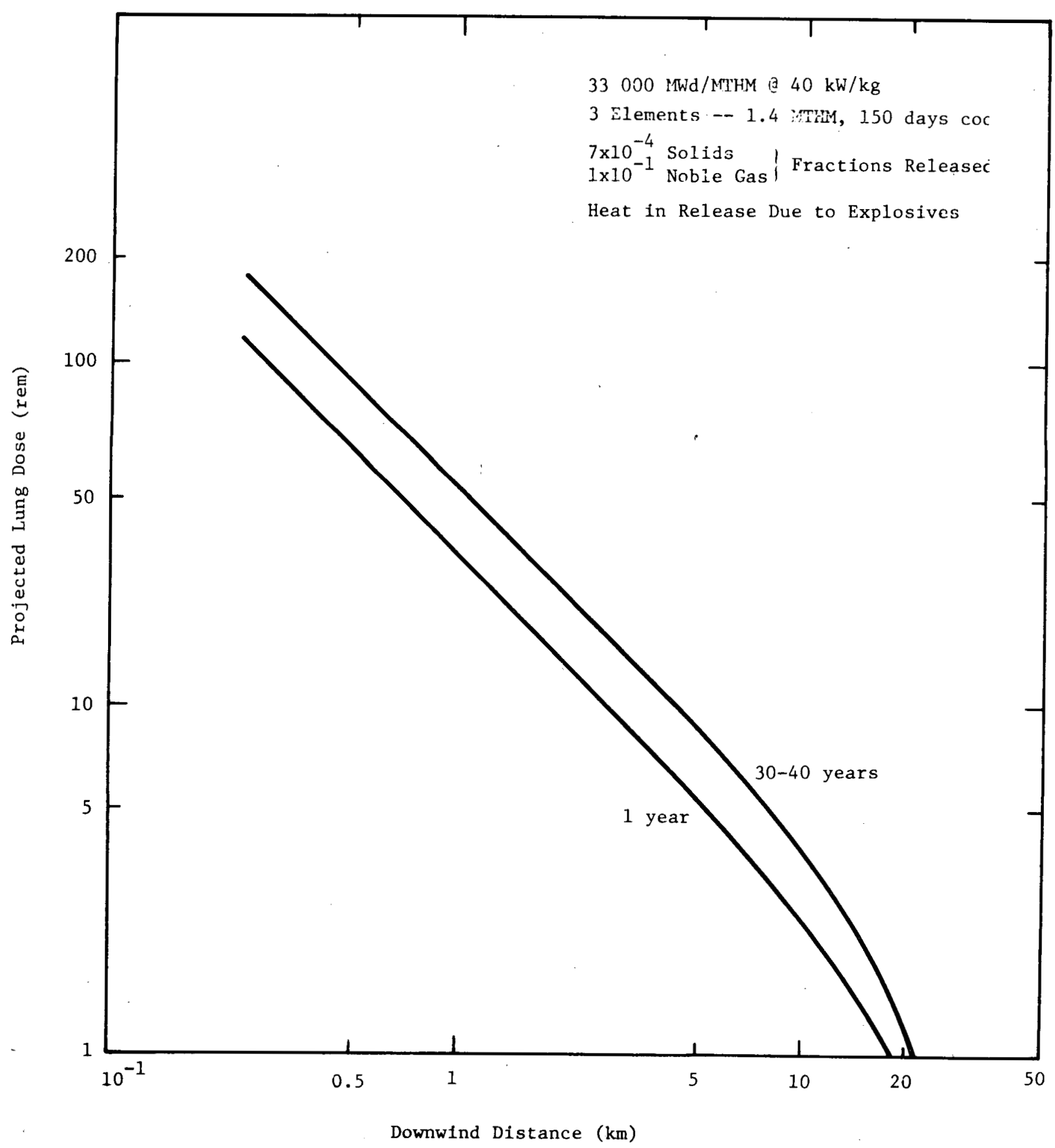

Figure 6-7. Projected Lung Dose versus Downwind Distance 
Another factor which should be considered is the question of chemical form of the materials shipped. Appendix $J$ of this document discusses the chemical toxicity of the commonly shipped materials. The conclusions of the analysis indicate major problems only with materials transported in large quantities. This is not to say that some of these materials could not be harmful (from chemical not radiological considerations) but that in general they are shipped in limited quantities so that the majority of a shipment would have to be inhaled or ingested by a single individual or a small group for significant effect. From the chemical point of view, changes in material form are probably not significant in altering the impacts of the several causative events described in Table 6-14.

Table 6-14

Effects from Changes in Material Form

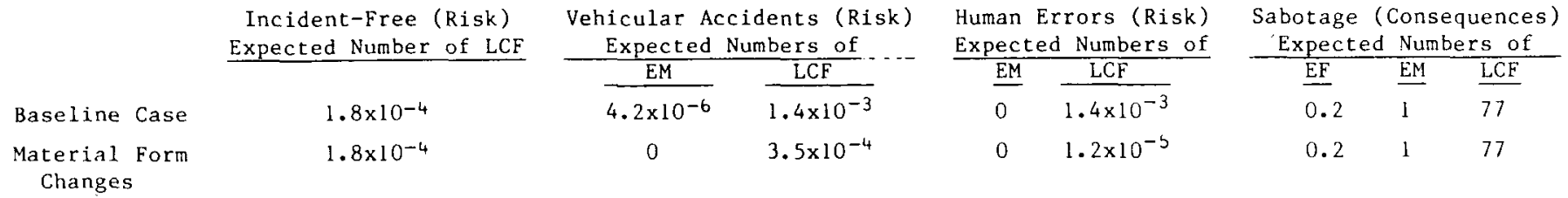

\subsection{Quantity Limits per Package or per Shipment}

Assuming that the total quantity of material shipped, i.e., total curies per year for a given radionuclide, remains constant, the impacts of this alternative can be discussed by examining the following:

- Changes in the quantity limits per package, thus altering the total number of packages shipped

- For total shipment quantity, changes in the current limits, necessitating a change in the overall numbers of shipments

For the incident-free case, as long as the total TI shipped per year does not change significantly, this alternative will have no effect. The question of changes in transport index has been addressed briefly in the section of this chapter concerned with the alternative of package type changes/overpack. As an example of the types of effects expected from this alternative, it is assumed that the TI per package does not change but that the quantity shipped is increased. To maintain a constant total for the amount of a material to be shipped, this results in a decrease in the total number of shipments (or packages) involved. Since the expressions used for calculating the integrated population dose are for the most part dependent linearly upon TI and total number of shipments, the implementation of this option would result in a decrease in the overall population exposure. Conversely, if the TI per package remains the same but quantity limits are decreased, the total number of shipments or packages would increase, thus increasing the population exposure. The overall effect of implementing this alternative for the incident-free transport case is expected to be small. 
For the accident and human errors cases, the important factor is total curies per package as well as total shipments per year. The effect of changes in the quantity limits per package for nondispersible materials would follow the same reasoning given above for the incident-free case. If a dispersible material is involved, the situation is more complex. The consequences of a given accident or human exror incident would be changed if the quantity limits are different. They would decrease for a lower quantity limit, increase for a larger limit. The second consideration here is the frequency of shipments (shipments per year) since the calculated values for expected number of health effects are dependent upon this factor as well as the accident rates and the total distance traveled.

Using the same examples as in the incident-free case, the quantity limit increase would probably result in larger consequences for a given accident or human error, but the decrease in the number of shipments tends to lower the overall accidentoccurrence probability. Conversely, a decrease in quantity limits produces smaller consequences but increases the probability that an event will occur. Especially important is the resultant increase in the probability that more severe accidents would occur.

The overall impacts of the implementation of this alternative are not in one direction. They depend primarily on the exact course of action chosen for the institution of quantity limits.

The sabotage event is different from the other cases in some ways. Here the limitations on quantities shipped per package or shipment are a discrete number of fuel elements (for spent fuel) or the necessary amount of material for a teletherapy source, as two examples. Since energetic attack of the transport vehicle is assumed, it is not clear that there is a decrease in the amount of material available for release since the amount of affected material is only a small fraction of the total shipment.

\subsection{Physica1 Protection of Large Quantity Shipments}

The most apparent impact of this alternative would be in the incident-free transport case, but only if it is assumed that those protecting the shipment are present in the vehicle cab. It is more probable that added physical protection would be accomplished by individuals riding in some type of escort vehicle. If this is the case, those individuals would be exposed as are individuals riding in vehicles adjacent to the shipment. Thus, their exposure has already been calculated as part of the total population exposure to people in vehicles. Assuming that the added crew member is in the vehicle with the drivers, the increase in exposure can be calculated. Since the only large quantity shipments in the limited New York City case are on Route 17, the effect of the change in number of crew members from two to three can be assessed. The increased exposure amounts to a value of $4.6 \times 10^{-3}$ person rem per shipment year for large quantity materials compared to a value of $3.1 \times 10^{-3}$ person rem per shipment year if only two crew members are present. The net effect of this alternative on the total incident-free population dose is negligible.

For vehicular accidents, the added physical protection for high curie shipments could result in a decrease in the probability of occurrence for all accidents involving these shipments since the added crew member as an additional observer could assist in the avoidance of problems. The amount of reduction in this probability 
cannot be quantified. However, the number of high curie shipments in the New York City model is not large, and the effect on the total population dose would be small if not negligible.

Added physical protection for high curie shipments is not expected to contribute any major risk reduction when considering human errors. In some instances, however, the added crew member might be able to see problems that were the result of human errors, thus averting major consequences (e.g., visual inspection indicates improper tiedown, loose bolts, etc.).

For sabotage, physical protection of the high curie shipments is likely to result in decreased availability of the materials, but beyond this, nothing conclusive about the effectiveness of added security can be stated.

\subsection{Summary of Alternatives}

Table 6-15 presents a summary of the effects on the baseline or reference case for each alternative. Comments in the final column indicate the authors opinions of the feasibility of implementing each alternative. In the presentation of these results, no synergisms between the alternatives have been considered. In the case of rerouting, the reader is referred to the details of this discussion within the chapter since the results of the analysis are too cumbersome for a tabular format. A general conclusion that can be drawn is that, for the most part, implementation of any single alternative produces less than an order of magnitude change in the calculated radiological risks. Many other factors are involved when a particular alternative is considered. These factors include the economic and social impacts of implementing the alternative. The social impacts of the transportation of radioactive materials in urban areas are addressed in Appendix $L$ of this report. Economic consequences are not quantified in this chapter; however, in the discussion of the feasibility of each alternative, a few words are included which indicate the general nature of these consequences. 


\section{Summary of Alternatives}

\begin{tabular}{|c|c|c|c|c|c|c|c|c|c|}
\hline \multirow[b]{2}{*}{ Alternative } & \multirow{2}{*}{$\begin{array}{c}\begin{array}{c}\text { Incident-Free } \\
\text { Transport }\end{array} \\
\text { Expected Number of } \\
\text { LCF } \\
\end{array}$} & \multicolumn{2}{|c|}{$\begin{array}{l}\text { Vehicular } \\
\text { Accidents }\end{array}$} & \multicolumn{2}{|c|}{ Human Error } & \multicolumn{2}{|c|}{ Sabotage } & \multirow{2}{*}{$\begin{array}{c}\text { Feasibility } \\
\text { of } \\
\text { Alternative } \\
\text { (yes/no) } \\
\end{array}$} & \multirow[b]{2}{*}{ Comments } \\
\hline & & $\begin{array}{l}\text { Expect } \\
\text { EM }\end{array}$ & $\begin{array}{c}\text { d Numbers of } \\
\text { LCF }\end{array}$ & EM & $\begin{array}{c}\text { Numbers of } \\
\text { LCF }\end{array}$ & $\begin{array}{l}\text { Expected } \\
\text { EF EM }\end{array}$ & $\begin{array}{l}\text { Numbers of } \\
\text { LCF }\end{array}$ & & \\
\hline Baseline Case & $\begin{array}{l}1.8 \times 10^{-4} \\
(\sim 7 \text { person rem })\end{array}$ & $4.2 \times 10^{-6}$ & $1.4 \times 10^{-3}$ & 0 & $1.4 \times 10^{-3}$ & $\begin{array}{l}0.2 \quad 1 \\
\text { (CRAC an }\end{array}$ & $\begin{array}{c}77 \\
\text { a1ys1s) }\end{array}$ & - & - \\
\hline $\begin{array}{l}\text { Transport Mode } \\
\text { Shifts }\end{array}$ & $\begin{array}{l}\text { 1. } 3 \times 10^{-4} \\
(\sim 5 \text { person rem })\end{array}$ & $4.2 \times 10^{-6}$ & $3.3 \times 10^{-3}$ & 0 & $\sim 1.4 \times 10^{-3}$ & $\begin{array}{l}2 \\
\text { (CRAC an }\end{array}$ & $\begin{array}{c}220 \\
\text { alysis) }\end{array}$ & Yes & $\begin{array}{l}\text { Usefulness is } \\
\text { questionable if } \\
\text { alternate mode } \\
\text { necessitates use of } \\
\text { secondary mode } \\
\text { transport to or from } \\
\text { urban destinations. } \\
\text { Costs might be high } \\
\text { for equipment for } \\
\text { other modes. }\end{array}$ \\
\hline $\begin{array}{l}\text { Package Type } \\
\text { Changes/Overpack }\end{array}$ & $\begin{array}{l}1.2 \times 10^{-4} \\
(<5 \text { person rem })\end{array}$ & 0 & $3.5 \times 10^{-4}$ & 0 & $-1.4 \times 10^{-3}$ & $\begin{array}{lr}0 & 0.3 \\
(\text { CRAC } & \text { an }\end{array}$ & $\begin{array}{c}24 \\
\text { alysis) }\end{array}$ & Yes & $\begin{array}{l}\text { This alternative is } \\
\text { quite feasible; how- } \\
\text { ever costs for shifts } \\
\text { from one package type } \\
\text { to another would te } \\
\text { to manufacturers and } \\
\text { distributors of } \\
\text { materials, not to } \\
\text { sh1ppers for trans- } \\
\text { port equipment } \\
\text { changes. Overpacks } \\
\text { would have to be de- } \\
\text { slgned, built, and } \\
\text { licensed. }\end{array}$ \\
\hline $\begin{array}{l}\text { Time of Shipment } \\
\text { Restriction } \\
\text { (Low and high } \\
\text { values are re- } \\
\text { ported. T1me of } \\
\text { day is also } \\
\text { spectfled.) }\end{array}$ & $\begin{array}{l}1.3 \times 10^{-4}(1800) \\
3.9 \times 10^{-4}(1130)\end{array}$ & $\begin{array}{l}3.0 \times 10^{-6} \\
2.5 \times 10^{-6}\end{array}$ & $\begin{array}{l}3.9 \times 10^{-4}(1200) \\
29 \times 10^{-3}(2400)\end{array}$ & 0 & $\begin{array}{l}1.4 \times 10^{-3}(2400) \\
1.8 \times 10^{-3}(1200)\end{array}$ & $\begin{array}{lr}0 & 31 \\
\text { (METRAN } \\
<1 \quad 21\end{array}$ & $\begin{array}{c}26(1200) \\
\text { analysis }) \\
34(2400)\end{array}$ & Yes & $\begin{array}{l}\text { Alternative } 1 \text { s } \\
\text { feasible. Convenience } \\
\text { of delivery for many } \\
\text { medical shipments } 1 \text { s } \\
\text { reduced. Costs for } \\
\text { staffing delivery } \\
\text { sites and rearrange- } \\
\text { ment of schedules for } \\
\text { use could be signifi- } \\
\text { cant. Economic conse- } \\
\text { quences must be } \\
\text { weighed against } \\
\text { radiological risk } \\
\text { reduction. }\end{array}$ \\
\hline
\end{tabular}


Table 6-15 (Continued)

Summary of Alternatives

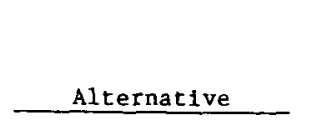

Baseline Case

Reduction or

Elimination of

Some Shipments
a) all through shipments

b) only large

quantity

shipments

eliminated.

Change in Form

of Material Shipped
Inc1dent-Free

$1.8 \times 10^{7}$

$1.3 \times 10^{-4}$

$1.7 \times 10^{-4}$ Expected Number of
Vehicular

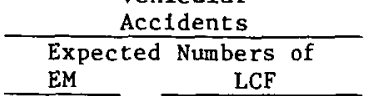

$4.2 \times 10^{-6} \quad \frac{1.4 \times 10^{-3}}{1.4}$

$7.7 \times 10^{74}$

$7.7 \times 10^{-4}$

0

4. $8 \times 10^{-5}$

$3.5 \times 10^{-4}$

$1.2 \times 10^{-5}$
Sabotage

Expected Numbers of

EF EM LCF

0.2

(CRAC analysis)

Removal of

certain shipment

may decrease thelr

the radiological con-

sequences if an

$\begin{array}{lll}0.2 & 1 & 77\end{array}$

(CRAC analysis) event occurs in an

Feasibility$$
\text { of }
$$

Al ternative (yes/no)

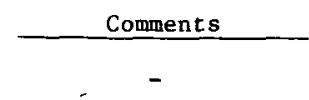

Yes

Choice of shipments to be eliminated will determine the feasibility of these alternatives. Costs resulting from reduction or elimination of certain shipments must be considered.

Requirements that material be shipped in nondispersible form would seriously affect radiopharmaceutical users. Also, manufacturing changes could result in different particle size distributions which could alter the calculated consequences. From a cost view-

point, this alterna-

tive is not effective and would have significant impacts on the industry, requiring major revisions in the techniques used to prepare these materials.

Varfable. Could decrease Variable as in Table 6-2 to

Unknown decrease as in Table 6-2 to $1.2 \times 10^{2}$

$3.5 \times 10^{-4}$

\section{0} Package or
Shipment
The possibilities for different sets of trade-offs are dis-
cussed in the text Lowering quantity 1 imits would increase costs. Increased quantity limits would result in increased consequences from single accident. 
Summary of Alternatives

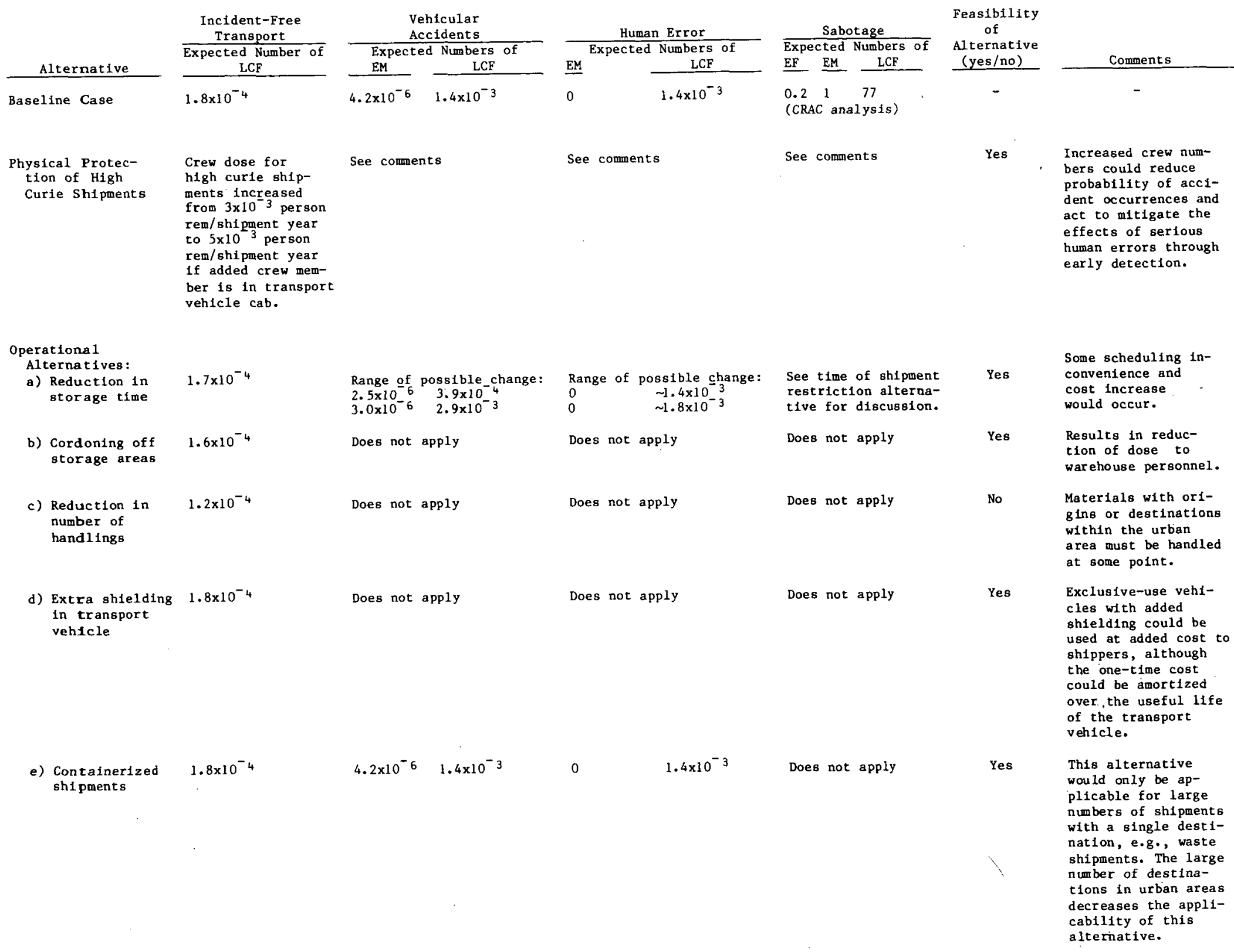


GENERIC EXTENSION

\subsection{Introduction}

The limited New York City area was chosen for the initial application of the METRAN methodology because of the unique characteristics of this particular urban area. Description of the selected area reveals large diurnal variations in population and traffic density as well as significant radioactive material transport patterns. Although this area possesses most of the features characteristic of any urban area, several notable exceptions were discovered. No appreciable rail or barge shipments have been observed (see Appendix A). The New York urban transport system also does not include features such as beltways which could carry traffic around the more densely populated sections of the city.

This chapter proposes methods for examining the radiological effects of radioactive material transportation in other urban areas without resorting to the complex models initially exercised. The 20 largest Standard Metropolitan Statistical Areas (SMSAs) as defined in 1975, were chosen to examine the radiological effects in other densely populated areas. Detailed analysis was performed on data for these SMSAs with wide geographical distribution and special characteristics (e.g., large rail facilities and significant amounts of water transport). The SMSAs examined are
1. New York
2. Los Angeles
3. Chicago
4. Philadelphia
5. Detroit
6. San Francisco/Oakland
7. Washington, D.C.
8. Boston
9. Nassau-Suffolk
10. Da1las

11. Houston

12. St. Louis

13. Pittsburgh

14. Baltimore

15. Minneapolis/St. Paul

16. Newark

17. Cleveland

18. Atlanta

19. Anaheim/Santa Ana/Garden Grove

20. San Diego

The majority of the SMSA designations refers to the major metropolitan center included in the area, although a few refer to other geographical areas.

The first step in applying the techniques in Appendices B and C to another area is a careful examination of the sensitivity and error analysis results discussed in Appendix D. A significant finding of this analysis is that demographic information 
(population densities, traffic counts, etc.) need not be as detailed as for the New York City case. That is to say, average values may be used for these variables without introducing large errors into the calculation. Also, in general, demographic variables appear as multiplicative factors in the equations, thus they can be easily varied from one city to the next. This is especially true in the incident-free transport case, where the simple equations given in Appendix $D$ can be used. If data are not available for a specific urban area, the average value for the limited New York City case is used.

The accident case is, however, more complicated. As indicated in Appendix D, only a rank ordering of important variables has been possible for the model equations. This is also the case for human errors or deviations from accepted quality assurance practices. Thus the analysis of shipment data for the SMSAs cannot be as detailed as in the incident-free case. For the occurrence of sabotage or malevolent act, the application of the CRAC and METRAN methodologies, or some adaptation of them to produce consequence estimates for other urban areas, is the approach used in this chapter, even though this application. is, at best, descriptive.

\subsection{Shipment Information for SMSAs}

The technique and data base described in Appendix A for development of the limited New York City standard shipments model have been applied to each of the 20 SMSAs. Original data from which Reference 1 was derived were obtained and tabulated by end use, transport mode, and package type. Where the end use of a specific material could not be easily defined, those shipments were included in each possible category (e.g., if the material could be either medical or industrial, the reported shipments have been included in both end-use categories). The authors recognize that this represents some double-counting of shipments, but do not think that this introduces significant error into the analysis. Since many of the SMSAs on the eastern seaboard overlap, it should be noted that the standard shipments models will also double-count some shipments. Therefore, comparison of these data with the summary tables in Reference 1 is not recommended. The exact procedures used to obtain the standard shipments data and detailed tabulations are described in Reference 2. Table 7-1 summarizes the shipment data for the 20 SMSAs on the bases of total shipments per year, total curies transported per year, and total TI transported per year. The corresponding values for the limited New York City case are included for reference.

\subsection{Demographic Data}

Reference 3 gives total population and land area for the major cities in the SMSAs. These data were converted to population densities for the areas (persons per square kilometre) for consistency and are summarized in the final column of Table 7-1. To account for nonresident travel in each urban area an average value of 3000 people $/ \mathrm{km}^{2}$ is assumed for consistency and in the absence of other available data. These people would move into and out of the urban centers for shopping, business, etc. This value is added to the previously determined population density to approximate the number of people in buildings in the urban centers during the day. The authors recognize that this assumption may underestimate the daytime population densities in the various urban areas. 
Little applicable data were available on vehicle densities in the SMSAs. Thus it is assumed that the urban vehicle density is a constant. For some vehicle-oriented SMSAs such as Los Angeles, this may well underestimate the true situation: conversely, in other areas, vehicle density may be significantly overestimated.

Information was requested from the primary urban center for each SMSA regarding pedestrian cordon counts.* Only Los Angeles provided applicable information in this area, from which it was possible to derive values for pedestrian density. The value for Los Angeles, $1.04 \times 10^{5}$ pedestrians $/ \mathrm{km}^{2}$, is used in the calculations. In all other cases, a value for pedestrian density $\left(2.0 \times 10^{5} / \mathrm{km}^{2}\right)$, derived from the limited New York City case, is used.

Table 7-1

Standard Shipments Summary for 20 Top SMSAs (1975)

\begin{tabular}{|c|c|c|c|c|}
\hline SMSA & $\begin{array}{c}\text { Total } \\
\text { Sh1pments } \\
\text { per Year }\end{array}$ & $\begin{array}{c}\text { Total } \\
\text { Curies Shipped } \\
\text { per Year } \\
\end{array}$ & $\begin{array}{l}\text { Total } \\
\text { TI Shipped } \\
\text { per Year } \\
\end{array}$ & $\begin{array}{c}\text { Population } \\
\text { Density } \\
\text { (persons } / \mathrm{km}^{2} \text { ) }\end{array}$ \\
\hline New York & $1.8 \times 10^{5}$ & $1.5 \times 10^{8}$ & $1.3 \times 10^{5}$ & $1.0 \times 10^{4}$ \\
\hline Los Angeles & $8.3 \times 10^{4}$ & $2.2 \times 10^{8}$ & $2.9 \times 10^{4}$ & $2.3 \times 10^{3}$ \\
\hline ChIcago & $1.8 \times 10^{5}$ & $2.3 \times 10^{8}$ & $9.9 \times 10^{4}$ & $5.8 \times 10^{3}$ \\
\hline Philadel phia & $2.0 \times 10^{5}$ & $1.5 \times 10^{6}$ & $2.3 \times 10^{5}$ & $5.9 \times 10^{3}$ \\
\hline Detroit & $3.7 \times 10^{4}$ & $2.7 \times 10^{7}$ & $3.3 \times 10^{4}$ & $4.2 \times 10^{3}$ \\
\hline San Francisco/Oakland & $6.7 \times 10^{4}$ & $7.8 \times 10^{5}$ & $2.7 \times 10^{4}$ & $6.1 \times 10^{3}$ \\
\hline Washington, D.C. & $5.1 \times 10^{4}$ & $3.6 \times 10^{6}$ & $1.0 \times 10^{5}$ & $4.8 \times 10^{3}$ \\
\hline Boston & $2.8 \times 10^{4}$ & $8.9 \times 10^{5}$ & $4.7 \times 10^{4}$ & $5.4 \times 10^{3}$ \\
\hline Nassau/Suffolk & $4.0 \times 10^{4}$ & $5.7 \times 10^{5}$ & $3.9 \times 10^{4}$ & $3.4 \times 10^{3}$ \\
\hline Dallas & $2.0 \times 10^{4}$ & $1.3 \times 10^{5}$ & $1.3 \times 10^{4}$ & $1.2 \times 10^{3}$ \\
\hline Houston & $3.5 \times 10^{4}$ & $7.7 \times 10^{6}$ & $1.2 \times 10^{4}$ & $1.1 \times 10^{3}$ \\
\hline St. Louis & $3.2 \times 10^{5}$ & $5.3 \times 10^{7}$ & $1.5 \times 10^{5}$ & $3.9 \times 10^{3}$ \\
\hline Pittsburgh & $1.1 \times 10^{5}$ & $6.6 \times 10^{6}$ & $1.0 \times 10^{5}$ & $3.6 \times 10^{3}$ \\
\hline Balt1more & $6.1 \times 10^{4}$ & $4.1 \times 10^{6}$ & $1.0 \times 10^{5}$ & $4.5 \times 10^{3}$ \\
\hline Minneapolis/St. Paul & $8.2 \times 10^{3}$ & $2.7 \times 10^{4}$ & $7.2 \times 10^{3}$ & $3.0 \times 10^{3}$ \\
\hline Newark & $2.3 \times 10^{5}$ & $1.5 \times 10^{8}$ & $1.9 \times 10^{5}$ & $6.3 \times 10^{3}$ \\
\hline Cleveland & $6.8 \times 10^{4}$ & $6.1 \times 10^{6}$ & $6.3 \times 10^{4}$ & $3.8 \times 10^{3}$ \\
\hline Atlanta & $2.6 \times 10^{4}$ & $2.4 \times 10^{5}$ & $2.3 \times 10^{4}$ & $1.5 \times 10^{3}$ \\
\hline $\begin{array}{l}\text { Anaheim/Santa Ana/ } \\
\text { Garden Grove }\end{array}$ & $2.6 \times 10^{4}$ & $4.2 \times 10^{4}$ & $1.1 \times 10^{4}$ & $1.9 \times 10^{3}$ \\
\hline San Dlego & $2.1 \times 10^{4}$ & $3.7 \times 10^{4}$ & $4.0 \times 10^{3}$ & $1.9 \times 10^{3}$ \\
\hline $\begin{array}{l}\text { Limited New York } \\
\text { City Area }\end{array}$ & $2.8 \times 10^{4}$ & $2.7 \times 10^{6}$ & $1.1 \times 10^{4}$ & $3.2 \times 10^{4}$ \\
\hline
\end{tabular}

\footnotetext{
${ }^{*}$ Cordon counts accumulate total numbers of people or vehicles entering or leaving a prescribed area as a function of time of day.
} 


\subsection{Incident-Free Transport}

Table 2-8 summarized the radiological impacts from incident-free transport in the limited New York City case. Examination of these results indicates that more than $96 \%$ of the population dose is accumulated by five groups: handlers, warehouse personnel, pedestrians, people in vehicles, and crew. Only small contributions arise from dose to people in air terminals and people in buildings. To examine the impacts from incident-free transport, the following assumptions are made.

- Impacts from the five major dose groups are the only ones to be evaluated.

- Materials shipped by modes other than truck remain in a storage area for 12 hours. Exposure distances for warehouse personnel are a minimum of 1.5 metres and a maximum of 100 metres.

- All materials having origins or destinations in the urban area are handled once.

- Transit through the urban area begins at 0700 hours except for fuel cycle materials, which begin at 0300 hours to minimize traffic complications and delays.

- Origin/destination shipments travel on two-way streets. For through shipments, travel is on freeways. (Velocity at 0700 hours on two-way streets = $3.34 \mathrm{~m} / \mathrm{s}$, freeways $=8.9 \mathrm{~m} / \mathrm{s}$; velocity at 0300 hours on two-way streets $=8.06 \mathrm{~m} / \mathrm{s}$, freeways $=24.4 \mathrm{~m} / \mathrm{s}$. )

- Each shipment travels a constant distance of $7 \mathrm{~km}$ across the urban area (a shipment-weighted length from the limited New York City case).

- Source-to-crew distance is nominally 3.1 metres.

- Street width is 20 metres and sidewalk width, 3 metres.

Other variables which appear in the scaled regression equations are set at nominal values to simplify the approximation of the various dose group impacts (e.g., vehicle count for 0700 hours is 1180 vehicles per cell, and at 0300 hours, the value used is 280 vehicles per cell).

For comparison, the New York City computation was performed a second time using the assumptions listed above. Results of this calculation and those for the other urban areas, as well as the results for incident-free transport from Chapter 2, are given in Table 7-2. Rank ordering of dose groups differs among the cities as the character of the shipments made through or into and out of the area change. In all cases, the predominant contributors to total integrated dose were pedestrians and people in vehicles.

There are rather wide variations among the results for the different urban areas. However, the expected numbers of latent cancer fatalities per year of shipping activity (calculated assuming $25 \mathrm{LCF} / 10^{6}$ person rem) are still quite small (from $\sim 10^{-4}$ LCF for the San Diego area to $\sim 10^{-3}$ LCF for Newark). In those SMSAs for which large numbers of origin/destination shipments were observed, the estimates of doses to people in vehicles, pedestrians and crew are probably upper bounds to the population doses. This is true because of the assumption that such shipments travel $7 \mathrm{~km}$ on city streets. In reality, some perhaps sizeable fraction of the travel for the shipments might be on freeways or beltways, thus reducing the overall population exposure. In interpreting the results in Table 7-2, the assumptions used in the analysis must be taken into account. 
Table 7-2

Incident-Free Generic Extension (Population Doses -- Person Rem)

\begin{tabular}{|c|c|c|c|c|c|c|}
\hline SMSA & $\begin{array}{l}\text { People } \\
\text { in } \\
\text { Vehicles }\end{array}$ & $\begin{array}{l}\text { Warehouse } \\
\text { Personne1 }\end{array}$ & Pedestrians & Handlers & Crew & Totals \\
\hline New York City (Iimited) & 1.6 & 1.2 & 1.0 & 2.5 & 0.6 & 7 \\
\hline $\begin{array}{l}\text { New York City (limited)* } \\
\text { (Generic extension } \\
\text { assumptions) }\end{array}$ & 3.8 & 1.2 & 3.3 & 2.5 & 1.7 & 13 \\
\hline New York & 37 & 4 & 22 & 16 & 20 & 99 \\
\hline Los Angeles & 13 & 5 & 6 & 7 & 5 & 36 \\
\hline Chicago & 9 & 20 & 34 & 22 & 1.7 & 102 \\
\hline Philadelphia & 14 & 2 & 13 & 19 & 13 & 61 \\
\hline Detroit & 8 & 2 & 3 & 2 & 3 & 18 \\
\hline San Francisco/Oakland & 11 & 6 & 10 & 7 & 4 & 38 \\
\hline Washington, D.C. & 23 & 1 & 3 & 2 & 6 & 23 \\
\hline Boston & 23 & 1 & 20 & 12 & 9 & 65 \\
\hline Nassau/Suffolk & 10 & $\ll<1$ & 3 & 2 & 6 & 21 \\
\hline Dallas & 5 & 3 & 4 & 3 & 2 & 17 \\
\hline Houston & 5 & 3 & 4 & 3 & 2 & 16 \\
\hline St. Louis & 58 & 14 & 45 & 31 & 20 & 168 \\
\hline Pittsburgh & 25 & 3 & 5 & 4 & 11 & 48 \\
\hline Baltimore & 13 & 1 & 3 & 2 & 6 & 25 \\
\hline Minneapolis/St. Paul & 3 & 2 & 2 & 1 & 1 & 9 \\
\hline Newark & 69 & 28 & 52 & 36 & 28 & 213 \\
\hline Cleveland & 12 & 1 & 3 & 2 & 4 & 22 \\
\hline Atlanta & 4 & 1 & 3 & 3 & 1 & 12 \\
\hline $\begin{array}{l}\text { Anaheim/Santa Ana/ } \\
\text { Garden Grove }\end{array}$ & 3 & 1 & 1 & 1 & l & 7 \\
\hline San Diego & 1 & 1 & 1 & 1 & $<<1$ & 4 \\
\hline
\end{tabular}

*Differences between the METRAN calculated values and those obtained using simplified equations arise from the assumptions of averaged vehicle counts across the urban area (people in vehicles) and uniform travel distance at an assumed time of day ( 0700 hours for all shipments except fuel cycle). 


\subsection{Vehicular Accidents}

Extension of the METRAN accident analysis is complicated by several factors. As with the incident-free case, the shipment patterns vary widely, and several materials appear in the shipment models for the other cities which are not in the New York City set. Many variables are used in the accident analysis which are city specific, and the importance of these must be evaluated before extending the analysis. These variables include meteorological patterns for the cities, fraction of building area, street area, etc. These are inputs not only to METRAN but also to the meteorological dispersion codes MICMET and PICMET (see Appendix E). Sensitivity analysis treatment of vehicular accidents produces only rank ordering of significant variables; thus the techniques employed earlier are not applicable here.

The approach chosen for extension of the vehicular accident case involves an examination of the major contributors to overall accident risk from 10 separate categories. The categories and a description of the selection process by which materials are placed in them follow and are also summarized in Table 7-3. For nondispersible materials, the selection process consists solely of subdividing on the basis of number of curies per package. Nondispersible shipments of less than or equal to 2 curies per package comprise Category 1. Category 2 contains all other nondispersible material shipments.

Dispersible material shipments are categorized based on three factors:

1. Curies per package--Shipments of less than or equal to 2 curies per package are classified as small and the remainder as large shipments.

2. Subdivision of materials is made on the basis of inhalation toxicity by examining the average rem per curie value for the material, as determined from the 50-year values listed in Appendix G. Materials with average rem per curie values less than $1 \times 10^{5}$ are considered to have low toxicity. All other materials are classified as having high toxicity.

3. Average photon energy per disintegration $\left(E_{d}\right)$--If a material has a value for $\mathrm{E}_{\mathrm{d}}$ less than or equal to $0.1 \mathrm{MeV}$, it is not considered to contribute as an external source (groundshine). All other materials are assumed to act as additional external sources, thus contributing to the overall groundshine consequences.

Tabie $7-3$

Description of Nisk Calceortes

\begin{tabular}{|c|c|}
\hline $\begin{array}{l}\text { Category } \\
\text { Number }\end{array}$ & Descrsytion \\
\hline 1 & Snall nothispersible shifients 42 euries \\
\hline 2 & Large nondisperstble mipents $>2$ curjes \\
\hline 3 & 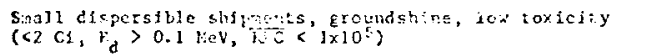 \\
\hline 4 & 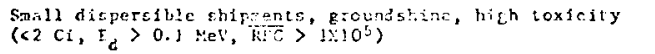 \\
\hline 5 & 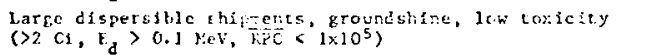 \\
\hline 6 & 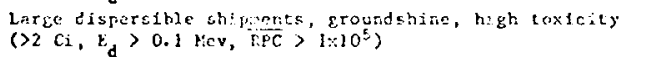 \\
\hline$\gamma$ & 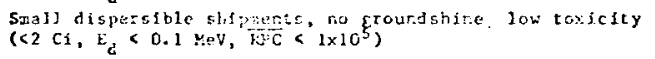 \\
\hline 8 & 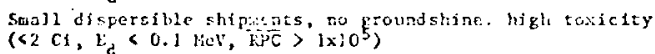 \\
\hline 9 & 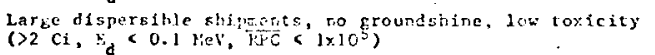 \\
\hline 10 & 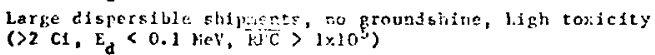 \\
\hline
\end{tabular}


The risk contributions for the New York City standard shipments model are given in Table 7-4. The major contributors to latent cancer fatalities and genetic effects are Categories 2 through 6 , which constitute $99 \%$ of the total. Category 6 materials contribute all of the observed early morbidities.

Further analysis of the information in Table 7-4 shows that pedestrians are the major dose group affected by vehicular accidents. For materials in Categories 1 and 2 , total expected latent cancer fatalities are divided between pedestrians and people in vehicles by a ratio of 7:3. For materials in Categories 3 through 6 , the breakdown of latent cancer fatalities is, on the average, a ratio of $7: 3$ (pedestrians to people in buildings). The majority of the risk from these categories arises from groundshine exposure. The risk from materials in Categories 7 through 10 is split among pedestrians, people in vehicles, and people in buildings (56\% pedestrians, $8 \%$ people in vehicles, and $36 \%$ people in buildings). This change in ratio comes from the fact that the inhalation pathway from material in the aerosol cloud predominates here and people in buildings are more vulnerable to this pathway. The fractional contribution of materials in Categories 7 through 10 is quite small for New York City. The trend does not continue, however, for cities in which a larger fraction of the materials shipped is within these categories. For fuel cycle materials in Categories 3 through 6 , the relative contribution to people in buildings predominates $(98 \%)$, and this is taken into account in the calculations.

The approach taken to estimate the accident risk. for other cities is to determine the fractional distribution of materials shipped by category for each urban area. For Categories 1 through 2 and 3 through 6 , the overall risk can be expressed as a function of the appropriate demographic parameters (pedestrian densities, population densities) and total curies shipped in that category. There is also a dependency on average total photon energy per disintegration. For Categories 7 through 10, there is the additional factor of average rem per curie value. This has been handled by separating out Categories 7 and 9 from Categories 8 and 10 with respect to quantity of material shipped (total curies).

The generic extension of accident results to other areas consisted of first separating the risk values obtained for New York City by category and dose group using the ratios discussed earlier. Second, these separated values were multiplied by the ratio of the appropriate demographic parameter (e.g., ratio of population densities for a given city to that for New York City). Finally, the quantity obtained above is multiplied by the ratio of total curies shipped for a city to total curies shipped for New York City (by category). An example of the technique is given in Equation 1 (for pedestrian contribution).

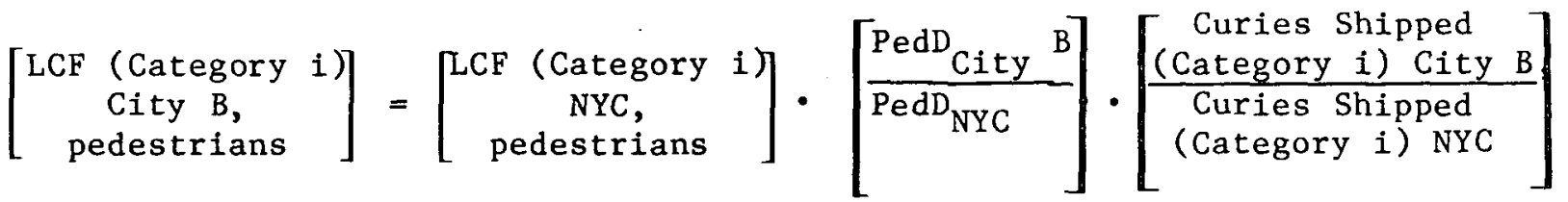

where $i=1,10$ and PedD = pedestrian density.

In the case of materials in Categories 1 and 2, the contribution to risk for people in vehicles, is assumed to be independent of the number of vehicles (vehicle count, as discussed earlier) but solely in total number of curies shipped. There are many other dependencies within the analysis which are not being considered here; thus these results are felt to be only a first-order approximation to the results for other urban areas. 
Table 7-4

Contribution of 10 Categories to Expected Risk Values (New York City)

\begin{tabular}{|c|c|c|c|c|c|}
\hline Category & Description & $\begin{array}{l}\text { Expected } \\
\text { Number of } \\
\text { Latent Cancer } \\
\text { Fatalities }\end{array}$ & $\underline{f}$ & $\begin{array}{c}\text { Expected } \\
\text { Number of } \\
\text { Early } \\
\text { Morbidities } \\
\end{array}$ & $\mathrm{f}$ \\
\hline 1 & Nondispersible Small & $2.6 \times 10^{-6}$ & 0.002 & - & - \\
\hline 2 & Nondispersible Large & $2.5 \times 10^{-4}$ & 0.18 & - & - \\
\hline 3 & $\begin{array}{l}\text { Dispersible Small, External Source } \\
\text { Low Toxicity }\end{array}$ & $1.2 \times 10^{-4}$ & 0.09 & - & - \\
\hline 4 & $\begin{array}{l}\text { Dispersible Small, External Source } \\
\text { High Toxicity }\end{array}$ & $2.1 \times 10^{-5}$ & 0.02 & - & - \\
\hline 5 & $\begin{array}{l}\text { Dispersible Large, External Source } \\
\text { Low Toxicity }\end{array}$ & $3.6 \times 10^{-4}$ & 0.26 & - & - \\
\hline 6 & $\begin{array}{l}\text { Dispersible Large, External Source } \\
\text { High Toxicity }\end{array}$ & $6.1 \times 10^{-4}$ & 0.44 & $2.7 \times 10^{-5}$ & 1.03 \\
\hline 7 & $\begin{array}{l}\text { Dispersible Small, No External Source } \\
\text { Low Toxicity }\end{array}$ & $2.6 \times 10^{-7}$ & $<0.001$ & - & - \\
\hline 8 & $\begin{array}{l}\text { Dispersible Small, No External Source } \\
\text { High Toxicity }\end{array}$ & $1.8 \times 10^{-5}$ & 0.01 & - & - \\
\hline 9 & $\begin{array}{l}\text { Dispersible Large, No External Source } \\
\text { Low Toxicity }\end{array}$ & $8.4 \times 10^{-8}$ & $<0.001$ & - & - \\
\hline 10 & $\begin{array}{l}\text { Dispersible Large, No External Source } \\
\text { High Toxicity }\end{array}$ & - & - & - & - \\
\hline & TOTALS & $1.4 \times 10^{-3}$ & 1.0 & $2.7 \times 10^{-5}$ & 1.0 \\
\hline
\end{tabular}


For this first-order approximation, the 10 material categories are partially recombined. All nondispersible materials are examined together (Categories 1 and 2 ) and the latent cancer fatalities for a given city are obtained, for pedestrians, as in Equation 1. For people in vehicles, the other major contributor, the LCFs are obtained by a ratio of total curies shipped. Materials having sizeable $(>0.1 \mathrm{MeV})$ average total photon energy per disintegration (Categories 3 through 6) are also combined. Latent cancer fatalities for pedestrians are obtained as described in Equation 1. For people in buildings, the pedestrian densities (PedD) in Equation 1 are replaced by the appropriate population densities. As described earlier, Categories 7 through 10 are subdivided by average rem per curie values. Except for this subdivision, the calculations are identical to those for materials in Categories 3 through 6 . Table 7-5 presents the results of the accident analysis for the 20 SMSA standard metropolitan statistical areas.

Several comments on interpretation of these results are necessary. No account is taken of route differences among the cities for origin/destination or through shipments. These differences could be significant, since one factor in determining the risk numbers is the actual distance traveled in the city. To illustrate this situation, Figures 7-1 and 7-2 are rough maps of the major freeway patterns for New York City and Houston, Texas (where most of the risk values can be attributed to large, nondispersible material shipments with origins or destinations in the city). It is reasonable to assume that carriers would attempt to remain on freeways as long as possible (there are fewer stops necessary, and freeways are usually in better condition than city streets, and allow for greater speed). In Houston, a loop surrounds the city with a number of interconnecting freeways for travel into the city. Only in outlying areas would there be the need for appreciable travel (more than 3 or $4, \mathrm{~km}$ ) on city streets to pick up or deliver materials. In New York City, on the other hand, there are some areas where freeway access is limited. It is not possible to estimate the relative effects of the city travel pattern without additional information on actual routes or delivery patterns. However, comparing the maps for the two cities indicates that the distance traveled off freeways for Houston will not be greater than that for New York City. If anything, the travel distances will probably be less. - The importance of this comparison is that for materials in Categories 1 through 6 , the major contribution to risk comes from pedestrians. Since it is assumed that no pedestrians are on freeways, the concern is with nonfreeway travel distance.

Another assumption used in the comparison is that vehicular accident rates do not differ significantly from city to city. This is probably valid for a first approximation. However, for freeway-oriented cities such as Los Angeles, the actual vehicular accident rate and fractional occurrences (by severity) could be markedly different.

Because pedestrian density plays a prominent role in the analysis, the quality of the information is quite important. In only three cases, other than New York City, has some information about total numbers of pedestrians for a specific area of the city been available (Los Angeles, Minneapolis/St. Paul, and Houston). Sketchy information on the fraction of city area occupied by streets, although the best available, increases the error in the calculated values for pedestrians per square kilometre of sidewalk. Thus, in one important variable necessary for extending the limited New York City analysis to other areas, there are several large uncertainties which must be considered in interpreting the results. Better information in this area is crucial to a more accurate analysis. Even for the limited New York City analysis, the pedestrian density values are derived from data for Manhattan, which are probably overestimates for certain sections of that study area, magnifying the errors in the base case used for the generic extension. 
Estimated Number of Latent Cancer

Fatalities from Vehicular Accidents for 20 SMSAs

\begin{tabular}{|c|c|c|c|c|c|c|c|c|}
\hline \multirow[b]{2}{*}{ SMSA } & \multicolumn{2}{|c|}{ Categories 1, 2} & \multicolumn{2}{|c|}{ Categories $3-6$} & \multicolumn{3}{|c|}{ Categories $7-10$} & \multirow[b]{2}{*}{$\begin{array}{l}\text { Total Estimated Numbers of } \\
\text { Latent Cancer Fatalities }\end{array}$} \\
\hline & Ped. & $\mathrm{PIV}^{\mathrm{a}}$ & Ped. & $\mathrm{PIB}^{\mathrm{b}}$ & Ped. & PIV & PIB & \\
\hline New York City & $1.8 \times 10^{-4}$ & $7.6 \times 10^{-5}$ & $3.6 \times 10^{-4}$ & $7.5 \times 10^{-4}$ & $1.0 \times 10^{-5}$ & $1.5 \times 10^{-6}$ & $6.6 \times 10^{-6}$ & $1.4 \times 10^{-3}$ \\
\hline New York & $5.6 \times 10^{-3}$ & $4.1 \times 10^{-3}$ & $2.2 \times 10^{-3}$ & $8.8 \times 10^{-4}$ & $9.4 \times 10^{-5}$ & $2.4 \times 10^{-5}$ & $4.3 \times 10^{-5}$ & $1.3 \times 10^{-2}$ \\
\hline Los Angeles & $4.5 \times 10^{-3}$ & $6.3 \times 10^{-3}$ & $1.7 \times 10^{-4}$ & $4.0 \times 10^{-5}$ & $1.1 \times 10^{-6}$ & $5.4 \times 10^{-7}$ & $4.0 \times 10^{-7}$ & $1.1 \times 10^{-2}$ \\
\hline Chicago & $8.9 \times 10^{-3}$ & $6.4 \times 10^{-3}$ & $9.4 \times 10^{-3}$ & $1.4 \times 10^{-1}$ & $3.5 \times 10^{-6}$ & $8.4 \times 10^{-7}$ & $1.0 \times 10^{-6}$ & $1.6 \times 10^{-1}$ \\
\hline Philadelphia & $5.0 \times 10^{-5}$ & $3.6 \times 10^{-5}$ & $5.9 \times 10^{-4}$ & $3.6 \times 10^{-4}$ & $1.1 \times 10^{-4}$ & $2.5 \times 10^{-5}$ & $3.1 \times 10^{-5}$ & $1.2 \times 10^{-3}$ \\
\hline Detroit & $1.1 \times 10^{-3}$ & $7.6 \times 10^{-4}$ & $9.4 \times 10^{-5}$ & $1.8 \times 10^{-5}$ & $6.5 \times 10^{-7}$ & $1.6 \times 10^{-7}$ & $1.6 \times 10^{-7}$ & $2.0 \times 10^{-3}$ \\
\hline San Francisco/Oakland & $1.7 \times 10^{-5}$ & $1.3 \times 10^{-5}$ & $4.1 \times 10^{-3}$ & $1.1 \times 10^{-3}$ & $5.5 \times 10^{-5}$ & $1.3 \times 10^{-5}$ & $1.7 \times 10^{-5}$ & $5.3 \times 10^{-3}$ \\
\hline Washington, D.C. & $1.3 \times 10^{-4}$ & $9.6 \times 10^{-5}$ & $1.2 \times 10^{-4}$ & $4.6 \times 10^{-4}$ & $7.1 \times 10^{-5}$ & $1.7 \times 10^{-5}$ & $1.8 \times 10^{-5}$ & $9.1 \times 10^{-4}$ \\
\hline Boston & $2.8 \times 10^{-5}$ & $2.0 \times 10^{-5}$ & $9.4 \times 10^{-4}$ & $2.1 \times 10^{-4}$ & $5.9 \times 10^{-5}$ & $1.4 \times 10^{-5}$ & $1.7 \times 10^{-5}$ & $1.3 \times 10^{-3}$ \\
\hline Nassau/Suffolk & $1.7 \times 10^{-5}$ & $1.3 \times 10^{-5}$ & $2.9 \times 10^{-4}$ & $5.3 \times 10^{-5}$ & $5.9 \times 10^{-5}$ & $1.4 \times 10^{-5}$ & $1.3 \times 10^{-5}$ & $4.6 \times 10^{-4}$ \\
\hline Dallas & $5.1 \times 10^{-6}$ & $3.7 \times 10^{-6}$ & $2.4 \times 10^{-5}$ & $2.3 \times 10^{-6}$ & $1.3 \times 10^{-7}$ & $3.1 \times 10^{-8}$ & $1.9 \times 10^{-8}$ & $3.5 \times 10^{-5}$ \\
\hline Houston & $3.0 \times 10^{-4}$ & $2.1 \times 10^{-4}$ & $6.6 \times 10^{-4}$ & $6.3 \times 10^{-5}$ & $1.5 \times 10^{-6}$ & $3.5 \times 10^{-7}$ & $2.0 \times 10^{-7}$ & $1.2 \times 10^{-3}$ \\
\hline St. Louis & $2.1 \times 10^{-3}$ & $1.5 \times 10^{-3}$ & $1.4 \times 10^{-3}$ & $8.9 \times 10^{-4}$ & $1.0 \times 10^{-5}$ & $2.5 \times 10^{-6}$ & $2.4 \times 10^{-6}$ & $5.9 \times 10^{-3}$ \\
\hline Pittsburgh & $2.0 \times 10^{-4}$ & $1.4 \times 10^{-4}$ & $6.5 \times 10^{-3}$ & $8.1 \times 10^{-2}$ & $1.2 \times 10^{-5}$ & $3.0 \times 10^{-6}$ & $2.9 \times 10^{-6}$ & $8.8 \times 10^{-2}$ \\
\hline Baltimore & $1.5 \times 10^{-4}$ & $1.1 \times 10^{-4}$ & $1.2 \times 10^{-4}$ & $1.8 \times 10^{-4}$ & $7.1 \times 10^{-5}$ & $1.7 \times 10^{-5}$ & $1.7 \times 10^{-5}$ & $6.7 \times 10^{-4}$ \\
\hline Minneapolis/St. Paul & $9.4 \times 10^{-7}$ & $7.0 \times 10^{-7}$ & $3.0 \times 10^{-5}$ & $3.7 \times 10^{-6}$ & $1.3 \times 10^{-7}$ & $3.1 \times 10^{-8}$ & $2.7 \times 10^{-8}$ & $3.6 \times 10^{-5}$ \\
\hline Newark & $5.6 \times 10^{-3}$ & $4.1 \times 10^{-3}$ & $1.2 \times 10^{-3}$ & $3.4 \times 10^{-4}$ & $9.4 \times 10^{-5}$ & $2.2 \times 10^{-3}$ & $3.0 \times 10^{-5}$ & $1.1 \times 10^{-2}$ \\
\hline Cleveland & $1.8 \times 10^{-4}$ & $1.3 \times 10^{-4}$ & $5.9 \times 10^{-3}$ & $8.3 \times 10^{-2}$ & $3.8 \times 10^{-6}$ & $9.3 \times 10^{-7}$ & $8.8 \times 10^{-7}$ & $8.9 \times 10^{-2}$ \\
\hline At lanta & $2.4 \times 10^{-6}$ & $1.7 \times 10^{-6}$ & $7.1 \times 10^{-4}$ & $7.3 \times 10^{-3}$ & $3.7 \times 10^{-7}$ & $9.0 \times 10^{-8}$ & $5.7 \times 10^{-8}$ & $8.0 \times 10^{-3}$ \\
\hline $\begin{array}{l}\text { Anaheim/Santa Ana/ } \\
\text { Garden Grove }\end{array}$ & $1.2 \times 10^{-6}$ & $8.4 \times 10^{-7}$ & $1.7 \times 10^{-4}$ & $1.7 \times 10^{-5}$ & $1.3 \times 10^{-6}$ & $3.1 \times 10^{-7}$ & $2.1 \times 10^{-7}$ & $1.9 \times 10^{-4}$ \\
\hline San Diego & $1.4 \times 10^{-6}$ & $9.7 \times 10^{-7}$ & $1.6 \times 10^{-5}$ & $1.3 \times 10^{-6}$ & $6.5 \times 10^{-7}$ & $1.6 \times 10^{-7}$ & $8.6 \times 10^{-8}$ & $2.1 \times 10^{-5}$ \\
\hline
\end{tabular}


$\Theta$

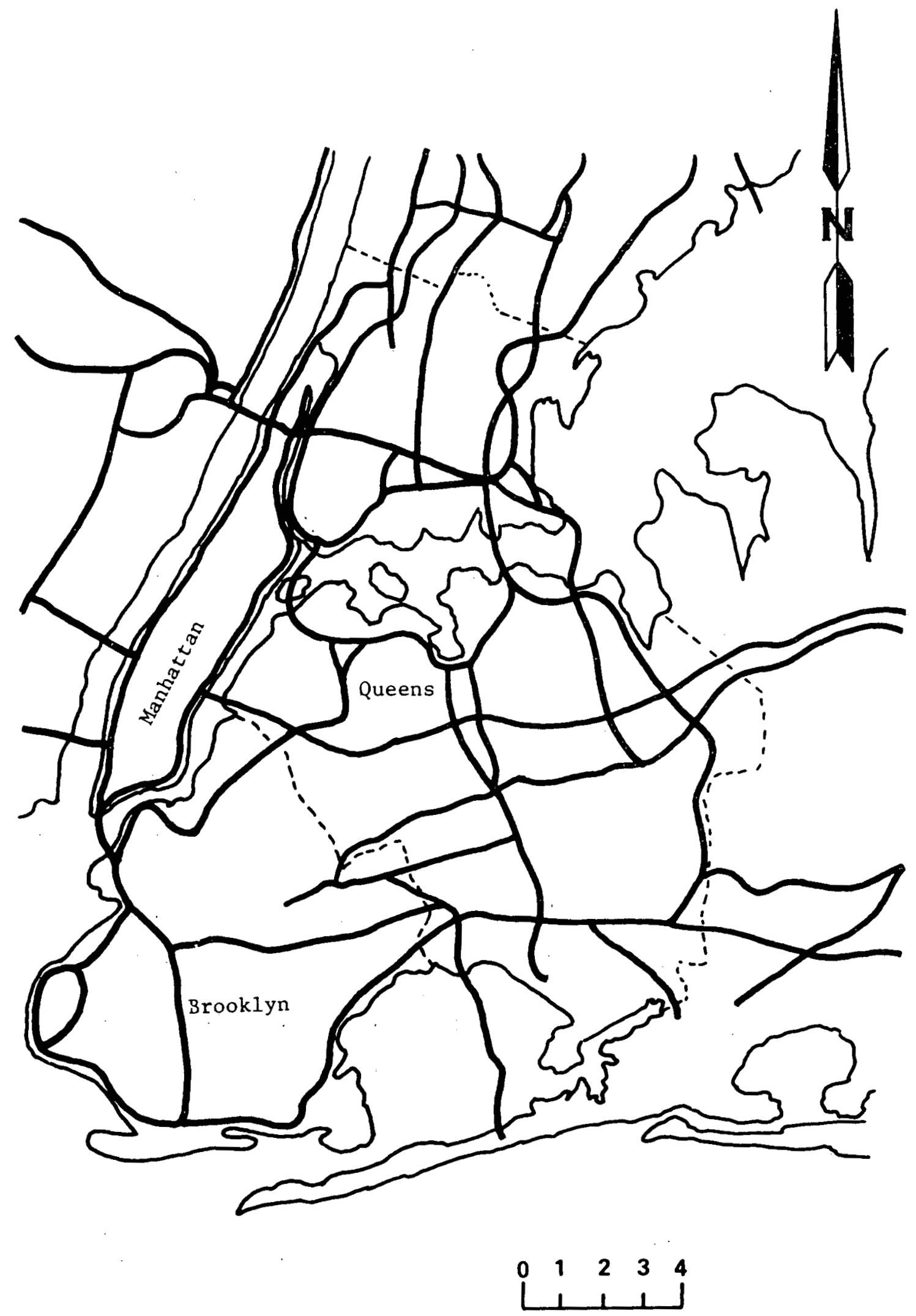

Figure 7-1. Major Freeway Patterns for New York City 


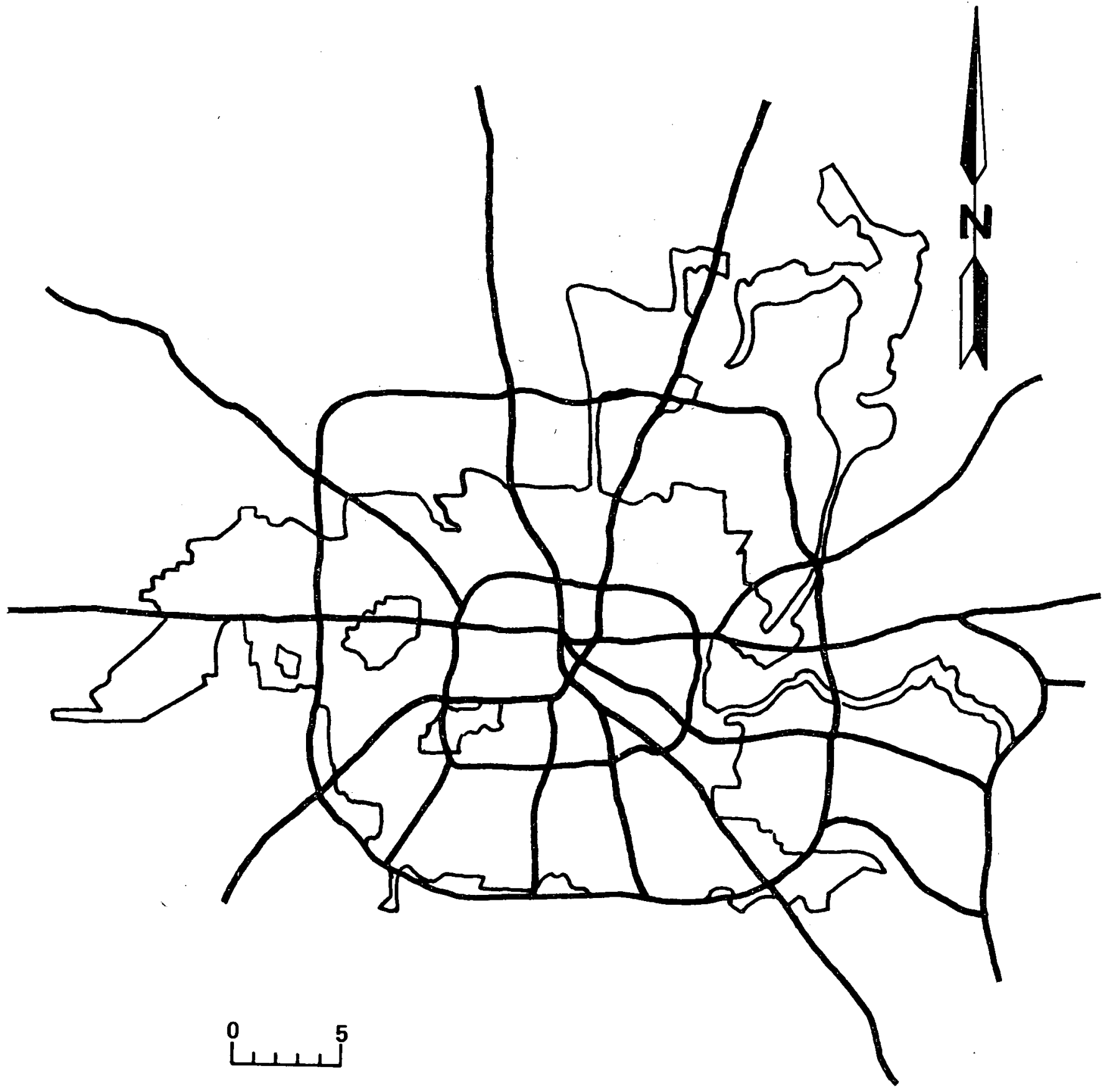

Figure 7-2. Major Freeway Patterns for Houston 
Table $7-6$

Estimated Number of Latent

Cancer Fatalities

from Human Errors for 20 SMSAs

\begin{tabular}{|c|c|c|c|c|c|c|c|c|}
\hline \multirow[b]{2}{*}{ SMSA } & \multicolumn{2}{|c|}{ Categories 1,2 } & \multicolumn{2}{|c|}{ Categories 3-6 } & \multicolumn{3}{|c|}{ Categories $7-10$} & \multirow{2}{*}{$\begin{array}{l}\text { Total Estimated Numbers of } \\
\text { Latent Cancer Fatalities }\end{array}$} \\
\hline & Ped. & PIV & Ped. & $\mathrm{PIB}$ & Ped. & PIV & PIB & \\
\hline New York City & $8.0 \times 10^{-6}$ & $3.4 \times 10^{-6}$ & $7.5 \times 10^{-5}$ & $1.3 \times 10^{-3}$ & $9.0 \times 10^{-8}$ & $1.3 \times 10^{-8}$ & $5.8 \times 10^{-8}$ & $1.4 \times 10^{-3}$ \\
\hline New York & $2.5 \times 10^{-4}$ & $1.9 \times 10^{-4}$ & $8.3 \times 10^{-5}$ & $6.0 \times 10^{-4}$ & $8.9 \times 10^{-7}$ & $2.0 \times 10^{-7}$ & $3.7 \times 10^{-7}$ & $1.1 \times 10^{-3}$ \\
\hline Los Angeles & $2.1 \times 10^{-4}$ & $2.9 \times 10^{-4}$ & $3.6 \times 10^{-6}$ & $1.2 \times 10^{-5}$ & $1.0 \times 10^{-8}$ & $4.7 \times 10^{-9}$ & $3.5 \times 10^{-9}$ & $5.2 \times 10^{-4}$ \\
\hline Chicago & $4.0 \times 10^{-4}$ & $2.9 \times 10^{-4}$ & $3.9 \times 10^{-2}$ & $3.2 \times 10^{-1}$ & $3.0 \times 10^{-8}$ & $7.3 \times 10^{-9}$ & $9.0 \times 10^{-9}$ & $3.6 \times 10^{-1}$ \\
\hline Philadelphia & $2.2 \times 10^{-6}$ & $1.6 \times 10^{-6}$ & $7.7 \times 10^{-5}$ & $5.5 \times 10^{-4}$ & $9.4 \times 10^{-7}$ & $2.2 \times 10^{-7}$ & $2.8 \times 10^{-7}$ & $6.3 \times 10^{-4}$ \\
\hline Detroit & $4.8 \times 10^{-5}$ & $3.5 \times 10^{-5}$ & $2.5 \times 10^{-6}$ & $7.3 \times 10^{-6}$ & $5.7 \times 10^{-9}$ & $1.4 \times 10^{-9}$ & $1.4 \times 10^{-9}$ & $9.3 \times 10^{-5}$ \\
\hline San Francisco/Oakland & $7.7 \times 10^{-7}$ & $5.7 \times 10^{-7}$ & $1.5 \times 10^{-4}$ & $8.1 \times 10^{-4}$ & $4.8 \times 10^{-7}$ & $1.2 \times 10^{-7}$ & $1.5 \times 10^{-7}$ & $9.6 \times 10^{-4}$ \\
\hline Washington, D.C. & $5.9 \times 10^{-6}$ & $4.4 \times 10^{-6}$ & $1.4 \times 10^{-4}$ & $9.8 \times 10^{-4}$ & $5.9 \times 10^{-7}$ & $1.5 \times 10^{-7}$ & $1.6 \times 10^{-7}$ & $1.1 \times 10^{-3}$ \\
\hline Boston & $1.2 \times 10^{-6}$ & $9.2 \times 10^{-7}$ & $1.8 \times 10^{-5}$ & $3.1 \times 10^{-6}$ & $5.3 \times 10^{-7}$ & $1.3 \times 10^{-7}$ & $1.5 \times 10^{-7}$ & $2.4 \times 10^{-5}$ \\
\hline Nassau/Suffolk & $7.7 \times 10^{-7}$ & $5.7 \times 10^{-7}$ & $1.0 \times 10^{-5}$ & $3.4 \times 10^{-5}$ & $5.2 \times 10^{-7}$ & $1.3 \times 10^{-7}$ & $1.1 \times 10^{-7}$ & $4.6 \times 10^{-5}$ \\
\hline Dallas & $2.4 \times 10^{-7}$ & $1.7 \times 10^{-7}$ & $4.7 \times 10^{-7}$ & $5.2 \times 10^{-7}$ & $1.2 \times 10^{-9}$ & $2.8 \times 10^{-10}$ & $1.7 \times 10^{-10}$ & $1.4 \times 10^{-6}$ \\
\hline Houston & $1.3 \times 10^{-5}$ & $9.7 \times 10^{-6}$ & $1.3 \times 10^{-5}$ & $1.2 \times 10^{-5}$ & $1.3 \times 10^{-8}$ & $3.1 \times 10^{-9}$ & $1.8 \times 10^{-9}$ & $4.8 \times 10^{-5}$ \\
\hline St. Louis & $9.4 \times 10^{-5}$ & $6.8 \times 10^{-5}$ & $2.7 \times 10^{-4}$ & $1.6 \times 10^{-3}$ & $8.9 \times 10^{-8}$ & $2.2 \times 10^{-8}$ & $2.1 \times 10^{-8}$ & $2.0 \times 10^{-3}$ \\
\hline Pittsburgh & $8.85 \times 10^{-6}$ & $6.6 \times 10^{-6}$ & $3.0 \times 10^{-2}$ & $1.8 \times 10^{-1}$ & $1.1 \times 10^{-6}$ & $2.6 \times 10^{-8}$ & $2.5 \times 10^{-8}$ & $2.1 \times 10^{-1}$ \\
\hline Baltimore & $7.1 \times 10^{-6}$ & $5.0 \times 10^{-6}$ & $5.4 \times 10^{-5}$ & $3.6 \times 10^{-4}$ & $5.9 \times 10^{-7}$ & $1.5 \times 10^{-7}$ & $1.5 \times 10^{-7}$ & $4.3 \times 10^{-4}$ \\
\hline Minneapolis/St. Paul & $4.4 \times 10^{-8}$ & $3.2 \times 10^{-8}$ & $4.5 \times 10^{-7}$ & $1.7 \times 10^{-7}$ & $1.2 \times 10^{-9}$ & $2.8 \times 10^{-10}$ & $2.4 \times 10^{-10}$ & $7.0 \times 10^{-7}$ \\
\hline Newark & $2.5 \times 10^{-4}$ & $1.9 \times 10^{-4}$ & $4.5 \times 10^{-5}$ & $2.3 \times 10^{-4}$ & $8.9 \times 10^{-7}$ & $2.0 \times 10^{-7}$ & $2.6 \times 10^{-7}$ & $7.2 \times 10^{-4}$ \\
\hline Cleveland & $8.3 \times 10^{-6}$ & $6.0 \times 10^{-6}$ & $3.0 \times 10^{-2}$ & $1.9 \times 10^{-1}$ & $3.4 \times 10^{-8}$ & $8.1 \times 10^{-9}$ & $7.8 \times 10^{-9}$ & $2.2 \times 10^{-1}$ \\
\hline At lanta & $1.1 \times 10^{-7}$ & $7.7 \times 10^{-8}$ & $3.9 \times 10^{-3}$ & $1.6 \times 10^{-2}$ & $3.3 \times 10^{-9}$ & $7.9 \times 10^{-10}$ & $5.0 \times 10^{-10}$ & $2.0 \times 10^{-2}$ \\
\hline $\begin{array}{l}\text { Anaheim/Santa Ana/ } \\
\text { Garden Grove }\end{array}$ & $5.3 \times 10^{-8}$ & $3.8 \times 10^{-8}$ & $2.5 \times 10^{-6}$ & $2.5 \times 10^{-7}$ & $1.2 \times 10^{-8}$ & $2.8 \times 10^{-9}$ & $1.9 \times 10^{-9}$ & $2.9 \times 10^{-6}$ \\
\hline San Diego & $5.9 \times 10^{-8}$ & $4.4 \times 10^{-8}$ & $2.7 \times 10^{-7}$ & $1.1 \times 10^{-7}$ & $5.8 \times 10^{-9}$ & $1.4 \times 10^{-9}$ & $7.6 \times 10^{-10}$ & $4.9 \times 10^{-7}$ \\
\hline
\end{tabular}




\subsection{Human Errors or Deviations from Accepted Quality Assurance Practices}

Generic extension of the radiological impacts from human errors is performed in a manner exactly parallel to that used above for vehicular accidents. As in that case, for materials in Categories 1 and 2, the total latent cancer fatalities can be apportioned between pedestrians and people in vehicles in the ratio of $7: 3$. In Categories 3 through 6 , the proportion is $7: 3$, pedestrians to people in buildings, except for fuel cycle materials, where the major contribution (95\%) is to people in buildings with the remainder to pedestrians. For Categories 7 through 10, the split is $56 \%$ to pedestrians, $8 \%$ to people in vehicles, and $36 \%$ to people in buildings. Using expressions similar to Equation 1, the estimates of radiological health effects from human errors can be determined. Table 7-6 presents the results of this analysis.

\subsection{Sabotage}

Application of the consequence model CRAC to releases of materials through sabotage or malevolent act of a shipment have been discussed in detail in Chapter 5. The difficulty with extrapolating the radiological consequences, calculated using CRAC, have been discussed in detail in Reference 4. In general, it is possible to scale, in an approximately linear fashion, the consequences of the sabotage calculations for areas in which the close-in population density ranges from approximately $1.6 \times 10^{4}$ to about $1.4 \times 10^{3}$ per $\mathrm{km}^{2}$. Since all urban areas addressed in this report have population densities within this range, a first-order approximation to the consequences of sabotage of a shipment could be obtained using a linear scaling factor with population density. Because the nature of individual shipments in each urban area is difficult to define, numerical values would not be particularly meaningful. Hence, the reader is referred to Chapter 5 of this report and to the details provided in Reference 4 for further information in this area.

\section{$7.8 \underline{\text { Summary }}$}

To perform a generic extension of the limited New York City area analysis to other cities, the simplifying equations produced using the sensitivity analysis for incident-free transport have been used to calculate dose contributions from the five major population subgroups. The incident-free analysis depends primarily on the total TI shipped in an area. These data, combined with the appropriate demographic parameters, were used to estimate the incident-free radiological impacts for the top 20 SMSAs in the U.S. The results indicate that people in vehicles and pedestrians are the major dose groups and that the total population dose can range from a low of 7 person rem per year of shipping activity for San Diego to a high of 350 person rem per year of shipping activity for Newark. The range arises from the widely different shipping patterns, both in terms of the modes by which materials are carried and the total TI transported. Calculations for the limited New York City case using the set of assumptions of the generic extension analysis are also reported for comparison.

In the accident and human error cases, the model complexities did not allow approximation of the dose expressions by simplifying equations. A technique was developed to allow for the estimation of expected numbers of latent cancer fatalities for other urban areas using a breakdown of the limited New York City shipment model and accident results (also human errors) into 10 categories. Detailed analysis of the dose group and dose pathway dependencies of the calculated health effects for materials in each of these categories produced a set of assumptions for 
extending the results to other urban areas. The range of estimated numbers of latent cancer fatalities from vehicular accidents for a year's shipping activity at the given level for each urban area was a low of $2.1 \times 10^{-5}$ for San Diego to a high of $1.6 \times 10^{-1}$ for Chicago. Using a similar technique, the range of estimated numbers of latent cancer fatalities from human errors was a low of $4.9 \times 10^{-7}$ for San Diego to a high of $3.6 \times 10^{-1}$ for Chicago. The wide ranges for other urban areas result from the set of assumptions required for accomplishing the analysis as well as the markedly different shipment patterns in the areas. An inherent assumption in this analysis is that dispersible materials become airborne without regard to any factors which might mitigate the situation. For example, shipments of uranium hexafluoride, when involved in an accident, might well release significant quantities of hydrofluoric acid and much smaller quantities of the available uranium. Thus, data about the chemical form of the materials shipped, especially large quantity shipments of certain kinds of fuel cycle materials, could strongly influence these results.

Recent information indicates that it may be possible to scale the consequences of a release of radioactive materials which might well result from the sabotage or malevolent act of a shipment. Too many complex factors must be taken into account to allow for meaningful generic extension of the sabotage calculations for the limited New York City case. However, references are provided to allow the interested reader to investigate this area in greater detail. 4

NOTES

$1_{\text {BNWL-1972. }}$

${ }^{2}$ S. F. Herreid, A Method for Determining Radioactive Material Shipment Patterns in Urban Areas, SAND79-2071, NUREG/CR-1117, Albuquerque: Sandia Laboratories, May 1980.

${ }^{3}$ U.S. Department of Commerce, Statistical Abstract of the United States, 1978.

${ }^{4}$ D. M. Ericson, Jr., "Observations on the Use of the Consequence Mode1 CRAC for Small Non-Reactor Atmospheric Releases," SAND80-0366, to be published. 
APPENDICES 
URBAN AREA DATA BASES

An extensive data base is required to analyze the environmental impact of transportation of radioactive material in urban areas. This appendix lists methods for derivation, assumptions, sources of information, and values for each parameter.

Section Al describes the division of the urban day into time spans. Section A2 discusses land-use parameters. These include

- The study area itself, how it was chosen, and division of the area into unit cells

- Amount of open area

- Amount of area occupied by buildings

- Amount of area occupied by streets and sidewalks

- Other related parameters such as street width and sidewalk width

Section A3 discusses building characteristics. These include wall thicknesses, construction materials, and ventilation systems.

Section A4 considers aspects of population peculiar to the urban area, such as the large diurnal population fluctuation. This section also concerns itself with population densities, pedestrian densities, and the number of people in the area for non-work-related reasons.

Section A5 addresses transportation parameters such as traffic densities, vehicle and pedestrian velocities, vehicle length, the number of people per vehicle, and distances between moving vehicles (separation distances). Parameters involved in rail, air, and water transport are also discussed.

Section A6 discusses shipment characteristics including route, roadway. type, mode, package type, number of packages, destinations, and transport indices.

Section A7 discusses the determination of severity-dependent accident rates. This section also discusses methods used to estimate the amount of time the shipment vehicle may be delayed by an accident.

\section{Al. Time Span Specification}

Because most data are not available on an hourly basis, the day has been divided along the lines of an urban day as shown in Table A-1. 
Table A-1

Daily Time Division

\begin{tabular}{|c|c|c|}
\hline Time Span & Hours & Description \\
\hline 1 & $1800-0700$ & Night \\
\hline 2 & $0700-0830$ & Morning rush \\
\hline 3 & $0830-1130$ & Morning work \\
\hline 4 & $1130-1300$ & Noon rush \\
\hline 5 & $1300-1630$ & Afternoon work \\
\hline 6 & $1630-1800$ & Afternoon rush \\
\hline
\end{tabular}

Time-dependent data are presented as hourly average values for each of the six time spans. The night time span could be divided into 1800-2400 and 0000-0700 for greater accuracy.

A2. Land-Use Data

A2.1 Basic Grid

The geographical area under consideration is a 100-cell, 10-km by 10-km grid encompassing portions of the New York City boroughs of Manhattan, Queens, and Brooklyn (Figure $A-1$ ). This grid has been selected to cover the maximum amount of land area with as much land-use diversity as possible.

\section{A2. 2 Open Area}

Open area is characterized on maps in Reference 2 by variations in shading. It has been necessary to convert much information on land use from this source to the grid by subdividing the grid squares with a square-counting technique; this facilitates the determination of the fraction of the grid which is open area.

\section{A2.3 Street Area, Building Area}

The fraction of each grid cell occupied by streets has been determined by combining information on New York City Community Planning Districts (CPDs)* with information on street areas in New York City Health Areas. A tabulation of street area (in acres) in each of the city's Health Areas is available at the office of the New York City Planning Commission. Health Area data on street area were converted to street areas for CPDs and then to the grid itself. This information was subsequently used to obtain the fraction of each grid square occupied by streets. Information on population and street areas for the New York City Health Areas reflects 1970 census information. ** CPDs range in size from $0.59 \mathrm{~km}^{2}$ to $10.52 \mathrm{~km}^{2}$.

*In 1968, the New York City Planning Commission delineated 62 "Community Planning Districts," which are profiled in Reference 1. Each CPD has an administrative planning board which advises the borough president and city agencies on planning.

**Health Areas are subdivisions of the city on the bases of health needs. 


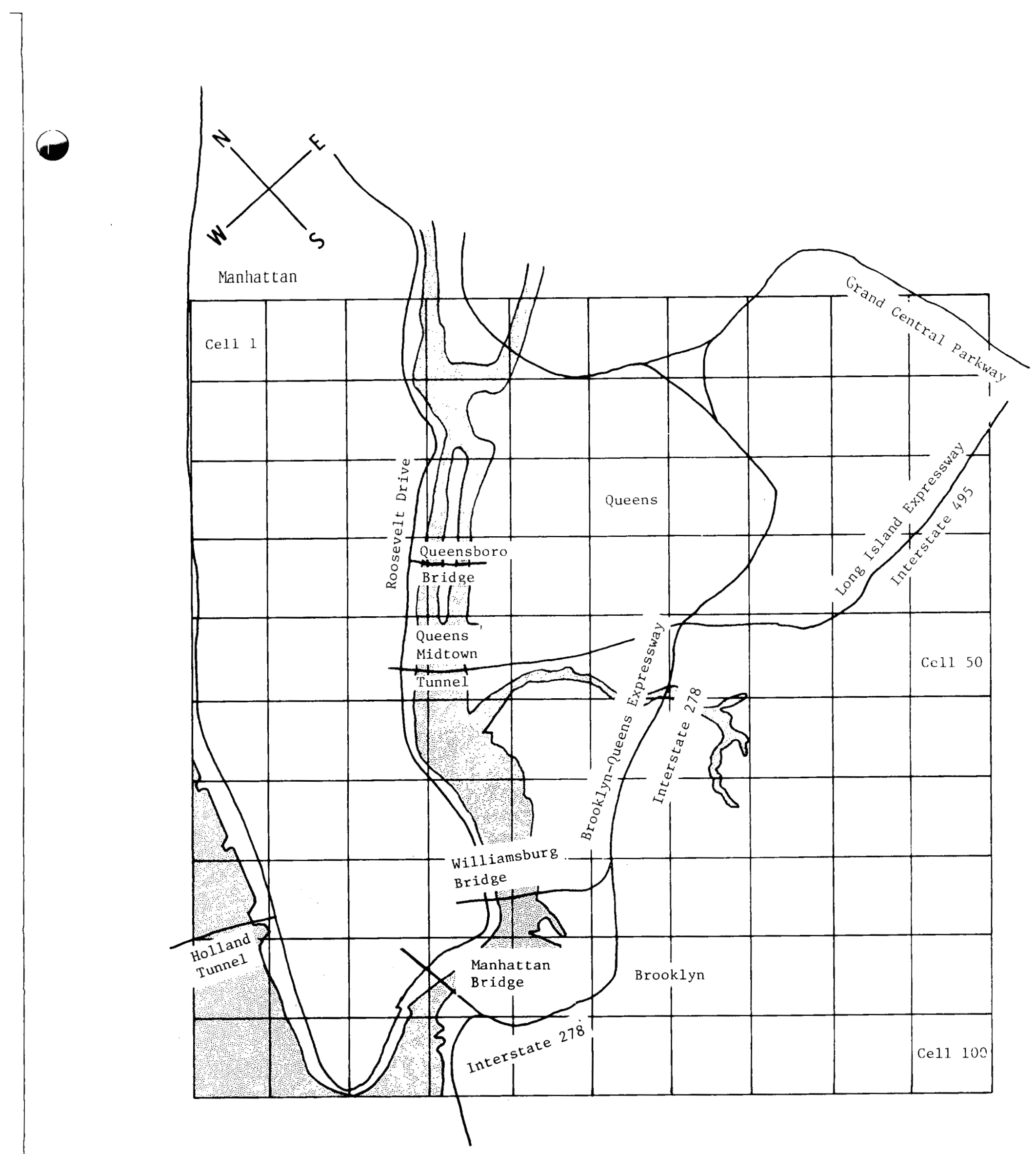

Figure A-1. Basic Grid for Portions of Manhattan, Queens, and Brooklyn 
Square-counting has determined the area of each affected CPD and the fraction of each grid cell occupied by a particular CPD.

The fraction of the grid square not occupied by open area or streets is assumed to be occupied by buildings. Values for open area, street area, and building area for each cell are tabulated in Table $\mathrm{A}-2$.

Table A-2

Cell Area Distribution

\begin{tabular}{|c|c|c|c|c|c|c|c|}
\hline & & & & & & & \\
\hline 1 & .000 & .243 & .757 & & 200 & & \\
\hline 2 & .700 & .135 & 165 & & 200 & .363 & .437 \\
\hline 3 & 200 & 319 & 481 & 53 & .000 & .433 & .567 \\
\hline 4 & 1.000 & 0.000 & 0.000 & & 200 & .342 & .458 \\
\hline 5 & .000 & $\begin{array}{l}.000 \\
.342\end{array}$ & .658 & 55 & .700 & 189 & 111 \\
\hline 6 & .000 & .289 & .711 & 56 & $\begin{array}{l}000 \\
000\end{array}$ & .408 & .592 \\
\hline 7 & .000 & .399 & 601 & $\begin{array}{l}30 \\
57\end{array}$ & 200 & .356 & .644 \\
\hline 8 & .000 & .334 & .666 & $\begin{array}{l}21 \\
58\end{array}$ & 2000 & 292 & .508 \\
\hline 9 & .000 & $\begin{array}{l}337 \\
.338\end{array}$ & $\begin{array}{l}.000 \\
.662\end{array}$ & $\begin{array}{l}58 \\
59\end{array}$ & .000 & 321 & .679 \\
\hline 10 & .000 & .309 & $\begin{array}{l}691 \\
691\end{array}$ & $\begin{array}{l}34 \\
60\end{array}$ & $\begin{array}{l}.000 \\
100\end{array}$ & .379 & .621 \\
\hline 11 & .000 & .200 & 800 & 61 & 500 & 231 & .669 \\
\hline 12 & .600 & .142 & .258 & 62 & .000 & 240 & 260 \\
\hline 13 & .100 & .404 & 496 & 63 & .000 & .461 & 539 \\
\hline 14 & .500 & 254 & 246 & 64 & .500 & $\begin{array}{r}401 \\
237\end{array}$ & 599 \\
\hline 15 & .000 & 385 & 615 & $\begin{array}{l}87 \\
65\end{array}$ & .000 & .399 & 263 \\
\hline 16 & .000 & .448 & 552 & 66 & .000 & .434 & .601 \\
\hline 17 & .000 & 411 & 589 & $\begin{array}{l}00 \\
67\end{array}$ & .000 & $\begin{array}{l}434 \\
328\end{array}$ & $\begin{array}{r}566 \\
672\end{array}$ \\
\hline 18 & .000 & .424 & .576 & 68 & .000 & $\begin{array}{l}328 \\
.418\end{array}$ & $\begin{array}{r}672 \\
582\end{array}$ \\
\hline 19 & .000 & 414 & 586 & 69 & .000 & .416 & \\
\hline 20 & .000 & 398 & 602 & 70 & 600 & 226 & $\begin{array}{l}584 \\
174\end{array}$ \\
\hline 21 & .000 & 331 & .669 & 71 & .700 & 105 & $\begin{array}{r}174 \\
195\end{array}$ \\
\hline 22 & .600 & 141 & 259 & 72 & .027 & 137 & $\begin{array}{r}195 \\
836\end{array}$ \\
\hline 23 & .100 & .405 & .495 & 73 & 000 & 474 & .836 \\
\hline 24 & .400 & .191 & 409 & 74 & .400 & 222 & $\begin{array}{r}526 \\
378\end{array}$ \\
\hline 25 & .000 & .405 & .595 & 75 & 200 & $\begin{array}{l}468 \\
468\end{array}$ & $\begin{array}{l}.318 \\
332\end{array}$ \\
\hline 26 & .500 & .402 & .098 & 16 & 000 & 485 & .515 \\
\hline 27 & .000 & .460 & .540 & 77 & 000 & 443 & 557 \\
\hline 28 & .000 & .467 & 533 & 78 & 000 & 419 & 581 \\
\hline 29 & .000 & 431 & 569 & 79 & .000 & 414 & .586 \\
\hline 30 & .000 & .365 & 635 & 80 & 600 & 336 & .064 \\
\hline 31 & .060 & .354 & 646 & 81 & 800 & 0.000 & 200 \\
\hline 32 & 200 & 213 & 587 & 82 & 100 & 367 & 533 \\
\hline 33 & 200 & 330 & 470 & 83 & .197 & $\begin{array}{l}301 \\
.335\end{array}$ & $\begin{array}{l}233 \\
468\end{array}$ \\
\hline 34 & .300 & 193 & 507 & 84 & 400 & .151 & 449 \\
\hline 35 & .300 & 368 & 332 & 85 & .077 & .147 & .776 \\
\hline 36 & .100 & .295 & 605 & 86 & .000 & 410 & $590^{\circ}$ \\
\hline 37 & .000 & .274 & 726 & 87 & .000 & 416 & 584 \\
\hline 38 & .000 & .368 & 632 & 88 & .000 & .407 & 593 \\
\hline 39 & .000 & .407 & 593 & 89 & .000 & 400 & .600 \\
\hline 40 & .000 & .339 & 661 & 90 & .000 & .439 & .561 \\
\hline 41 & .000 & .427 & 573 & 91 & 1.000 & 0.000 & 0.000 \\
\hline 42 & .000 & 393 & 607 & 92 & .300 & .262 & .438 \\
\hline 43 & .300 & .240 & 460 & 93 & .500 & 210 & 290 \\
\hline 44 & .300 & .271 & 429 & 94 & 500 & 399 & 101 \\
\hline 45 & .200 & .352 & 448 & 95 & .000 & 402 & 598. \\
\hline 46 & .000 & .296 & .704 & 96 & .000 & 435 & 565 \\
\hline 47 & .000 & .239 & .761 & 97 & .000 & 413 & 587 \\
\hline 48 & 000 & 317 & 683 & 98 & .000 & 398 & $60 ?$ \\
\hline 49 & .000 & 303 & 697 & 99 & .000 & .410 & 590 \\
\hline & .000 & $3 ! 9$ & 681 & 100 & .000 & .399 & 601 \\
\hline
\end{tabular}

\section{A2.4 Street Width, Sidewalk Width}

Constant values of 20 metres (street width) and 3 metres (sidewalk width) are used for street and sidewalk widths since they reflect reasonable standards for metropolitan areas. 2 


\section{A3. Building Characteristics}

Average building heights have been estimated on the basis of personal observation

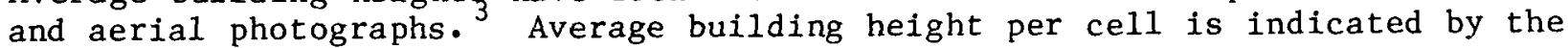
number of floors per building, assuming a constant floor height of 3 metres. (e.g., a 20-floor building is 60 metres high). An architect has estimated that an older residential building with exterior supporting walls would have an average wall thickness of 0.38 metre, and a newer building with support from the skeleton, not the walls alone, would have a wall thickness of 0.20 metre. 4 If a grid cell has average building heights of less than 24 metres, it is assumed to be mostly residential and to have wall thicknesses of 0.38 metre. Grid cells with buildings taller than 24 metres are assumed to be commercial, containing structures with wall thicknesses of 0.20 metre. Data for building height and wall thickness ss for each cell are given in Table A-3.

Table A-3

\section{Building Characteristics}
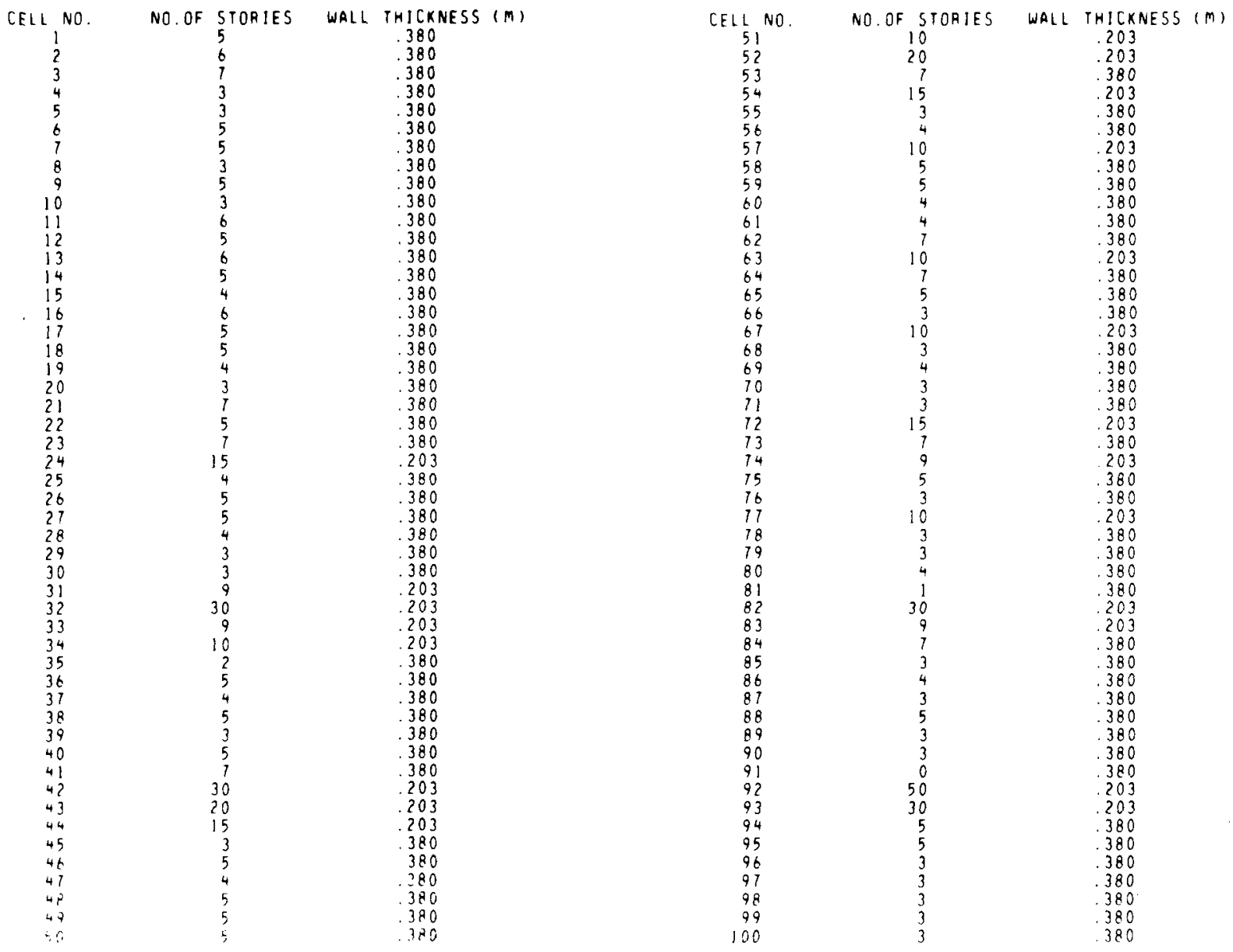

Most buildings in the study area have either concrete or brick facing. Although the capability to analyze other materials exists, the absorption of radiation by building materials for the entire study area is assumed to be that of concrete. 
A4. Population Parameters

A4.1 Population Density

One of the unique characteristics of an urban area is the large diurnal variation in population density. Population densities are calculated by determining the area of each pertinent CPD (Table A-4).* The population density of a cell entirely within a single CPD is set equal to the CPD population density.

Table A-4

Population Densities of Selected Community Planning Districts

$\begin{array}{ccrrr}\begin{array}{c}\text { CPD } \\ \text { Manhattan }\end{array} & \begin{array}{c}\text { Area }\left(\mathrm{km}^{2}\right) \\ \text { Population }\end{array} & \begin{array}{c}\text { Pop. Density } \\ \left.\text { (persons } / \mathrm{km}^{2}\right)\end{array} \\ 1 & 2.64 & 7034 & 2967 \\ 2 & 2.36 & 85357 & 35750 \\ 3 & 2.94 & 182171 & 61963 \\ 4 & 3.25 & 83857 & 25802 \\ 5 & 2.86 & 31458 & 10997 \\ 6 & 2.36 & 121886 & 51646 \\ 7 & 3.33 & 212316 & 63629 \\ 8 & 3.50 & 200189 & 54194 \\ 11 & 2.64 & 154450 & 58504\end{array}$

Brooklyn

$\begin{array}{crrrrr}1 & 7.94 & 179 & 458 & 23 & 736 \\ 2 & 3.04 & 73 & 609 & 24 & 213 \\ 3 & 6.08 & 216 & 983 & 35 & 688 \\ 4 & 3.33 & 137 & 895 & 41 & 369 \\ \text { BNY (Brook1yn } & 0.59 & 1 & 134 & 1 & 902 \\ \text { Naval Yard) } & & & & & \\ \text { Queens } & & & & & \\ 1 & 10.52 & 194 & 384 & 18 & 097 \\ 2 & 9.59 & 124 & 146 & 13 & 994 \\ 3 & 5.11 & 123 & 598 & 24 & 101 \\ 4 & 3.66 & 107 & 961 & 27 & 694 \\ 5 & 6.10 & 125 & 167 & 20 & 519 \\ 6 & 5.80 & 119 & 019 & 20 & 521\end{array}$

\footnotetext{
* Information on total populations for the CPDs was obtained from New York City Planning Commission data in Reference 1.
} 
If the cell overlaps more than one CPD or part of some body of water, the population density for the cell is computed by taking the fraction of the square occupied by each CPD and multiplying by the appropriate population density. The fractional population densities are then summed to obtain the average value for the cell. Table A-5 shows how this calculation has been done for cell 62 .

Table A-5

Sample Calculation of Population Density for Grid Cell 62

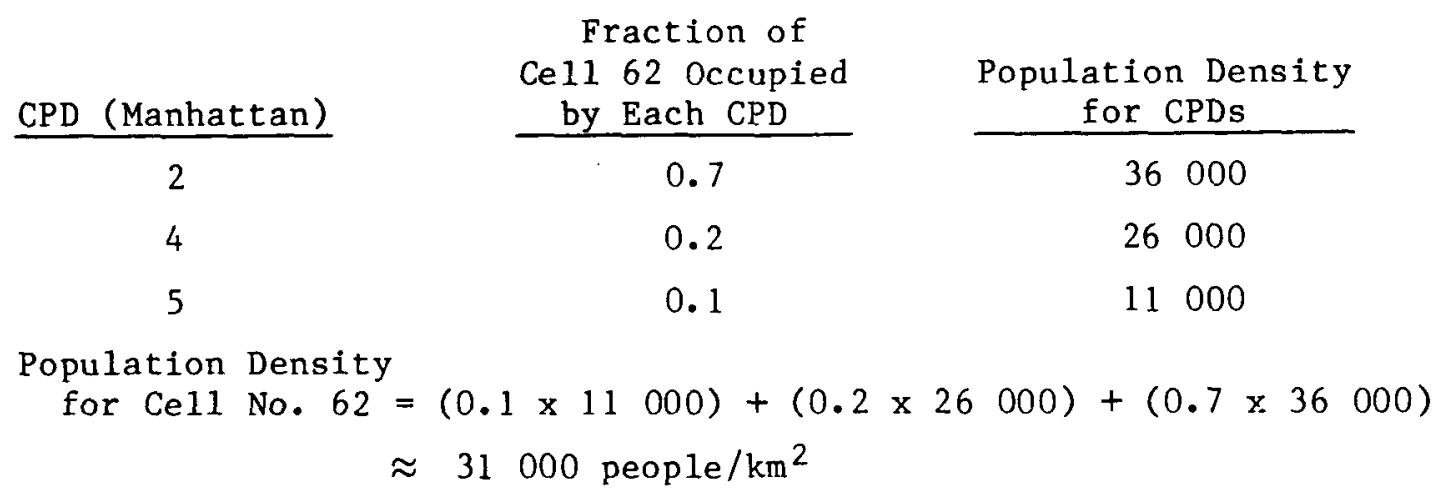

The calculation in Table A-5 reflects only the resident (nighttime) population densities of the cell. Values for the number of people over 16 years of age employed in each CPD, information on the percentage of workers residing in a borough who work in that borough, and information on the number of workers who commute to a borough to work have been used to calculate daytime population densities. ${ }^{5}$ Reference 3 provides information on the designation of a grid cell as residential, commercial, industrial, or mixed. The designations are used to estimate the number of resident workers who remain in the cell and the number who commute out of the cell to work. For example:

Ce11 No. 1 (Manhattan)

- Average population density: 64000

- Resident workers: 36000

- Percentage of workers 1iving and working in Manhattan: $71.7 \%$

- Residents living in cell and working in Manhattan: $0.717 \times 36000 \approx 26000$

It is arbitrarily assumed that $20 \%$ of the residents of any cell also work in that same cell. Thus 5100 residents remain in cell 1 . This cell is principally residential; hence, most of the residents who work leave the cell during the day. The number of resident workers remaining in the cell is added to the nonworking population to obtain values for daytime resident population. 
Census data provides information on the total number of commuters coming into the borough to work. 5 The total number of people commuting into the grid is distributed among the cells by borough. This net commuter flux is added to, or subtracted from, the daytime resident population to obtain a more realistic figure for total average daytime population. Table A-6 lists values for population density in different time spans for each cell.

\section{A4.2 Pedestrian Density}

Determination of pedestrian densities requires a different approach. Table 3.8 in Reference 6 lists characteristics of pedestrian flow in terms of effective space occupied by a single pedestrian ( $\mathrm{m}^{2} /$ pedestrian). The six time spans are associated with degrees of pedestrian flow, as shown in Table A-7. Pedestrian densities per $\mathrm{km}^{2}$ of sidewalk are calculated by inverting the effective space per pedestrian and multiplying by the units conversion factor of $10^{6} \mathrm{~m}^{2} / \mathrm{km}^{2}$. These values are also given in Table A-7. More accurate information from cordon counts in the area was not available; however, the information is not inconsistent with that acquired in performing the generic extension to other cities described in Chapter 7.

\section{A4.3 Transient Population}

In addition to residents and pedestrians, there are people in each cell whose purpose may not be directly work-related. It is initially assumed that this "transient" population is significant only during the day. In the Manhattan central business district, 4.3 daily one-way trips are made for every $93 \mathrm{~m}^{2}$ of floor space, regardless of floor space utilization and trip purpose. ${ }^{6}$ This value is assumed to be constant across the grid so that the number of one-way trips for each cell can be estimated using the expression

$$
\text { No. of one-way trips }=\frac{f_{b} \cdot A \cdot n \cdot 10^{6} \mathrm{~m}^{2} / \mathrm{km}^{2} \cdot 1 \mathrm{~km}^{2} \cdot 4.3 \mathrm{trips}}{93 \mathrm{~m}^{2}} \text {, }
$$

where

$$
\begin{aligned}
\mathrm{f}_{\mathrm{b}} & =\text { fraction of grid squares occupied by buildings } \\
\mathrm{n} & =\text { average number of floors per building } \\
\mathrm{A} & =\text { cell area }\left(=1 \mathrm{~km}^{2}\right)
\end{aligned}
$$

For example, in cell No. $1, \mathrm{f}_{\mathrm{b}}=0.76$ and $\mathrm{n}=5$. Thus, $1.8 \times 10^{5}$ one-way trips per day are associated with cell no. 1 .

Reference 2 ( $p$ 157) suggests that $19 \%$ of all daily trips in urban areas are specifically for nonwork purposes $(7.5 \%$ for shopping and $11.8 \%$ for miscellaneous reasons). The total number of one-way trips is multiplied by this fraction to obtain the figure for transient population. This is the total value for the 11 hours of the urban work day. An hourly figure is obtained by dividing the result by 11 . Therefore 3100 trips per hour are made to grid cell No. 1 for nonworking purposes. Since most of these trips are to buildings, it is assumed that all the transient population is in buildings during the daytime hours. This may count some people twice since the values for traffic count and the pedestrian density clearly include some of the transient population. A summary of transient population data is shown in Table A-8. This calculation does not account for people who would come into the area in the evening for entertainment. 
Table A-6

Population Density (persons $/ \mathrm{km}^{2}$ ); Time Span

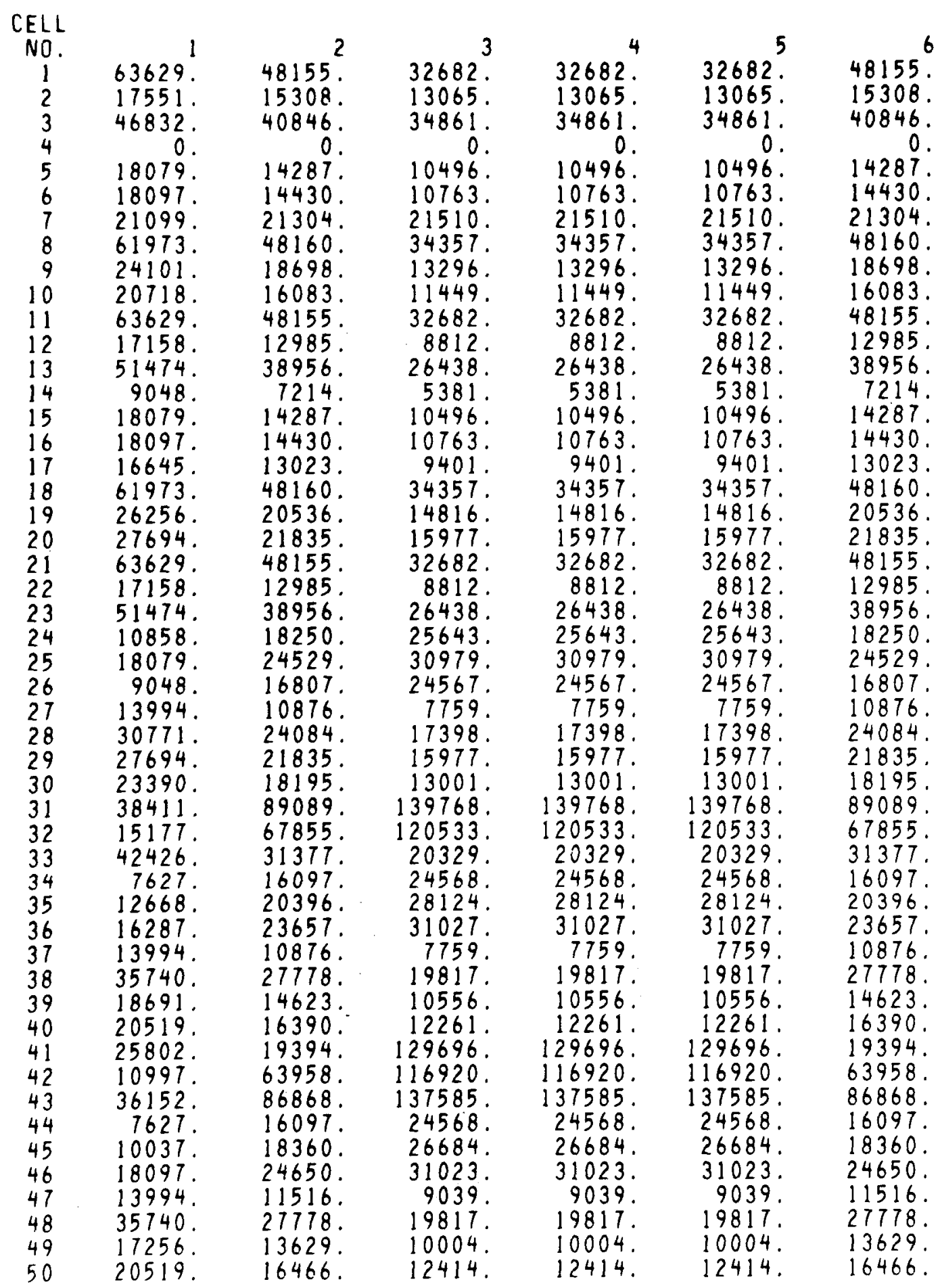


Table A-6 (Continued)

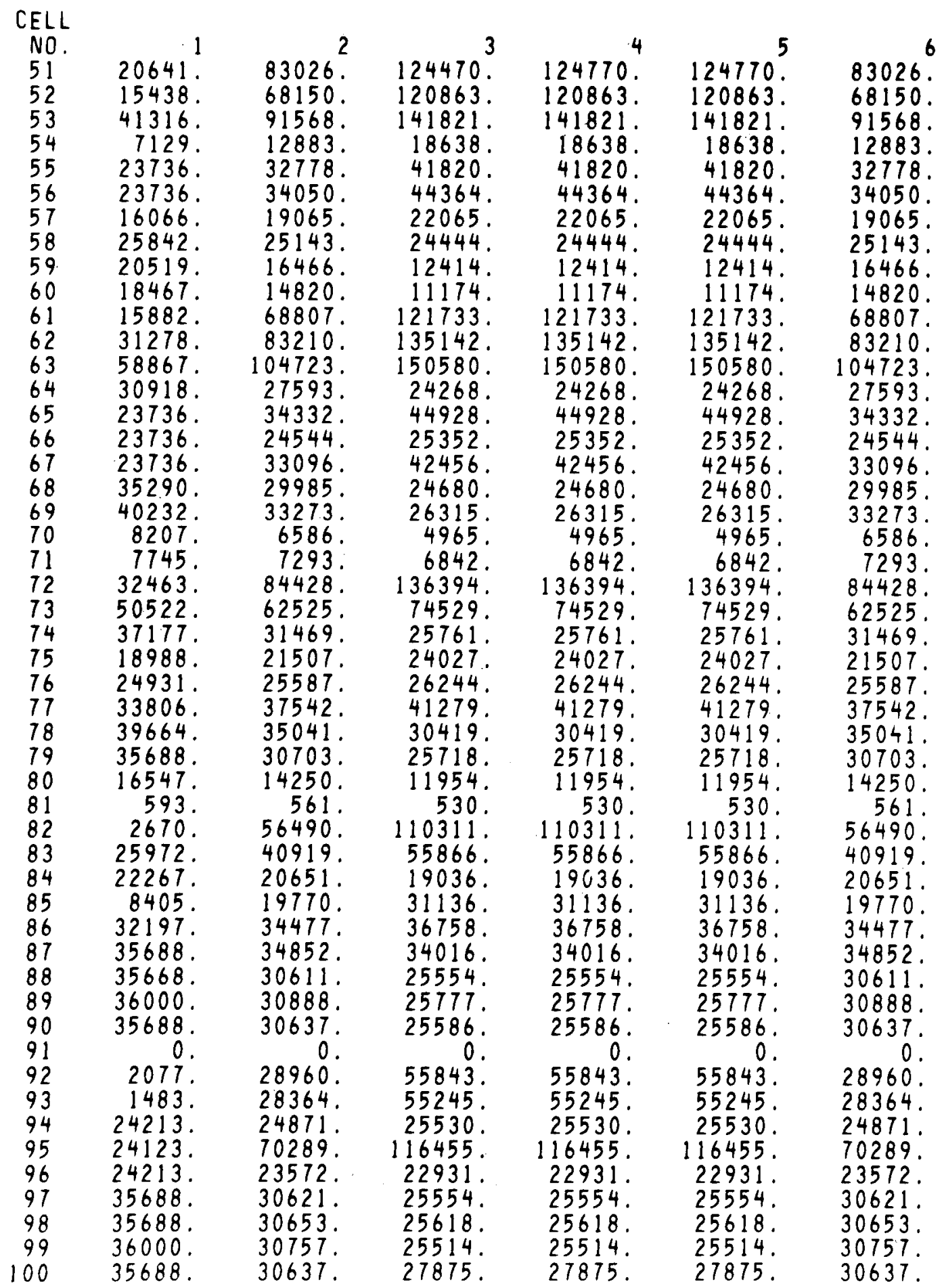


Table A-8

Transient Population (persons $/ \mathrm{km}^{2}$ )

Time Span

\begin{tabular}{|c|c|c|c|c|c|c|}
\hline $\begin{array}{c}\text { CELL } \\
\text { NO. }\end{array}$ & 1 & 2 & 3 & 4 & 5 & 6 \\
\hline 1 & 0. & 3075 . & 3075. & 3075 . & $3075^{\circ}$ & 3075 . \\
\hline 2 & 0. & 803. & 803 & 803. & 803 & 803. \\
\hline $\begin{array}{l}3 \\
4\end{array}$ & 0. & 2734. & 2734. & 2734. & 2734. & 2734. \\
\hline 5 & 0. & 1598. & 1598. & $1598^{\circ}$ & 1598. & 1598. \\
\hline 6 & 0. & 2888 . & 2888 & 2888 . & 2888 . & 2888 . \\
\hline 7 & 0. & 2442 . & 2442 . & 2442 . & 2442 . & 2442 . \\
\hline 8 & 0. & 1622. & 1622. & 1622 & 1622. & 1622 \\
\hline $\begin{array}{r}9 \\
10\end{array}$ & 0. & 2685. & 2685 . & 2685 . & 2685. & $\begin{array}{l}2685 . \\
1679\end{array}$ \\
\hline 11 & 0. & $3894^{\circ}$ & $3894^{\circ}$ & 3894. & $3894^{\circ}$ & 3894. \\
\hline 12 & 0. & 1046. & 1046. & 1046. & 1046. & 1046. \\
\hline 13 & 0. & 2418. & 2418. & 2418. & 2418. & 2418. \\
\hline 14 & 0. & 998. & 998. & 998. & 998. & 998. \\
\hline $\begin{array}{l}15 \\
16\end{array}$ & 0. & 1996. & 1996. & 1996. & 1996. & $\begin{array}{l}1996 . \\
2685 .\end{array}$ \\
\hline 17 & 0. & 2393. & 2393. & 2393. & 2393. & $2393^{\circ}$ \\
\hline 18 & 0. & 2336. & 2336 & 2336. & 2336. & 2336. \\
\hline $\begin{array}{l}19 \\
20\end{array}$ & 0. & $\begin{array}{l}1898 . \\
1468\end{array}$ & $\begin{array}{l}1898 . \\
1468\end{array}$ & 1898. & 1898. & 1898. \\
\hline $\begin{array}{l}20 \\
21\end{array}$ & 0. & $\begin{array}{l}1468 . \\
3797 .\end{array}$ & $\begin{array}{l}1468 . \\
3797\end{array}$ & $\begin{array}{l}1468 . \\
3797 .\end{array}$ & $\begin{array}{l}1468 . \\
3797 .\end{array}$ & $\begin{array}{l}1468 . \\
3797 .\end{array}$ \\
\hline 22 & 0 & $1055^{\circ}$ & 1055. & 1055. & 1055. & 1055. \\
\hline 23 & 0 . & 2815 . & 2815 . & 2815 & 2815 . & 2815 \\
\hline 24 & 0. & 4981. & 4981. & 4981. & 4981. & 4981. \\
\hline 25 & 0. & 1931. & 1931. & 1931. & 1931. & 1931. \\
\hline 26 & 0. & 397. & 397. & 397 & 397. & 397. \\
\hline 27 & 0. & 2190. & 2190. & $2190^{\circ}$ & 2190. & 2190. \\
\hline $\begin{array}{l}28 \\
29\end{array}$ & 0. & $\begin{array}{l}1728 \\
1387\end{array}$ & 387 & 1387. & 387. & 387 \\
\hline 30 & 0. & $1549^{\circ}$ & 1549. & 1549. & 1549. & $549^{\circ}$ \\
\hline 31 & 0. & 4713. & 4713. & 4713. & 4713. & 4713. \\
\hline 32 & 0. & 14286 & 14286 & 14286 & 14286 & 14286 \\
\hline 33 & 0. & 3432 & 3432 & 3432 & 3432 . & 3432 \\
\hline 34 & 0. & 4113. & 4113 & 4113. & 4113. & 4113. \\
\hline $\begin{array}{l}35 \\
36\end{array}$ & 0. & & $\begin{array}{r}53 \\
245\end{array}$ & 5. & 5. & $\begin{array}{r}535 \\
.\end{array}$ \\
\hline $\begin{array}{l}30 \\
37\end{array}$ & 0. & $\begin{array}{l}24 \\
23\end{array}$ & $\begin{array}{l}2438 . \\
2353 .\end{array}$ & $\begin{array}{l}2438 . \\
2353 .\end{array}$ & $\begin{array}{l}8 . \\
3 .\end{array}$ & $\begin{array}{l}24 \\
23\end{array}$ \\
\hline 38 & 0. & 25 & 256 & & & \\
\hline 39 & 0. & & 146 & 1444. & & \\
\hline 40 & 0. & 5. & 2685 . & 2685 . & & . \\
\hline 41 & 0. & & 3253 . & 3253. & & \\
\hline 42 & 0. & 147 & 14773 & 14773 & 147 & 14773 \\
\hline 43 & 0. & 74 & 7463 & 7463. & & 7463 \\
\hline 44 & 0. & & 5224. & 5224. & & 5224. \\
\hline 45 & 0. & 10 & 1087 & 1087. & & \\
\hline 46 & 0. & & 2855 . & 2855 . & & 2855 \\
\hline 47 & 0. & & & & & \\
\hline 48 & $v$ & & 2 & & & 2775. \\
\hline 49 & U. & 28 & & & & \\
\hline 50 & 0 & & & & & \\
\hline
\end{tabular}


Table A-8 (Continued)

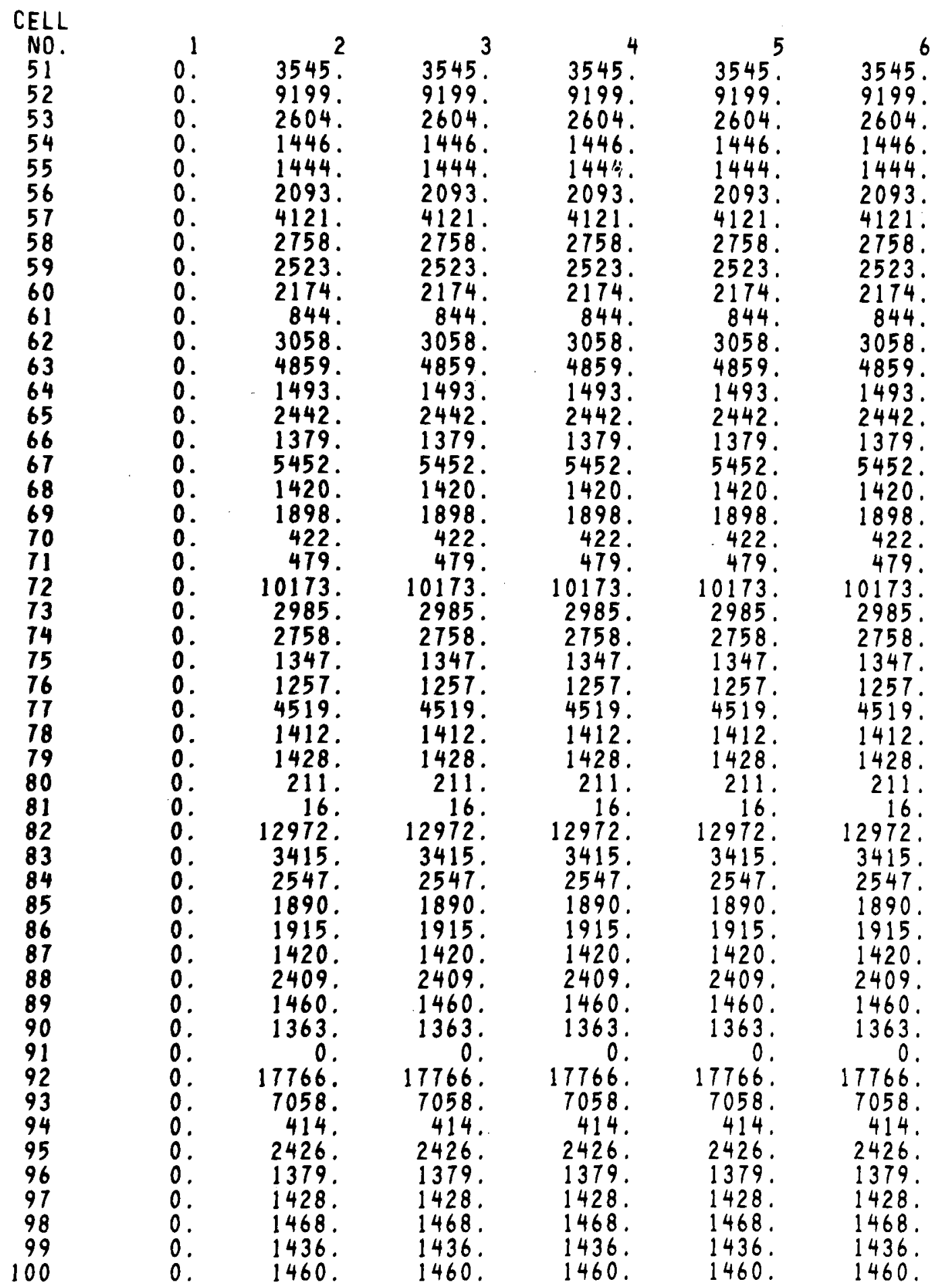


Table A-7

Characteristics of Pedestrian Flow

\begin{tabular}{|c|c|c|c|c|}
\hline & Time Span & Pedestrian Flow & $\begin{array}{c}\begin{array}{c}\text { Area/Pedestrian } \\
\left(\mathrm{m}^{2}\right)\end{array} \\
\end{array}$ & $\begin{array}{l}\text { Pedestrians/ } \\
\mathrm{km}^{2} \text { of sidewalk }\end{array}$ \\
\hline 1 & Night & Unlimited* & 24.7 & 40500 \\
\hline 2 & Morning rush & Constrained & 2.95 & 339000 \\
\hline 3 & Morning work & Impeded & 4.65 & 215000 \\
\hline 4 & Noon & Congested & 1.25 & $800 \quad 000$ \\
\hline 5 & Afternoon work & Impeded & 4.65 & 215000 \\
\hline 6 & Afternoon rush & Constrained & 2.95 & 339000 \\
\hline
\end{tabular}

*The value for this time span is based on weighted averages reflecting changes in pedestrian flow for evening, late night, and early morning hours.

\section{A5. Transportation Parameters}

\section{A5.1 Traffic Density}

Information from Reference 6 provides the effective street area and instantaneous vehicle count for a significant portion of downtown Manhattan. These data are considered typical for cell 42 in the limited New York City study area. Additional data from this reference allow for further discrimination of the vehicular pattern with time of day. To extend these data to other cells, the appropriate vehicle density for cell 42 is adjusted for street area fraction in each cell, compared with that for cel1 42 and also for the time-weighted average population densities for the cells. Because this places too much emphasis on the character of the cell (residential, commercial, etc.), a third correction is used for the time-span dependent population density for each cell compared to its time-weighted average population density, resulting in the following expression:

$$
N_{i}(t)=N_{c e 1142}(t) \cdot \frac{P_{i}(t)}{\overline{P D}_{42}} \cdot \frac{\left(f_{s t}\right)_{i}}{\left(f_{s t}\right)_{42}}
$$

where

$$
\begin{aligned}
& N_{i}(t)=\text { vehicle density in cell } i \text { at time } t \\
& N_{\text {cell } 42}(t)=\text { vehicle density in cell } 42 \text { at time } t \\
& \mathrm{PD}_{i}(t)=\text { population density in cell } i \text { at time } t \\
& \overline{\mathrm{PD}}_{42}=\text { time-weighted average population } \\
& \text { density in cell } 42 \\
& \left(f_{s t}\right)_{i}=\text { street area fraction in cell } i \\
& \left(f_{s t}\right)_{42}=\text { street area fraction in cell } 42
\end{aligned}
$$

Table A-9 summarizes the data for instantaneous vehicle numbers. 
Table A-9

Instantaneous Vehicle Numbers

Cell

\begin{tabular}{|c|c|c|c|c|c|c|}
\hline Number & TS 1 & TS 2 & TS 3 & TS 4 & TS 5 & TS 6 \\
\hline 1 & 480 & 1250 & 960 & 1040 & 1010 & 1880 \\
\hline 2 & 73 & 220 & 210 & 230 & 225 & 330 \\
\hline 3 & 460 & 1390 & 1350 & 1460 & 1420 & 2090 \\
\hline 4 & 0 & 0 & 0 & 0 & 0 & 0 \\
\hline 5 & 190 & 520 & 440 & 470 & 460 & 780 \\
\hline 6 & 160 & 450 & 380 & $410^{\circ}$ & 400 & 670 \\
\hline 7 & 260 & 910 & 1040 & 1120 & 1090 & 1360 \\
\hline 8 & 640 & 1720 & 1390 & 1500 & 1460 & 2580 \\
\hline 9 & 250 & 680 & 550 & 590 & 570 & 1010 \\
\hline 10 & 200 & 530 & 430 & 460 & 450 & 800 \\
\hline 11 & 390 & 1030 & 790 & 860 & 830 & 1540 \\
\hline 12 & 75 & 200 & 150 & 160 & 160 & 300 \\
\hline 13 & 640 & 1660 & 1300 & 1400 & 1360 & 2500 \\
\hline 14 & 71 & 200 & 170 & 180 & 170 & 290 \\
\hline 15 & 220 & 590 & 490 & 530 & 520 & 880 \\
\hline 16 & 250 & 690 & 580 & 630 & 620 & 1040 \\
\hline 17 & 210 & 570 & 470 & 510 & 490 & 860 \\
\hline 18 & 810 & 2200 & 1770 & 1900 & 1860 & 3300 \\
\hline 19 & 340 & 910 & 740 & 800 & 780 & 1360 \\
\hline 20 & 340 & 930 & 770 & 830 & 810 & 1390 \\
\hline 21 & 650 & 1700 & 1310 & 1410 & 1380 & 2550 \\
\hline 22 & 75 & 200 & 150 & 160 & 160 & 290 \\
\hline 23 & 640 & 1690 & 1300 & 1400 & 1370 & 2530 \\
\hline 24 & 64 & 370 & 590 & 640 & 620 & 560 \\
\hline 25 & 230 & 1060 & 1520 & 1640 & 1600 & 1590 \\
\hline 26 & 110 & 720 & 1200 & 1290 & 1260 & 1080 \\
\hline 27 & 200 & 540 & 430 & 470 & 460 & 800 \\
\hline 28 & 440 & 1200 & 980 & 1060 & 1040 & 1800 \\
\hline 29 & 370 & 1010 & 830 & 900 & 880 & 1510 \\
\hline 30 & 260 & 710 & 580 & 620 & 610 & 1060 \\
\hline 31 & 420 & 3400 & 6000 & 6500 & 6300 & 5100 \\
\hline
\end{tabular}


Table A-9 (continued)

Instantaneous Vehicle Numbers

Cell

Number

32

33

34

35

36

37

38

39

40

41

42

43

44

45

46

47

48

49

50

51

52

TS

$\frac{1}{100}$

TS 2

TS

430

1540

3110

TS 4

$\frac{\text { TS } 5}{3270} \quad \frac{\text { TS } 6}{2320}$

1100

810

880

$860 \quad 1660$

45

330

580

620

610

500

140

800

1250

1350

1320

1200

150

75

1110

1200

1170

1120

120

320

260

280

$270 \quad 480$

410

1100

880

950

930

1640

240

640

520

560

550

950

220

590

500

540

530

890

340

4100

6700

7200

7100

6100

640

2220

2520

2720

2650

3300

270

2230

4000

4300

4200

3340

64

470

810

870

850

700

110

690

1140

1230

1200

1040

170

780

1110

1200

1170

1170

100

$290 \quad 260$

280

280

440

350

$940 \quad 760$

820

800

1400

160

$440 \quad 370$

400

390

660

200

560

480

520

510

840

230

3220

5480

5910

5760

4830

$3150 \quad 6340$

6850

6670

4730

53

440

3340

5880

6340

6180

5020

54

42

260

430

460

450

380

55

300

1430

2070

2230

2180

2140

56

260

1290

1910

2070

2010

1940

57

150

600

780

840

820

890

58

260

86

950

1030

1000

1300

59

240

670

570

620

$600 \quad 1000$

60

130

370

310

340

330

550

61

120

$1760 \quad 3540$

3820

3720

2650 
Table A-9 (continued)

Instantaneous Vehicle Numbers

Cel1

\begin{tabular}{|c|c|c|c|c|c|c|}
\hline Number & TS 1 & TS 2 & TS 3 & TS 4 & TS 5 & TS 6 . \\
\hline 62 & 450 & 4100 & 7550 & 8150 & 7940 & 6150 \\
\hline 63 & 730 & 4480 & 7320 & 7900 & 7700 & 6730 \\
\hline 64 & 230 & 700 & 700 & 750 & 730 & 1050 \\
\hline 65 & 290 & 1460 & 2170 & 2340 & 2290 & 2200 \\
\hline 66 & 320 & 1140 & 1330 & 1440 & 1400 & 1710 \\
\hline 67 & 240 & 1160 & 1690 & 1820 & 1780 & 1740 \\
\hline 68 & 460 & 1340 & 1250 & 1350 & 1320 & 2010 \\
\hline 69 & 520 & 1480 & 1330 & 1430 & 1400 & 2220 \\
\hline 70 & 57 & 160 & 140 & 150 & 140 & 240 \\
\hline 71 & 25 & 82 & 87 & 94 & 92 & 120 \\
\hline 72 & 140 & 1240 & 2260 & 2440 & 2380 & 1850 \\
\hline 73 & 740 & 3170 & 4280 & 4620 & 4500 & 4750 \\
\hline 74 & 260 & 750 & 690 & 750 & 730 & 1120 \\
\hline 75 & 270 & 1080 & 1360 & 1470 & 1430 & 1610 \\
\hline 76 & 370 & 1330 & 1540 & 1670 & 1620 & 1990 \\
\hline 77 & 460 & 1780 & 2220 & $2390^{\circ}$ & 2330 & 2660 \\
\hline 78 & 510 & 1570 & 1550 & 1670 & 1630 & 2350 \\
\hline 79 & 460 & 1360 & 1290 & 1390 & 1360 & 2040 \\
\hline 80 & 170 & 510 & 490 & 530 & 510 & 770 \\
\hline 81 & 0 & 0 & 0 & 0 & 0 & 0 \\
\hline 82 & 30 & 2214 & 4910 & 5300 & 5160 & 3320 \\
\hline 83 & 270 & 1460 & 2270 & 2450 & 2390 & 2200 \\
\hline 84 & 100 & 330 & 350 & 380 & 370 & 500 \\
\hline 85 & 38 & 310 & 560 & 600 & 580 & 470 \\
\hline 86 & 410 & 1510 & 1830 & 1970 & 1920 & 2270 \\
\hline 87 & 460 & 1550 & 1720 & 1850 & 1800 & 2320 \\
\hline 88 & 450 & 1330 & 1260 & 1360 & 1330 & 2000 \\
\hline 89 & 450 & 1320 & 1250 & 1350 & 1320 & 1980 \\
\hline 90 & 480 & 1440 & 1360 & 1470 & 1430 & 2160 \\
\hline 91 & 0 & 0 & 0 & 0 & 0 & 0 \\
\hline 92 & 17 & 810 & 1770 & 1910 & 1870 & 1220 \\
\hline
\end{tabular}


Table A-9 (continued)

Instantaneous Vehicle Numbers

Ce11

\begin{tabular}{|c|c|c|c|c|c|c|}
\hline Number & TS 1 & TS 2 & TS 3 & TS 4 & TS 5 & TS 6 \\
\hline 93 & 10 & 640 & 1410 & 1520 & 1480 & 950 \\
\hline 94 & 300 & 1060 & 1240 & 1330 & 1300 & 1590 \\
\hline 95 & 300 & 3020 & 5680 & 6130 & 5970 & 4530 \\
\hline 96 & 330 & 1100 & 1210 & 1310 & 1270 & 1640 \\
\hline 97 & 460 & 1350 & 1280 & 1380 & 1350 & 2030 \\
\hline 98 & 440 & 1300 & 1240 & 1330 & 1300 & 1950 \\
\hline 99 & 460 & 1350 & 1270 & 1370 & 1330 & 2020 \\
\hline 100 & 440 & 1310 & 1350 & 1460 & 1420 & 1960 \\
\hline
\end{tabular}

A5.2 Vehicle Speeds, Intersection Delay, and Stopped Separation Distance

Vehicular speeds have been extrapolated from Table 4.5 of Reference 6 and are given in Table A-10.

Table A-10

Average Vehicle Speed

\begin{tabular}{cc} 
Time Span & $\begin{array}{c}\bar{V} \\
\text { (average speed } \\
\text { including delays, } \mathrm{m} / \mathrm{s} \text { ) }\end{array}$ \\
\cline { 1 - 2 } 1 & 8.1 \\
2 & 3.3 \\
3 & 4.1 \\
4 & 3.6 \\
5 & 3.8 \\
6 & 3.3
\end{tabular}

Another necessary parameter is the fraction of intersections at which a vehicle stops while traveling in a cell $(\xi)$. Values for $\xi$ for different time spans are shown in Table A-11. 
Table A-11

Fraction of Intersections at Which Vehicle Must Stop

\begin{tabular}{cc} 
Time Span & \multicolumn{2}{c}{$\begin{array}{c}\xi \text { (fraction of int } \\
\text { which vehicle is re }\end{array}$} \\
\cline { 2 - 2 } 1 & 0 \\
2 & 1 \\
3 & 0.5 \\
4 & 0.75 \\
5 & 0.5 \\
6 & 1
\end{tabular}

Also required is the average length of time a vehicle is delayed by stopping at an intersection $(\Omega)$. The value assumed for $\Omega$ is 25 seconds. Traffic engineers in Albuquerque, New Mexico, indicate that this is a reasonable value for the average duration of an urban red light. Note that this does not account for multiple delays at the same intersection resulting from extremely congested conditions. That factor is partially absorbed in the average speed calculation.

A third intersection-related parameter is the distance between vehicles stopped at an intersection $(\sigma)$. Experience indicates a wide variation in values for this distance. As a first approximation, an arbitrary value of 1 metre is used for $\sigma$. Information from Albuquerque, New Mexico, traffic engineers and observations of local patterns were the basis for this assumption.

\section{A5.3 Pedestrian Speed}

Pedestrians also travel at varying speeds. Information is readily available (see Reference 2) for degrees of pedestrian congestion. These, coupled with urban pedestrian patterns and times of day, yield the values in Table A-12.

\section{A5.4 People per Vehicle}

Since urban traffic consists of several vehicle types, a value for people per vehicle, averaged over all vehicle types, has been calculated. The calculation uses data from Reference 2 which indicate the relationship between total person-miles of travel by autos (including trucks and taxis) and total person-miles of travel for buses on an hourly basis for midtown Manhattan. Person-miles of travel are converted to vehicle-miles of travel by dividing by the assumed vehicle occupancies listed in Table A-13.

Ratios of autos to buses for each time interval are then calculated by dividing vehicle-miles for autos by the vehicle-miles for buses. (This assumes that vehicle-miles for buses and autos are equivalent.) Values thus obtained are given in Table A-14. 
Table A-12

Pedestrian Speeds

\begin{tabular}{cc} 
Time Span & Pedestrian Speed (m/s) \\
\cline { 2 - 2 } 1 & 1.4 \\
2 & 1.3 \\
3 & 1.3 \\
4 & 1.1 \\
5 & 1.3 \\
6 & 1.3
\end{tabular}

Table A-13

Vehicle Occupancy ${ }^{2}$

Vehicle Type

Auto

Bus

Rush hour

off peak hours

Night time
Number of Occupants

1.5

77

43

24

Table A-14

Ratio of Autos to Buses

\begin{tabular}{cc} 
Time Span & Cars/Bus \\
\hline 1 & 50 \\
2 & 69 \\
3 & 42 \\
4 & 89 \\
5 & 44 \\
6 & 61
\end{tabular}


From these ratios, it is possible to calculate average number of people per vehicle by summing the percentage of each vehicle type times its occupancy. (It is not necessary to discriminate between trucks and cars at this point since the occupancies are assumed to be the same for both.) Results are given in Table A-15.

Table A-15

Average Number of People per Vehicle (PPV)

\begin{tabular}{|c|c|c|c|c|c|}
\hline Time Span & Cars (\%) & $\begin{array}{c}\text { Car } \\
\text { Occupancy } \\
\end{array}$ & Buses (\%) & $\begin{array}{c}\text { Bus } \\
\text { Occupancy } \\
\end{array}$ & PPV \\
\hline 1 & 98 & 1.5 & 2.0 & 24 & 2.0 \\
\hline 2 & 98.6 & 1.5 & 1.4 & 77 & 2.6 \\
\hline 3 & 97.7 & 1.5 & 2.3 & 43 & 2.5 \\
\hline 4 & 98.9 & 1.5 & 1.1 & 77 & 2.3 \\
\hline 5 & 97.8 & 1.5 & 2.2 & 43 & 2.4 \\
\hline 6 & 98.4 & 1.5 & 1.6 & 77 & 2.7 \\
\hline
\end{tabular}

\section{A5.5 Vehicle Length}

Standard design vehicle lengths are available in Reference 2 . Of interest are those for trucks ( 9.1 metres), autos ( 5.8 metres), and buses (12.2 metres).

A5.6 Vehicle Distribution

The distributions of vehicles calculated for each time span are summarized in Table A-16.

The average vehicle length for each time span is obtained by multiplying each percentage by the appropriate length, then summing this value. The variation in vehicle length from one time span to another is slight. An average value of 6.4 metres is used.

Table A-16

Distribution of Vehicle Trave1

\begin{tabular}{|c|c|c|c|}
\hline Time Span & Autos (\%) & Trucks (\%) & Buses (\%) \\
\hline 1 & 83.0 & 15 & 2.0 \\
\hline 2 & 83.6 & 15 & 1.4 \\
\hline 3 & 82.7 & 15 & 2.3 \\
\hline 4 & 83.9 & 15 & 1.1 \\
\hline 5 & 82.8 & 15 & 2.2 \\
\hline 6 & 83.4 & 15 & 1.6 \\
\hline
\end{tabular}

A-20 


\section{A5.7 Vehicle Separation Distances}

Vehicle separation distances are calculated using the following analytical expression:

$$
\begin{aligned}
& \text { Vehicle separation } \\
& \text { distance }
\end{aligned}=\frac{\frac{A \cdot\left(f_{s t}\right)_{i}}{w_{s t}} \cdot(\# \text { lanes of street })}{N_{i}(t)}-\left(\begin{array}{l}
\text { Average } \\
\text { Vehicle } \\
\text { Length }
\end{array}\right)
$$

where

$$
\begin{aligned}
A & =\text { cell area }\left(\mathrm{m}^{2}\right) \\
(\mathrm{f} \mathrm{st})_{i} & =\text { street area fraction for cell } \mathrm{i} \\
\mathrm{w}_{\mathrm{st}} & =\text { street width (metres) } \\
\# \text { lanes of street } & =\frac{\text { street width }}{\text { lane width }} \\
\mathrm{N}_{\mathrm{i}}(\mathrm{t}) & =\text { vehicle density for cell } i \text { at time } t
\end{aligned}
$$

Average vehicle length $=6.4$ matres

Calculated values are given in Table A-17.

\section{A5.8 Freeway Traffic Parameters}

Freeway traffic parameters include speeds, separation distances, and width.

Freeway Speeds

Information on freeway speeds was obtained from Reference 6 . Values for average speed as a function of time are shown in Table A-18.

Miscellaneous Freeway Parameters

The value assumed for freeway width, including right-of-way, is 71 metres. Values for freeway vehicle separation distance are estimated from a curve fit to data from Reference 6 (Table 4.6) which gives vehicle headways (from which separation distances can be derived) as a function of travel speed. The expression used for calculating the freeway vehicle separation distances is

$$
\text { Separation distance }=0.17 \mathrm{v}_{\mathrm{f}}^{2}-1.7 \mathrm{v}_{\mathrm{f}}+6.4
$$

where

$$
v_{f}=\text { freeway velocity (a function of time of day). }
$$


Table A-17

Vehicle Separation Distances (in Metres)

Veh. Sep., Veh. Sep., Veh. Sep., Veh. Sep., Veh. Sep., Veh. Sep.,

\begin{tabular}{|c|c|c|c|c|c|c|}
\hline Ce11 Number & TS 1 & TS 2 & TS 3 & TS 4 & TS 5 & TS 6 \\
\hline 1 & 132 & 47 & 63 & 57 & 59 & 29 \\
\hline 2 & 500 & 161 & 170 & 154 & 158 & 105 \\
\hline 3 & 183 & 56 & 58 & 53 & 55 & 35 \\
\hline 4 & 0 & 0 & 0 & 0 & 0 & 0 \\
\hline 5 & 485 & 173 & 206 & 192 & 197 & 113 \\
\hline 6 & 487 & 169 & 201 & 186 & 191 & 112 \\
\hline 7 & 413 & 113 & 98 & 91 & 94 & 74 \\
\hline 8 & 136 & 47 & 59 & 54 & 56 & 29 \\
\hline 9 & 363 & 129 & 162 & 150 & 156 & 85 \\
\hline 10 & 416 & 153 & 190 & 177 & 181 & 99 \\
\hline 11 & 134 & 47 & 63 & 57 & 59 & 29 \\
\hline 12 & 511 & 188 & 252 & 236 & 236 & 123 \\
\hline 13 & 166 & 60 & 79 & 72 & 75 & 38 \\
\hline 14 & 971 & 341 & 402 & 379 & 402 & 232 \\
\hline 15 & 472 & 172 & 208 & 192 & 196 & 113 \\
\hline 16 & 483 & 171 & 205 & 188 & 191 & 111 \\
\hline 17 & 528 & 191 & 233 & 214 & 223 & 124 \\
\hline 18 & 137 & 46 & 59 & 55 & 56 & 29 \\
\hline 19 & 326 & 118 & 147 & 135 & 139 & 77 \\
\hline 20 & 313 & 111 & 135 & 125 & 128 & 72 \\
\hline 21 & 133 & 47 & 63 & 58 & 59 & 29 \\
\hline 22 & 507 & 186 & 250 & 234 & 234 & 126 \\
\hline 23 & 167 & 59 & 79 & 73 & 74 & 37 \\
\hline 24 & 809 & 135 & 82 & 75 & 78 & 87 \\
\hline 25 & 475 & 98 & 66 & 61 & 63 & 63 \\
\hline 26 & 992 & 146 & 85 & 79 & 81 & 95 \\
\hline 27 & 622 & 226 & 286 & 261 & 267 & 151 \\
\hline 28 & 284 & 100 & 124 & 114 & 116 & 65 \\
\hline 29 & 312 & 110 & 136 & 124 & 127 & 72 \\
\hline 30 & 377 & 134 & 166 & 154 & 157 & 88 \\
\hline 31 & 224 & 22 & 9.7 & 8.5 & 9.0 & 12.6 \\
\hline
\end{tabular}


Table A-17 (continued)

\begin{tabular}{|c|c|c|c|c|c|c|}
\hline Ce11 Number & $\begin{array}{r}\text { Veh. Sep., } \\
\text { TS } 1 \\
\end{array}$ & $\begin{array}{r}\text { Veh. Sep., } \\
\text { TS } 2 \\
\end{array}$ & $\begin{array}{r}\text { Veh. Sep., } \\
\text { TS } 3 \\
\end{array}$ & $\begin{array}{l}\text { Veh. Sep., } \\
\text { TS } 4 \\
\end{array}$ & $\begin{array}{l}\text { Veh. Sep., } \\
\text { TS } 5 \\
\end{array}$ & $\begin{array}{c}\text { Veh. Sep. } \\
\text { TS } 6 \\
\end{array}$ \\
\hline 32 & 576 & 31 & 12.3 & 10.9 & 11.4 & 18.7 \\
\hline 33 & 203 & 76 & 105 & 96 & 98 & 48 \\
\hline 34 & 1170 & 153 & 85 & 79 & 80 & 99 \\
\hline 35 & 712 & 119 & 74 & 68 & 70 & 77 \\
\hline 36 & 531 & 101 & 66 & 61 & 62 & 66 \\
\hline 37 & 618 & 228 & 282 & 261 & 271 & 150 \\
\hline 38 & 239 & 85 & 108 & 99 & 102 & 55 \\
\hline 39 & 456 & 167 & 208 & 192 & 196 & 111 \\
\hline 40 & 415 & 151 & 179 & 165 & 168 & 98 \\
\hline 41 & 337 & 22 & 11 & 9.8 & 10.0 & 12.7 \\
\hline 42 & 161 & 42 & 36 & 33 & 34 & 26 \\
\hline 43 & 237 & 23 & 10.0 & 8.9 & 9.2 & 13.2 \\
\hline 44 & 1150 & 151 & 85 & 79 & 81 & 99 \\
\hline 45 & 868 & 133 & 78 & 72 & 74 & 86 \\
\hline 46 & 469 & 97 & 66 & 61 & 63 & 63 \\
\hline 47 & 647 & 219 & 245 & 227 & 227 & 142 \\
\hline 48 & 241 & 86 & 108 & 99 & 102 & 56 \\
\hline 49 & 510 & 182 & 217 & 201 & 206 & 119 \\
\hline 50 & 429 & 149 & 175 & 161 & 165 & 97 \\
\hline 51 & 425 & 24 & 11.7 & 10.4 & 10.8 & 14.1 \\
\hline 52 & 557 & 31 & 12.3 & 10.9 & 11.3 & 18.6 \\
\hline 53 & 206 & 22 & 9.5 & 8.3 & 8.7 & 12.2 \\
\hline 54 & 1220 & 192 & 114 & 106 & 108 & 130 \\
\hline 55 & 365 & 72 & 48 & 44 & 45 & 46 \\
\hline 56 & 368 & 80 & 52 & 47 & . 49 & 51 \\
\hline 57 & 526 & 127 & 96 & 89 & 91 & 83 \\
\hline 58 & 331 & 96 & 86 & 79 & 81 & 61 \\
\hline 59 & 425 & 148 & 175 & 161 & 166 & 97 \\
\hline 60 & 479 & 164 & 197 & 179 & 185 & 108 \\
\hline 61 & 540 & 31 & 12.1 & 10.8 & 11.2 & 18.3 \\
\hline 62 & 274 & 24 & 10.3 & 9.1 & 9.5 & 14.1 \\
\hline 63 & 144 & 18.1 & 8.6 & 7.5 & 7.8 & 9.9 \\
\hline
\end{tabular}


Table A-17 (continued)

\begin{tabular}{|c|c|c|c|c|c|c|}
\hline Ce11 Number & $\begin{array}{r}\text { Veh. Sep., } \\
\text { TS } 1 \\
\end{array}$ & $\begin{aligned} & \text { Veh. Sep., } \\
& \text { TS } 2 \\
&\end{aligned}$ & $\begin{array}{r}\text { Veh. Sep., } \\
\text { TS } 3 \\
\end{array}$ & $\begin{aligned} & \text { Veh. Sep., } \\
& \text { TS } 4 \\
&\end{aligned}$ & $\begin{array}{r}\text { Veh. Sep., } \\
\text { TS } 5 \\
\end{array}$ & $\begin{aligned} & \text { Veh. Sep., } \text { TS } 6 \\
&\end{aligned}$ \\
\hline 64 & 275 & 86 & 86 & 80 & 82 & 55 \\
\hline 65 & 370 & 68 & 44 & 40 & 41 & 43 \\
\hline 66 & 364 & 98 & 83 & 76 & 78 & 63 \\
\hline 67 & 367 & 71 & 47 & 43 & 44 & 45 \\
\hline 68 & 242 & 79 & 85 & 78 & 80 & 50 \\
\hline 69 & 212 & 70 & 79 & 73 & 75 & 45 \\
\hline 70 & 1080 & 380 & 435 & 405 & 435 & 251 \\
\hline 71 & 1140 & 344 & 323 & 299 & 305 & 233 \\
\hline 72 & 261 & 24 & 10.2 & 8.9 & 9.3 & 13.8 \\
\hline 73 & 169 & 35 & 24 & 22 & 22 & 21 \\
\hline 74 & 227 & 75 & 82 & 75 & 77 & 48 \\
\hline 75 & 467 & 112 & 88 & 81 & 83 & 73 \\
\hline 76 & 352 & 93 & 80 & 73 & 75 & 60 \\
\hline 77 & 257 & 62 & 48 & 44 & 46 & 39 \\
\hline 78 & 218 & 67 & 68 & 62 & 64 & 42 \\
\hline 79 & 240 & 77 & 81 & 75 & 77 & 49 \\
\hline 80 & 534 & 174 & 181 & 167 & 174 & 113 \\
\hline 81 & 0 & 0 & 0 & 0 & 0 & 0 \\
\hline 82 & 3340 & 39 & 14 & 12.5 & 13 & 24 \\
\hline 83 & 333 & 56 & 34 & 31 & 32 & 35 \\
\hline 84 & 406 & 119 & 112 & 102 & 105 & 76 \\
\hline 85 & 1050 & 123 & 65 & 61 & 63 & 79 \\
\hline 86 & 267 & 68 & 55 & 51 & 52 & 43 \\
\hline 87 & 241 & 67 & 60 & 55 & 57 & 43 \\
\hline 88 & 241 & 77 & 82 & 75 & 77 & 49 \\
\hline 89 & 237 & 76 & 81 & 75 & 76 & 49 \\
\hline 90 & 244 & 77 & 82 & 75 & 78 & 49 \\
\hline 91 & 0 & 0 & 0 & 0 & 0 & 0 \\
\hline 92 & 4200 & 82 & 34 & 31 & 32 & 52 \\
\hline 93 & 5730 & 83 & 34 & 31 & 32 & 54 \\
\hline 94 & 357 & 97 & 82 & 76 & 78 & 62 \\
\hline
\end{tabular}


Table A-17 (continued)

\begin{tabular}{|c|c|c|c|c|c|c|}
\hline Ce11 Number & $\begin{aligned} \text { Veh. Sep., } \\
\text { TS } 1 \\
\end{aligned}$ & $\begin{aligned} \text { Veh. Sep., } \\
\text { TS } 2 \\
\end{aligned}$ & $\begin{aligned} & \text { Veh. Sep., } \text { SS } 3 \\
&\end{aligned}$ & $\begin{aligned} & \text { Veh. Sep., } \\
& \text { TS } 4 \\
&\end{aligned}$ & $\begin{array}{r}\text { Veh. Sep., } \\
\text { TS } 5 \\
\end{array}$ & $\begin{aligned} \text { Veh. Sep. } \\
\text { TS } 6 \\
\end{aligned}$ \\
\hline 95 & 360 & 30 & 13 & 11.5 & 12 & 18 \\
\hline 96 & 354 & 102 & 92 & 84 & 87 & 66 \\
\hline 97 & 239 & 77 & 82 & 75 & 77 & 49 \\
\hline 98 & 241 & 77 & 81 & 75 & 77 & 49 \\
\hline 99 & 237 & 77 & 82 & 75 & 78 & 49 \\
\hline 100 & 241 & 77 & 74 & 68 & 70 & 49 \\
\hline
\end{tabular}

Table A-18

Freeway Speeds

Time Span

$V_{f}$ (freeway speed)

1

24.4

2

8.9*

3

9.7

4

9.7

5

9.7

6

$8.9 *$

*Lower values can occur during periods of extremely heavy traffic.

\section{A5.9 Rai1 Parameters}

Information on all rail parameters was obtained through private communications with people in the rail transit industry in the New York City area.

\section{Population Densities in Terminal Areas}

Information on mass transit characteristics indicates that approximately 70000 persons use the maijor urban räil facilities in New York during rush hours and that, in addition, there are approximately 2000 off-peak users of the facility. Grand Central Station, the major rail transit facility in the study area, occupies about $0.0255 \mathrm{~km}^{2}$ (measured from maps in Reference 3). Values for people per square kilometre for each time span are obtained by apportioning the off-peak travelers as indicated in Table A-19. 
Table A-19

Population Densities for Rail Transit Facilities

\begin{tabular}{|c|c|c|c|c|}
\hline $\begin{array}{l}\text { Time } \\
\text { Span }\end{array}$ & $\begin{array}{l}\text { Total People } \\
\text { in Terminal Area } \\
\text { during Entire } \\
\text { Time Span } \\
\end{array}$ & $\begin{array}{l}\text { Totals for } \\
\text { Time Span } \\
\left(\text { per } \mathrm{km}^{2} \text { ) }\right.\end{array}$ & Totals $/ \mathrm{km}^{2} / \mathrm{h}$ & $\begin{array}{l}\text { Totals } / \mathrm{km}^{2} \\
\text { at any time } \\
\text { (assuming } \\
10 \text {-min visit) }\end{array}$ \\
\hline 1 & $2 \times 10^{3}$ & $7.8 \times 10^{4}$ & $6 \times 10^{3}$ & $10^{3}$ \\
\hline 2 & $7 \times 10^{4}$ & $2.7 \times 10^{6}$ & $1.8 \times 10^{6}$ & $3.1 \times 10^{5}$ \\
\hline 3 & $5 \times 10^{3}$ & $2 \times 10^{5}$ & $6.5 \times 10^{4}$ & $1.1 \times 10^{4}$ \\
\hline 4 & $8 \times 10^{3}$ & $3.1 \times 10^{5}$ & $2.1 \times 10^{5}$ & $3.5 \times 10^{4}$ \\
\hline 5 & $5 \times 10^{3}$ & $2 \times 10^{5}$ & $5.6 \times 10^{4}$ & $9.3 \times 10^{3}$ \\
\hline 6 & $7 \times 10^{4}$ & $2.7 \times 10^{6}$ & $1.8 \times 10^{6}$ & $3.1 \times 10^{5}$ \\
\hline
\end{tabular}

Miscellaneous Rail Parameters

Other parameter values are

- Length of time a train remains in terminal area $\left(\Delta \mathrm{T}_{\text {depot }}\right)$ by time span:

\begin{tabular}{cc} 
Time Span & In Depot (sec \\
\cline { 2 - 2 } 1 & $7.2 \times 10^{3}$ \\
2 & $6.0 \times 10^{2}$ \\
3 & $7.2 \times 10^{3}$ \\
4 & $1.8 \times 10^{3}$ \\
5 & $7.2 \times 10^{3}$ \\
6 & $6.0 \times 10^{2}$
\end{tabular}

- Minimum exposure radius $r_{3}=2.4$ metres

- Maximum exposure radius $\mathrm{r}_{4}=6.1$ metres

- Distance between passing trains $r_{5}=6.1$ metres

- Width of right-of-way (outside terminal area) $\mathrm{RW}=3$ metres

- Average train speed (within terminal area) $\mathrm{v}_{r}=6.7 \mathrm{~m} / \mathrm{s}$

- Train traffic count $\left(\mathrm{N}_{\mathrm{T}}\right.$, one way, per second):

$\begin{array}{cr}\text { Time Span } & \frac{\text { Trains } / \mathrm{s}}{1.25 \times 10^{-3}} \\ 2 & 1.5 \times 10^{-2} \\ 3 & 1.25 \times 10^{-3} \\ 4 & 5 \times 10^{-3} \\ 5 & 1.25 \times 10^{-3} \\ 6 & 1.5 \times 10^{-2}\end{array}$


- People per train (PPT): Values are obtained assuming occupancies of $10 \%$ of seating capacity at night, $50 \%$ at of f-peak hours, and $100 \%$ at peak times. An average train has eight cars with a seating capacity per car of 120 .

\begin{tabular}{cr} 
Time Span & $\frac{\text { PPT }}{96}$ \\
\hline 1 & 960 \\
3 & 480 \\
4 & 480 \\
5 & 480 \\
6 & 960
\end{tabular}

\section{A5.10 Air Transport Parameters}

Information on air transport parameters has been obtained through private communication with air transit experts in the New York City area. All of these values are dependent on the type and location of the airport under consideration.

La Guardia Airport is the only airport facility in the study area. No cargo terminal exists at this facility, so values for $\Delta \mathrm{T}_{\text {cterm }}$ and $\mathrm{PD}$ cterm are set to zero.

other information which has proven useful in its original form includes

- Average time a passenger aircraft remains in the terminal area: $\Delta \mathrm{T}_{\text {pterm }}=$ 3300 seconds

- Minimum exposure radius $r_{6}=120$ metres (distance of closest approach for occupants of the terminal before boarding)

- Maximum exposure radius $r_{7}=240$ metres (approximate maximum distance from aircraft for terminal occupants before boarding)

Air Terminal Population Densities

Air terminal population density information, available on an hourly basis, has been converted to the time-span basis discussed earlier (see Table A-20). These data consider the population to be only in the passenger waiting areas of the terminal building and not in the areas restricted to employees. It has been determined that the area of interest is $0.043 \mathrm{~km}^{2}$ (see Reference 3 ). An approximate length of stay of 45 minutes is assumed for persons in the air terminal. Since the annulus of integration for air terminals is $0.14 \mathrm{~km}^{2}$, the calculated values for population density are reduced by a factor of $0.043 / 0.14$ to spread the population over the entire area of integration. 
Table A-20

Air Terminal Population Densities

\begin{tabular}{|c|c|c|c|}
\hline Time Span & Persons/h & Persons $/ \mathrm{h} / \mathrm{km}^{2}$ & Persons $/ \mathrm{km}^{2}$ \\
\hline 1 & $1.3 \times 10^{3}$ & $2.9 \times 10^{4}$ & $6.8 \times 10^{3}$ \\
\hline 2 & $1.6 \times 10^{3}$ & $3.8 \times 10^{4}$ & $8.7 \times 10^{3}$ \\
\hline 3 & $2.2 \times 10^{3}$ & $5.2 \times 10^{4}$ & $1.2 \times 10^{4}$ \\
\hline 4 & $2.5 \times 10^{3}$ & $5.9 \times 10^{4}$ & $1.4 \times 10^{4}$ \\
\hline 5 & $3.2 \times 10^{3}$ & $7.4 \times 10^{4}$ & $1.7 \times 10^{4}$ \\
\hline 6 & $3.6 \times 10^{3}$ & $8.5 \times 10^{4}$ & $1.9 \times 10^{4}$ \\
\hline
\end{tabular}

It must be stressed that these values depend upon the particular airport being considered.

\section{A5.11 Water Transport Parameters}

The last mode to be considered is water transport. Information on shipping data has been obtained from Task Group member William Luch and dock officials in the New York City area. Parameters of interest are

- $\Delta \mathrm{T}_{\text {dock }}$-- time spent at dock (for containerized shipments) 24 to 30 hours, depending upon vessel size

- Minimum and maximum exposure radii (12 and 91 metres, respectively)

- $\mathrm{PD}$ dock -- population densities at the dock $/ \mathrm{km}^{2}$ by time interval (total PD for entire dock area is 7000 persons). If $1 / 10$ of dock personnel are arbitrarily assumed to be within the annulus of integration, this would make the population density equal to $5400 / \mathrm{km}^{2}$. (This calculation parallels that used for air terminals.)

The major facility for cargo shippers in the New York City area is at Port Elizabeth, New Jersey. For this study, $P D_{\text {dock }}$ is assumed to be zero since all water transport is assumed to pass through without stopping.

A6. Shipment Information

The transportation of radioactive materials into, around, and through a major urban area involves such a diversity of materials, package types, quantities, package radiation levels, and transport modes that detailed consideration of every shipments is impractical. Therefore, realistic assessment of the radiological risk associated with transportation requires selection of a smaller number of shipment types for analysis. This representative set of shipments is referred to as the standard shipments model.

The shipment model used in this document is similar to that used in Reference 7 and is based on the same shipper survey. 8 This discussion outlines the basic mechanics used to reduce the overall survey data to a workable set of New York City "standard shipments." 
In the 1975 shippers' survey mentioned above, certain shippers completed detailed questionnaires, while others completed summary questionnaires. The detailed questionnaires requested information based upon actual shipping records, while data requested by the summary questionnaires were based upon shipper estimates. Most major shippers, i.e., those known to ship large numbers of packages annually, and all special nuclear material licensees completed detailed questionnaires, although a few were missed and sent only summary questionnaires. Summary questionnaires sent to a cross section of licensees were intended to represent all licensees for sampling purposes. Thus, the summary questionnaire data base was divided into two separate groups: one for minor shippers and the other for apparent major shippers. Therefore, three data bases exist: one from the detailed questionnaires, one from the summary questionnaires completed by minor shippers, and one from the summary questionnaires completed by apparent major shippers. Each of these data bases was extrapolated differently to account for the entire shipper population. The set of standard shipments upon which this risk assessment is based was determined from these three data bases.*

Each standard shipment is specified by the isotope or material being shipped, the package type, the number of packages shipped per year, the average number of packages per shipment, the average quantity of material per package, the average transport index (TI) per package, the transport modes, and the specific urban route followed.

The standard shipments model uses a subset of the total shipment data base compiled by merging data from Reference 8 with a Geographic Data File. ${ }^{9}$ In addition to information on shipment characteristics, Reference 8 contains information on shippers' Zip Codes and shipment destinations by city and state.

Zip Code data from Reference 8 have been checked against a file of the latitude and longitude of Zip Codes found in Reference 9. Government organizations whose Zip Codes appeared in Reference 8 , but not in the Geographic Data Base, have been assigned the latitude and longitude for downtown Washington, D.C.

A second file has been prepared from Reference 9 with all cities within each state arranged alphabetically. About $1 / 3$ of the city/state pairs listed in Reference 8 are not in the Geographic Data Base. The observed anomalies are of three types:

1. Typographic or transcription errors, e.g.,

BIEM INGHAM, AL ) BIRHAMGTON, AL BIRMHINGHA, AL BIRMINGHAN, AL

all thought to be BIRMINGHAM, AL BIRMINGHAM, AK )

\footnotetext{
*Although there is a question about the validity of some entries in the data obtained in Reference 8 , the survey has been accepted as the best available set of data.
} 
2. An abbreviation, shortened name, or familiar name used in place of a given name, e.g.,

LA, CA: thought to be LOS ANGELES, CA (max. city field is 10 characters) LASL, NM: thought to be LOS ALAMOS, NM

TRAVIS, CA

TRAVIS AF, CA

AFB TRAVER, CA

al1 thought to be TRAVIS AIR FORCE BASE, CA

TRAVIS AFB, CA

3. Unresolved, e.g.,

MIC RIDER, CT

ROSIN, IL

[blank], CA (or most other state codes)

Of approximately $2.6 \times 10^{4}$ city/state pairs in the Reference 8 data, 3600 are unique. After consulting the Geographic Data Base, the Zip Code directory, and a geographic place name directory, fewer than $0.1 \%$ of the names have remained unresolved. Most of the unresolved names represent only one package or shipment per year (a small

fraction of the total number of packages in the data base).

A latitude and longitude have been assigned to each corrected city/state pair in the Geographic Data Base. Knowledge of the latitude and longitude of both the origin and destination permits the choice of a subset of data which fits any one (or all) of the following criteria:

- The shipment origin is in the vicinity of a given latitude and longitude

- The shipment destination is in the vicinity of a given latitude and longitude

- An imaginary line joining the origin and destination passes through the vicinity of a given latitude and longitude

The expression "vicinity of a given latitude and longitude" is used to caution the potential user. The program assumes that the latitude and longitude are at the center of a circle of radius $R$ measured in degrees. It further assumes that the origin and destination are on this planar grid. The distance on the earth's surface represented by one degree of latitude is independent of a given latitude. A longitude degree represents a distance on the earth's surface that varies with latitude. Hence, the "vicinity" described is more elliptical than circular (see Figure A-2).

For the New York City study area, a circle of $7-\mathrm{km}$ radius has been drawn centered on the $100-\mathrm{km}^{2}$ grid. The data base was accessed to give shipping information within this circle. The $7-\mathrm{km}$ radius totally encompasses the study area. Using this approach, only one shipment had either an origin or destination within the grid. This appeared to be simply an artifact of the data base since Zip Codes for particular boroughs in New York were not common in shipment information--usually only New York City was given as the destination, hence all shipments were given citycenter destination or origin Zip Codes. To more fully examine the New York City shipments, the center of the $7-\mathrm{km}$ radius circle was placed at the geographical center of New York City. This approach provides much information on shipments with origins and destinations within the city. (Although the circle surrounding the geographic city center does not intersect the grid area, the types of shipments reaching or originating within this circle are arbitrarily assumed to be typical 
for the study area.) In preparing routing information, typical routes have been designated for each kind of isotope appearing in the output for the city center circle. No attempt has been made to determine the fraction of the total observed shipments which specifically applies to the study area. Shipments contained in the grid-centered circle are also included and routed as through shipments. Although this approach may double-count some shipments, the fraction so counted is considered small.

\section{A6.1 Development of a Standard Shipments Mode1}

Once the shipment data base had been reduced so that only New York City shipments were included, it was necessary to reduce the remaining $3 \times 10^{5}$ shipments to a workable set of "standard shipments" as discussed in Appendix A of Reference 7. This reduction process involved three steps: elimination of shipments, combination of remaining shipments, and calculation of shipment parameters.

\section{A6.2 Elimination Phase}

Six categories of shipments have been eliminated from the NYC shipment data base: limited or exempt shipments, other extremely small shipments, mail shipments, shipments were no mode was specified, government shipments, and miscellaneous shipments.

Limited shipments have been shown to contribute a negligible amount to the overall radiological impact under both incident and incident-free circumstances, even when large numbers of packages are shipped (see Reference 7). There are very few limited quantity shipments listed in the NYC data base $(2.21 \%$ of the total shipments, $0.0061 \%$ of total curies, and $7 \times 10^{-4 \%}$ of total TI).

Because only small quantities of radioactive material can be mailed, these shipments are treated by 49CFR173.391 10 in the same manner as limited shipments.* By excluding these shipments, $0.71 \%$ of the total shipments, $2.7 \times 10^{-4 \%}$ of total activity, and $0.014 \%$ of the total TI are eliminated.

Certain shipments are smail enough to be considered negligible from both incident and incident-free points of view, even though they are not shipped under the 1 imited quantity regulations. Using a criterion of $10^{-4}$ curie per package for dispersible materials and $10^{-3}$ curie per package for nondispersible materials, an additional $3 \%$ of the total shipments, $2.4 \%$ of the total curies, and $1.5 \%$ of the total TI are eliminated.

Shipments for which no mode has been specified account for only $0.57 \%$ of the total shipments, $0.08 \%$ of the total curies, and $0.41 \%$ of the total $T I$. Rather than assign these to an arbitrary mode, they are excluded from the standard shipments model.

Government shipments are outside the scope of this study_and are also excluded. These account for $0.0068 \%$ of the total shipments, $1.0 \times 10^{-3 \%}$ of total curies, and $0.0014 \%$ of total TI.

\footnotetext{
* See U.S. Postal Service Publication 6, April 1971.
} 


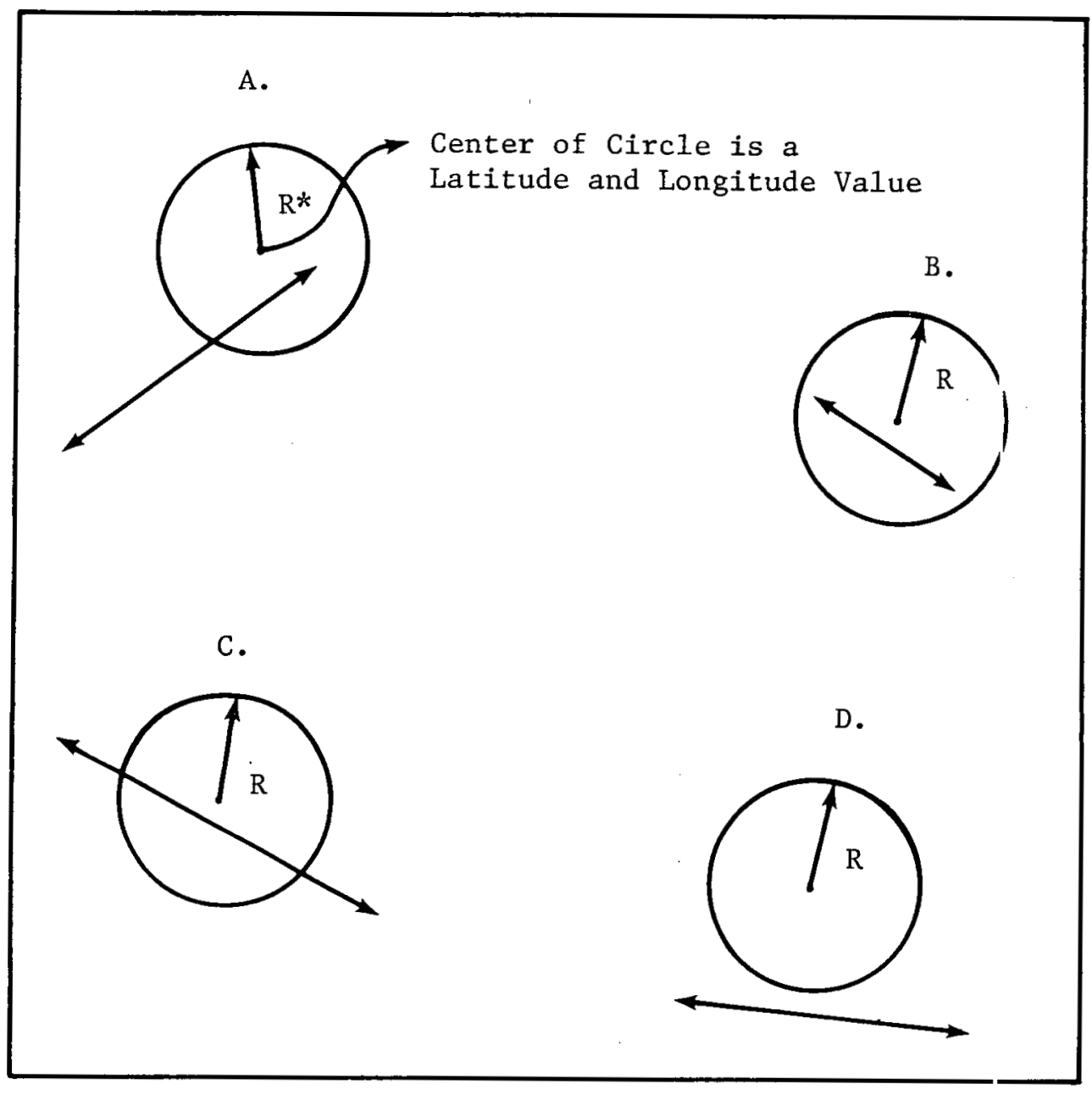

Types of information which can be acquired:

A. Either origin or destination is within circle,

B. Both origin and destination are within circle,

C. Neither origin nor destination is within circle but path between passes through circle, or

D. Neither origin nor destination is within circle and path between excludes circle.

$*_{R}$ is the radius of the circle within which shipment information is requested.

Figure A-2. Differing Criteria for Shipment Routes into, out of, through, and in the Vicinity of a Given Latitude and Longitude 
In summary, $1.9 \times 10^{4}$ shipments ( $6.4 \%$ of the tota 1$)$ are eliminated from the reduced New York City shipment data base. This amounts to $2.4 \%$ of the total activity and $2 \%$ of the total TI. Shipments have been subdivided by end use, and typical routes have been established for each end-use category. Values for curies per package, packages per year, and TI per package have been obtained by averaging overall shipments in each end-use category by each mode. Methods for specifying a given route are given in Section A6.3.

\section{A6.3 Route Specification}

Information on shipping routes in the New York City area was made available through Dr. Calvin Brantley of New England Nuclear Corporation. A secondary carrier used by New England Nuclear also supplied transport information from the major airport facilities into New York (specifically to Sloane-Kettering Cancer Research Center) and to Long Island (used as an out-of-grid area destination for some through shipments).

Possible direction of travel within a cell is restricted to eight vector directions with either origin or destination at the center of a cell, as shown in Figure A-3. This restriction forces some approximation of the actual route followed.

Transport modes include truck, rail, passenger and cargo aircraft, and watercraft. Roadway types are 1) one-way streets, 2) two-way streets, 3) freeway, and 4) nonroad (used for all transport except truck or van). A typical route description is given in Table A-2l.

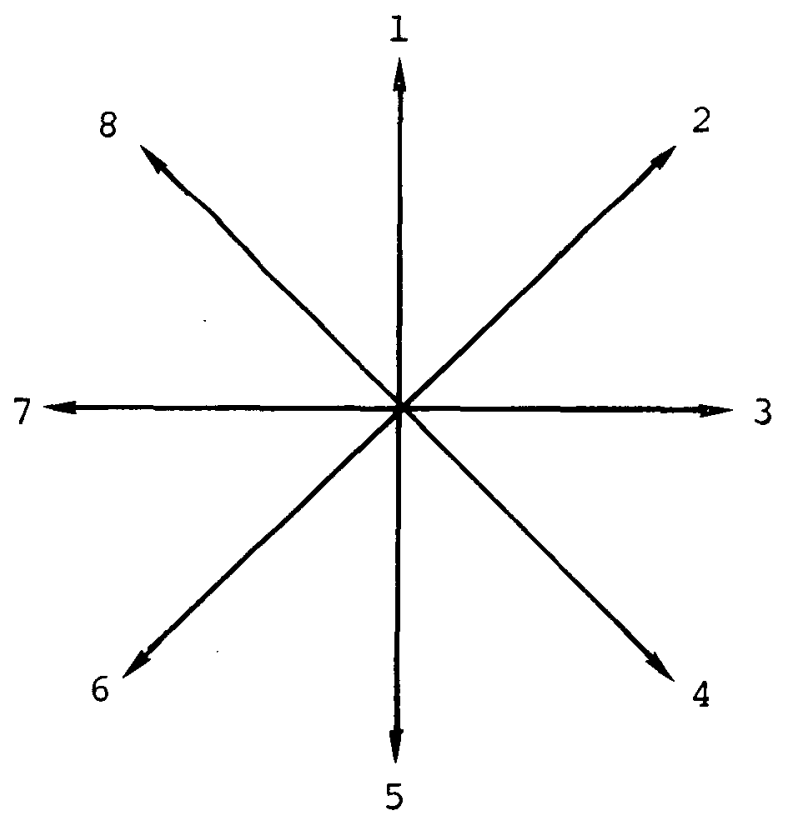

Figure A-3. Vector Directions of Travel within a Cell 
Table A-21

Typical Description of Route

\begin{tabular}{|c|c|c|c|c|}
\hline $\begin{array}{c}\text { Ce } 11 \\
\text { Sequence } \\
\end{array}$ & $\begin{array}{c}\text { In } \\
\text { Direction }\end{array}$ & $\begin{array}{c}\text { Out } \\
\text { Direction }\end{array}$ & $\begin{array}{c}\text { Transport } \\
\text { Mode } \\
\end{array}$ & $\begin{array}{l}\text { Road } \\
\text { Type } \\
\end{array}$ \\
\hline 3 & 5 & 5 & 1 & 2 \\
\hline 13 & 5 & 5 & 1 & 2 \\
\hline 23 & 5 & 0 & 1 & 2 \\
\hline
\end{tabular}

Although noise abatement ordinances restrict aircraft overflights, some possible routes to each major airport facility are included. Through shipment routes on a single transport vehicle, secondary mode transport from an air facility back into the study area, and transfer from one vehicle or mode to another are allowed to give the model increased flexibility. Quite frequently, shipments are stored for a time before secondary mode transport is begun. This time delay, called storage time, is route dependent. Different types of shipments are assumed to reach the grid at various start times, resulting in time dependencies within the standard shipments mode1.

All shipments and routes are described in Tables A-22 through A-38 and may be traced using Figure A-1. 
Table A-22

Route No. 1; End Use: Medical

$\begin{array}{ccccc}\text { CELL } & \text { IN } & \text { OUT } & \text { TRANSPORT } & \text { ROAD } \\ \text { SE OUENCE } & \text { DIRECTION } & \text { OIRECTION } & \text { MODE } & \text { TYPE } \\ 61 & 3 & 3 & 4 & 0 \\ 62 & 3 & 3 & 4 & 0 \\ 63 & 3 & 3 & 4 & 0 \\ 64 & 3 & 3 & 4 & 0 \\ 65 & 3 & 3 & 4 & 0 \\ 66 & 3 & 3 & 4 & 0 \\ 67 & 3 & 3 & 4 & 0 \\ 68 & 3 & 3 & 4 & 0 \\ 69 & 3 & 3 & 4 & 0 \\ 76 & 3 & 3 & 1 & 0 \\ 20 & 7 & 5 & 1 & 3 \\ 30 & 5 & 6 & 1 & 3 \\ 39 & 6 & 6 & 1 & 3 \\ 48 & 6 & 7 & 1 & 3 \\ 47 & 7 & 7 & 1 & 2 \\ 46 & 7 & 8 & 1 & 2 \\ 35 & 8 & 7 & 1 & 3 \\ 34 & 7 & 7 & 1 & 2 \\ 33 & 7 & 1 & 1 & 2 \\ 23 & 1 & 0 & & \end{array}$

\begin{tabular}{|c|c|c|c|c|c|c|c|}
\hline I SOTOPE & $\begin{array}{c}\text { PACKAGE } \\
\text { TYPE }\end{array}$ & $\begin{array}{l}\text { CURIES PER } \\
\text { PACKAGE }\end{array}$ & $\begin{array}{l}\text { TRANSPOR I } \\
\text { INDEX/PKG }\end{array}$ & $\begin{array}{c}\text { PACKAGES PER } \\
\text { SHIPMENT }\end{array}$ & $\begin{array}{l}\text { SHIPMENTS } \\
\text { PER YEAR }\end{array}$ & $\begin{array}{l}\text { HANDLINGS } \\
\text { PER SHIPHENT }\end{array}$ & $\begin{array}{l}\text { STORAGE TIME } \\
\text { (SECONDS) }\end{array}$ \\
\hline $\begin{array}{c}A U-198 \\
C O-60 \\
C R-51 \\
C-14 \\
H G-197 \\
H G-203 \\
I-125 \\
I-131 \\
H O-99 \\
N A-24 \\
P-32 \\
X E-133\end{array}$ & $\begin{array}{r}\text { A } \\
A \\
A \\
A \\
A \\
\text { D } \\
\text { DRU } \\
\text { A } \\
\text { A } \\
\text { A } \\
\text { A } \\
\text { A } \\
\text { A }\end{array}$ & $\begin{array}{l}1.30 E+01 \\
1.80 E+01 \\
5.40 E-03 \\
4 \cdot 30 E-01 \\
6.50 E-02 \\
2.00 E-03 \\
2.70 E-01 \\
1.40 E-02 \\
1.20 E+00 \\
6.00 E-03 \\
2.80 E-02 \\
2.80 E+01\end{array}$ & $\begin{array}{r}4.8800 \\
.9561 \\
.0006 \\
.5163 \\
.3000 \\
.4000 \\
.0413 \\
.6713 \\
.2100 \\
1.8750 \\
.0750 \\
.6331\end{array}$ & $\begin{array}{l}1 . \\
1 . \\
1 . \\
1 . \\
1 . \\
1 . \\
1 . \\
1 . \\
1 . \\
1 . \\
1 . \\
1 .\end{array}$ & $\begin{array}{r}64.1 \\
11.4 \\
21.7 \\
506.0 \\
52.0 \\
2.0 \\
486.0 \\
529.0 \\
1140.0 \\
48.4 \\
34.7 \\
168.0\end{array}$ & $\begin{array}{l}1 . \\
1 . \\
1 . \\
1 . \\
1 . \\
1 . \\
1 . \\
1 . \\
1 . \\
1 . \\
1 . \\
1 .\end{array}$ & $\begin{array}{l}43200 . \\
43200 . \\
43200 . \\
43200^{\circ} \\
43200^{\circ} \\
43200 . \\
43200^{\circ} \\
43200 . \\
43200 . \\
43200 . \\
43200 . \\
43200^{\circ}\end{array}$ \\
\hline
\end{tabular}




$\begin{array}{ccccc}\text { CELL } & \text { IN } & \text { OUT } & \text { TRANSPORT } & \text { ROAD } \\ \text { SEQUENCE } & \text { DIRECTION } & \text { DIRECTICN } & \text { MODE } & \text { TYPE } \\ 3 & 5 & 5 & 1 & 2 \\ 13 & 5 & 5 & 1 & 2 \\ 23 & 5 & 0 & 1 & 2\end{array}$

I SOTOPE
AU-198
$C O-57$
$C O-6 C$
$C P-51$
$C-14$
$F E-59$
$G A-67$
$H G-197$
$I N-111$
IN-114M
$I-123$
$I-125$
$I-131$
$I-131$
$M O-97$
$P-32$
$I C-99 M$
$X E-133$

\begin{tabular}{|c|c|}
\hline $\begin{array}{l}\text { PACKAGE } \\
\text { TYPE }\end{array}$ & $\begin{array}{l}\text { CURIES PE } \\
\text { PACKAGE }\end{array}$ \\
\hline A & $1.60 E-02$ \\
\hline A & $2.60 E-05$ \\
\hline$B$ & $4.70 E+C .3$ \\
\hline A & $1.30 E-03$ \\
\hline A & $7.50 E-05$ \\
\hline A & $5.00 E-04$ \\
\hline A & $1.40 E-02$ \\
\hline A & $2.30 E-01$ \\
\hline A & $7.30 E-03$ \\
\hline A & $3.0 \cup E-03$ \\
\hline A & $2.62 E-03$ \\
\hline A & $4.40 E-03$ \\
\hline A & $8.96 E-03$ \\
\hline A & $2.60 E-03$ \\
\hline A & $1.00 E+00$ \\
\hline A & $4.3 \cup E-03$ \\
\hline A & $9.70 E-i 2$ \\
\hline A & $1.40 E-01$ \\
\hline
\end{tabular}

TFANSPORT
INDEX/PKG

PACKAGES PER
SHIPMENT

SHIPMENTS

.8500

.2333

1.3000

.2400

0.0000

.6000

.0500

.2500

0.0000

.4000

.0600

.0632

.5875

.7804

2.4000

.2286

.3146

.0625

$\begin{array}{rr}1 . & 104.0 \\ 1 . & 104.0 \\ 1 . & 6.0 \\ 1 . & 260.0 \\ 1 . & 104.0 \\ 1 . & 52.0 \\ 1 . & 104.0 \\ 1 . & 154.0 \\ 1 . & 121.0 \\ 1 . & 260.0 \\ 1 . & 249.0 \\ 1 . & 610.0 \\ 1 . & 62.0 \\ 1 . & 22.1 \\ 1 . & 5360.0 \\ 1 . & 116.0 \\ 1 . & \end{array}$

HANOLINGS PER SHIPMENT PER YEAR

1.
1.
1.
1.
1.
1.
1.
1.
1.
1.
1.
1.
1.
1.
1.
1.
1.
1.

STORAGE TIME (SECONDS)

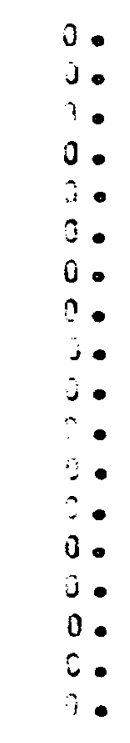


Table A-24

Route No. 3; End Use: Medical

$\begin{array}{ccccc}\text { CELL } & \text { IN } & \text { OUT } & \text { TRANSPORT } & \text { ROAD } \\ \text { SEQUENCE } & \text { DIRECTION } & \text { DIRECTION } & \text { MODE } & \text { TYPE } \\ 61 & 3 & 3 & 4 & 0 \\ 62 & 3 & 3 & 4 & 0 \\ 63 & 3 & 3 & 4 & 0 \\ 54 & 3 & 3 & 4 & 0 \\ 65 & 3 & 3 & 4 & 0 \\ 66 & 3 & 3 & 4 & 0 \\ 67 & 3 & 3 & 4 & 0 \\ 68 & 3 & 3 & 4 & 0 \\ 69 & 3 & 3 & 4 & 0 \\ 70 & 3 & 3 & 4 & 0\end{array}$

$$
\begin{gathered}
\text { ISOTOPE } \\
\text { CO-60 } \\
C-14 \\
C-14 \\
6 A-57 \\
\text { I-125 } \\
M O-99 \\
P-32
\end{gathered}
$$

\section{PACKAGE}

CURIES PER

PACKAGE

\section{TRANSPORT \\ INDEX/PKG}

$$
\begin{aligned}
& 1.50 E+00 \\
& 3 \cdot 70 E-03 \\
& 1.50 E-03 \\
& 1 \cdot 10 E-02 \\
& 5 \cdot 30 E-03 \\
& 5.70 E-01 \\
& 5.30 E-03
\end{aligned}
$$

PACKAGES PER SHIPMEN T

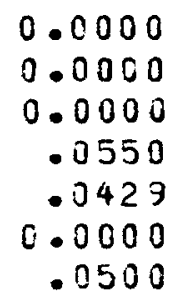

SHIPMENTS PER YEAR

1.
1.
1.
1.
1.
1.

20.8
91.0
8.7
8.7
30.3
21.7
26.0

HANDLINGS PER SHIPMENT
STORAGE TIME

(SECONDS)

- indicates nondispersible shipments 


$\begin{array}{ccccc}\text { CELL } & \text { IN } & \text { OUT } & \text { TRANSPORT } & \text { ROAD } \\ \text { SEQUENCE } & \text { DIRECTION } & \text { DIRECTION } & \text { MODE } & \text { TYPE } \\ 3 & 5 & 5 & 1 & 2 \\ 13 & 5 & 5 & 1 & 2 \\ 23 & 5 & 5 & 1 & 2 \\ 33 & 3 & 3 & 1 & 3 \\ 34 & 3 & 3 & 1 & 3 \\ 35 & 3 & 4 & 1 & 2 \\ 45 & 4 & 2 & 1 & 2 \\ 47 & 4 & 3 & 1 & 2 \\ 48 & 3 & 2 & 1 & 3 \\ 35 & 2 & 2 & 1 & 3 \\ 30 & 2 & 2 & 1 & 3\end{array}$

I SOTOPE
AU-198
$C O-57$
$C R-51$
$C-14$
$G A-67$
$I-123$
$I-125$
$I-131$
$I-131$
$I N-111$
$M O-99$
$T C-99 M$
$X E-133$

$\begin{array}{cc}\begin{array}{c}\text { PACKAGE } \\ \text { TYPE }\end{array} & \begin{array}{c}\text { CURIES PER } \\ \text { PACKAGE }\end{array} \\ \text { A } & 5.10 E-02 \\ \text { A } & 9.50 E-05 \\ \text { A } & 1.00 E-04 \\ \text { A } & 1.00 E-04 \\ A & 1.20 E-02 \\ \text { A } & 1.00 E-03 \\ A & 2.20 E-05 \\ A & 6.60 E-03 \\ \text { A } & 3.00 E-03 \\ A & 9.00 E-03 \\ \text { A } & 8.50 E-01 \\ \text { A } & 3.70 E-02 \\ \text { A } & 4.00 E-02\end{array}$

TRANSPORT
INDEX/PKE

1.0000
0.0000
.1000
0.0000
.1000
.0500
.0333
.6900
1.1100
0.0000
2.3300
.1972
0.0000

PACKAGES PER SHIPMENTS SHIPMENT

PER YEAR

$$
\begin{array}{r}
104.0 \\
208.0 \\
52.0 \\
52.0 \\
52.0 \\
104.0 \\
156.0 \\
1040.0 \\
160.0 \\
104.0 \\
156.0 \\
1670.0 \\
104.0
\end{array}
$$

STORAGE TIME (SECONDS) 
Table A-26

Route No. 5; End Use: Industrial

$\begin{array}{ccccc}\text { CELL } & \text { IN } & \text { OUT } & \text { TRANSPORT } & \text { ROAD } \\ \text { SEQUENCE } & \text { DIRECTION } & \text { DIRECTION } & \text { MODE } & \text { TYPE } \\ 3 & 5 & 5 & 1 & 2 \\ 13 & 5 & 5 & 1 & 2 \\ 23 & 5 & 5 & 1 & 2 \\ 33 & 5 & 3 & 1 & 2 \\ 34 & 3 & 3 & 1 & 3 \\ 35 & 3 & 5 & 1 & 2 \\ 45 & 5 & 7 & 1 & 2 \\ 44 & 7 & 0 & 1 & 2\end{array}$

I SOTOPE
CO-6I
CR-51
$E U-152$
$F E-55$
$H-3$
$K R-85$
$S E-75$
$X E-133$
$C S-137$

$\begin{array}{cc}\begin{array}{c}\text { PACKAGE } \\ \text { TYPE }\end{array} & \begin{array}{c}\text { CURIES PER } \\ \text { PACKAGE }\end{array} \\ & \\ \text { B } & 4.70 E+03 \\ \text { A } & 1.30 E-03 \\ \text { A } & 2.00 E-03 \\ \text { A } & 1.50 E-01 \\ \text { A } & 9.30 E-03 \\ \text { A } & 5.00 E-03 \\ \text { A } & 5.80 E-04 \\ \text { A } & 1.40 E-01 \\ \text { B } & 2.40 E+03\end{array}$

TRANSPORT

PACKAGES PER

SHIPMENTS

MANDLINGS

STORAGE TIME INDEXIPK SHIPHENT

PER YEAR PER SHIPMENT (SECONDS)

CS -137 .

1.3000
.2400
.8000
0.0 .000
.0370
.1000
.2333
.0625
0.0000

1.
1.
1.
1.
1.
1.
1.
1.
6.0
260.0
12.0
1.0
54.0
2.0
156.0
116.0
2.0

1.
1.
1.
1.
1.
1.
1.
1.

- INOICATES NONDISPERSIELE SHIPMENTS 
Table A-27

Route No. 6; End Use: Industrial

$\begin{array}{ccccc}\text { CELL } & \text { IN } & \text { OUT } & \text { TRANSPORT } & \text { ROAD } \\ \text { SEQUENCE } & \text { DIRECTION } & \text { DIRECTION } & \text { MODE } & \text { TYPE } \\ 61 & 2 & 2 & 3 & 0 \\ 52 & 2 & 2 & 3 & 0 \\ 43 & 2 & 2 & 3 & 0 \\ 34 & 2 & 2 & 3 & 0 \\ 25 & 2 & 2 & 3 & 0 \\ 16 & 2 & 2 & 3 & 0 \\ 7 & 2 & 2 & 3 & 0\end{array}$

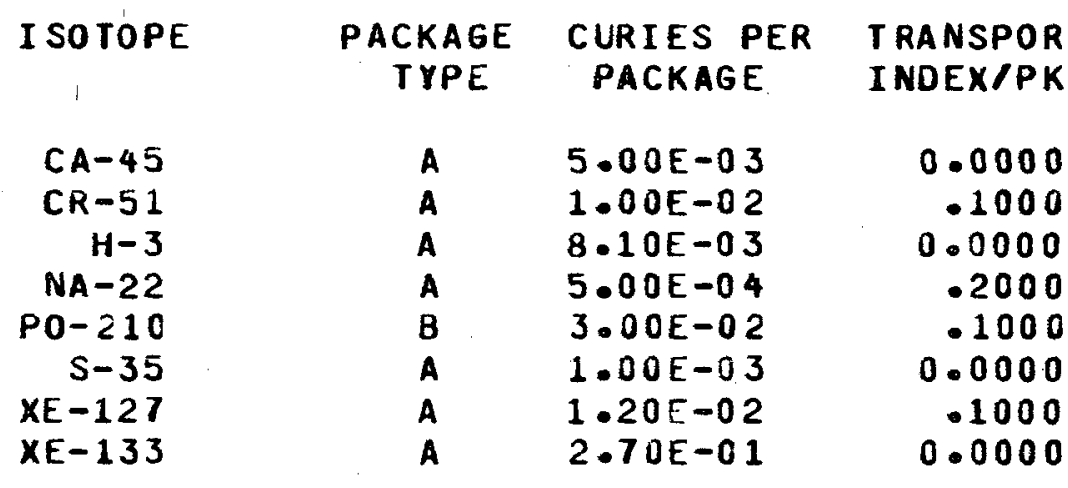

$\begin{array}{cccc}\text { ISOTOPE } & \begin{array}{c}\text { PACKAGE } \\ \text { TYPE }\end{array} & \begin{array}{c}\text { CURIES PER } \\ \text { PACKAGE }\end{array} & \begin{array}{r}\text { TRANSPORT } \\ \text { INDEX/PKG }\end{array} \\ \text { CA-45 } & \text { A } & 5.00 E-03 & 0.0000 \\ \text { CR-51 } & \text { A } & 1.00 E-02 & .1000 \\ H-3 & \text { A } & 8.10 E-03 & 0.0000 \\ N A-22 & \text { A } & 5.00 E-04 & .2000 \\ \text { PO-210 } & \text { B } & 3.00 E-02 & .1000 \\ S-35 & \text { A } & 1.00 E-03 & 0.0000 \\ \text { XE }-127 & \text { A } & 1.20 E-02 & .1000 \\ \text { XE }-133 & \text { A } & 2.70 E-01 & 0.0000\end{array}$

PACKAGES PER

SHIPMENTS

HANDLINGS SHIPMENT PER YEAR PER SHIPMENT

STORAGE TIME (SECONOS)

1.
1.
1.
1.
1.
1.

$4 \cdot 3$
$21 \cdot 7$
$73 \cdot 7$
$4 \cdot 3$
$2 \cdot 0$
$4 \cdot 3$
$2 \cdot 0$
$8 \cdot 7$

0.
0.
0.
0.
0.
0.
0.
0.

0
0
0
0
0
0
0
0
0

- INDICATES NONDISPERSIBLE SHIPMENTS 
Table A-28

Route No. 7; End Use: Industrial

$\begin{array}{ccccc}\begin{array}{c}\text { CELL } \\ \text { SEOUEMCE }\end{array} & \begin{array}{c}\text { IH } \\ \text { OIRECTION }\end{array} & \begin{array}{c}\text { OUT } \\ \text { OIRECTION }\end{array} & \begin{array}{c}\text { TRARSPORT } \\ \text { MODE }\end{array} & \begin{array}{c}\text { ROAD } \\ \text { TYPE }\end{array} \\ 61 & 3 & 3 & 4 & 0 \\ 62 & 3 & 3 & 4 & 0 \\ 63 & 3 & 3 & 4 & 0 \\ 64 & 3 & 3 & 4 & 0 \\ 65 & 3 & 3 & 4 & 0 \\ 66 & 3 & 3 & 4 & 0 \\ 67 & 3 & 3 & 4 & 0 \\ 68 & 3 & 3 & 4 & 0 \\ 69 & 3 & 3 & 4 & 0 \\ 70 & 3 & 3 & 4 & 0 \\ 20 & 7 & 5 & 1 & 3 \\ 30 & 5 & 6 & 1 & 3 \\ 39 & 6 & 6 & 1 & 3 \\ 48 & 6 & 7 & 1 & 3 \\ 47 & 6 & 7 & 1 & 2 \\ 46 & 7 & 8 & 1 & 2 \\ 35 & 7 & 5 & 1 & 2 \\ 45 & 8 & 7 & 1 & 2 \\ 44 & 5 & 0 & 1 & 2\end{array}$

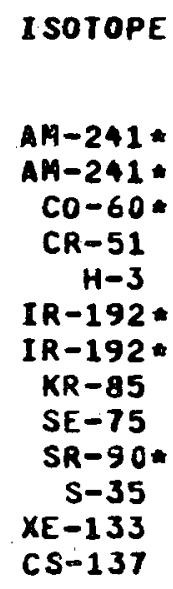

$\begin{array}{cc}\begin{array}{c}\text { PACKAGE } \\ \text { TYPE }\end{array} & \begin{array}{c}\text { CURIES PER } \\ \text { PACKAGE }\end{array} \\ \text { A } & 1.00 E-01 \\ \text { B } & 1.30 E+01 \\ \text { A } & 1.80 E+01 \\ \text { A } & 5.40 E-03 \\ \text { A } & 1.60 E-02 \\ \text { A } & 8.00 E+01 \\ \text { B } & 1.00 E+02 \\ \text { A } & 2.50 E+00 \\ \text { A } & 1.60 E-02 \\ \text { A } & 8.50 E-02 \\ \text { A } & 4.60 E-03 \\ \text { A } & 2.80 E+01 \\ \text { ORUA } & 1.00 E-04\end{array}$

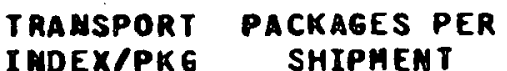

SHIPAEHTS PER YEAR

HANDLINGS PER SHIPMENT

110.0
48.0
11.4
21.7
303.0
52.0
263.0
82.1
52.0
34.0
60.7
168.0
2.0

1.
1.
1.
1.
1.
1.
1.
1.
1.
1.

STORAGE TIME (SECONOS)

$\$ 3200$

43200 .

43200 .

43200.

43200 .

43200 .

43200 .

43200.

43200 .

43200 .

43200 .

43200 .

43200 . 
Route No. 8; End Use: Industrial

$\begin{array}{ccccc}\text { CELL } & \text { IN } & \text { OUT } & \text { TRANSPORT } & \text { ROAD } \\ \text { SEQUENCE } & \text { DIRECIION } & \text { DIRECTION } & \text { MODE } & \text { TYPE } \\ 3 & 5 & 5 & 1 & 2 \\ 13 & 5 & 5 & 1 & 2 \\ 23 & 5 & 5 & 1 & 2 \\ 33 & 5 & 3 & 1 & 3 \\ 34 & 3 & 3 & 1 & 3 \\ 35 & 3 & 4 & 1 & 2 \\ 45 & 4 & 2 & 1 & 2 \\ 47 & 4 & 3 & 1 & 2 \\ 48 & 3 & 2 & 1 & 3 \\ 35 & 2 & 2 & 1 & 3 \\ 30 & 2 & 2 & 1 & 3\end{array}$

\begin{tabular}{|c|c|c|c|c|c|c|c|}
\hline I SOTOPE & $\begin{array}{l}\text { PACKAGE } \\
\text { TYPE }\end{array}$ & $\begin{array}{l}\text { CURIES PER } \\
\text { PACKAGE }\end{array}$ & $\begin{array}{l}\text { TRANSPORT } \\
\text { I NDE X/PKG }\end{array}$ & $\begin{array}{l}\text { PACKAGES PER } \\
\text { SHIPMENT }\end{array}$ & $\begin{array}{l}\text { SHIPMENTS } \\
\text { PER YEAR }\end{array}$ & $\begin{array}{l}\text { HANDLINGS } \\
\text { PER SHIPMENT }\end{array}$ & $\begin{array}{l}\text { STORAGE TIME } \\
\text { (SECONDS) }\end{array}$ \\
\hline $\begin{array}{r}C R-51 \\
H-3 \\
X E-133\end{array}$ & $\begin{array}{l}A \\
A \\
A\end{array}$ & $\begin{array}{l}1.00 E-04 \\
5.00 E-03 \\
4.00 E-02\end{array}$ & $\begin{array}{r}.1000 \\
0.0000 \\
0.0000\end{array}$ & $\begin{array}{l}1 . \\
1 \\
1\end{array}$ & $\begin{array}{r}52.0 \\
52.0 \\
104.0\end{array}$ & $\begin{array}{l}0 . \\
0 . \\
0 .\end{array}$ & $\begin{array}{l}0 . \\
0 . \\
0 .\end{array}$ \\
\hline
\end{tabular}

* INoicates nonoispersible shipments 
Table A-30

Route No. 9; End Use: Fuel Cycle

$\begin{array}{ccccc}\text { CELL } & \text { IN } & \text { OUT } & \text { TRANSPORT } & \text { ROAD } \\ \text { SEQUENCE } & \text { DIRECTION } & \text { DIRECTION } & \text { MODE } & \text { TYPE } \\ 91 & 8 & 1 & 5 & 0 \\ 81 & 1 & 1 & 5 & 0 \\ 71 & 1 & 8 & 5 & 0\end{array}$

I SO TOPE

ENRICHED U

NATURAL U

\section{PACKAGE TYPE}

\section{CURIES PER}

PACKAGE

TRANSPORT

I NDEX/PKG

$2.00 E-03$

$8.50 E-03$

.2499

.2000
PACKAGES PER SHIPMENTS SHIPMENT

PER YEAR

HANDLINGS PER SHIPMENT

2270.0

36.3
1.
0 .

0.
STORAGE TIME (SECONDS)
0 .

0 .

- INDICATES NONDISPERSIBLE SHIPMENTS 


\begin{tabular}{|c|c|c|c|c|}
\hline $\begin{array}{l}\text { CELL } \\
\text { SEQUENCE }\end{array}$ & $\begin{array}{c}\text { IN } \\
\text { DIRECII ON }\end{array}$ & $\begin{array}{c}\text { OUT } \\
\text { DIRECTION }\end{array}$ & $\begin{array}{l}\text { TRANSPORT } \\
\text { MODE }\end{array}$ & $\begin{array}{l}\text { ROAD } \\
\text { TYPE }\end{array}$ \\
\hline 61 & 2 & 2 & 3 & 0 \\
\hline 52 & 2 & 2 & 3 & 0 \\
\hline 43 & 2 & 2 & 3 & 0 \\
\hline 34 & 2 & 2 & 3 & 0 \\
\hline 25 & 2 & 2 & 3 & 0 \\
\hline 16 & 2 & 2 & 3 & 0 \\
\hline 7 & 2 & 2 & 3 & 0 \\
\hline 7 & 5 & 3 & 1 & 3 \\
\hline 8 & 3 & 2 & 1 & 3 \\
\hline 9 & 4 & 6 & 1 & 2 \\
\hline 18 & 6 & 6 & 1 & 2 \\
\hline 27 & 6 & 6 & 1 & 2 \\
\hline 36 & 6 & 7 & 1 & 3 \\
\hline 35 & 7 & 5 & 1 & 2 \\
\hline 45 & 5 & 7 & 1 & 2 \\
\hline 44 & 7 & 0 & 1 & 2 \\
\hline
\end{tabular}

I SOTOPE
AH -241 *
CF-252*
$C R-51$
FE -55
$H-3$
$K R-85$
$R A-226$
$S E-75$
SN-113*
$S R-89$
$X E-133$

$\begin{array}{cr}\begin{array}{c}\text { PACKAGE } \\ \text { TYPE }\end{array} & \begin{array}{r}\text { CURIES PER } \\ \text { PACKAGE }\end{array} \\ \text { A } & 9.30 E-01 \\ \text { A } & 2.70 E-02 \\ \text { A } & 2.20 E-01 \\ \text { A } & 2.00 E-03 \\ \text { A } & 1.10 E-02 \\ \text { A } & 5.00 E-01 \\ \text { A } & 2.00 E-04 \\ \text { A } & 2.50 E-04 \\ \text { A } & 2.20 E-02 \\ \text { A } & 4.50 E-02 \\ \text { A } & 1.60 E+00\end{array}$

\section{TRANSPORT} I NDEX/PKG

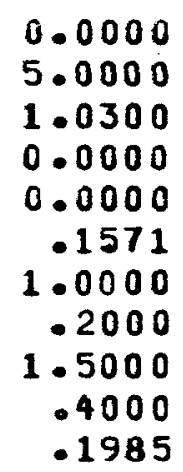

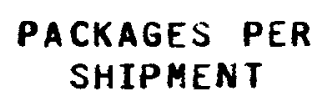

$$
\begin{aligned}
& 1 . \\
& 1 . \\
& 1 . \\
& 1 . \\
& 1 . \\
& 1 . \\
& 1 . \\
& 1 . \\
& 1 . \\
& 1 .
\end{aligned}
$$

SHIPMENTS PER YEAR

30.1
2.0
36.3
4.3
232.0
14.0
12.1
52.0
48.4
12.1
241.0

HANDLINGS PER SHIPMENT

1.
1.
1.
1.
1.
1.
1.
1.

STORAGE TIME (SECONDS)

43200 .

43200 。

43200 .

43200 .

43200 .

43200 .

43200 .

43200 .

43200 .

43200 .

43200 .

* INDICATES NONDISPERSIBLE SHIPMENTS 
Table A-32

Route No. 11; End Use: Waste

$\begin{array}{ccccc}\text { CELL } & \text { IN } & \text { OUT } & \text { TRANSPORT } & \text { ROAD } \\ \text { SEQUENCE } & \text { DIRECTION } & \text { DIRECTION } & \text { MODE } & \text { TYPE } \\ 3 & 5 & 5 & 1 & 2 \\ 13 & 5 & 5 & 1 & 2 \\ 23 & 5 & 5 & 1 & 2 \\ 33 & 5 & 3 & 1 & 2 \\ 34 & 3 & 3 & 1 & 3 \\ 35 & 3 & 4 & 1 & 2 \\ 45 & 4 & 2 & 1 & 2 \\ 47 & 4 & 3 & 1 & 3 \\ 48 & 3 & 2 & 1 & 3 \\ 39 & 2 & 2 & 1 & 3 \\ 39 & 2 & 2 & 1 & 3\end{array}$

I SO TOFE

WASTE
PACKAGE TYPE

A

CURIES PER PACKAGE

TRANSPORT I NDE X/PKG

$4 \cdot 20 E-0 E$

1.0000

PACKAGES PER SHIPMENT

SHIPMENTS PER YEAR

HANDLINGS PER SHIPMENT

STORAGE TIME (SECONDS)

* INDICATES NONDISPERSIBLE SHIPMENTS 
Table A-33

Route No. 12; End Use: Fuel Cycle

$\begin{array}{ccccc}\text { CELL } & \text { IN } & \text { OUT } & \text { TRANSPORT } & \text { ROAD } \\ \text { SEQUENCE } & \text { DIRECTION } & \text { DIRECTION } & \text { MOOE } & \text { TYPE } \\ 3 & 5 & 5 & 1 & 2 \\ 13 & 5 & 5 & 1 & 2 \\ 23 & 5 & 5 & 1 & 2 \\ 33 & 5 & 3 & 1 & 2 \\ 34 & 3 & 3 & 1 & 3 \\ 35 & 3 & 4 & 1 & 2 \\ 45 & 4 & 2 & 1 & 2 \\ 47 & 4 & 3 & 1 & 3 \\ 48 & 3 & 2 & 1 & 3 \\ 39 & 2 & 2 & 1 & 3 \\ 30 & 2 & 2 & 1 & 3\end{array}$

I SOTOPE

PACKAGE TYPE

CURIES PER PACKAGE

TRANSPORT I NDEX/PKG

PACKAGES PER SHIPMENT

SHIPMENTS PER YEAR

HANDLINGS PER SHIPMENT

9.0 0 .
STORAGE TIME (SECONDS)

* INDICATES NONDISPERSiele shipMENTS 
Table A-34

Route No. 13; End Use: Medical

$\begin{array}{ccccc}\text { CELL } & \text { IN } & \text { OUT } & \text { TRANSPORT } & \text { ROAD } \\ \text { SEQUENCE } & \text { DIRECTION } & \text { DIRECTION } & \text { MODE } & \text { TYPE } \\ 61 & 2 & 2 & 3 & 0 \\ 52 & 2 & 2 & 3 & 0 \\ 43 & 2 & 2 & 3 & 0 \\ 34 & 2 & 2 & 3 & 0 \\ 25 & 2 & 2 & 3 & 0 \\ 16 & 2 & 2 & 3 & 0 \\ 7 & 2 & 2 & 3 & 0\end{array}$

$\begin{array}{ccr}\begin{array}{c}\text { PACKAGE } \\ \text { TYPE }\end{array} & \begin{array}{c}\text { CURIES PER } \\ \text { PACKAGE }\end{array} & \begin{array}{r}\text { TRANSPORT } \\ \text { INDEX/PKG }\end{array} \\ \text { A } & 1.50 E-03 & 0.0000 \\ \text { A } & 1.00 E-02 & .1000 \\ \text { A } & 8.00 E-02 & .5000 \\ \text { A } & 1.50 E-03 & 0.0000 \\ \text { A } & 2.20 E-04 & 1.0000 \\ \text { A } & 1.40 E+00 & 2.0000 \\ \text { A } & 1.60 E-03 & .0143 \\ \text { A } & 1.00 E-02 & 0.0000 \\ \text { A } & 5.70 E-03 & .1000 \\ \text { A } & 1.20 E-02 & .1000 \\ \text { A } & 2.70 E-01 & 0.0000\end{array}$

$X E-127$

$X E-133$
PACKAGES PER SHIPMENTS SHIPMENT
PER YEAR
HANDLINGS PER SHIPMENT
STORAGE TIME (SECONDS)

$\begin{array}{rr}1 \cdot & 34.7 \\ 1 . & 21.7 \\ 1 . & 2 \cdot 0 \\ 1 . & 147.0 \\ 1 . & 12 \cdot 0 \\ 1 . & 4.3 \\ 1 . & 30.3 \\ 1 . & 4.3 \\ 1 . & 6.0 \\ 1 . & 2.0 \\ 1 . & 8.7\end{array}$

0.
0.
0.
0.
0.
0.
0.
0.
0.
0.
0. 
Table A-35

Route No. 14; End Use: Industrial

$\begin{array}{ccccc}\begin{array}{c}\text { CELL } \\ \text { SEQUENCE }\end{array} & \text { IN } & \text { OUT } & \text { TRANSPORT } & \text { ROAD } \\ 61 & \text { DIRECIION } & \text { DIRECTION } & \text { MODE } & \text { TYPE } \\ 62 & 3 & 3 & 4 & 0 \\ 63 & 3 & 3 & 4 & 0 \\ 64 & 3 & 3 & 4 & 0 \\ 65 & 3 & 3 & 4 & 0 \\ 66 & 3 & 3 & 4 & 0 \\ 67 & 3 & 3 & 4 & 0 \\ 68 & 3 & 3 & 4 & 0 \\ 69 & 3 & 3 & 4 & 0 \\ 70 & 3 & 3 & 4 & 0\end{array}$

I SO TOPE
CO-60
$C R-51$
$H-3$
IR -192
$S-35$

$\begin{array}{cc}\begin{array}{c}\text { PACKAGE } \\ \text { TYPE }\end{array} & \begin{array}{c}\text { CURIES PER } \\ \text { PACKAGE }\end{array} \\ & \\ \text { A } & 1.50 E+00 \\ \text { A } & 1.10 E-02 \\ \text { A } & 8.80 E-03 \\ \text { A } & 6.00 E+01 \\ \text { A } & 4.00 E-03\end{array}$

TRANSPORT
INDEXIPKE
0.0000
.1000
0.0000
1.0000
0.0000

PACKAGES PER SHIPMENT

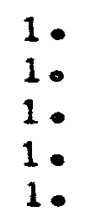

SHIPMENTS PER YEAR

HANDLINGS PER SHIPMENT

20.8
21.7
191.0
20.8
13.0

0 .

0 .

0 .

0 .
STORAGE TIME (SECONDS)

- INDICATES NONDISPERSIBLE SHIPMENTS 
Table A-36

Route No. 15; End Use: Medica1

$\begin{array}{ccccc}\begin{array}{c}\text { CELL } \\ \text { SECUEMCE }\end{array} & \begin{array}{c}\text { IN } \\ \text { DIRECTION }\end{array} & \begin{array}{c}\text { OUT } \\ \text { DIRECTIOM }\end{array} & \begin{array}{c}\text { TRANSPORT } \\ \text { MOOE }\end{array} & \begin{array}{c}\text { ROAD } \\ \text { TYPE }\end{array} \\ 61 & 2 & 2 & 3 & -0 \\ 52 & 2 & 2 & 3 & 0 \\ 43 & 2 & 2 & 3 & 0 \\ 34 & 2 & 2 & 3 & 0 \\ 25 & 2 & 2 & 3 & 0 \\ 16 & 2 & 2 & 3 & 0 \\ 7 & 2 & 2 & 3 & 0 \\ 7 & 5 & 3 & 1 & 3 \\ 8 & 3 & 2 & 1 & 3 \\ 9 & 4 & 6 & 1 & 2 \\ 18 & 6 & 6 & 1 & 2 \\ 27 & 6 & 6 & 1 & 2 \\ 36 & 6 & 7 & 1 & 3 \\ 35 & 7 & 7 & 1 & 3 \\ 34 & 7 & 7 & 1 & 3 \\ 33 & 7 & 1 & 1 & 2 \\ 23 & 1 & 0 & 1 & 2\end{array}$

I SOTOPE
AU-198
$C O-57$
$C R-51$
$C-14$
$F E-52$
$M G-203$
$I-125$
$I-131$
$K-43$
$M G-28$
$M O-99$
$P-32$
$M O-99$
$T L-201$
$X E-133$

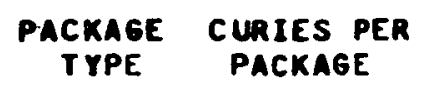

TRANSPORT

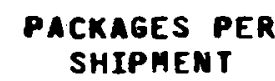

SHIPMENTS I NDEX/PKG SHIPMEN T PER PEAR

HANDLINGS PER SHIPHENT

STORAGE TIME

$\begin{array}{rrrr}A & 1.80 E-03 & .2000 & 1 . \\ A & 6.00 E-01 & 2.6000 & 1 . \\ A & 2.20 E-01 & 1.0330 & 1 . \\ A & 8.00 E-05 & 0.0000 & 1 . \\ A & 2.52 E+00 & 4.0000 & 1 . \\ A & 1.00 E-02 & .3000 & 1 . \\ A & 1.90 E-03 & .0046 & 1 . \\ A & 4000-02 & .4845 & 1 . \\ A & 2.00 E-03 & .4000 & 1 . \\ A & 5.20 E+01 & 1.1100 & 1 . \\ A & 1.30 E+00 & .1318 & 1 . \\ A & 1.30 E-01 & .436 E & 1 . \\ B & 9.10 E+01 & 6.1960 & 1 . \\ A & 3.10 E-03 & .4250 & 1 . \\ A & 1.60 E+00 & .1985 & 1 .\end{array}$

48.4
24.2
36.3
181.0
4.0
52.0
1580.0
856.0
24.2
46.0
1470.0
253.0
48.4
8.0
241.0

1.
1.
1.
1.
1.
1.
1.
1.
1.
1.
1.
1.

43200 .

43200

43200 .

43200 .

43200 .

43200 .

43200 .

43200 .

43200

43200 .

43200 .

43200 .

43200

43200 .

43200 . 
Table A-37

Route No. 16; End Use: Waste

$\begin{array}{ccccc}\text { CELL } & \text { IN } & \text { OUT } & \text { TRANSPORT } & \text { ROAD } \\ \text { SEQUENCE } & \text { DIRECTION } & \text { DIRECTION } & \text { MODE } & \text { TYPE } \\ 3 & 5 & 5 & 1 & 2 \\ 13 & 5 & 5 & 1 & 2 \\ 23 & 5 & 5 & 1 & 2 \\ 33 & 5 & 3 & 1 & 2 \\ 34 & 3 & 3 & 1 & 3 \\ 35 & 3 & 4 & 1 & 2 \\ 45 & 4 & 2 & 1 & 2 \\ 47 & 4 & 3 & 1 & 3 \\ 48 & 3 & 2 & 1 & 3 \\ 39 & 2 & 2 & 1 & 3 \\ 30 & 2 & 2 & 1 & 3\end{array}$

I SOTOPE

PACKAGE

CUR IES PER

TRANSPORT PACKAGE I NOE X/PKG
PACKAGES PER SHIPMENT

SHIPMENTS PER SHIPMENT

STORAGE TIME TYPE

$8 \cdot 40 E-05$

.2394

1.

66.0

0 .

(SECONDS)

MCP

DRUM

$8.40 E-05$

$1 \cdot$

* INDICATES NONDISPERSIBLE SHIPMENTS 
Table A-38

Route No. 17; End Use: Fuel Cycle

$\begin{array}{ccccc}\text { CELL } & \text { IN } & \text { OUT } & \text { TRANSPORT } & \text { ROAD } \\ \text { SEQUENCE } & \text { DIRECTION } & \text { DIRECTION } & \text { MODE } & \text { TYPE } \\ 30 & 6 & 6 & 1 & 3 \\ 39 & 6 & 6 & 1 & 3 \\ 48 & 6 & 7 & 1 & 3 \\ 47 & 7 & 7 & 1 & 3 \\ 46 & 7 & 8 & 1 & 3 \\ 35 & 8 & 7 & 1 & 3 \\ 34 & 7 & 7 & 1 & 3 \\ 33 & 7 & 7 & 1 & 2 \\ 32 & 7 & 8 & 1 & 2 \\ 21 & 8 & 1 & 1 & 3 \\ 11 & 1 & 1 & 1 & 3 \\ 1 & 1 & 1 & 1 & 3\end{array}$

$\begin{array}{lcccccc}\text { ISOTOPE } & \begin{array}{c}\text { PACKAGE } \\ \text { TYPE }\end{array} & \begin{array}{c}\text { CLRIES PER } \\ \text { PACKAGE }\end{array} & \begin{array}{c}\text { IRANSPORT } \\ \text { INDEX/PKG }\end{array} & \begin{array}{c}\text { PACKAGES PER } \\ \text { SHIPMENT }\end{array} & \begin{array}{c}\text { SHIPMENTS } \\ \text { PER YEAR PER SHIPMENT }\end{array} \\ \text { SPF-IH } & \text { CASK-1 } & 1.54 E+02 & 0.0000 & 1 . & 12.0 & 0 . \\ \text { SPF-EX } & \text { CASK-2 } & 2.17 E+05 & 1.0000 & 1 . & 12.0 & 0 .\end{array}$

- INDICATES NONDISPERSIBLE ShIPMENTS 
A7. Accident Rates and Delay Times

\section{A7.1 Motor Vehicles}

Reference 11 reported $3.8 \times 10^{6}$ total truck accidents during 1975 . Additionally data from Reference 7 indicate that of this number of accidents, $76 \%$ or $2.9 \times 10^{6}$ truck accidents occurred in urban areas. Using data from Reference 10, it is possible to subdivide these accidents by time of occurrence. Table A-39 presents the results of this subdivision.

Table A-39

Calculation of Motor Vehicle Accident Rates

\begin{tabular}{|c|c|c|c|c|}
\hline Time Span & $\begin{array}{c}\text { Fraction of Total } \\
\text { Accidents } \\
\text { Occurring During } \\
\text { Time Span } \\
\end{array}$ & $\begin{array}{c}\text { Number of } \\
\text { Urban } \\
\text { Accidents }\end{array}$ & $\begin{array}{c}\text { Urban } \\
\text { Vehicle } \\
\text { Kilometres } \\
\text { Traveled } \\
\text { by Time Span } \\
\end{array}$ & $\begin{array}{l}\text { Motor Vehicle } \\
\text { Accident } \\
\text { Rates } \\
\text { (accidents } / \mathrm{km} \text { ) }\end{array}$ \\
\hline 1 & 0.42 & $1.2 \times 10^{6}$ & $1.3 \times 10^{10}$ & $\sim 9.1 \times 10^{-5}$ \\
\hline 2 & 0.062 & $1.8 \times 10^{5}$ & $2.3 \times 10^{10}$ & $\sim 7.7 \times 10^{-6}$ \\
\hline 3 & 0.16 & $4.6 \times 10^{5}$ & $6.4 \times 10^{10}$ & $7.2 \times 10^{-6}$ \\
\hline 4 & 0.085 & $2.5 \times 10^{5}$ & $2.8 \times 10^{10}$ & $8.8 \times 10^{-6}$ \\
\hline 5 & 0.20 & $5.8 \times 10^{5}$ & $5.3 \times 10^{10}$ & $1.1 \times 10^{-5}$ \\
\hline 6 & 0.075 & $2.2 \times 10^{5}$ & $8.5 \times 10^{9}$ & $2.5 \times 10^{-5}$ \\
\hline
\end{tabular}

The fractional breakdown of urban truck travel by time of day has been obtained from Figure 5-7 of Reference 2 using a square counting technique. These data are combined with a value of $1.9 \times 10^{11}$ total kilometres of truck travel in urban areas. 11 These total truck travel data are also reported in Table A-39. The final column in this table lists the calculated accident rates, obtained by dividing the number of urban accidents by the urban vehicle kilometres traveled. A further subdivision of these accident rates is made on the basis of severity-dependent fractional occurrences for inclusion in the risk calculations. The urban-specific fractional occurrences for truck accidents are given in Table A-40, and the method used for their determination follows.

The fractional occurrences by accident severity category are taken from average values for property damage accidents in major urban areas* and values for injury and fatality accidents. It is assumed that no fatalities occur in anything less

*Information was obtained through telephone conversations with police departments in Philadelphia, San Francisco, and Los Angeles since New York City data were unavailable. 
severe than Category $\mathrm{V}$ accidents (see Reference 7, Chapter 5) and that injuries occur predominantly in Category II - Category IV accidents. A value of 0.714 represents the average for accidents involving only property damage. The total for all accidents involving injuries is 0.282 , leaving 0.004 as the fraction of accidents with fatalities. Fractional occurrences are listed in Table $\mathrm{A}-40$.

Table A-40

Fractional Occurrence for Urban Truck Accidents

\begin{tabular}{cc}
$\begin{array}{c}\text { Accident Severity } \\
\text { Category }\end{array}$ & $\begin{array}{c}\text { Fractional } \\
\text { Occurrence }\end{array}$ \\
\cline { 1 - 2 } I & 0.71 \\
II & 0.23 \\
III & 0.044 \\
IV & 0.010 \\
V & 0.0021 \\
VI & $8.3 \times 10^{-4}$ \\
VII & $6.4 \times 10^{-5}$ \\
VIII & $1.1 \times 10^{-5}$
\end{tabular}

\section{A7.2 Aircraft}

In the New York City study area, $33 \mathrm{~km}^{2}$ are streets, $52 \mathrm{~km}^{2}$ are occupied by buildings, and the remaining $15 \mathrm{~km}^{2}$ are open area (water, parks, etc.). A previous study adjusted the aircraft accident rates to account for real surface--as distinguished from unylelding surface--effects. 7 Since the data in Reference 7 are for the entire nation and reflect large amounts of open area, an adaption of these data is necessary. Designations of surface type from Reference 7 have been adapted to the urban area. The probabilitiy of occurrence of a particular surface type has been multiplied by the appropriate urban area in $\mathrm{km} 2$ (open, streets, buildings). The resulting numbers have been standardized to yield a set of revised probabilities for the urban area. These values are listed in Table A-41.

Using the probability information in Table A-41, the severity-dependent aircraft accident occurrence probabilities can be adjusted as in Appendix $\mathrm{H}$ of Reference 7. If the comparison of urban open space with water/soft soil and urban street/buildings with hard soil/soft rock/hard rock is extended to the values for Young's modulus and Poisson's ratio, then the values for $\mathrm{V} / \mathrm{V}$ can be extracted from Table $\mathrm{H}-1$ of Reference 7 and used directly. When this is done, the values given in Table A-42 can be computed.

\section{A7.3 Rail Transport}

Information on rall transport accident rates has been taken from Table 5-5 of Reference 7. Values for accident rates to be applied to this study are listed in Table A-43. 
Table A-41

Fractional Occurrences for Aircraft Accidents

in Urban Areas

\begin{tabular}{|c|c|c|c|}
\hline Surface Type & Urban Area Example & $\begin{array}{l}\text { Occurrences in } \\
\text { Urban Areas }\end{array}$ & $v / v_{s}^{a}$ \\
\hline Water & Water & 0.055 & 4.5 \\
\hline Yielding surface & $\begin{array}{l}\text { Parks, cemeteries, } \\
\text { other open space }\end{array}$ & 0.085 & 7.1 \\
\hline $\begin{array}{l}\text { Slightly unyield- } \\
\text { ing surface }\end{array}$ & $\begin{array}{l}\text { Streets, small resi- } \\
\text { dential buildings }\end{array}$ & 0.80 & $3.0^{\mathrm{b}}$ \\
\hline $\begin{array}{l}\text { Moderately unyield- } \\
\text { ing surface }\end{array}$ & Other buildings & 0.051 & 2.2 \\
\hline Unyielding surface ${ }^{c}$ & $\begin{array}{l}\text { Abutments, steel } \\
\text { reinforcements }\end{array}$ & 0.01 & 1.0 \\
\hline $\begin{array}{l}{ }_{\text {Ratio of impact }} \\
\text { similar damage onto } \\
{ }^{b} \text { Arithmetic mean } \\
{ }^{c_{A} 1 \% \text { value for }} \\
\text { Reference } 7 \text {. }\end{array}$ & $\begin{array}{l}\text { ocity onto a real su } \\
\text { nyielding surface. } \\
\text { values for hard soil } \\
\text { elding surface has }\end{array}$ & $\begin{array}{l}\text { to the impact } \\
\text { oft rock in } R \\
\text { lded for conse }\end{array}$ & nce \\
\hline
\end{tabular}

Table A-42

Adjusted Scheme for Urban Aircraft Accidents

\begin{tabular}{|c|c|c|c|c|c|c|c|c|c|}
\hline \multirow{2}{*}{$\begin{array}{l}\text { Accident } \\
\text { Severity }\end{array}$} & \multirow{2}{*}{$\begin{array}{c}\text { Fractional } \\
\text { Occurrence } \\
\text { Unyielding } \\
\text { Surface } \\
\end{array}$} & \multirow{2}{*}{$\begin{array}{c}\text { Fraction } \\
\text { Deleted } \\
\text { by } \\
\text { Ad justment }\end{array}$} & \multirow{2}{*}{$\begin{array}{c}\text { Fraction } \\
\text { Unyielding }\end{array}$} & \multicolumn{5}{|c|}{ Fraction Added by Adjustment } & \multirow[t]{2}{*}{$\begin{array}{c}\text { Fractional } \\
\text { Occurrence } \\
\text { Rea1 } \\
\text { Surfaces } \\
\end{array}$} \\
\hline & & & & $w$ & ys & sus & mus & total & \\
\hline VIII & 0.03 & 0.0297 & 0.0003 & 0 & 0 & 0 & 0 & 0 & 0.0003 \\
\hline VII & 0.04 & 0.0396 & 0.0004 & 0 & 0 & 0 & 0.0015 & 0.0015 & 0.0019 \\
\hline VI & 0.03 & 0.0297 & 0.0003 & 0 & 0 & 0.024 & 0 & 0.024 & 0.0243 \\
\hline v & 0.03 & 0.0297 & 0.0003 & 0.0016 & 0 & 0.031 & 0.002 & 0.035 & 0.0353 \\
\hline IV & 0.05 & 0.0495 & 0.0005 & 0.0022 & 0.0025 & 0.024 & 0.003 & 0.032 & 0.0325 \\
\hline III & 0.09 & 6.0891 & 0.0009 & 0.0016 & 0.0034 & 0.024 & 0.0015 & 0.031 & 0.0319 \\
\hline I, II & 0.73 & b & b & 0.024 & 0.017 & 0.11 & 0.0071 & 0.16 & 0.888 \\
\hline
\end{tabular}

averall accident rate, all categories $=1.44 \times 10^{-8}$ accident $/ \mathrm{km}$.

${ }^{b}$ Categories I and II not adjusted. 
Table A-43

Fractional Occurrences for Urban Train Accidents by Accident Severity Category

\begin{tabular}{cc}
$\begin{array}{c}\text { Accident } \\
\text { Severity } \\
\text { Category }\end{array}$ & $\begin{array}{c}\text { Fractional } \\
\text { Occurrences* }\end{array}$ \\
\cline { 2 - 2 } I & 0.50 \\
II & 0.30 \\
III & 0.18 \\
IV & 0.018 \\
V & 0.0018 \\
VI & $1.3 \times 10^{-4}$ \\
VII & $6.0 \times 10^{-5}$ \\
VIII & $1.0 \times 10^{-5}$ \\
& *0verall accident rate $=$ \\
$0.93 \times 10^{-6}$ railcar accident/ \\
railcar-kilometre.
\end{tabular}

\section{A7.4 Accident Rates for Water Transport}

Rates for ship or barge accidents in the study area are identical to those used in Reference 7. Pertinent values from Table 57 of Reference 7 are given in Table A-44.

Table A-44

Fractional Occurrences for Urban Water Transport Accidents

Accident

Severity

Category

Fractional

I Occurrence*

0.897

II

0.0798

III

0.00113

IV

0.0186

$\mathrm{V}$

$5.2 \times 10^{-6}$

VI

$7.2 \times 10^{-5}$

VII

$1.95 \times 10^{-4}$

VIII

$1.3 \times 10^{-5}$

* Overall accident rate

$=6.06 \times 10^{-6}$ accidents $/ \mathrm{km}$. 


\section{A7.5 Delay Time}

Accident delay time is defined as the length of time a carrier vehicle (and, therefore, the transported material) does not move following an accident.

Basic information on delay time for various accidents has been obtained from a carrier of radioactive materials who frequently operates in the New York area. officials have indicated that the time following a minor traffic accident before the vehicle begins to move again is from 30 minutes to 4 hours, with an average of around 2 hours. On the other hand, severe accidents could cause delays of 6 to 8 hours, with a maximum of about 24 hours. It has also been indicated that geographical location and time of day are not significant factors in these delay times. 12

The small quantity of data available in NRC and DOT accident records support the estimates for Categories II through $\mathrm{V}$ accidents.* Values may be quite high for more severe accidents, but the paucity of available data makes the number difficult to obtain. The values in Table A-45 are used for all modes under the assumption that the delay time is more dependent on the overall accident severity than on the mode of transport.

Table A-45

Estimated Accident Delay Times for Trucks

$\begin{array}{rc}\begin{array}{c}\text { Severity } \\ \text { Class }\end{array} & \begin{array}{c}\text { Estimated Accident } \\ \text { Delay Time (seconds) }\end{array} \\ \text { I } & 1.8 \times 10^{3} \\ \text { II } & 3.6 \times 10^{3} \\ \text { III } & 7.2 \times 10^{3} \\ \text { IV } & 1.4 \times 10^{4} \\ \text { V } & 2.9 \times 10^{4} \\ \text { VI } & 4.3 \times 10^{4} \\ \text { VII } & 6.5 \times 10^{4} \\ \text { VIII } & 8.6 \times 10^{4}\end{array}$

\section{A7.6 Release Fractions}

In order to assess the risk of a transportation accident, it is necessary to predict the fraction of the total package contents which would be released from an accident of a given severity. The actual releases for a particular package type would not necessarily be the same for a number of accidents of the same severity class. In some cases there may be no release, while in others there may be, for example, a $10 \%$ release. Indeed, in an accident involving a number of radioactive

\footnotetext{
${ }^{\star}$ See NRC and DOT incident reports in Appendices $H$ and $J$ of this report.
} 
material packages transported together, some of the packages may release part of their contents while others have no release at all. The approach taken in this assessment is identical to that used in Reference 7, where point estimates are used for the average release fraction for each severity category and package type, and where it is assumed that all such packages, including each package in a multipackage shipment, respond to such an accident in the same way regardless of transport mode or package contents. In addition, it is assumed that an accident of a given severity produces the same release fraction for a specific package type regardless of transport mode. Although this assumption appears to directly equate crush force, impact, and puncture, it actually assumes that the release from a specific impact accident will equal the release from an equivalent crush or puncture accident. This equivalency is made by appropriate assignment of the fractional occurrence by severity for each mode.

Packaging standards do not require testing to the point of package failure; the paucity of data on package responses to severe accidents makes it difficult to predict even the average release fraction, much less a distribution. Therefore, until recently, there has been little information relating the response of packages to accident environments. A series of severe impact tests was carried out using several types of containers commonly used to ship plutonium and spent fuels. 1391415 Tests of plutonium containers revealed structural damage to the inner container after impact onto unyielding targets at speeds up to those typical of a Category $\mathrm{V}$ impact accident. Several containers exhibited some minor structural damage and cracking in Category VI impacts, but no verified release occurred. Tests of typical commercial containers showed the failure of a nonspecification cast-iron plug, material loss, and compromise of the overall integrity of the inner containers. In one set of tests, a container was estimated to have 10 st $6 \%$ of its contents (magnesium oxide powder, a radioactive material surrogate) in a Category VII impact, while others survived Category VIII impacts with no loss of contents. Although none of the containers in this test series was subjected to fire, others of the same type survived less severe impacts followed by a $1300^{\circ} \mathrm{C}$ environment lasting for 30 minutes with no release.

The responses of packages are estimated using either this test information or assuming that packaging begins to fail at levels just above those they are required by regulations to survive. The release fraction estimates for all package types evaluated are shown in Table A-46. A more detailed derivation of these values for each package type is contained in Chapter 5 of Reference 7.

Table A-46

Release Fractions

\begin{tabular}{|c|c|c|c|c|c|c|}
\hline \multirow[b]{2}{*}{$\begin{array}{l}\text { Severity } \\
\text { Category }\end{array}$} & \multirow[b]{2}{*}{$\begin{array}{l}\text { LSA } \\
\text { Drum }\end{array}$} & \multirow[b]{2}{*}{$\begin{array}{c}\text { Type } \\
\mathrm{A}\end{array}$} & \multicolumn{2}{|c|}{ Ty } & \multirow[b]{2}{*}{$\begin{array}{c}\text { Cask } \\
\text { (exposure) }\end{array}$} & \multirow[b]{2}{*}{$\begin{array}{c}\text { Cask } \\
\text { (release } \\
\end{array}$} \\
\hline & & & No $\mathrm{Pu}$ & $\begin{array}{l}1975 \\
\mathrm{Pu} \\
\end{array}$ & & \\
\hline I & 0 & 0 & 0 & 0 & 0 & 0 \\
\hline II & 0.01 & 0.01 & 0 & 0 & 0 & 0 \\
\hline III & 0.1 & 0.1 & 0.01 & 0 & 0 & 0.01 \\
\hline IV & 1.0 & 1.0 & 0.1 & 0 & 0 & 0.1 \\
\hline $\mathrm{v}$ & 1.0 & 1.0 & 1.0 & 0 & 0 & 1.0 \\
\hline VI & 1.0 & 1.0 & 1.0 & 0.01 & $3.2 \times 10^{-7}$ & 1.0 \\
\hline VII & 1.0 & 1.0 & 1.0 & 0.05 & $3.2 \times 10^{-5}$ & 1.0 \\
\hline VIII & 1.0 & 1.0 & 1.0 & 0.1 & $3.2 \times 10^{-3}$ & 1.0 \\
\hline
\end{tabular}


NOTES

${ }^{1}$ New York City Planning Commission, Community Planning District Profiles, September 1974.

2J. E. Baerwald, ed, Transportation and Traffic Engineering Handbook (Englewood Cliffs, NJ: Prentice-Hal1, 1976).

${ }^{3}$ New York City Planning Commission, Plan for New York City (Cambridge: MIT Press, 1969).

${ }^{4}$ Personal Communications with Dr. B. Bunting, University of New Mexico, an architect familiar with construction of older buildings in the northeast United States.

5 Tri-State Regional Planning Commission, 1970 County to County Travel: Census Journey to Work by Mode; Person Travel by Purpose, Interim Technical Report 45491302, New York, December 1975. $1975)$.

$6_{\mathrm{B} .}$ Pushkarev and M. Zupan, Urban Space for Pedestrians (Cambridge: MIT Press,

${ }^{7}$ NUREG-0170.

8NUREG-0073.

${ }^{9}$ Centre Mark Company, CENTRE-US: The Data Base of Geography, Elmhurst, IL. Latitudes and longitudes are given to the nearest 0.01 degree; place names are fully spelled out to a maximum to 32 characters.

${ }^{10}$ U.S. Department of Transportation, Federal Highway Administration, Bureau of Motor Carrier Safety, Accidents of Motor Carriers of Property (Washington: USDOT, 1975).

${ }^{1 l_{U}}$.S. Department of Commerce, Statistical Abstract of the United States, 1978.

12 Personal communication with officials at Tristate Motor Transit, 1978.

${ }^{13_{L}}$. Bonzon and R. McWhirter, "Special Tests of Plutonium Shipping Containers," IAEA-SR-10/22, presented at International Atomic Energy Agency Seminar on Radioactive Materials Packaging and Transportation, August 1976.

$14 \mathrm{~L}$. Bonzon and J. T. Schamaun, "Container Damage Correlation with Impact Velocity and Target Hardness," IAEA-SR-10/21, presented at International Atomic Energy Agency Seminar on Radioactive Materials Packaging and Transportation, August 1976.

15R. M. Jefferson and H. R. Yoshimura, Crash Testing of Nuclear Fuel Shipping Containers, SAND77-1962 (Albuquerque: Sandia Laboratories, February 1978). 
DOSE MODEL FOR INCIDENT-FREE TRANSPORT OF RADIOACTIVE MATERIALS

Since virtually all packages of radioactive material emit some external penetrating radiation, a person adjacent to a transportation route will receive some radiation dose as a consequence of the shipment. This dose, referred to as incident-free transport dose, is defined as that which results from transport involving no vehicular accident, packaging or handling abnormalities (e.g., improper labeling, crushing by fork lifts, omission of o-rings), or malevolent attack.

The model used to calculate dose due to incident-free transport is based on the transport index (TI), defined in the Code of Federal Regulations as the dose rate in $\mathrm{mrem} / \mathrm{h}$ measured at a distance of 0.9 metre ( $3 \mathrm{feet}$ ) from a package (see Reference 1).* Since the measured value for this parameter is noted on each package, it provides a convenient benchmark for computing incident-free dose.

The entire development of the dose computation is based on the following formula for dose rate from a point source of ionizing radiation:

$$
D R=\frac{K e^{-\mu r} B(r)}{r^{2}}
$$

where

$$
\begin{aligned}
D R & =\text { dose rate }(\mathrm{mrem} / \mathrm{h}) * * \\
K & =\text { dose rate factor }\left(\mathrm{mrem} \cdot \mathrm{m}^{2} / \mathrm{h}\right) \\
\mu & =\text { attenuation coefficient }\left(\mathrm{m}^{-1}\right) \\
\mathrm{r} & =\text { distance from source (metres) } \\
B(r) & =\text { dose rate buildup factor (dimensionless) }
\end{aligned}
$$

* The Code of Federal Regulations addresses two types of TI. Radiation TI is equal to the dose rate measured 0.9 metre from the package; fissile TI is computed based on criticality considerations. Since the TI rating of a package is the larger of the two, this assessment assumes that the quoted $T I$ is radiation $T I$. This assumption will overestimate dose effects for some packages of fissile material.

A complete list of variables, constants, and functions used in the equations in this appendix is in Addendum 1. 
In this case, $e^{-\mu r}$ accounts for the attenuation of photons in the absorbing medium which surrounds the receptor, $1 / \mathrm{r}^{2}$ accounts for the inverse square dose rate reduction, and $B(r)$ accounts for the dose buildup caused by inelastic photon scattering in the attenuating medium. Attenuation coefficient and $B(r)$ are energy dependent (see section B1.2). If $K$ is redefined to incorporate package and shipment characteristics, it can be replaced by $\mathrm{K}_{0} \cdot \mathrm{TI} \cdot \mathrm{PPS}$, as described below.

A package shape factor, $K_{0}$, is defined to account for the actual package dimensions.* The dose rate at a distance $r$ from a shipment of radioactive materials containing a certain number of packages, PPS, can be expressed as

$$
\mathrm{DR}=\frac{\mathrm{K}_{\mathrm{o}} \cdot \mathrm{TI} \cdot \mathrm{PPS} \cdot \mathrm{e}^{-\mu \mathrm{r}} \cdot \mathrm{B}(\mathrm{r})}{\mathrm{r}^{2}}
$$

where PPS = number of packages per shipment.

Figure B-1 diagrams the most important manipulation in determining integrated population exposures. The questions to be answered are (1) what integrated dose will be received by an individual at a specified perpendicular distance d from the path of a vehicle carrying radioactive material? and (2) what integrated dose will be received by a uniform set of stationary individuals in the strip of length $L$ as the vehicle passes that strip?

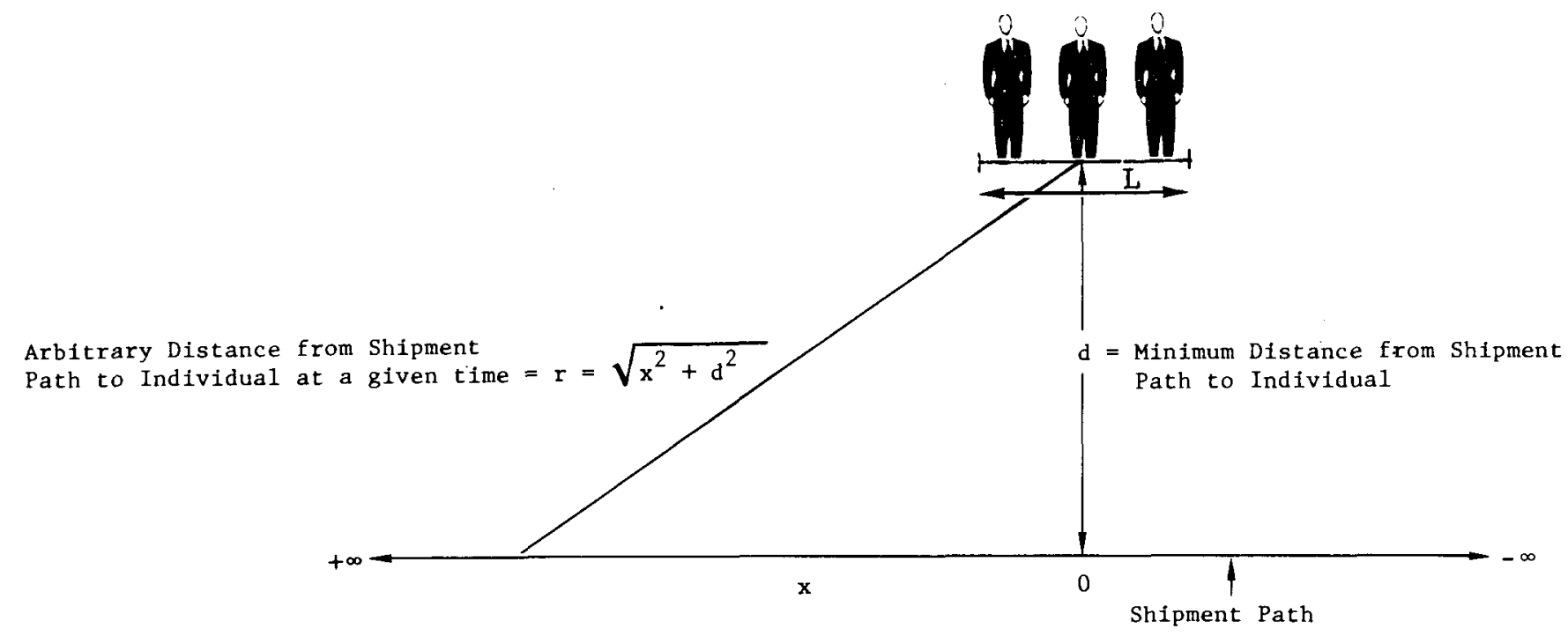

Figure B-1. Basic Geometry for Integrated Dose Calculations

* Note that the $\mathrm{K}_{0}$ values used in the model include a factor of 0.84 to allow for the use of TI in terms of metres rather than feet. Values for $\mathrm{K}$ are 1.25 for Type A packages, 1.49 for Type B, BPu, LSA, and drums, and 110 for casks. 
Initially it is noted that

$$
D R=\frac{d D}{d t}
$$

where $D=$ total dose.

By manipulating a definition,

$$
\mathrm{dt}=\frac{\mathrm{dx}}{\mathrm{V}}
$$

where $\mathrm{V}=$ speed (assumed to be constant). Combining Eqs. (1), (3), and (4), and integrating an expression for total dose from a shipment traveling from $-\infty$ to $+^{\infty}$ past the individual, the following expression is obtained:

$$
D=\frac{K}{V} \int_{-\infty}^{\infty} \frac{e^{-\mu r} B(r) d x}{r^{2}}
$$

Integrating over $r$ instead of $x$ and noting that this function is symmetric about the origin results in the following expression for individual dose:

$$
D=\frac{2 K}{V} \int_{d}^{\infty} \frac{e^{-\mu r} B(r) d r}{r \sqrt{r^{2}-d^{2}}}
$$

This expression, derived in more detail in Reference 2, Appendix D, forms the basis for many of the dose expressions used in the incident-free analysis.

The unique characteristics of each of the various transport modes with regard to incident-free transport are considered in sections B1 through B5.

\section{B1. Dose Due to Incident-Free Truck Transport}

This dose can vary significantly depending on the nature of the transport link. Three principal transport links are analyzed: two-way streets, one-way streets, and freeways. The population exposed as a result of shipment by truck is divided into four groups: pedestrians, people in buildings, people in vehicles, and transport crew. Each of these groups and transport links will be analyzed in detail in the following subsections.

\section{B1.1 Dose Due to Incident-Free Truck Transport on Two-Way Streets}

\section{Pedestrians}

Assume a shipment is moving on a street of width $w_{s t}$, with sidewalks on each side of width $w_{s}$, at a speed $\bar{v}$ (averaged over cruising periods and stopped periods), as shown in Figure B-2. Assume as well that equal numbers of pedestrians are moving in each direction at speed $v_{p}$ at a density given by PedD (persons $/ \mathrm{km}^{2}$ of sidewalk). 


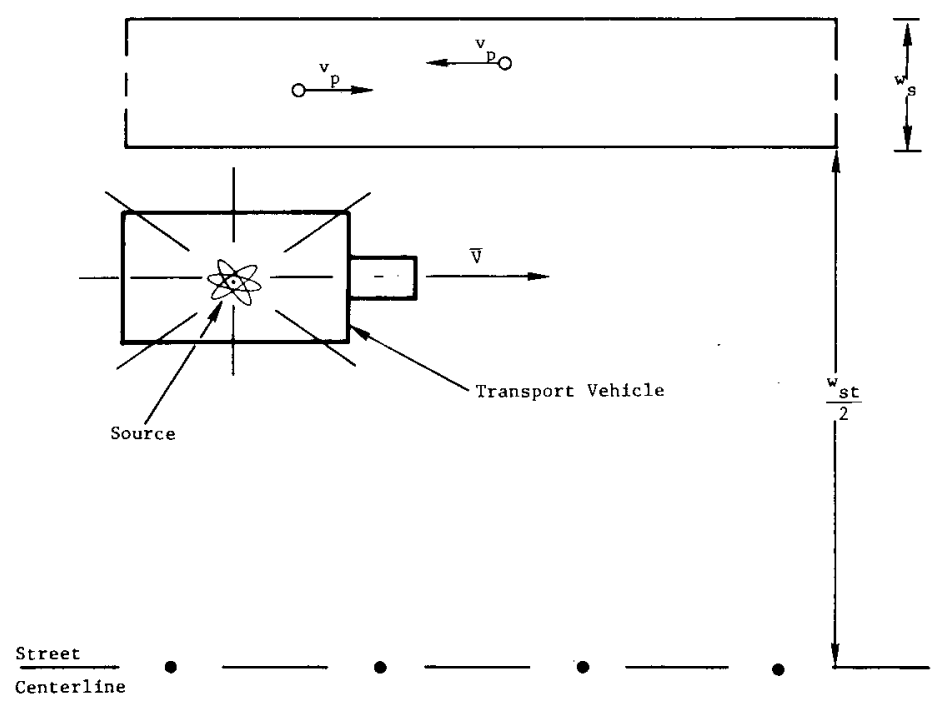

Figure B-2. Geometry for Incident-Free Dose to Pedestrians

The dose received by pedestrians, resulting from these assumptions, has four components :

1. Dose to pedestrians moving in the same direction as the shipment on the same side of the street,

2. Dose to pedestrians moving in a direction opposite the shipment on the same side of the street,

3. Dose to pedestrians moving in the same direction as the shipment on the far side of the street, and

4. Dose to pedestrians moving in a direction opposite the shipment on the far side of the street.

Relative motion between shipment and pedestrians is accounted for by considering that pedestrians are stationary and that the shipment is moving past them at a speed of $\vec{v}+v_{p}$ (components 2 and 4 ), or $\bar{v}-v_{p}$ (components 1 and 3 ).* In general, shipment velbcity is much greater than pedestrian velocity. However, under congested traffic conditions $v_{p}$ may be significant with respect to $\bar{v}$.

If it is assumed that the truck travels in the center of either half of the street and that truck width and package width are small compared with street width,** the distance from the center of the package to the edge of the street at the far sidewalk is $0.75 \mathrm{w}_{\mathrm{st}}$, and the distance from the center of the package to the edge of the street at the near sidewalk is $0.25 \mathrm{w}_{\text {st: }}$ Using Eq. (6), the dose received by an individual as the shipment passes can be given by

\footnotetext{
* This effectively lengthens or shortens the strip of length $L$ shown in Figure $\mathrm{B}-1$.

**Average truck width is 2.6 metres, average package width 0.7 metre, and average street width is 20 metres.
} 


$$
D(x)=\frac{2 K}{V} I(x)
$$

where

$$
\begin{aligned}
D(x)= & \text { total integrated exposure dose to an individual at a distance } \\
& \quad \text { from the path of a radioactive shipment (effect of shield- } \\
& \text { ing by truck is not included) } \\
\mathrm{K}= & \text { dose rate factor for the shipment } \\
\mathrm{V}= & \text { shipment speed } \\
\mathrm{x}= & \text { perpendicular distance from individual to shipment (replacing } \\
& \text { D as previously used); and }
\end{aligned}
$$

where

$$
\begin{aligned}
\mu_{a i r} & =\text { attenuation coefficient (for air) } \\
B_{a i r}(r) & =\text { dose buildup function (for air) }
\end{aligned}
$$

When this general expression for $D(x)$ is multiplied by pedestrian density and integrated over the area of exposure, an expression for total pedestrian exposure per shipment on each side of the road is obtained:

$$
I D=Q \cdot \operatorname{PedD} \cdot L \cdot \int_{d}^{d+w_{s}} D(x) d x
$$

where

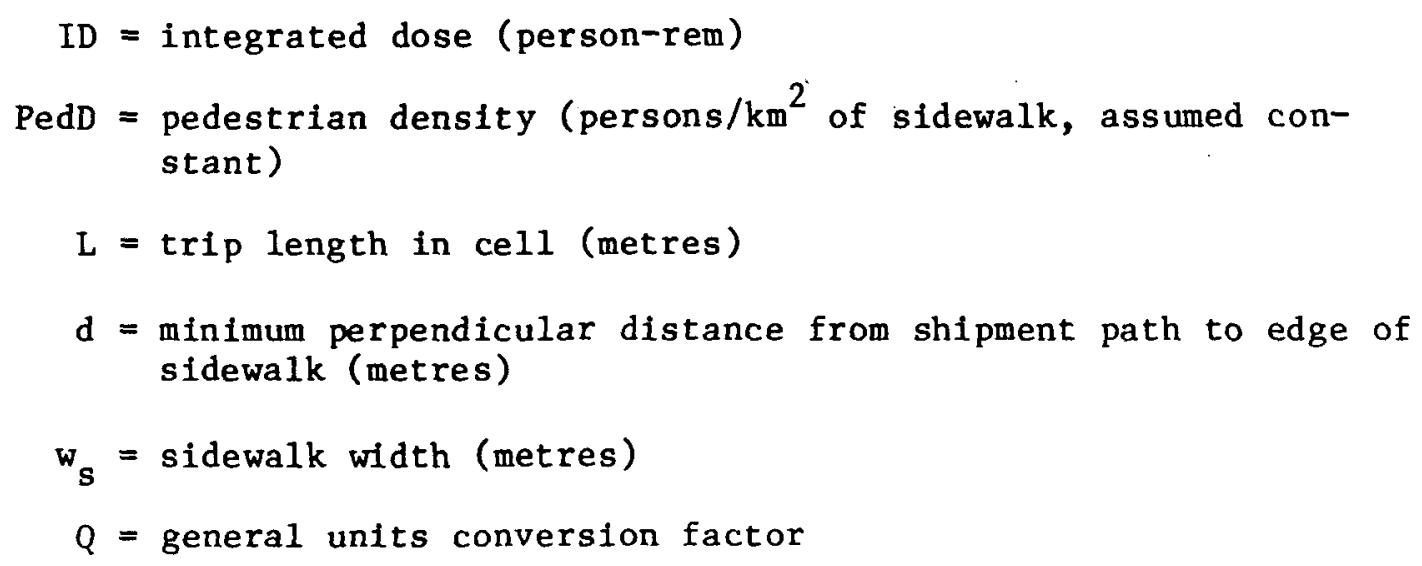


The dose received by pedestrians from the ground and/or adjacent structures is called albedo dose and has three components, as shown in Figure B-3. The magnitude of these components relative to free-air exposure is analyzed in Reference 4. The experimental relationship between groundscatter albedo and height-to-distance ratio $(\mathrm{h} / \mathrm{d})$ is shown in Figure B-4. A typical value of $\mathrm{h} / \mathrm{d}$ for urban transport geometries is 0.1 , so a value of 0.2 from Figure $B-4 b$ for groundscatter albedo dose is assumed, even though differences exist between the experimental data and the present case. There is some variation of this factor with photon energy so an energy considered typical for transported radionuclides, $0.4 \mathrm{MeV}$ ( $\mathrm{Ir}-192$ decay), was selected. The total albedo dose from building backscatter as a function of energy is shown in Figure $\mathrm{B}-5$. Using $0.4 \mathrm{MeV}$, a value of 0.16 for normal backscatter is obtained. If it is assumed that each person subtends a finite portion of the reflection given in Figure B-5 (0.67 steradians, assuming a 0.9-metre standoff and a 1.8-metre by 0.3 -metre profile), the total albedo of 0.16 becomes 0.017 . The backscatter of groundscattered radiation is the product of 0.2 and 0.017 , or $3.4 \times 10^{-3}$.

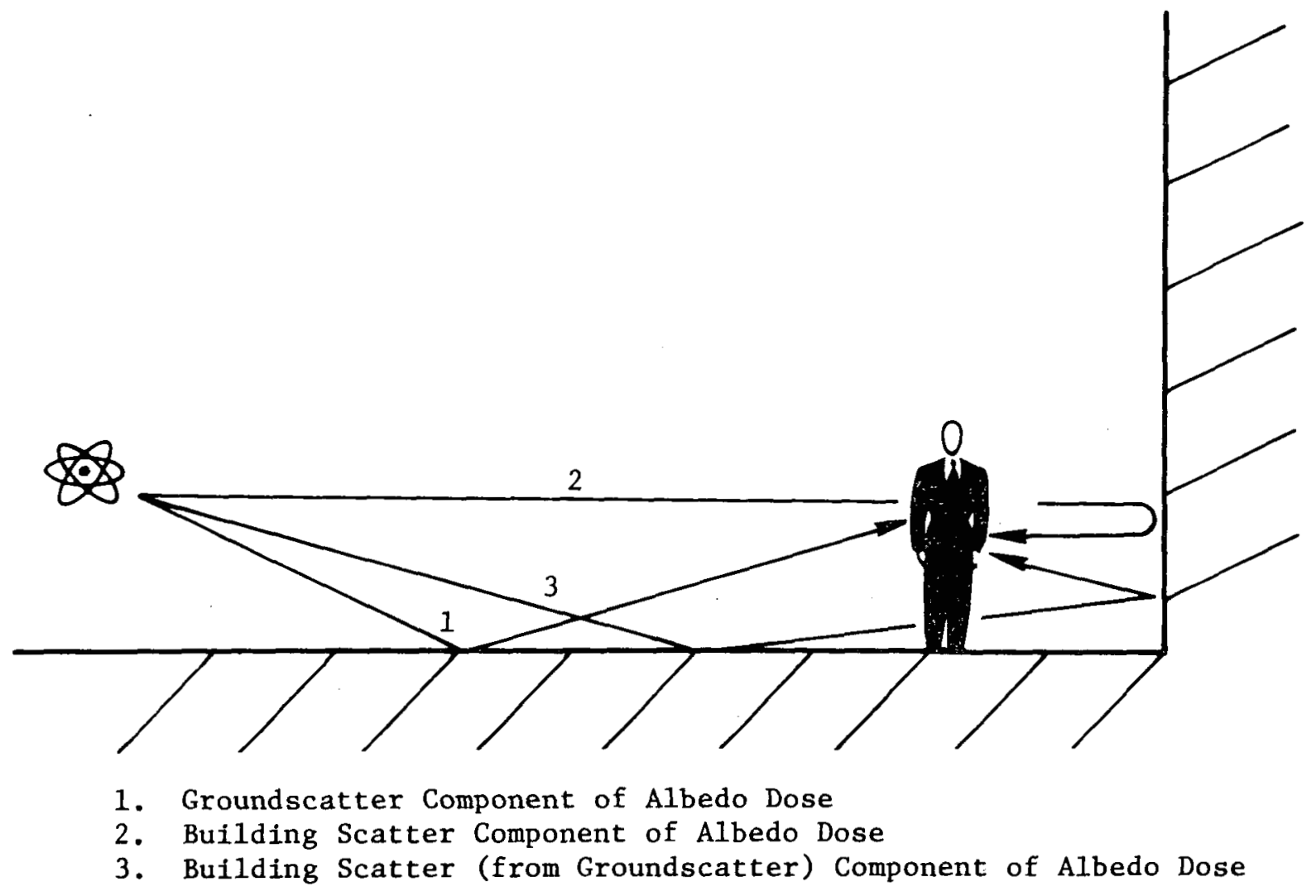

Figure B-3. Albedo Dose Contributions 


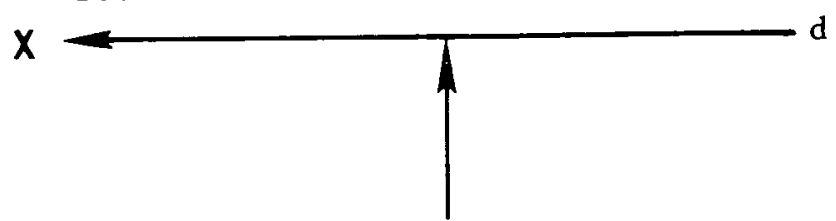

d

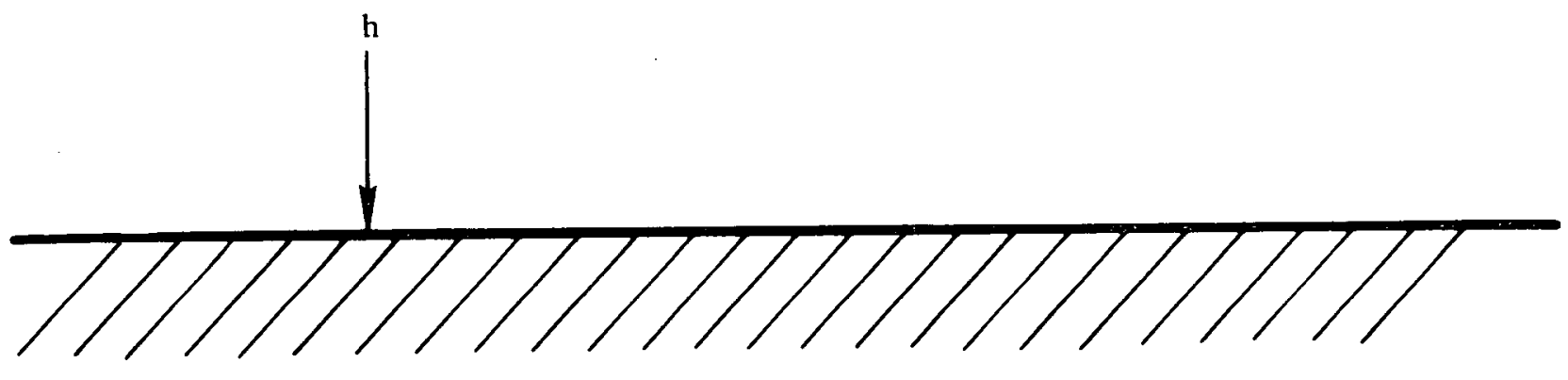

Concrete

B-4a. Geometry for Gamma-Ray Scattering Experiments Performed by Clarke and Batter ${ }^{4}$

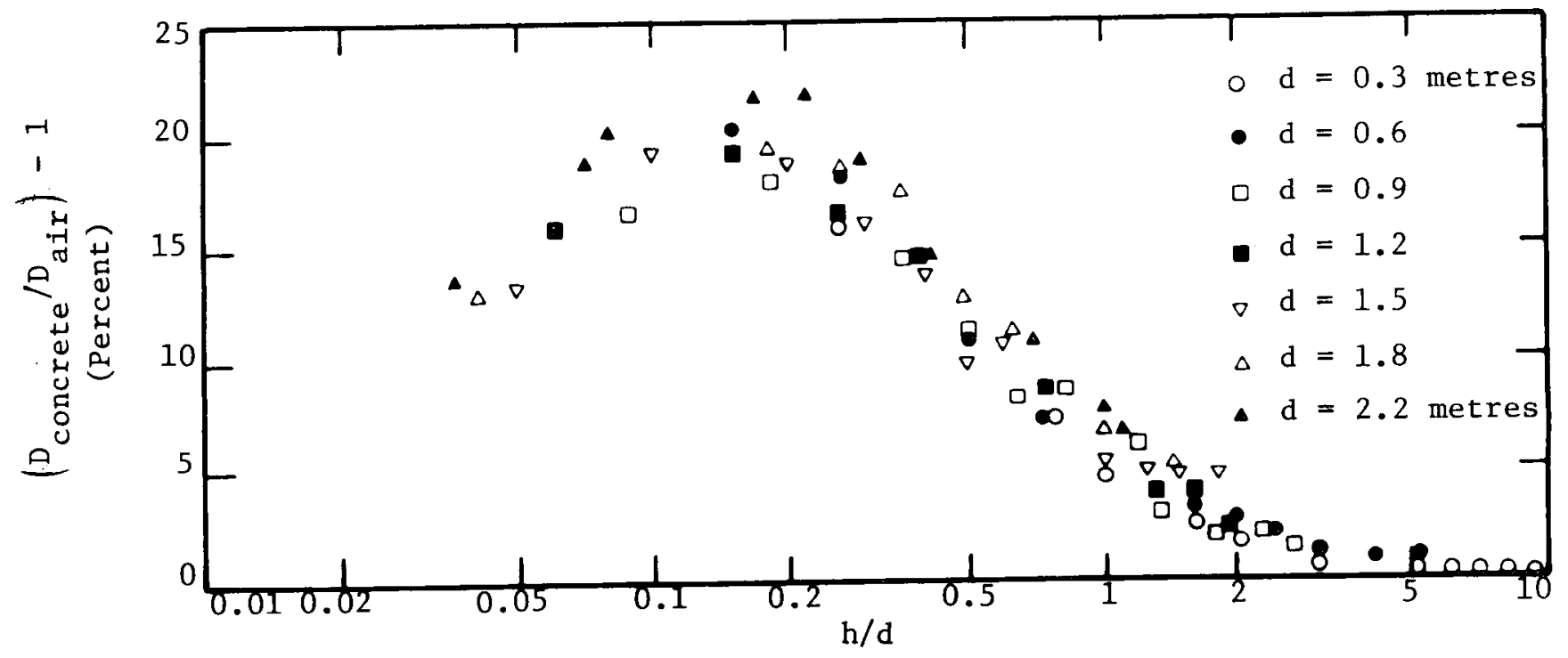

B-4b. Increase in Gamma-Ray Dose Rate from an $\mathrm{Ir}-192$ Source Due to Scattering from a Concrete Surface 4

Figure $B-4$. 


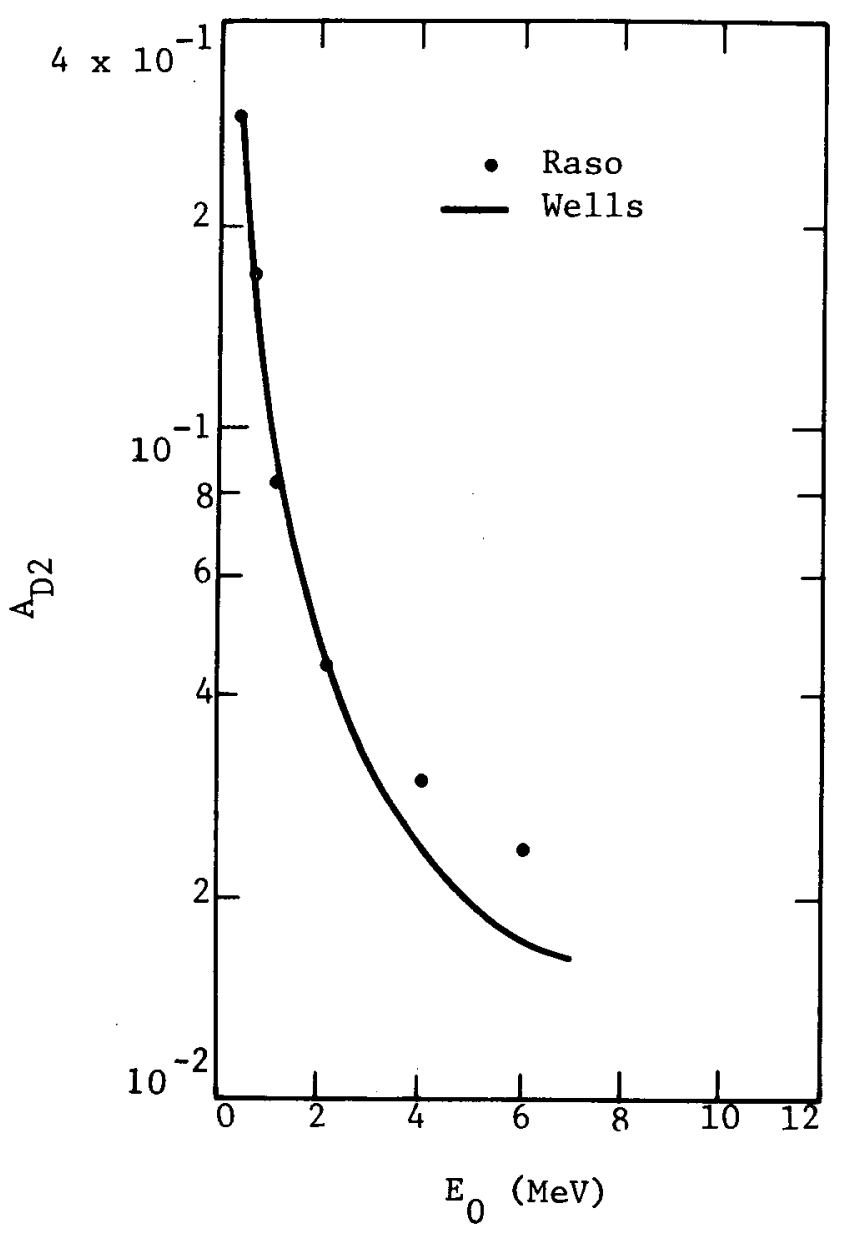

Figure B-5. Total Dose Albedos for Gamma Rays Normally Incident on Concrete ${ }^{4}$

When both albedo dose and direct dose are considered, the integrated dose to pedestrians becomes

$$
\begin{aligned}
& \mathrm{ID}=\mathrm{Q}_{1} \cdot \operatorname{PedD} \cdot \mathrm{L} \cdot\left(2 \cdot \mathrm{K}_{\mathrm{O}} \cdot \mathrm{TI} \cdot \mathrm{PPS}\right) \cdot \mathrm{ABD} \cdot \\
& \frac{1}{2}\left[\begin{array}{cccc}
0.75 w_{s t}{ }^{+w} s & 0.75 w_{s t}{ }^{+w} s & 0.25 w_{s t}{ }^{+w_{s}} & 0.25 w_{s t}{ }^{+w} s \\
\int \frac{I(x) d x}{\bar{v}-v_{p}}+\int \frac{I(x) d x}{\bar{v}+v_{p}}+\int \frac{I(x) d x}{\bar{v}-v_{p}}+\int \frac{I(x) d x}{\bar{v}+v_{p}} \\
0.75 w_{s t} & 0.75 w_{s t} & 0.25 w_{s t} & 0.25 w_{s t}
\end{array}\right]
\end{aligned}
$$

where

$$
\begin{aligned}
& Q_{1}=\text { units conversion factor, } 2.78 \times 10^{-13} \frac{\mathrm{rem} \cdot \mathrm{h} \cdot \mathrm{km}^{2}}{\mathrm{mrem} \cdot \mathrm{s} \cdot \mathrm{m}^{2}} \\
& A B D=\text { albedo dose factor }(=1.22)
\end{aligned}
$$


It should be noted that as $\bar{V}$ approaches $v_{p}$, the integrated dose approaches infinity. This is clearly unrealistic and an artifaet of the modeling. In the numerical calculation, a default value is assumed when $\bar{v}=\mathrm{v}_{\mathrm{p}}$.

The integrals in Eq. (9) can be evaluated using zero-order Bessel functions.* No pedestrian self-shielding is evaluated, and no structural shielding from the transport vehicle, other cargo, or other intervening vehicles is assumed.

Two additional factors will be considered: dose to people moving at right angles to the shipment direction on sidestreet sidewalks and dose to people moving in crosswalks in front of the shipment while it is stopped at intersections.

The geometry for calculating dose to pedestrians on sidestreets is shown in Figure B-6.** If building shielding is ignored, the dose received by a person in the cross-hatched area at an arbitrary distance $x$ from the shipment can be calculated using

$$
D(x)=\frac{2 K}{\bar{y}} \int_{x}^{\infty-\mu a i r^{r} \cdot B_{a i r}(r) d r}
$$

The integrated dose received by all people on the sidewalk (shaded areas in Figure $B-6)$ as the shipment passes is given by

$$
\mathrm{ID}_{\perp}=\frac{2 \mathrm{~K}}{\overline{\mathrm{V}}} \cdot \operatorname{PedD} \cdot \mathrm{w}_{\mathrm{s}} \cdot \int_{\mathrm{d}+\mathrm{w}_{\mathrm{s}}}^{\infty}\left[\int_{\mathrm{x}}^{\infty} \frac{\mathrm{e}^{-\mu}{ }_{\mathrm{air}}^{\mathrm{r}} \cdot \mathrm{B}_{\mathrm{air}}(\mathrm{r}) \mathrm{dr}}{\mathrm{r}^{\sqrt{r^{2}-x^{2}}}}\right] \mathrm{dx}
$$

If it is assumed that people are stationary as the shipment passes, the expression for integrated dose to people moving parallel to the shipment for one block is

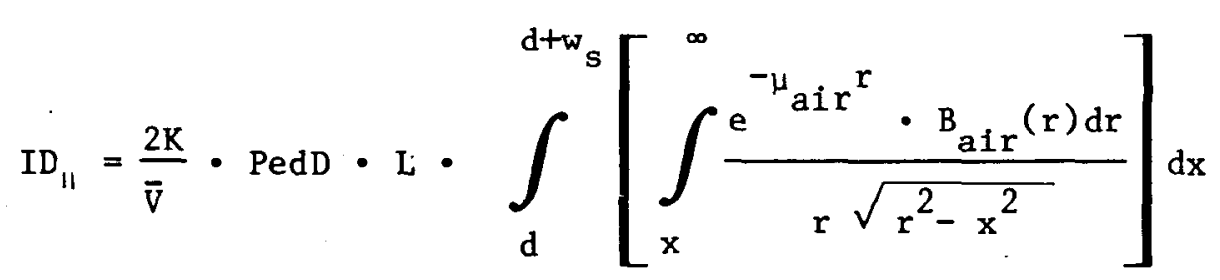

* The numerical analysis used to evaluate complex functions and integrals is discussed in Addendum 2 to this appendix.

**

Note that pedestrians moving parallel to the shipment in the crosswalk area are implicitly included in Eq. (9). 


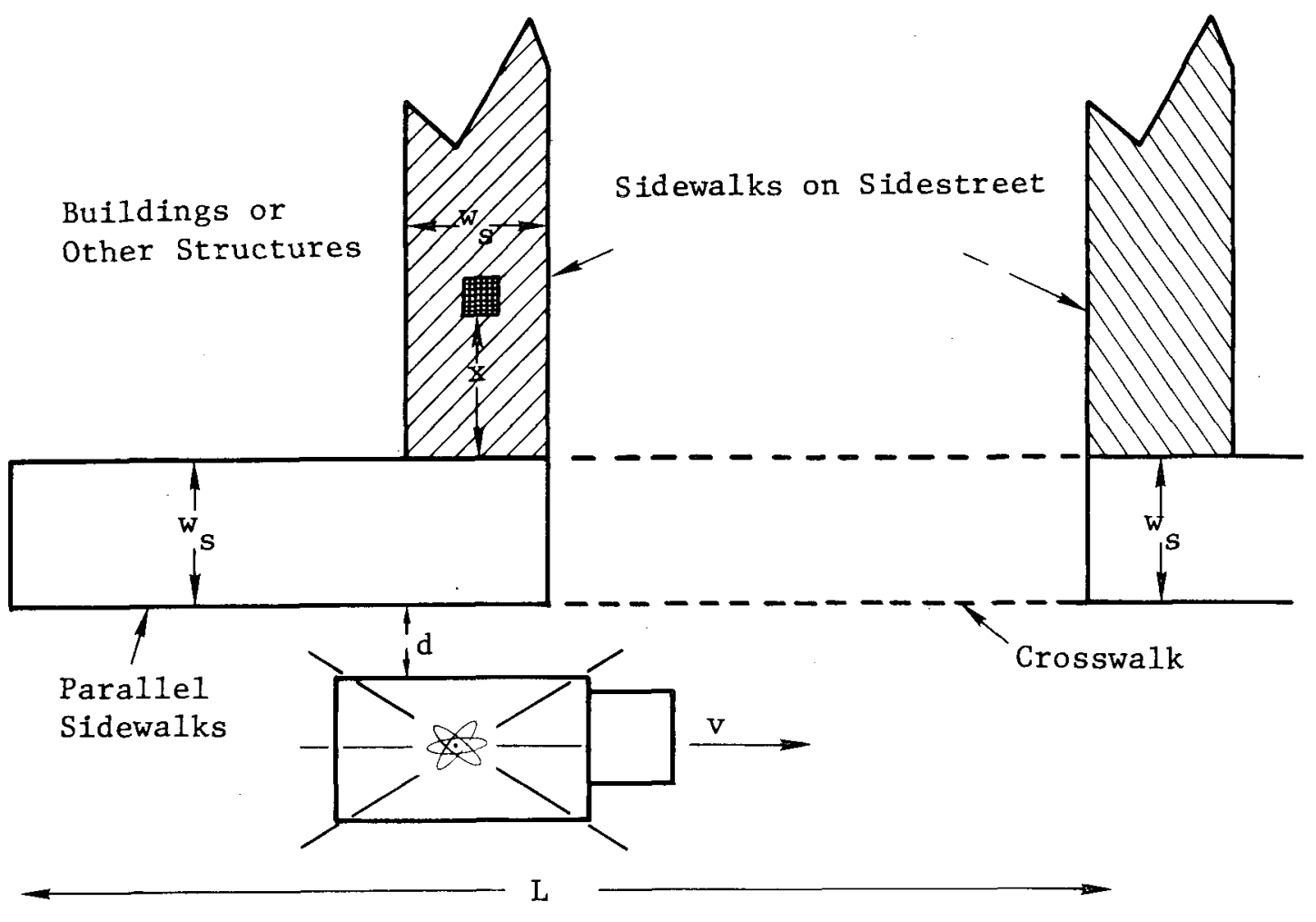

Figure B-6. Geometry for Incident-Free Dose to Pedestrians on Side Street

The ratio of the perpendicular and parallel components of pedestrian dose is formed by

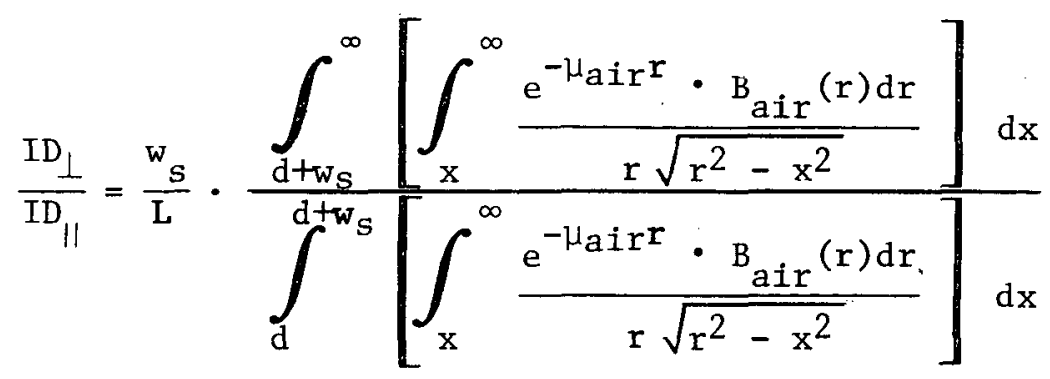

Assuming that an average block is 200 metres long, a sidewalk is 3 metres wide, and the closest distance of approach of a vehicle to the sidewalk is 3 metres, this ratio is approximately equal to 0.07 . Since shielding due to intervening structures will further decrease the dose received by pedestrians on sidestreets as the distance from vehicle to the intersection increases, ignoring perpendicular pedestrian flow on sidestreets does not introduce a significant underestimate of the dose to pedestrians.

The second aspect of the perpendicular pedestrian dose calculation is illustrated in Figure B-7. Here, the source is stationary and the people are moving at a speed $v_{p}$ across the intersection in front of the stopped vehicle. It can be shown that the ${ }^{p}$ dose received by a person walking past the stationary source at a perpendicular distance $x$ at a speed $v_{p}$ is the same as the dose received at the position of the vehicle if the source were moving by at a perpendicular distance $x$ at a speed $v_{0}$. If it is assumed that the vehicle is located at the center of the street, the tbtal dose received by pedestrians who walk across the street in the crosswalk nearest the vehicle can be expressed as 


$$
\mathrm{ID}_{\perp, 1}=\frac{2 \mathrm{~K}}{\mathrm{v}_{\mathrm{p}}} \cdot \mathrm{w}_{\mathrm{st}} \cdot \operatorname{PedD} \cdot \int_{\mathrm{d}}^{\mathrm{d}+\mathrm{w}_{\mathrm{s}}}\left[\int_{\mathrm{x}}^{\alpha_{1} \frac{\mathrm{e}^{-\mu} \operatorname{air}^{\mathrm{r}} \cdot \mathrm{B}_{\mathrm{air}}(\mathrm{r}) \mathrm{dr}}{\mathrm{r}^{2}-\mathrm{x}^{2}}}\right] \mathrm{dx}
$$

where

$$
\alpha_{1}=\sqrt{\left(w_{s t} / 2\right)^{2}+x^{2}} .
$$

Similarly, the dose to people in crosswalks on the far side of the street is

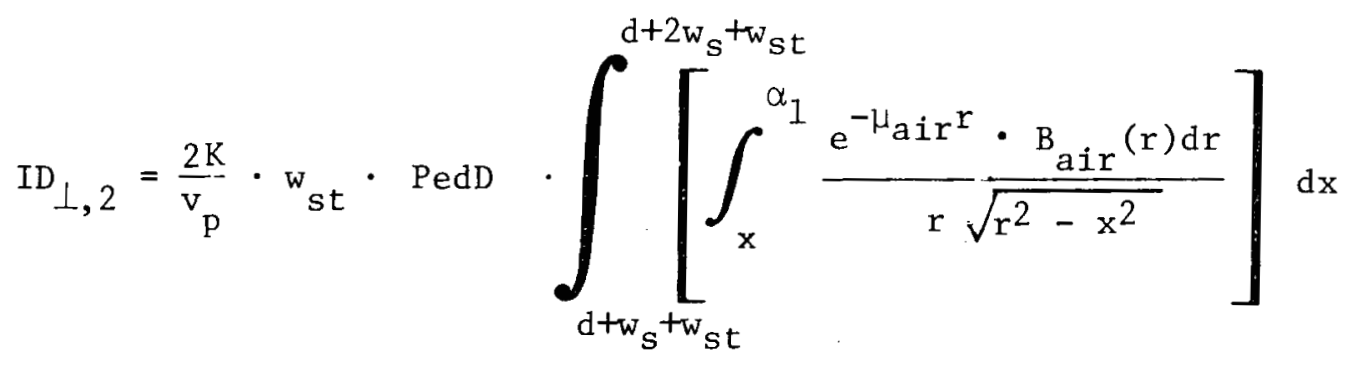

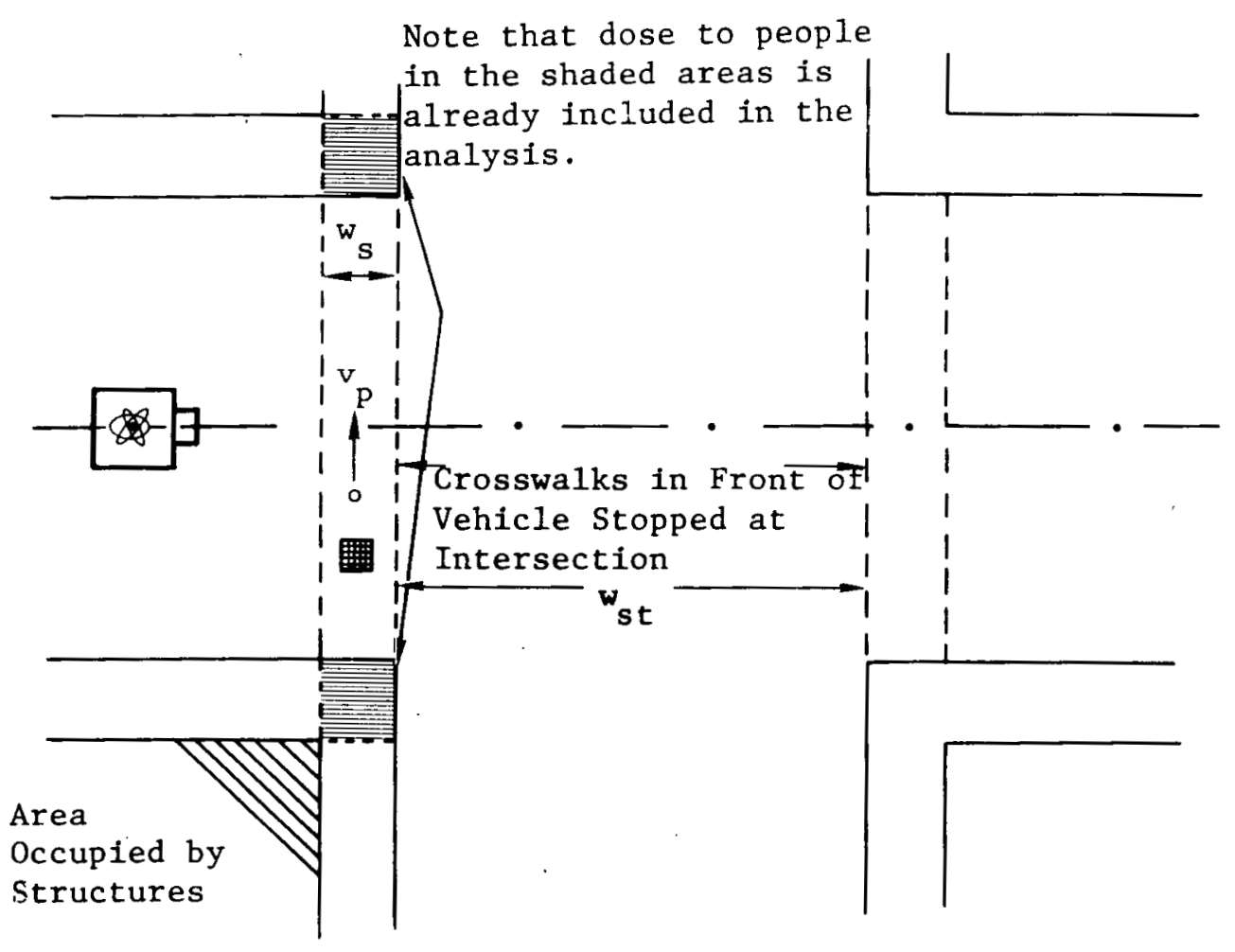

Figure B-7. Geometry for Incident-Free-Dose to Pedestrians in Crosswalks Ahead of Vehicle 
A ratio of these values to the dose received during parallel passage can now be formed:

$$
\frac{\mathrm{ID}_{\perp, 1+2}}{\mathrm{ID}_{\|}}=\frac{\overline{\mathrm{V}}}{\mathrm{v}_{\mathrm{p}}} \cdot \frac{\mathrm{w}_{\mathrm{st}}}{\mathrm{L}} \cdot \frac{\mathrm{ID}_{\perp, 1}+\mathrm{ID}_{\perp, 2}}{\mathrm{ID}_{\|}}
$$

If the previously mentioned values for $L$ and $w_{S}$ are used, and if values of 20 metres for $w_{s t}, 10$ metres for $d, 0.8 \mathrm{~m} / \mathrm{s}$ for $v_{p}$, and $6.7 \mathrm{~m} / \mathrm{s}$ for $\bar{v}$ are used, the ratio is approximately 0.1 . Note that this assumes that the truck stops adjacent to the crosswalk each time it reaches an intersection. This is conservative since the truck can stop anywhere along the block and probably will not be stopped at each intersection. The ratio drops off rapidly as the distance from the crosswalk increases ( 0.07 for 12 metres, 0.01 for 30 metres). Because of these factors, dose received by pedestrians in crosswalks is not included.

People in Buildings

Dose to people inside buildings is computed in a similar fashion to dose to pedestrians. Significant differences are (1) structural shielding is considered and (2) people are considered stationary inside buildings. The basic geometry is shown in Figure $B-8$.

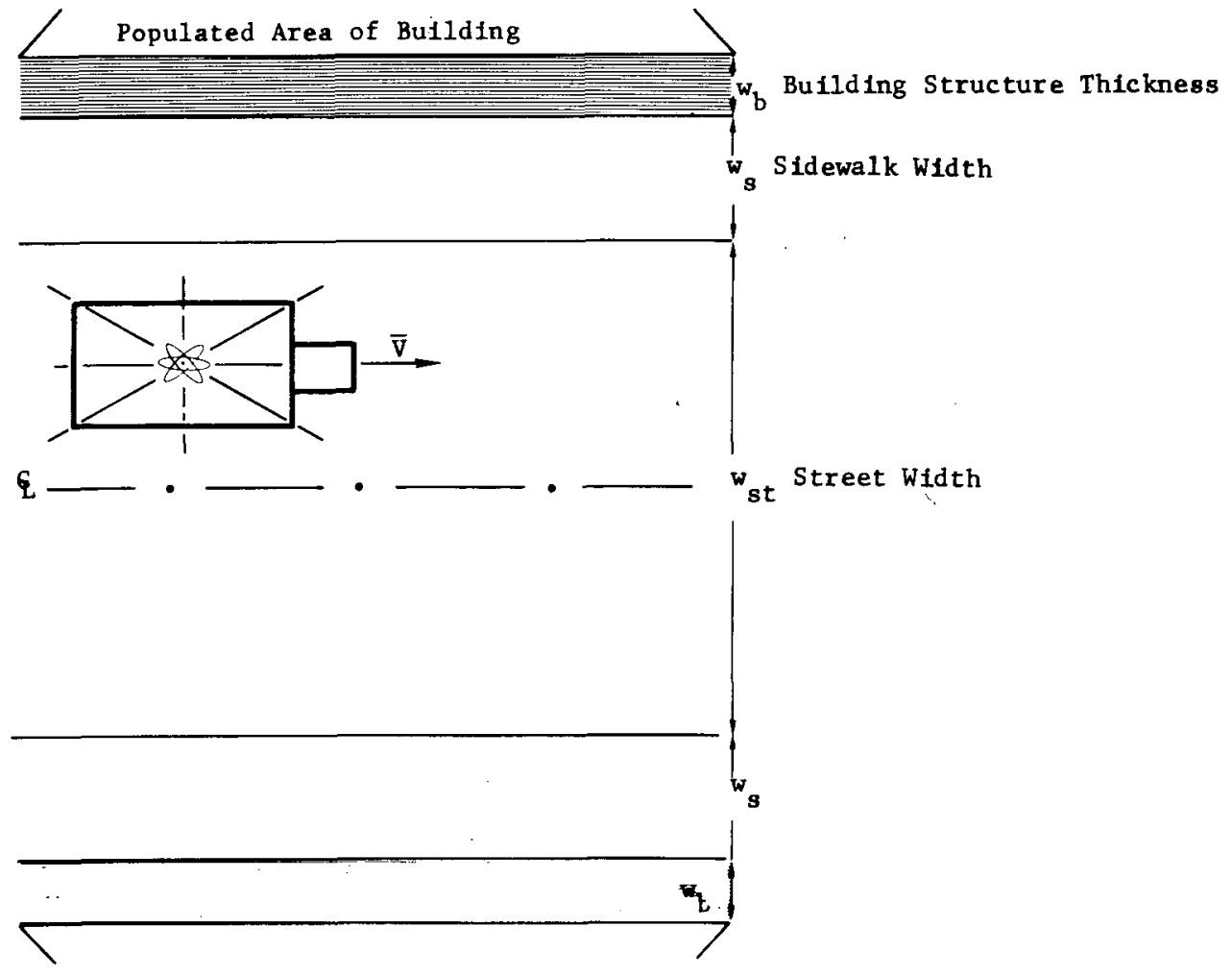

Figure B-8. Geometry for Incident-Free Dose to People in Buildings 
Building Characterization - In order to detail the development of this dose model, building types and materials must be considered. Initially, buildings are characterized by three parameters: principal construction material, wall thickness, and height.

Many single-family residences are either frame/stucco, using wall board mounted on 2 by 4 studs; or masonry with brick or concrete block and associated cosmetic finishings. This model assumes that single-family residences have shielding equivalent to either $20 \mathrm{~cm}$ of brick/concrete or 10 to $20 \mathrm{~cm}$ of wood and/or insulating materials. These dimensions are also typical of older, frame tenement dwellings or low-rise buildings (i.e., 3 to 5 floors) where the structural support requirements are minimal. In larger buildings with increased structural requirements, reinforced concrete is generally used. Large, older buildings probably have exterior walls of reinforced concrete, while new, high-rise buildings usually have structural support at the center or at corners, and large expanses of plate glass as the fronting material.

The model considers four thickness/material combinations:

1. 10 to $20 \mathrm{~cm}$ of wood,

2. 20 to $25 \mathrm{~cm}$ of brick/concrete,

3. $61 \mathrm{~cm}$ of concrete, and

4. $2 \mathrm{~cm}$ of plate glass. 5

Attenuation Coefficient - The linear absorption coefficient describes the attenuation of radiation in a given material by photoelectric effect, Compton scattering, and pair production. Values for this coefficient as a function of energy are shown in Table $B-1$ and plotted in Figure $B-9$.

Dose Buildup Factor - When high energy electromagnetic radiation passes through an attenuating medium, secondary gamma radiation may be produced by Compton scattering. Thus, a dose buildup (caused by the additional dose from secondary radiation) can occur. Reference 6 suggests the following mathematical expression to describe this dose buildup:

$$
B\left(E, \mu, w_{b}\right)=A_{1} e^{-\alpha{ }^{\mu w_{b}}}+A_{2} e^{-\alpha{ }_{3} \mu w_{b}}
$$

where

$$
\begin{aligned}
E= & \text { incident photon energy }(\mathrm{MeV}) \\
\mu= & \text { attenuation coefficient }\left(\mathrm{m}^{-1}\right)\left(\text { the product } \mu \mathrm{w}_{\mathrm{b}}\right. \text { is } \\
& \text { called relaxation length) } \\
\mathrm{A}_{1}, \mathrm{~A}_{2}, \alpha_{2}, \alpha_{3}= & \text { empirical constants } \\
\mathrm{w}_{\mathrm{b}}= & \text { wall thickness (metres) }
\end{aligned}
$$


Table $\mathrm{B}-1$

Attenuation Coefficients for Various Materials $\left(\mathrm{m}^{-1}\right)$

Photon

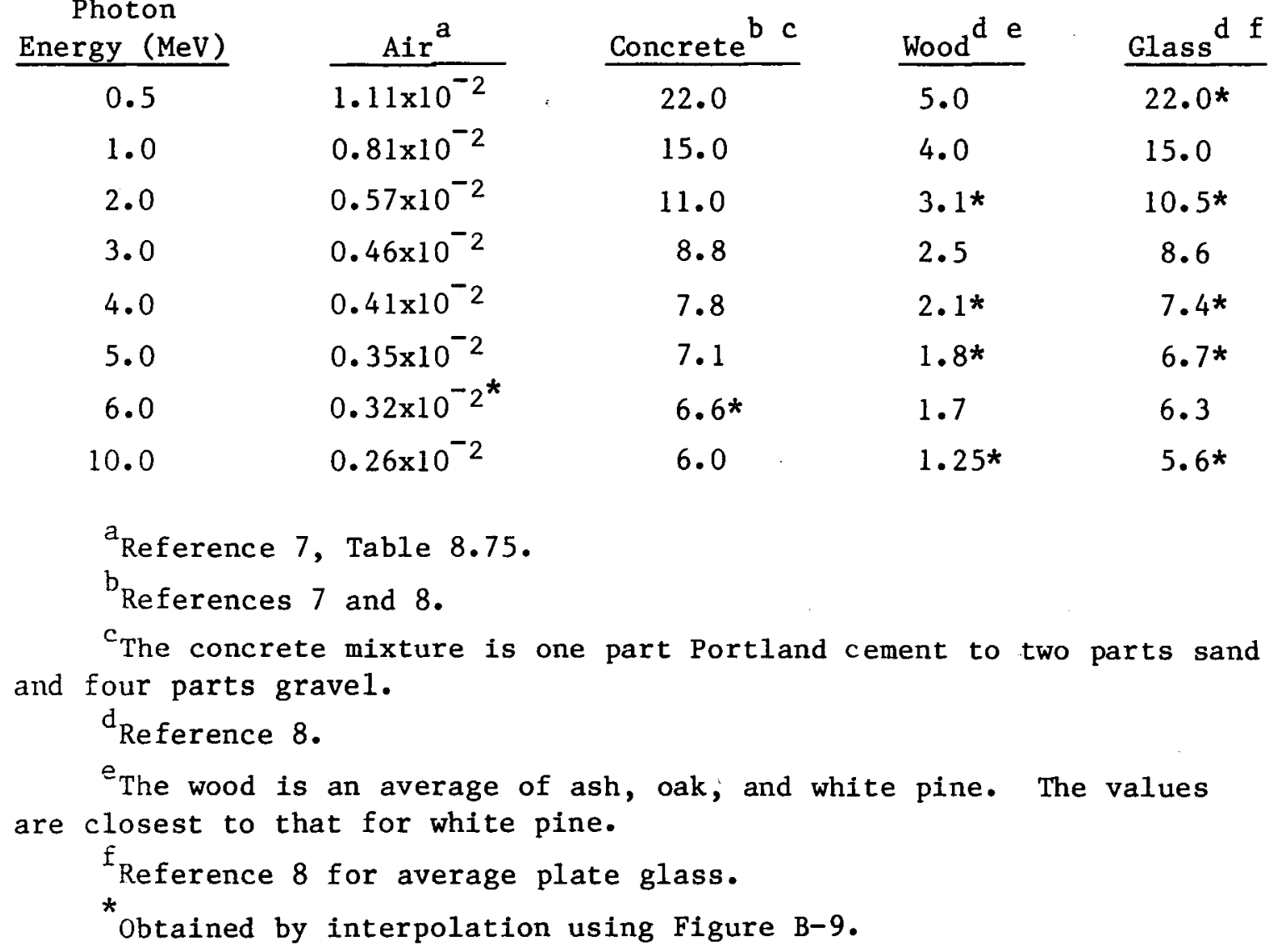

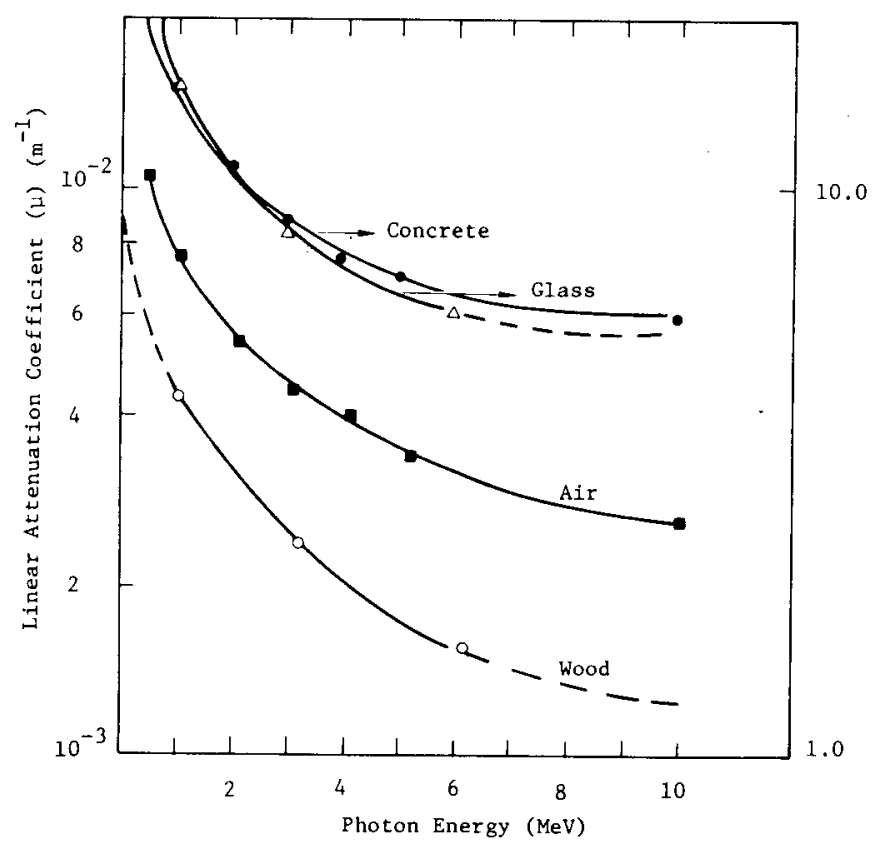


Reference 6 provides curves for $A_{1}, A_{2}, \alpha_{2}$, and $\alpha_{3}$ for some materials. The curves for concrete are included in Figure B-10. Reference 5 provides buildup factor data for different types of glass and various relaxation lengths. Using Type-8365 glass and $\mu \mathrm{w}_{b}=2$, buildup factors for thin barriers of glass can be estimated.

Buildup factors for wood are taken from Reference 6 using $\mu_{\mathrm{w}}=1.0$. Buildup factors for air are of the form

$$
B(r)=1+C(E) r
$$

where $C(E)$ is an energy-dependent factor. Values for $C(E)$ have been chosen to make the values for $B(r)$ compatible with those suggested in Reference 6 .

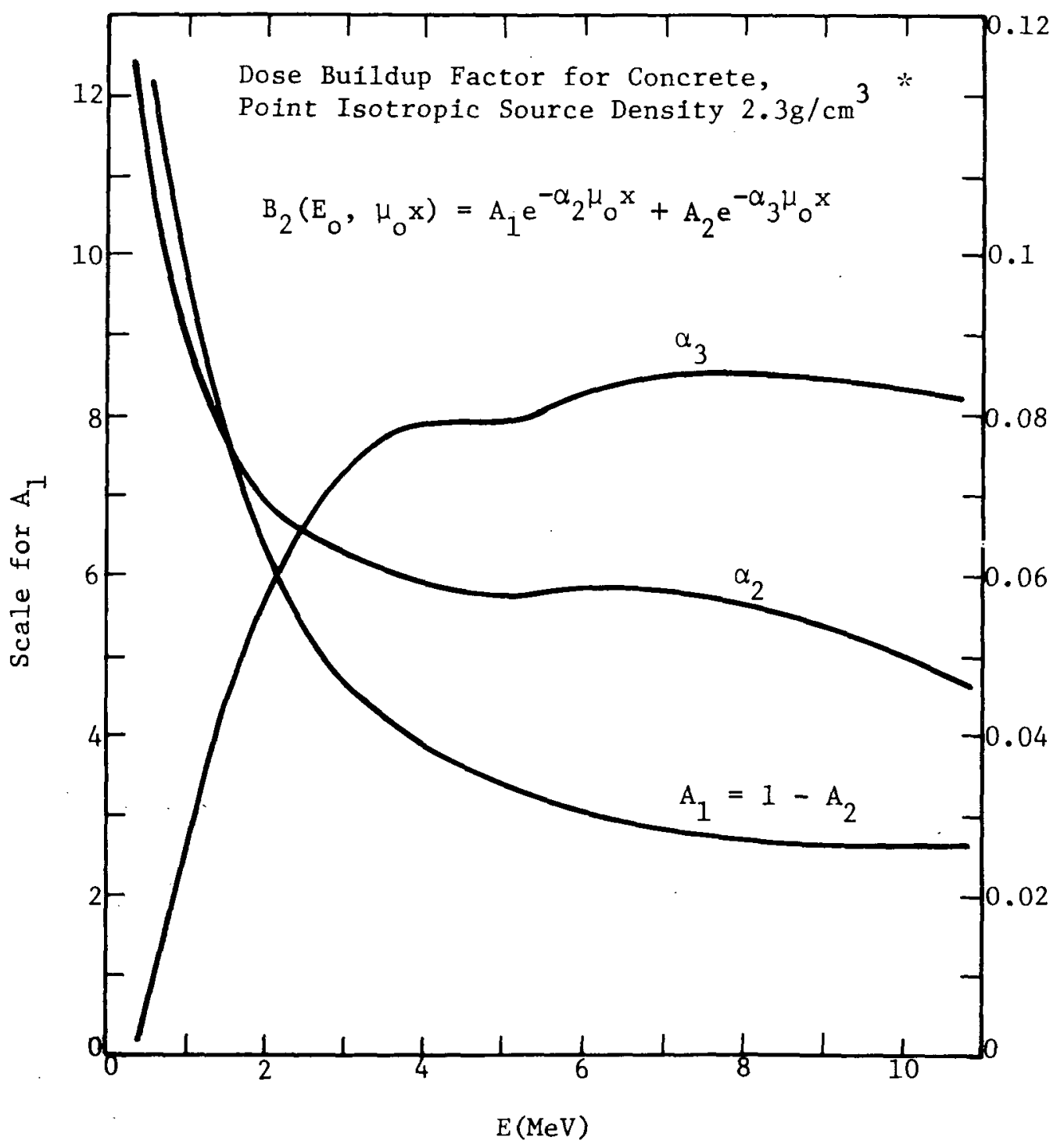

*Obtained from Reference 6

Figure B-10. Dose Buildup Factor for Concrete 
Multiple Slab Geometry -- A multiple slab geometry arises because the photon flux must penetrate a "slab" of air and then a "slab" of building material before it reaches the population of concern, as shown in Figure B-11. Reference 6 suggests that for two slabs of a light material (e.g., air) followed by a heavier material (e.g., wall) it is most accurate to use the buildup factor for the heavier material alone, regardless of slab thickness. Thus this technique is used.

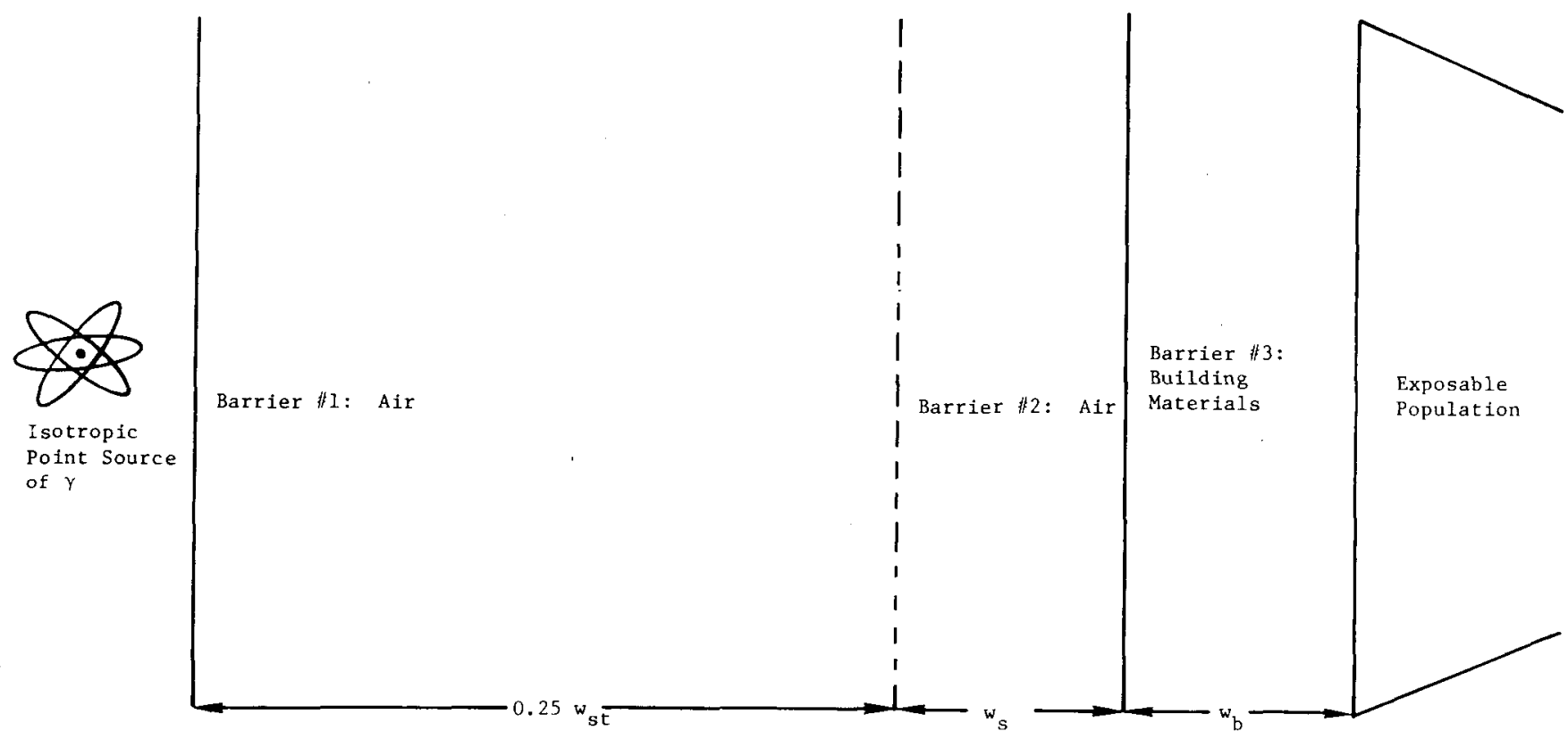

Figure B-11. Multiple Slab Geometry

Attenuation Due to Oblique Impingement -- When radiation impinges perpendicularly on a shield, the scattered radiation which penetrates the shield always travels farther in the shield than the remaining uncollided flux. The assumption that radiation obliquely incident on a shield is attenuated as if it were normally incident on a shield whose thickness is equal to the effective slant distance may lead to significant dose underestimates since scattered radiation may play an important role. Using a technique described in Reference 6, a factor can be derived for the buildup/ attenuation tradeoff for oblique impingement: Figure B-12 shows a parametric plot of the Peebles dose buildup factor $(D B F)$ as a function of relaxation length and angle of incidence. By taking the ratio of the Peebles DBF for an arbitrary angle to that for the straight-through path $(\cos \theta=1.0)$, the effect of oblique impingement can be evaluated. If it is assumed that multifloor buildings have concrete walls with an average wall thickness of $61 \mathrm{~cm}$, a relaxation length of approximately 4 can be computed. By using this value on Figure B-12 and replotting the ratio discussed above as a function of incidence angle, an effective attenuation factor, called the obliqueness factor (OF), can be plotted as shown on Figure B-13. The basic information on Figure $\mathrm{B}-12$ is derived from $6-\mathrm{MeV}$ photon buildup in iron. However, the use of the technique described above is assumed to minimize the dependence of the derived obliqueness factor on both the energy and medium used to derive Figure B-12. 


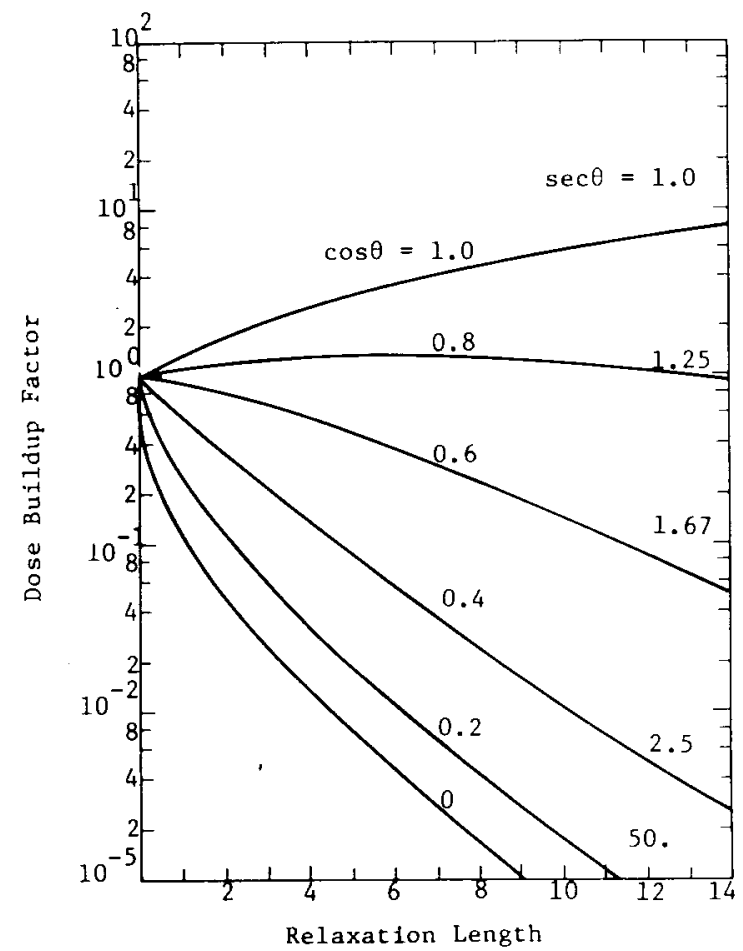

Figure B-12. Peebles Buildup Factor (Iron Buildup Used)

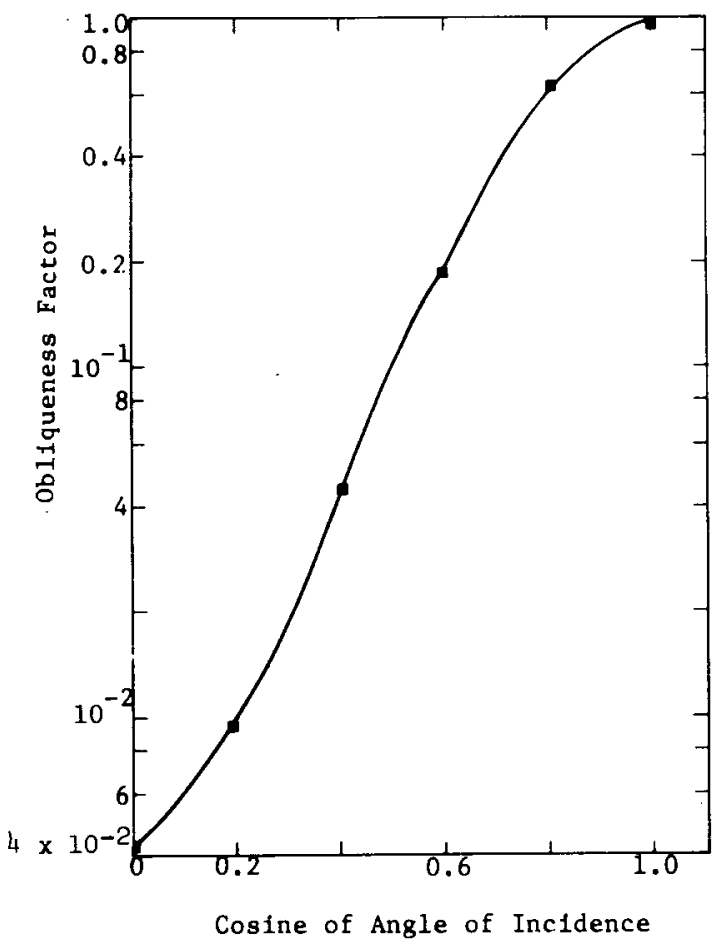

Figure B-13. Ob1iqueness Factor 
The angle between the center of a particular floor and a point in the center of the street directly in front of the building would be used if the vehicle always stayed in front of the building. In fact, the angle subtended by a ray path must also account for the distance of the vehicle down the street from the point directly in front of the building, as shown in Figure B-14. Based on this geometry, the oblique angle of impingement between the ray path and a line normal to the building at the particular floor is given by

$$
\theta=\frac{\pi}{2}-\tan ^{-1}\left(\frac{\mathrm{w}_{s t} / 2+\mathrm{w}_{s}}{\sqrt{\left(r^{\prime}\right)^{2}+((n-0.5) h)^{2}}}\right)
$$

where

$$
\begin{aligned}
& \mathrm{n}=\text { number of floors in buildings in cell } \\
& \mathrm{h}=\text { height per floor (metres) }
\end{aligned}
$$

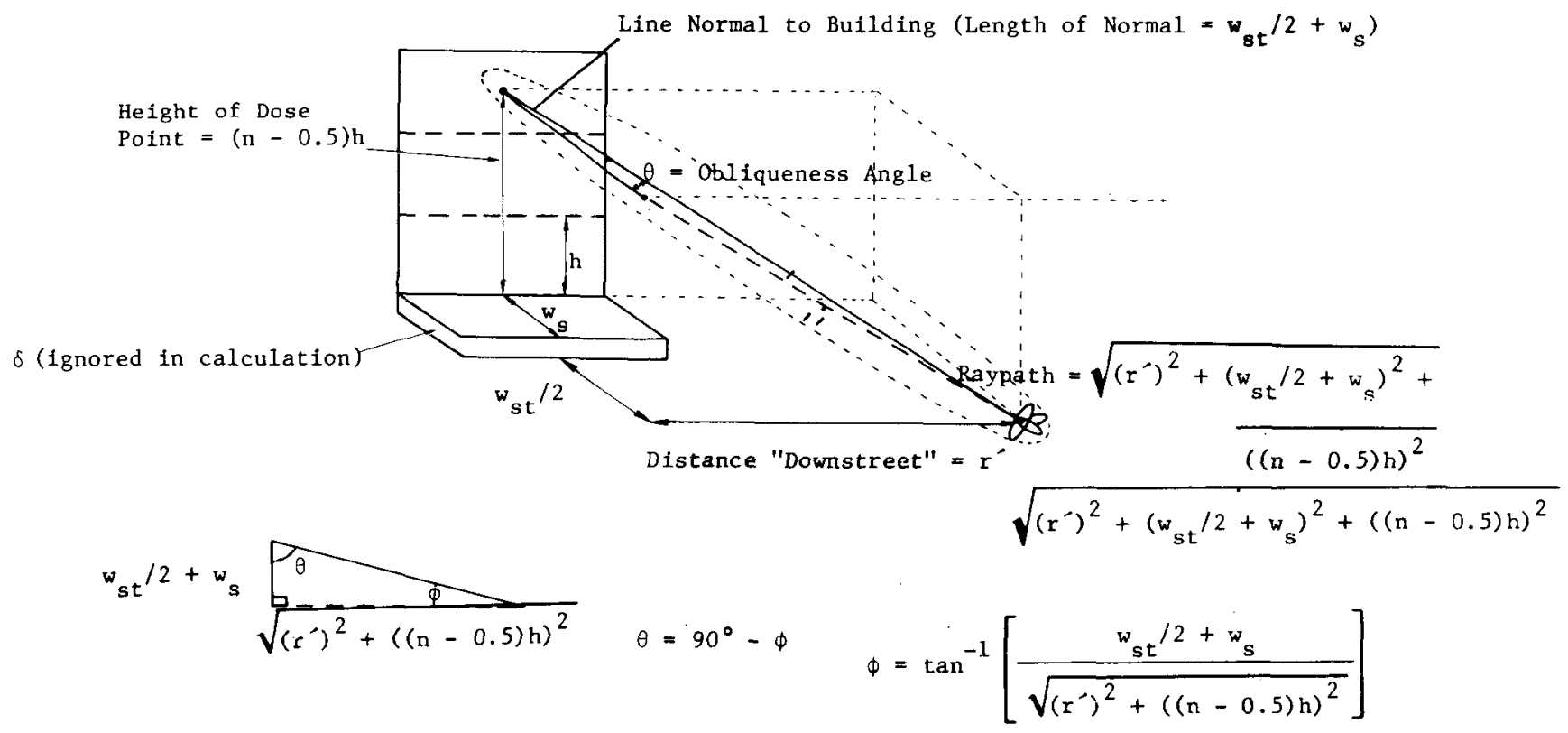

Figure B-14. Obliqueness Geometry

The slant distance from the vehicle to the point of oblique impingement is given by

$$
r=\sqrt{\left(r^{\prime}\right)^{2}+((n-0.5) h)^{2}+\left(w_{s t} / 2+w_{s}\right)^{2}}
$$

The obliqueness factor plotted in Figure B-13 can be replotted as a function of slant distance $r$. The result is a family of curves for various values of the sum $\left(w_{s t} / 2+w_{s}\right)$ which provides the obliqueness factors used in the dose computations. 


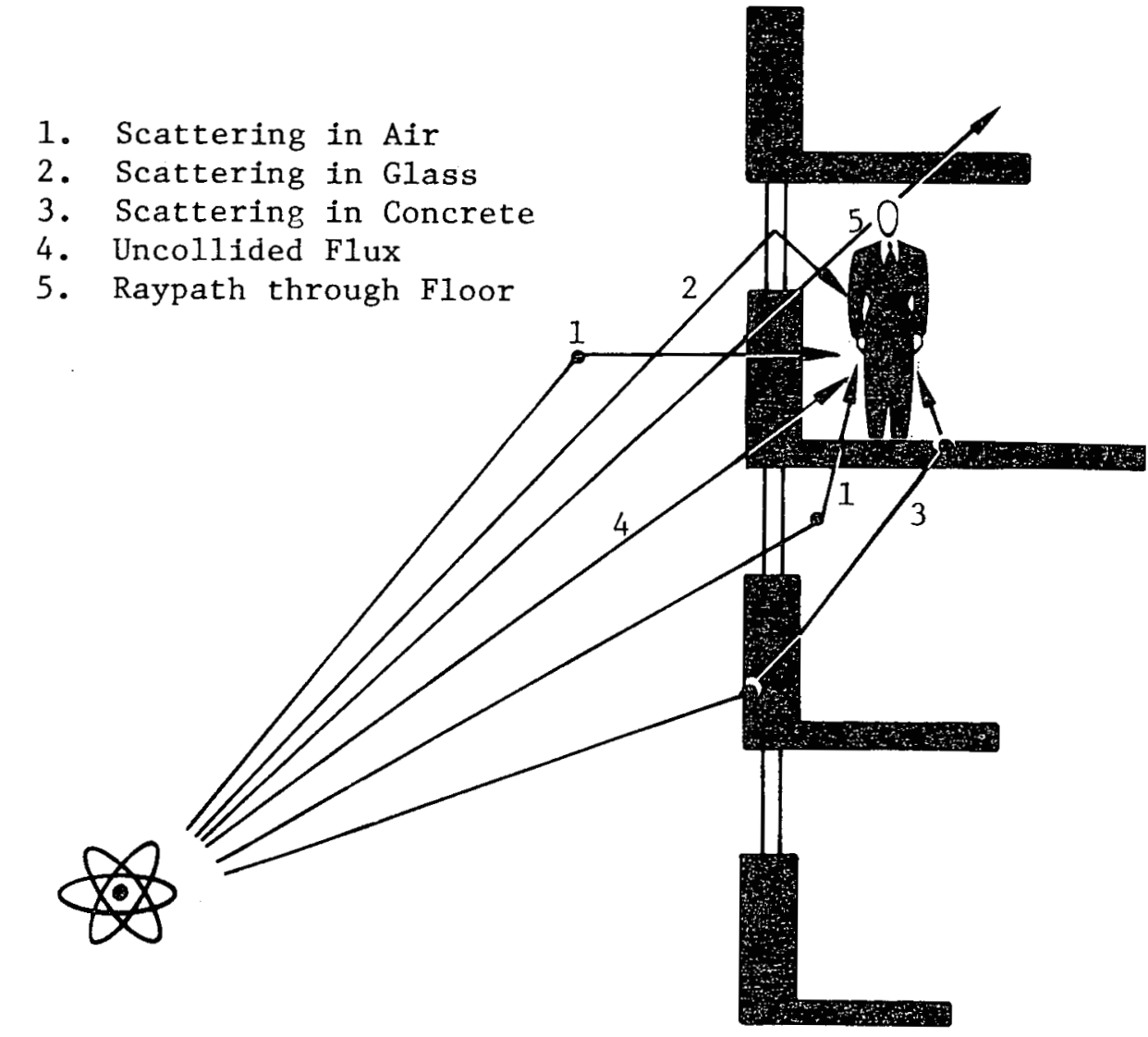

Figure B-15. Raypaths to People in Buildings

In addition, total cell area can be specified by

$$
A=k^{2}\left(\Delta b+w_{s t}+2 w_{s}\right)^{2}
$$

Solving these equations for $\mathrm{k}^{2}$ and $\Delta \mathrm{b}$, and substituting values into

$$
P_{T}=4 \Delta b^{2}
$$

for total building perimeter in the cell, it can be shown that

$$
P_{T}=\frac{4 A \sqrt{f_{b}}}{\left(w_{s t}+2 w_{s}\right)}\left(1-\sqrt{f_{b}}\right)
$$

Thus, the equivalent linear population density of people in buildings on each floor is given by 


$$
\left(\begin{array}{c}
\text { linear } \\
\begin{array}{c}
\text { population } \\
\text { density }
\end{array}
\end{array}\right)=\frac{\frac{(P D+T C)}{n} \cdot A}{\frac{4 A \sqrt{f_{b}}\left(1-\sqrt{f_{b}}\right)}{\left(w_{s t}+2 w_{s}\right)}}=\frac{(P D+T C)\left(w_{s t}+2 w_{s}\right)}{4\left(\sqrt{f_{b}}-f_{b}\right) n}
$$

Using Eq. (27) and the integration technique in Eq. (6), the following expression for integrated dose to people in buildings can be derived:

$$
\begin{gathered}
I D=\frac{4 \cdot Q_{1} \cdot K_{o} \cdot T I \cdot P P S}{\bar{V}} \cdot \frac{(P D+T C)\left(w_{s t}+2 w_{s}\right)}{4\left(\sqrt{f_{b}}-f_{b}\right) n} \cdot L \cdot \\
e^{-\mu_{b} w_{b}} \cdot B_{b}\left(w_{b}\right) \cdot \sum_{i=1}^{n}\left[\int_{\beta_{1}}^{\infty} \frac{e^{-\mu_{a i r} r} \cdot O F_{i}(r) d r}{r^{2} \sqrt{r^{2}-\beta_{1}^{2}}}\right]
\end{gathered}
$$

where

$$
\beta_{1}=\sqrt{(h(i-0.5))^{2}+\left(w_{s t} / 2+w_{s}\right)^{2}}
$$

The integral in Eq. (28) is evaluated numerically in Addendum 2 of this appendix. The derivation assumes that the shipment moves from $+\infty$ to $-\infty$ while exposing people in the cell. This approximates the condition where people in one cell may receive some dose while the source is in an adjacent cell.

People in Vehicles

During a shipment, people in vehicles sharing the link with the transport vehicle will be exposed to external penetrating radiation from the package. Two subgroups may be exposed: people in vehicles moving in the same direction as the shipment, and people in vehicles moving in the direction opposite the shipment (Figure B-16). The dose to people moving at right angles to the shipment can be shown to be negligible using arguments similar to those used in analyzing dose received by pedestrians moving perpendicular to the shipment.

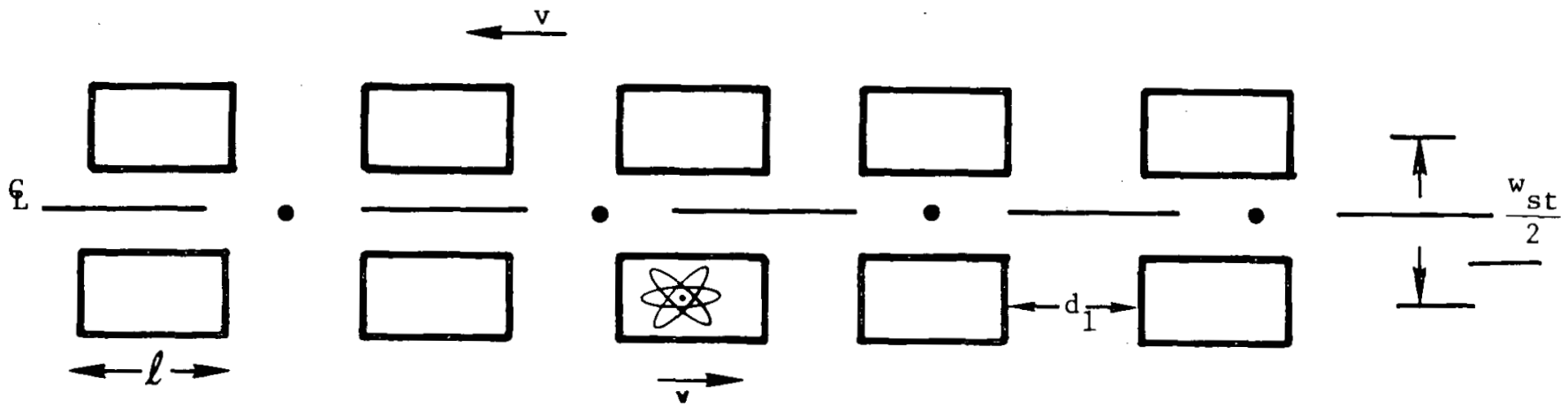

Figure B-16. Incident-Free Dose to People in Vehicles 
Before these radiological factors can be used to compute population dose, the distribution of people in buildings must be considered. It is assumed that all people who are residents, workers, or transient population are inside buildings. This is a conservative assumption since some are clearly accounted for by estimates of pedestrian density and people in vehicles. The expression used to calculate the population density in buildings is given by

$$
P P B=(P D+T C) \frac{A}{A f_{b}}
$$

where

$$
\begin{aligned}
& \mathrm{PPB}= \text { population density inside building in cell } \\
& \mathrm{PD}= \text { overall population density in cell (residents } \pm \text { commuters, } \\
&\text { persons } \left./ \mathrm{km}^{2}\right) \\
& \mathrm{A}= \text { area of cell }\left(\mathrm{km}^{2}\right) \\
& \mathrm{TC}= \text { transient population density (persons } / \mathrm{km}^{2} \text { ) } \\
& \mathrm{f}_{\mathrm{b}}=\text { fraction of cell area occupied by buildings }
\end{aligned}
$$

If it is assumed that people are uniformly distributed on a floor-to-floor basis, the number of people per floor is given by

$$
P P F=\frac{P P B}{n}\left({A f_{b}}_{b}\right)
$$

Actual ray-path geometry can be complex, as shown in Figure B-15. Radiation can pass directly through building materials or can have multiple scatterings in air, windows, or wall materials. To account for the population distribution on each floor and the heterogeneity of the outer wall, it is assumed that all people are immediately adjacent to the inside of the outer wall of the building. Because no allowance is made for shielding by interior partitions, furnishings, or floor materials, the analysis is conservative.

The number of people passed by a shipment in an incremental length of travel $\Delta r$ on a given floor in the cell can be obtained by multiplying the linear population density per floor (that is, people passed per kilometre of travel) by the incremental distance traveled. The equivalent linear population density per floor is obtained by dividing the total number of people on a specific floor in the cell by the total building perimeter of the cell. A value of total building perimeter can be derived from the cell geometry if it is assumed that buildings are uniform throughout the cell and have square cross sections.

If the cell contains $\mathrm{k}^{2}$ buildings of width $\Delta \mathrm{b}$, then the fraction of total cell area occupied by buildings is given by

$$
f_{b}=\frac{k^{2}(\Delta b)^{2}}{k^{2}\left(\Delta b+w_{s t}+2 w_{s}\right)^{2}}
$$


The model for dose to people moving in the same direction as the shipment assumes that the transport vehicle moves between intersections at the same average velocity as the rest of the traffic. Under this assumption, traffic can be modeled as a stationary set of vehicles with some specific vehicle separation distance, $d_{1}$, lined up adjacent to, ahead of, and behind the shipment vehicle. Depending upon traffic conditions, the transport vehicle will stop at some or all of the intersections along its route. When traffic stops under these circumstances, the vehicle pattern can again be modeled as a stationary set of vehicles with a shorter separation distance, $\delta$.

The following additional assumptions are made:

- Vehicles accelerate and decelerate instantaneously so that the separation distance changes from $d_{1}$ to $\delta$ instantaneously.

- No shielding is provided by vehicles.

- People are located at the center of vehicles.

- Traffic is uniform across lanes on each side of street.

Using these assumptions, the total integrated dose to each person in a vehicle at a distance $d_{l}$ from the transport vehicle during the cruising phase is given by

$$
I D\left(d_{1}\right)=\left[K_{0} \cdot \operatorname{TI} \cdot \operatorname{PPS} \cdot \frac{e^{-\mu \operatorname{air}\left(d_{1}+l\right)} \cdot B_{\operatorname{air}}\left(d_{1}+\ell\right)}{\left(d_{1}+\ell\right)^{2}}\right] \cdot\left[\frac{L}{V_{c}}\right]
$$

where the first factor represents dose rate at a distance $d_{1}+\ell$ and the second represents the time spent at that distance. By noting that for values less than 100 metres, the product $e^{-\mu_{a i r}\left(d_{1}+l\right) \cdot B}\left(d_{i}+l\right)$ is approximately equal to 1.0 , the expression given in Eq. (27) can be simplified by substituting 1.0 for this product for the remainder of the derivation. This results in less than a $10 \%$ overestimate of integrated dose in all cases. The value for cruising speed $V$ can be derived from average velocity, $\bar{v}$, knowing certain cell and traffic parameters. Initially, it is noted that

$$
\overline{\mathrm{V}}=\frac{\mathrm{L}}{\frac{\mathrm{L}}{\mathrm{V}_{\mathrm{c}}}+\left(\xi \cdot \frac{\mathrm{L}}{\mathrm{D}^{\prime}} \cdot \Omega\right)}=\frac{1}{\frac{1}{\mathrm{~V}_{\mathrm{c}}}+\frac{\xi \Omega}{\mathrm{D}^{\prime}}}
$$

where

$$
\begin{aligned}
\overline{\mathrm{V}} & =\text { average traffic velocity }(\mathrm{m} / \mathrm{s}) \\
\mathrm{L} & =\text { total distance traveled in cell (metres) } \\
\mathrm{V}_{\mathrm{c}} & =\text { crusing velocity (metres per second) } \\
\mathrm{L} / \mathrm{V}_{\mathrm{c}} & =\text { time required to drive across cell (seconds) } \\
\xi & =\text { fraction of intersections at which vehicle stops } \\
\Omega & =\text { delay time at intersections (seconds) }
\end{aligned}
$$




$$
\begin{aligned}
D^{-} & =\text {average block length (metres) } \\
\xi \Omega / D^{-} & =\text {time spent stopped at intersections (seconds) }
\end{aligned}
$$

By noting that average block length is equal to the sum of building width, street width, and twice sidewalk width and substituting for $\Delta \mathrm{b}$ in Eq. (23), average block length can be shown to be equal to

$$
\frac{w_{s t}+2 w_{s}}{1-\sqrt{f_{b}+f_{o}}}
$$

where $f_{0}=$ fraction of open area in the cell and where $f_{b}$ in Eq. (23) is assumed to include all nonstreet area.

Substituting Eq. (31) into Eq. (30) and solving the resulting expression for $\mathrm{V}_{\mathrm{C}}$ yields

$$
\mathrm{v}_{\mathrm{c}}=\frac{1}{\frac{1}{\overline{\mathrm{V}}}-\frac{\xi \cdot \Omega \cdot\left(1-\sqrt{f_{b}+\mathrm{f}_{\mathrm{o}}}\right)}{\mathrm{w}_{\mathrm{st}}+2 \mathrm{w}_{\mathrm{s}}}}
$$

In order to more accurately calculate dose to people in vehicles, the phenomenon of platooning must be considered. Taking into account the limitation imposed by average street width, it is assumed that the dose to people in vehicles is only accumulated by people in vehicles in the two lanes on each side linearly adjacent to the lane in which the transport vehicle is traveling. In addition, up to two vehicles directly ahead of and behind the transport vehicle are also included. With this in mind, the intersection situation can be visualized as shown in Figure B-17, with a maximum of 24 vehicles "at risk." A "platooning factor" can be derived to adjust the maximum possible dose for the actual number of vehicles present. This is done by first computing the maximum integrated dose for a total cluster of five full lanes:

$$
\begin{aligned}
\mathrm{ID}= & \mathrm{Q} \cdot \mathrm{K}_{\mathrm{O}} \cdot \mathrm{TI} \cdot \mathrm{PPS} \cdot \mathrm{PPV} \cdot \Delta \mathrm{T} \cdot \\
& {\left[\left(\begin{array}{c}
\text { inner group } \\
\text { distance and } \\
\text { attenuation factor }
\end{array}\right)+\left(\begin{array}{c}
\text { middle group } \\
\text { distance and } \\
\text { attenuation factor }
\end{array}\right)+\left(\begin{array}{c}
\text { outer group } \\
\text { distance and } \\
\text { attenuation factor }
\end{array}\right)\right] }
\end{aligned}
$$

where

$$
\begin{aligned}
Q & =\text { general units conversion factor } \\
\Delta T & =\text { generalized time } \\
P P V & =\text { number of people per vehicle }
\end{aligned}
$$




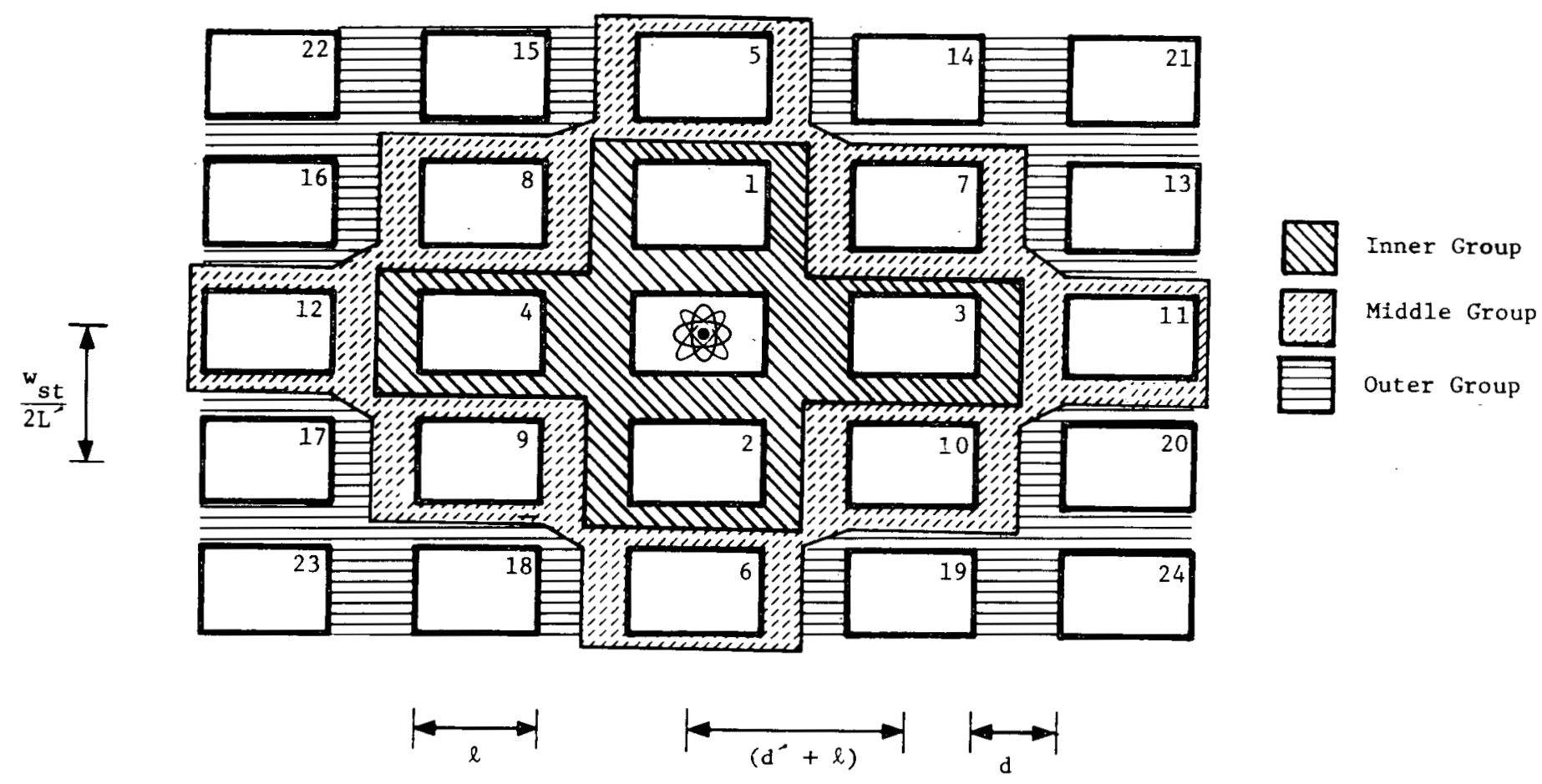

Figure B-17. Model of Dose to People in Vehicles Including the Phenomenon of Platooning

Using the simplified version of Eq. (29) and Figure B-17, the three distance and attenuation factors can be specified as

$$
\begin{aligned}
& (\text { IGDAF })=\frac{2}{\left(d_{1}+l\right)^{2}}+\frac{2}{\left(\frac{w_{s t}}{2 L}\right)^{2}} \\
& (\text { MGDAF })=\frac{2}{\left(\frac{w_{s t}}{L}\right)^{2}}+\frac{2}{\left(2\left(d_{1}+\ell\right)\right)^{2}}+\frac{4}{\left(d_{1}+\ell\right)^{2}+\left(\frac{w_{s t}}{L}\right)^{2}} \\
& (\text { OGDAF })=\frac{4}{\left(d_{1}+l\right)^{2}+\left(\frac{w_{s t}}{L}\right)^{2}}+\frac{4}{\left(2\left(d_{1}+l\right)\right)^{2}+\left(\frac{w_{s t}}{2 L}\right)^{2}}+ \\
& \frac{4}{\left(2\left(d_{1}+l\right)\right)^{2}+\left(\frac{w_{s t}}{L}\right)^{2}}
\end{aligned}
$$


If $R$ is defined as $\frac{\left(w_{s t} / L^{-}\right)^{2}}{\left(d_{1}+\ell\right)^{2}}$, then

$$
\begin{aligned}
(I+M+0)= & \frac{2}{\left(d_{1}+\ell\right)^{2}}+\frac{2}{\frac{R}{4}\left(d_{1}+\ell\right)^{2}}+\frac{2}{R\left(d_{1}+l\right)^{2}}+\frac{0.5}{\left(d_{1}+l\right)^{2}}+ \\
& \frac{4}{\left.\left(d_{1}+\ell\right)^{2}+\frac{R}{4} d_{1}+\ell\right)^{2}}+\frac{4}{\left(d_{1}+l\right)^{2}+R\left(d_{1}+l\right)^{2}}+ \\
& \frac{4}{4\left(d_{1}+\ell\right)^{2}+\frac{R\left(d_{1}+\ell\right)^{2}}{4}+\frac{4}{4\left(d_{1}+\ell\right)^{2}+R\left(d_{1}+\ell\right)^{2}}} \\
= & \frac{1}{\left(d_{1}+\ell\right)^{2}}\left(2.5+\frac{10}{R}+\frac{20}{(4+R)}+\frac{4}{(1+R)}+\frac{16}{(16+R)}\right)
\end{aligned}
$$

Eq. (33) can now be modified as follows:

$$
\begin{aligned}
\mathrm{ID}= & \mathrm{Q} \cdot \mathrm{K}_{\mathrm{O}} \cdot \mathrm{TI} \cdot \mathrm{PPS} \cdot \mathrm{PPV} \cdot \Delta \mathrm{T} \cdot \frac{1}{\left(\mathrm{~d}_{1}+\ell\right)^{2}} . \\
& {\left[2.5+\frac{10}{\mathrm{R}}+\frac{4}{\mathrm{R}+1}+\frac{20}{\mathrm{R}+4}+\frac{16}{\mathrm{R}+16}\right] }
\end{aligned}
$$

This equation gives the maximum dose possible assuming that the platoon of vehicles contains 24 vehicles as shown in Figure B-17. If there is less than the maximum number of vehicles present, the integrated dose of Eq. (34) should be reduced accordingly. This is modeled by assuming that the available vehicles fill the spaces in the order shown on Figure B-17.

The number of vehicles available to fill space is computed by evaluating the number of one-way vehicles moving in each block in the cell:

$$
\begin{aligned}
& \left(\begin{array}{c}
\text { one-way } \\
\text { vehicles per } \\
\text { block }
\end{array}\right)=\left[\frac{\left(\begin{array}{c}
\text { one-way vehicles } \\
\text { per cel1 }
\end{array}\right)}{\left(\begin{array}{c}
\text { total one-way } \\
\text { street length } \\
\text { per cell }
\end{array}\right)} \cdot\left(\begin{array}{c}
\text { average } \\
\text { velocity }
\end{array}\right)\right] \cdot\left[\frac{\text { block length }}{\text { average velocity }}\right] \\
& =Q_{2} \cdot\left[\frac{\frac{N}{2}}{\frac{A f}{2 w_{s t}}} \cdot \bar{V}\right] \cdot\left[\frac{\frac{w_{s t}+2 w_{s}}{1-\sqrt{f_{b}+f_{o}}}}{\bar{V}}\right] \\
& =\frac{Q_{2} \cdot N \cdot w_{s t} \cdot\left(w_{s t}+2 w_{s}\right)}{A \cdot f_{s t} \cdot\left(1-\sqrt{f_{b}+f_{o}}\right)}
\end{aligned}
$$


where

$$
\begin{aligned}
& N=\text { total number of vehicles in a cell at any given time } \\
& Q_{2}=\text { units conversion factor }\left(10^{-6} \mathrm{~km}^{2} / \mathrm{m}^{2}\right)
\end{aligned}
$$

The reduced integrated dose resulting from a reduced number of available vehicles is related to the maximum dose (with 24 vehicles present) by the platooning factor $f$. If the number of available vehicles exceeds $24, \mathrm{f}$ is set equal to 1 . Note that $\mathrm{f}$ will take on different values for stopped vehicles and moving vehicles and will vary from cell to cell and time span to time span as values of $\delta, d_{1}, w_{s t}$, and $L$ ' change. General expressions for platooning factors for varying numbers of available vehicles are given in Table B-2. As might be suspected, most of the dose is received by people in adjacent vehicles, particularly those in laterally adjacent vehicles. This becomes more significant as vehicle separation increases.

By incorporating these assumptions into both the cruising and stopped phases, the final form used to compute the "same direction" dose is obtained:

$$
\begin{aligned}
\mathrm{ID}= & \mathrm{Q}_{3} \cdot \mathrm{K}_{\mathrm{o}} \cdot \mathrm{TI} \cdot \mathrm{PPS} \cdot \mathrm{PPV} \cdot \\
& \left(\frac{\mathrm{f}_{2} \cdot \mathrm{Y} \cdot \mathrm{L}}{\mathrm{v}_{\mathrm{c}}\left(\mathrm{d}_{1}+\ell\right)^{2}}+\frac{\xi \cdot \mathrm{L} \cdot \Omega \cdot\left(1-\sqrt{\left.\mathrm{f}_{\mathrm{b}}+\mathrm{f}_{\mathrm{o}}\right) \cdot \mathrm{f}_{1} \cdot \mathrm{X}}\right.}{\left(\mathrm{w}_{\mathrm{st}}+2 \mathrm{w}_{\mathrm{s}}\right)(\delta+\ell)^{2}}\right)
\end{aligned}
$$

where

$$
\begin{aligned}
& \mathrm{f}_{1}=\text { stopped platooning factor } \\
& \mathrm{X}=\text { stopped dose factor } \\
& \mathrm{f}_{2}=\text { moving platooning factor } \\
& \mathrm{Y}=\text { moving dose factor } \\
& \mathrm{Q}_{3}=\text { units conversion factor }\left(2.78 \times 10^{-7} \frac{\mathrm{rem} \cdot \mathrm{h}}{\mathrm{mrem} \cdot \mathrm{s}}\right) \\
& \text { See Table } \mathrm{B}-2
\end{aligned}
$$

If it is assumed that traffic velocity is the same in both directions, as indicated in Figure B-16, people traveling in the opposite direction can be modeled as stationary with the shipment passing by at a speed of $2 \mathrm{~V}_{c^{\circ}}$ By noting (1) that the linear population density (LPD) in vehicles can be specified by

$$
\mathrm{LPD}=\frac{\mathrm{N} \cdot \mathrm{PPV}}{\left(\frac{2 \cdot \mathrm{A} \cdot \mathrm{f}_{\text {st }}}{\mathrm{w}_{\text {st }}}\right)}
$$

(2) that the separation distance is $w_{s t} / 2$, and (3) that the total exposure time in each cell is given by $\mathrm{L} / 2 \mathrm{~V}_{\mathrm{c}}$, the integrated dose to people moving opposite to the shipment can be expressed as 
Table B-2

Platooning Factor
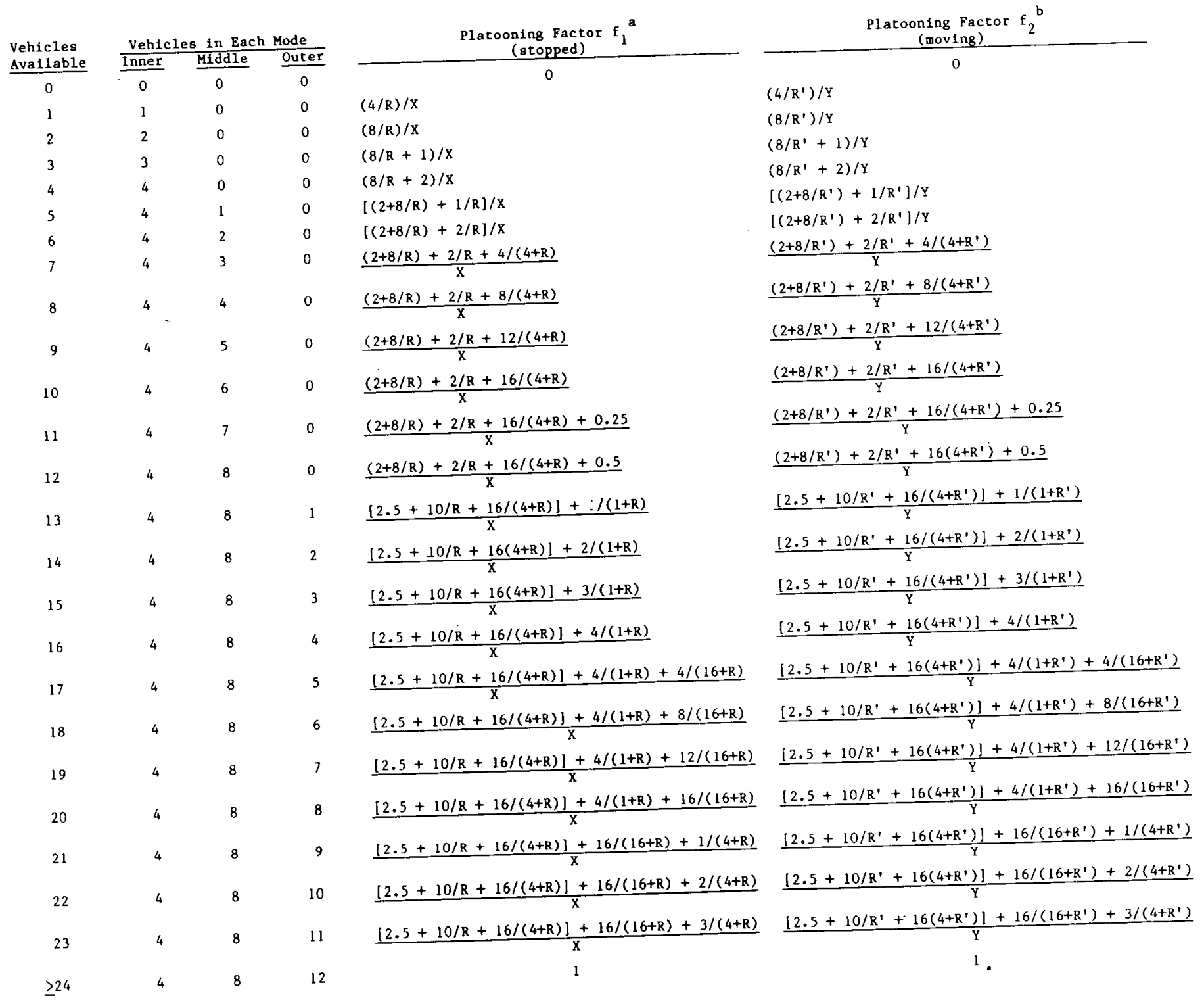

$$
\begin{aligned}
& a_{X}=2.5+10 / R+4 /(1+R)+20 /(4+R)+16(16+R) ; R=\frac{\left(w_{s t^{\prime}} / L^{\prime}\right)^{2}}{(\delta+l)^{2}} \\
& b_{Y}=2.5+10 / R^{\prime}+4\left(1+R^{\prime}\right)+20\left(4+R^{\prime}\right)+16\left(16+R^{\prime}\right) ; R^{\prime}=\frac{\left(w_{s t^{\prime}} / L^{\prime}\right)^{2}}{\left(d_{1}+\ell\right)^{2}}
\end{aligned}
$$

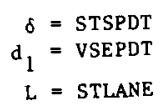




$$
\begin{gathered}
\mathrm{ID}=\mathrm{Q}_{1} \cdot 2 \cdot \mathrm{K}_{\mathrm{o}} \cdot \mathrm{TI} \cdot \mathrm{PPS} \cdot \frac{\mathrm{L}}{2 \mathrm{~V}_{\mathrm{c}}} \cdot \frac{{ }^{N w_{\mathrm{st}}}}{2 \cdot \mathrm{A} \cdot \mathrm{f}_{\mathrm{st}}} \cdot \mathrm{PPV} \\
\int_{\mathrm{w}_{\mathrm{st}} / 2}^{\infty} \frac{\mathrm{e}^{-\mu \mathrm{air} r} \cdot \mathrm{B}_{\mathrm{air}}(\mathrm{r}) \mathrm{dr}}{\mathrm{r} \sqrt{\mathrm{r}^{2}-\left(\mathrm{w}_{\mathrm{st}} / 2\right)^{2}}}
\end{gathered}
$$

By combining Eqs. (35) and (40), the following expression for dose to people in vehicles sharing the transport link with the transport vehicle can be obtained:

$$
\begin{aligned}
\mathrm{ID}= & \mathrm{K}_{\mathrm{o}} \cdot \mathrm{TI} \cdot \mathrm{PPS} \cdot \mathrm{PPV} \cdot \mathrm{L} \cdot \\
& \left\{\mathrm{Q}_{3} \cdot\left[\frac{\mathrm{f}_{2} \cdot \mathrm{Y}}{\mathrm{V}_{\mathrm{c}}\left(\mathrm{d}_{1}+\ell\right)^{2}}+\frac{\mathrm{f}_{1} \cdot \mathrm{X} \cdot \xi \cdot \Omega \cdot\left(1-\sqrt{\mathrm{f}_{\mathrm{b}}+\mathrm{f}_{\mathrm{o}}}\right)}{\left(\mathrm{w}_{\mathrm{st}}+2 \mathrm{w}_{\mathrm{s}}\right)(\delta+\ell)^{2}}\right]+\right. \\
& {\left.\left[\mathrm{Q}_{1} \cdot \frac{\mathrm{Nw}_{\mathrm{st}}}{2 \cdot \mathrm{A} \cdot \mathrm{f}_{\mathrm{st}} \cdot \mathrm{V}_{\mathrm{c}}} \int_{\mathrm{w}_{\mathrm{st}} / 2}^{\infty} \frac{\mathrm{e}^{-\mu_{\mathrm{air}} \mathrm{r}^{\mathrm{r}} \cdot \mathrm{B}_{\mathrm{air}}(\mathrm{r}) \mathrm{dr}}}{\mathrm{r} \sqrt{\mathrm{r}^{2}-\left(\mathrm{w}_{\mathrm{st}} / 2\right)^{2}}}\right]\right\} }
\end{aligned}
$$

Crew

The crew aboard a vehicle transporting radioactive material will be exposed to some low level of external penetrating radiation for the duration of the trip. If a characteristic source-to-crew distance $d_{2}$ is assigned for the particular mode, an expression for the dose received by the crew during travel through a cell can be written

$$
I_{\text {crew }}=Q_{3} \cdot \frac{\mathrm{L}}{\bar{V}} \cdot \mathrm{N}_{\mathrm{c}} \cdot \frac{\mathrm{K}_{\mathrm{o}} \cdot \mathrm{TI} \cdot \mathrm{PPS} \cdot \mathrm{e}^{-\mu_{\mathrm{air}} \mathrm{d}_{2}} \cdot \mathrm{B}_{\mathrm{air}}\left(\mathrm{d}_{2}\right)}{\mathrm{d}_{2}{ }^{2}}
$$

where $\mathrm{N}_{\mathrm{C}}=$ number of crew.

This development assumes no structural shielding from the vehicle or from other cargo in the vehicle. Since transport vehicle materials and construction vary from vehicles with shielded cabs to delivery vans with no partition between driver and source, and since cargo distribution schemes can also vary considerably, a conservative assumption of no vehicle shielding for crew is made. This approach is supported by a tabulation of shielding factors in Reference 13 which suggests that vehicles provide negligible shielding from the external penetrating radiation of a cloud of airborne radioactive material.*

*In the case of exclusive-use vehicles, the dose rate in any continuouslyoccupied portion of the vehicle (i.e., the cab) is limited by law to $2 \mathrm{mrem} / \mathrm{h}$. The model limits cab dose rate to this value. 
B2.2 Modifications to Account for Truck Travel on One-Way Streets

If it is assumed that vehicles on one-way streets are traveling at the center of the street, two equations must be modified. The dose to pedestrians given in Eq. (9) becomes

$$
\begin{aligned}
\mathrm{ID}= & \mathrm{Q}_{1} \cdot \operatorname{PedD} \cdot \mathrm{L} \cdot\left(2 \cdot \mathrm{K}_{0} \cdot \mathrm{TI} \cdot \mathrm{PPS}\right) \cdot \mathrm{ABD} \cdot \\
& {\left[\left(\frac{1}{\left(\overline{\mathrm{V}}+\mathrm{v}_{\mathrm{p}}\right)}+\frac{1}{\left(\overline{\bar{V}}-\mathrm{v}_{\mathrm{p}}\right)}\right) \cdot \int_{0.5 \mathrm{w}_{\mathrm{st}}}^{0.5 \mathrm{w}_{\mathrm{st}}+\mathrm{w}_{s}}\right] }
\end{aligned}
$$

and the dose to people in vehicles (Eq. (41)) becomes

$$
\begin{aligned}
I D= & Q_{3} \cdot \mathrm{K}_{\mathrm{o}} \cdot \mathrm{TI} \cdot \mathrm{PPS} \cdot \mathrm{L} \cdot \mathrm{PPV} \cdot \\
& {\left[\frac{\mathrm{f}_{2} \cdot \mathrm{Y}}{\mathrm{V}_{\mathrm{c}}\left(\mathrm{d}_{1}+\ell\right)^{2}}+\frac{\mathrm{f}_{1} \cdot \mathrm{X} \cdot \xi \cdot \Omega \cdot\left(1-\sqrt{\mathrm{f}_{\mathrm{b}}+\mathrm{f}_{\mathrm{o}}}\right)}{\left(\mathrm{w}_{\mathrm{st}}+2 \mathrm{w}_{\mathrm{s}}\right)(\delta+\ell)^{2}}\right] }
\end{aligned}
$$

The dose to people traveling in the opposite direction is automatically zero, and the formulations for dose to people in buildings and dose to crew remain unaffected.

B3. Modifications to Account for Truck Travel on Freeways

Four dose expressions must be modified to allow for trave1 on freeways: First, pedestrian dose is set to zero; second, dose to crew is modified by substituting freeway velocity $V_{f}$ for average velocity $\vec{V}$ in Eq. (42); third, the dose to people in vehicles $(\mathrm{Eq} \cdot(41) \mathrm{f}$ is modified as follows:

$$
\begin{aligned}
& \mathrm{ID}=\mathrm{K}_{\mathrm{o}} \cdot \mathrm{TI} \cdot \mathrm{PPS} \cdot \mathrm{PPV} \cdot \mathrm{L} \cdot\left\{\mathrm{f}_{\mathrm{f}} \cdot \mathrm{Y}_{\mathrm{f}} \cdot \mathrm{Q}_{3} \cdot\left[\frac{1}{\mathrm{~V}_{\mathrm{f}}\left(\mathrm{d}_{\mathrm{f}}+\ell\right)^{2}}\right]+\right.
\end{aligned}
$$

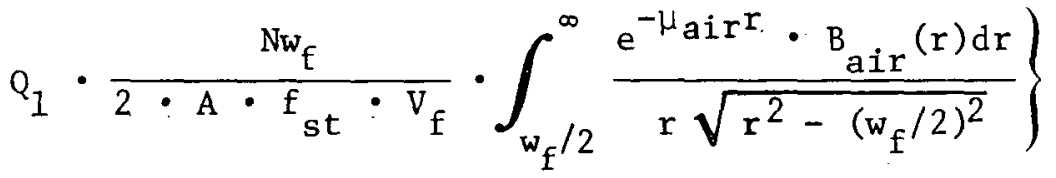

where

$$
\begin{aligned}
\mathrm{f}_{\mathrm{f}}= & \text { freeway platooning factor, computed in a similar way to } \mathrm{f}_{2} \text { and } \\
& \mathrm{Y}_{2} \text { using freeway separation distance in place of } \mathrm{d}_{1} \text { in the } \\
& \text { definition of } \mathrm{R} \\
\mathrm{Y}_{\mathrm{f}}= & \text { freeway dose factor } \\
\mathrm{v}_{\mathrm{f}}= & \text { average freeway velocity (metres per second) } \\
\mathrm{w}_{\mathrm{f}}= & \text { freeway width, including right-of-way (metres) }
\end{aligned}
$$


Finally, dose to people in buildings (Eq. (28)) is modified as follows:

$$
\begin{aligned}
& \mathrm{ID}=\frac{\mathrm{Q}_{1} \cdot 4 \cdot \mathrm{K}_{\mathrm{o}} \cdot \mathrm{II} \cdot \mathrm{PPS}}{\mathrm{V}_{\mathrm{f}}} \cdot \frac{(\mathrm{PD}+\mathrm{TC}) \cdot\left(\mathrm{w}_{\mathrm{st}}+2 \mathrm{w}_{\mathrm{s}}\right) \cdot \mathrm{L}}{4 \cdot \mathrm{n} \cdot\left(\sqrt{\mathrm{f}_{\mathrm{b}}}-\mathrm{f}_{\mathrm{b}}\right)} \cdot \\
& e^{-\mu_{b} w_{b}} \cdot B_{b}\left(w_{b}\right) \cdot \sum_{i=1}^{n} \int_{\beta_{2}}^{\infty} \frac{e^{-\mu_{a i r} r} \cdot o F_{i}(r) d r}{r \sqrt{r^{2}-\beta_{2}^{2}}}
\end{aligned}
$$

where

$$
\beta_{2}=\sqrt{(h(i-0.5))^{2}+\left(w_{f} / 2\right)^{2}}
$$

and the transport vehicle is assumed to be in the center of the freeway.

B4. Dose Due to Incident-Free Rail Transport

Dose due to incident-free rail transport is similar to that for trucks, with four important differences:

1. Since sidewalks are not situated along railroad tracks, dose to pedestrians is not expicitly computed.

2. Since freight trains may either pass through or stop in passenger terminal areas, a "depot dose" is calculated.

3. Because of the large amounts of structural material continuously interposed between source and crew in the form of engines, cars, etc., crew dose is considered negligible and is not computed.

4. Because the areas along railroad rights-of-way contain people in buildings, people in vehicles, and pedestrians, a "right-of-way dose" is computed.

Dose Accumulated in Railroad Terminals

If the populated terminal area is modeled as an annular area with no one closer to the package than some distance $r_{1}$ and with people uniformly distributed between that radius and some maximum radius $r_{2}$, Eq. (2) can be integrated using an annular differential element to yield the following expression for integrated dose:

$$
\begin{aligned}
\mathrm{ID}= & \mathrm{Q}_{1} \cdot \mathrm{K}_{\mathrm{o}} \cdot \mathrm{TI} \cdot \mathrm{PPS} \cdot \Delta \mathrm{T}_{\text {depot }} \cdot \mathrm{PD}_{\mathrm{depot}} \cdot \\
& \int_{\mathrm{r}_{1}}^{\mathrm{r}_{2}} \frac{(2 \pi \mathrm{r}) \cdot \mathrm{e}^{-\mu_{\mathrm{air}} \mathrm{r}^{2} \cdot \mathrm{B}_{\mathrm{air}}(\mathrm{r}) \mathrm{dr}}}{\mathrm{r}^{2}}
\end{aligned}
$$


where

$$
\begin{aligned}
\Delta \mathrm{T}_{\mathrm{depot}} & =\text { average stop time in depot area (seconds) } \\
\mathrm{PD}_{\text {depot }} & =\text { population density in depot area (persons } / \mathrm{km}^{2} \text { ) } \\
\mathrm{r}_{1} & =\text { minimum radius (metres) } \\
\mathrm{r}_{2} & =\text { maximum radius (metres) }
\end{aligned}
$$

Persons Sharing the Transport Link

Although commercial passenger trains are disappearing on a national level, many urban areas still have extensive commuter rail service to suburban areas, and some population exposure could be accumulated by people in these trains. The development and assumptions are similar to those for dose to people in vehicles traveling in the direction opposite to a truck shipment (Eq - (40)). When factors appropriate to rail transport are substituted, the expression becomes

$$
\begin{gathered}
\mathrm{ID}=2 \cdot \mathrm{Q}_{3} \cdot \mathrm{K}_{\mathrm{o}} \cdot \mathrm{TI} \cdot \mathrm{PPS} \cdot \frac{\mathrm{LN}_{\mathrm{T}}}{\mathrm{V}_{\mathrm{T}}^{2}} \cdot \mathrm{PPT} \cdot \\
\int_{\mathrm{r}_{3}}^{\infty} \frac{\mathrm{e}^{-\mu \mathrm{air}^{\mathrm{r}} \cdot \mathrm{Bair}^{(\mathrm{r}) \mathrm{dr}}}}{\mathrm{r} \sqrt{\mathrm{r}^{2}-\mathrm{r}_{3}^{2}}}
\end{gathered}
$$

where

$$
\begin{aligned}
\mathrm{N}_{\mathrm{T}} & =\text { one-way train traffic count (vehicles per second) } \\
\mathrm{V}_{\mathrm{T}} & =\text { average train velocity (metres per second) } \\
\mathrm{PPT} & =\text { number of persons per train } \\
\mathrm{r}_{3} & =\text { distance between passing trains (metres) }
\end{aligned}
$$

People Along the Right-of-Way

The location of railroad tracks in relation to streets, buildings, and pedestrian areas is highly variable. Therefore, an average value for population density along the railroad right-of-way is computed using the average overall population density given by

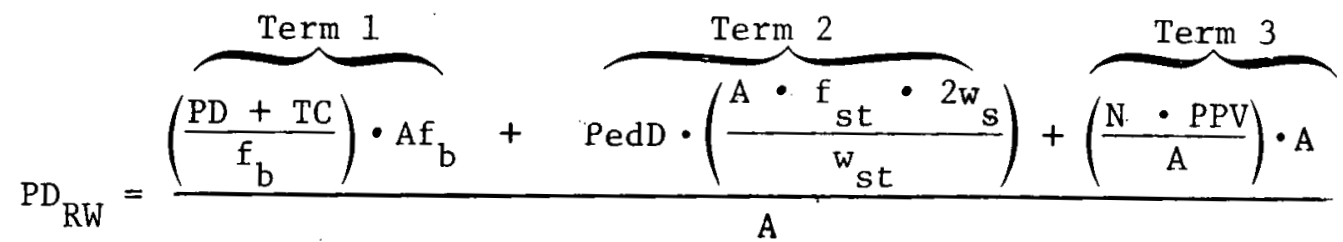

$$
\begin{aligned}
& =(P D+T C)+\operatorname{PedD} \cdot\left(\frac{f s t \cdot 2 w_{s}}{w_{s t}}\right)+\left(\frac{N \cdot P P V}{A}\right)
\end{aligned}
$$


where

$$
\begin{aligned}
& \text { Term } 1=\text { total people in buildings in cell } \\
& \text { Term } 2=\text { total pedestrians in cell } \\
& \text { Term } 3=\text { total people in vehicles in cell }
\end{aligned}
$$

This expression is used in an expression similar to Eq. (9), in which the integration is carried out across a strip from the minimum right-of-way distance to approximately 400 metres on both sides of the track. The value of 400 metres was subjectively chosen assuming that intervening building and terrain factors not explicitly included in the formulation will act to reduce the dose. The resulting equation is

$$
\mathrm{ID}=2 \cdot \mathrm{PD}_{\mathrm{RW}} \cdot \mathrm{Q}_{1} \cdot \mathrm{K}_{\mathrm{o}} \cdot \mathrm{TI} \cdot \mathrm{PPS} \cdot \frac{\mathrm{L}}{\mathrm{V}_{\mathrm{T}}} \cdot \int_{\mathrm{R}_{\mathrm{W}}}^{400} \mathrm{I}(\mathrm{x}) \mathrm{dx}
$$

where $R_{x}=$ minimum right-of-way distance (metres).

No shielding is considered and no obliqueness effects are included for people in buildings.

B5. Dose Due to Incident-Free Air Transport

Doses from passenger aircraft service and from cargo aircraft service are considered. Only the dose to people in the terminal area is considered applicable to the urban area. The dose accumulated by crew, flight attendants, or onboard passengers is dominated by that fraction accumulated in flight rather than in the urban area per se and is not evaluated.

The fundamental mathematical form for dose to people in terminals is the same as that derived for rail depot doses in Eq. (48). By substituting air parameters for rail parameters, the following expression is obtained:

$$
\begin{gathered}
\mathrm{ID}=\mathrm{Q}_{1} \cdot \mathrm{K}_{\mathrm{O}} \cdot \mathrm{TI} \cdot \mathrm{PPS} \cdot \Delta \mathrm{T}_{\text {term }} \cdot \mathrm{PD}_{\text {term }} \cdot \\
\int_{\mathrm{r}_{4}}^{\mathrm{r}_{5}} \frac{(2 \pi r) \mathrm{e}^{-\mu} \mathrm{air}^{\mathrm{r}} \cdot \mathrm{B}_{\mathrm{air}}(\mathrm{r}) \mathrm{dr}}{\mathrm{r}^{2}}
\end{gathered}
$$

where

$$
\begin{aligned}
\Delta \mathrm{T}_{\text {term }}= & \text { the average stop time in air terminals (seconds) (different } \\
& \text { for passenger and cargo aircraft) } \\
\mathrm{PD}_{\text {term }}= & \text { terminal area population density (persons } / \mathrm{km}^{2} \text { ) (different } \\
& \text { for passenger and cargo terminals) }
\end{aligned}
$$




$$
\begin{aligned}
& r_{4}=\text { minimum radius (metres) } \\
& r_{5}=\text { maximum radius (metres) }
\end{aligned}
$$

B6. Dose Due to Incident-Free Water Transport

Like air transport, the urban component of transport by water is principally accumulated in the locality where the vehicle stops, i.e., the dock area. Ships and barges are massive steel structures, and shipping lanes in navigable rivers are generally in the channel at some distance from occupied shoreline. Therefore, neither crew nor people along the riverbank would receive appreciable doses. The dose received by persons in the dock area is developed similarly to that for rail depots and air terminals, with appropriate changes in nomenclature:

$$
\begin{gathered}
\mathrm{ID}=0.5 \cdot \mathrm{Q}_{1} \cdot \mathrm{K}_{\mathrm{o}} \cdot \mathrm{TI} \cdot \mathrm{PPS} \cdot \Delta \mathrm{T}_{\mathrm{dock}} \cdot \mathrm{PD}_{\text {dock }} \cdot \\
\int_{\mathrm{r}_{6}}^{\mathrm{r}_{7}} \frac{(2 \pi \mathrm{r}) \mathrm{e}^{-\mu_{\mathrm{air}} \mathrm{r}} \cdot \mathrm{B}_{\mathrm{air}}(\mathrm{r}) \mathrm{dr}}{\mathrm{r}^{2}}
\end{gathered}
$$

where

$$
\begin{aligned}
\Delta \mathrm{T}_{\mathrm{dock}} & =\text { time spent in dock area (seconds) } \\
\mathrm{PD}_{\text {dock }} & =\text { person-density in dock area (persons } / \mathrm{km}^{2} \text { ) } \\
\mathrm{r}_{6} & =\text { minimum exposure radius (metres) } \\
\mathrm{r}_{7} & =\text { maximum exposure radius (metres) }
\end{aligned}
$$

A factor of 0.5 is included since half the annular area around the vessel is arbitrarily assumed to be open water.

Incident-Free Dose To Handlers

The expression for dose to handlers is based on two previous assessments of package handling: one which considers the handling of small packages and one which considers the handling of large packages such as casks. This exposure dose is independent of the mode of transport.

A study was conducted on the handling of small radioactive packages by cargo handlers at aixport freight terminals. 14 Using the method discussed in Reference 14, the dose to cargo handlers can be specified as

$$
\mathrm{ID}=\mathrm{Q}_{4} \cdot \mathrm{N}_{\mathrm{H}} \cdot \mathrm{PPS} \cdot \mathrm{TI}
$$

where

$$
\begin{aligned}
& \mathrm{ID}=\text { integrated dose to handlers per shipment (person rem) } \\
& \mathrm{N}_{\mathrm{H}}=\text { number of handlings } \\
& \mathrm{Q}_{4}=2.5 \times 10^{-4} \text { person rem/handling/TI }
\end{aligned}
$$


In the derivation of the empirical constant in this equation, a handling is defined as all terminal operations associated with a single package.

In the case of casks, a general formulation based on References 2, 15, and 21 is used. It is assumed that a person handling a large cask will probably be close to the radioactive source only while attaching or detaching rigging equipment or otherwise preparing the cask for transfer. Since the radiation field around a large cask is heterogeneous, particularly close to the cask, a dose rate based on some standard (such as a spent fuel cask) is used. Additionally, there may be a wide variation in handling capability for a particular cask at a particular location, so a standard length of handling time is chosen. Two other variables must also be specified: the number of handlers and the number of transfers per shipment. By combining all these factors, the following general expression is obtained:

$$
\mathrm{ID}=\mathrm{K}_{1} \cdot \mathrm{K}_{2} \cdot \mathrm{K}_{3} \cdot \mathrm{K}_{4}
$$

\section{where}

$$
\begin{aligned}
& \mathbf{K}_{1}=\text { dose rate at } 0.9 \text { metre from cask (typical value, } 0.02 \mathrm{rem} / \mathrm{h} \text { ) } \\
& \mathbf{K}_{2}=\text { length of time spent in dose field (typical value, } 0.5 \text { hour) } \\
& \mathbf{K}_{3}=\text { number of handlers required (typical value, 2.0) } \\
& \mathbf{K}_{4}=\text { number of transfers per shipment (typical value, } 1.0 \text { ) }
\end{aligned}
$$

It should be noted that large casks, especially those containing irradiated fuel, are not expected to be handled in urban areas.

Dose to Warehouse Personne1

If a radioactive package is placed in storage during a shipment, the warehouse personnel (other that handlers previously discussed) will be exposed to radiation from the package during their normal routine. If it is assumed that the package is stored so that no one except handlers will get within a certain exclusion radius $r_{8}$ of the package, and that the warehouse personnel are distributed uniformly throughout the warehouse to some maximum radius $r_{9}$ at some density $\mathrm{PD}_{\text {stor, }}$, the integrated dose received during storage can be predicted by integrating a form of Eq. (2) using an annular differential element as discussed earlier:

$$
\begin{gathered}
\mathrm{ID}=\mathrm{Q}_{1} \cdot \mathrm{K}_{\mathrm{o}} \cdot \mathrm{TI} \cdot \mathrm{PPS} \cdot \Delta \mathrm{T}_{\mathrm{stor}} \cdot \mathrm{PD}_{\text {stor }} \cdot \\
\int_{\mathrm{r}_{8}}^{\mathrm{r}_{9}} \frac{(2 \pi r) \mathrm{e}^{-\mu} \mathrm{air} \mathrm{r}^{\mathrm{r}} \cdot \mathrm{B}_{\mathrm{air}}(\mathrm{r}) \mathrm{dr}}{\mathrm{r}^{2}}
\end{gathered}
$$

where $\Delta \mathrm{T}_{\text {stor }}=$ storage time (seconds).

Note that no credit is taken for shielding due to other stored items or internal warehouse structural materials. 
ADDENDUM 1: VARIABLES, CONSTANTS, AND FUNCTIONS

Table Ad-1

List of Variables

\begin{tabular}{|c|c|c|c|}
\hline Symbol & Description & Units & $\begin{array}{l}\text { Equation in Which } \\
\text { Variable Appears }\end{array}$ \\
\hline $\mathrm{DR}$ & Dose rate & mrem/h & $1,2,3$ \\
\hline $\mathrm{K}$ & Dose rate factor & $\mathrm{mrem} \cdot \mathrm{m}^{2} / \mathrm{h}$ & $1,5,6,7,10,11,12,14,15$ \\
\hline$\mu$ & $\begin{array}{l}\text { Linear attenuation } \\
\text { coefficient }\end{array}$ & $\mathrm{m}^{-1}$ & $1,2,5,6,17,29$ \\
\hline $\mathbf{r}$ & Distance & metres & $\begin{array}{l}1,2,5,6,7 a, 10,11,12,13 \\
14,15,18,20,28,40,41,45 \\
46,47,48,51,52,55\end{array}$ \\
\hline $\mathrm{K}_{\mathrm{o}}$ & Package shape factor & $\mathrm{m}^{2}$ & $\begin{array}{l}2,9,28,29,33,36,38,40 \\
41,42,43,44,45,46,47,48 \\
50,51,52,55\end{array}$ \\
\hline $\mathrm{TI}$ & Transport Index & $\mathrm{mrem} / \mathrm{h}$ & $\begin{array}{l}2,9,28,29,33,34,36,38, \\
40,41,42,43,44,45,46,47 \\
48,50,51,53\end{array}$ \\
\hline PPS & $\begin{array}{l}\text { Number of packages } \\
\text { per shipment }\end{array}$ & - & $\begin{array}{l}2,9,28,29,33,34,36,38, \\
39,40,41,42,43,44,45,46, \\
47,48,50,51,52,53,55\end{array}$ \\
\hline $\mathrm{D}$ & Total individual dose & mrem & $3,5,6,7,8,10$ \\
\hline $\mathrm{t}$ & Time (general) & hours & 3,4 \\
\hline $\mathrm{x}$ & Distance (general) & metres & $\begin{array}{l}4,5,7,7 a, 8,10,11,12,13 \\
14,15,43,50\end{array}$ \\
\hline $\mathrm{V}$ & Velocity (genera1) & $\mathrm{m} / \mathrm{h}$ & $4,5,6,7$ \\
\hline $\mathrm{d}$ & $\begin{array}{l}\text { Minimum distance from } \\
\text { shipment path to } \\
\text { individual }\end{array}$ & metres & $6,8,11,12,13,14,15$ \\
\hline$\mu_{\text {air }}$ & $\begin{array}{l}\text { Linear attenuation } \\
\text { coefficient for air }\end{array}$ & $\mathrm{m}^{-1}$ & $\begin{array}{l}7 a, 10,11,12,13,14,15,28 \\
40,41,42,45,46,47,48,51 \\
52,55\end{array}$ \\
\hline ID & $\begin{array}{l}\text { Integrated population } \\
\text { exposure per shipment }\end{array}$ & person rem & $\begin{array}{l}8,9,28,33,36,40,41,42 \\
43,44,45,46,47,48,50 \\
51,52,53,54,55\end{array}$ \\
\hline
\end{tabular}


Table Ad-1 (Continued)

\begin{tabular}{|c|c|c|c|}
\hline Symbol & Description & Units & $\begin{array}{l}\text { Equation in Which } \\
\text { Variable Appears }\end{array}$ \\
\hline PedD & Pedestrian density & $\begin{array}{l}\text { persons per } \\
\text { square kilo- } \\
\text { metre of } \\
\text { sidewalk }\end{array}$ & $8,9,11,12,14,15,43,49$ \\
\hline $\mathrm{L}$ & $\begin{array}{l}\text { Distance traveled in } \\
\text { cell }\end{array}$ & metres & $\begin{array}{l}8,9,12,13,16,28,29,30,34 \\
38,40,41,42,43,44,45,46 \\
48,50\end{array}$ \\
\hline $\mathrm{w}_{\mathrm{s}}$ & Sidewalk width & metres & $\begin{array}{l}8,9,11,12,13,14,15,19 \\
20,23,24,26,27,28,31,32 \\
37,38,41,43,44,46,49\end{array}$ \\
\hline Q & $\begin{array}{l}\text { General units con- } \\
\text { version factor }\end{array}$ & various & $8,33,36$ \\
\hline $\mathrm{ABD}$ & Albedo dose factor & - & 9,43 \\
\hline$w_{\text {st }}$ & Street width & metres & $\begin{array}{l}9,14,15,16,19,20,23,24, \\
26,27,28,31,32,34,35,37, \\
38,39,40,41,43,44,46,49\end{array}$ \\
\hline$\overline{\mathrm{V}}$ & $\begin{array}{l}\text { Average shipment } \\
\text { velocity }\end{array}$ & $\mathrm{m} / \mathrm{s}$ & $\begin{array}{l}9,10,11,12,16,28,30,32 \\
37,42,43\end{array}$ \\
\hline$v_{p}$ & Pedestrian velocity & $\mathrm{m} / \mathrm{s}$ & $9,14,15,16,43$ \\
\hline $\mathrm{ID}_{\perp}$ & $\begin{array}{l}\text { Integrated pedestrian } \\
\text { exposure on side- } \\
\text { streets per shipment }\end{array}$ & person rem & 11,13 \\
\hline$I D_{11}$ & $\begin{array}{l}\text { Integrated pedestrian } \\
\text { exposure on parallel } \\
\text { sidewalks for one } \\
\text { block }\end{array}$ & person rem & $12,13,16$ \\
\hline $\mathrm{ID}_{\perp, 1}$ & $\begin{array}{l}\text { Integrated pedestrian } \\
\text { exposure in near } \\
\text { crosswalk }\end{array}$ & person rem & 14,16 \\
\hline$\alpha_{1}$ & Surrogate variable & metres & 14 \\
\hline $\mathrm{ID}_{\perp, 2}$ & $\begin{array}{l}\text { Integrated pedestrian } \\
\text { exposure in far } \\
\text { crosswalk }\end{array}$ & person rem & 15,16 \\
\hline $\mathrm{ID}_{1,1+2}$ & $\begin{array}{l}\text { Integrated pedestrian } \\
\text { exposure in crosswalks }\end{array}$ & person rem & 16 \\
\hline$A_{1}, A_{2}, \alpha_{2}, \alpha_{3}$ & $\begin{array}{l}\text { Empirical constants in } \\
\text { dose buildup factor } \\
\text { calculation }\end{array}$ & - & 17 \\
\hline
\end{tabular}


Table Ad-1 (Continued)

\begin{tabular}{|c|c|c|c|}
\hline Symbol & Description & Units & Variable Appears \\
\hline$w_{b}$ & Building wall thickness & metres & $17,28,46$ \\
\hline $\mathrm{E}$ & Photon energy & $\mathrm{MeV}$ & 17 \\
\hline$\theta$ & $\begin{array}{l}\text { oblique angle of } \\
\text { impingement }\end{array}$ & radians & 19 \\
\hline $\mathbf{r}^{\prime}$ & $\begin{array}{l}\text { Distance of source from } \\
\text { point in street dir- } \\
\text { ectly in front of } \\
\text { building }\end{array}$ & metres & 19,20 \\
\hline $\mathbf{n}$ & $\begin{array}{l}\text { Number of floors in } \\
\text { buildings in a cell }\end{array}$ & - & $19,20,22,27,28,46$ \\
\hline $\mathrm{h}$ & Height per floor & metres & $19,20,28,46$ \\
\hline $\mathrm{PPB}$ & $\begin{array}{l}\text { Population density in } \\
\text { bufldings }\end{array}$ & $\begin{array}{l}\text { persons per } \\
\text { square kilo- } \\
\text { metre of } \\
\text { building }\end{array}$ & 21,22 \\
\hline PD & $\begin{array}{l}\text { Population density } \\
\text { in cell (residents } \\
\pm \text { commuters) }\end{array}$ & $\begin{array}{l}\text { persons per } \\
\text { square kilo- } \\
\text { metre of cell }\end{array}$ & $21,27,28,46,49$ \\
\hline $\mathrm{TC}$ & $\begin{array}{l}\text { Transient population } \\
\text { density }\end{array}$ & $\begin{array}{l}\text { persons per } \\
\text { square kilo- } \\
\text { metre of cell }\end{array}$ & $21,27,28,46,49$ \\
\hline A & Cell area & $\mathrm{km}^{2}$ & $\begin{array}{l}21,22,24,26,37,39,40,41, \\
45,49\end{array}$ \\
\hline$f_{b}$ & $\begin{array}{l}\text { Fraction of cell area } \\
\text { occupied by buildings }\end{array}$ & - & $\begin{array}{l}21,22,23,26,27,28,31,32, \\
37,38,41,44,46\end{array}$ \\
\hline PPF & $\begin{array}{l}\text { Number of people on } \\
\text { each floor of butldings } \\
\text { in cell }\end{array}$ & persons & 22 \\
\hline$k$ & $\begin{array}{l}\text { Square root of number } \\
\text { of buildings in ceil }\end{array}$ & - & $23,24,25$ \\
\hline$\Delta \mathrm{b}$ & Building width & metres & $23,24,25$ \\
\hline $\mathrm{P}_{\mathrm{T}}$ & $\begin{array}{l}\text { Total building perimeter } \\
\text { in cell }\end{array}$ & metres & 25,26 \\
\hline$\mu_{b}$ & $\begin{array}{l}\text { Linear attenuation } \\
\text { coefficient for building } \\
\text { material }\end{array}$ & $\mathrm{m}^{-1}$ & 28,46 \\
\hline 1 & $\begin{array}{l}\text { Summation variable } \\
\text { over floors }\end{array}$ & - & 28,46 \\
\hline$\beta_{1}$ & Surrogate variable & metres & 28 \\
\hline
\end{tabular}




\begin{tabular}{|c|c|c|c|}
\hline Symbol & Description & Units & $\begin{array}{l}\text { Equation in Which } \\
\text { Variable Appears }\end{array}$ \\
\hline $\mathrm{ID}_{\mathrm{c}}$ & $\begin{array}{l}\text { Integrated dose to } \\
\text { people in vehicles } \\
\text { during the cruising } \\
\text { phase }\end{array}$ & person rem & 29 \\
\hline$d_{1}$ & $\begin{array}{l}\text { Vehicle separation } \\
\text { distance during } \\
\text { cruising phase }\end{array}$ & metres & $29,38,41,44$ \\
\hline$\ell$ & Vehicle length & metres & $29,34,35,36,38,41,44,45$ \\
\hline $\mathrm{v}_{\mathrm{C}}$ & Cruising velocity & $\mathrm{m} / \mathrm{s}$ & $29,30,32,38,40,41,44$ \\
\hline$\xi$ & $\begin{array}{l}\text { Fraction of inter- } \\
\text { sections stopped at }\end{array}$ & - & $30,32,38,41,44$ \\
\hline$\Omega$ & $\begin{array}{l}\text { Time spent stopped at } \\
\text { each intersection }\end{array}$ & seconds & $30,32,38,41,44$ \\
\hline$D^{\prime}$ & Block length & metres & 30 \\
\hline$f_{0}$ & $\begin{array}{l}\text { Fraction of cell area } \\
\text { which is open area }\end{array}$ & - & $31,32,37,38,41,44$ \\
\hline PPV & $\begin{array}{l}\text { Number of people per } \\
\text { vehicle }\end{array}$ & - & $33,36,38,39,40,41,44,45$ \\
\hline$\Delta \mathrm{T}$ & $\begin{array}{l}\text { Time interval } \\
\text { (general) }\end{array}$ & seconds & 33,36 \\
\hline $\mathrm{N}$ & $\begin{array}{l}\text { Total vehicles in } \\
\text { a cell at any time }\end{array}$ & - & $37,39,40,41,45,49$ \\
\hline$f_{\text {st }}$ & $\begin{array}{l}\text { Fraction of cell area } \\
\text { occupied by streets }\end{array}$ & - & $37,39,40,41,45,49$ \\
\hline $\mathbf{f}$ & $\begin{array}{l}\text { Vehicle platooning } \\
\text { factor }\end{array}$ & - & $38,41,44$ \\
\hline$\delta$ & $\begin{array}{l}\text { Vehicle separation } \\
\text { distance during } \\
\text { stopped phase }\end{array}$ & metres & $38,41,44$ \\
\hline LPD & $\begin{array}{l}\text { Linear population } \\
\text { density }\end{array}$ & persons $/ \mathrm{km}$ & 39 \\
\hline $\mathrm{ID}_{\text {crew }}$ & Integrated dose to crew & person rem & 42 \\
\hline${ }^{N_{C}}$ & $\begin{array}{l}\text { Number of crew per } \\
\text { vehicle }\end{array}$ & - & 42 \\
\hline $\mathrm{d}_{2}$ & $\begin{array}{l}\text { Source-to-crew } \\
\text { distance }\end{array}$ & metres & 42 \\
\hline$f_{f}$ & $\begin{array}{l}\text { Vehicle platooning } \\
\text { (freeways) }\end{array}$ & - & 45 \\
\hline
\end{tabular}


Table Ad-1 (Continued)

\begin{tabular}{|c|c|c|c|}
\hline Symbol & Description & Units & $\begin{array}{l}\text { Equation in Which } \\
\text { Variable Appears }\end{array}$ \\
\hline$v_{f}$ & $\begin{array}{l}\text { Average freeway } \\
\text { velocity }\end{array}$ & $\mathrm{m} / \mathrm{s}$ & 45,46 \\
\hline$d_{f}$ & $\begin{array}{l}\text { Vehicle separation } \\
\text { distance - freeways }\end{array}$ & metres & 45 \\
\hline$w_{f}$ & Freeway width & metres & 45,46 \\
\hline$\beta_{2}$ & Surrogate variable & metres & 46 \\
\hline$\Delta \mathrm{T}_{\text {depot }}$ & $\begin{array}{l}\text { Stop time in railway } \\
\text { depot }\end{array}$ & seconds & 47 \\
\hline $\mathrm{PD}_{\text {depot }}$ & $\begin{array}{l}\text { Train depot population } \\
\text { density }\end{array}$ & persons $/ \mathrm{km}^{2}$ & 47 \\
\hline$r_{1}$ & $\begin{array}{l}\text { Minimum rail exposure } \\
\text { distance }\end{array}$ & metres & 47 \\
\hline $\mathrm{r}_{2}$ & $\begin{array}{l}\text { Maximum rail exposure } \\
\text { distance }\end{array}$ & metres & 47 \\
\hline$r_{3}$ & $\begin{array}{l}\text { Distance between } \\
\text { passing trains }\end{array}$ & metres & 48 \\
\hline $\mathrm{N}_{\mathrm{T}}$ & $\begin{array}{l}\text { One-way train traffic } \\
\text { count }\end{array}$ & vehicles/s & 48 \\
\hline $\mathrm{V}_{\mathrm{T}}$ & Average train velocity & $\mathrm{m} / \mathrm{s}$ & 48,50 \\
\hline PPT & $\begin{array}{l}\text { Number of persons per } \\
\text { train }\end{array}$ & - & 48 \\
\hline $\mathrm{PD}_{\mathrm{RW}}$ & $\begin{array}{l}\text { Population density } \\
\text { adjacent to rail right- } \\
\text { of-way }\end{array}$ & people $/ \mathrm{km}^{2}$ & 49,50 \\
\hline $\mathrm{R}_{\mathrm{w}}$ & $\begin{array}{l}\text { Rail right-of-way } \\
\text { distance }\end{array}$ & metres & 50 \\
\hline$\Delta \mathrm{T}_{\text {term }}$ & $\begin{array}{l}\text { Airport terminal } \\
\text { stop time }\end{array}$ & seconds & 51 \\
\hline $\mathrm{PD}_{\text {term }}$ & $\begin{array}{l}\text { Air terminal population } \\
\text { density }\end{array}$ & persons $/ \mathrm{km}^{2}$ & 51 \\
\hline $\mathbf{r}_{4}$ & $\begin{array}{l}\text { Minimum air terminal } \\
\text { exposure distance }\end{array}$ & metres & 51 \\
\hline$r_{5}$ & $\begin{array}{l}\text { Maximum air terminal } \\
\text { exposure distance }\end{array}$ & metres & 51 \\
\hline$\Delta \mathrm{T}_{\mathrm{dock}}$ & Stop time in dock area & seconds & 52 \\
\hline $\mathrm{PD}_{\text {dock }}$ & $\begin{array}{l}\text { Population density in } \\
\text { dock area }\end{array}$ & persons $/ \mathrm{km}^{2}$ & 52 \\
\hline$r_{6}$ & $\begin{array}{l}\text { Minimum dock exposure } \\
\text { radius }\end{array}$ & metres & 52 \\
\hline
\end{tabular}


Table Ad-1 (Continued)

\begin{tabular}{|c|c|c|c|}
\hline Symbol & Description & Units & $\begin{array}{l}\text { Equation in which } \\
\text { Variable Appears }\end{array}$ \\
\hline$r_{7}$ & $\begin{array}{l}\text { Maximum dock exposure } \\
\text { radius }\end{array}$ & metres & 52 \\
\hline $\mathrm{N}_{\mathrm{H}}$ & Number of handlings & - & 53 \\
\hline $\mathrm{K}_{1}, \mathrm{~K}_{2}, \mathrm{~K}_{3}, \mathrm{~K}_{4}$ & $\begin{array}{l}\text { Empirical constants for } \\
\text { dose to cask handlers }\end{array}$ & - & 54 \\
\hline$\Delta \mathrm{T}_{\text {stor }}$ & $\begin{array}{l}\text { Time spent in warehouse } \\
\text { storage }\end{array}$ & seconds & 55 \\
\hline $\mathrm{PD}_{\text {stor }}$ & $\begin{array}{l}\text { Population density } \\
\text { in warehouse }\end{array}$ & persons $/ \mathrm{km}^{2}$ & 55 \\
\hline$r_{8}$ & $\begin{array}{l}\text { Minimum warehouse } \\
\text { exposure distance }\end{array}$ & metres & 55 \\
\hline$r_{9}$ & $\begin{array}{l}\text { Maximum warehouse } \\
\text { exposure distance }\end{array}$ & metres & 55 \\
\hline
\end{tabular}


ADDENDUM 2: NUMERICAL ANALYSIS FOR APPENDIX B

Evaluation of Integrals

I. Consider

$$
\int_{c}^{\infty} \frac{e^{-\mu s} \cdot O F\left(w_{s t} / 2+w_{s}, \theta\right) d s}{s \sqrt{s^{2}-c^{2}}}
$$

where

$$
\begin{aligned}
& c=\sqrt{\left(w_{s t} / 2+w_{s}\right)^{2}+[h(n-0.5)]^{2}}, \\
& s=\sqrt{r^{2}+\left(w_{s t} / 2+w_{s}\right)^{2}+[h(n-0.5)]^{2}} \text {, and } \\
& \theta=\pi / 2-\tan ^{-1}\left[\left(w_{s t} / 2+w_{s}\right) / \sqrt{r^{2}+[h(n-0.5)]^{2}}\right]
\end{aligned}
$$

In order to remove the singularity at $s=c$, we substitute $s=c \cdot \cosh (t)$. Then we have

$$
\frac{1}{c} \int_{0}^{\infty} \frac{e^{-\mu c \cdot \cosh (t)} \cdot O F\left(w_{s t} / 2+w_{s}, \theta(t)\right) d t}{\cosh (t)}
$$

A curve-fitting routine was used to obtain a B-spline representation of of vs $\theta$. The numerical integration of the above integral was approached in the following manner using GAUS816, an adaptive routine based on the eight-point Gauss-Legendre formula:

1. Compute $\theta_{c}$ :

$$
\theta_{c}=\pi / 2-\tan ^{-1}\left(\frac{\mathrm{w}_{s t} / 2+\mathrm{w}_{s}}{\mathrm{~h}(\mathrm{n}-0.5)}\right)
$$

(when $s=c, r=0$ ) 
Table Ad-2

Constants

\begin{tabular}{|c|c|c|c|}
\hline Symbol & Value & Units & $\begin{array}{l}\text { Equations in Which } \\
\text { Constants Appear }\end{array}$ \\
\hline$Q_{1}$ & $2.78 \times 10^{-13}$ & $\frac{\mathrm{rem} \cdot \mathrm{h} \cdot \mathrm{km}^{2}}{\mathrm{mrm} \cdot \mathrm{s} \cdot \mathrm{m}^{2}}$ & $\begin{array}{l}9,28,40,41,43,45,46,47,50 \\
51,52,55\end{array}$ \\
\hline $\mathrm{Q}_{2}$ & $10^{-6}$ & $\frac{\mathrm{km}^{2}}{\mathrm{~m}^{2}}$ & 37 \\
\hline$Q_{3}$ & $2.78 \times 10^{-7}$ & $\frac{r e m \cdot h}{m r m \cdot s}$ & $38,41,42,44,45,48$ \\
\hline$Q_{4}$ & $2.5 \times 10^{-4}$ & $\begin{array}{l}\text { person rem/ } \\
\text { handling/TI }\end{array}$ & 53 \\
\hline
\end{tabular}


Table Ad-3

Functions

$\frac{\text { Symbol }}{B(r), B_{a i r}(r), B_{b}(r)}$
$I(x)$
$B\left(E, \mu, w_{b}\right)$
$O F(r)$

Description
Dose rate buildup factor
(general, air, building
material)
Specified integral (see
Eq. ( $7 a)$ )
Generalized dose buildup
factor

Equations in Which Functions Appear

$1,2,5,6,7 \mathrm{a}, 10,11,12,13$, $14,15,18,28,40,41,42,45$, $46,47,48,51,52,55$

$7,7 a, 9,43,50$

obliqueness factor

28,46 
2. Locate $\theta_{i}$, where $\theta_{i}$ is the first spline fit knot $>\theta_{c}$.

3. Compute $r_{j}$ 's for $\theta_{j}$ 's, $j \geq i$ :

$$
r_{j}=\sqrt{\left[\frac{w_{s t}+w_{s}}{\tan \left(\pi / 2-\theta_{j}\right)}\right]-(h(n-0.5))^{2}}
$$

4. Compute $s_{j}$ 's from $r_{j}$ 's:

$$
s_{j}=\left(w_{s t} / 2+w_{s}\right) \cdot \csc \left(\pi / 2-\theta_{j}\right)
$$

5. Sum quadratures of length $\Delta t$ between spline knots:

$$
\int_{0}^{t} 1+\int_{t_{1}}^{t 2}+\int_{t_{2}}^{t}+\cdots \int_{t n-2}^{t n-1}
$$

where

$$
\Delta t=\min \left(\cosh ^{-1} 1 / \mu c, 1 .\right)
$$

and terminate on an estimated truncation error equal to

$$
\frac{\pi \cdot O F\left(w_{s c} / 2+w_{s}, \bar{\theta}\right) \cdot e^{-\mu c \cdot \cosh (\bar{t})}}{c}
$$

by means of

$$
\frac{\text { estimated truncation error }}{\text { total quadrature sum }} \leq \text { TOL, TOL }=1 . \mathrm{E}-4
$$

where $\bar{t}$ is the endpoint of integrations and $\bar{\theta}$ corresponds to $\bar{t}$.

6. If the integration does not terminate in step 6 , then for $\boldsymbol{S}_{\mathrm{t}}^{\infty}$,
sum the integrals

$$
\int_{t_{n-1}}^{\Delta t_{1}}+\int_{\Delta t_{1}}^{\Delta t_{2}}+\cdots \int_{\Delta t m-1}^{\Delta t}
$$


where

$$
\begin{aligned}
& \Delta t_{i}=\min \left(\frac{1}{\mu c \cdot \sinh (t)}, 1 .\right), \quad i=1,2, \cdots \\
& \text { until }\left|\frac{\text { estimated error }}{\text { total quadrature sum }}\right|<\text { ETOL, ETOL }=5 . \mathrm{E}-6
\end{aligned}
$$

The truncation error for steps 6 and 7 is estimated by means of

$$
\begin{aligned}
R & =\frac{1}{c} \int_{\bar{t}}^{\infty} \frac{e^{-\mu c \cdot \cosh (t)} \cdot O F\left(w_{s t} / 2+w_{s}, \theta\right) d t}{\cosh (t)} \\
& \leq \frac{O F\left(w_{s t} / 2+w_{s}, \bar{\theta}\right)}{c} \int_{\bar{t}}^{\infty} \frac{e^{-\mu c \cdot \cosh (t)}}{\cosh (t)} d t
\end{aligned}
$$

since $O F$ is monotone decreasing in $t$ ( $s$ increases with $t$, $r$ increases with $s$, and $\theta$ increases with $r$ ). Then

$$
R \leq \frac{O F\left(w_{s t} / 2+w_{s}, \bar{\theta}\right)}{c} e^{-\mu c \cdot \cosh (\bar{t})} \int_{\bar{t}}^{\infty} \frac{d t}{\cosh (t)}
$$

so,

$$
R \leq \frac{O F\left(w_{s t} / 2+w_{s}, \bar{\theta}\right)}{c} e^{-\mu c \cdot \cosh (\bar{t})}\left(2 \cdot \int_{0}^{\infty} \frac{e^{t} d t}{e^{2 t}+1}\right)
$$

and

$$
R \leq \frac{O F\left(w_{s t} / 2+w_{s}, \bar{\theta}\right)}{c} e^{-\mu c \cdot \cosh (\bar{t})}\left(\left.2 \tan ^{-1} e^{t}\right|_{0} ^{\infty}\right)
$$

and

$$
R \leq \pi \frac{O F\left(w_{s t} / 2+w_{s}, \bar{\theta}\right)}{c} e^{-\mu c \cdot \cosh (\bar{t})}
$$

II. Consider

$$
\int_{a}^{b} d y \int_{a}^{\infty} \frac{e^{-\mu \sqrt{x^{2}+y^{2}}}\left(c \sqrt{x^{2}+y^{2}}+1 .\right)}{x^{2}+y^{2}} d x
$$


where $a=w_{s t} / 2$ and $b=w_{s t} / 2+w_{s}$.

Converting to polar form, Expression 10 can be rewritten as

$$
\int_{\theta_{1}}^{\theta}\left[\int_{r_{1}(\theta)}^{r_{2}(\theta)} \frac{e^{-\mu r}(c r+1) r d r}{r^{2}}\right] d \theta
$$

where

$$
\theta_{1}=0, \theta_{2}=\pi / 4, r_{1}(\theta)=\frac{a}{\sin \theta}, r_{2}(\theta)=\frac{b}{\sin \theta}
$$

and

$$
F\left(r_{1}, r_{2}\right)=\int_{r_{1}(\theta)}^{r_{2}(\theta)} \frac{e^{-\mu r}(c r+1) r d r}{r^{2}}
$$

Thus,

$$
\int_{\theta_{1}}^{\theta_{2}}\left[\int_{r_{0}(\theta)}^{r_{2}(\theta)} \frac{e^{-\mu r}(c r+1) r d r}{r^{2}}\right]=\int_{\theta_{1}}^{\theta_{2}} F\left(r_{1}, r_{2}\right) d \theta
$$

and

$$
F(\alpha, \beta)=\int_{\alpha}^{\beta} e^{-\mu}(c+1 / r) d r
$$

so

$$
F(\alpha, \beta)=c \int_{\alpha}^{\beta} e^{-\mu r} d r+\int_{\alpha}^{\beta} e^{-\mu r} / r d r
$$

Evaluating Equation 13,

$$
F(\alpha, \beta)=\frac{c}{\mu}\left(-e^{-\mu \beta}+e^{-\mu \alpha}\right)+\left(\frac{1}{\alpha^{0}} E_{1}(\mu \alpha)-\frac{1}{\beta^{0}} E_{1}(\mu \beta)\right)
$$

or

$$
F(\alpha, \beta)=\frac{c}{\mu}\left(e^{-\mu \alpha}-e^{-\mu \beta}\right)+\left(E_{1}(\mu \alpha)-E_{1}(\mu \beta)\right)
$$


The exponential integral was evaluated using the routing MMDEI from IMSL (International Mathematics and Statistical Libraries, Inc.). 17

III. Consider

$$
I(d, \mu)=\int_{d}^{\infty} \frac{e^{-\mu r}\left(c_{2} r+c_{1}\right)}{r \sqrt{r^{2}-d^{2}}} d r
$$

Let

$$
I_{1}=c_{2} \int_{d}^{\infty} \frac{e^{-\mu r}}{\sqrt{r^{2}-d^{2}}} d r=c_{2} k_{0}(\mu d)
$$

where $K_{0}(x)$ is the modified Bessel function of the second kind, and

$$
I_{2}=c_{1} \int_{d}^{\infty} \frac{1}{r} \frac{e^{-\mu r} d r}{\sqrt{r^{2}-d^{2}}}=\int_{d}^{\infty} \frac{\int_{\mu}^{\infty} e^{-t r} d t}{\sqrt{r^{2}-d^{2}}} d r
$$

This can be rewritten as

$$
I_{2}=\int_{\mu}^{\infty}\left[\int_{d}^{\infty} \frac{e^{-t r} d r}{\sqrt{r^{2}-d^{2}}}\right] d t=\int_{\mu}^{\infty} k_{0}(t d) d t
$$

or

$$
I_{2}=\frac{1}{d} \int_{\mu d}^{\infty} K_{0}(w) d w, \quad t d=w
$$

Then,

$$
I(d, \mu)=c_{2} K_{0}(\mu d)+\frac{c_{1}}{d} \int_{\mu d}^{\infty} K_{0}(w) d w
$$

The routines BESKOI $^{18}$ and INTKO ${ }^{19}$ were used to evaluate the $\mathrm{K}_{0}$ Bessel function and its integral, respectively. 
IV. Consider

$$
\int_{a}^{B} \int_{d}^{\infty} \frac{e^{-\mu r}\left(c_{2} r+c_{1}\right)}{r \sqrt{r^{2}-d^{2}}} d r d(d)
$$

Applying the conclusions of Section III above,

$$
\begin{aligned}
& \int_{a}^{b}\left[c_{2} k_{0}(\mu d)+\frac{c_{1}}{d} \int_{\mu d}^{\infty} k_{0}(w) d w\right] d(d) \\
= & \frac{c_{2}}{\mu} \int_{\mu a}^{\mu b} k_{0}(t) d t+\int_{a}^{b} \frac{c_{1}}{d} \int_{\mu d}^{\infty} k_{0}(w) d w d(d) \\
= & \frac{c_{2}}{\mu} \int_{\mu a}^{\mu b} k_{0}(t) d t+\int_{\mu a}^{\mu b}\left[\frac{c_{1}}{d} \int_{t}^{\infty} k_{0}(w) d w\right] d t
\end{aligned}
$$

To retain significance, we compute

$$
\int_{x_{1}}^{x_{2}} k_{0}(t) d t=\int_{0}^{x_{2}}-\int_{0}^{x_{1}} \text { if } x_{2}<1
$$

and

$$
\int_{x_{1}}^{x_{2}} x_{0}(t) d t=\int_{x_{1}}^{\infty}-\int_{x_{2}}^{\infty} \text { if } x_{2}>1
$$

where the forms on $[0, \mathrm{x}]$ and $[\mathrm{x}, \infty]$ are available from INTK0. ${ }^{19}$

The outer double integral form can be evaluated using GAUS8 (see Reference 16) with INTKO on $[x, \infty]$.

Determine $t$ based on $s$ value:

Since $\cosh (t)=s / c$, let $D=s / c$; 
then

$$
\begin{aligned}
& \frac{e^{t}+e^{-t}}{2}=D \\
& e^{2 t}-2 D e^{t}+1 .=0 \\
& e^{t}=\left(2 D \pm \sqrt{\left.4 D^{2}-4\right) / 2} .\right. \\
& e^{t}=D+\sqrt{D^{2}+1}
\end{aligned}
$$

and

$$
\cosh ^{-1}(D)=t=\ln (D+\sqrt{(D+1)(D-1)})
$$

V. Consider

$$
\int_{a}^{b} d y \int_{0}^{\infty} \frac{e^{-\mu \sqrt{x^{2}+y^{2}}}\left(c_{2} \sqrt{x^{2}+y^{2}}+1 .\right)}{x^{2}+y^{2}} d x
$$

Let

$$
I_{1}=\int_{a}^{b} d y \int_{0}^{\infty} \frac{c_{2} e^{-\mu \sqrt{x^{2}+y^{2}}}}{\sqrt{x^{2}+y^{2}}} d x
$$

Using the substitution $v^{2}+x^{2}+y^{2}$,

$$
I_{1}=\int_{a}^{b} d y \int_{y}^{\infty} \frac{c_{2} e^{-\mu v} d v}{\sqrt{v^{2}-y^{2}}}
$$

If $v=y w$, then, by Equation 17,

$$
I_{1}=c_{2} \int_{a}^{b} k_{0}(\mu y) d y
$$

Let

$$
I_{2}=\int_{a}^{b} d y \int_{0}^{\infty} \frac{e^{-\mu \sqrt{x^{2}+y^{2}}}}{x^{2}+y^{2}} d x
$$


Substituting $\mathrm{v}^{2}=\mathrm{x}^{2}+\mathrm{y}^{2}$ gives

$$
I_{2}=\int_{a}^{b} d y \int_{y}^{\infty} \frac{e^{-\mu v} d v}{\sqrt{v^{2}-y^{2}}}
$$

If $\mathrm{v}=\mathrm{yw}$, then, from Equations 19 and 20 ,

$$
I_{2}=\int_{a}^{b}\left[\frac{1}{y} \int_{\mu y}^{\infty} k_{o}(t) d t\right] d y
$$

Thus, $I_{1}$ and $I_{2}$ are of the same form as Section IV above and can be computed in the same manner.

VI. Consider

$$
\int_{c}^{\infty} \frac{e^{-\mu r} O F_{i, f}(\theta, r) d r}{r^{2}}
$$

where

$$
\begin{aligned}
& c=\sqrt{\left(w_{f} / 2\right)^{2}+[h(i-0.5)]^{2}} \\
& \theta=\frac{\pi}{2}-\tan ^{-1}\left[\frac{\dot{w}_{f} / 2}{\sqrt{z^{2}+[h(i-0.5)]^{2}}}\right]
\end{aligned}
$$

and

$$
z^{2}=r^{2}-[h(i-0.5)]^{2}-\left(w_{f} / 2\right)^{2}
$$

This integral was evaluated using GAUS8 (see Reference 16 ), taking $\Delta \mathrm{r}=3 . / \mu$ and terminating integration when the amount being added to the accumulated sum relative to the sum became less than a specified tolerance.

VII. Consider

$$
\int_{a}^{b} \frac{e^{-\mu r}\left(c_{2} r+c_{1}\right)}{r} d r
$$

Expression 30 can be shown to be equal to

$$
c_{2} \int_{a}^{b} e^{-\mu r} d r+c_{1} \int_{a}^{b} \frac{e^{-\mu r}}{r} d r
$$


which, when evaluated, equals

$$
\frac{c_{2}}{\mu}\left(e^{-\mu a}-e^{-\mu b}\right)+c_{1}\left[\left(E_{1}(\mu a)-E_{1}(\mu b)\right]\right.
$$

The exponential integral was evaluated using the routine MMDEI (see Reference 17). 
NOTES

149 CFR173.389(i) (1).

${ }^{2}$ U.S. Atomic Energy Commission, Directorate of Regulatory Standards, Environmental Survey of Transportation of Radioactive Material to and from Nuclear Power Plants, WASH 1238 (Washington: USAEC, 1972).

${ }^{3}$ U.S. Nuclear Regulatory Commission, Office of Standards Development, Final Environmental Statement on the Transportation of Radioactive Materials by Air or Other Modes, NUREG-0170 (Washington: USNRC, December 1977).

${ }^{4}$ W. E. Selph, Neutron and Gamma-Ray Albedos, ORNL-RSIC-21, (DASA-1892-2) (Oak Ridge, TN: Oak Ridge National Laboratory, February 1968).

${ }^{5}$ Libby-Owens Ford, Glass for Construction, LOF 8.26/Li, January 1976.

${ }^{6}$ T. Rockwel1, Reactor Shielding Design Manual (Cincinnati: D. Von Nostrand Company, Inc., 1956).

${ }^{7}$ S. Glasstone, ed, The Effects of Nuclear Weapons (Washington: U.S. Atomic Energy Commission, April 1962).

${ }^{8}$ R. G. Jaeger et al, Engineering Compendium on Radiation Shielding (New York: Springer-Verlag, 1975).

${ }^{9} \mathrm{H}$. Goldstein, Fundamental Aspects of Reactor Shielding (Reading, MA: Add i son-Wesley, 1959).

${ }^{10}$ U.S. Department of Commerce, Bureau of the Census, Journey to Work, 1974.

1lJ. E. Baerwald, ed., Transportation and Traffic Engineering Handbook (Englewood Cliffs, NJ: Prentice-Ha11, 1976).

12 B. Pushkarev and J. M. Zupan, Urban Space for Pedestrians (Cambridge, MA: MIT Press, 1975).

13 Appendix VI to WASH-1400.

$14 \mathrm{~J}$. Shapiro, "Exposure of Airport Workers to Radiation from Shipments of Radioactive Materia1," draft report to U.S. Nuclear Regulatory Commission, January 1976.

15U.S. Nuclear Regulatory Commission, Final Generic Environmental Impact Statement on Use of Recycle Plutonium in Mixed Oxide Fuel Light Water Reactors, NUREG-0002 (August 1976).

${ }^{16}$ R. E. Jones and C. B. Bailey, Brief Instruction for Using MATHLIB (Version 6.0), SAND75-0545 (Albuquerque: Sandia Laboratories, February 1976).

17 International and Statistical Libraries, Inc., The IMSL Library, IMSL LIB03-0006, IMSL Library 3, Edition 6, (Fortran) CDC 6000/7000, CYBER $7 \overline{0 / 170}$ Series, July 1977. 
${ }^{18}$ D. E. Amos and S. L. Daniel, CDC 6600 Subroutines for Bessel Functions $I_{u}(x)$, $v=0,-\infty<x<\infty$ and $k_{v}(x), v=0,1, x>0$, SAND75-0149 (Albuquerque: Sandia Laboratories, September 1975).

${ }^{19}$ D. E. Amos and S. L. Danie1, CDC 6600 Subroutines for Integrals of (sin $\left.t\right) / t$, $(\cos t) / t, K_{0}(t)$, SAND76-0062 (Albuquerque: Sandia Laboratories, February 1976).

20 F. H. Clark, "Gamma-Ray Buildup Factors for Sand, Air, and Wood (Ce1lulose)," Nuclear Applications, Vo1. 6, June 1969, pp 588-593. 
ANALYSIS OF THE RADIOLOGICAL CONSEQUENCES OF ACCIDENTS

INVOLVING RADIOACTIVE MATERIALS

Shipments of radioactive material may be involved in vehicular accidents which result in package damage. If this occurs, radioactive material may be dispersed or become unshielded and expose the surrounding population. If the accident is not severe enough to damage the package but is severe enough to delay the transporting vehicle, some additional exposure can result.

Accidents which compromise package integrity can be divided into those involving shipments of dispersible materials and those involving shipments of nondispersible materials. In the first case, both the inhalation and direct exposure aspects are important and should be evaluated. In the second case, only direct exposure is of interest. The various combinations of material types and accident consequences can be visualized as shown in Figure $C-1$.

\section{C1. Material Characterization}

The consequences of an accident involving release of or exposure to a particular radioactive material depend upon certain dosimetric parameters. These include material toxicity parameters, the particular physiological systems affected, the fraction of released material which becomes aerosolized, the respirable fraction, and the resuspension dose factor. The method of obtaining values for each of these factors for each radionuclide and the method by which they are used are discussed in Appendix G.

C2. Accident Environment/Package Release Mode1

The model used to describe the severity of accident environments and to relate package response to those environments is essentially the same as that used in Reference $l$ and is only summarized in this appendix. Detailed data for accident occurrence probabilities by severity and mode are provided in Appendix A.

\section{C2.1 Accident Environment Severity Classification}

The fraction of contained radioactive material released to the environment in an accident depends upon both the severity of the accident and the accident resistance of the package. Very severe accidents could release a large fraction of the contained radioactive material, while minor accidents are unlikely to result in any release. 


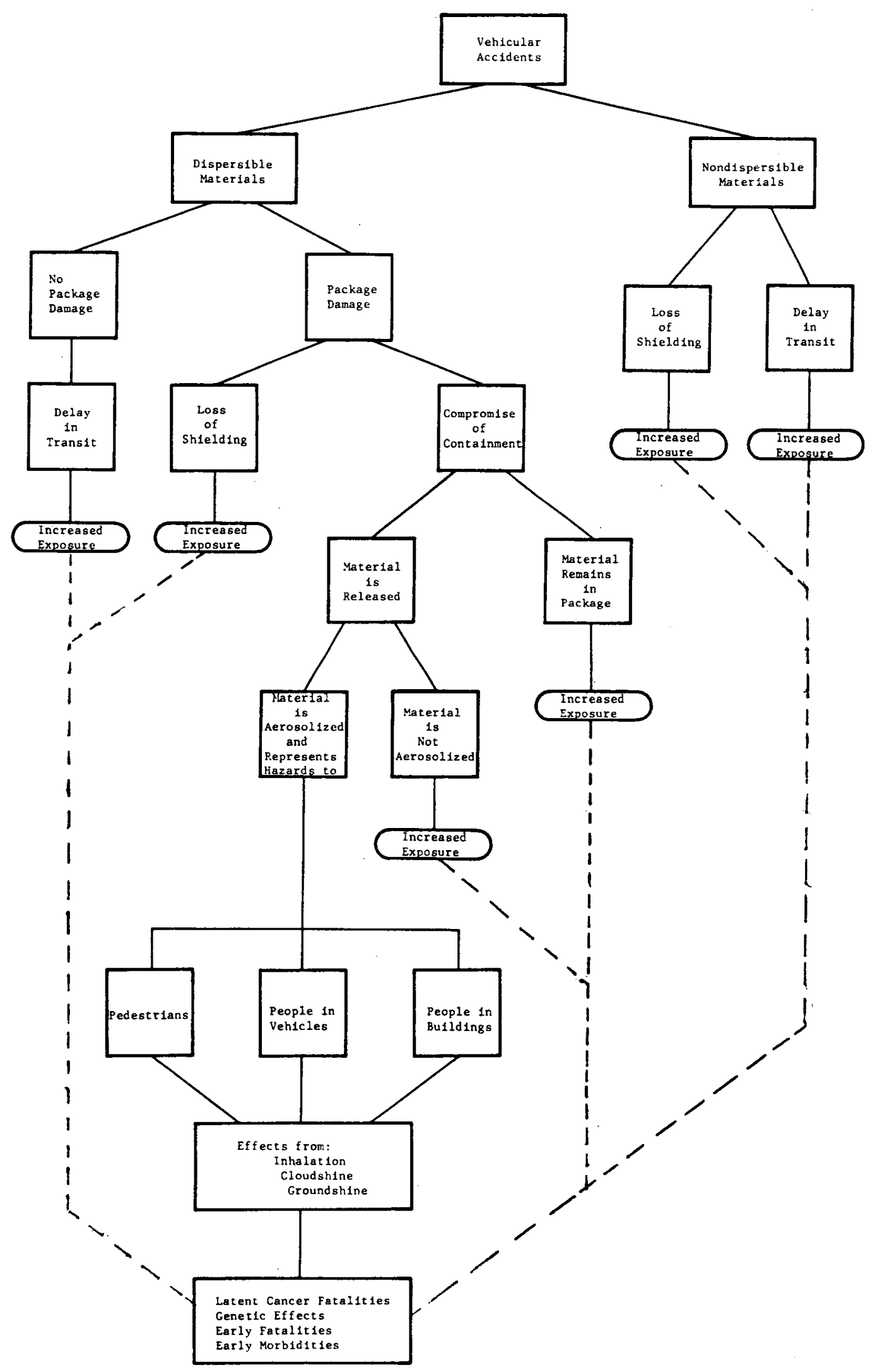

Figure C-1. Possible Accidents and Their Consequences 
In addition to the overall accident rate for each mode, the distributions of accidents according to severity must be determined. The eight-severity classification scheme used in this report assigns a principal accident damage mechanism (impact, crush, puncture, or fire) to each transport mode, and assigns to each of the eight severity categories a mode-dependent occurrence probability. Synergistic effects resulting from combinations of two or more damage mechanisms in the same accident have been examined elsewhere, and as a result of these studies, the only combinations considered in this model involve a single deformation force and fire. ${ }^{2}$ Once the occurrence probabilities for environments of each severity have been determined, an urban area accident rate by severity, mode, and time span is obtained.

\section{C2.2 Package Response Model}

To assess the risk of a transportation accident, the response of a particular package type to an accident of given severity must be predicted. In particular, it is necessary to know the fraction of the total package contents which might be released, although the actual releases for a given package type would not necessarily be the same for a number of accidents of the same severity class. In some cases, there may be no release, while in others there may be a release of some or all of the package contents. Indeed, in any given accident involving a number of radioactive material packages transported together, some of the packages may release part of their contents while others remain unaffected. The approach taken in Reference 1 and in this assessment is to derive a single value representing the average release fraction for each severity category and package type (independent of mode) and to assume that all such packages, including separate packages in a multipackage shipment, respond to the accident in the same way.

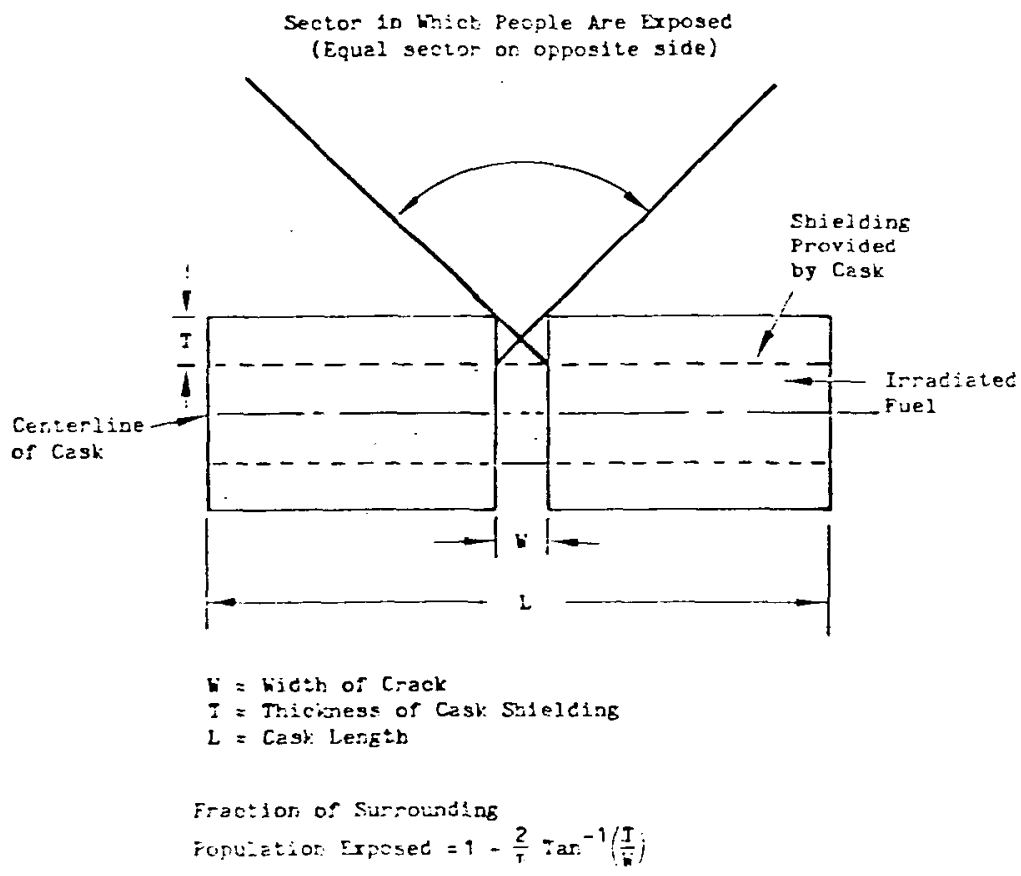

F-Eve C-2. Feicase Fráctior 
Two terms must be explained further: packages containing dispersible material and packages containing nondispersible materials. For dispersible materials, the concept of "release" is straightforward: released material is that which ends up beyond the outer boundary of the package after an accident. It is more complicated in the case of nondispersible materials. As discussed earlier, the radiological consequence of an accident involving a nondispersible shipment would result from a loss of shielding which would expose people more directly to some fraction of the package contents. For small packages, this fraction is taken to be identical to the release fraction for a dispersible material carried in that same package. For a large package, such as a spent fuel cask, the effect of loss of shielding is modeled by assuming that the accident forces produce a circumferential crack in the container. "Release fraction" in this case is the product of the fraction of the source exposing the surrounding population and the fraction of the surrounding area within the sector being exposed, as shown in Figure $\mathrm{C}-2$. The computation of the integrated population dose is then carried out assuming an effective point source, the strength of which is the total curies multiplied by the release fraction.

The values used for release fractions for the various package types are given in Table C-1. These values are model variables and can be modified as desired by the analyst.

In the following sections, the mathematical basis for the radiological health effect impacts shown in Figure $\mathrm{C}-3$ is provided and actual expressions used to calculate impacts are derived.

\section{C3. Accident Doses from Nondispersible Materials}

If a shipment of a particular nondispersible material is involved in an accident of sufficient severity to compromise packaging integrity, the effectiveness of the shielding around the source is assumed to be reduced.

Tab1e C-1

Release Fractions for Package Types

(Cask releases are fractions of source term hypothetically released to the environment)

\begin{tabular}{|c|c|c|c|c|c|c|}
\hline \multirow[b]{2}{*}{$\begin{array}{l}\text { Severity } \\
\text { Category }\end{array}$} & \multirow[b]{2}{*}{$\begin{array}{l}\text { LSA } \\
\text { Drum }\end{array}$} & \multirow[b]{2}{*}{$\begin{array}{c}\text { Type } \\
\mathrm{A} \\
\end{array}$} & \multicolumn{2}{|c|}{ Type B } & \multirow[b]{2}{*}{$\begin{array}{c}\text { Cask } \\
\text { (exposure) } \\
\end{array}$} & \multirow[b]{2}{*}{$\begin{array}{c}\text { Cask } \\
\text { (release) } \\
\end{array}$} \\
\hline & & & No $\mathrm{Pu}$ & $\begin{array}{c}1975 \\
\mathrm{Pu} \\
\end{array}$ & & \\
\hline I & 0 & 0 & 0 & 0 & 0 & 0 \\
\hline II & 0.01 & 0.01 & 0 & 0 & 0 & 0 \\
\hline III & 0.1 & 0.1 & 0.01 & 0 & 0 & 0.01 \\
\hline IV & 1.0 & 1.0 & 0.1 & 0 & 0 & 0.1 \\
\hline v & 1.0 & 1.0 & 1.0 & 0 & 0 & 1.0 \\
\hline VI & 1.0 & 1.0 & 1.0 & 0.01 & $3.2 \times 10^{-7}$ & 1.0 \\
\hline VII & 1.0 & 1.0 & 1.0 & 0.05 & $3.2 \times 10^{-5}$ & 1.0 \\
\hline VIII & 1.0 & 1.0 & 1.0 & 0.1 & $3.1 \times 10^{-3}$ & 1.0 \\
\hline
\end{tabular}



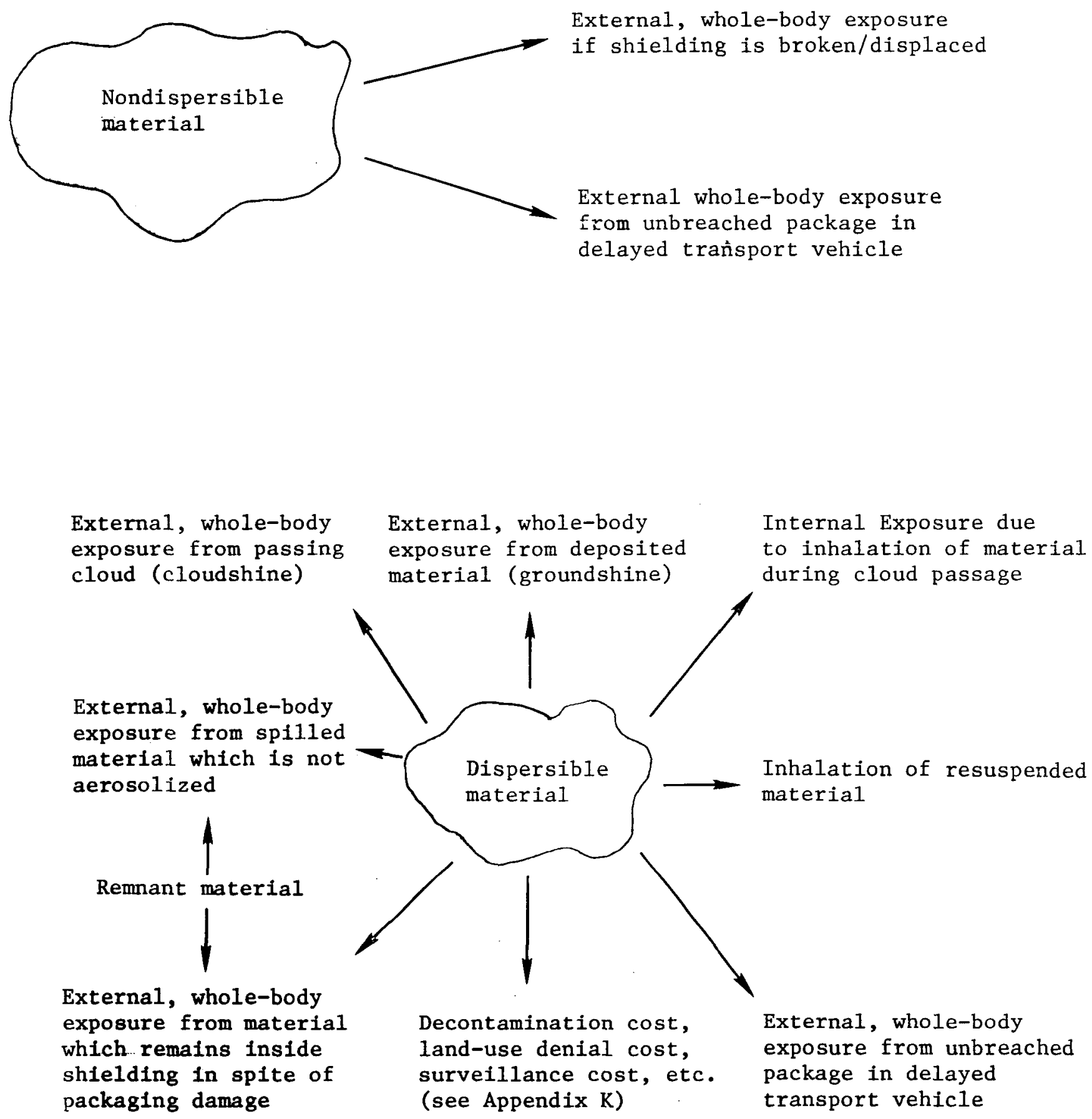

Figure C-3. Radiological Health-Effect Hazard Categories 
The basic expression* for dose rate at a distance $r$ from the source under these circumstances is derived from the information in Reference 3:

$$
\mathrm{DR}(\mathrm{r})=\frac{\mathrm{Q}_{5} \cdot \mathrm{n}_{1} \cdot \mathrm{RF} \cdot \mathrm{PPS} \cdot \mathrm{E}_{\mathrm{d}} \cdot \mu_{\mathrm{r}} \cdot \mathrm{e}^{-\mu_{a i r}} \cdot \mathrm{B}_{\mathrm{air}}(\mathrm{r})}{\mathrm{r}^{2}}
$$

where

$$
\begin{aligned}
Q_{5}= & \text { units conversion factor }\left(4.8 \times 10^{-5} \frac{\mathrm{rem} \cdot \mathrm{m}^{3}}{\mathrm{~s} \cdot \mathrm{Ci} \cdot \mathrm{MeV}}\right) \\
\mathrm{n}_{1}= & \text { amount of material per package (curies) } \\
\mathrm{RF}= & \text { release fraction } \\
\mathrm{PPS}= & \text { number of packages per shipment } \\
\mathrm{E}_{\mathrm{d}}= & \text { photon energy released per disintegration }(\mathrm{MeV}) \\
\mu_{\mathrm{R}}= & \text { attenuation coefficient of receptor }\left(3.1 \mathrm{~m}^{-1}\right. \text { for } \\
& \text { body tissue) } \\
\mu_{\text {air }}= & \text { attenuation coefficient of air }\left(\mathrm{m}^{-1}\right) \\
\mathrm{r}= & \text { distance from source (metres) } \\
\mathrm{B}_{\text {air }}(\mathrm{r})= & \text { dose buildup factor }
\end{aligned}
$$

This equation is similar to that used in Appendix B to derive expressions for incident-free dose rate and can be similarly manipulated.

\section{C3.1 Dose from Truck Transport}

Dose to Pedestrians

The individual dose, $\phi(r)$, received by a pedestrian at a distance $r$ from an accident involving an exposure source is given by

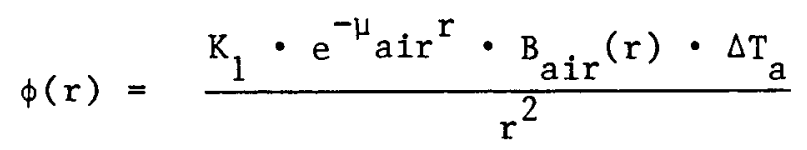

where

$$
\begin{aligned}
\Delta \mathrm{T}_{\mathrm{a}} & =\text { severity dependent accident delay time (seconds) } \\
\mathrm{K}_{1} & =\text { source term }\left(\frac{\mathrm{rem} \cdot \mathrm{m}^{2}}{\mathrm{~s}} ;\right. \text { see following discussion) }
\end{aligned}
$$

*A complete list of variables, constants, and functions used in the development is given in the addendum to this appendix. 
In addition, material which remains shielded will continue to expose people while initial emergency action is being taken to remove exposed material. Unshielded material will continue to expose people in the area throughout the accident delay time $\Delta \mathrm{T}_{\mathrm{a}}$. In this case, the source term is simply $\mathrm{K}_{\mathrm{o}} \cdot \mathrm{TI} \cdot \mathrm{PPS} \cdot(1-\mathrm{RF})$. Thus, a general form for a source term from an accident involving a nondispersible shipment can be written as

$$
\begin{aligned}
\mathrm{K}_{1}= & \left(Q_{5} \cdot \mathrm{n}_{1} \cdot \mathrm{PPS} \cdot \mu_{\mathrm{r}} \cdot \mathrm{E}_{\mathrm{d}} \cdot \mathrm{RF}\right)+ \\
& {\left[\mathrm{Q}_{3} \cdot \mathrm{K}_{\mathrm{o}} \cdot \mathrm{TI} \cdot \mathrm{PPS} \cdot(1-\mathrm{RF})\right] }
\end{aligned}
$$

where

$$
\begin{aligned}
& \mathrm{K}_{0}=\text { shape factor for specific package type }\left(\frac{\mathrm{mrem} \cdot \mathrm{m}^{2}}{\mathrm{~h}}\right) \\
& \mathrm{TI}=\text { transport index for package (equivalent to mrem/h) } \\
& \mathrm{Q}_{3}=\text { units conversion factor }\left(2.78 \times 10^{-7} \frac{\mathrm{rem} \cdot \mathrm{h}}{\mathrm{mrem} \cdot \mathrm{s}}\right)
\end{aligned}
$$

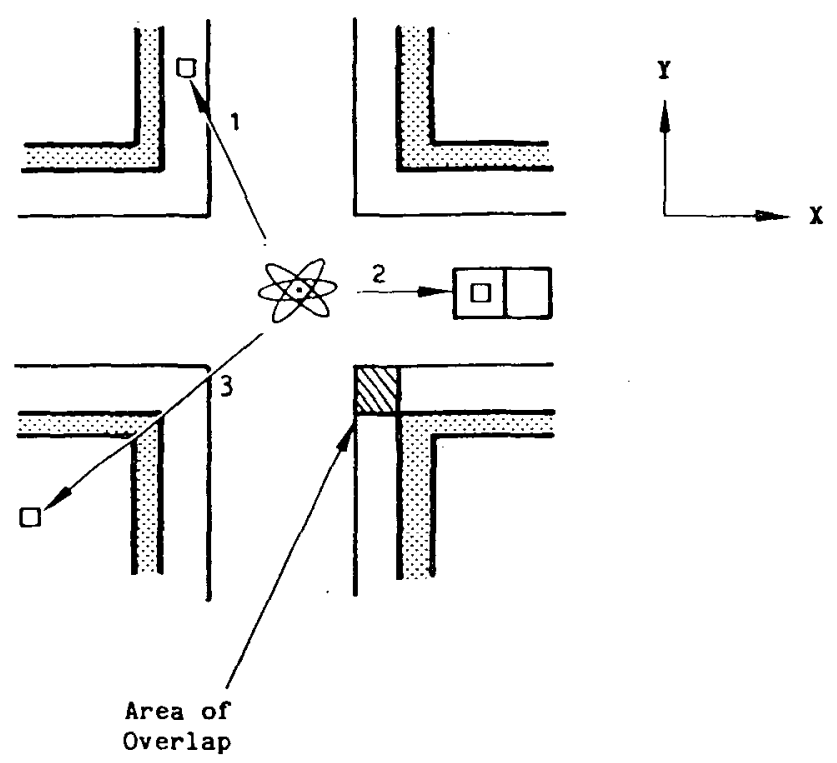

1. Dose to Pedestrians

2. Dose to People in Vehicles

3. Dose to People in Buildings

Figure C-4. Dose Due to Nondispersible Sources 


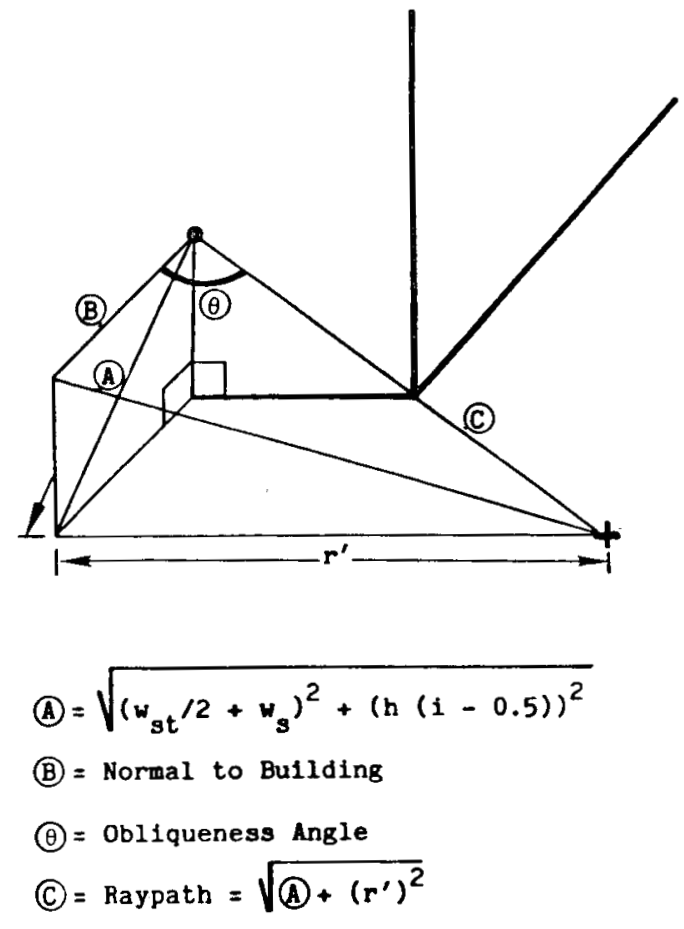

Figure C-5. Nondispersible Accident Geometry (Intersection Shown)

The integrated dose geometry is illustrated in Figures $\mathrm{C}-4$ and $\mathrm{C}-5$. Both intersection and midblock geometries are considered, and an accident location occurrence probability is assigned to each geometry based on data from Reference 4. Pedestrians are exposed on eight sidewalk segments in the intersection case; therefore the expression for integrated dose can be written as

$$
\begin{aligned}
& \mathrm{ID}=\mathrm{Q}_{2} \cdot 8 \cdot \operatorname{PedD} \cdot \mathrm{K}_{1} \cdot \Delta \mathrm{T}_{\mathrm{a}} \cdot \\
& \int^{\frac{w_{s t}}{2}+w_{s}} \int_{y}^{\infty} \frac{e^{-\mu_{a i r} \sqrt{x^{2}+y^{2}}} \cdot B_{a i r}\left(\sqrt{x^{2}+y^{2}}\right) d x}{x^{2}+y^{2}} \\
& \frac{w_{\text {st }}}{2} \quad \frac{w_{s t}}{2}
\end{aligned}
$$

where

$$
\begin{aligned}
\mathrm{Q}_{2} & =\text { units conversion factor }\left(10^{-6} \frac{\mathrm{km}^{2}}{\mathrm{~m}^{2}}\right) \\
\mathrm{K}_{1} & =\text { source term (see } \mathrm{Eq} \cdot(3)) \\
\text { PedD } & =\text { pedestrian density (persons } / \mathrm{km}^{2} \text { of sidewalk) } \\
\mathrm{w}_{\mathrm{st}} & =\text { street width (metres) } \\
\mathrm{w}_{\mathrm{s}} & =\text { sidewalk width (metres) }
\end{aligned}
$$


There is some overlap on the corners of the sidewalks. However, this overlap can be removed by changing to polar coordinates and modifying the limits of integration.

In the case of midblock pedestrian exposure, the limits of integration on the inner integral of Eq. (4) are modified to run from 0 to infinity, and the factor of 8 is replaced by a factor of 4 (since there are four 0 to $\infty$ segments). This yields

$$
\begin{aligned}
& \mathrm{ID}=\mathrm{Q}_{2} \cdot 4 \cdot \operatorname{PedD} \cdot \mathrm{K}_{1} \cdot \Delta \mathrm{T}_{\mathrm{a}} \cdot \\
& \int_{w}^{\frac{w}{2}+w_{s t}} \int^{\infty} \frac{e^{-\mu} a i r^{\sqrt{x^{2}+y^{2}}} \cdot B_{a i r}\left(\sqrt{x^{2}+y^{2}}\right) d x}{x^{2}+y^{2}} \\
& \frac{w_{\text {st }}}{2} \quad 0
\end{aligned}
$$

Eqs. (4) and (5) can be combined and weighted by their intersection and midblock accident occurrence factors to give the expression for integrated pedestrian exposure:

$$
\begin{aligned}
& \mathrm{ID}=\mathrm{Q}_{2} \cdot \operatorname{PedD} \cdot \mathrm{K}_{1} \cdot \Delta \mathrm{T} \mathrm{a} \cdot \\
& \left\{8 \cdot \operatorname{INTF} \cdot\left[\int_{w_{s t} / 2}^{w_{s t} / 2+w_{s}} d y \int_{w_{s t} / 2}^{\infty} \frac{e^{-\mu_{a i r} \sqrt{x^{2}+y^{2}} \cdot B_{a i r}\left(\sqrt{x^{2}+y^{2}}\right) d x}}{x^{2}+y^{2}}\right\}+\right. \\
& \left.4 \cdot \operatorname{MBF} \cdot\left[\int_{w_{s t} / 2}^{w_{s t} / 2+w_{s}} d y \int_{0}^{\infty} \frac{e^{-\mu_{a i r} \sqrt{x^{2}+y^{2}}} \cdot B_{a i r}\left(\sqrt{x^{2}+y^{2}}\right) d x}{x^{2}+y^{2}}\right]\right\}
\end{aligned}
$$

where

$$
\begin{aligned}
\text { INTF } & =\text { intersection accident occurrence factor }(0.4) \\
\mathrm{MBF} & =\text { midblock accident occurrence factor }(0.6)
\end{aligned}
$$

In using this analysis, several conservative points should be noted. First, the sidewalk length is assumed to be infinite. This allows dose to pedestrians in adjacent cells to be included but is clearly an overestimate. Second, streets (and hence, sidewalks) are assumed to be straight. Third, no terrain variation is considered. 
Dose to People in Buildings

The individual dose to a person in a building will depend upon the floor that person is on and his distance "down the block" from the shipment. With those constraints on $r$, the individual dose expression can be written

$$
\phi(r)=K_{1} \cdot \Delta T_{a} \cdot e^{-\mu_{b} w_{b}} \cdot B_{b}\left(w_{b}\right) \cdot O F(r) \cdot \frac{e^{-\mu_{a i r} r}}{r^{2}}
$$

where

$$
\begin{aligned}
& \mathrm{OF}(\mathbf{r})=\text { obliqueness factor (see Appendix } B \text { ) } \\
& w_{b}=\text { building wall thickness (metres) } \\
& \mu_{b}=\begin{array}{l}
\text { linear attenuation coefficient for building } \\
\text { material }\left(\mathrm{m}^{-1}\right)
\end{array} \\
& \mathrm{B}_{\mathrm{b}}=\text { dose rate buildup factor (see Appendix } B \text { ) }
\end{aligned}
$$

In order to compute integrated dose to people in buildings, it is first necessary to compute the fraction of all people in buildings in a particular cell who will be exposed to radiation from the accident. Assuming that buildings are uniform with a square cross section, a linear population density can be obtained (per $\mathrm{km}$ of building perimeter) by dividing the total number of people in the cell by the total building perimeter in the cell as in Appendix B. This linear population density is given by

$$
\left[\begin{array}{l}
\text { people per } \\
\text { kilometre } \\
\text { of building } \\
\text { perimeter }
\end{array}\right]=\frac{(P D+T C) \cdot\left(w_{s t}+2 w_{s}\right) \cdot Q_{7}}{4\left(\sqrt{f_{b}}-f_{b}\right)}
$$

where

$$
\begin{aligned}
& \left.\mathrm{PD}=\text { population density (people } / \mathrm{km}^{2}\right) \\
& \left.\mathrm{TC}=\text { transient population (people } / \mathrm{km}^{2}\right) \\
& \mathrm{f}_{\mathrm{b}}=\text { fraction of cell area occupied by buildings } \\
& \mathrm{Q}_{7}=\text { units conversion factor }\left(10^{-3} \frac{\mathrm{km}}{\mathrm{m}}\right)
\end{aligned}
$$

If this expression is divided by the number of floors, $n$, in each building in the cell, the density per floor is obtained. If an incremental distance $\Delta r$ at a distance $r$ down any street segment from the accident site is examined, the total exposure on the ith floor in that increment is given by

$$
\begin{array}{r}
I D=Q_{2} \cdot \frac{(P D+T C) \cdot\left(w_{s t}+2 w_{s}\right)}{4\left(\sqrt{f_{b}}-f_{b}\right) \cdot n} \cdot \Delta r \cdot \Delta T_{a} \\
\quad e^{-\mu_{b} w_{b}} \cdot B_{b}\left(w_{b}\right) \cdot k_{1} \cdot \frac{e^{-\mu_{a i r} r} \cdot O F_{i}(r)}{r^{2}}
\end{array}
$$


Both the intersection and midlbock geometries must now be separately evaluated to compute integrated exposure. Figure $\mathrm{C}-5$ shows that the only difference between the intersection and midblock geometries is the minimum value of $r^{\prime}$. In the case of a midblock accident, $r^{\prime}$ can run from 0 to $\infty$; in the intersection case, $r^{\prime}$ can run from $\left(w_{s t} / 2+w_{s}\right)$ to $\infty$.

The final expression for integrated exposure to people in buildings due to nondispersible source accidents can now be formed by integrating the dose over the exposed distance on each floor, summing the doses accumulated on each floor, accounting for the number of exposed building segments (eight for intersections, four for midblock), and separating the accidents into occurrence locations. This yields

$$
\begin{aligned}
& \mathrm{ID}=\frac{\mathrm{Q}_{2} \cdot(\mathrm{PD}+\mathrm{TC}) \cdot\left(\mathrm{w}_{\mathrm{st}}+2 \mathrm{w}_{\mathrm{s}}\right) \cdot \Delta \mathrm{T}_{\mathrm{a}} \cdot \mathrm{e}^{-\mu_{\mathrm{b}} \mathrm{w}_{\mathrm{b}} \cdot \mathrm{B}_{\mathrm{b}}\left(\mathrm{w}_{\mathrm{b}}\right) \cdot \mathrm{K}_{1}}}{4 \cdot \mathrm{n} \cdot\left(\sqrt{\mathrm{f}_{\mathrm{b}}}-\mathrm{f}_{\mathrm{b}}\right)} \cdot \\
& \left\{\left[\operatorname{INTF} \cdot 8 \cdot \sum_{i=1}^{n}\left(\int_{\beta_{4}}^{\infty} \frac{e^{-\mu a i r^{r} \cdot O F_{i}(r) d r}}{r^{2}}\right)\right]+\right. \\
& \left.\left[\operatorname{MBF} \cdot 4 \cdot \sum_{i=1}^{n}\left(\int_{B_{5}}^{\infty} \frac{e^{-\mu_{a i r}} \cdot O F_{i}(r) d r}{r^{2}}\right)\right]\right\}
\end{aligned}
$$

where

$$
\begin{aligned}
& B_{4}=\sqrt{\left(\sqrt{\left.2\left(w_{s t} / 2+w_{s}+w_{b}\right)\right)^{2}+(h(i-0.5))^{2}}\right.} \\
& B_{5}=\sqrt{\left(w_{s t} / 2+w_{s}+w_{b}\right)^{2}+(h(i-0.5))^{2}}
\end{aligned}
$$

Dose to People in Vehicles

Because it is assumed that vehicles provide no shielding, individual dose to people in vehicles is identical to dose to pedestrians (developed in Eq. (2)).

The integrated dose received by people in vehicles in the vicinity of an intersection accident can be visualized by thinking of cars stopped at discrete distances from the accident, as shown in Figure C-6. If, as in the figure, the lines of vehicles extended all the way to the accident site, an individual in a vehicle located $j$ vehicles away from the site, would receive a dose equal to

$$
\phi=K_{1} \cdot \Delta T_{a} \cdot \frac{e^{-\mu_{a i r}(\delta+\ell) j} \cdot B_{a i r}((\delta+\ell) j)}{((\delta+\ell) j)^{2}}
$$

where

$$
\begin{aligned}
& \delta=\text { vehicle separation distance (metres) } \\
& \ell=\text { vehicle length (metres) }
\end{aligned}
$$


If an infinite row of vehicles on each of the four radiating streets is assumed, the general expression for integrated exposure is

$$
\mathrm{ID}=4 \cdot \mathrm{K}_{1} \cdot \Delta \mathrm{T}_{\mathrm{a}} \cdot \mathrm{L}^{\prime} \cdot \mathrm{PPV} \cdot \sum_{j=1}^{\infty} \frac{\mathrm{e}^{-\mu} \mathrm{air}^{(\delta+\ell) j} \cdot \mathrm{B}_{\mathrm{air}}((\delta+\ell) j)}{j^{2}(\delta+\ell)^{2}}
$$

where $L^{\prime}=$ number of lanes per side of street.

The numerator inside the summation is approximately equal to 1.0 for all values of $\mu_{a}, j$, and $(\delta+\ell)$. With this in mind, Eq. (12) can be simplified to

$$
\mathrm{ID}=4 \cdot \mathrm{K}_{1} \cdot \Delta \mathrm{T}_{\mathrm{a}} \cdot \mathrm{L}^{\prime} \cdot \mathrm{PPV} \cdot \frac{\mathrm{I}}{(\delta+\ell)^{2}} \sum_{j=1}^{\infty} \frac{1}{j^{2}}
$$

The equality overestimates the dose by about $7 \%$. In addition, the summation can be shown to be equal to 1.64 . Thus, Eq. (13) reduces to

$$
\mathrm{ID}=4 \cdot \mathrm{K}_{1} \cdot \Delta \mathrm{T}_{\mathrm{a}} \cdot \mathrm{L}^{\prime} \cdot \mathrm{PPV} \cdot \frac{1.64}{(\delta+\ell)^{2}}
$$

A portion of this dose is actually accumulated by people in vehicles within the intersection. Since it is assumed that only response and recovery vehicles are allowed in the intersection area (see Figure C-6), this contribution to the dose must be subtracted from that predicted by Eq. (14).*

where

$$
\begin{aligned}
\mathrm{ID} & =4 \cdot \mathrm{K}_{1} \cdot \Delta \mathrm{T}_{\mathrm{a}} \cdot \mathrm{L}^{\prime} \cdot \mathrm{PPV} \cdot\left[\frac{1.64}{(\delta+\ell)^{2}}-\sum_{j=1}^{N^{\prime}} \frac{1}{(j(\delta+\ell))^{2}}\right] \\
& =\frac{4 \cdot \mathrm{K}_{1} \cdot \Delta \mathrm{T}_{\mathrm{a}} \cdot \mathrm{L}^{\prime} \cdot \mathrm{PPV}}{(\delta+\ell)^{2}}\left[1.64-\sum_{j=1}^{N^{\prime}} \frac{1}{j^{2}}\right]
\end{aligned}
$$

$$
\begin{aligned}
\mathrm{N}^{\prime}= & \text { number of cars in intersection in each lane }= \\
& \frac{\text { intersection half-width }}{\text { distance per car }}=\frac{{ }_{\mathrm{w} t} / 2}{\delta+\ell} \text { (rounded to next highest integer) }
\end{aligned}
$$

*It is also assumed that the vicinity of an accident will be characterized by congested traffic flow. No credit is taken for the less congested conditions which might occur during periods of reduced traffic flow. 


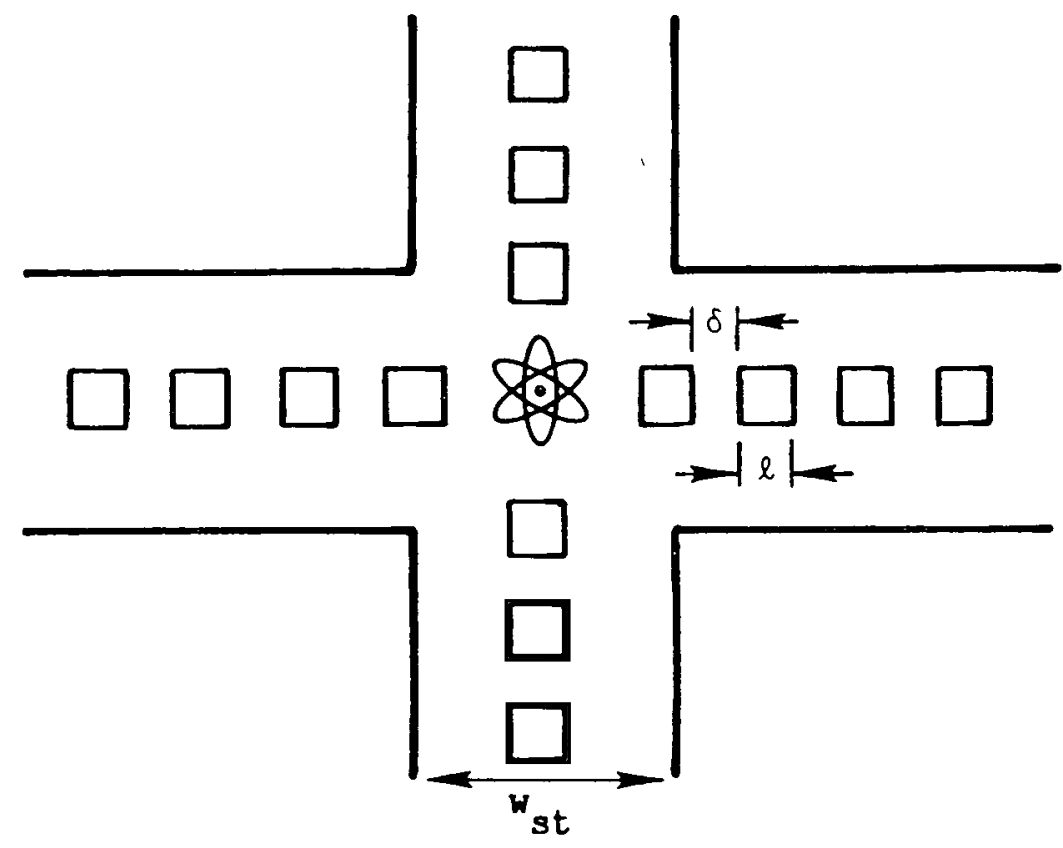

a. Cars Extending into Intersection

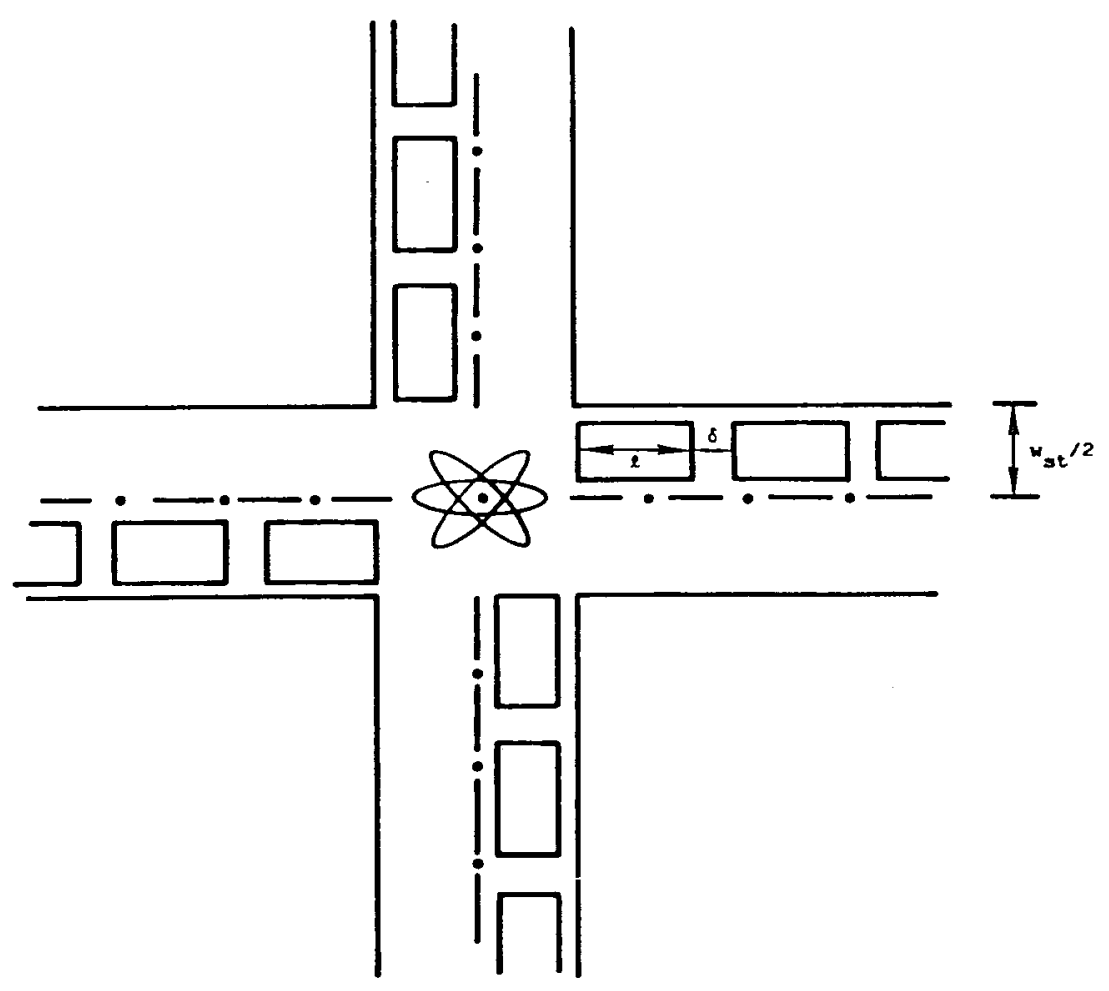

b. Cars Kept Out of Intersection

Figure C-6. Exposure Geometry for Direct Exposure to People in Vehicles Resulting from an Intersection Accident 
To account for dose to people in vehicles from nondispersible source accidents occurring midblock, it is assumed that traffic continues to move in the opposite direction at half the normal cruising velocity and that traffic moving in the same direction as the shipment is essentially stopped. Therefore, the dose expression has two components: "same-direction dose" and "opposite-direction dose." Samedirection dose is analogous to the intersection dose derived in Eq. (13). In this case, however, there are two radiating street segments (one ahead of the accident and one behind the accident), and the lane of stopped vehicles extends only to the next intersection. The expression thus becomes

$$
\mathrm{ID}=2 \cdot \mathrm{K}_{1} \cdot \Delta \mathrm{T}_{\mathrm{a}} \cdot \mathrm{L}^{\prime} \cdot \mathrm{PPV} \cdot \frac{1}{(\delta+\ell)^{2}} \cdot \sum_{j=\mathrm{N}^{\prime}}^{\mathrm{N}^{\prime \prime}} \frac{1}{j^{2}}
$$

where

$$
N^{\prime \prime}=\frac{w_{s t}+2 w_{s}}{(\delta+\ell) \cdot 2\left(1-\sqrt{f_{b}+f_{o}}\right)}=\left[\begin{array}{c}
\text { number of vehicles in each lane } \\
\text { in } 1 / 2 \text { block length }
\end{array}\right]
$$

In the case of opposite-direction dose, an expressions analogous to that developed in Appendix $B$ can be used. In this situation, $L / V_{C}$ is replaced by $\Delta T_{a}$ since traffic is assumed to be exposed for the entire time period during which the accident is being cleared.

$$
\begin{gathered}
I D=Q_{2} \cdot \mathrm{K}_{1} \cdot \Delta \mathrm{T}_{\mathrm{a}} \cdot \frac{\mathrm{N} \cdot \mathrm{w}_{\text {st }}}{2 \cdot \mathrm{A} \cdot \mathrm{f}_{\text {st }}} \\
\int_{\mathrm{w}_{\mathrm{st}} / 2}^{\infty} \frac{\mathrm{e}^{-\mu_{\mathrm{air}} \mathrm{r} \cdot \mathrm{B}_{\mathrm{air}}(\mathrm{r}) \mathrm{dr}}}{\mathrm{r} \sqrt{\mathrm{r}^{2}-\left(\mathrm{w}_{\mathrm{st}} / 2\right)^{2}}}
\end{gathered}
$$

When the expressions in Eqs. (16) and (17) are combined using the appropriate accident location weighting factor, the final expression for dose to people in vehicles from nondispersible source accidents becomes

$$
\begin{gathered}
I D=\mathrm{K}_{1} \cdot \Delta \mathrm{T}_{\mathrm{a}} \cdot \mathrm{PPV} \cdot\left\{\operatorname{INTF}\left[\frac{4 \mathrm{~L}^{\prime}}{(\delta+\ell)^{2}}\left(1.64-\sum_{j=1}^{N^{\prime}} \frac{1}{j^{2}}\right)\right]+\right. \\
\operatorname{MBF}\left[\frac{2 \mathrm{~L}^{\prime}}{(\delta+\ell)^{2}} \cdot \sum_{j=\mathrm{N}^{\prime}}^{N^{\prime \prime}}\left(\frac{1}{j^{2}}\right)\right)+\left(\frac{Q_{2} \cdot \mathrm{N} \cdot \mathrm{w}_{\mathrm{st}}}{2 \cdot \mathrm{A} \cdot \mathrm{f}_{\mathrm{st}}} \cdot\right. \\
\left.\left.\left.\int_{\mathrm{w}_{\mathrm{st}} / 2} \frac{\mathrm{e}^{-\mu_{\mathrm{air}} \mathrm{r}} \cdot \mathrm{B}_{\mathrm{air}}(\mathrm{r}) \mathrm{dr}}{\mathrm{r} \sqrt{\mathrm{r}^{2}-\left(\mathrm{w}_{\mathrm{st}} / 2\right)^{2}}}\right)\right]\right\}
\end{gathered}
$$




\section{C3.2 Nondispersible Source Accidents on Freeways}

Since a relatively large amount of truck travel in urban areas is expected to occur on freeways, unique aspects of this roadway type with respect to nondispersible source accidents need to be considered.

Dose to Pedestrians

Since it is assumed that there are no pedestrians on freeways, pedestrian dose from nondispersible source accidents is zero.

Dose to People in Buildings

The geometry for dose to people in buildings from a freeway accident is similar to the geometry which would result from a midblock accident on a very wide street. The linear population density is identical to that computed in Eq. (6), but both the minimum exposure distance (i.e., the lower limit of integration) and the number of exposed building faces (i.e., total exposed perimeter) change. In addition, the expression used for computing the angle of oblique impingement changes as shown below:

$$
\theta_{f}=\pi / 2-\tan ^{-1}\left[\frac{{ }_{f} / 2}{\sqrt{\left(r^{\prime}\right)^{2}+((n-0.5) h)^{2}}}\right]
$$

where

$$
\begin{aligned}
& \theta_{f}=\text { angle of oblique impingement (freeways) } \\
& \mathrm{w}_{\mathrm{f}}=\text { freeway width, including median and right-of-way on each side } \\
& r^{\prime}=\text { distance from the accident site parallel to the freeway } \\
& \text { (metres) }
\end{aligned}
$$

With this revised obliqueness expression and the incorporation of other changes, the equation for integrated exposure to people in buildings becomes

$$
\begin{aligned}
& I D=Q_{2} \cdot \frac{(P D+T C) \cdot\left(w_{s t}+2 w_{s}\right) \cdot \Delta_{a} \cdot e^{-\mu_{b} w_{b}} \cdot B_{b}\left(w_{b}\right) \cdot K_{1}}{4 \cdot n \cdot\left(\sqrt{f_{b}}-f_{b}\right)} \\
& 4 \cdot \sum_{i=1}^{n}\left[\int_{\beta_{6}}^{\infty} \frac{e^{-\mu_{a i r^{r}}} \cdot O F_{i, f}(r) d r}{r^{2}}\right]
\end{aligned}
$$

where $\beta_{6}=\sqrt{\left(w_{f} / 2\right)^{2}+(h(i-0.5))^{2}}$

Dose to People in Vehicles

Accidents on freeways seldom disable traffic in both directions. The model considers that the side of the freeway involving the shipment is essentially stopped, and that other traffic continues to move normally, as shown in Figure $\mathrm{C}-7$. It is 


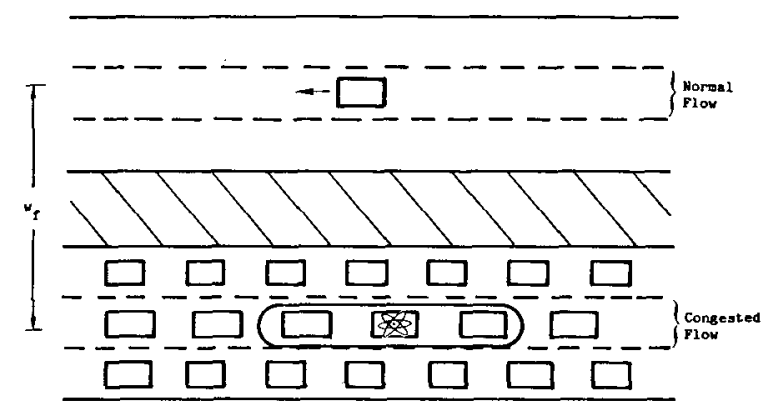

Figure C-7. Nondispersible Source Dose to People in Vehicles for Freeway Accidents

assumed that the area around the vehicle is congested and vehicle separation in the area immediately surrounding the damaged vehicle is equal to the value for intersections, $\delta$, used in Eqs. (13) through (18). The flow in the area of congestion is modeled by assuming a "platoon" of vehicles around the accident site, as discussed in Appendix B. The expression for dose to people traveling the same direction as the shipment therefore becomes

$$
\mathrm{ID}=\mathrm{K}_{\mathrm{i}} \cdot \operatorname{PPV} \cdot \frac{\overline{\mathrm{x}} \cdot \Delta \mathrm{T}_{\mathrm{a}} \cdot \mathrm{f}_{\mathrm{f}}}{(\delta+\ell)^{2}}
$$

where

$$
\begin{aligned}
f_{f}= & \text { freeway platooning factor } \\
X= & \text { total freeway platoon dose factor (see Appendix B for } \\
& \text { details) }
\end{aligned}
$$

The dose to people traveling in the direction opposite the shipment, derived in Appendix B, is modified to account for the fact that the shipment is stopped instead of moving. The net result for total exposure to people in vehicles on freeways is

$$
\begin{aligned}
\mathrm{ID}= & \mathrm{K}_{1} \cdot \mathrm{PPV} \cdot \Delta \mathrm{T}_{\mathrm{a}} \cdot\left[\frac{\mathrm{X} \cdot \mathrm{f}_{\mathrm{f}}}{(\delta+\ell)^{2}}+\frac{\mathrm{Q}_{2} \cdot \mathrm{N} \cdot \mathrm{w}_{\mathrm{f}}}{2 \cdot \mathrm{A} \cdot \mathrm{f}_{\mathrm{st}}}\right. \\
& \left.\int_{\mathrm{w}_{\mathrm{f}} / 2}^{\infty} \frac{\mathrm{e}^{-\mu_{\mathrm{air}} \mathrm{r}} \cdot \mathrm{B}_{\mathrm{air}}(\mathrm{r}) \mathrm{dr}}{r \sqrt{\mathrm{r}^{2}-\left(\mathrm{w}_{\mathrm{f}} / 2\right)^{2}}}\right]
\end{aligned}
$$

where $N=$ total number of vehicles in cell at any time. 


\section{C3.3 Nondispersible Source Accidents on One-Way Streets}

Because most urban areas have a significant number of one-way streets, the model is equipped to analyze transport on this type of arterial. The only expression which changes is dose to people in vehicles in that in the case of one-way streets, there is no opposite direction traffic stream. Instead, a midblock accident is basically surrounded by " $L '-1$ " streams of traffic moving past it in the same direction.

$$
\begin{aligned}
& \mathrm{ID}=\mathrm{K}_{1} \cdot \Delta \mathrm{T}_{\mathrm{a}} \cdot \mathrm{PPV} \cdot\left\{\mathrm{INTF}\left[\frac{3 \cdot \mathrm{L}^{\prime}}{(\delta+\ell)^{2}}\left(1.64-\sum_{j=1}^{\mathrm{N}^{\prime}} \frac{1}{\mathrm{j}^{2}}\right)\right]+\right. \\
& \operatorname{MBF}\left[\left(\frac{1}{(\delta+\ell)^{2}} \sum_{j=N^{\prime}}^{N^{\prime \prime}} \frac{1}{j^{2}}\right)+\left(\frac{Q_{2} \cdot N \cdot w_{S t}}{2 \cdot A \cdot f_{S t}} \cdot \frac{\left(L^{\prime}-1\right)}{L^{\prime}} \cdot\right.\right. \\
& \left.\left.\left.\int_{w_{s t} / 2}^{\infty} \frac{e^{-\mu_{a i r} r} \cdot B_{a i r}(r) d r}{r \sqrt{r^{2}-\left(w_{s t} / 2\right)^{2}}}\right)\right]\right\}
\end{aligned}
$$

\section{C3.4 Air Transport}

Since aircraft accidents in urban areas can occur at any place, the dose equations used are identical to those for truck transport on two-way streets. This will probably overestimate actual doses because the exclusion area around an aircraft accident site in a city will probably not allow pedestrians or people in vehicles as close as one-half of a street width.

\section{C3.5 Rail Transport}

A rail accident of sufficient severity to damage cargo is more likely to occur along the rail right-of-way than in the depot area because of higher velocities and larger number of grade crossings. Therefore, a development analogous to the incident-free dose to people along the-right-of-way (Appendix B) is used.

$$
I D=K_{1} \cdot \Delta T_{a} \cdot P D_{R W} \cdot \int_{R_{W}}^{\infty} I(x) d x
$$

where

$$
\begin{aligned}
& \mathrm{PD}_{\mathrm{RW}}=\begin{array}{l}
\text { population density along right-of-way (people } / \mathrm{km}^{2} ; \\
\text { see Appendix } \mathrm{B}, \mathrm{Eq} \cdot(47))
\end{array} \\
& I(\mathrm{x})=\int_{\mathrm{x}}^{\infty} \frac{\mathrm{e}^{-\mu_{\mathrm{a} i \mathrm{r}} \mathrm{r} \cdot \mathrm{B}_{\mathrm{air}}(\mathrm{r}) \mathrm{dr}}}{\mathrm{r} \sqrt{\left(\mathrm{r}^{2}-\mathrm{x}^{2}\right)}}
\end{aligned}
$$

\section{C3.6 Water Transport}

The dock area is the only place where nondispersible source accidents during waterborne transport can expose significant numbers of people. Hence, an equation analogous to Eq. (50) of Appendix B is used. 


$$
\mathrm{ID}=\mathrm{K}_{1} \cdot \mathrm{PD}_{\text {dock }} \cdot \Delta \mathrm{T}_{\mathrm{a}} \cdot \int_{\mathrm{r}_{6}}^{\mathrm{r}_{7}} \frac{2 \pi \mathrm{r} \cdot \mathrm{e}^{-\mu_{\mathrm{air}} \mathrm{r}} \cdot \mathrm{B}_{\mathrm{air}}(\mathrm{r}) \mathrm{dr}}{\mathrm{r}^{2}}
$$

where

$$
\begin{aligned}
\mathrm{PD}_{\text {dock }} & =\text { population density in dock area (persons } / \mathrm{km}^{2} \text { ) } \\
\mathrm{r}_{6} & =\text { minimum dock exposure distance (metres) } \\
\mathrm{r}_{7} & =\text { maximum dock exposure distance (metres) }
\end{aligned}
$$

\section{C4. Accidents Involving Dispersible Materials}

An accident involving a dispersible material can result in up to five different doses (see Figure C-1). Each of these will be explored separately. Doses from "nonrelease" accidents, common to both nondispersible and dispersible materials, are discussed in Section C5.

\section{C4.1 Doses Due to Inhalation of Radioactive Material}

\section{Inhalation Dose to Pedestrians}

The individual critical organ dose received by a pedestrian who inhales a given concentration of airborne radioactivity from a particular radionuclide is given by

$$
\phi=\mathrm{n}_{1} \cdot \mathrm{PPS} \cdot \mathrm{RF} \cdot \mathrm{AER} \cdot \mathrm{RPC} \cdot \mathrm{DF} \cdot \mathrm{BR} \cdot \Delta \mathrm{T}
$$

where

$$
\begin{aligned}
\phi= & \text { dose to specific organ (rem) } \\
\mathrm{n}_{1}= & \text { amount of material per package (curies) } \\
\mathrm{PPS}= & \text { packages per shipment } \\
\mathrm{RF}= & \text { release fraction } \\
\mathrm{AER}= & \text { fraction of released material which becomes } \\
& \text { aerosolized } \\
\mathrm{RPC}= & \text { radiological dose factor for a specific organ } \\
& \text { (rem to specific organ per curie of material } \\
& \text { deposited in pulmonary portion of lung) } \\
\mathrm{DF}= & \text { atmospheric dillution factor at street level } \\
& \text { (curies per m per curie released) } \\
\mathrm{BR}= & \text { breathing rate (m } 3 / \mathrm{h}) * \\
\Delta \mathrm{T}= & \text { total exposure time (hour)* }
\end{aligned}
$$

*In general, times used are in seconds. However, program compatability requirements necessitate the use of hours instead of seconds for time-step length and breathing rate. 
The dilution factor DF changes as the cloud of debris disperses from the accident site. If it is assumed that a pedestrian remains within a specific cell in the grid for the duration of the cloud passage through the grid, the dose received by that pedestrian during a cloud transit consisting of $t$ time steps, each of length $\Delta \mathrm{T}$, would be

$$
\phi=\mathrm{K}_{2} \cdot \Delta \mathrm{T} \cdot \sum_{\mathrm{k}=1}^{\mathrm{t}_{\mathrm{N}}} \mathrm{DF}_{\mathrm{k}}
$$

where

$$
\begin{aligned}
\mathrm{K}_{2} & =\mathrm{n}_{1} \cdot \mathrm{PPS} \cdot \mathrm{RF} \cdot \mathrm{AER} \cdot \mathrm{RPC} \cdot \mathrm{BR}\left(\frac{\mathrm{rem} \cdot \mathrm{m}^{3}}{\mathrm{~s}}\right) \\
\mathrm{DF}_{\mathrm{k}} & =\text { dilution factor in given cell for time-step } \mathrm{k}\left(\frac{\mathrm{Ci} / \mathrm{m}^{3}}{\mathrm{Ci} \text { released }}\right) \\
\Delta \mathrm{T} & =\text { length of each time step (seconds) } \\
\mathrm{t}_{\mathrm{N}} & =\text { total number of steps }
\end{aligned}
$$

Because this expression uses the time-step length rather than the actual cloud passage time past a point, individual doses are overestimated. In situations where doses are large enough to result in early morbidities or early fatalities, these numbers may also be overestimated.

The integrated inhalation dose received by pedestrians in a single cell during one time step is computed by multiplying the dose each pedestrian receives by the number of exposed pedestrians in the cell:

$$
\mathrm{ID}=\phi \cdot \operatorname{PedD} \cdot \frac{2 \cdot \mathrm{w}_{s} \cdot \mathrm{f}_{s t} \cdot \mathrm{A}}{{ }_{\mathrm{s}} \mathrm{st}} \cdot \gamma \cdot \mathrm{RDF}
$$

where

$$
\begin{aligned}
& \mathrm{f}_{\mathrm{st}}=\text { fraction of cell area occupied by streets } \\
& \mathrm{A}=\text { cell area }\left(\mathrm{km}^{2}\right) \\
& \mathrm{Y}=\text { fraction of area of cell covered by } \\
& \text { cloud during the specified time step } \\
& \mathrm{RDF}=\text { resuspension dose factor* } \\
& \frac{2 \cdot \mathrm{w}_{\mathrm{s}} \cdot \mathrm{f}_{\mathrm{st}} \cdot \mathrm{A}}{\mathrm{w}_{\mathrm{st}}}=\text { total sidewalk area in cell }\left(\mathrm{km}^{2}\right)
\end{aligned}
$$

In order to combine doses absorbed during both short and long time steps, integrated doses for both the intersection and midblock release cases are summed over

*The resuspension dose factor is the dimensionless ratio of the total dose (cloud passage plus resuspension) to cloud passage dose and accounts for additional dose accrued as material which is initially deposited is resuspended and inhaled. It is included in Eq. (28) and not Eq. (27) because the impact of resuspension is significant only for long-term integrated doses. 
all time steps in each cell and over all cells affected by the cloud. The pedestrian density is varied as required to simulate diurnal variation. The result is the integrated dose to pedestrians due to an accident of specific severity occurring in a specific cell along the route:

$$
\begin{aligned}
& I D=\left(\mathrm{K}_{2} \cdot \Delta \mathrm{T}_{\mathrm{s}} \cdot\left(\frac{\mathrm{w}_{1} \mathrm{f}_{\mathrm{st}} \cdot \mathrm{A}}{\mathrm{w}_{\mathrm{st}}{ }_{1}}\right) \cdot \mathrm{RDF} \cdot \sum_{\mathrm{u}=1}^{\mathrm{m}}\left(\mathrm{DF}_{1, \mathrm{u}} \cdot \operatorname{PedD_{1,u}} \cdot \gamma_{\mathrm{u}}\right)\right)+ \\
& \left(\mathrm{K}_{2} \cdot \Delta \mathrm{T}_{\mathrm{L}} \cdot \mathrm{RDF} \cdot \sum_{\mathrm{w}=1}^{\mathrm{q}} \sum_{\mathrm{z}=1}^{\mathrm{q}}\left(\mathrm{DF}_{\mathrm{w}, \mathrm{z}} \cdot\left(\frac{2 \cdot \mathrm{w}_{\mathrm{s}} \cdot \mathrm{f}_{\mathrm{st}} \cdot \mathrm{A}}{\mathrm{w}_{\mathrm{st}} \mathrm{z}_{\mathrm{z}}}\right) \cdot \operatorname{PedD}_{\mathrm{w}, \mathrm{z}} \cdot \gamma_{\mathrm{w}, \mathrm{z}}\right)\right.
\end{aligned}
$$

where

$$
\begin{aligned}
\mathrm{m}= & \text { number of short time steps (short-term atmospheric } \\
\text { transport) } & \\
\mathrm{u}= & \text { summation index for short time steps } \\
\mathrm{w}= & \text { summation index for long time steps } \\
\mathrm{q}= & \text { number of long time steps (long-term atmospheric } \\
& \text { transport) } \\
z= & \text { summation index for grid cells } \\
\mathrm{p}= & \text { number of cells in grid } \\
\Delta \mathrm{T}_{\mathrm{s}}= & \text { length of small time steps (hours) } \\
\Delta \mathrm{T}_{\mathrm{L}}= & \text { length of long time step (hours) }
\end{aligned}
$$

The integrated dose is split into that accumulated during small-scale atmospheric transport analysis in the cell where the accident occurs and that accumulated during large-scale atmospheric transport analysis in all cells (including the ce11 where the accident occurs). This approach has been chosen because of distance constraints on the small-scale atmospheric transport analysis. Although there may be large differences in the dilution factors at a given place and time as a result of intersection or midblock releases, the basic form given in Eq. (29) applies to both cases. The subscripted 1 in the short time portion refers to the cell in which the release occurred, and $\gamma$ is the area fraction for the cloud in the volume segment of interest.

\section{Inhalation Dose to People in Vehicles}

The dose received by people in vehicles as the cloud of debris from an accident passes is analogous to the dose received by pedestrians. The individual dose for one accident severity, one time step, and one cell is given by Eq. (26). Since it is not possible to predict if drivers will have windows up or down, or if they will have vents open or closed, no allowance is made for possible filtration of the cloud of debris as it enters the vehicle. As was the case for pedestrians, the total individual dose for a given accident can be given by Eq. (27), using ground level dilution factors. The density of people in vehicles in each cell in the grid is required for the integrated dose computation. If this density is incorporated, 
the general expression for integrated dose to people in vehicles from an accident of a given severity occurring in a specific cell becomes

$$
\begin{aligned}
\mathrm{ID}= & {\left[\Delta \mathrm{T}_{\mathrm{S}} \cdot \mathrm{K}_{2} \cdot \mathrm{RDF} \cdot \mathrm{PPV} \cdot \sum_{\mathrm{u}=1}^{\mathrm{m}}\left(\mathrm{N}_{1, \mathrm{u}} \cdot \mathrm{DF}_{1, \mathrm{u}} \cdot \gamma_{1, \mathrm{u}}\right)\right] } \\
& {\left[\Delta \mathrm{T}_{\mathrm{L}} \cdot \mathrm{K}_{2} \cdot \mathrm{RDF} \cdot \mathrm{PPV} \cdot \sum_{\mathrm{w}=1}^{\mathrm{q}} \sum_{\mathrm{z}=1}^{\mathrm{p}}\left(\mathrm{N}_{\mathrm{w}, \mathrm{z}} \cdot \mathrm{DF} \mathrm{w}_{\mathrm{w}, \mathrm{z}} \cdot \gamma_{\mathrm{w}, \mathrm{z}}\right)\right] }
\end{aligned}
$$

where $\mathrm{N}=$ total vehicles in the cell at given time.

Inhalation Dose to People in Buildings

The concentration of radioactive material inside a building depends on the rate of exchange of outside air with the air inside the building. As airborne material enters the building, either through a ventilation system or by diffusion processes through windows, doors, cracks in walls, etc., some fraction is filtered out. Radioactive decay, deposition on surfaces internal to the building, and recirculation through ventilating filters and ducts could also act to reduce the inside concentration. An equation which relates the dose received by an individual inside a building to that received outside the building has been developed in Reference 5 . This ratio, referred to as Building Dose Factor (BDF), is given by

$$
\mathrm{BDF}=\frac{\phi_{\text {inside }}}{\phi_{\text {outside }}}=\frac{\mathrm{F}}{1+\frac{\mathrm{D}_{1}+\lambda+\mathrm{K}^{\prime} \varepsilon_{\mathrm{r}}}{\mathrm{R}}}
$$

where

$$
\begin{aligned}
F= & \text { ingress fraction ( } 1 \text { minus fraction removed by } \\
& \text { filtration upon entry) } \\
D_{1}= & \text { deposition factor }\left(s^{-1}\right) \\
\lambda= & \text { radioactive decay constant for material }\left(s^{-1}\right) \\
\mathrm{K}^{\prime}= & \text { recirculating air turnover rate }\left(\mathrm{s}^{-1}\right) \\
\varepsilon_{\mathrm{r}}= & \text { recirculation loop filter efficiency } \\
\mathrm{R}= & \text { building air change rate }\left(\mathrm{s}^{-1}\right)
\end{aligned}
$$

Building ventilation systems can be divided into two main categories: continuous intake systems and central air conditioning systems. Continuous intake systems are those which have direct air exchange with the outside through window-mounted intake systems or through natural wind processes. This type of system would be found primarily in older structures or small, single-family dwellings. Central airconditioning systems occur in larger, high-rise office buidings and typically include a relatively limited amount of air intake through a small number of specific intake locations coupled with a large amount of conditioning and recirculation. Central air-conditioning systems normally use a system of filters, precipitators, and dehumidifying coils. Information has been extracted from References 5 and 6 to evaluate the BDF for the two systems described above. Initially the decay constant is set at zero since the largest value of a decay constant for a shipped radioactive material is $3.21 \times 10^{-5} \mathrm{~s}^{-1}$ ( Tc-99m) and the smallest is $4.9 \times 10^{-18}$

( $U-238)$. Values for air change rate $R$ for homes vary upward from $2.8 \times 10^{-5} \mathrm{~s}^{-1}$. 
Central air-conditioning systems have typical air change rate values of from 0.001 to $0.002 \mathrm{~s}^{-1}$. Filtration is a strong function of the number and type of filtration devices as well as of the particle size and electrostatic nature of the aerosol. In the case of the noble gases, the ingress fraction $F$ is assumed to be 1.0 . This is conservative, because even noble gas molecules may agglomerate onto particles in the ambient aerosol and be removed. For other gases and/or particulates, a range of values from 0.5 to 1.0 , with an average of 0.85 , is suggested for homes (see Reference 5). Central air-conditioning systems are much more efficient, with values for the ingress fraction to rooms and corridors from 0.002 to 0.25 per pass for particles with diameters between 1 and 30 microns.

Deposition rates are a function of particle size and air velocity. In a room where wall and furnishing surface area is 2.5 times the floor area and where wall deposition is $20 \%$ of floor deposition, a value for $D$ of $8.3 \times 10^{-5} \mathrm{~s}^{-1}$ with a range of $5.6 \times 10^{-6}$ to $5.6 \times 10^{-4} \mathrm{~s}^{-1}$ is suggested. Noble gases are considered to have a zero deposition rate.

The last two parameters, $\mathrm{K}^{\prime}$ and $\varepsilon_{r}$, relate to recirculation of air in the system and the filter efficiency in the recirculation mode. Almost by definition, there is no recirculation in continuous intake systems. For these situations, $\mathrm{K}^{\prime} \cdot \varepsilon_{r}=0$. Large central air-conditioning systems have significant recirculation: Reference 6 suggests that filter efficiencies of 0.75 to 0.998 for 1 - to 30-micron particles are currently installed and/or commercially available. Reference 5 suggests that central systems have recirculation rates of between $1.4 \times 10^{-3}$ and $2.8 \times 10^{-3} \mathrm{~s}^{-1}$.

Table C-2 summarizes the BDF information and indicates values chosen for this analysis.

Table C-2

Building Dose Determination

A. Continuous Intake Systems

Material Type

Noble gases

Other gases

and particulates

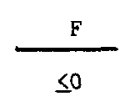

$0.5-1.0$

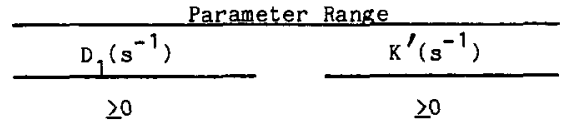

$5.6 \times 10^{-6}--5.6 \times 10^{-4}$

0

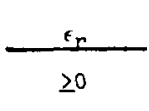

0

$\geq 0$

$0.75--0.998$

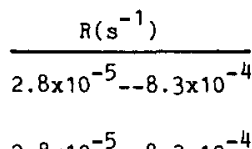

$2.8 \times 10^{-5}--8.3 \times 10^{-4}$

$0.001--0.002$

$0.001--0.002$ $\frac{\text { BDF Chosen }}{1.0}$

.65

1.0

0.0086
Other gases

and particulates
$1.4 \times 10^{-3}--2.8 \times 10^{-3}$

$1.4 \times 10^{-3}--2.8 \times 10^{-3}$
$0.002--0.25 \quad 5.6 \times 10^{-6}--5.6 \times 10^{-4}$ 
Individual Doses -- The dispersion codes which give values for DF and $\gamma$ provide a vertical distribution of material in each of four 30-metre-thick zones. With this information, a dose outside the building can be computed for each time step using Eq. (26). By applying the BDF for the appropriate type of ventilation system, the dose received inside the building in each vertical zone can be calculated.

Integrated Doses - The number of people exposed to a given concentration is equal to the number of people in the particular building in each of the vertical zones. Thus, for continuous intake systems, the total integrated exposure to people in buildings is given by

$$
\begin{aligned}
\mathrm{ID}= & {\left[\frac{10 \cdot \mathrm{PPB}_{1}}{\mathrm{n}} \cdot \Delta \mathrm{T}_{\mathrm{s}} \cdot \mathrm{K}_{2} \cdot \mathrm{RDF} \cdot \sum_{\mathrm{nz}=1}^{z}\left(\mathrm{DF}_{1, \mathrm{nz}} \cdot \gamma_{1, \mathrm{nz}} \cdot \mathrm{BDF}_{\text {cont }}\right)\right]+} \\
& {\left[\mathrm { \Delta T } _ { \mathrm { L } } \cdot \mathrm { K } _ { 2 } \cdot \mathrm { RDF } \cdot \frac { 1 0 } { \mathrm { n } } \sum _ { \mathrm { w } = 1 } ^ { \mathrm { q } } \sum _ { \mathrm { nz } = 1 } ^ { \mathrm { z } } \sum _ { \mathrm { z } = 1 } ^ { \mathrm { p } } \left(\mathrm{PPB}_{\mathrm{w}, \mathrm{z}} \cdot \gamma_{\mathrm{w}, \mathrm{nz}, \mathrm{z}} \cdot\right.\right.} \\
& \left.\left.\mathrm{DF}_{\mathrm{w}, \mathrm{nz}, \mathrm{z}} \cdot \mathrm{BDF}_{\text {cont }}\right)\right]
\end{aligned}
$$

where

$$
\begin{aligned}
\mathrm{nz}= & \text { summation index over vertical dispersion zones } \\
z= & \text { number of vertical dispersion zones (assumed to be } \\
& 4 \text { in current analysis) } \\
10= & \text { number of floors per zone }(30 \mathrm{~m} / \text { zone } \div 3 \mathrm{~m} / \mathrm{f} 100 \mathrm{r})
\end{aligned}
$$

The expression for central air-conditioning systems is identical to that in $\mathrm{Eq}$. (32) with the single replacement of $\mathrm{BDF}_{\mathrm{CAC}}$ for $\mathrm{BDF}_{\text {cont }}{ }^{\circ}$

\section{C4.2 Cloudshine Doses from Accidents Involving Dispersible Sourcés}

For each isotope, the dose received due to immersion in the cloud for the duration. of its passage is directly related to the concentration of the material and the length of the exposure. The cloudshine dose received by an individual can be specified by

$$
\phi=\mathrm{Q}_{8} \cdot \mathrm{CDF} \cdot \mathrm{DF} \cdot \Delta \mathrm{T} \cdot \mathrm{N}_{1} \cdot \mathrm{PPS} \cdot \mathrm{RF}
$$

where

$$
\begin{aligned}
Q_{8} & =\text { units conversion factor }\left(3.2 \times 10^{-17} \frac{\mathrm{rem} \cdot \mathrm{yr} \cdot \mathrm{m}^{3}}{\mathrm{mrem} \cdot \mathrm{s} \cdot \mathrm{cm}^{3}}\right) \\
\mathrm{CDF} & =\text { cloudshine dose factor }\left(\frac{\mathrm{mrem} \cdot \mathrm{cm}^{3}}{\mathrm{C} i \cdot \mathrm{yr}}\right)
\end{aligned}
$$

By incorporating the building dose factor and the appropriate dilution factor, individual doses recelved by each population subgroup can be determined. Integrated doses are computed as before by summing over time steps and including population density and cloud area fraction. No allowance for shielding is included 
in computing cloudshine doses to people in vehicles. ${ }^{7}$ Values for CDF are given'in Appendix $\mathrm{H}$.

As was the case for inhalation exposure, the use of time-step length rather than cloud passage time results in overestimation of individual doses for cloudshine exposure. However, this is counterbalanced by the fact that only doses to persons in the cloud are computed.

\section{C4.3 Groundshine Doses from Accidents Involving Dispersible Sources}

For each specific isotope, the dose recelved from exposure to contaminated ground is related to the level of contamination, the length of exposure, and the exposure geometry.

The expressions used to compute this "groundshine" dose are all based on the formula for photon flux at a distance $x$ (metres) above a uniformly contaminated infinite plane (see References 3 and 8 ):

$$
\operatorname{flux}_{\mathrm{f} \text { lut }}\left(\frac{\text { photons }}{\mathrm{cm}^{2} \cdot \mathrm{s}}\right)=\frac{\mathrm{s}}{2} \cdot \mathrm{E}_{1}(\mu \mathrm{x})
$$

where

$$
\begin{aligned}
S= & \text { photon source per unit area (photons per second } \\
& \text { per } \left.\mathrm{cm}^{2}\right) \\
\mathrm{E}_{1}(\mu \mathrm{x})= & 1 \text { st order exponential integral } \\
\mu= & \text { linear absorption coefficient }\left(\mathrm{m}^{-1}\right)
\end{aligned}
$$

Because dose is a measure of the energy absorbed by a material (in this case, body tissue) and since each photon in a multiphoton decay scheme deposits some fraction of its energy in a given amount of tissue (described by $\mu$ ), an exact expression for dose requires a detailed description of the decay scheme of each radionuclide. To simplify this analysis, the relative flatness of the $\mu$-versus-energy curve in the energy region of interest is used by assuming that each decay, be it single photon or cascade, behaves as if it were a single photon decay. Thus, the energy deposited per unit mass per disintegration becomes the total photon energy per disintegration, $E_{d}$, times the effective attenuation coefficient. For the purposes of this analysis, body tissue, the target of groundshine dose, is comparable to dry air in terms of energy absorption within the applicable photon energy range. Thus the definition of roentgen as the absorption of $7.1 \times 10^{4} \mathrm{MeV}$ in $1 \mathrm{~cm}^{3}$ of dry air is considered applicable and can be combined with a conversion of $S$ from photons per $\mathrm{cm}^{2}$ per second to microcuries per $\mathrm{m}^{2}$. If $\mathrm{E}_{1}(\mathrm{x})$ is evaluated at a height of 1 metre above the surface, dose rate (in rem per day) becomes

$$
\mathrm{DR}=3.04 \times 10^{-4} \cdot \mathrm{CULVL} \cdot \mathrm{E}_{\mathrm{d}}
$$

where $C U L V L=$ cleanup level $\left(\mu \mathrm{Ci} / \mathrm{m}^{2}\right)$. 
Eq. (35) must be further modified to account for the radioactive decay of isotopes with half lives on the order of a few hours to a few days:

$$
\operatorname{DR}(t)=3.04 \times 10^{-4} \cdot \operatorname{CULVL} \cdot \mathrm{E}_{\mathrm{d}} \cdot \mathrm{e}^{-\left(\frac{0.693 t}{t_{1 / 2}}\right)}
$$

This equation forms the basis for the remainder of the groundshine analysis.

The basic exposure scenario is assumed to be as follows:

1. People are exposed for the first full day following the accident to unmodified contamination levels. During this day, it is assumed that surveys are being conducted and a determination made of the need for evacuation, interdiction, decontamination, etc.

2. If the decontamination factor DF (see Appendix $K$ ) is less than or equal to 1.0, no remedial action is required, and persons are assumed to be exposed for 50 years to radiation from an area contamined to the level initially deposited, as modified by radioactive decay.

3. If the decontamination factor is greater than 1.0 , a 10-day survey/cleanup period is assumed during which no exposure is accrued. This 10-day period is followed by 50 years of exposure to the cleaned-up level.

There are some exceptions to this basic scenario which are detailed in the discussion below.

Groundshine Doses to Pedestrians

Pedestrian groundshine doses are computed using the contamination level on streets and sidewalks (CULVLDFS, where DFS is the street decontamination factor), with a pedestrian density computed by a time-weighted average of pedestrian densities for each time span in each affected cell ( $\overline{\text { PedD }})$.

$$
\begin{aligned}
& \text { ID }=Q_{9} \cdot E_{d} \cdot t_{1 / 2} \cdot \text { CULVL } \cdot A \cdot \\
& \sum_{z=1}^{p}\left[\frac { 2 \cdot w _ { s } \cdot f _ { s t } } { \operatorname { P e d D } _ { z } } \cdot \gamma _ { \operatorname { m a x } _ { z } } \cdot \frac { w _ { z } } { w _ { s t } } \cdot \left\{D_{z S_{z}}\left(1-e^{-\left(\frac{0.693}{t_{1 / 2}}\right)}\right)+\right.\right. \\
& \left.\left.\left(e^{-\left(\frac{6.93}{t_{1 / 2}}\right)}-e^{-\left(\frac{1.26 \times 10^{4}}{t_{1 / 2}}\right)}\right)\right\}\right]
\end{aligned}
$$

where $Q_{9}=$ units conversion factor $\left(=4.39 \times-10^{-5} \mathrm{rem} / \mathrm{MeV} \cdot \mu \mathrm{Ci} \cdot \mathrm{d}\right)$.

The term $\gamma_{\max }$ in Eq. (37) describes the maximum fraction of the cell covered by the cloud during its passage. The first term in the brackets describes the initial exposure to undecontaminated ground, and the second term describes the 50-year exposure to decontaminated area.

In the case of pedestrians, if the decontamination factor is greater than a fixed value determined by the analyst (in this analysis the value is 2 ), the bracketed expression is replaced by the 1-day exposure alone since the cleanup technique for that level of contamination is assumed to be outright replacement of all pavement. 
Groundshine doses to people in bufldings are computed using the contamination level on building horizontal area (CULVL·DFB, where DFB is the building decontamination factor), with a population density computed by a time-weighted average of population and transient clientele densities for each time span ( $\overline{\mathrm{PD}+\mathrm{TC}}$ ) in each affected cell.

$$
\begin{aligned}
& \mathrm{ID}=\mathrm{Q}_{9} \cdot \mathrm{E}_{\mathrm{d}} \cdot \mathrm{t}_{\frac{1}{2}} \cdot \mathrm{CULVL} \cdot \mathrm{A} \cdot \\
& \sum_{z=1}^{\mathrm{p}}\left[(\overline{\mathrm{PD}+\mathrm{TC}})_{z} \cdot \gamma_{\max _{z}} \cdot\left\{\mathrm{DFB}\left(1-\mathrm{e}^{-\left(\frac{0.693}{\mathrm{t}} 1 / 2\right.}\right)\right)+\right. \\
& \left.\left.\left(e^{-\left(\frac{6.93}{t_{1 / 2}}\right)}-e^{-\left(\frac{1.26 \times 10^{4}}{t_{1 / 2}}\right)}\right)\right\}\right]
\end{aligned}
$$

If the building decontamination factor, $\mathrm{DFB}$, is greater than a fixed value (in this analysis, the value is 40 ), the bracketed expression is replaced by the 1-day exposure term alone since the area is assumed to be interdicted if the contamination is that severe.

Groundshine Doses to People in Vehicles

Because of the relatively large amount of metal shielding provided by vehicle chassis, no groundshine dose is computed for people in vehicles.

\section{C4.4 Dose Due to Material Remaining at the Scene of the Accident}

The fraction of released material which remains at the accident site, referred to as the remnant material, can be split into two groups: material which remains inside package shielding and material which escapes from the package but does not become airborne. The fraction of material which remains shielded inside the package is given by "1-RF;" the portion of material which is released from the package but does not become airborne is given by "RF・(1-AER)." These two groups act as an exposure source identical to that discussed in Section C3, with a source term given by

$$
\begin{aligned}
\mathrm{K}_{3}= & \left\{\mathrm{Q}_{6} \cdot \mathrm{n}_{1} \cdot \mathrm{PPS} \cdot \mathrm{E}_{\mathrm{d}} \cdot \mathrm{\mu}_{\mathrm{r}} \cdot[\mathrm{RF} \cdot(1-\mathrm{AER})]\right\}+ \\
& {\left[\mathrm{Q}_{3} \cdot \mathrm{TI} \cdot \mathrm{PPS} \cdot(1-\mathrm{RF})\right] }
\end{aligned}
$$

Subsitution of this source term for $\mathrm{K}_{1}$ in the equations in Section $\mathrm{C} 3$ gives the dose due to the material which remains at the site of an accident.

C5. Nonrelease Vehicular Accidents

Many vehicular accidents are not severe enough to displace package shielding or release package contents. Even so, these accidents may still be severe enough to cause the vehicle to remain at the accident site for an extended period, exposing people in the area. This situation is analogous to the nondispersible source accident discussed earlier, except that the source term does not involve the curie content since the package shielding is not affected. Instead, the source term can be expressed as 


$$
\mathrm{K}_{4}=\mathrm{Q}_{3} \cdot \mathrm{K}_{\mathrm{O}} \cdot \mathrm{TI} \cdot \mathrm{PPS}
$$

The basic dose rate equations used to derive expressions for incident-free exposures in Appendix $B$ can be used to determine integrated doses to pedestrians, people in buildings, and people in vehicles. In addition to these groups, crew would receive an additional dose by virtue of the delay time. This dose is specified as

$$
\operatorname{ID}_{c r e w}\left(d_{2}\right)=K_{4} \cdot N_{c} \cdot \frac{e^{-\mu_{a i r} d_{2}} \cdot B_{a i r}\left(d_{2}\right)}{\left(d_{2}\right)^{2}} \cdot \Delta T_{a}
$$

where

$$
\begin{aligned}
& \mathrm{N}_{\mathrm{c}}=\text { number of crew } \\
& \mathrm{d}_{2}=\text { source-to-crew distance (metres) }
\end{aligned}
$$

C6. Summation of Direct Radiological Effects Resulting from Accidents

Previous sections have discussed the determination of individual and population exposure consequences of specific accidents. For comparisons between various routes or with incident-free impact, some further operations are necessary:

1. Combination of groundshine, cloudshine, inhalation, and direct exposure doses;

2. Conversion of doses to expected number of health effects;

3. Combination of release and nonrelease accident consequences; and

4. Expression of consequences in terms of annual expected value, i.e., in risk terms.

As discussed in Appendix $\mathrm{H}$, the "common denominators" for health effects comparison are early fatalities, early morbidities, latent cancer fatalities, and genetic effects. Use of these parameters allows quantification of population doses to various organs resulting from inhalation of radioactive material and/or external whole-body radiation doses.

Individual doses can be converted to expected numbers of early fatalities by multiplying the doses received by individuals by the probability of fatality given a specific dose and then summing over the various computed individual doses received:

$$
\left[\begin{array}{c}
\text { Expected number of } \\
\text { early fatalities } \\
\text { per accident }
\end{array}\right]=\sum^{\eta_{1}} \sum^{g} N\left(\phi_{n, g}\right) \cdot P_{\text {ef }}\left(\phi_{n, g}\right)
$$

where

$$
\begin{aligned}
n_{1}= & \text { number of organs which can be sufficiently } \\
& \text { radiosensitive and physiologically critical } \\
& \text { to cause acute fatality if significantly } \\
& \text { overexposed }
\end{aligned}
$$




$$
\begin{aligned}
& \mathrm{g}=\text { number of dose subgroups evaluated } \\
& \mathrm{N}\left(\phi_{g}\right)=\begin{array}{l}
\text { number of people receiving dose } \phi_{g} \text { to organ } n \\
\text { in the given accident }
\end{array} \\
& \mathrm{P}_{\text {ef }}\left(\phi_{g}\right)=\underset{\substack{\text { probability of early fatality, given dose } \phi_{g} \\
\text { to organ } \eta}}{ }
\end{aligned}
$$

Early fatality probabilities are provided for a set of discrete dose intervals, and the people exposed in each of these intervals are assigned the probability of early fatality associated with the lowest dose in the interval.

Early morbidities are handled slightly differently because they are evaluated by assuming $100 \%$ occurrence of morbidity above a specified dose threshold.

$$
\left[\begin{array}{c}
\text { Expected number of } \\
\text { early morbidities } \\
\text { per accident }
\end{array}\right]=\sum_{\mathrm{m}=1}^{n_{2}} \mathrm{~N}\left(\phi_{\mathrm{m}}\right)
$$

where

$$
\begin{aligned}
n_{2}= & \text { number of organs which can be exposed sufficiently } \\
& \text { to cause morbidity } \\
\mathrm{N}\left(\phi_{\mathrm{m}}\right)= & \text { number of people receiving dose } \phi_{\mathrm{m}} \text { or greater in } \\
& \text { the given accident, where } \phi_{\mathrm{m}} \text { is the threshold } \\
& \text { dose for the specific physiological morbidity }
\end{aligned}
$$

Each computed integrated dose can be converted to a value for expected number of latent cancer fatalities or genetic effects by multiplying the integrated organ dose by its appropriate coefficient. First, however, the integrated exposure associated with persons who suffer early fatalities is subtracted from the total. The remaining integrated exposure can then be converted to expected numbers of long-term effects as shown:

$$
\left[\begin{array}{c}
\text { Expected number of } \\
\text { long-term effects } \\
\text { per accident }
\end{array}\right]=\sum_{s}\left[\left(\mathrm{ID}_{\text {Total,s }}-\mathrm{ID}_{\mathrm{ef}, \mathrm{s}}\right) \cdot \mathrm{CF}_{\mathrm{s}} \cdot \mathrm{DEF}\right]
$$

where

$$
\begin{aligned}
& \mathbf{s}=\text { index over various organs } \\
& \begin{aligned}
\mathrm{ID}_{\text {Total, }}= & \text { total integrated population dose to the } \mathrm{s}^{\text {th }} \\
& \text { organ received in a specific accident (person }
\end{aligned} \\
& \text { rem) } \\
& \begin{aligned}
& \mathrm{ID}_{\mathrm{ef}, \mathrm{s}}= \text { integrated population dose accumulated in } \\
& \text { persons who will become early fatalities }
\end{aligned} \\
& \text { (person rem) } \\
& \mathrm{CF}_{\mathrm{S}}=\text { health effect coefficient for } \mathrm{s}^{\text {th }} \text { organ for } \\
& \text { long-term health effect of interest (number } \\
& \text { of health effects per person rem) (see } \\
& \text { Appendix H) } \\
& \mathrm{DEF}=\text { dose-rate effectiveness factor (see Appendix } \mathrm{H} \text { ) }
\end{aligned}
$$


Note that, as discussed earlier, the number of early fatalities may be overestimated because of the time-step technique used to calculate individual dose. If this is the case, long-term effects may be underestimated.

Once the expected number of consequences per accident has been evaluated, a value for risk can be computed. The expected values for health effects computed in Eqs. (42), (43), and (44) are only for accidents of a specific severity occurring in a specific cell along the route. Accidents of other severities occurring in other cells must now be considered. Since consequence per route is expressed in terms of expected numbers of health effects, the occurrence rate for each severity category must be considered. Thus, the expected number of accidents of severity $c$ in cell $z, \varepsilon_{z, c}$, is given by

$$
\varepsilon_{z, c}=L_{z} \cdot A R_{z} \cdot e_{c, z}
$$

where

$$
\begin{aligned}
\mathrm{c}= & \text { index over accident severities } \\
\mathrm{L}_{z}= & \text { the distance traveled in cell } \mathrm{z}(\mathrm{km}) \\
\mathrm{AR}_{z}= & \text { accident rate per unit distance } \\
& \text { (dependent upon time span when } \\
& \text { transport occurred) (acc/km) } \\
\mathrm{e}_{c, z}= & \text { fraction of accidents of severity } \\
& \text { c in cell } \mathrm{z}
\end{aligned}
$$

mode

dependent

This can be combined with earlier expressions to give the total expected number of health effects of any type from transport using a specific mode along a specific route:

$$
\left[\begin{array}{l}
\text { Total expected } \\
\text { health effects }
\end{array}\right]=\sum_{z=1}^{H} \sum_{c=1}^{C} \text { Effects }_{z, c} \cdot \varepsilon_{z, c}
$$

where

$$
\begin{aligned}
\mathrm{H}= & \text { number of cells on route } \\
\mathrm{C}= & \text { number of accident severity categories } \\
\text { Effects }_{\mathrm{z}, \mathrm{C}=} & \text { expected number of effects from accident } \\
& \text { of severity } \mathrm{c} \text { in cell } \mathrm{z} \text { during time span } \\
& \text { of interest (sum of values from Eqs. (42), } \\
& (43),(44)) \\
\varepsilon_{z, c}= & \text { expected number of accidents of severity c } \\
& \text { in cell } \mathrm{z} \text { per trip through grid (Eq. (45)). }
\end{aligned}
$$

These values can, in turn, be summed over all modes of transport and all routes to get the final expected value of health effects for a specific set of standard shipments. 
ADDENDUM: VARIABLES, CONSTANTS, AND FUNCTIONS

Table Ad-1

Variables

\begin{tabular}{|c|c|c|c|}
\hline Variable & Description & Units & $\begin{array}{l}\text { Equations In Which } \\
\text { Variable Appears }\end{array}$ \\
\hline $\mathrm{n}_{1}$ & $\begin{array}{l}\text { number of curies } \\
\text { per package }\end{array}$ & Ci & $1,3,26,33$ \\
\hline RF & release fraction & - & $1,3,26,33,39$ \\
\hline PPS & $\begin{array}{l}\text { number of packages } \\
\text { per shipment }\end{array}$ & - & $1,3,26,33,39,40$ \\
\hline $\mathrm{E}_{\mathrm{d}}$ & $\begin{array}{l}\text { photon energy } \\
\text { per disintegration }\end{array}$ & $\mathrm{MeV}$ & $1,3,35,36,37,38$, \\
\hline$\mu$ & attenuation coefficient & $\mathrm{m}^{-1}$ & $1,3,34,39$ \\
\hline$\mu_{\text {air }}$ & $\begin{array}{l}\text { attenuation coefficient } \\
\text { of air }\end{array}$ & $\mathrm{m}^{-1}$ & $\begin{array}{l}1,2,4,5,6,7,11, \\
12,17,18,20,22, \\
23,25,41\end{array}$ \\
\hline $\mathbf{r}$ & $\begin{array}{l}\text { distance from source to } \\
\text { exposable population }\end{array}$ & metres & $\begin{array}{l}1,2,7,9,10,17, \\
18,19,20,22,23\end{array}$ \\
\hline$\phi$ & individual dose & & $\begin{array}{l}2,7,9,11,26,27, \\
28,31,33\end{array}$ \\
\hline $\mathrm{K}_{1}$ & $\begin{array}{l}\text { source term for external } \\
\text { exposure }\end{array}$ & $\frac{\mathrm{rem} \cdot \mathrm{m}^{2}}{\mathrm{~s}}$ & $\begin{array}{l}2,3,4,5,6,7,9 \\
10,11,12,13,14, \\
15,16,17,18,20 \\
21,22,23,24,25\end{array}$ \\
\hline$\Delta \mathrm{T}_{\mathrm{a}}$ & accident delay time & $\mathbf{s}$ & $\begin{array}{l}2,4,5,6,7,9,10, \\
11,12,13,14,15, \\
16,17,18,21,22, \\
23,24,25,41\end{array}$ \\
\hline $\mathrm{K}_{\mathrm{O}}$ & package shape factor & $\frac{\mathrm{mrem} \cdot \mathrm{m}^{2}}{\mathrm{~h}}$ & 3,40 \\
\hline $\mathrm{TI}$ & package transport index & - & $3,39,40$ \\
\hline ID & integrated dose & person-rem & $\begin{array}{l}4,9,10,12,13,14, \\
16,17,18,20,21, \\
22,23,24,25,28, \\
29,30,32,37,38, \\
41,44\end{array}$ \\
\hline PedD & pedestrian density & $\begin{array}{l}\text { persons per } \\
\text { square kilome- } \\
\text { tre of sidewalk }\end{array}$ & $4,5,6,28,29,37$ \\
\hline
\end{tabular}


Table Ad-1 (Continued)

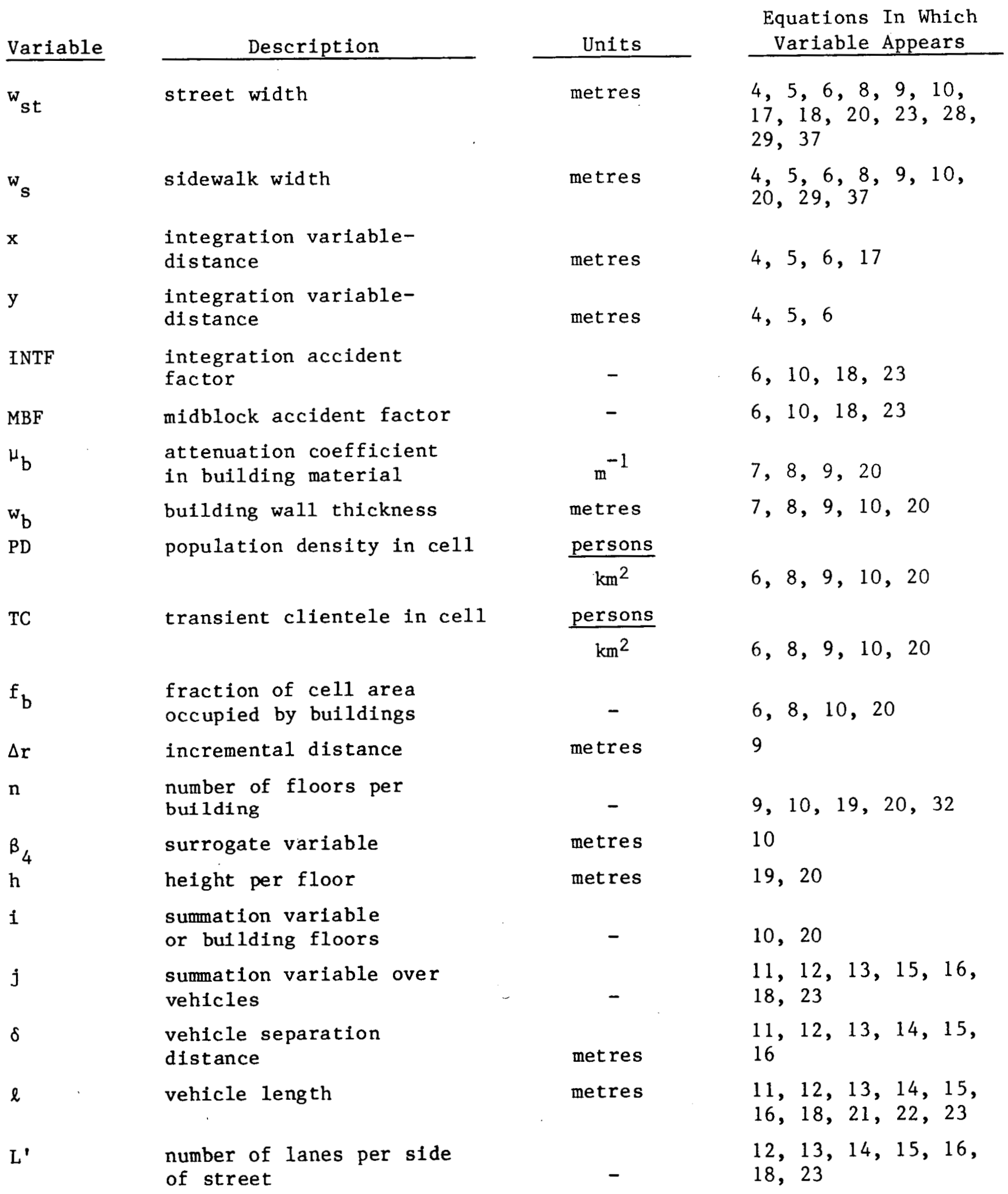


Table Ad-1 (Continued)

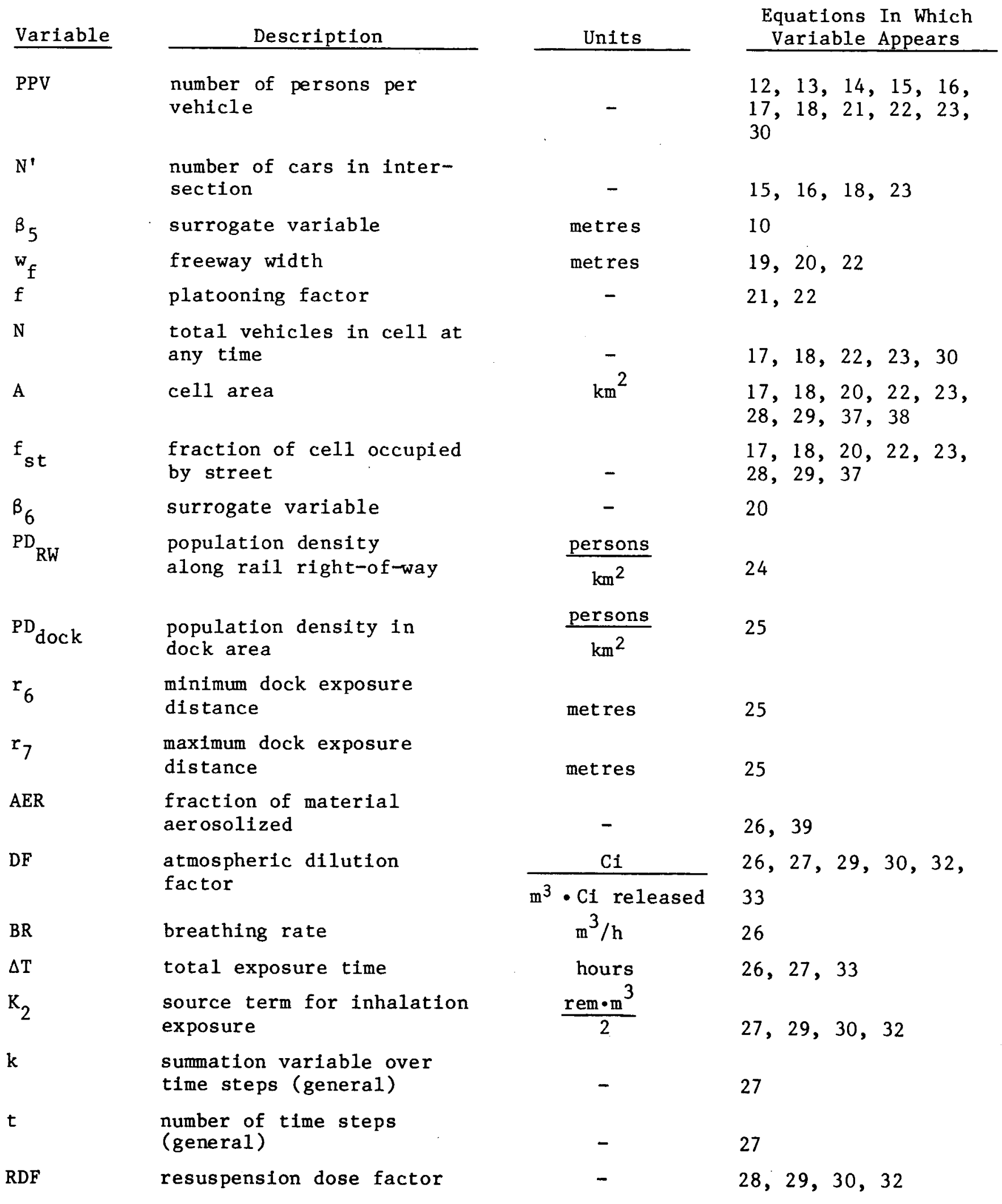




\begin{tabular}{|c|c|c|c|}
\hline Variable & Description & Units & $\begin{array}{c}\text { Equations In Which } \\
\text { Variable Appears }\end{array}$ \\
\hline$\gamma$ & $\begin{array}{l}\text { fraction of cell area } \\
\text { covered by cloud }\end{array}$ & - & $28,29,30,32$ \\
\hline$\gamma_{\max }$ & maximum value of gamma & - & 37,38 \\
\hline$\Delta \mathrm{T}_{\mathbf{s}}$ & length of short time step & hours & $29,30,32$ \\
\hline $\mathbf{u}$ & $\begin{array}{l}\text { summation variable over } \\
\text { short time steps }\end{array}$ & - & 29,30 \\
\hline m & number of short time steps & - & 29,30 \\
\hline$\Delta \mathrm{T}_{\mathrm{L}}$ & length of long time steps & hours & $29,30,32$ \\
\hline w & $\begin{array}{l}\text { summation variable over } \\
\text { long time steps }\end{array}$ & - & 29,30 \\
\hline$q$ & number of long time steps & - & 29,30 \\
\hline $\mathbf{z}$ & $\begin{array}{l}\text { summation variable over } \\
\text { cells in grid }\end{array}$ & - & $\begin{array}{l}29,30,32,37,38, \\
45,46\end{array}$ \\
\hline $\mathrm{p}$ & number of cells in grid & - & $29,30,32,37,38$ \\
\hline $\mathrm{F}$ & building filtration factor & - & 31 \\
\hline $\mathrm{D}_{1}$ & deposition factor & $\mathrm{m}^{-1}$ & 31 \\
\hline$\lambda$ & decay constant for material & $s^{-1}$ & 31 \\
\hline $\mathrm{K}^{\prime} \varepsilon_{\mathrm{r}}$ & $\begin{array}{l}\text { recirculation loop } \\
\text { parameter }\end{array}$ & $s^{-1}$ & 31 \\
\hline R & building air change rate & $s^{-1}$ & 31 \\
\hline BDF & building dose factor & - & 32 \\
\hline $\mathrm{PPB}$ & people per building & $\frac{\text { persons }}{\mathrm{km}^{2}}$ & 32 \\
\hline $\mathrm{nz}$ & $\begin{array}{l}\text { sumation variable over } \\
\text { vertical dispersion zones }\end{array}$ & - & 32 \\
\hline $\mathrm{Z}$ & $\begin{array}{l}\text { number of vertical disper- } \\
\text { sion zones }\end{array}$ & - & 32 \\
\hline $\mathrm{CDF}$ & cloudshine dose factor & $\frac{\mathrm{mrem} \cdot \mathrm{m}^{3}}{\mathrm{Ci} \cdot \mathrm{yr}}$ & 33 \\
\hline$S$ & photon source strength & $\begin{array}{c}\text { photons } / \mathrm{cm}^{2} \cdot \mathrm{s} \\
2\end{array}$ & 34 \\
\hline $\mathrm{K}_{3}$ & remnant dose source term & $\frac{\mathrm{rem} \cdot \mathrm{m}^{2}}{\mathrm{~s}}$ & 39 \\
\hline $\mathrm{K}_{4}$ & $\begin{array}{l}\text { nonrelease accident source } \\
\text { term }\end{array}$ & $\frac{\mathrm{rem} \cdot \mathrm{m}^{2}}{\mathrm{~s}}$ & 40,41 \\
\hline $\mathrm{N}_{\mathrm{c}}$ & number of crewmen & - & 41 \\
\hline
\end{tabular}


Table Ad-1 (Continued)

\begin{tabular}{|c|c|c|c|}
\hline Variable & Description & Units & $\begin{array}{l}\text { Equations In Which } \\
\text { Variable Appears }\end{array}$ \\
\hline$d_{2}$ & source-to-crew distance & metres & 41 \\
\hline CULVL & $\begin{array}{l}\text { assumed decontamination } \\
\text { level }\end{array}$ & $\mu \mathrm{Ci} / \mathrm{m}^{2}$ & $35,36,37,38$ \\
\hline$t$ & half-1ife of isotope & days & $36,37,38$ \\
\hline PedD & $\begin{array}{l}\text { time-weighted average } \\
\text { pedestrian density }\end{array}$ & $\begin{array}{l}\text { persons } \\
\text { per } \mathrm{km}^{2} \text { of } \\
\text { sidewalk }\end{array}$ & 37 \\
\hline DFS & $\begin{array}{l}\text { street decontamination } \\
\text { factor }\end{array}$ & - & 37 \\
\hline $\mathrm{PD}+\mathrm{TC}$ & $\begin{array}{l}\text { time-weighted average } \\
\text { density of people in } \\
\text { buildings }\end{array}$ & $\frac{\text { people }}{\mathrm{km}^{2}}$ & 38 \\
\hline DFB . & $\begin{array}{l}\text { decontamination factor - } \\
\text { buildings }\end{array}$ & - & 38 \\
\hline$n_{1}$ & $\begin{array}{l}\text { number of early fatality } \\
\text { types }\end{array}$ & - & 42 \\
\hline g & number of dose groups & - & 42 \\
\hline $\mathrm{N}(\phi)$ & $\begin{array}{l}\text { number of persons receiving } \\
\text { dose } \phi\end{array}$ & - & 42,43 \\
\hline$P_{\text {ef }}(\phi)$ & $\begin{array}{l}\text { probability of early } \\
\text { fatality after receiving } \\
\text { dose }\end{array}$ & - & 42 \\
\hline$n_{2}$ & $\begin{array}{l}\text { number of early morbidity } \\
\text { types }\end{array}$ & - & 43 \\
\hline$s$ & $\begin{array}{l}\text { summation variable over } \\
\text { organ types }\end{array}$ & - & 44 \\
\hline $\mathrm{CF}$ & $\begin{array}{l}\text { long-term health effect } \\
\text { coefficient }\end{array}$ & $\begin{array}{l}\text { number of } \\
\text { effects per } \\
\text { person-rem }\end{array}$ & 44 \\
\hline $\mathrm{DEF}$ & $\begin{array}{l}\text { dose-rate effectiveness } \\
\text { factor }\end{array}$ & - & 44 \\
\hline c & $\begin{array}{l}\text { summation variable over } \\
\text { accident severities }\end{array}$ & - & 45,46 \\
\hline$\varepsilon$ & $\begin{array}{l}\text { expected number of } \\
\text { accidents }\end{array}$ & - & 45,46 \\
\hline $\mathrm{L}$ & distance traveled in cell & metres & 45 \\
\hline AR & accident rate & accidents $/ \mathrm{km}$ & 45 \\
\hline
\end{tabular}


$\underline{\text { Variable }}$

e

C

H

\section{Table Ad-1 (Continued)}


Table Ad-2

Constants

\begin{tabular}{|c|c|c|c|}
\hline Constant & Value & Units & $\begin{array}{c}\text { Equations In Which } \\
\text { Constant Appears }\end{array}$ \\
\hline $\mathrm{Q}_{2}$ & $10^{-6}$ & $\frac{\mathrm{km}^{2}}{\mathrm{~m}^{2}}$ & $\begin{array}{l}4,6,8,9,10,14, \\
17,18,20,22\end{array}$ \\
\hline$Q_{3}$ & $2.78 \times 10^{-7}$ & $\frac{\mathrm{rem} \cdot \mathrm{h}}{\mathrm{mrem} \cdot \mathrm{s}}$ & $3,39,40$ \\
\hline$Q_{5}$ & $4.8 \times 10^{-5}$ & $\frac{\mathrm{rem} \cdot \mathrm{m}^{3}}{\mathrm{~s} \cdot \mathrm{C} i \cdot \mathrm{MeV}}$ & 1,3 \\
\hline$Q_{6}$ & 0.041 & $\frac{\mathrm{rem} \cdot \mathrm{m}^{3}}{\mathrm{~s} \cdot \mathrm{C} i \cdot \mathrm{MeV}}$ & 39 \\
\hline$Q_{7}$ & $10^{-3}$ & $\frac{\mathrm{km}}{\mathrm{m}}$ & 8 \\
\hline$Q_{8}$ & $3.2 \times 10^{-17}$ & $\frac{\mathrm{rem} \cdot \mathrm{yr} \cdot \mathrm{m}^{3}}{\mathrm{mrem} \cdot \mathrm{s} \cdot \mathrm{cm}^{3}}$ & 33 \\
\hline$Q_{9}$ & $4.39 \times 10^{-5}$ & $\frac{\mathrm{rem}}{\mathrm{MeV} \cdot \mu \mathrm{Ci} \cdot \mathrm{d}}$ & 37,38 \\
\hline
\end{tabular}


Table Ad-3

Functions

\begin{tabular}{|c|c|c|}
\hline Function & Description & $\begin{array}{c}\text { Equation In Which } \\
\text { Function Appears } \\
\end{array}$ \\
\hline$B_{\text {air }}(r)$ & $\begin{array}{l}\text { dose rate buildup factor } \\
\text { in ai } r\end{array}$ & $\begin{array}{l}1,2,4,5,6,11 \\
17,18,22,23,41\end{array}$ \\
\hline $\mathrm{B}_{\mathrm{b}}$ & $\begin{array}{l}\text { dose rate buildup factor } \\
\text { in building material }\end{array}$ & $7,8,9,20$ \\
\hline OF & obliqueness factor & $7,8,9,10,20$ \\
\hline$I(x)$ & $\int_{x}^{\infty} \frac{e^{-\mu} a i r^{r} \cdot B_{a i r}(r) d r}{r \sqrt{\left(r^{2}-x^{2}\right)}}$ & 24,41 \\
\hline$E_{1}(\mu x)$ & $\begin{array}{l}\text { first-order exponential } \\
\text { integral evaluated at } \mu \mathrm{x}\end{array}$ & 34 \\
\hline
\end{tabular}

NOTES

$1_{\text {NUREG-0 }} 0170$.

2 R. K. Clarke et al, Severities of Transportation Accidents, SAND74-0001 (Albuquerque: Sandia Laboratories, July 1975).

${ }^{3}$ S. Glasstone and A. Sesonske, Nuclear Reactor Engineering (Cincinnati: Van Nostrand, 1967).

"National Safety Council, "Accident Facts," pamphlet (Chicago: NSC, 1977).

5. C. Aldrich and D. M. Ericson, Jr., Public Protection in the event of a Nuclear Reactor Accident: Multi-Compartment Ventilation Model for Shelters, SAND77-1555 (Albuquerque: Sandia Laboratories, 1977).

${ }^{6}$. I. Weiner, "The Potential of Nuclear Pacemakers as a Terrorist Tool," Hazard Prevention, Nov/Dec 1978, p 23.

7 WASH 1400-Appendix VI.

${ }^{8}$ K. Z. Morgan and J. E. Turner, eds, Principles of Radiation Protection, (Huntington, N.Y.: Robert E. Krieger Publishing Co., 1973). 
SENSITIVITY AND ERROR ANALYSES

This appendix describes the methods used to perform the sensitivity and error analyses of the mathematical models used in this study. These models are for

- Incident-free transport

- Vehicular accidents

- Deviations from accepted quality assurance practices

- Sabotage or malevolent attack

The incident-free and accident models described in Appendices $B$ and $C$ can each be thought of as a single model with multiple responses or as a set of submodels. They are treated here as though composed of a number of single response submodels in order to simplify the sensitivity and error analyses and their discussion. Each submodel, then, calculates the dose from external penetrating radiation received by a particular population. The dose units are person rem or expected health effects: ear1y fatalities, ear1y morbidities, latent cancer fatalities, and genetic effects.

\section{D1. The Incident-Free Transport Model}

\section{D1.1 Sensitivity Analysis}

The objectives of the sensitivity analysis are to

- Determine the order of importance of the input variables in characterizing the response variables

- Provide simplified, closed-form equations for calculating doses and on which to perform error analyses

Achieving the above goals will make it possible to use the mathematical models and the simplified equations in anylurban area. Knowing.the relative importance of the input variables required to simulate the transport of radioactive materials through urban areas will permit the use of the models with data bases less detailed than those obtained for New York City.

The mathematical models used to assess the impacts of transporting radioactive materials through urban areas are deterministic simulation models derived from physical principles. They include all the input variables considered necessary to calculate the various doses of interest. The values of the input variables relating to characteristics of an urban area are peculiar to New York City. 
The computer code for the incident-free model, for example, simulates the travel of a shipment along a specified route through the 100-cell grid covering portions of New York City. Integrated radiation doses received by different populations exposed, such as people in vehicles and people in buildings, are accumulated cell-by-cell as the shipment traverses the grid on its specified route. The dose calculated for each cell depends on a number of variables that vary with time of day and cell location. The variables for a truck shipment, classified by their dependency, for the incident-free model are listed below.

Time-Span Dependent Variables

1. Fraction of intersections at which vehicles stop

2. Freeway traffic count

3. Separation distance between vehicles on freeway

4. Freeway vehicle velocity

5. Persons per vehicle

Ce11-Dependent Variables

1. Fraction of grid area occupied by streets

2. Street width

3. Sidewalk width

4. Building wall thickness

5. Building material

6. Number of floors

7. Story height

8. Fraction of grid area occupied by buildings

Time-Span and Ce11-Dependent Variables

1. Separation distance between vehicles

2. Vehicle speed

3. Pedestrian density

4. Pedestrian speed

5. Traffic count

6. Population density

7. Shopper population (elsewhere called transient population)

To evaluate the effects of these variables, the sensitivity analysis is performed for each dose group on a single cell basis, i.e., for a unit distance traveled. The model equations for incident-free transport typically consist of the product of a number of variables and parameters, and a term usually made up of one or more integrals. The equation of dose to warehousemen (Eq. (54), Appendix B) for example, is

$$
\begin{aligned}
& I D=Q_{1} \cdot K_{0} \cdot T I \cdot P P S \cdot \Delta T_{\text {stor }} \cdot P_{\text {stor }} \cdot \\
& \int_{8} \int^{r_{9}} \frac{(2 \pi r) e^{-\mu} a i r^{r} B_{a i r}(r) d r}{r^{2}} \text {, }
\end{aligned}
$$


where

$$
\begin{aligned}
\mathrm{Q}_{1} & =\text { scaling factor } \\
\mathrm{K}_{0} & =\text { package shape factor } \\
\mathrm{TI} & =\text { transport index } \\
\mathrm{PPS} & =\text { number of packages per shipment } \\
\mathrm{T}_{\text {stor }} & =\text { storage time } \\
\mathrm{PD}_{\text {stor }} & =\text { population density in storage area } \\
\mu_{\text {air }} & =\text { linear attenuation coefficient for air } \\
\mathrm{B}_{\mathrm{air}}(\mathrm{r}) & =\text { buildup factor for air } \\
\mathrm{r}_{8} & =\text { minimum exposure distance } \\
\mathrm{r}_{9} & =\text { maximum exposure distance }
\end{aligned}
$$

$\mu_{\text {air }}$ and $B_{\text {air }}(r)$ are functions of the photon energy, $E$, of the radionuclide being
transported.

Parameters in the mathematical models, such as stop-time at intersections, crew separation distance, and distance between stopped vehicles, are fixed at their nominal values in the data base for the sensitivity analysis since use of these values does not result in doses significantly different from the doses calculated at extreme values.

The sensitivity of the response variable to the multiplicative factors is obvious, i.e., doubling TI doubles the dose, so these factors are fixed and the effects of the remaining variables are investigated. In.Eq. (1), the variables investigated are $r_{8}, r_{9}$, and $E$.

The sensitivity analysis is accomplished by calculating doses with the mathematical model as the input variables under investigation are permitted to vary over plausible ranges and multiplicative factors are set at nominal values. The values of the input variables used in the calculations are selected according to appropriate response surface designs.l The data thus obtained are fitted by linear least squares. The resulting regression equations are then rescaled to include the multiplicative factors. Ranges for input variables and their limits of variation are

\begin{tabular}{l} 
Variable \\
\hline Photon energy, $E$ \\
Sidewalk width, ${ }_{s}$ \\
Street width, $w_{s t}$ \\
Vehicle velocity, $\bar{v}$ \\
Vehicle velocity on freeway, $v_{f}$ \\
Pedestrian velocity, $v_{p}$ \\
Building wall thickness, $w_{b}$ \\
Number of floors, $n$
\end{tabular}

Minimum/Maximum Values

$0.5 / 1.5 \mathrm{MeV}$

$2 / 4$ metres

$10 / 30$ metres

$3.34 / 8.06 \mathrm{~m} / \mathrm{s}$

$9 / 24.4 \mathrm{~m} / \mathrm{s}$

$1.1 / 1.4 \mathrm{~m} / \mathrm{s}$

$0.2 / 0.4$ metres

$1 / 29$ 


$\begin{array}{ll}\text { Minimum exp. dist., warehouse, } r_{8} & .7 / 2.3 \text { metres } \\ \text { Maximum exp. dist., warehouse, } r_{9} & 50 / 150 \text { metres } \\ \text { Freeway width, } w_{f} & 40 / 100 \text { metres } \\ \text { Minimum exp. dist., airports, } r_{4} & 82 / 162 \text { metres } \\ \text { Maximum exp. dist., airports, } r_{5} & 204 / 284 \text { metres } \\ \text { Minimum exp. dist., depot, } r_{1} & 1 / 3.8 \text { metres } \\ \text { Maximum exp. dist., depot, } r_{2} & 4 / 10 \text { metres } \\ \text { Dist. between passing trains, } r_{3} & 3 / 9 \text { metres } \\ \text { Rail right-of-way dist., } r_{w} & 2 / 5 \text { metres } \\ \text { Minimum exp. dist., docks, } r_{6} & 5 / 19.4 \text { metres } \\ \text { Maximum exp. dist., docks, } r_{7} & 20 / 200 \text { metres }\end{array}$

The fitted regression equations are then used to calculate the percent change in each input variable that will produce a $100 \%$ change (doubling) in the response variable, keeping all other input variables at their nominal values. This process permits the ranking of the input variables according to their influence on the response variable.

The data are fitted with a stepwise regression program. ${ }^{2}$ A regression model is postulated, and the program obtains a set of equations by successively examining the contribution of each input variable. The program first admits the input variable in the postulated regression model that explains the most variation in the response variable and then admits other variables in order of their additional contribution to the overall fit as measured by their partial correlation with the response variable. As each variable is added, all the variables already admitted are evaluated according to their current contribution, and any time their contribution is not significant at some specified probability level, the variable contributing least is deleted. The procedure continues until only variables that are not significant at some specified level are excluded from the model. From the equations thus obtained, the one with the least number of input variables that accounts for at least $90 \%$ of the variation in the response variable is chosen.

The equation to be fitted to the results obtained with the simulation models are of the type:

$$
y=\beta_{0}+\sum_{i=1}^{k} \beta_{i} x_{i}+\sum_{i=1}^{k} \sum_{j=1}^{k} \beta_{i j} x_{i} x_{j}+\varepsilon
$$

where

$$
\begin{aligned}
y & =\text { the response variable } \\
x_{1} & =\text { the } i \text { th input variable } \\
k & =\text { the number of input variables }
\end{aligned}
$$


$\varepsilon=$ the devation due to lack of fit

$\beta_{i}=$ the average change in $y$ for a unit change in $x_{i}$ (The $\beta_{i}$ 's are estimated from the data.)

A complete equation for two input variables, for example, is

$$
y=\beta_{0}+\beta_{1} x_{1}+\beta_{2} x_{2}+\beta_{11} x_{1}^{2}+\beta_{22} x_{2}^{2}+\beta_{12} x_{1} x_{2}+\varepsilon .
$$

Addendum 1 to this appendix gives a table of the nominal values of the parameters and variables not treated in the regression analysis, and Addendum 2 gives the regression equations obtained by fitting the mathematical model calculations by least squares. For completeness, raw data used for regression analysis are in Addendum 8. The relative importance of the input variables for each population is shown in Table $\mathrm{D}-1$.

As can be seen, the list of important variables is different for each population at risk and no overall ranking of variables is possible. There are, however, some commonalities among the results for a given population at risk. For example, average velocity, $\bar{v}$, is among the most influential variable for dose to pedestrians, and wall thickness, $w_{s}$, ranks first for doses to persons in buildings for one- and two-way streets and second for doses to persons in buildings for freeways.

For most populations at risk, the most influential variables are easily ascertainable or can be given conservative values in dose calculations (see Addendum 1).

The 15 equations derived from the sensitivity analysis can be combined to calculate total dose for a given set of circumstances (Addendum 2). Manipulation of 15 equations which must be evaluated repeatedly as values of input variables change along a route is a cumbersome and tedious process. However, the analysis of the standard shipments model reported in Chapter 2 reveals that about $96 \%$ of the yearly person rem accumulated in New York City is received by five populations: pedestrians, people in vehicles, handlers, crew, and warehouse personnel. of the equations for these dose groups, two are for static situations which are independent of the route traveled. The dose to pedestrians and people in vehicles are the only ones that need to be evaluated for a given route. Dose to crew requires only the length of the route and the average vehicle velocity. The only urban-specific variables needed to evaluate these doses are average vehicle velocity, $\bar{v}$, pedestrian density, PedD, sidewalk width, $w_{b}$, and street width, $w_{s+}$. Therefore, given the shipping experience of a particular city, the grid can'se superimposed over that city, the pertinent data collected, and the impacts estimated with relative ease.

\section{D1.2 Error Analysis}

The purpose of error analyses is to determine the uncertainty or error produced in the response variables by inexact knowledge of the input variables. Error analyses should provide guidelines as to how precisely input variables need to be known in generic applications.

The error analyses are performed on the regression equations obtained in the sensitivity analyses. Error propagation formulas are derived from Taylor expansions of the regression equations. ${ }^{3}$ Since the equations consist mainly of a series of product terms, and the distributions of the $x$ 's are not known, the error equations are expressed in terms of relative errors. 


\section{Table D-1}

\section{Input Variables Ranked According to Percent \\ Change that Results in $100 \%$ Change in the \\ Response Variable for Incident-Free Transport}

1. Dose to Handlers

TI 100

2. Dose to Warehousemen

$$
\begin{array}{lr}
\mathrm{TI} & 100 \\
\mathrm{PD}_{\text {stor }} & 100 \\
\Delta \mathrm{T}_{\text {stor }} & 100 \\
\mathrm{r}_{8} & -355 \\
\mathrm{r}_{9} & 414
\end{array}
$$

3. Dose to Crew

$$
\begin{array}{lr}
\text { TI } & 100 \\
\mathrm{~L} & 100 \\
\mathrm{~N}_{\mathrm{C}} & 100 \\
\overline{\mathrm{V}} & -100
\end{array}
$$

4. Dose to Pedestrians
a) One-way Streets
$\overline{\mathrm{V}}$
$-75$
$w_{\text {st }} \quad-92$
TI $\quad 100$
L $\quad 100$
PedD $\quad 100$
$\mathrm{w}_{\mathrm{S}} \quad 102$

b) Two-way Streets

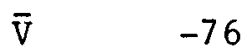

$w_{\text {s }} \quad 84$

$w_{\text {st }} \quad-99$

TI $\quad 100$

PedD $\quad 100$

L $\quad 100$
5. Dose to Persons in Vehicles
a) One-way Streets $\overline{\mathrm{V}} \quad-89$
TI $\quad 100$
L $\quad 100$
$w_{\text {st }}-117$

b) Two-way Streets

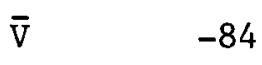

TI $\quad 100$

L $\quad 100$

N $\quad 100$

$w_{\text {st }} \quad-118$

c) \begin{tabular}{ll} 
Freeways \\
\hline $\mathrm{V}_{\mathrm{f}}$ & -69 \\
$\mathrm{TI}$ & 100 \\
$\mathrm{~L}$ & 100 \\
$\mathrm{~N}$ & 100
\end{tabular}

6. Dose to Persons in Buildings

a) One- and Two-way Streets

$\begin{array}{lr}\mathrm{W}_{\mathrm{ST}} & 17 \\ \mathrm{E} & 41 \\ \overline{\mathrm{V}} & -57 \\ \mathrm{w}_{\mathrm{b}} & -73 \\ \mathrm{TI} & 100 \\ \mathrm{PIB} & 100 \\ \mathrm{~L} & 100 \\ \mathrm{n} & -153\end{array}$


6. Dose to Persons in Bldgs (cont)

b) \begin{tabular}{lr}
\multicolumn{2}{l}{ Freeways } \\
TI & 100 \\
PIB & 100 \\
L & 100 \\
E & -104 \\
$\mathrm{w}_{\mathrm{st}}$ & -104 \\
$\mathrm{n}$ & -264 \\
$\mathrm{w}_{\mathrm{b}}$ & -272
\end{tabular}

7. Dose to Persons in Air Terminals

$\begin{array}{lll}\mathrm{r}_{6} & -56 \\ \mathrm{TI} & 100 \\ \Delta \mathrm{T} & 100 \\ \mathrm{PD} & 100 \\ \mathrm{r}_{5} & 201 \\ \mathrm{E} & 201\end{array}$

8. Dose to Persons in Railroad Terminals

$\begin{array}{ll}\mathrm{r}_{1} & -98 \\ \mathrm{TI} & 100 \\ \Delta \mathrm{T} & 100 \\ \mathrm{PD} & 100 \\ \mathrm{r}_{2} & 106\end{array}$

9. Dose to Persons along Transportation Link

$\begin{array}{lr}\mathrm{V}_{\mathrm{T}} & -50 \\ \mathrm{TI} & 100 \\ \mathrm{~L} & 100 \\ \mathrm{~N}_{\mathrm{T}} & 100 \\ \mathrm{PPT} & 100 \\ \mathrm{r}_{3} & -141\end{array}$

10. Dose to Persons along Railroad Right-of-way

$\begin{array}{lr}\text { TI } & 100 \\ \mathrm{PD} & 100 \\ \mathrm{~L} & 100 \\ \mathrm{~V}_{\mathrm{T}} & 100 \\ \mathrm{r}_{\mathrm{rW}} & -392 \\ \mathrm{E} & 611\end{array}$

11. Dose to Persons in Wharf Area

$\begin{array}{rr}\mathrm{r}_{7} & 68 \\ \mathrm{TI} & 100 \\ \Delta \mathrm{T} & 100 \\ \mathrm{PD} & 100 \\ \mathrm{r}_{6} & -197\end{array}$


For a function $y=f\left(x_{1}, x_{2}, \ldots, x_{n}\right)$, the linear terms in Taylor's series can be used to express the effect on $y$ of small errors in the $x^{\prime} s$. If $\Delta x_{i}$ is the error in $x_{i}$, the error in $y, \Delta y$, can accurately be expressed as

$$
\Delta y=\sum_{i=1}^{n}\left(\frac{\partial y}{\partial x_{i}}\right) \Delta x_{i}
$$

where $\partial y / \partial x_{i}$ is the first partial derivative of $y$ evaluated at the point $\left(\mathrm{X}_{1}, \mathrm{X}_{2}, \ldots, \mathrm{x}_{\mathrm{n}}\right)$. Eq. (4) is appropriate provided that the $\Delta \mathrm{x}_{i}$ or the higher derivatives are small.

Dividing (1) by $\hat{y}=f\left(x_{1}, x_{2}, \ldots x_{n}\right)$ results in the relative error in $y$,

$$
\frac{\Delta y}{\hat{y}}=\sum_{i=1}^{n}\left(\frac{\partial y}{\partial x_{i}}\right) \frac{\Delta x_{i}}{\hat{y}}
$$

Multiplying and dividing the right hand side of $\mathrm{Eq} \cdot(5)$ by $\mathrm{x}_{i}$ results in

$$
\operatorname{RE}(y)=\frac{\Delta y}{\hat{y}}=\sum_{n=1}^{n}\left(\frac{\partial y}{\partial x_{i}}\right)_{\hat{i}}^{x_{i}} \operatorname{RE}\left(x_{i}\right)
$$

which expresses the relative error in $y$ in terms of the relative errors of the $x_{i}$.

The equations thus obtained are evaluated at nominal values of the input variables, and the coefficients for the various relative error terms measure their contribution to the relative error in the calculated doses.

The error equations appear in Addendum 3 to this appendix in a form which permits the direction of the errors in the input variables to be taken into account. When the direction of the errors is not known, these equations can be expressed as a sum of the absolute values of the contributions of the individual input variables.

Examination of the coefficients in the equations in Addendum 3 shows that most of the coefficients are at most equal to 1 . The input variables for which relative errors are magnified, i.e., those with coefficients $\geq 1.05$, are as follows:

\section{Population}

\section{Pedestrians}

2. Persons in Vehicles
a) One- and two-way streets
b) Freeway

Variable
Average velocity, $\overline{\mathrm{V}}$
Average velocity, $\overline{\mathrm{V}}$
Street width, $\mathrm{w}_{\mathrm{st}}$
Freeway velocity, $\mathrm{v}_{\mathrm{f}}$


3. Persons in Buildings

a) One- and two-way streets

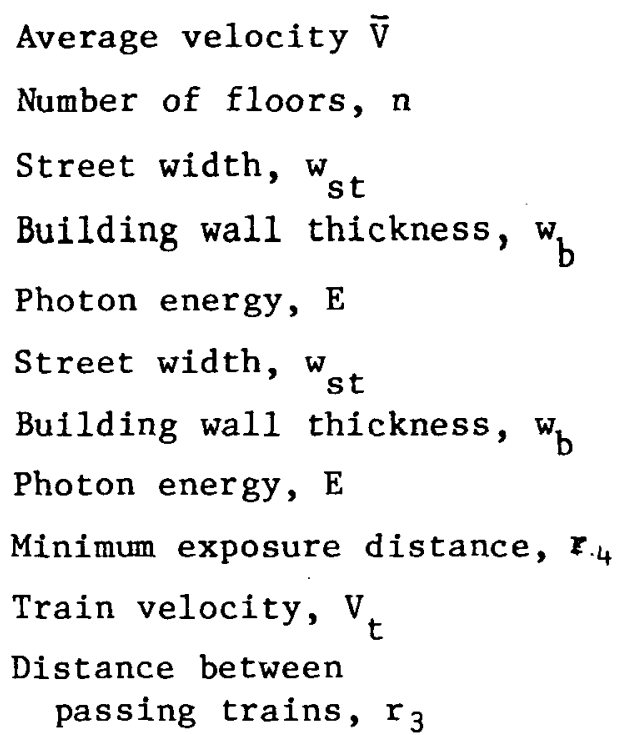

These input variables are shown to be important in calculating the corresponding doses (see Addendum 2). Of the five dose groups, only dose to crew contributes significantly to the total person rem for New York City. This indicates that the shipping experience for a particular city--i.e., end use, mode, and route--is perhaps as important as the values of the urban variables.

As indicated in the previous section, dose to pedestrians is a major contributor to the total dose for New York City. The error equation for dose to pedestrians has six variables, each with a coefficient approximately equal to 1.0 . Assuming a $10 \%$ error $\left(\operatorname{RE}\left(x_{1}\right)=0.1\right)$ in each of these variables, the maximum absolute relative error in dose to pedestrians is 0.6 . Thus, an upper limit for dose to pedestrians is obtained by multiplying the estimated dose by 1.6. This means, for this example, that the dose may be $60 \%$ higher than calculated due to $10 \%$ errors in the input variables; this does not lend itself to a probabilistic interpretation.

\section{D2. Vehicular Accident Mode1}

The accident models calculate the consequences of accidents involving nondispersible and dispersible materials. The models for these two cases differ in several respects and require different approaches for the sensitivity and error analyses. The consequences for accidents involving nondispersible materials are integrated whole-body external doses to populations at risk such as pedestrians, people in vehicles, and people in buildings. The sensitivity and error analyses are performed as for the incident-free model; i.e., simplifying equations are obtained, the input variables are ranked in order of their effects on the response variables, and error propagation is studied by means of the simplifying equations.

In doing the generic extension of the accident analysis to other urban areas, it was learned that pedestrians and persons in vehicles account for essentially $100 \%$ of the radiological consequences of a vehicular accident. Consequently, only these two dose groups are treated in the sensitivity analysis for nondispersible materials.

Accidents involving dispersible materials can result in both internal and external exposure. The consequences evaluated are early fatalities, early morbidities, latent cancer fatalities, and genetic effects. Further, the model for dispersible 
materials incorporates meteorological variables, and numerous implicit input variables such as the radiological dose factor (RPC) and the fraction of inhaled material deposited in the pulmonary compartment (RESP). RPC is a function of the radionuclide transported, and RESP is a function of the particle size. The meteorological and implicit variables preclude the fitting of simplifying equations that can be used to mimic the mathematical model, and to study error propagation. Therefore, the sensitivity analysis for dispersible materials is limited to studying the effects variations in the nonmultiplicative variables have on the response variables.

\section{D2.1 Nondispersible Materials}

Sensitivity Analysis

The two components for doses from nondispersible material--the dose from the breached package and that from the unbreached package--are accounted for in the model equations by means of the multiplicative factor, $K_{l}$, which is a function of release fraction, $\mathrm{RF}$ (Eq. (3), Appendix $\mathrm{C}$ ), where

$$
\mathrm{K}_{1}=\left[\left(\mathrm{Q}_{5} \cdot \mathrm{N}_{1} \cdot \mathrm{PPS} \cdot \mathrm{\mu}_{\mathrm{r}} \cdot \mathrm{E}_{\mathrm{d}} \cdot(\mathrm{RF})\right)+\mathrm{q}_{3} \cdot \mathrm{K}_{\mathrm{o}} \cdot \mathrm{TI} \cdot \mathrm{PPS} \cdot(1-\mathrm{RF})\right]
$$

When $R F=0$, the doses for nonrelease accidents are obtained, and when $0<R F<1$, the combined dose from material that is released and from material remaining in the package is obtained. For $R F=1$, the entire dose is due to released materia1. $R e-$ lease fraction and other multiplicative factors are set constant at nominal values for the calculations with the model (Addendum 4). The regression equations are then rescaled to incorporate these multiplicative factors.

The input variables that are permitted to change, and their limits of variation are

Variable

Sidewalk width, $w_{s}$
Street width, $w_{s t}$
Freeway width, ${ }_{f}$
Photon energy, E
Photon energy/disintegration, $E_{d}$
Building wall thickness, $w_{b}$
No. of floors/building, $n$

\begin{tabular}{l} 
Minimum/Maximum Values \\
\hline $2 / 4$ metres \\
$10 / 30$ metres \\
$40 / 100$ metres \\
$0.5 / 1.5 \mathrm{MeV}$ \\
$0 / 2.5 \mathrm{MeV}$ \\
$0.2 / 0.4$ metres \\
$1 / 29$
\end{tabular}

$2 / 4$ metres

$10 / 30$ metres

Addendum 8 contains the raw data analyzed for the nondispersible case.

The equations obtained for nondispersible material are given in Addendum 5 . Table D-2 ranks the input variables in order of their importance. As can be seen in Table D-2, all the variables for pedestrians are approximately of equal importance, except for TI, which has a minimal effect on the integrated dose. For persons in vehicles on one-way and two-way streets, street width, $w_{s f}$, is the most important variable; Transport Index, TI, the least important; and afi others are of about equal importance. Along freeways, the photon energy, $\mathrm{E}$; freeway width, $\mathrm{w}_{\mathrm{f}}$, and TI are the least important input variables, with the other, multiplicative variables having equal effects. 


\section{Table D-2}

\section{Input Variables Ranked According to Percent Change that Results in $100 \%$ Change in the Response Variable for Nondispersible Materials Accidents}

1. Dose to Pedestrians; One- and Two-Way Streets

$\begin{array}{ll}\Delta \mathrm{T} & 100 \\ \text { PedD } & 100 \\ \mathrm{n}_{\mathrm{I}} & 117 \\ \mathrm{E}_{\mathrm{d}} & 117 \\ \mathrm{RF} & 117 \\ \mathrm{E} & 123 \\ \mathrm{w}_{\text {st }} & 132 \\ \text { TI } & 9.5 \times 10^{5}\end{array}$

2. Dose to Persons in Vehicles
a) One-way Streets
89
wst
92
$\mathrm{n}_{1}$
92
$\mathrm{E}_{\mathbf{d}}$
92
$\mathrm{RF}$
100
TI

\section{$7.5 \times 10^{5}$}
b) Two-way Streets

$\begin{array}{ll}\mathrm{w}_{\text {st }} & 89 \\ \mathrm{n}_{\mathrm{l}} & 92 \\ \mathrm{E}_{\mathrm{d}} & 92 \\ \mathrm{RF} & 92 \\ \Delta \mathrm{T} & 100 \\ \mathrm{~N} & 100\end{array}$
TI
c) Freeways

$\begin{array}{ll}\mathrm{n}_{\mathrm{l}} & 100 \\ \mathrm{E}_{\mathrm{d}} & 100 \\ \mathrm{RF} & 100 \\ \Delta \mathrm{T} & 100 \\ \mathrm{~N} & 100 \\ \mathrm{w}_{\mathrm{f}} & 1.2 \times 10^{5} \\ \mathrm{E} & 1.2 \times 10^{5} \\ \mathrm{TI} & 4.0 \times 10^{5}\end{array}$


Error Analysis

The error equations for the nondispersible case are shown in Addendum 6 . The variables for which the errors are amplified, i.e., the variables with coefficients greater than 1.05 , are

Population

1. Persons in Vehicles

(a) One-way streets

(b) Two-way streets

(c) Freeway

\section{Variable}

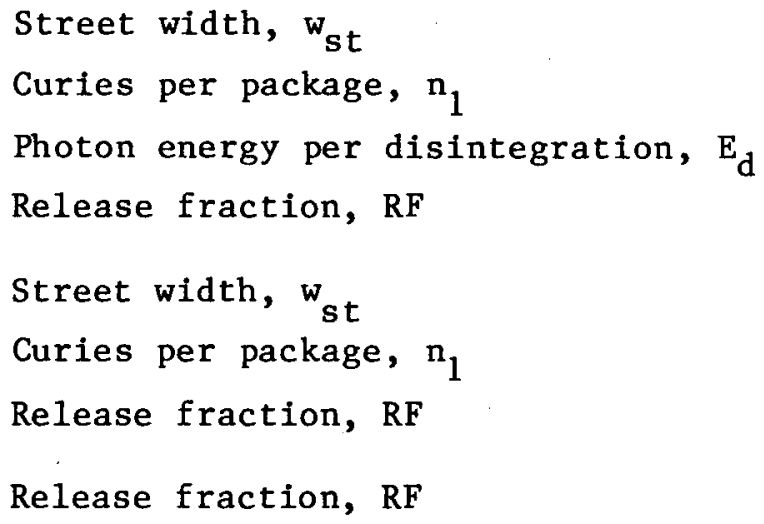

\section{D2.2 Dispersible Materia1}

Sensitivity Analysis

The response variables for accidents involving shipments of dispersible material are

- Remnant LCF (1atent cancer fatalities)

- Cloudshine LCF

- Nonrelease LCF

- Groundshine LCF

- Total LCF

- Inhalation LCF

- Remnant GE (genetic effects)

- Cloudshine GE

- Nonrelease GE

- Groundshine GE

- Total GE

- Inhalation GE

- Early fatalities

- Early morbidities

These responses were calculated for the two types of ventilation systems, 1.e., air conditioning and continuous intake. Of the above 14 consequences, the following 6 were analyzed explicity:

- Groundshine LCF

- Groundshine GE

- Inhalation LCF 
- Inhalation GE

- Early fatalities

- Early morbidities

Remnant, cloudshine, and nonrelease consequences contribute on the order of $0.1 \%$ or less of the total LCF and GE, and are therefore not analyzed. Tables Ad-20 and Ad-21 of Addendum 8 present the data analyzed for the dispersible material case.

The sensitivity analysis for dispersible materials was carried out by postulating a severity-VIII accident in cell $46 \mathrm{f}$ or a spent fuel shipment. The curies per package were set at artificially high levels in order to allow for analysis of fatalities and morbidities.

The input variables studies and the limits within which they were varied are listed below.

Variable
Wind speed
Street width, ${ }_{\text {st }}$
Sidewalk width, $w_{s}$
Fraction of cell area in buildings, $f_{b}$
Curies per package, $\mathrm{n}_{1}$
Reflectivity coefficient

Minimum/Maximum Values

$5 / 17^{\circ} \mathrm{m} / \mathrm{s}$

$11 / 28$ metres

$2 / 4$ metres

$0.15 / 0.65$

$10^{6} / 3 \times 10^{6} \mathrm{Ci}$

$.01 / 1.0$

Preliminary calculations with the meteorological models provided evidence that the reflectivity coefficient, fraction of cell area occupied by buildings, and wind speed should be included in the sensitivity analysis. At the same time, it was learned that variations in the eddy diffusivity, building height, and wind direction had minimal effects on the consequences, so these variables were not studied further.

The variables and parameters kept constant for this portion of the study and their nominal values are presented in Addendum 7 of this appendix.

Table D-3 presents the input variables ranked in order of their strength in producing variation in the response variables. Examination of this table shows that the groundshine consequences are influenced by the same variables for both ventilation systems: "curies per package is a function of the shipment, while the other important variables, $w_{s t}, w_{s}$, and $f_{b}$, are space variables. These space variables determine the number and types of populations at risk, i.e., pedestrians and persons in buildings.

It has not been possible to fit the data on early fatalities and early morbidities adequately. The rank ordering is done by examining the linear change of the response variable versus the change in each input variable, independent of all other input variables, in the neighborhood of the nominal, i.e., one point on either side of the nominal value of the input variable. The failure to obtain an adequate regression equation is attributed to the complexities introduced by the use of thresholds in the probability distributions relating early fatalities and early morbidities to individual exposure levels. 
Table D-3

Input Variables Ranked According to Percent Change that Results in $100 \%$ Change in the Response Variable for Dispersible Material Accidents

1. Continuous Intake

Groundshine

\begin{tabular}{cccccc}
\hline \multicolumn{2}{c}{ LCF } & & \multicolumn{2}{c}{ GE } \\
\cline { 1 - 3 } \cline { 5 - 6 } Variable & $\frac{\text { Percent }}{94}$ & & $\frac{\text { Variable }}{\mathrm{n}_{1}}$ & & $\frac{\text { Percent }}{94}$ \\
$\mathrm{f}_{\mathrm{b}}$ & -129 & & $\mathrm{n}_{1}$ & 94 \\
${ }_{\text {w }}$ & -189 & & $\mathrm{f}_{\mathrm{b}}$ & -130 \\
w $_{\mathrm{s}}$ & 242 & & ${ }_{\text {w }}$ & -189
\end{tabular}

Inhalation

\begin{tabular}{|c|c|c|c|}
\hline \multicolumn{2}{|c|}{ EM* } & \multicolumn{2}{|c|}{$E F^{*}$} \\
\hline Variable & Percent & Variable & Percent \\
\hline Wind Speed & -40 & $w_{s}$ & 8 \\
\hline $\mathbf{w}_{\mathbf{s}}$ & 84 & Wind Speed & -16 \\
\hline $\mathrm{n}_{1}$ & 108 & $\mathrm{n}_{1}$ & 17 \\
\hline$w_{s t}$ & -150 & $f_{b}$ & -18 \\
\hline$f_{b}$ & -236 & $w_{\text {st }}$ & -1133 \\
\hline
\end{tabular}

Total Inhalation

\begin{tabular}{|c|c|c|c|}
\hline \multicolumn{2}{|c|}{ LCF } & \multicolumn{2}{|c|}{ GE } \\
\hline Variable & Percent & Variable & Percent \\
\hline $\mathrm{n}_{1}$ & 63 & Wind Speed & -58 \\
\hline$f_{b}$ & 96 & $w_{s t}$ & -58 \\
\hline${ }^{w_{s t}}$ & -181 & $f_{b}$ & 117 \\
\hline & & $\mathrm{n}_{1}$ & 117 \\
\hline
\end{tabular}

\section{Air Conditioning}

Groundshine

\begin{tabular}{|c|c|c|c|}
\hline \multicolumn{2}{|c|}{ LCF } & \multicolumn{2}{|c|}{ GE } \\
\hline$\frac{\text { Variable }}{\mathbf{w}_{\mathrm{s}}}$ & $\frac{\text { Percent }}{93}$ & $\frac{\text { Variable }}{w_{\text {st }}}$ & $\frac{\text { Percent }}{-49}$ \\
\hline $\mathrm{n}_{1}$ & 94 & $w_{s}$ & 93 \\
\hline$f_{b}$ & 137 & $n_{1}$ & 94 \\
\hline$w_{s t}$ & -146 & $f_{b}$ & 137 \\
\hline
\end{tabular}

Inhalation

\begin{tabular}{|c|c|c|c|}
\hline \multicolumn{2}{|c|}{ EM* } & \multicolumn{2}{|c|}{$\mathrm{GE}^{*}$} \\
\hline Variable & Percent & Variable & Percent \\
\hline Wind Speed & -107 & $\mathbf{w}_{\mathbf{s}}$ & 4 \\
\hline $\mathrm{w}_{\mathrm{s}}$ & 131 & $w_{s}$ & -9 \\
\hline $\mathrm{n}_{1}$ & 146 & $\mathrm{n}_{1}$ & 9 \\
\hline$w_{s t}$ & -162 & $f_{b}$ & -10 \\
\hline$f_{b}$ & -295 & $w_{\text {st }}$ & -28 \\
\hline
\end{tabular}

Total Inhalation

\begin{tabular}{|c|c|c|c|}
\hline \multicolumn{2}{|c|}{ LCF } & \multicolumn{2}{|c|}{ GE } \\
\hline Variable & Percent & Variable & Percent \\
\hline Wind Speed & -64 & $\mathrm{n}_{1}$ & 103 \\
\hline$f_{b}$ & 83 & $f_{b}$ & 108 \\
\hline $\mathrm{n}_{1}$ & 86 & Wind Speed & -119 \\
\hline$w_{s t}$ & -87 & ${ }_{\alpha}^{w_{s t}}$ & $\begin{array}{r}-136 \\
304\end{array}$ \\
\hline
\end{tabular}

*As discussed in the text, the method used in rank ordering the input variables for these consequences is different from that used for the other consequences. 
The variables affecting total inhalation consequences are essentially the same for both ventilation systems. The differences are that wind speed does not appear important for total inhalation latent cancer fatalities for continuous intake and it does appear important for other consequences; and that the reflectivity coefficient, $\alpha$, appears in the 1ist of variables for total inhalation genetic effects for air-conditioning and not for other consequences.

D3. Deviations from Accepted Quality Assurance Practices

The vehicular accident models are also used for the human error and sabotage ana1yses, with adaptions of the accident rates and release fractions to the particular situation. Thus, the sensitivity analysis treatment of both nondispersible and dispersible material accidents can be applied to either human errors or the sabotage event since radiological consequences and not risk are treated explicitly. No attempt has been made to perform a sensitivity and error analysis on the CRAC model used in part in Chapter 5 since this model was developed independently and has been used simply to compare with the METRAN calculations.

D4. Summary and Conclusions

The major conclusions resulting from the sensitivity and error analysis are

- The methods used to perform the sensitivity analyses, i.e., fitting model results with simplified regression models, demonstrate the generic nature of the math models. The 100-cell grid can be superimposed on any urban area for which pertinent data are avallable, and the models can be used to calculate the impacts from shipments of radionuclides through that area.

- Information has been developed regarding which urban variables need to be known in detail in applying the models. Guidance as to how accurately these variables have to be known 18 provided by the error equations.

- The model data were successfully fitted for all response variables except for early morbidities and early fatalities in accidents involving dispersible material.

- A number of variables and parameters such as health effect coefficients and accident rates were not investigated explicitly. These variables were not investigated because either

1. The variables, e.g., accident rates, are multiplicative factors in determining expected values or risk, or

2. The variables are not treated explicitly in the model equations; $1 . e .$, they are functions of other variables. Several parameters, for example, are determined by the radionuclide involved. 
ADDENDUM 1: NOMINAL VALUES OF PARAMETERS AND VARIABLES USED IN THE SENSITIVITY AND ERROR ANALYSIS EQUATIONS, INCIDENT-FREE TRANSPORT

$\frac{\text { Variable }}{\Delta \mathrm{T}_{\text {stor }}}$
$\Delta \mathrm{T}_{\text {air term }}$,
$\Delta \mathrm{T}_{\text {dock }}$
$\Delta \mathrm{T}_{\text {depot }}$
$\xi$

$\Omega$

PPS

Shipments/yr.

TI

E

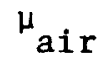

$\mathrm{B}_{\text {air }}(\mathrm{r})$

$\delta$

$\ell$

$\mathrm{d}_{1}$

$d_{2}$

$\overrightarrow{\mathrm{v}}^{2}$

$\mathrm{v}_{\mathrm{c}}$

$v_{\mathrm{f}}$

$v_{p}=V E L P$

$\mathrm{V}_{\mathrm{T}}$

$f_{b}$

$f_{\text {st }}$

n

h
Definition

Time spent in warehouse storage

Stop time in air terminal

Stop time in dock area

Stop time in rail depot

Fraction of intersections at which vehicles stop

Delay time at intersections

No. of packages per shipment

Transportation Index

Photon energy

Linear attenuation, air

Dose buildup factor, air

Vehicle separation distance during stops

Vehicle length

Vehicle separation distance while cruising

Source-to-crew distance

Average shipment velocity

Cruising velocity

Freeway velocity

Pedestrian velocity

Train velocity

Fraction of cell area occupied by buildings

Fraction of cell area occupied by streets

Number of floors in buildings in cell

Height per floor
Nominal Value

$43200 \mathrm{~s}(12 \mathrm{~h})$

$3300 \mathrm{~s}(0.92 \mathrm{~h})$

$36000 \mathrm{~s}(10 \mathrm{~h})$

$3600 \mathrm{~s}(1 \mathrm{~h})$

0.5

$25 \mathrm{~s}$

1

1000

1

$1 \mathrm{MeV}$

0.0081

$(0.00197 r)+1$

$1 \mathrm{~m}$

$6.4 \mathrm{~m}$

$80 \mathrm{~m}$

$3 \mathrm{~m}$

$5.7 \mathrm{~m} / \mathrm{s}$

$6.2 \mathrm{~m} / \mathrm{s}$

$16.7 \mathrm{~m} / \mathrm{s}$

$1.25 \mathrm{~m} / \mathrm{s}$

$5 \mathrm{~m} / \mathrm{s}$

0.3

0.7

15

$3 \mathrm{~m}$ 
Variable

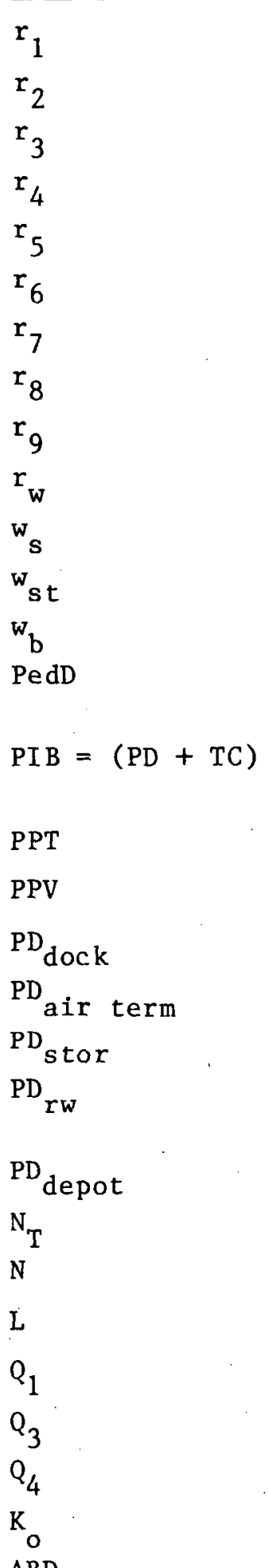

Definition

Minimum rail exposure distance

Maximum rail exposure distance

Distance between passing trains

Minimum air terminal exposure distance

Maximum air terminal exposure distance

Minimum dock exposure radius

Maximum dock exposure radius

Minimum warehouse exposure distance

Maximum warehouse exposure distance

Rail right-of-way distance

Sidewalk width

Street width

Building wall thickness

Pedestrian density

People in buildings (population density + transient clientele)

No. of persons per train

No. of persons per vehicle

Population density in dock area

Population density in air terminal

Population density in warehouse

Population density along rail right-ofway

Population density in train depot

One-way train traffic count

One-way traffic count

Distance traveled in cell

Scaling factor

Scaling factor

Scaling factor

Package shape factor

Albedo dose factor
Nominal Value

$2.4 \mathrm{~m}$

$7.0 \mathrm{~m}$

$6 \mathrm{~m}$

$122 \mathrm{~m}$

$244 \mathrm{~m}$

$12.2 \mathrm{~m}$

$110 \mathrm{~m}$

$1.5 \mathrm{~m}$ $100 \mathrm{~m}$

$3 \mathrm{~m}$

$3 \mathrm{~m}$

$20 \mathrm{~m}$

$0.3 \mathrm{~m}$

$10^{6}$ persons $/ \mathrm{km}^{2}$

sidewalk

110000 persons $/ \mathrm{km}^{2}$

1000

2

1000 persons $/ \mathrm{km}^{2}$

$10^{4}$ persons $/ \mathrm{km}^{2}$

800 persons $/ \mathrm{km}^{2}$

$14.356 \times 10^{6}$

1000 persons $/ \mathrm{km}^{2}$

0.00556 vehicle/s

1000 vehicles

$1000 \mathrm{~m}$

$2.78 \times 10^{-13}$

$2.78 \times 10^{-7}$

$2.5 \times 10^{-4}$

1.05

1.22 
ADDENDUM 2: SENSITIVITY ANALYSIS EQUATIONS FOR INCIDENT-FREE TRANSPORT

(Expressions given here are for a single shipment. To calculate integrated doses for a specific level of shipping activity, multiply these expressions by the total shipments per year.)

1. Dose to Handlers

(Closed form--Eq. (51), App. B)

$$
\mathrm{ID}=\mathrm{Q}_{4} \cdot \mathrm{N}_{\mathrm{H}} \cdot \mathrm{PPS} \cdot \mathrm{TI}
$$

2. Dose to Warehousemen

$$
\begin{aligned}
\mathrm{R}^{2}= & 0.9562 \\
\mathrm{ID}= & \mathrm{Q}_{1} \cdot \mathrm{K}_{\mathrm{o}} \cdot \Delta \mathrm{T}_{\text {stor }} \cdot \mathrm{PD}_{\text {stor }} \cdot \mathrm{TI} \cdot \mathrm{PPS} \cdot \\
& {\left[28.11-5.047\left(\mathrm{r}_{8}\right)+0.06492\left(\mathrm{r}_{9}\right)\right] }
\end{aligned}
$$

3. Dose to Crew*

(Closed form--Eq. (40), App. B)

$$
\mathrm{ID}=\mathrm{Q}_{3} \cdot \frac{\mathrm{L}}{\overline{\mathrm{V}}} \cdot \mathrm{N}_{\mathrm{c}} \cdot \frac{\mathrm{K}_{\mathrm{o}} \cdot \mathrm{TI} \cdot \mathrm{PPS} \cdot \mathrm{e}^{-\mu \mathrm{air} \mathrm{d}_{2}} \cdot \mathrm{B}_{\mathrm{air}\left(\mathrm{d}_{2}\right)}}{\mathrm{d}_{2}{ }^{2}}
$$

4. Dose to Pedestrians

a) One-way streets

$$
\begin{aligned}
\mathrm{R}^{2}= & 0.9404 \\
\mathrm{ID}= & 2 \times 10^{-3} \cdot \mathrm{Q}_{1} \cdot \operatorname{PedD} \cdot \mathrm{L} \cdot \mathrm{K}_{\mathrm{o}} \cdot \mathrm{TI} \cdot \mathrm{PPS} \cdot \mathrm{ABD} \cdot \\
& {\left[191.5+156.8\left(\mathrm{w}_{\mathrm{s}}\right)-18.97\left(\mathrm{w}_{\mathrm{s}}\right)(\overline{\mathrm{V}})-14.41\left(\mathrm{w}_{\mathrm{st}}\right)+1.096\left(\mathrm{w}_{\mathrm{st}}\right)(\overline{\mathrm{v}})\right] }
\end{aligned}
$$

b) Two-way streets

$$
\begin{aligned}
R^{2}= & 0.9445 \\
I D= & 10^{-1} \cdot Q_{1} \cdot \operatorname{PedD} \cdot I \cdot K_{o} \cdot T I \cdot P P S \cdot A B D \cdot \\
& {\left[4.572+3.740\left(w_{s}\right)-0.4502\left(w_{s}\right)(\bar{V})-0.3272\left(w_{s t}\right)+0.02417\left(w_{s t}\right)(\bar{V})\right] }
\end{aligned}
$$

*The value for calculated dose rate to crew is truncated at the regulatory limit of $2 \mathrm{mR} / \mathrm{h}$ in the cab. If a particular shipment produces a dose rate greater than this, it is assumed that shlelding reduces the dose rate to $2 \mathrm{mR} / \mathrm{h}$. This fact must be remembered when using the crew dose simplified equation. 
5. Dose to Persons in Vehicles

a) One-way streets

$R^{2}=0.9862$

$\mathrm{ID}=10^{-3} \cdot \mathrm{Q}_{3} \cdot \mathrm{K}_{\mathrm{o}} \cdot \mathrm{TI} \cdot \mathrm{PPS} \cdot \mathrm{L} \cdot \mathrm{PPV} \cdot$

$\left[402.6-20.76\left(w_{s t}\right)+0.2893\left(w_{s t}\right)^{2}-24.90(\bar{v})+0.7799(\bar{v})\left(w_{s t}\right)\right]$

b) Two-way streets

$\mathrm{R}^{2}=0.9872$

$\mathrm{ID}=10^{-12} \cdot \mathrm{K}_{\mathrm{o}} \cdot \mathrm{TI} \cdot \mathrm{PPS} \cdot \mathrm{PPV} \cdot \mathrm{L} \cdot \mathrm{N} \cdot$

$\left[117.9-6.025\left(w_{s t}\right)+0.0821\left(w_{s t}\right)^{2}-7.717(\bar{v})+0.2459(\bar{v})\left(w_{s t}\right)\right]$

c) Freeways

$$
\begin{aligned}
& \mathrm{R}^{2}=0.9963 \\
& \mathrm{ID}=10^{-12} \cdot \mathrm{K}_{\mathrm{o}} \cdot \mathrm{TI} \cdot \mathrm{PPS} \cdot \mathrm{PPV} \cdot \mathrm{L} \cdot \mathrm{N} \cdot \\
& {\left[17.38-1.244\left(\mathrm{v}_{\mathrm{f}}\right)+0.02665\left(\mathrm{v}_{\mathrm{f}}\right)^{2}\right]}
\end{aligned}
$$

6. Dose to Persons in Buildings

a) One- and two-way streets

$$
\mathrm{R}^{2}=0.9323
$$

$$
\mathrm{ID}=10^{-3} \cdot \frac{\mathrm{K}_{\mathrm{o}} \cdot \mathrm{TI}}{\left(\sqrt{\mathrm{f}_{b}}-\mathrm{f}_{\mathrm{b}}\right)} \cdot \mathrm{PPS} \cdot(\mathrm{PD}+\mathrm{TC}) \cdot \mathrm{L} \cdot
$$

$$
\left[389.8-10.47(\bar{v})-6457(n)-0.07138\left(w_{s t}\right)(n)+\right.
$$

$\left.0.3733(\bar{v})(n)+0.1216(n)^{2}-1590\left(w_{b}\right)+2031\left(w_{b}\right)^{2}+1.922\left(w_{s t}\right)(E)\right]$

b) Freeway

$$
\begin{aligned}
\mathrm{R}^{2}= & 0.9384 \\
\mathrm{ID}= & 10^{-3} \cdot \frac{\mathrm{K}_{0} \cdot T I}{\left(\sqrt{\mathrm{f}_{b}}-\mathrm{f}_{\mathrm{b}}\right)} \cdot \mathrm{PPS} \cdot(\mathrm{PD}+\mathrm{TC}) \cdot \mathrm{L} \cdot \\
& {\left[162.9-5.504(\mathrm{n})+0.3815\left(\mathrm{w}_{\mathrm{st}}\right)(E)+19.60(E)^{2}-599.2\left(\mathrm{w}_{\mathrm{b}}\right)+\right.} \\
& \left.17.77(\mathrm{n})\left(\mathrm{w}_{\mathrm{b}}\right)+665.4\left(\mathrm{w}_{\mathrm{b}}\right)^{2}-202.0\left(\mathrm{w}_{\mathrm{b}}\right)(E)\right]
\end{aligned}
$$


7. Dose to Persons in Air Terminals

$$
\begin{aligned}
\mathrm{R}^{2}= & 0.9555 \\
\mathrm{ID}= & \mathrm{Q}_{1} \cdot \mathrm{K}_{\mathrm{o}} \cdot \mathrm{TI} \cdot \mathrm{PPS} \cdot \Delta \mathrm{T}_{\text {term }} \cdot \mathrm{PD}_{\text {term }} \\
& {\left[7.134-0.04534\left(\mathrm{r}_{4}\right)+0.006316\left(\mathrm{r}_{5}\right)(\mathrm{E})\right] }
\end{aligned}
$$

8. Dose to Persons in Railroad Terminals

$$
\begin{aligned}
\mathrm{R}^{2}= & 0.9760 \\
\mathrm{ID}= & \mathrm{Q}_{1} \cdot \mathrm{K}_{\mathrm{o}} \cdot \mathrm{TI} \cdot \mathrm{PPS} \cdot \Delta \mathrm{T}_{\mathrm{depot}} \cdot \mathrm{PD}_{\mathrm{depot}} \cdot \\
& {\left[7.614-2.954\left(\mathrm{r}_{1}\right)+0.9392\left(\mathrm{r}_{2}\right)\right] }
\end{aligned}
$$

9. Dose to Persons Sharing Transportation Link

$$
\begin{aligned}
\mathrm{R}^{2}= & 0.9981 \\
\mathrm{ID}= & \mathrm{Q}_{3} \cdot \mathrm{K}_{\mathrm{o}} \cdot \mathrm{TI} \cdot \mathrm{PPS} \cdot \mathrm{L} \cdot \mathrm{PPT} \cdot \frac{\mathrm{N}_{\mathrm{T}}}{\mathrm{v}_{\mathrm{T}}^{2}} \cdot \\
& {\left[1.991-0.3729\left(\mathrm{r}_{3}\right)+0.02119\left(\mathrm{r}_{3}\right)^{2}\right] }
\end{aligned}
$$

10. Dose to Persons along Railroad Right-of-Way

$$
\begin{aligned}
\mathrm{R}^{2}= & 0.9741 \\
\mathrm{ID}= & 10^{-3} \cdot \mathrm{Q}_{1} \cdot \mathrm{K}_{\mathrm{o}} \cdot \mathrm{TI} \cdot \mathrm{PPS} \cdot \mathrm{PD}_{\mathrm{rw}} \cdot \frac{\mathrm{L}}{\mathrm{V}_{\mathrm{T}}} \\
& {\left[213.2-15.30\left(\mathrm{r}_{\mathrm{rw}}\right)+32.68(\mathrm{E})\right] }
\end{aligned}
$$

11. Dose to Persons in Wharf Area

$$
\begin{aligned}
\mathrm{R}^{2}= & 0.9750 \\
\mathrm{ID}= & \mathrm{Q}_{1} \cdot \mathrm{K}_{\mathrm{O}} \cdot \mathrm{TI} \cdot \mathrm{PPS} \cdot \Delta \mathrm{T}_{\text {wharf }} \cdot \mathrm{PD}_{\text {wharf }} \cdot \\
& {\left[4.472-0.2896\left(\mathrm{r}_{6}\right)+0.08315\left(\mathrm{r}_{7}\right)-0.0002268\left(\mathrm{r}_{7}\right)^{2}\right] }
\end{aligned}
$$


ADDENDUM 3: ERROR EQUATIONS FOR INCIDENT-FREE TRANSPORT MODEL

1. Dose to Handlers

$$
\operatorname{RE}(\mathrm{ID})=\mathrm{RE}(\mathrm{TI})
$$

2. Dose to Warehousemen

$$
\operatorname{RE}(I D)=\operatorname{RE}(T I)+\operatorname{RE}\left(\Delta \mathrm{T}_{\text {stor }}\right)+\operatorname{RE}\left(\mathrm{PD}_{\text {stor }}\right)-0.28 \mathrm{RE}\left(\mathrm{r}_{8}\right)+0.24 \operatorname{RE}\left(\mathrm{r}_{9}\right)
$$

3. Dose to Crew

$$
R E(I D)=R E(T I)+R E(L)+R E\left(N_{c}\right)-R E(\bar{V})
$$

4. Dose to Pedestrians

a) One-way streets

$$
R E(I D)=R E(T I)+R E(P e d D)+R E(L)+0.84 R E\left(w_{s}\right)-1.15 R E(\bar{V})-0.94 R E\left(w_{s t}\right)
$$

b) Two-way streets

$$
R E(I D)=R E(T I)+R E(\operatorname{PedD})+R E(L)+0.82 R E\left(w_{s}\right)-1.15 R E(\bar{V})-0.88 R E\left(w_{s t}\right)
$$

5. Dose to Persons in Vehicles

a) One-way streets

$$
R E(I D)=R E(T I)+R E(L)-1.89 R E\left(w_{s t}\right)-1.06 R E(\bar{V})
$$

b) Two-way streets

$$
R E(I D)=R E(T I)+R E(L)+R E(N)-1.88 R E\left(w_{s t}\right)-1.12 R E(\bar{V})
$$

c) Freeway

$$
R E(I D)=R E(T I)+R E(L)+R E(N)-1.46 R E\left(V_{f}\right)
$$

6. Dose to Persons in Buildings

a) One- and two-way streets

$$
\begin{aligned}
R E(I D)= & R E(T I)+\operatorname{RE}(P I B)+R E(L)-1.81 R E(\bar{v})-2.06 R E(n)+1.11 R E\left(w_{S t}\right)- \\
& 7.25 R E\left(w_{b}\right)+2.50 R E(E)
\end{aligned}
$$




\section{b) Freeway}

$$
\begin{aligned}
R E(I D)= & R E(T I)+R E(P I B)+R E(L)-0.39 R E(n)+1.16 R E\left(w_{s t}\right)-2.09 R E(E)- \\
& 6.16 R E\left(w_{b}\right)
\end{aligned}
$$

7. Dose to Persons in Air Terminals

$$
\begin{aligned}
\mathrm{RE}(\mathrm{ID})= & \mathrm{RE}(\mathrm{TI})+\mathrm{RE}\left(\Delta \mathrm{T}_{\text {term }}\right)+\mathrm{RE}\left(\mathrm{PD}_{\text {term }}\right)-1.76 \mathrm{RE}\left(\mathrm{r}_{4}\right)+ \\
& 0.49 \mathrm{RE}\left(\mathrm{r}_{5}\right)+0.49 \mathrm{RE}(\mathrm{E})
\end{aligned}
$$

8. Dose to Persons in Railroad Terminals

$$
R E(I D)=R E(T I)+R E\left(\Delta T_{\text {depot }}\right)+R E\left(P D_{\text {depot }}\right)-R E\left(r_{1}\right)+0.93 R E\left(r_{2}\right)
$$

9. Dose to Persons along Transportation Link

$$
R E(I D)=R E(T I)+R E(L)+R E(P P T)+R E\left(N_{T}\right)-2 R E\left(V_{T}\right)+1.38 R E\left(r_{3}\right)
$$

10. Dose to Persons along Railroad Right-of-Way

$$
R E(I D)=R E(T I)+R E\left(P D_{r W}\right)+R E(L)-R E\left(V_{T}\right)-0.26 R E\left(r_{w}\right)+0.17 R E(E)
$$

11. Dose to Persons in Wharf Area

$$
\mathrm{RE}(\mathrm{ID})=\mathrm{RE}(\mathrm{TI})+\mathrm{RE}\left(\mathrm{PD}_{\mathrm{w}}\right)+\mathrm{RE}\left(\Delta \mathrm{T}_{\mathrm{w}}\right)-0.48 \mathrm{RE}\left(\mathrm{r}_{6}\right)+0.50 \mathrm{RE}\left(\mathrm{r}_{7}\right)
$$


ADDENDUM 4:- NOMINAL VALUES OF PARAMETERS AND VARIABLES USED IN THE SENSITIVITY AND ERROR ANALYSIS EQUATIONS, NONDISPERSIBLE MATERIAL

\begin{tabular}{|c|c|c|}
\hline Variable & Definition & Nominal Value \\
\hline PedD & Pedestrian density & $\begin{array}{l}10^{6} \text { persons } / \mathrm{km}^{2} \\
\text { sidewalk }\end{array}$ \\
\hline PD & Population density & $10^{5}$ persons $/ \mathrm{km}^{2}$ \\
\hline $\mathrm{TC}$ & Transient clientele & 50000 persons $/ \mathrm{km}^{2}$ \\
\hline PPV & Number of persons per vehicle & 2 \\
\hline PPS & No. of packages per shipment & 1 \\
\hline Shipments/yr. & & 1000 \\
\hline $\mathrm{TI}$ & Transportation index & 1 \\
\hline$n_{1}$ & Curies per package & $10^{6} \mathrm{Ci}$ \\
\hline$\mu_{\text {air }}$ & Linear attenuation coefficient, air & $0.0081 \mathrm{~m}^{-1}$ \\
\hline$\underset{\mathrm{f}}{\mathrm{Bir}}$ & $\begin{array}{l}\text { Dose buildup factor } \\
\text { Attenuation coefficient for body tissue }\end{array}$ & $\begin{array}{l}0.00197 \mathrm{r}+1 \\
3.1 \mathrm{~m}^{-1}\end{array}$ \\
\hline $\mathrm{k}_{\mathrm{o}}$ & Package shape factor & $257.88 \mathrm{mrem} \cdot \mathrm{m}^{2} / \mathrm{h}$ \\
\hline S & Vehicle separation distance & $80 \mathrm{~m}$ \\
\hline$\ell$ & Vehicle length & $6.4 \mathrm{~m}$ \\
\hline $\mathrm{T}_{\mathrm{a}}$ & Accident delay time & $86400 \mathrm{~s}$ \\
\hline $\mathrm{N}$ & Traffic count & $\begin{array}{l}100 \text { vehicles per } \\
\text { cell }\end{array}$ \\
\hline $\mathrm{Q}_{2}$ & Scaling factor & $10^{-6}$ \\
\hline$Q_{3}$ & Scaling factor & $2.78 \times 10^{-7}$ \\
\hline$Q_{5}$ & Scaling factor & $4.8 \times 10^{-5}$ \\
\hline
\end{tabular}


ADDENDUM 5: SENSITIVITY ANALYSIS EQUATIONS FOR NONDISPERSIBLE MATERIALS ACCIDENTS

1. Dose to Pedestrians; One- and Two-Way Streets

$$
\begin{aligned}
& \mathrm{R}^{2}=0.9469 \\
& \mathrm{ID}=\mathrm{Q}_{2} \cdot \operatorname{PedD} \cdot \mathrm{K}_{1} \cdot \Delta \mathrm{T}_{\mathrm{a}} \cdot\left[1.575-0.07462\left(\mathrm{w}_{\mathrm{st}}\right)+1.598(\mathrm{E})\right]
\end{aligned}
$$

2. Dose to Persons in Vehicles

a) One-way streets

$$
\mathrm{R}^{2}=0.9465
$$$$
\mathrm{ID}=\mathrm{K}_{1} \cdot \Delta \mathrm{T}_{\mathrm{a}} \cdot \mathrm{PPV} \cdot\left[0.3644-0.02491\left(\mathrm{w}_{\mathrm{st}}\right)+0.4862 \times 10^{-3}\left(\mathrm{w}_{\mathrm{st}}\right)^{2}\right]
$$

b) Two-way streets

$$
\begin{aligned}
& \mathrm{R}^{2}=0.9464 \\
& \mathrm{ID}=10^{-3} \cdot \mathrm{K}_{1} \cdot \Delta_{\mathrm{a}} \cdot \mathrm{PPV} \cdot \mathrm{N} \cdot\left[6.375-0.4360\left(\mathrm{w}_{\mathrm{st}}\right)+0.008513\left(\mathrm{w}_{\mathrm{st}}\right)^{2}\right] \\
& \frac{\text { Freeway }}{\mathrm{R}^{2}=0.7712} \\
& \mathrm{ID}=10^{-6} \cdot \mathrm{K}_{1} \cdot \mathrm{PPV} \cdot \Delta \mathrm{T}_{\mathrm{a}} \cdot \mathrm{N} \cdot\left[905.6-0.02149\left(\mathrm{w}_{\mathrm{f}}\right)+0.01034\left(\mathrm{w}_{\mathrm{f}}\right)(\mathrm{E})\right]
\end{aligned}
$$

c) Freeway 
ADDENDUM 6: ERROR EQUATIONS FOR NONDISPERSIBLE MATERIALS ACCIDENTS

1. Dose to Pedestrians; One- and Two-Way Streets

$\operatorname{RE}(I D)=\operatorname{RE}(\operatorname{PedD})+\operatorname{RE}\left(\Delta \mathrm{T}_{\mathrm{a}}\right)+0.85 \mathrm{RE}\left(\mathrm{n}_{1}\right)+0.85 \mathrm{RE}\left(\mathrm{E}_{\mathrm{d}}\right)+0.85 \mathrm{RE}(\mathrm{RF})-$ $0.76 \operatorname{RE}\left(w_{s t}\right)+0.81 R E(E)+0.0001 R E(T I)$

2. Dose to Persons in Vehicles

a) One-way streets

$$
\begin{aligned}
\operatorname{RE}(\mathrm{ID})= & \operatorname{RE}\left(\Delta_{\mathrm{a}}\right)-1.94 \mathrm{RE}\left(\mathrm{w}_{\mathrm{st}}\right)+1.08 \mathrm{RE}\left(\mathrm{n}_{1}\right)+1.08 \mathrm{RE}\left(\mathrm{E}_{\mathrm{d}}\right)+1.08 \mathrm{RE}(\mathrm{RF})+ \\
& 0.0001 \mathrm{RE}(\mathrm{TI})
\end{aligned}
$$

b) Two-way streets

$$
\begin{aligned}
\operatorname{RE}(\mathrm{ID})= & \operatorname{RE}\left(\Delta_{\mathrm{a}}\right)+\operatorname{RE}(\mathrm{N})+1.08 \mathrm{RE}\left(\mathrm{n}_{1}\right)+\operatorname{RE}\left(\mathrm{E}_{\mathrm{d}}\right)+1.08 \mathrm{RE}(\mathrm{RF})-1.95 \mathrm{RE}\left(\mathrm{w}_{\mathrm{st}}\right)+ \\
& 0.0003 \operatorname{RE}(\mathrm{TI})
\end{aligned}
$$

c) Freeway

$$
\begin{aligned}
\operatorname{RE}(I D)= & R E\left(\Delta T_{a}\right)+R E(N)+R E\left(n_{1}\right)+R E\left(E_{d}\right)+1.34 R E(R F)+0.0002 R E(T I)- \\
& 0.0009 R E\left(w_{f}\right)+0.0008 R E(E)
\end{aligned}
$$


ADDENDUM 7: NOMINAL VALUES OF PARAMETERS AND VARIABLES USED IN THE SENSITIVITY ANALYSIS OF ACCIDENTS INVOLVING DISPERSIBLE MATERIALS

\begin{tabular}{|c|c|c|}
\hline Variable & Definition & Nominal Value \\
\hline PedD & Pedestrian density & $10^{5}$ persons $/ \mathrm{km}^{2}$ \\
\hline $\mathrm{N}$ & Traffic count & 1000 vehicles/cell \\
\hline $\mathrm{PD}$ & Population density & 50000 persons $/ \mathrm{km}^{2}$ \\
\hline $\mathrm{TC}$ & Transient clientele & 50000 persons $/ \mathrm{km}^{2}$ \\
\hline $\mathrm{f}_{\text {st }}$ & Fraction of cell area in streets & 0.3 \\
\hline $\mathrm{RF}$ & Release fraction & 1.0 \\
\hline AER & Fraction of material aerosolized & 1.0 \\
\hline $\mathrm{h}$ & Height per floor & $3 \mathrm{~m}$ \\
\hline BR & Breathing rate & $1.2 \mathrm{~m}^{3} / \mathrm{h}$ \\
\hline TI & Transportation index & 1.0 \\
\hline RDF & Resuspension dose factor & 1.6 \\
\hline PPV & Average number of persons/vehicle & 2.33 \\
\hline $\mathrm{CDF}$ & Cloudshine dose factor & $1.6 \times 10^{10}$ \\
\hline$t_{1 / 2}$ & Isotope ha1f life & $1910 \mathrm{~d}$ \\
\hline$w_{b}$ & Building wall thickness & $0.3 \mathrm{~m}$ \\
\hline
\end{tabular}


ADDENDUM 8: SENSITIVITY ANALYSIS DATA

Table Ad-1

Data for Sensitivity Analysis, Incident-Free Model

DOSE TO HAREMOUSEMEN

$\begin{array}{rrrc}\text { WSPMIN } & \text { USPMAX } & E & \begin{array}{c}\text { DOSE } \\ \text { (PERSON-REM) }\end{array} \\ 2.2 & & & .27 \\ . .8 & 141 . & 1.4 & .34 \\ 2.2 & 141 . & 1.4 & .21 \\ .8 & 59 . & 1.4 & .28 \\ 2.2 & 59 . & 1.4 & .26 \\ .8 & 141 . & .6 & .34 \\ 2.2 & 141 . & .6 & .21 \\ . .8 & 59 . & .6 & .28 \\ 1.5 & 59 . & .6 & .34 \\ .7 & 100 . & 1.0 & .24 \\ 2.3 & 100 . & 1.0 & .23 \\ 1.5 & 100 . & 1.0 & .30 \\ 1.5 & 50 . & 1.0 & .27 \\ 1.5 & 150 . & 1.0 & .27 \\ 1.5 & 100 . & .5 & \end{array}$


Data for Sensitivity Analysis, Incident-Free Model

DOSE TO PEDESTRIANS

ONE-HAY STREETS

$\begin{array}{llll}\text { US } & \text { HST } & \text { BVEL } & \text { VELP } \\ & & & \\ 2.4 & 13.5 & 4.17 & 1.15 \\ 3.6 & 26.5 & 4.17 & 1.15 \\ 3.6 & 13.5 & 7.23 & 1.15 \\ 2.4 & 26.5 & 7.23 & 1.15 \\ 3.6 & 13.5 & 4.17 & 1.35 \\ 2.4 & 26.5 & 4.17 & 1.35 \\ 2.4 & 13.5 & 7.23 & 1.35 \\ 3.6 & 26.5 & 7.23 & 1.35 \\ 3.6 & 13.5 & 4.17 & 1.15 \\ 2.4 & 26.5 & 4.17 & 1.15 \\ 2.4 & 13.5 & 7.23 & 1.15 \\ 3.6 & 26.5 & 7.23 & 1.15 \\ 2.4 & 13.5 & 4.17 & 1.35 \\ 3.6 & 26.5 & 4.17 & 1.35 \\ 3.6 & 13.5 & 7.23 & 1.35 \\ 2.4 & 26.5 & 7.23 & 1.35 \\ 3.0 & 20.0 & 5.70 & 1.25 \\ 2.0 & 20.9 & 5.70 & 1.25 \\ 4.0 & 20.0 & 5.70 & 1.25 \\ 3.0 & 10.0 & 5.70 & 1.25 \\ 3.0 & 30.0 & 5.70 & 1.25 \\ 3.0 & 20.0 & 3.34 & 1.25 \\ 3.0 & 20.0 & 8.06 & 1.25 \\ 3.0 & 20.0 & 5.70 & 1.10 \\ 3.0 & 20.0 & 5.70 & 1.40 \\ 3.0 & 20.0 & 5.70 & 1.25 \\ 3.0 & 20.0 & 5.70 & 1.25 \\ & & & \end{array}$

$E$

.7

.7

.7

. .7

.7

.7

1.5

1.5

1.5

1. 5

1.5

1.5

1.5

1.5

1. 0

1.0

1.0

1.0

$1 \cdot 0$

1. 0

1.0

1. 0

1.0

$1 \cdot 5$
DOSE

(PERSON-REM)

.018

.013

.013

.005

.025

.010

.010

.008

.025

.010

.010

.008

.018

.014

.014

.005

.011

.008

.014

.020

.007

.020

.007

.011

.011

.010 
Table Ad-3

Data for Sensitivity Analysis, Incident-Free Model

DOSE TO PEDESTRIANS

THO- MAY STREETS

US

\section{UST}

$$
\text { BVEL }
$$

VELP

$2.4 \quad 13.5$

3.6

$3 \cdot 6$

$2 \cdot 4$

3.6

2.4

2.4

3.6

2.4

2.4

3.6

2.4

3.6

3.6

$2 \cdot 4$

3.0
2.0

4.0

3.0

$3 \cdot 0$

3.0

3.0

$3 \cdot 0$

3.0

$3 \cdot 0$

$\begin{array}{ll}4.17 & 1.15 \\ 4.17 & 1.15 \\ 7.23 & 1.15 \\ 7.23 & 1.15 \\ 4.17 & 1.35 \\ 4.17 & 1.35 \\ 7.23 & 1.35 \\ 7.23 & 1.35 \\ 4.17 & 1.15 \\ 4.17 & 1.15 \\ 7.23 & 1.15 \\ 7.23 & 1.15 \\ 4.17 & 1.35 \\ 4.17 & 1.35 \\ 7.23 & 1.35 \\ 7.23 & 1.35 \\ 5.70 & 1.25 \\ 5.70 & 1.25 \\ 5.70 & 1.25 \\ 5.70 & 1.25 \\ 5.70 & 1.25 \\ 3.34 & 1.25 \\ 8.06 & 1.25 \\ 5.70 & 1.10 \\ 5.70 & 1.40 \\ 5.70 & 1.25 \\ 5.70 & 1.25\end{array}$

E

- 7

.7

.7

. 7

.7
.7

.7

.7

1. 5

1.5

1.5

1.5

1.5

1. 5

1.5

1.5

1.0

1.0

1. 0

1. 0

1. 0

1.0

1.0

1.0

1. 0

1.5
1.5
DOSE

(PERSON-REM)

.22

17

.16

.07

.31

.13

.12

.09

.30

.12

.12

.09

.23

.18

.17

.07

.13

.09

.17

.23

.09

.25

.09

.13

.14

.13 
Data for Sensitivity Analysis, Incident-Free Model

DOSE TO PERSONS IN VEHICLES

\section{ONE-HAY STREETS}

$\begin{array}{ll}\text { US } & \text { UST } \\ & \\ 2.4 & 13.5 \\ 3.6 & 26.5 \\ 3.6 & 13.5 \\ 2.4 & 26.5 \\ 3.6 & 13.5 \\ 2.4 & 26.5 \\ 2.4 & 13.5 \\ 3.6 & 26.5 \\ 3.6 & 13.5 \\ 2.4 & 26.5 \\ 2.4 & 13.5 \\ 3.6 & 26.5 \\ 2.4 & 13.5 \\ 3.6 & 26.5 \\ 3.6 & 13.5 \\ 2.4 & 26.5 \\ 3.0 & 20.0 \\ 2.0 & 20.0 \\ 4.0 & 20.0 \\ 3.0 & 10.0 \\ 3.0 & 30.0 \\ 3.0 & 20.0 \\ 3.0 & 20.0 \\ 3.0 & 20.0 \\ 3.0 & 20.0 \\ 3.0 & 20.0 \\ 3.0 & 20.0\end{array}$

$\begin{array}{ll}\text { BVEL } & \text { VELP } \\ & \\ 4.17 & 1.15 \\ 4.17 & 1.15 \\ 7.23 & 1.15 \\ 7.23 & 1.15 \\ 4.17 & 1.35 \\ 4.17 & 1.35 \\ 7.23 & 1.35 \\ 7.23 & 1.35 \\ 4.17 & 1.15 \\ 4.17 & 1.15 \\ 7.23 & 1.15 \\ 7.23 & 1.15 \\ 4.17 & 1.35 \\ 4.17 & 1.35 \\ 7.23 & 1.35 \\ 7.23 & 1.35 \\ 5.70 & 1.25 \\ 5.70 & 1.25 \\ 5.70 & 1.25 \\ 5.70 & 1.25 \\ 5.70 & 1.25 \\ 3.34 & 1.25 \\ 8.06 & 1.25 \\ 5.70 & 1.10 \\ 5.70 & 1.40 \\ 5.70 & 1.25 \\ 5.70 & 1.25\end{array}$

$E$
.7
.7
.7
.7
.7
.7
.7
1.5
1.5
1.5
1.5
1.5
1.5
1.5
1.5
1.0
1.0
1.0
1.0
1.0
1.0
1.0
1.0
1.0
.05
1.5

DOSE

(PERSON-REM)

.064

.023

.043

.016

.070

.023

.040

.015

.070

.023

.040

.015

.064

.023

.043

.016

.028

.028

.027

.076

.014

.046

.021

.028

.028

.028

.028 
Table Ad-5

Data for Sensitivity Analysis, Incident-Free Model
DOSE TO PERSONS IN VEHICLES

\section{THO- WAY STREETS}
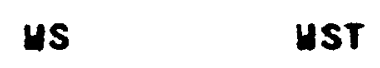

BVEL

VELP

13.5

3.4

3.6

2.4

3.6

2.4

2.4

3.6

3.6

2.4

2.4

3.6

2.4

3.6

$2 \cdot 4$

3.0

$2 \cdot 0$

4.0

$3 \cdot 0$

3.0

3.0

3.0

3.0

3.0

3.0

4.17
4.17
7.23
7.23
4.17
4.17
7.23
7.23
4.17
4.17
7.23
7.23
4.17
4.17
7.23
7.23
5.70
5.70
5.70
5.70
5.70
3.34
8.06
5.70
5.70
5.70
5.70

1.15

1.15

1.15

1.15

1.35

1.35

1.35

1.35

1.15

1.15

1.15

1.15

1.35

1.35

1.35

1.35

1.25

1.25

1.25

1.25

1.25

1.25

1.25

1.10

1.40

1.25

1.25
E

- 7

.7

.7

.7
.7

.7
.7

.7
.7

1.5

1.5

1.5

1.5

1.5

1.5

1.5

1.5

1. 0

1. 0

1.0

1. 0

1. 0

1. 0

1. 0

1.0
1.0

.5
1.5
DOSE

\section{(PERSON-REM)}

.067

.024

.043

.016

.073

.024

.040

.016

.073

.024

.040

.016

.067

.024

.043

.016

.028

.029

.028

.076

.015

.046

.021

.028

.028

.028 
Table Ad-6

Data for Sensitivity Analysis, Incident-Free Model

\section{DOSE TO PERSONS IN VEHICLES}

\section{FREEHAYS}

\section{FREVEL}

23.0

10.4

23.0

10.4

23.0

10.4

23.0

10.4

16.7

9.0

24.4

16.7

16.7

16.7

16.7
FREUDT

$$
\begin{array}{r}
94.7 \\
94.7 \\
45.3 \\
45.3 \\
94.7 \\
94.7 \\
45.3 \\
45.3 \\
70.0 \\
70.0 \\
70.0 \\
40.0 \\
100.0 \\
70.0 \\
70.0
\end{array}
$$

$E$

1.4

1.4

1.4

.6

.6

.6

1.0

1.0

1.0

1.0

1.3

.5

$1 \cdot 5$
DOSE

(PERSON-REA)

.006

.015

.006

.015

.006

.015

.006

.015

.009

.018

.0 .06

.009

.009

.009

.009 
Table Ad-7

Data for Sensitivity Analysis, Incident-Free Model

DOSE TO PERSONS IN BUILDINGS

OME - AND THO-WAY STREETS

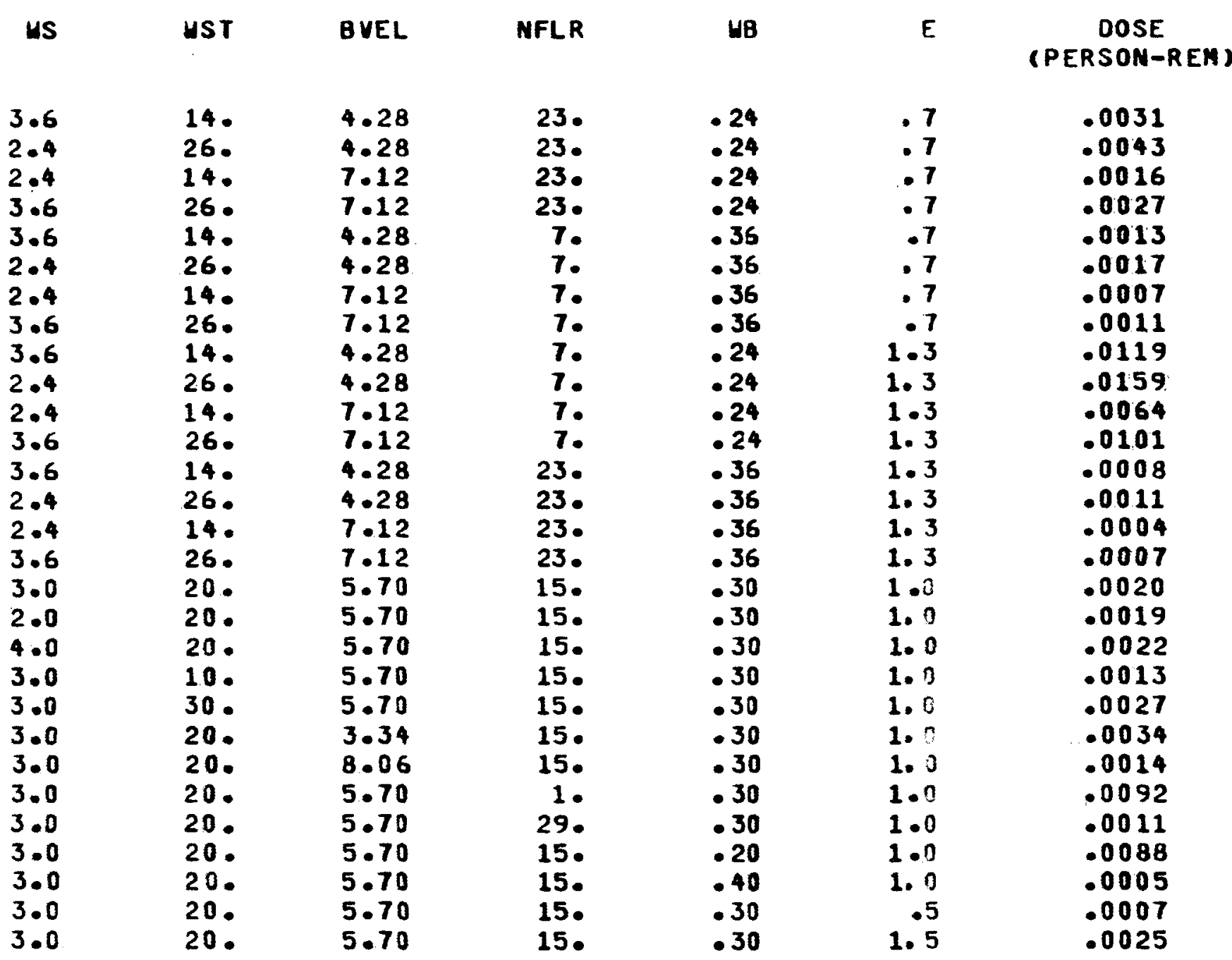


DOSE TO PERSONS IN BUILDINGS

\section{FREEUAYS}

\begin{tabular}{|c|c|c|c|c|c|c|}
\hline WS & UST & BVEL & NFLR & UB & $E$ & $\begin{array}{c}\text { DOSE } \\
\text { (PERSON-REM) }\end{array}$ \\
\hline $\begin{array}{l}3.6 \\
2.4 \\
2.4 \\
3.6 \\
3.6 \\
2.4 \\
2.4 \\
3.6 \\
3.6 \\
2.4 \\
2.4 \\
3.6 \\
3.6 \\
2.4 \\
2.4 \\
3.6 \\
3.0 \\
2.0 \\
4.0 \\
3.0 \\
3.0 \\
3.0 \\
3.0 \\
3.0 \\
3.0 \\
3.0 \\
3.0 \\
3.0 \\
3.0\end{array}$ & 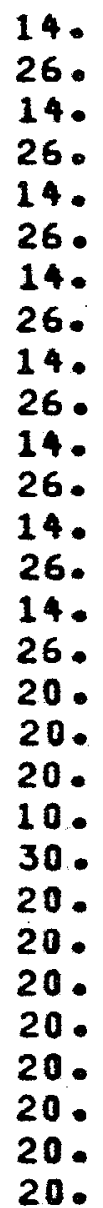 & $\begin{array}{l}12.1 \\
12.1 \\
21.3 \\
21.3 \\
12.1 \\
12.1 \\
21.3 \\
21.3 \\
12.1 \\
12.1 \\
21.3 \\
21.3 \\
12.1 \\
12.1 \\
21.3 \\
21.3 \\
16.7 \\
16.7 \\
16.7 \\
16.7 \\
16.7 \\
9.0 \\
24.4 \\
16.7 \\
16.7 \\
16.7 \\
16.7 \\
16.7 \\
16.7\end{array}$ & 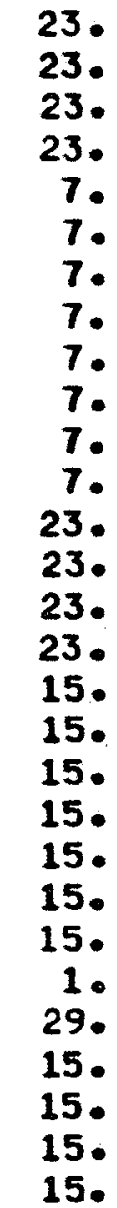 & $\begin{array}{l}.24 \\
.24 \\
.24 \\
.24 \\
.36 \\
.36 \\
.36 \\
.36 \\
.34 \\
.24 \\
.24 \\
.24 \\
.36 \\
.36 \\
.36 \\
.36 \\
.30 \\
.30 \\
.30 \\
.30 \\
.30 \\
.30 \\
.30 \\
.30 \\
.30 \\
.30 \\
.20 \\
.40 \\
.30 \\
.30\end{array}$ & $\begin{array}{l}.7 \\
.7 \\
.7 \\
.7 \\
.7 \\
.7 \\
.7 \\
.7 \\
1.3 \\
1.3 \\
1.3 \\
1.3 \\
1.3 \\
1.3 \\
1.3 \\
1.3 \\
1.0 \\
1.0 \\
1.0 \\
1.0 \\
1.0 \\
1.0 \\
1.0 \\
1.0 \\
1.0 \\
1.0 \\
1.0 \\
.5 \\
1.5\end{array}$ & $\begin{array}{l}.00101 \\
.00146 \\
.00088 \\
.00156 \\
.00028 \\
.00043 \\
.00025 \\
.00046 \\
.00284 \\
.00427 \\
.00253 \\
.00458 \\
.00026 \\
.00037 \\
.00023 \\
.00040 \\
.00088 \\
.00082 \\
.00095 \\
.00055 \\
.00122 \\
.00088 \\
.00088 \\
.00165 \\
.00049 \\
.00397 \\
.00020 \\
.00303 \\
.00113\end{array}$ \\
\hline
\end{tabular}


Table Ad-9

Data for Sensitivity Analysis, Incident-Free Model

DOSE TO PERSONS IN AIR TERMINALS

\begin{tabular}{|c|c|c|c|}
\hline AIRMIN & AIRMAX & $E$ & $\begin{array}{c}\text { DOSE } \\
\text { (PERSON-REM) }\end{array}$ \\
\hline $\begin{array}{r}155^{\circ} \\
89^{\circ} \\
155^{\circ} \\
89^{\circ} \\
155^{\circ} \\
89^{\circ} \\
155^{\circ} \\
89^{\circ} \\
122^{\circ} \\
82^{\circ} \\
162^{\circ} \\
122^{\circ} \\
122^{\circ} \\
122^{\circ} \\
122^{\circ}\end{array}$ & 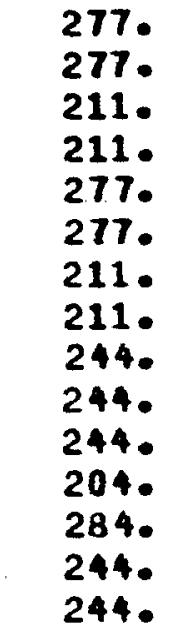 & $\begin{array}{r}1.4 \\
1.4 \\
1.4 \\
1.4 \\
.6 \\
.6 \\
.6 \\
.6 \\
1.0 \\
1.0 \\
1.0 \\
1.0 \\
1.0 \\
. .5 \\
1.5\end{array}$ & $\begin{array}{l}.025 \\
.058 \\
.015 \\
.046 \\
.016 \\
.040 \\
.010 \\
.037 \\
.030 \\
.052 \\
.016 \\
.024 \\
.034 \\
.021 \\
.034\end{array}$ \\
\hline
\end{tabular}


Table Ad-10

Data for Sensitivity Analysis, Incident-Free Mode1

DOSE TO PERSONS IN RAILROAD TERMINAL;

$\begin{array}{rrrc}\text { DEPHIN } & \text { DEPMAX } & E & \begin{array}{c}\text { DOSE } \\ \text { (PERSON-REM) }\end{array} \\ 1.2 & & & .0088 \\ 3.6 & 4.5 & .6 & .0015 \\ 1.2 & 4.5 & .6 & .0137 \\ 3.6 & 9.5 & .6 & .0064 \\ 1.2 & 9.5 & .6 & .0088 \\ 3.6 & 4.5 & 1.4 & .0015 \\ 1.2 & 4.5 & 1.4 & .0137 \\ 3.6 & 9.5 & 1.4 & .0064 \\ 2.4 & 9.5 & 1.4 & .0073 \\ 1.0 & 7.0 & 1.0 & .0131 \\ 3.8 & 7.0 & 1.0 & .0040 \\ 2.4 & 7.0 & 1.0 & .0034 \\ 2.4 & 4.0 & 1.0 & .0095 \\ 2.4 & 10.0 & 1.0 & .0070 \\ 2.4 & 7.0 & .5 & .0070\end{array}$




\section{Table Ad-11}

Data for Sensitivity Analysis, Incident-Free Mode1

\section{DOSE TO PERSONS SHARING THE TRANSPORT - INK}

$\begin{array}{ccc}\text { DBPTRN } & \text { E } & \begin{array}{c}\text { DOSE } \\ \text { (PES SON-REM) }\end{array} \\ 3 . & & .067 \\ 3 . & .5 & .070 \\ 3 . & 1.0 & .070 \\ 6 . & 1.5 & .034 \\ 6 . & .5 & .034 \\ 6 . & 1.0 & .034 \\ 9 . & 1.5 & .022 \\ 9 . & .5 & .023 \\ 9 . & 1.0 & .023\end{array}$


Table Ad-12

Data for Sensitivity Analysis, Incident-Free Model

DOSE TO PERSONS ALONG THE RIGHT-OF-HAY

RRTHAY

2
2
2
3
3
3
5
5
5

2 .

$2 \cdot$

$2 \cdot$

3.

3.

5.

5.
E

.5

1.0

1.5

.5

$1 \cdot 0$

1.5

.5

1.0

1.5
DOSE

(PER SON-REM)

.17

.19

.20

.15

.17

.18

.13

.15

.16 
Data for Sensitivity Analysis, Incident-Free Model

\section{DOSE TO PERSONS IN THE DOCK AREA}

\begin{tabular}{|c|c|c|c|}
\hline DOCMIN & DOCMAX & $E$ & $\begin{array}{c}\text { DOSE } \\
\text { (PERSON-REM) }\end{array}$ \\
\hline $\begin{array}{r}6.3 \\
18.1 \\
6.3 \\
18.1 \\
6.3 \\
18.1 \\
6.3 \\
18.1 \\
12.2 \\
5.0 \\
19.4 \\
12.2 \\
12.2 \\
12.2 \\
12.2\end{array}$ & 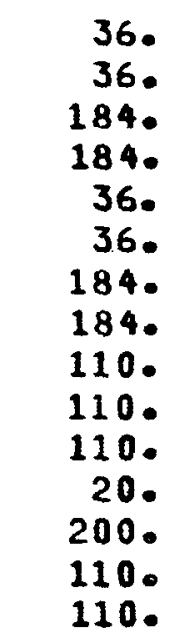 & $\begin{array}{r}.6 \\
.6 \\
.6 \\
.6 \\
1.4 \\
1.4 \\
1.4 \\
1.4 \\
1.0 \\
1.0 \\
1.0 \\
1.0 \\
1.0 \\
.05 \\
1.5\end{array}$ & $\begin{array}{l}.058 \\
.023 \\
.104 \\
.070 \\
.061 \\
.024 \\
.113 \\
.076 \\
.073 \\
.104 \\
.058 \\
.017 \\
.088 \\
.070 \\
.076\end{array}$ \\
\hline
\end{tabular}


Data for Sensitivity Analysis, Accident Model, Nondispersible Material

DOSE TO PEDESTRIANS

\section{ONE- AND THO- GAY STREETS}

\section{US}

3.7

$2 \cdot 3$

3.7

3.7

2.3

2.3

3.7

3.0

2.0

4.0

3.0

3.0

3.0

3.0

3.0

3.0
UST

27.4

ED

12.6

27.4

12.6

27.4

12.6

27.4

12.6

20.0

20.0

$20 . ?$

10.0

30.0

20.3

20.0

20.0

20.3
2. 17

2.17

.33

.33

2.17

$2 \cdot 17$

.33

.33

1.25

1.25

1.25

1.25

1.25

0.00

2. 50

1.25

1.25

\section{E}

1.4
1.4

1.4

1.4

.6

.6

.6

.6

1.0

1.0

1.0

1.0

1.0

1.0

1.0

.5

1.5
DOSE

\section{(PERSON-REM)}

\section{0}

207000.0

15400.0

46600.0

147000.0

202000.0

14600.0

45400.0

98300.0

68400.0

126000.0

1790.00 .0

66800.0

12.2

197000.0

94500.0

9960.0 .0 
Table Ad-15

Data for Sensitivity Analysis, Accident Model, Nondispersible Material

DOSE TO PERSONS IN VEHICLES

THO-MAY STREETS

\begin{tabular}{|c|c|c|c|}
\hline US & UST & $E$ & $\begin{array}{c}\text { DOSE } \\
\text { CPERSON-REM }\end{array}$ \\
\hline $\begin{array}{l}3.8 \\
2.2 \\
3.8 \\
2.2 \\
3.8 \\
2.2 \\
3.8 \\
2.2 \\
3.0 \\
2.0 \\
4.0 \\
3.0 \\
3.0 \\
3.0 \\
3.0\end{array}$ & 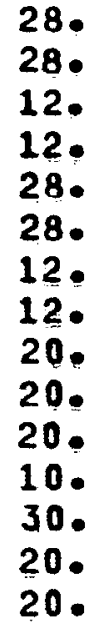 & $\begin{array}{l}1.4 \\
1.4 \\
1.4 \\
1.4 \\
.6 \\
.6 \\
.6 \\
.6 \\
1.0 \\
1.0 \\
1.0 \\
1.0 \\
1.0 \\
. .5 \\
1.5\end{array}$ & $\begin{array}{r}9790^{\circ} \\
9780^{\circ} \\
25000^{\circ} \\
25000^{\circ} \\
9790^{\circ} \\
9780^{\circ} \\
25000^{\circ} \\
25000^{\circ} \\
9770^{\circ} \\
9760^{\circ} \\
9770^{\circ} \\
25000^{\circ} \\
5970^{\circ} \\
9760^{\circ}\end{array}$ \\
\hline
\end{tabular}


Table Ad-16

Data for Sensitivity Analysis, Accident Model, Nondispersible Material

\section{DOSE TO PERSONS IN VEHICLES}

\section{ONE- MAY STREETS}

HS
3.8
2.2
3.8
2.2
3.8
2.2
3.8
2.2
3.0
2.0
4.0
3.0
3.0
3.0
3.0

WST

28.

28.

12.

12.

28.

28.

12.

12.

20.

20.

20.

10.

30 .

20.

20.
E

1.4

1.4

1.4

1.4

.6

.6

.6

.6

1.0

1.0

$1 \cdot n$

1.0

.5

1.5
DOSE

\section{(PERSON-REM)}

5600.

5600 .

14300 .

14300 .

5600 .

5600 .

14300 .

14300 .

5590 .

5590 .

5600 .

14300 .

3420 .

5590 .

5600 . 
Table Ad-17

Data for Sensitivity Analysis, Accldent Model, Nondispersible Material

\section{DOSE TO PERSONS IN VEHICLES}

\section{FREEMAYS}

MF

$$
\begin{aligned}
& 40 . \\
& 40 . \\
& 40 . \\
& 70 . \\
& 70 . \\
& 70 . \\
& 100 . \\
& 100 \\
& 100
\end{aligned}
$$

$\mathbf{E}$

$$
\begin{array}{r}
.5 \\
1.0 \\
1.5 \\
.5 \\
1.0 \\
1.5 \\
.5 \\
1.0 \\
1.5
\end{array}
$$

DOSE (PER SON-REM)

$$
\begin{aligned}
& 9020 . \\
& 9020 \\
& 9020 \\
& 7010 \\
& 9020 \\
& 9020 \\
& 9010 . \\
& 9010 . \\
& 9020 .
\end{aligned}
$$


Data for Sensitivity Analysis, Accident Model, Nondispersible Material

DOSE TO PERSONS IN BUILDINGS

ONE- AND THO-HAY STREETS

\begin{tabular}{|c|c|c|c|c|c|c|}
\hline us & UST & NFLR & 48 & ED & $E$ & $\begin{array}{c}\text { DOSE } \\
\text { (PERSON-REH) }\end{array}$ \\
\hline $\begin{array}{l}3.6 \\
2.4 \\
2.4 \\
3.6 \\
3.6 \\
2.4 \\
2.4 \\
3.6 \\
3.6 \\
2.4 \\
2.4 \\
3.6 \\
3.6 \\
2.4 \\
2.4 \\
3.6 \\
3.0 \\
2.0 \\
4.0 \\
3.0 \\
3.0 \\
3.0 \\
3.0 \\
3.0 \\
3.0 \\
3.0 \\
3.0 \\
3.0 \\
3.0\end{array}$ & 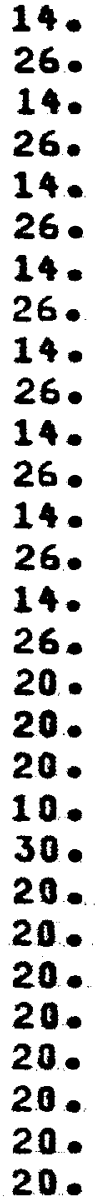 & 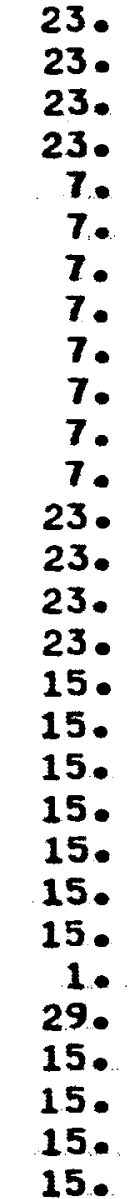 & $\begin{array}{l}.24 \\
.24 \\
.24 \\
.24 \\
.36 \\
.36 \\
.36 \\
.36 \\
.24 \\
.24 \\
.24 \\
.24 \\
.36 \\
.36 \\
.36 \\
.36 \\
.30 \\
.30 \\
.30 \\
.30 \\
.30 \\
.30 \\
.30 \\
.30 \\
.30 \\
.20 \\
.40 \\
.30 \\
.30\end{array}$ & $\begin{array}{l}.50 \\
.50 \\
2.00 \\
2.00 \\
.50 \\
.50 \\
2.00 \\
2.00 \\
.50 \\
.50 \\
2.00 \\
2.00 \\
.50 \\
.50 \\
2.00 \\
2.00 \\
1.25 \\
1.25 \\
1.25 \\
1.25 \\
1.25 \\
0.00 \\
2.50 \\
1.25 \\
1.25 \\
1.25 \\
1.25 \\
1.25 \\
1.25\end{array}$ & $\begin{array}{l}.7 \\
.7 \\
.7 \\
: 7 \\
.7 \\
.7 \\
.7 \\
1.3 \\
1.3 \\
1.3 \\
1.3 \\
1.3 \\
1.3 \\
1.3 \\
1.3 \\
1.0 \\
1.0 \\
1.0 \\
1.0 \\
1.0 \\
1.0 \\
1.0 \\
1.0 \\
1.0 \\
1.0 \\
1.0 \\
.5 \\
1.5\end{array}$ & $\begin{array}{r}.1970000 \\
.4840000 \\
.5920000 \\
2.3500000 \\
.0451000 \\
.0957000 \\
.1410000 \\
.4430000 \\
.2880000 \\
.6250000 \\
.8970000 \\
2.9100000 \\
.0400000 \\
.0974000 \\
.1210000 \\
.4720000 \\
.4690000 \\
.3960000 \\
.5470000 \\
.1660000 \\
.9160000 \\
.0000581 \\
.9370000 \\
.5470000 \\
.2720000 \\
2.0400000 \\
.1070000 \\
.2190000 \\
.4660000\end{array}$ \\
\hline
\end{tabular}


Table Ad-19

Data for Sensitivity Analysis, Accident Model, Nondispersible Material

DOSE TO PERSONS IN BUILDINGS

\section{FREEHAYS}

$\begin{array}{ccccc}\text { UF } & \text { MB } & \text { NFLR } & E & \begin{array}{c}\text { DOSE } \\ \text { (PERSON-REM) }\end{array} \\ 92 . & & & & .351 \\ 48 . & .37 & 25 . & 1.4 & .984 \\ 48 . & .23 & 25 . & 1.4 & .226 \\ 92 . & .37 & 5 . & 1.4 & 2.400 \\ 92 . & .23 & 5 . & 1.4 & .140 \\ 48 . & .37 & 25 . & .6 & .938 \\ 48 . & .23 & 25 . & .6 & .109 \\ 92 . & .37 & 5 . & .6 & 2.510 \\ 70 . & .23 & 5 . & .6 & .726 \\ 40 . & .30 & 15 . & 1.0 & .415 \\ 100 . & .30 & 15 . & 1.0 & .961 \\ 70 . & .30 & 15 . & 1.0 & .190 \\ 70 . & .20 & 15 . & 1.0 & .165 \\ 70 . & .40 & 15 . & 1.0 & .801 \\ 70 . & .30 & 1 . & 1.0 & .570 \\ 70 . & .30 & 29 . & .5 & .328 \\ 70 . & .30 & 15 . & 1.5 & .745\end{array}$


Data for Sensitivity Analysis, Accident Model, Dispersible Material

AIR CONDITIONING

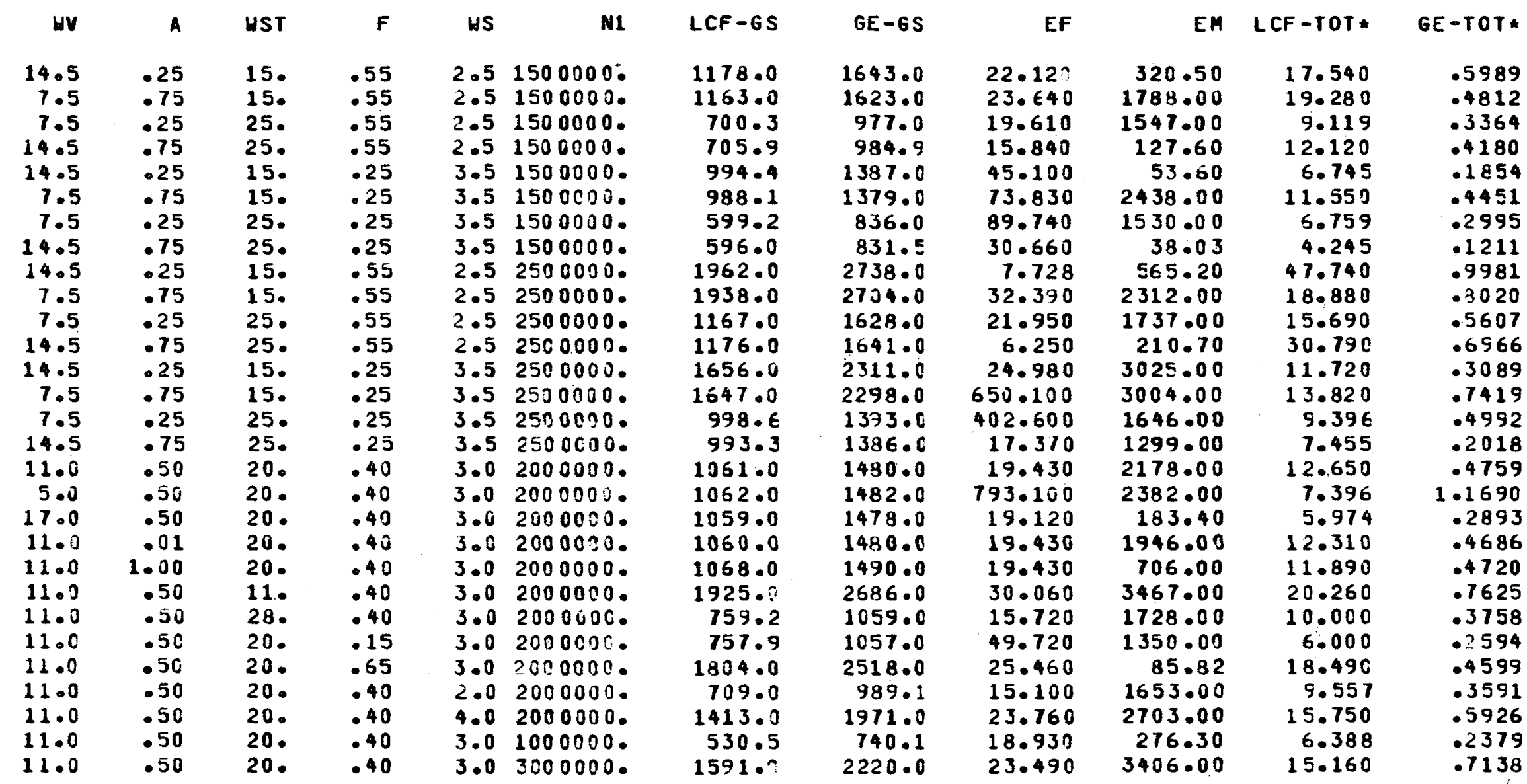


Table Ad-21

Data for Sensitivity Analysis, Accident Mode1, Dispersible Material

CONTINUOUS INTAKE

\begin{tabular}{|c|c|c|c|c|c|c|c|c|c|c|c|}
\hline UV & A & HST & $F$ & US & N1 & LCF-GS & $G E-G S$ & $E F$ & $E M$ & LCF -TOT * & GE-TOT* \\
\hline $\begin{array}{r}14.5 \\
7.5\end{array}$ & $\begin{array}{l}.25 \\
.75\end{array}$ & $\begin{array}{l}15 . \\
15 .\end{array}$ & $\begin{array}{l}.55 \\
.55\end{array}$ & $\begin{array}{l}2.5 \\
2.5\end{array}$ & $\begin{array}{l}1500000 . \\
1500000 .\end{array}$ & $\begin{array}{l}1853 . \\
1822 .\end{array}$ & $\begin{array}{l}2 \vdots 85 . \\
2543 .\end{array}$ & $\begin{array}{l}64.68 \\
82.25\end{array}$ & $\begin{array}{r}311.5 \\
4549.0\end{array}$ & $\begin{array}{l}27.240 \\
33.995\end{array}$ & $\begin{array}{l}1.3160 \\
1.0520\end{array}$ \\
\hline 7.5 & .25 & 25. & .55 & 2.5 & 1500000 & 1351. & $\begin{array}{l}25830 \\
1899 .\end{array}$ & 80.74 & 4033.0 & 23.590 & $\begin{array}{r}.0083 \\
.9083\end{array}$ \\
\hline 14.5 & .75 & 25. & .55 & 2.5 & 1500000 . & 1381 . & 1926. & 58.41 & 108.6 & 21.680 & 1.1350 \\
\hline 14.5 & .25 & 15. & .25 & 3.5 & 1500000. & $245 \%$. & 3430 & 108.40 & 116.3 & 12.220 & .3451 \\
\hline 7.5 & .75 & 15. & .25 & 3.5 & 1500000. & 2451 . & 3420 . & 114.80 & 5807.0 & 19.720 & .8366 \\
\hline 7.5 & .25 & 25. & .25 & 3.5 & 1500030. & 2068 . & 2885 . & 149.80 & 4666.0 & 14.950 & .6892 \\
\hline 14.5 & .75 & 25. & .25 & 3.5 & 1500000. & 2061 . & 2875. & 94.00 & $10 \circlearrowleft .7$ & 8.609 & .2767 \\
\hline 14.5 & .25 & 15. & .55 & 2.5 & 2500000 & 3088 . & 4308 . & 43.56 & 867.2 & 78.220 & 2.1930 \\
\hline 7.5 & .75 & 15. & .55 & 2.5 & 2500000 & 3037. & 4238. & 62.53 & 5465.0 & 58.160 & 1.7530 \\
\hline 7.5 & .25 & 25. & .55 & 2.5 & 2500000. & 2268 . & 3265 . & 55.25 & 5665.0 & 42.250 & 1.5140 \\
\hline 24.5 & .75 & 25. & .55 & 2.5 & 2500000. & 2301. & 3211. & $42 \cdot c 8$ & 186.2 & 61.090 & 1.8910 \\
\hline 14.5 & .25 & 15. & .25 & 3.5 & 2500000 . & 4097 & 5716. & 57.39 & 4559.0 & $21.60 \mathrm{C}$ & .5752 \\
\hline 7.5 & .75 & 15. & .25 & 3.5 & 2500000. & 4085 & 5700. & 850.10 & 6233.0 & 30.770 & 1.3940 \\
\hline 7.5 & .25 & 25. & .25 & 3.5 & 2500000. & 3446. & 4808. & 834.60 & $4488 \cdot 0$ & 27.420 & 1.1490 \\
\hline 14.5 & .75 & 25. & .25 & 3.5 & 2500000. & $343 \equiv$ & 4772 & 49.78 & 1761.0 & 16.920 & .4611 \\
\hline 11.0 & .50 & 20. & .40 & 3.0 & 2000000 . & $229=$ & 3198. & 53.92 & 2580.0 & 26.630 & 1.0830 \\
\hline 5.0 & .50 & 20. & .40 & 3.0 & 2000000. & 2297. & 3205 . & 1446.00 & 5753.0 & 18.750 & $2.6 \in 30$ \\
\hline 17.0 & .50 & 20. & .40 & 3.0 & 2000000. & $229 \mathrm{C}$. & 3195 . & 58.31 & 188.7 & 13.220 & .0585 \\
\hline 11.0 & .01 & 20. & .40 & 3.0 & 2000000 & 2292 . & 3198 . & 53.92 & 2504.0 & 26.970 & 1.2680 \\
\hline 11.0 & 1.00 & 20. & .40 & 3.0 & 2000000 . & 2306 . & 3217 . & 53.92 & 1163.0 & 25.690 & 1.0740 \\
\hline 11.0 & .50 & 11. & .40 & 3.0 & 2000000. & $315 \epsilon$. & 4404. & 64.55 & 3869.0 & 34.240 & i. .3690 \\
\hline 11.0 & .53 & 28. & .40 & 3.0 & 2000000. & 1991. & 2777 & 50.21 & 2130.0 & 23.980 & .9828 \\
\hline 11.0 & .50 & 20. & .15 & 3.0 & $200000^{\circ}$ & 3945. & $5: 09$. & 143.90 & 2757.0 & 12.350 & .5755 \\
\hline $12 \cdot 0$ & - 50 & 20. & - 65 & 3.0 & 2000000. & 2557. & 3568 . & 98.45 & 141.2 & 32.670 & 1.0520 \\
\hline 11.0 & .50 & 20. & .40 & 2.0 & 2306000. & 1942. & 2707 . & 49.59 & 2055.0 & 23.540 & .7661 \\
\hline 11.0 & .50 & 20. & .40 & 4.0 & 2000000. & 2644 . & 3689. & 58.25 & 3105.0 & 29.730 & 1.2000 \\
\hline 11.0 & .50 & 20. & .40 & 3.0 & 1000000. & $114=0$ & 1599 . & 58.64 & 514.2 & 11.080 & .5414 \\
\hline 11.0 & .50 & 20. & .40 & 3.0 & 3000000. & $343 j$ & 4797. & 56.22 & 1199.0 & 41.890 & $1.0<40$ \\
\hline
\end{tabular}




\section{NOTES}

${ }^{\mathrm{l}}$. H. Myers, Response Surface Methodology (Boston: Allyn and Bacon, Inc., 1971).

${ }^{2}$ R. L. Iman, STEPWISE Regression, SAND76-0364 (A1buquerque: Sandia Laboratories, 1976).

${ }^{3}$ J. W. Tukey, The Propagation of Errors, Fluctuations, and Tolerances, Basic Generalized Formulas, Statistical Research Group, Technical Report No. 10

(Princeton, NJ: Princeton University, 1958). 
The atmospheric dispersion and transport of pollutants in cities are influenced by several features that are generally not present in a nonurban environment. 1 Increased surface roughness and enhanced aerodynamic drag caused by buildings and other urban features enhances dispersion and results in reduced mean wind speeds. The large urban heat island results in a more frequent occurrence of neutral stability conditions. Polutant dispersion and transport can also be significantly influenced by street canyon effects. ${ }^{2}$ For the purposes of this study, two models have been developed to estimate the atmospheric transport and diffusion of radioactive material released in a puff as the result of an accident.* The two models examine, in a predominantly qualitative manner, the urban features described above.**

The initial evolution of the puff release is analyzed using the sma11-scale atmospheric transport model, MICMET. After the cloud has moved a few hundred metres from the release point, MICMET results are supplied to PICMET, the urban-regional atmospheric transport model, which follows the dispersal of the released material over distances of the order of tens of kilometres, or to the defined boundaries of the urban region under study. Both MICMET and PICMET supply estimates of the normalized air concentration of the radioactive material (concentration per unit of material released) and the area of cloud coverage to the radiological consequence model, METRAN, at the end of prescribed time intervals. A similar approach, using a combination of small-scale and regional transport models, has been recently applied by Sheih to the study of pollutant transport over an urban area. ${ }^{4}$ MICMET and PICMET are briefly described in the remainder of this appendix. The meteorological data and approach used in this study are described in Appendix F.

E1. MICMET -- Sma11-Scale Atmospheric Transport Model

MICMET uses a layered Gaussian or plume element technique to predict the air concentrations of radioactive material within a short distance (one or two city blocks) of the point of release. 5 The cloud of radioactive material resulting from the postulated incident is initially assumed to be stabilized in a ground-level cylindrical shape with 10-metre height and 2-metre diameter. The stabilized cloud is subdivided into five horizontal layers, or plume elements, to allow for variations of wind speed with height. Each layer is transported by the mean wind velocity corresponding to the initial height of the layer, and allowed to grow in the downwind, crosswind, and vertical directions. The expressions used to estimate the vertical variation of mean horizontal wind speed are presented in subsection El.1.1

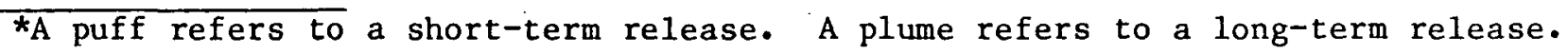

**A detailed quantitative analysis of atmospheric transport and dispersion in an urban area on the scale of individual streets has been undertaken in the past, ${ }^{3}$ but the techniques employed are too complex and costly to be used in the present study. 
Gaussian standard deviations ( $\sigma^{\prime} s$ ) are calculated as a function of downwind distance and turbulence intensity using a relationship provided by Wandel and Kofoed-Hansen: 67

$$
\sigma_{j}=(2 / 9) i_{j} x
$$

where

$$
\begin{aligned}
x & =\text { the downwind travel distance } \\
i & =\text { the turbulence intensity in the jth coordinate direction } \\
\sigma_{j} & =\text { the standard deviation of cloud concentration in the } j \text { th } \\
& \text { coordinate direction }
\end{aligned}
$$

Values of turbulence intensity corresponding to the Pasquill atmospheric stability categories are presented in a paper by Luna and Church ${ }^{8}$ who used the above relationship in their computer program, DIFOUT. 9

MICMET allows particles to fall with a velocity based on their assumed aerodynamic diameter. Only interaction with horizontal surfaces is considered; these surfaces may be specified as perfectly reflecting, perfectly absorbing, or any intermediate situation. A $50 \%$ reflection surface has been assumed in this study. Wet deposition (also termed rainout or washout) is not included in the model.

In some instances, restrictions are placed on the direction of travel and rate of growth of the cloud. These are based upon the size and orientation of the street canyon relative to the initial stabilized cloud. The restrictions and the cases for which they are assumed to apply are discussed in subsection El.2.

MICMET calculates normalized airborne concentrations (concentration per unit of radioactive material released) as a function of time and position. At specified time steps, the spatial airborne concentrations in all five horizontal layers are integrated to obtain the centroid and standard deviations of the resultant cloud. In the present configuration, MICMET is only used for the first 300 metres of travel of the centroid of the resultant cloud. The time required for the cloud to reach this position is divided into four approximately equal intervals. At the end of each of these intervals, the centroid and standard deviations of the cloud (or clouds) are provided to METRAN for direct use in the health effects model. At the end of the last step, the centroid and standard deviations of the cloud (or clouds) are used as initial conditions to load particles into PICMET.

\section{E1.1 Vertical Velocity Profile Calculations}

The vertical variation in mean horizontal wind speed is estimated using the input building height, the fraction of area covered by buildings, and the wind speed at a height of 30 metres for each cell. A logarithmic velocity profile is assumed above the average building height. 210 The velocity profile below the average building is described using an exponential relationship. ${ }^{2}$ The logarithmic expression assumed in Reference 2 for the velocity profile above building height has the following form:

$$
\bar{u}(z)=\frac{u_{*}}{k} \ln \left(\frac{z+z_{0}+d}{z_{0}}\right)
$$


where

$$
\begin{aligned}
\bar{u}(z) & =\text { mean horizontal velocity at height } z(\mathrm{~m} / \mathrm{s}) \\
u_{\star} & =\text { frictional (or shear) velocity }(\mathrm{m} / \mathrm{s}) \\
\mathrm{k} & =\text { von Karman constant }(0.38) \\
z & =\text { vertical coordinate }(\mathrm{m}) \\
z_{0} & =\text { surface roughness length }(\mathrm{m}) \\
\mathrm{d} & =\text { displacement height }(\mathrm{m})
\end{aligned}
$$

Variations in the logarithmic profile due to atmospheric stability effects have not been included.

Values of $z_{0}$ and $d$ are computed for each urban cell using expressions developed by Lettau: ${ }^{11}$

$$
\begin{aligned}
z_{0} & =0.5 h_{b} s / s \\
d & =z_{o} x-h_{b}-z_{o} \\
x \ell n x & =0.1 h_{b}^{2} / z_{o}^{2}
\end{aligned}
$$

where

$$
\begin{aligned}
\mathrm{h}_{\mathrm{b}}= & \text { average building height in grid cell }(\mathrm{m}) \\
\mathrm{s}= & \text { silhouette area* of average building }\left(\mathrm{m}^{2}\right) \\
\mathrm{s}= & \text { average building lot area, i.e., if there are } \mathrm{N} \text { buildings } \\
& \text { distributed over an area } \mathrm{A} \text {, then } \mathrm{S}=\mathrm{A} / \mathrm{N}\left(\mathrm{m}^{2}\right) \\
\mathrm{x}= & \text { intermediate variable used in the calculation of } \\
& \text { displacement height } \mathrm{d} \text { (dimensionless) }
\end{aligned}
$$

If $A$ is assumed to be the cell area $\left(1 \mathrm{~km}^{2}\right), f_{b}$ is the fraction of area $A$ that is occupied by buildings, and $\mathrm{N}$ is the number of buildings in $\mathrm{A}$, then the silhouette area of an average building in $A$ can be estimated by:

$$
s \simeq h_{b} \sqrt{f_{b} A / N}
$$

Using this estimate, plus $\mathrm{S}=\mathrm{A} / \mathrm{N}$, gives

$$
z_{0}=\frac{0.5 h_{b}^{2} \sqrt{f_{b}}}{\sqrt{A / N}}
$$

Data are not available for $N$, the number of buildings in each grid element. However, if it is assumed that each of the horizontal dimensions of a building is proportional to its height, then the area of a building is proportional to $h_{b}{ }^{2}$. It therefore follows that

*Vertical surface area "seen" by wind, i.e., building width $\mathrm{x}$ building height. 


$$
\frac{f_{b} A}{N} \propto h_{b}^{2}
$$

and

$$
\sqrt{\frac{A}{N}}=\frac{1}{C} \frac{h_{b}}{\sqrt{f_{b}}}
$$

where $C=$ some constant. Substituting this expression into Eq. (7) leads to

$$
z_{o}=0.5 \mathrm{Cl}_{b} \mathbf{f}_{b}
$$

To determine a value for the constant $C$, surface roughness length data, presented by Counihan, 12 are used where values of $z_{\text {o }}$ ranging from 1 metre to approximately 7 metres are given for urban areas. In this study, the grid cell with the largest surface roughness has an average $h_{b}$ of 100 metres and $f_{b}$ equal to 0.44 . Assuming a

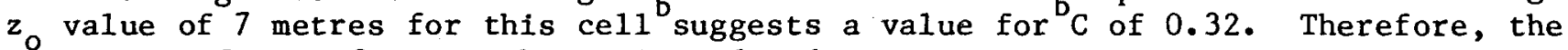
expression for surface roughness is reduced to

$$
z_{0}=0.16 h_{b} f_{b}
$$

For the application of the expressions_above, a minimum value for $z$ of 0.2 metre is assumed. The values of $z_{o}, d$, and $\bar{u}(30)$ are used in Eq. (2) to calculate $u_{*}$. Eq. (2) can then be used to evaluate the mean velocity at any height above building level.

For elevations below the average building height, an exponential expression is applied : ${ }^{2}$

$$
\vec{u}(z)=U_{0} e^{z / A^{\prime}}
$$

where

$$
A^{\prime}=\frac{0.1 h_{b}{ }^{2}}{z_{0}}
$$

If in a particular cell $\mathrm{h}_{\mathrm{b}}$ is less than 30 metres, $\mathrm{U}_{0}$ is evaluated by equating $\bar{u}\left(h_{b}\right)$ in Eqs. (2) and (12). When $h_{b}$ is greater than 30 metres, $U_{0}$ is computed from the input data for that cell, and $u_{*}$ is computed by equating Eqs. ${ }^{\circ}$ (2) and (12) at $z=h_{b}$. This change is made since the wind speeds are given at 30 metres.

\section{E1.2 Street Canyon Effects}

MICMET has two modes of operation based upon the stabilized height of the initial cloud relative to the average height of the buildings in the cell of release. If the height of the initial stabilized cloud is greater than the average building height, the cloud is allowed to travel in the direction of the mean wind. If, however, the initial cloud height is less than the mean building height, two release locations are considered: a release at an intersection or a release midblock.

For an intersection release, the cloud is constrained to travel along the streets downwind of the intersection. Either one or two clouds may result. The number of clouds is determined by the relative orientation of the direction of the mean wind 
and the streets in the region of interest. If there is more than a 10-degree difference between the mean wind direction and the nearest downwind street direction, two clouds are used in the calculation. The amount of material allocated to each cloud is proportional to the complement of the angle between the wind direction and the street of interest. For the first block of travel, the lateral growth of the cloud is limited by the width of the street. After the first block of travel, the lateral growth of the cloud is allowed to resume, although the direction of travel remains constrained by the orientation of the street. These assumptions involving direction of cloud travel and restriction of lateral cloud growth are considered to be valid only a short distance from the release point (approximately two or three blocks). In the present application of the model, the cloud is only allowed to travel approximately 300 metres before the transport calculation is transferred to PICMET.

If the release is postulated to occur at the center of the block, two situations are considered. The first occurs when the mean wind direction is within 30 degrees of the street in which the release occurs. In this situation, the cloud is constrained to travel along the street as discussed above. When there is more than a 30-degree difference between the mean wind direction and the street, full-scale measurements indicate that a vortex flow is developed in the street canyon. 13 In such a case, a flushing time is computed based on a technique developed by Nicholson.2 A single cloud the size of the street canyon, one block long and of uniform concentration, is provided as input to PICMET after the flushing time has elapsed.

\section{E2.2 PICMET -- Urban-Regiona1 Atmospheric Transport Mode1}

The PICMET code estimates the atmospheric transport and diffusion of the released cloud of radioactive material by numerically solving the atmospheric diffusion equation ${ }^{34}$ with a modified particle-in-cell technique. ${ }^{5}$ The mathematical basis for solving this equation by PIC techniques is summarized by Sklarew et al.15 16

The atmospheric diffusion equation is solved on a three-dimensional array of cells that cover the urban region. All cells must have the same dimensions, but cell dimensions and the number of cells used to cover the region can be specified by the user. A mean wind velocity (speed and direction) and three components of eddy diffusivity must be prescribed at the center of each cell. In addition, the boundary conditions that apply at the vertical sides, the top surface, and the bottom surface (ground level) of the cell array must be specified.

Within the boundaries of the celi array, PICMET follows the motion of a large number $N$ of Lagrangian particles, each of which is assumed to carry a fraction $1 / \mathrm{N}$ of the released material. These Lagrangian particles are initially positioned in the cell array with a density proportional to the normalized concentration of airborne material provided by the MICMET model. The particles are subsequently moved in short time steps along trajectories calculated using the mean wind and turbulent flux velocity fields. The simulated transport is terminated when all but three of the Lagrangian particles have passed through the boundaries of the region or are lost through surface deposition.

\section{E2.1 Calculation of Wind Fields and Eddy Diffusivities}

The mean wind field and eddy diffusivities are constructed from the input data for the horizontal mean wind field (assumed for a fixed reference height of 30 metres above the ground), the mean building height, and the fraction of land occupied by 
buildings. These data are included in the data bases discussed in Appendices $A$ and G.

A vertical profile of horizontal mean wind velocity is calculated for the surface layer overlying each base cell, using the same formulae as described in Section El.l for use in MICMET. The stratified horizontal winds obtained are then made divergence-free at each cell center by the addition of an appropriate, usually small, vertical velocity component. Finally, a free-fall velocity is added to the vertical component of wind in all the cells. The free-fall velocity depends on the average size of the small particles that may constitute the released material, i.e.,

$$
\mathrm{w}_{\text {free-fall }}=-1.26 \times 10^{-3} \mathrm{D}^{2} \mathrm{~km} / \mathrm{h}
$$

where $D$ is the particle aerodynamic diameter in $\mu \mathrm{m}$. The same free-fall velocity is assumed for gaseous and particulate releases.

The data on horizontal mean wind at reference height, the average building height, and the fraction of land occupied by structures are also used to determine the profile of the vertical component of eddy diffusivity $\mathrm{K}_{z}$ by formulae given in Ragland and Peirce. ${ }^{17}$ An adiabatic atmospheric surface layer is assumed in the model so that

$$
\mathrm{K}_{\mathrm{z}}=0.4 \mathrm{u}_{*} \mathrm{z}
$$

where $\mathrm{K}_{\mathrm{z}}$ is in $\mathrm{m}^{2} / \mathrm{s}, \mathrm{u}_{\star}$ is the frictional (or shear) velocity (computed in the same way employed for MICMET; see subsection El.1), and $z$ is the height above ground level. In the absence of empirical information on the relative magnitudes of the horizontal eddy diffusivities $\left(\mathrm{K}_{x}\right.$ and $\mathrm{K}_{\mathrm{y}}$ ) in an urban setting, $\mathrm{K}_{\mathrm{x}}$ and $\mathrm{K}_{\mathrm{y}}$ have arbitrarily been assumed to be equal to $\mathrm{K}_{\mathrm{z}} \cdot \mathrm{y}^{\mathrm{y}}$

\section{E2.2 Boundary Conditions and Surface Depositions}

There are several kinds of boundary conditions in the PICMET mode1. The vertical sides and top surface of the cell array are assumed to be transmitting boundaries; that is, material is allowed to flow freely across the boundary and is subsequently removed from the cell array. The lower boundary of the cell array (ground level) can be anything between a reflecting boundary and a completely absorbing boundary, depending upon a coefficient of surface absorption, $\alpha$, that is assigned to each ground level cell. To assure conservation of mass in the implementation of these boundary conditions and to calculate surface deposition, the concept of particle weighting* is used. A particle weight $P$, initially $=1 / n$, is assigned to each of the $n$ Lagrangian particles employed in the simulation. The particle weight never changes unless the particle either is dropped from the set after crossing a transmitting or perfectly absorbing $(\alpha=1)$ boundary or crosses a partially absorbing boundary $(0<\alpha<1)$. In the latter case, the particle is not dropped, but is

*Both particle weighting and volume weighting are used in PICMET. Volume weighting is a standard technique in PIC calculations. See Reference 15, for example. 
physically reflected as though the boundary were perfectly reflecting $(\alpha=0)$. However, the reflected particle's weight is multiplied by $(1-\alpha)$, and it is assumed that a fraction, $\alpha P$, of the released material has been deposited on horizontal surfaces during the time step and within the cell where the particle crossed

the boundary. The particle weights are used to calculate the normalized, volumetric concentrations of the airborne material in a mass-conserving way.

\section{E2.3 Special Features of PICMET}

When applied to the atmospheric diffusion equation, the standard PIC techniques (see References 15 and 16) underestimate the rate of turbulent diffusion in material clouds whose characteristic sizes are small compared to cell dimensions. The source of trouble is the finite-difference approximations made for computing the three components of turbulent flux velocity:

$$
\begin{aligned}
& u_{f}=-\frac{k_{x}}{C} \frac{\delta C}{\delta x} \\
& v_{f}=-\frac{k_{y}}{C} \frac{\delta C}{\delta y} \\
& w_{f}=-\frac{k_{z}}{C} \frac{\delta C}{\delta z}
\end{aligned}
$$

Until material has spread through several cells, a finite-difference approximation to the concentration derivatives of Eq. (16) will give unphysical, small turbulent flux velocities, thereby inhibiting expansion of the cloud.

There are several techniques that can be used instead of finite differences that give better approximations to the field of turbulent flux velocity in small clouds. Two of these techniques used in PICMET are described below.

Treatment of Horizontal Turbulent Flux Velocity

As an alternative to the finite-difference approximation of the horizontal turbulent flux velocities, $u_{f}$ and $v_{f}$, of $\mathrm{Eq}$. (16), an analytic calculation of these quantities is made in which the instantaneous concentration field of suspended material is assumed to have a horizontal $(x, y)$ Gaussian distribution. The centroid coordinates $(\bar{x}, \bar{y})$ and the standard deviations $\left(\sigma_{x}, \sigma_{y}\right)$ of the Gaussian are computed from the positions of the weighted particles. For lnstance,

$$
\begin{aligned}
\vec{x} & =\frac{\sum_{i} P_{i} x_{i}}{\sum_{i} P_{i}} \\
\sigma_{x}{ }^{2} & =\frac{\sum_{i} P_{i}\left(\bar{x}-x_{i}\right)^{2}}{\sum_{i} P_{i}}
\end{aligned}
$$


where $x_{i}$ is the $x$-coordinate of the $i^{\text {th }}$ particle in the mesh, $P_{i}$ is the weight of the $i^{\text {th }}$ particle, and the sums are over all particles in a singte cloud. The turbulent flux velocities are obtained by analytical differentiation of the Gaussian, according to the expressions for $u_{f}$ and $v_{f}$ given in Eq. (16). The results for a particle at position $\left(x_{i}, y_{i}\right)$ are

$$
u_{f}=\frac{K_{x}}{\sigma_{x}}\left(x_{i}-\bar{x}\right)
$$

and

$$
v_{f}=\frac{k_{y}}{\sigma_{y}^{2}} \cdot\left(y_{i}-\bar{y}\right)
$$

These velocity components are added to the corresponding components of the mean horizontal wind $(\bar{u}, \bar{v})$, taken at the position of the $i$ particle, to get the total horizontal velocity of that Lagrangian particle. The technique requires that the particles comprising each cloud be distinguished so that the centroids and standard deviations for each cloud may be calculated.

Figure E-1 shows results of the two different treatments of horizontal diffusion for a single cloud. The standard deviation of cloud concentration the Y-direction, $\sigma$, is plotted as a function of distance from the release point. The curve marked "Y (18)" is calculated using the approximation leading to Eq. (18). The dashed curve, marked "(FD)," is calculated using the finite-difference approximation for the turbulent flux velocities. In each case, the empirical standard deviation of particle concentration, given in Eq. (17), is plotted against the absolute displacenent distance of the cloud centroid after handover by MICMET. For the comparison calculations, a wind speed of $1 \mathrm{~m} / \mathrm{s}$ at reference height ( 30 metres) and wind direction along the $X$-axis have been assumed.

1lso shown in Figure $\mathrm{E}-1$ are the curves of $\sigma$ versus distance expected for the cange of Pasquill turbulence types. ${ }^{7}$ Note the unphysical behavior of the Einite-difference approximation when the characteristic horizontal size of the sloud is less than one cell dimension.

Treatment of Vertical Turbulent Diffusion

The vertical distribution of material in the cloud is not likely to be Gaussian because of constraining effects of the ground surface. Thus, a treatment of the vertical component of turbulent flux velocity in a manner similar to the one described for the horizontal component in the preceding subsection is not always possible. Instead, PICMET uses a technique employed by Schwartzl ${ }^{18}$ to numerically solve an equation of the same form as the atmospheric diffusion equation. In each time step, each Lagrangian particle is displaced by an amount w, where

$$
w=\bar{w}(i) \Delta t+\xi_{i}
$$




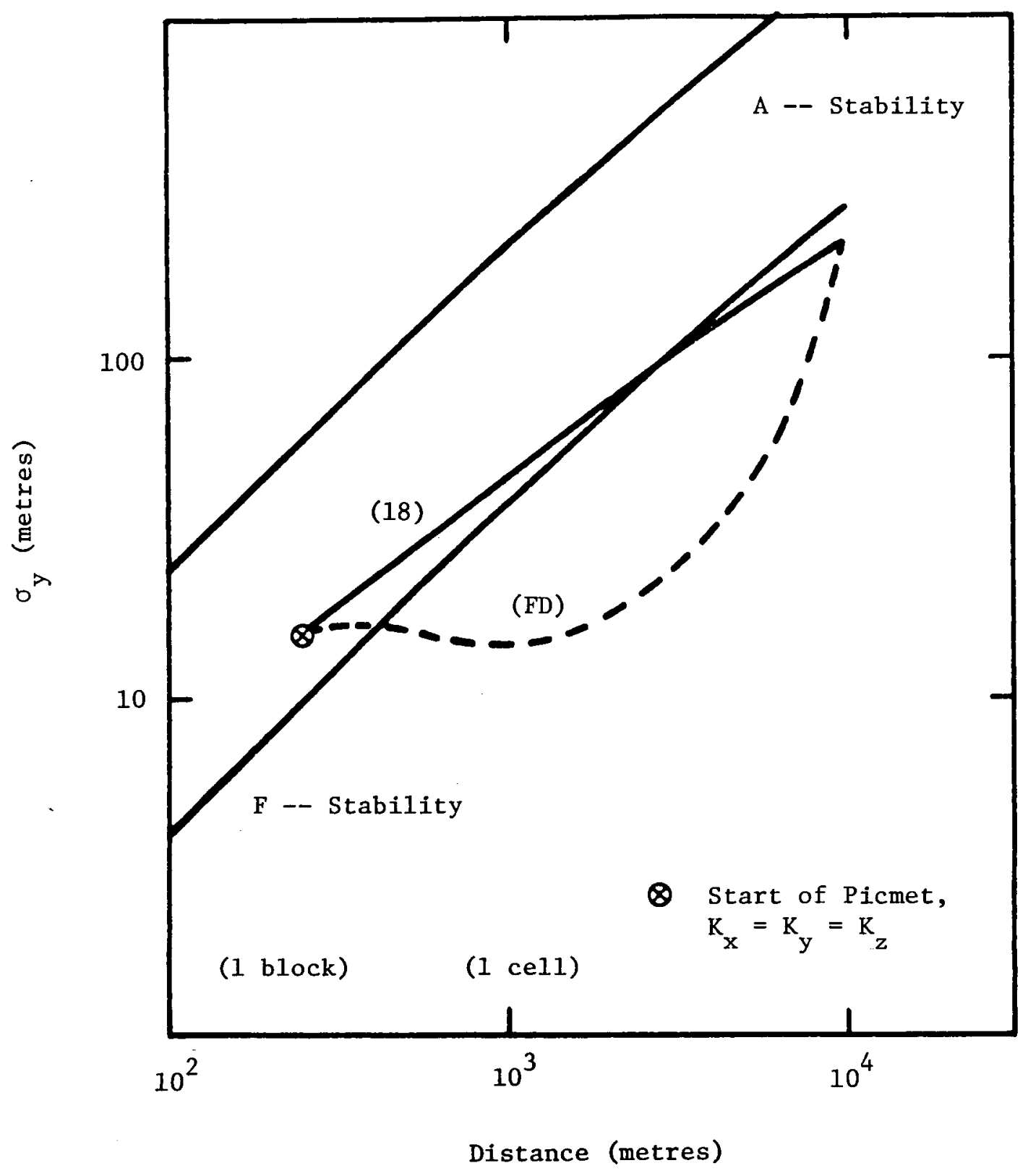

Figure E-1. Comparison of Different Treatments of Horizontal Diffusion 
$\bar{w}(i)$ is the mean vertical wind speed (plus constant particle settling velocity) measured at the position of the $i^{\text {th }}$ particle, $\Delta t$ is the length of the time-step, and $\xi_{j}$ is a random displacement drawn from a normal distribution whose mean is zero and whose standard deviation is $\sqrt{2 K_{z}(i) \Delta t} . K_{z}(i)$ is the vertical component of eddy diffusivity measured at the location of the $i^{z}$ particle. Because this technique can be computationally expensive, it is only applied in PICMET to the vertical component of turbulent diffusion.

NOTES

${ }^{1}$ F. A. Gifford, "Atmospheric Transport and Dispersion over Cities," Nuclear Safety, Vo1. 13, No. 5, September-October 1972.

${ }^{2}$ S. E. Nicholson, "A Pollution Model for Street-Leve1 Air," Atmospheric Environment, 9:13-31, 1975 .

${ }^{3}$ B. D. Nichols and C. W. Hirt, "Transient Three-Dimensional Fluid Flow in the Vicinity of Large Structures," LA-DC-72-711 (Los Alamos: Los Alamos Scientific Laboratory, 1972).

${ }^{4}$ C. M. Sheih, "A Puff-Grid Model for Predicting Pollutant Transport Over an Urban Area," Journal of the Air Pollution Control Association, 27:784-785, August 1977.

${ }^{5}$ U.S. Nuclear Regulatory Commission, "Methods for Estimating Atmospheric Transport and Dispersion of Gaseous Effluents in Routine Releases from Light-WaterCooled Reactors," Regulatory Guide 1.11, Revision 1 (Washington: USNRC, July 1977).

${ }^{6} \mathrm{C}$. F. Wandel and 0 . Kofoed-Hansen, "On the Eulerian-Lagrangian Transform in the Statistical Theory of Turbulence," J. Geophys. Res., 67:3089-3094, 1962.

${ }^{7}$ U.S. Nuclear Regulatory Commission, Reactor Safety Study, Appendix VI: Calculation of Reactor Accident Consequences, WASH-1400(NUREG-75/014) (Washington: USNRC, October 1975) p A-3.

${ }^{8}$ R. E. Luna and H. W. Church, "A Comparison of Turbulence Intensity and Stability Ratio Measurements to Pasquil1 Stability Classes," J. App1. Meteor., $11: 663-669,1972$.

${ }^{9}$ R. E. Luna and H. W. Church, DIFOUT: A Model for Computation of Aerosol Transport and Diffusion in the Atmosphere, SC-RR-68-555 (Albuquerque: Sandia Laboratories, 1969).

10 E. J. Plate, Aerodynamic Characteristics of Atmospheric Boundary Layers, AEC Critical Review Series (U.S. Atomic Energy Commission, May 1971), p 44.

$1 \mathrm{l}_{\mathrm{H}}$. H. Lettau, "Physical and Meteorological Basis for Mathematical Models of Urban Diffusion Processes," Proceedings of a Symposium on Multiple-Source Urban Diffusion Models, U.S. Air Pollution Office, Publication AP-86, 1970.

$12 \mathrm{~J}$. Counihan, "Adiabatic Atmospheric Boundary Layers," Atmospheric Environment, $9: 885,1975$. 
13 W. F. Dabbert, F. L. Ludwig, and W. B. Johnson, "Validation and Applications of an Urban Diffusion Model for Vehicle Pollutants," Atmospheric Environment, F: $603-618,1973$.

$14 \mathrm{~J}$. H. Seinfeld, Air Pollution - Physical and Chemical Fundamentals, (New York: McGraw-Hill, Inc., 1975), p 294.

${ }^{15}$ R. C. Sklarew, A. J. Fabrick, and J. E. Prager, "A Particle-in-Cell Method for Numerical Solution of the Atmospheric Diffusion Equation and Application to Air Pollution Problems," Systems, Science and Software, Vol. 1, 3SRP44 (La Jolla, CA, $1971)$.

${ }^{16}$ R. C. Sklarew, A. J. Fabrick, and J. E. Prager, "Mathematical Modeling of Photochemical Smog Using the PICK Method," Journal of the Air Pollution Contro1 Association, 25:48-51, January 1975.

${ }^{17}$ K. W. Ragland and J. J. Peirce, "Boundary Layer Model for Air Pollutant Concentrations Due to Highway Traffic," Journal of the Air Pollution Control Association, 25:48-51, January 1975.

18F. W. Schwartz, "A Probabilistic Mass Transfer Mode1," Canadian Hydrology Symposium-75 Proceedings, 11-14 August 1975 (published 1976), pp 408-412. 
METEOROLOGICAL DATA AND APPROACH

The meteorological models used in this study require as input an assumed wind speed and direction at a height of 30 metres for each $1-\mathrm{km}^{2}$ grid cell in the urban area. Wind speeds at heights other than 30 metres are determined using vertical velocity profile expressions given in Appendix E. Calculations used to estimate public risk from accidentally released pollutants are performed assuming a selection of weather situations spanning the range of possible wind speeds and directions over the study area.

Initially, this study made use of wind speed and direction data gathered during a study conducted at New York University to develop an air pollution model for the distribution of sulfur dioxide in New York City. ${ }^{1}$ Data in this study were collected during 12 test periods of 3 to 5 days each and consisted of hourly averaged wind speeds and directions from numerous recording stations scattered throughout New York. Three of these test periods were selected for documentation by Bornstein. The Bornstein data are useful because they were gathered at a sufficient number of points to allow interpolation of wind speed and direction values for each $1-\mathrm{km}^{2} \mathrm{grid}$ cel1. However, because of the limited number of observation days in this data base, the probability and representativeness of the observed weather conditions for the New York urban area can not be adequately determined.

A series of preliminary calculations was made to determine the impact on calculated consequences of the spatial variation of wind speed and direction across the urban grid. A large release was postulated in the center of the grid (cell 46). Consequences* were estimated using a number of actual, spatially varying, hourly wind-field situations from the Bornstein data. Calculations were then repeated using constant wind speeds and directions across the grid, equal to the values in cell 46. Early fatalities and early morbidities calculated by the two sets of data were identical in each case.**. Calculated latent cancer fatalities and genetic effects differed by less than $10 \%$. Therefore, spatial variations of wind field across the grid do not have a significant impact on estimated consequences.

Because of the limited number of observations in the Bornstein data base and the results of the preliminary calculations described above, surface wind data (speed and direction) from Kennedy Airport, located in southeastern Queens, were used in

\footnotetext{
*Early fatalities, early morbidities, latent cancer fatalities, and genetic effects.

**Note that early effects are predicted to occur only in the cell of release for the magnitude of releases addressed in this study.
} 
this study. These data were gathered over a period of approximately 17 years and are tabulated in Reference 2. The data and their appropriateness for the New York urban area are discussed in Section F1.

\section{F1. Kennedy Airport (NY) Surface Wind Data}

The tables summarizing surface wind data at John F. Kennedy International Airport (JFK) for the period from July 1948 to June 1965 present wind speed and direction joint frequency statistics (1) for the entire observation period, (2) individually for each month of the year, and (3) by month for eight 3-hour periods corresponding to the following sets of hourly observations: 0000-0200, 0300-0500, 0600-0800, $0900-1100,1200-1400,1500-1700,1800-2000,2100-2300$ (1ocal time).2

The height above ground at which the airport wind measurements were taken varied during the observation period. From July 1948 through December 1957, the recording instruments were about 16 metres above ground. They were repositioned at approximately 7 metres from January 1958 to March 1958, at 10 metres from March 1958 to November 1962, and at $\sim 6$ metres from November 1962 until June 1965.

The meteorological models used in this present study (MICMET and PICMET) require wind speeds and directions at a height of 30 metres as input. Expressions for the variation of wind speed with height, presented in Appendix E, depend on the surface roughness characteristics of the area under study. The following simple analysis was made to determine what, if any, correction to the JFK data was required for use as input data in this study: the surface roughness characteristics at JFK are significantly different from those in the highly populated cells of the urban area. The vertical profiles of wind velocity at these locations will therefore differ. At some height above the ground, however, surface frictional effects will no longer have any influence on the wind field, i.e., geostrophic wind. 3 It can therefore be assumed that the geostrophic wind speeds (here assumed at a height of 1000 metres) over the airport and the rest of the urban area are equal. Assuming that the JFK data were measured at a height of 10 metres, that the area surrounding the airport has an average building height of 6 metres, and that the fraction of land occupied by buildings is equal to 0.1 , Eqs. (2), (3), (4), (5), and (11) in Appendix E (and a minimum value of $z_{0}=0.2$ metres) can be used to determine the geostrophic wind speed:

$$
\mathrm{u}(1000 \mathrm{~m}) \approx 2.2 \mathrm{u}_{\mathrm{k}}(10 \mathrm{~m})
$$

where $u_{k}(10 \mathrm{~m})$ is the measured wind speed at JFK. Assuming typical building heights of 15 metres and a value of 0.6 for the fraction of land occupied by buildings in the populated urban area, and working down from the 1000-metre height using the same equations,

$$
\mathrm{u}_{\mathrm{c}}(30 \mathrm{~m}) \approx 0.43 \mathrm{u}(1000 \mathrm{~m}) \approx 0.95 \mathrm{u}(10 \mathrm{~m})
$$

where $u_{c}(30 \mathrm{~m})$ is the wind speed at a 30-metre height over the city. For all practical purposes, $u_{c}(30 \mathrm{~m}) \approx u_{k}(10 \mathrm{~m})$, showing that the JFK data can be used without adjustment in this study without introducing a significant error.

Surface wind data for the entire 17-year observation period are summarized in Table F-1. Joint frequency statistics and summary statistics are presented for 16 wind directions and 11 wind speed categories. A plot of the total observation period 
Table F-1

Kennedy Alrport Surface Wind Data, 1948-1965 (A11 Hours, Al1 Months)

Wind Speed* $(\mathrm{m} / \mathrm{s})$

\begin{tabular}{|c|c|c|c|c|c|c|c|c|c|c|c|c|}
\hline \multirow{2}{*}{$\begin{array}{c}\text { Wind } \\
\text { Direction } \\
\end{array}$} & \multicolumn{12}{|c|}{ Wind Speed* (m/B) } \\
\hline & $0.5-1.8$ & $1.8-3.3$ & $3.3-5.4$ & $5.4-8.5$ & $8.5-11$ & $11-14$ & $14-17$ & $\underline{17-21}$ & $21-24$ & $24-29$ & 29 & $\%$ \\
\hline $\mathbf{N}$ & 0.4 & 1.3 & 2.6 & 1.9 & 0.5 & 0.1 & 0 & 0 & 0 & 0 & 0 & 6.8 \\
\hline $\mathrm{NNE}$ & 0.4 & 1.2 & 2.1 & 1.5 & 0.5 & 0.1 & 0 & 0 & 0 & 0 & 0 & 5.8 \\
\hline $\mathrm{NE}$ & 0.5 & 1.3 & 1.8 & 1.4 & 0.4 & 0.1 & 0 & 0 & 0 & 0 & 0 & 5.5 \\
\hline ENE & 0.4 & 1.1 & 1.3 & 0.9 & 0.3 & 0.1 & 0 & 0 & 0 & 0 & 0 & 4.1 \\
\hline $\mathbf{E}$ & 0.4 & 0.7 & 1.0 & 0.7 & 0.2 & 0.1 & 0 & 0 & 0 & 0 & 0 & 3.1 \\
\hline ESE & 0.2 & 0.7 & 1.0 & 0.6 & 0.1 & 0 & 0 & 0 & 0 & 0 & 0 & 2.7 \\
\hline SE & 0.3 & 0.6 & 1.2 & 0.8 & 0.2 & 0.1 & 0 & 0 & 0 & 0 & 0 & 3.1 \\
\hline SSE & 0.2 & 0.8 & 1.9 & 1.6 & 0.4 & 0.1 & 0 & 0 & 0 & 0 & 0 & 5.1 \\
\hline $\mathbf{S}$ & 0.3 & 1.5 & 3.9 & 3.2 & 0.8 & 0.2 & 0 & 0 & 0 & 0 & 0 & 9.9 \\
\hline SSW & 0.4 & 1.6 & 3.4 & 2.5 & 0.5 & 0.1 & 0 & 0 & 0 & 0 & 0 & 8.5 \\
\hline SW & 0.5 & 1.8 & 3.7 & 2.1 & 0.3 & 0.1 & 0 & 0 & 0 & 0 & 0 & 8.5 \\
\hline WSW & 0.3 & 1.2 & 2.7 & 2.2 & 0.7 & 0.2 & 0 & 0 & 0 & 0 & 0 & 7.5 \\
\hline $\mathbf{W}$ & 0.3 & 0.8 & 1.7 & 1.9 & 0.9 & 0.3 & 0 & 0 & 0 & 0 & 0 & 5.9 \\
\hline WNW & 0.2 & 0.7 & 2.0 & 2.7 & 1.3 & 0.4 & 0.1 & 0 & 0 & 0 & 0 & 7.4 \\
\hline NW & 0.3 & 0.9 & 2.3 & 3.0 & 1.3 & 0.4 & 0.1 & 0 & 0 & 0 & 0 & 8.2 \\
\hline NNW & 0.3 & 0.9 & 2.2 & 2.2 & 0.7 & 0.2 & 0 & 0 & 0 & 0 & 0 & 6.5 \\
\hline Calm & - & - & - & - & - & - & - & - & - & - & - & 1.4 \\
\hline$\%$ & 5.4 & 17.1 & 34.8 & 29.0 & 9.1 & 2.7 & 0.4 & 0 & 0 & 0 & 0 & 100.0 \\
\hline
\end{tabular}

${ }^{*}$ Wind speed converted from knots in Reference 2 to metres per second 
probability versus wind direction is presented as Figure F-1. In general, the probabilities do not vary greatly with wind direction (max/min probability $\approx 3$ ), although there is some tendency for winds to blow from the south to southwest.*

Five distinct population distributions representative of the following times of day are addressed in the study: 1800-0700, 0700-0830, 0830-1100,1100-1330, 1330-1630, and 1630-1800. To correspond to these time intervals, JFK surface wind data are presented in Tables F-2 through F-6 for the following 3-hour time periods: 0000-0200, 0600-0800, 0900-1100, 1200-1400, and 1500-1700 (assumed for both the 1330-1630 and 1630-1800 populations).

To further summarize, the data in Tables $\mathrm{F}-1$ through $\mathrm{F}-6$ have been collapsed into three wind speed categories $(0 .-3.3, * * 3.3-5.4$, and $>5.4 \mathrm{~m} / \mathrm{s})$ and eight wind direction categories ( $N, \mathrm{NE}, \mathrm{E}, \mathrm{SE}, \mathrm{S}, \mathrm{SW}, \mathrm{W}, \mathrm{NW}) . \dagger$ Probabilities for these categories, by time of day, are presented in Tables F-7 and F-8. Table F-9 presents wind speed and direction joint-frequency probabilities for the same categories. In general, wind speed and direction do not appear to be strongly interdependent, so independent probabilities can be used with little error. Probabilities for wind direction are taken directly from Table F-8. The probabilities for the three wind speed categories in Table F-7 are assumed for three representative wind speeds: 2,4 , and 8 $\mathrm{m} / \mathrm{s}$. For example, at 0100 the probability of a low wind speed $(2 \mathrm{~m} / \mathrm{s})$ from the southwest $\approx 0.34 \times 0.19=0.065$.

The use of JFK data for the entire New York urban area may lead to some error in estimated wind-field probabilities. Results should therefore be interpreted with caution.

This may be due in some part to sea breeze effects at the Kennedy Airport site. They may or may not be representative of the New York urban area.

** Includes data for calm, $0.5-1.8$, and $1.8-3.3 \mathrm{~m} / \mathrm{s}$ wind conditions.

tExample: From Table F-1, probability of wind in $45^{\circ}$ sector centered on south $=$ $P(S)+0.5 P(S S E)+0.5 P(S S W)=0.099+0.0255+0.0425=0.167$. 


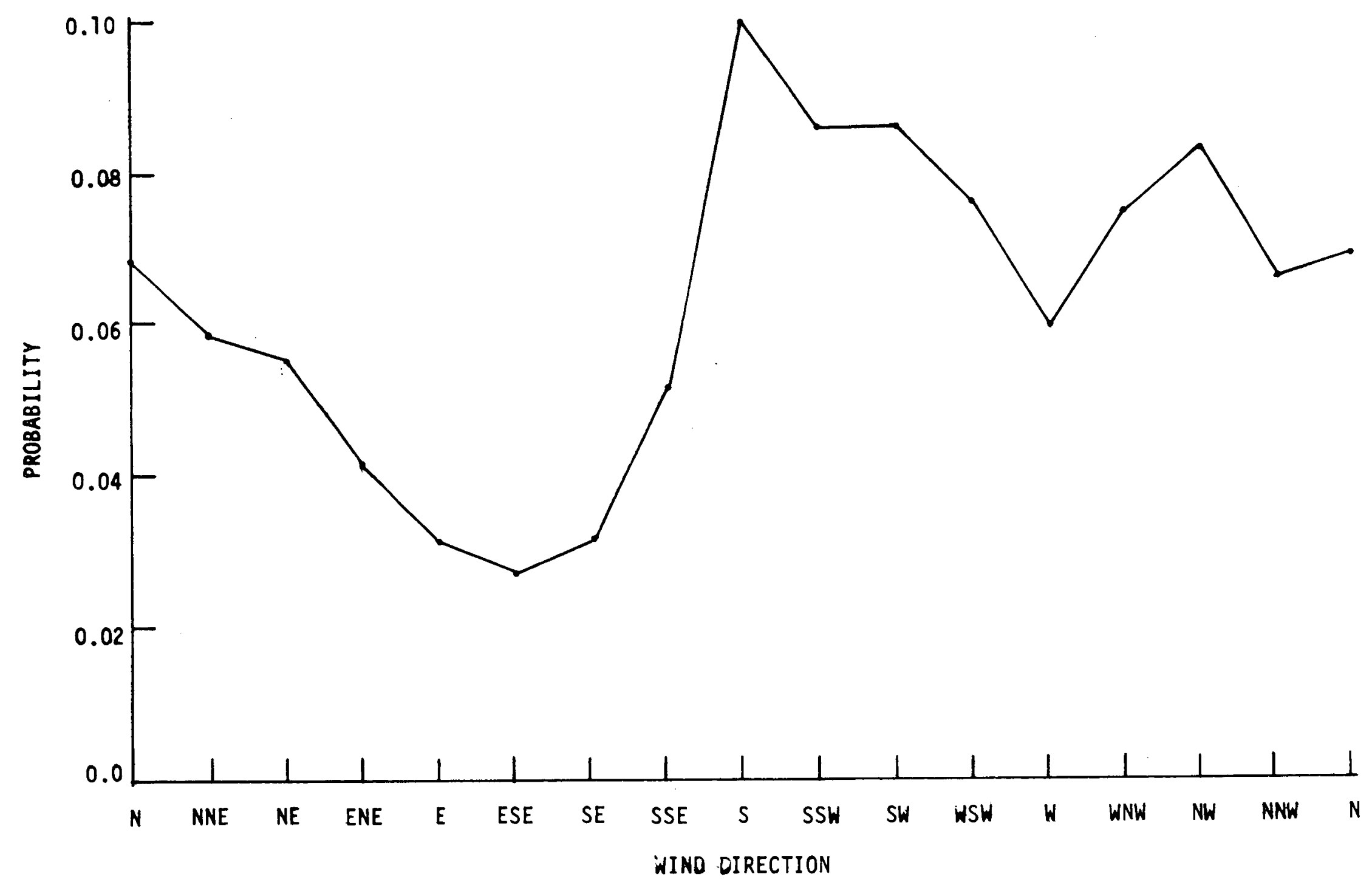

Figure F-1. Kennedy Airport Surface Wind Data, 1948-1965 (Al1 Months, All Hours): Probability versus Wind Direction 
Table F-2

Kennedy Airport Surface Wind Data, 0000-0200 (A11 Months)

Wind Speed $(\mathrm{m} / \mathrm{s})$

\begin{tabular}{|c|c|c|c|c|c|c|c|c|c|c|c|c|c|}
\hline \multirow{2}{*}{$\begin{array}{c}\text { Wind } \\
\text { Direction }\end{array}$} & \multicolumn{13}{|c|}{ Wind Speed ${ }^{\star}(\mathrm{m} / \mathrm{s})$} \\
\hline & $\underline{0.5-2}$ & $2-3$ & $3-5.4$ & $5.4-9$ & $9-11$ & $11-14$ & $14-17$ & $17-21$ & $21-24$ & $\underline{24-29}$ & 29 & $\%$ & Mean \\
\hline $\mathrm{N}$ & 0.56 & 2.03 & 3.24 & 1.93 & 0.34 & 0.09 & 0.03 & 0.01 & 0.00 & 0.00 & 0.00 & 8.18 & 8.92 \\
\hline NNE & 0.66 & 1.71 & 2.55 & 1.50 & 0.44 & 0.08 & 0.04 & 0.00 & 0.00 & 0.00 & 0.00 & 6.98 & 9.04 \\
\hline $\mathrm{NE}$ & 0.72 & 1.91 & 1.78 & 1.08 & 0.28 & 0.08 & 0.02 & 0.00 & 0.01 & 0.00 & 0.00 & 5.85 & 8.22 \\
\hline ENE & 0.63 & 1.58 & 1.18 & 0.73 & 0.13 & 0.11 & 0.02 & 0.00 & 0.00 & 0.00 & 0.00 & 4.37 & 7.76 \\
\hline E & 0.47 & 0.86 & 0.78 & 0.40 & 0.13 & 0.05 & 0.03 & 0.01 & 0.01 & 0.00 & 0.00 & 2.70 & 8.12 \\
\hline ESE & 0.33 & 0.69 & 0.50 & 0.28 & 0.07 & 0.04 & 0.01 & 0.00 & 0.00 & 0.00 & 0.00 & 1.90 & 7.96 \\
\hline SE & 0.29 & 0.62 & 0.67 & 0.20 & 0.11 & 0.07 & 0.00 & 0.00 & 0.00 & 0.00 & 0.00 & 1.88 & 8.22 \\
\hline SSE & 0.29 & 0.75 & 1.07 & 0.50 & 0.23 & 0.05 & 0.02 & 0.00 & 0.00 & 0.00 & 0.00 & 2.87 & 9.22 \\
\hline $\mathbf{s}$ & 0.37 & 1.61 & 2.58 & 1.15 & 0.26 & 0.09 & 0.06 & 0.00 & 0.00 & 0.00 & 0.00 & 6.07 & 9.22 \\
\hline SSW & 0.53 & 2.17 & 3.40 & 1.38 & 0.15 & 0.11 & 0.01 & 0.00 & 0.00 & 0.00 & 0.00 & 7.73 & 8.70 \\
\hline SW & 0.78 & 2.71 & 4.65 & 1.76 & 0.25 & 0.07 & 0.01 & 0.00 & 0.00 & 0.00 & 0.00 & 10.17 & 8.47 \\
\hline WSW & 0.53 & 2.18 & 3.85 & 1.95 & 0.48 & 0.15 & 0.04 & 0.00 & 0.00 & 0.00 & 0.00 & 9.17 & 9.24 \\
\hline w & 0.49 & 1.47 & 2.25 & 1.79 & 0.65 & 0.21 & 0.01 & 0.00 & 0.00 & 0.00 & 0.00 & 6.86 & 9.75 \\
\hline WNW & 0.37 & 1.18 & 2.51 & 2.42 & 0.97 & 0.23 & 0.04 & 0.01 & 0.01 & 0.00 & 0.00 & 7.73 & 10.30 \\
\hline NW & 0.42 & 1.24 & 2.68 & 2.68 & 1.06 & 0.32 & 0.03 & 0.00 & 0.00 & 0.00 & 0.00 & 8.40 & 10.48 \\
\hline \multirow[t]{2}{*}{ NNW } & 0.41 & 1.29 & 2.68 & 1.97 & 0.58 & 0.15 & 0.02 & 0.00 & 0.00 & 0.00 & 0.00 & 7.05 & 9.87 \\
\hline & & & & & & & & & & & CALM & 2.09 & \\
\hline$\%$ & 7.72 & 23.95 & 36.33 & 21.67 & 6.07 & 1.83 & 0.31 & 0.03 & 0.02 & 0.00 & 0.00 & 100.00 & 9.05 \\
\hline
\end{tabular}

* See footnote, Table F-1 
Table F-3

Kennedy Airport Surface Wind Data, 0600-0800 (AlI Months)

\begin{tabular}{|c|c|c|c|c|c|c|c|c|c|c|c|c|c|}
\hline \multirow{2}{*}{$\begin{array}{c}\text { Wind } \\
\text { Direction } \\
\end{array}$} & \multicolumn{13}{|c|}{ W1nd Speed* $(\mathrm{m} / \mathrm{s})$} \\
\hline & $0.5-2$ & $2-3$ & $3-5.4$ & $5.4-9$ & $9-11$ & $11-14$ & $14-17$ & $\underline{17-21}$ & $21-24$ & $\underline{24-29}$ & 29 & $\%$ & Mean \\
\hline $\mathbf{N}$ & 0.54 & 1.87 & 3.33 & 2.27 & 0.56 & 0.14 & 0.02 & 0.00 & 0.00 & 0.00 & 0.00 & 8.71 & 9.58 \\
\hline NNE & 0.62 & 1.64 & 3.23 & 2.02 & 0.50 & 0.13 & 0.01 & 0.00 & 0.00 & 0.00 & 0.00 & 8.16 & 9.44 \\
\hline $\mathrm{NE}$ & 0.77 & 1.83 & 2.73 & 1.90 & 0.41 & 0.09 & 0.02 & 0.00 & 0.00 & 0.00 & 0.00 & 7.73 & 8.98 \\
\hline ENE & 0.66 & 1.53 & 1.59 & 0.98 & 0.28 & 0.15 & 0.05 & 0.00 & 0.00 & 0.00 & 0.00 & 5.23 & 8.54 \\
\hline $\mathrm{E}$ & 0.50 & 0.77 & 0.82 & 0.42 & 0.21 & 0.07 & 0.02 & 0.02 & 0.01 & 0.01 & 0.00 & 2.81 & 8.49 \\
\hline ESE & 0.23 & 0.57 & 0.48 & 0.36 & 0.13 & 0.04 & 0.02 & 0.00 & 0.00 & 0.00 & 0.00 & 1.82 & 8.83 \\
\hline $\mathrm{SE}$ & 0.24 & 0.46 & 0.59 & 0.33 & 0.12 & 0.06 & 0.02 & 0.00 & 0.00 & 0.00 & 0.00 & 1.78 & 9.10 \\
\hline SSE & 0.18 & 0.60 & 0.90 & 0.58 & 0.13 & 0.13 & 0.01 & 0.01 & 0.00 & 0.00 & 0.00 & 2.52 & 9.99 \\
\hline S & 0.30 & 1.28 & 2.38 & 1.02 & 0.34 & 0.09 & 0.01 & 0.00 & 0.00 & 0.00 & 0.00 & 5.41 & 9.39 \\
\hline SSW & 0.38 & 1.49 & 2.66 & 1.03 & 0.19 & 0.12 & 0.04 & 0.00 & 0.00 & 0.00 & 0.00 & 5.86 & 8.96 \\
\hline SW & 0.49 & 2.34 & 3.83 & 1.72 & 0.29 & 0.08 & 0.02 & 0.00 & 0.00 & 0.00 & 0.00 & 8.78 & 8.77 \\
\hline WSW & 0.49 & 1.88 & 3.41 & 2.30 & 0.58 & 0.22 & 0.03 & 0.01 & 0.00 & 0.00 & 0.00 & 8.89 & 9.75 \\
\hline $\mathrm{W}$ & 0.48 & 1.22 & 2.03 & 2.02 & 0.71 & 0.21 & 0.03 & 0.00 & 0.00 & 0.00 & 0.00 & 6.63 & 10.25 \\
\hline WNW & 0.36 & 0.96 & 2.21 & 2.59 & 0.93 & 0.29 & 0.05 & 0.01 & 0.01 & 0.00 & 0.00 & 7.38 & 10.94 \\
\hline NW & 0.41 & 1.18 & 2.76 & 2.98 & 1.25 & 0.30 & 0.05 & 0.00 & 0.00 & 0.00 & 0.00 & 8.88 & 11.07 \\
\hline NNW & 0.38 & 1.22 & 2.58 & 2.18 & 0.59 & 0.19 & 0.03 & 0.02 & 0.00 & 0.00 & 0.00 & 7.19 & 10.34 \\
\hline & & & & & & & & & & & CALM & 2.23 & \\
\hline$\%$ & 7.01 & 20.79 & 35.50 & 24.73 & 7.15 & 2.25 & 0.32 & 0.05 & 0.02 & 0.01 & 0.00 & 100.00 & 9.52 \\
\hline
\end{tabular}

*See footnote, Table F-1 
Table F-4

Kennedy Alrport Surface Wind Data, 0900-1100 (Al1 Months)

Wind Speed* $(\mathrm{m} / \mathrm{s})$

\begin{tabular}{|c|c|c|c|c|c|c|c|c|c|c|c|c|c|}
\hline \multirow{2}{*}{$\begin{array}{c}\text { Wind } \\
\text { Direction }\end{array}$} & \\
\hline & $0.5-2$ & $2-3$ & $3-5.4$ & $5.4-9$ & $9-11$ & $11-14$ & $14-17$ & $17-21$ & $21-24$ & $24-29$ & 29 & $\%$ & Mean \\
\hline$N$ & 0.30 & 1.05 & 2.61 & 2.41 & 0.69 & 0.16 & 0.01 & 0.00 & 0.00 & 0.00 & 0.00 & 7.23 & 10.75 \\
\hline NNE & 0.29 & 0.97 & 2.43 & 2.18 & 0.57 & 0.18 & 0.03 & 0.01 & 0.00 & 0.00 & 0.00 & 6.63 & 10.69 \\
\hline $\mathrm{NE}$ & 0.32 & 1.03 & 2.08 & 2.13 & 0.58 & 0.17 & 0.04 & 0.00 & 0.00 & 0.00 & 0.00 & 6.33 & 10.66 \\
\hline ENE & 0.37 & 0.81 & 1.48 & 1.12 & 0.37 & 0.10 & 0.03 & 0.01 & 0.00 & 0.00 & 0.00 & 4.27 & 9.79 \\
\hline E & 0.25 & 0.62 & 1.22 & 0.85 & 0.28 & 0.10 & 0.01 & 0.00 & 0.00 & 0.00 & 0.01 & 3.33 & 9.87 \\
\hline ESE & 0.18 & 0.65 & 1.13 & 0.75 & 0.16 & 0.03 & 0.02 & 0.00 & 0.00 & 0.01 & 0.00 & 2.90 & 9.54 \\
\hline SE & 0.20 & 0.53 & 1.13 & 0.78 & 0.14 & 0.06 & 0.00 & 0.00 & 0.00 & 0.00 & 0.00 & 2.81 & 9.63 \\
\hline SSE & 0.15 & 0.65 & 2.00 & 1.27 & 0.36 & 0.17 & 0.03 & 0.00 & 0.00 & 0.00 & 0.00 & 4.58 & 10.93 \\
\hline s & 0.29 & 1.12 & 4.44 & 2.41 & 0.53 & 0.13 & 0.03 & 0.00 & 0.00 & 0.00 & 0.00 & 8.95 & 10.22 \\
\hline SSW & 0.35 & 1.22 & 3.63 & 2.33 & 0.33 & 0.10 & 0.04 & 0.00 & 0.00 & 0.00 & 0.00 & 7.98 & 9.95 \\
\hline SW & 0.31 & 1.46 & 3.97 & 3.01 & 0.43 & 0.07 & 0.03 & 0.00 & 0.00 & 0.00 & 0.00 & 9.26 & 10.08 \\
\hline WSW & 0.20 & 0.74 & 2.17 & 2.45 & 0.84 & 0.29 & 0.04 & 0.01 & 0.00 & 0.00 & 0.00 & 6.74 & 11.77 \\
\hline W & 0.13 & 0.52 & 1.28 & 2.00 & 1.10 & 0.29 & 0.08 & 0.01 & 0.00 & 0.00 & 0.00 & 5.27 & 12.97 \\
\hline WNW & 0.12 & 0.52 & 1.62 & 3.01 & 1.53 & 0.48 & 0.11 & 0.01 & 0.00 & 0.00 & 0.00 & 7.36 & 13.13 \\
\hline NW & 0.18 & 0.55 & 2.13 & 3.53 & 1.43 & 0.43 & 0.09 & 0.01 & 0.00 & 0.00 & 0.00 & 8.33 & 12.70 \\
\hline \multirow[t]{2}{*}{ NNW } & 0.20 & 0.66 & 2.31 & 2.79 & 0.88 & 0.28 & 0.03 & 0.00 & 0.00 & 0.00 & 0.00 & 7.15 & 11.80 \\
\hline & & & & & & & & & & & CALM & 0.88 & \\
\hline$\%$ & 3.78 & 12.91 & 35.59 & 33.08 & 10.17 & 2.95 & 0.58 & 0.05 & 0.00 & 0.01 & 0.01 & 100.00 & 11.04 \\
\hline
\end{tabular}

* See footnote, Table F-1 
Table F-5

Kennedy Airport Surface Wind Data, 1200-1400 (Al1 Months)

Wind Speed* $(\mathrm{m} / \mathrm{s})$

\begin{tabular}{|c|c|c|c|c|c|c|c|c|c|c|c|c|c|}
\hline \multirow{2}{*}{$\begin{array}{c}\text { Wind } \\
\text { Direction } \\
\end{array}$} & \multicolumn{13}{|c|}{ Wind Speed* $(\mathrm{m} / \mathrm{s})$} \\
\hline & $0.5-2$ & $2-3$ & $3-5.4$ & 5.4-9 & $9-11$ & $11-14$ & $14-17$ & $17-21$ & $21-24$ & 24-29 & 29 & $\%$ & Mean \\
\hline $\mathbf{N}$ & 0.18 & 0.48 & 1.89 & 1.96 & 0.47 & 0.26 & 0.03 & 0.00 & 0.00 & 0.00 & 0.00 & 5.24 & 11.53 \\
\hline NNE & 0.13 & 0.43 & 1.36 & 1.43 & 0.60 & 0.15 & 0.05 & 0.02 & 0.00 & 0.00 & $0: 00$ & 4.13 & 11.74 \\
\hline $\mathrm{NE}$ & 0.16 & 0.49 & 1.48 & 1.33 & 0.31 & 0.20 & 0.03 & 0.01 & 0.01 & 0.00 & 0.00 & 4.03 & 11.17 \\
\hline ENE & 0.10 & 0.36 & 1.02 & 0.99 & 0.34 & 0.13 & 0.01 & 0.01 & 0.00 & 0.00 & 0.00 & 2.92 & 11.26 \\
\hline $\mathbf{E}$ & 0.09 & 0.39 & 0.96 & 1.03 & 0.21 & 0.07 & 0.03 & 0.00 & 0.00 & 0.02 & 0.00 & 2.77 & 11.06 \\
\hline ESE & 0.08 & 0.39 & 1.45 & 1.22 & 0.20 & 0.07 & 0.00 & 0.00 & 0.00 & 0.00 & 0.00 & 3.36 & 10.27 \\
\hline SE & 0.13 & 0.38 & 1.82 & 1.74 & 0.20 & 0.08 & 0.01 & 0.01 & 0.00 & 0.00 & 0.00 & 4.33 & 10.77 \\
\hline SSE & 0.08 & 0.48 & 3.06 & 3.67 & 0.83 & 0.23 & 0.04 & 0.00 & 0.00 & 0.00 & 0.00 & 8.41 & 11.53 \\
\hline $\mathbf{s}$ & 0.17 & 0.82 & 5.78 & 6.51 & 1.32 & 0.39 & 0.04 & 0.01 & 0.00 & 0.00 & 0.00 & 15.02 & 11.50 \\
\hline SSW & 0.15 & 0.73 & 3.56 & 3.86 & 0.59 & 0.18 & 0.04 & 0.00 & 0.00 & 0.00 & 0.00 & 9.09 & 11.21 \\
\hline SW & 0.24 & 0.74 & 2.83 & 3.25 & 0.67 & 0.14 & 0.03 & 0.00 & 0.00 & 0.00 & 0.00 & 7.87 & 11.49 \\
\hline WSW & 0.13 & 0.39 & 1.43 & 2.48 & 1.18 & 0.34 & 0.08 & 0.02 & 0.00 & 0.00 & 0.00 & 5.99 & 13.43 \\
\hline $\mathbf{W}$ & 0.08 & 0.19 & 1.05 & 2.22 & 1.53 & 0.53 & 0.11 & 0.00 & 0.01 & 0.00 & 0.00 & 5.68 & 14.63 \\
\hline WNW & 0.09 & 0.33 & 1.33 & 2.92 & 1.95 & 0.56 & 0.13 & 0.03 & 0.00 & 0.00 & 0.00 & 7.28 & 14.28 \\
\hline NW & 0.09 & 0.48 & 1.82 & 2.83 & 1.63 & 0.73 & 0.13 & 0.01 & 0.00 & 0.00 & 0.00 & 7.66 & 13.68 \\
\hline NNW & 0.12 & 0.60 & 1.67 & 2.33 & 0.90 & 0.23 & 0.05 & 0.00 & 0.00 & 0.00 & 0.00 & 5.84 & 12.24 \\
\hline & & & & & & & & & & & CALM & 0.38 & \\
\hline$\%$ & 1.90 & 7.70 & 32.49 & 39.72 & 12.78 & 4.18 & 0.71 & 0.09 & 0.02 & 0.02 & 0.00 & 100.00 & 12.22 \\
\hline
\end{tabular}

* See footnote, Tabie F-1 
Table F-6

Kennedy A1rport Surface Wind Data, 1500-1700 (Al1 Months)

Wind Speed* $(\mathrm{m} / \mathrm{s})$

\begin{tabular}{|c|c|c|c|c|c|c|c|c|c|c|c|c|c|}
\hline \multirow{2}{*}{$\begin{array}{l}\text { Wind } \\
\text { Direction }\end{array}$} & \multicolumn{13}{|c|}{ Wind Speed $*(\mathrm{~m} / \mathrm{s})$} \\
\hline & $\underline{0.5-2}$ & $2-3$ & $3-5.4$ & $5.4-9$ & $9-11$ & $11-14$ & $14-17$ & $17-21$ & $21-24$ & $24-29$ & 29 & $\%$ & Mean \\
\hline $\mathbf{N}$ & 0.17 & 0.60 & 1.72 & 1.66 & 0.45 & 0.09 & 0.01 & 0.00 & 0.00 & 0.00 & 0.00 & 4.65 & 11.06 \\
\hline NNE & 0.12 & 0.43 & 1.16 & 1.16 & 0.41 & 0.15 & 0.00 & 0.00 & 0.00 & 0.00 & 0.00 & 3.44 & 11.45 \\
\hline $\mathrm{NE}$ & 0.10 & 0.49 & 1.27 & 1.11 & 0.38 & 0.20 & 0.03 & 0.01 & 0.01 & 0.00 & 0.00 & 3.54 & 11.41 \\
\hline ENE & 0.12 & 0.43 & 0.98 & 0.88 & 0.28 & 0.08 & 0.01 & 0.02 & 0.00 & 0.00 & 0.00 & 2.78 & 10.86 \\
\hline E & 0.14 & 0.43 & 1.20 & 1.26 & 0.23 & 0.08 & 0.03 & 0.00 & 0.00 & 0.02 & 0.00 & 3.38 & 10.86 \\
\hline ESE & 0.13 & 0.70 & 1.76 & 1.12 & 0.18 & 0.06 & 0.01 & 0.00 & 0.00 & 0.00 & 0.00 & 3.96 & 9.38 \\
\hline SE & 0.15 & 0.61 & 2.33 & 1.67 & 0.33 & 0.05 & 0.00 & 0.00 & 0.00 & 0.00 & 0.00 & 5.08 & 10.09 \\
\hline SSE & 0.14 & 0.72 & 3.21 & 3.91 & 1.08 & 0.22 & 0.02 & 0.00 & 0.00 & 0.00 & 0.00 & 9.29 & 11.36 \\
\hline$s$ & 0.22 & 1.21 & 5.38 & 6.79 & 2.16 & 0.74 & 0.06 & 0.01 & 0.00 & 0.00 & 0.00 & 16.53 & 12.06 \\
\hline SSW & 0.25 & 0.99 & 2.97 & 3.86 & 1.13 & 0.28 & 0.04 & 0.00 & 0.00 & 0.00 & 0.00 & 9.48 & 11.57 \\
\hline SW & 0.26 & 0.65 & 2.29 & 2.03 & 0.42 & 0.05 & 0.02 & 0.00 & 0.00 & 0.00 & 0.00 & 5.68 & 10.89 \\
\hline WSW & 0.16 & 0.35 & 1.64 & 2.28 & 0.99 & 0.31 & 0.07 & 0.03 & 0.00 & 0.00 & 0.00 & 5.81 & 13.14 \\
\hline $\mathbf{W}$ & 0.08 & 0.24 & 1.10 & 2.35 & 1.26 & 0.42 & 0.06 & 0.01 & 0.00 & 0.00 & 0.00 & 5.48 & 13.98 \\
\hline WNW & 0.08 & 0.24 & 1.48 & 3.21 & 1.89 & 0.51 & 0.12 & 0.01 & 0.00 & 0.00 & 0.00 & 7.53 & 14.17 \\
\hline NW & 0.10 & 0.33 & 1.71 & 3.28 & 1.61 & 0.55 & 0.12 & 0.00 & 0.00 & 0.00 & 0.00 & 7.70 & 13.87 \\
\hline NNW & 0.08 & 0.38 & 1.53 & 2.19 & 0.74 & 0.28 & 0.03 & 0.00 & 0.00 & 0.00 & 0.00 & 5.28 & 12.78 \\
\hline & & & & & & & & & & & CALM & 0.38 & \\
\hline$\%$ & 2.22 & 8.78 & 31.72 & 38.74 & 13.48 & 4.02 & 0.56 & 0.05 & 0.01 & 0.02 & 0.00 & 100.00 & 12.14 \\
\hline
\end{tabular}

*See footnote, Table F-1 
Table $F-7$

Probability of Wind Speed Categories versus Time of Day

Time of Day
A11 Hours
$0000-0200$
$0600-0800$
$0900-1100$
$1200-1400$
$1500-1700$

\begin{tabular}{lll} 
& Wind Speed $(\mathrm{m} / \mathrm{s})$ \\
\hline $0-3.3$ & $\frac{3.3-5.4}{0.35}$ & $\frac{\geq 5.4}{0.41}$ \\
0.24 & 0.36 & 0.30 \\
0.34 & 0.36 & 0.34 \\
0.30 & 0.36 & 0.54 \\
0.18 & 0.33 & 0.57 \\
0.10 & 0.32 & 0.57 \\
0.11 & &
\end{tabular}

Table $F-8$

Probability of Wind Speed Directions versus Time of Day

Wind Direction

\begin{tabular}{|c|c|c|c|c|c|c|c|c|}
\hline Time of Day & $\mathrm{N}$ & $\mathrm{NE}$ & $\mathrm{E}$ & SE & $\mathrm{S}$ & SW & $\mathrm{W}$ & NW \\
\hline A11 Hours & 0.13 & 0.11 & 0.07 & 0.07 & 0.17 & 0.17 & 0.13 & 0.15 \\
\hline $0000-0200$ & 0.15 & 0.12 & 0.06 & 0.04 & 0.12 & 0.19 & 0.16 & 0.16 \\
\hline $0600-0800$ & 0.17 & 0.14 & 0.06 & 0.04 & 0.10 & 0.17 & 0.15 & 0.17 \\
\hline $0900-1100$ & 0.14 & 0.12 & 0.07 & 0.07 & 0.15 & 0.17 & 0.12 & 0.16 \\
\hline $1200-1400$ & 0.10 & 0.08 & 0.06 & 0.10 & 0.24 & 0.15 & 0.12 & 0.15 \\
\hline $1500-1700$ & 0.09 & 0.07 & 0.07 & 0.12 & 0.26 & 0.13 & 0.12 & 0.14 \\
\hline
\end{tabular}

Table F-9

Approximate Joint Frequency Probabilities for Wind Speed and Direction (All Months, All Hours)

Wind Direction

\begin{tabular}{|c|c|c|c|c|c|c|c|c|}
\hline Wind Speed $(\mathrm{m} / \mathrm{s})$ & $\mathbf{N}$ & $\mathrm{NE}$ & $\mathbf{E}$ & SE & $\mathrm{S}$ & SW & $\mathrm{W}$ & $\mathrm{NW}$ \\
\hline $0-3.3$ & 0.033 & 0.036 & 0.024 & 0.019 & 0.036 & 0.043 & 0.024 & 0.024 \\
\hline $3.3-5.4$ & 0.049 & 0.035 & 0.020 & 0.028 & 0.066 & 0.066 & 0.042 & 0.042 \\
\hline$>5.4$ & 0.050 & 0.037 & 0.025 & 0.025 & 0.070 & 0.058 & 0.070 & 0.083 \\
\hline
\end{tabular}




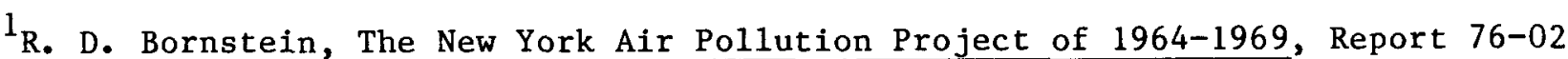
(San Jose, CA: San Jose State Univ. Department of Meteorology, 1976).

${ }^{2}$ Uniform Summary of Surface Weather Observations (July 48-June 65) for Kennedy Airport, New York, Part C-Surface Winds (Asheville, NC: Air Weather Service).

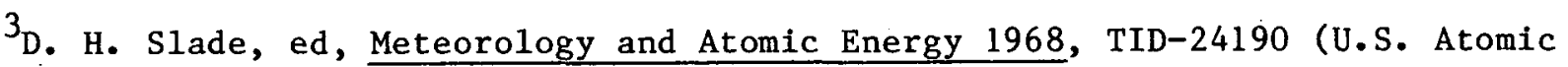
Energy Commission, 1968). 
Biological effects of radiation are manifestations of the localized deposition of energy in molecules along the path traveled by the radiation. The ionizations and excitations caused by this deposition can directly or indirectly alter both the chemical composition and the chemical equilibrium within tissue cells along the path of the radiation. 1 The possible effects of this energy deposition range from undetectable changes to acute physiological changes, carcinogenesis, or genetic effects, depending on the amount and type of incident radiation, the type of cells irradiated, and the time span over which irradiation occurs. These effects have been the subject of considerable research since the early part of the 20th century. This appendix will not attempt to discuss in detail the extensive literature which has been developed but will summarize those aspects of radiological health effects which bear directly on this environmental assessment.

\section{G1. Specific Radiological Health Effects}

\section{G1.1 Acute Physiological Changes}

Acute physiological changes due to radiation exposure are normally associated with relatively large absorbed doses received over a short period of time. Data on these effects in humans are derived primarily from studies of Japanese atomic bomb casualties, ${ }^{2}$ studies of some radiation therapy patients, ${ }^{3}$ and studies of a few recipients of high acute doses from industrial accidents in the early days of the nuclear weapons development program. 4

The acute physiological changes of interest can be divided into two groups: early morbidities and early fatalities. These effects are defined somewhat arbitrarily as occurring within 1 year of the radiation exposure in question. 5 . Two mechanisms are considered for early fatality: acute bone marrow irradition and acute pulmonary irradiation.* Dose-response curves for the two early fatality possibilities evaluated are shown in Figures G-1 and G-2. The derivation of the curves, including experiments and uncertainties, is discussed in Reference 5.

Early morbidities are analyzed somewhat more qualitatively because the effects of sublethal doses are not as well understood. 5 The approach taken here is to compute the number of people recelving greater than some threshold dose to a particular organ or body system (e.g., bone marrow). These thresholds represent acute doses

\footnotetext{
*Acute gastrointestinal exposure could also cause early fatalities but, as explained in subsection G3.1, this early fatality mechanism is not considered.
} 


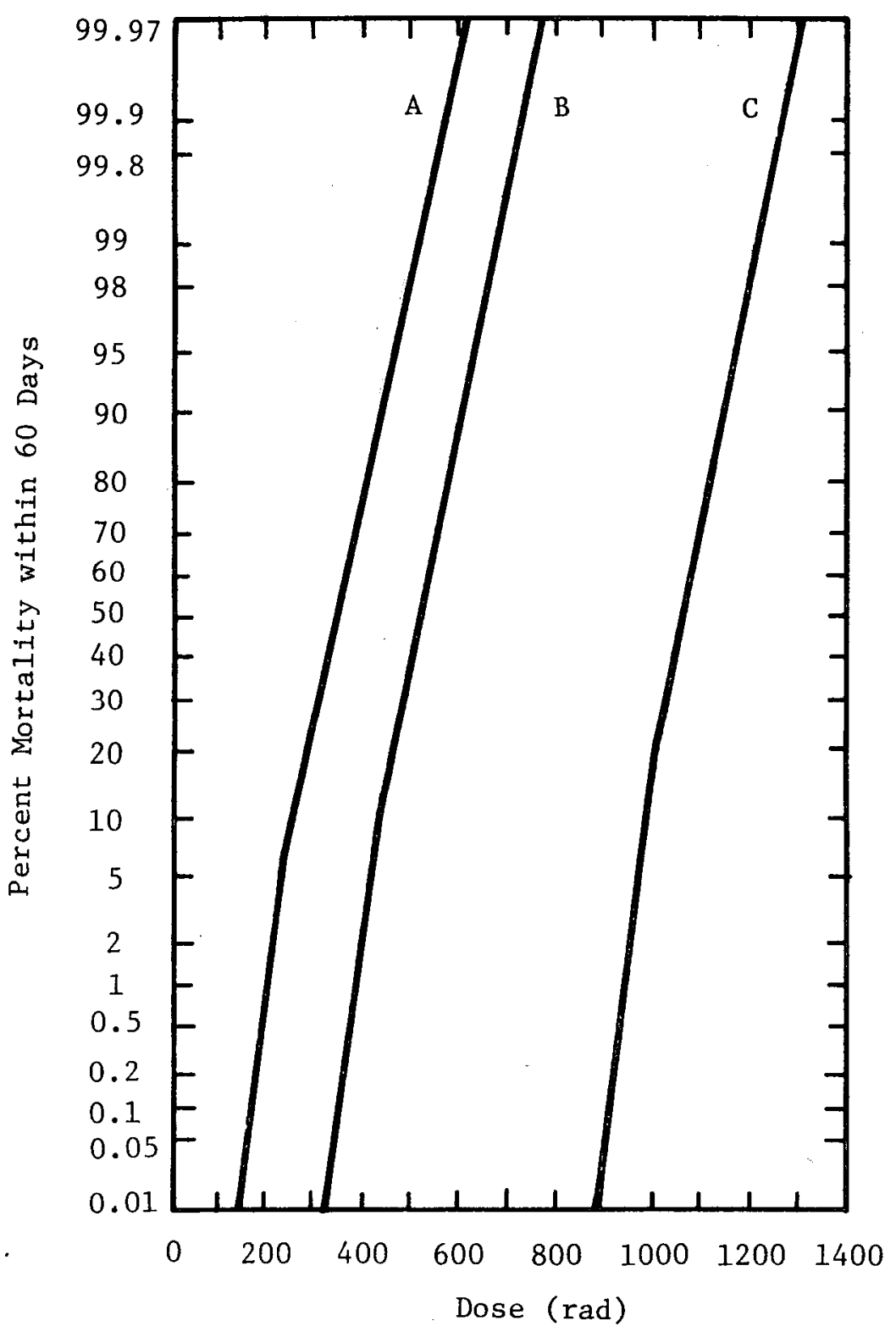

Figure G-1. Estimated Dose-Response Curves. The percentage of mortality within 60 days of acute bone marrow irradiation is plotted for doses requiring (A) minimal treatment (basic hospitalization), (B) supportive treatment (barrier nursing, antibiotics, transfusion), and $(C)$ heroic treatment (bone marrow transplants, etc.). (From References 5 and 27.) 


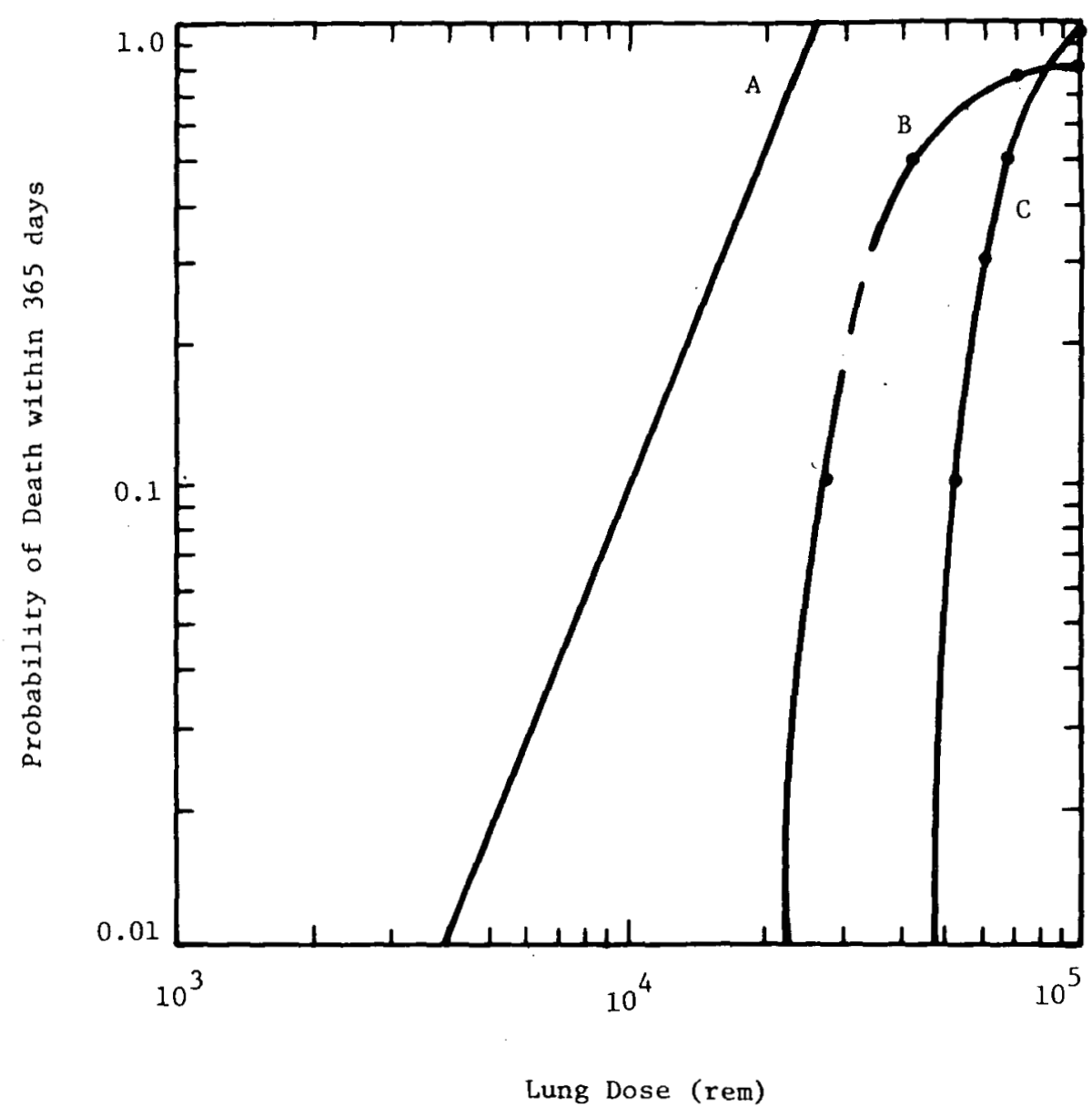

A. Yttrium-90 and -91 were the isotopes used to obtain this curve. It is equally valid for other short half-life beta or garma emitting isotopes which result in approximately the same dose rate. This curve is used for all short half-life materials potentially encountered in transportation accidents. 5

B. This curve is based on data using $5 r-90 / Y-90$ inhalation by beagles, and is used for long half-life, low-LET* radiation. 37

C. This curve is based on data from Pu-239 inhalation by beagles, and is used for long half-life, high-LET* radiation. 37

*LET (Linear Energy Transport) is a measure of the energy deposited per unit distance traveled in a particular medium. High LEF radiation includes $\alpha$-particles and fast neutrons; low LET radiation includes $x$-rays, $\gamma$-rays, and $\beta$-particles.

Figure G-2. Dose response curves for mortality due to acute pulmonary effects of radiation 
above which some type of debilitating physiological response is possible. This group of people could be referred to as "potential radiation-induced morbidities." Latent cancer fatalities which might occur in this group are not considered separately, and synergistic effects between doses to separate organs or between radiation exposures and other effects, such as old age or poor health, are not considered. This clearly produces some "double counting," but it should have a small effect on the final estimates of early morbidities. Values of morbidity thresholds for various organs are given in Table G-1.

Table G-1

Morbidity Thresholds 5

$\begin{array}{lcll}\text { Organ } & \begin{array}{c}\text { Threshold Dose } \\ \text { (rem) }\end{array} & & \text { Physiologica1 Result } \\ \text { Marrow } & 75 & & \text { Radiation Syndrome* } \\ \text { Lung } & 3000 & & \text { Radiation Pneumonitis } \\ \text { GI Tract } & 1000 & & \text { Stem-Cell Loss } \\ \text { Gonads } & 50 & \text { Transient Sterility }\end{array}$

*Radiation syndrome is a group of symptoms which are normally associated with large acute whole-body exposures, such as nausea, vomiting, etc.

\section{G1.2 Long-Term Somatic and Genetic Effects}

Both long-term somatic and genetic effects are evaluated. These effects are quantified using statistical data from Reference 4 which relate expected occurrences to overall integrated population exposure.

There is considerable controversy surrounding the appropriate choice of risk models for the evaluation of radiation carcinogenesis. The choice involves the use of either a relative risk model or an absolute risk model. Relative risk is defined as the ratio of the risk in those exposed to the risk to those not exposed (incidence in exposed populations to incidence in control populations). 6 Absolute risk is defined as the "product of assumed risk times the total population at risk." The absolute risk model is used here since it provides a better indication than the relative risk model of the impact in terms of the total number of deaths in a population due to a disease. Cancer fatalities which occur in a given population are variable and may be influenced by many factors. In addition, a 30-year plateau is assumed for the period of risk, as compared to the lifetime plateau suggested by some individuals and organizations. These points of controversy will not be settled in the near future and are acknowledged as important issues warranting further discussion and investigation. The net effect of using the relative risk model 
and a lifetime plateau would be to increase the estimates of latent cancer fatalities by less than an order of magnitude (roughly a factor of 7 ).

\section{G1.3 Carcinogenesis}

Fatal cancers account for between $16 \%$ and $20 \%$ of all deaths in the U.S. ${ }^{7} 8$ These cancers are divided into three broad groups: carcinomas, sarcomas, and leukemias or lymphomas. Within these groups, there are 100 or so distinct varieties of disease based on the original site of the malignancy.

There are many theories of carcinogenesis, but most researchers acknowledge that a statistical correlation can be established between certain environmental factors and cancer induction. Examples include the correlation between smoking and lung cancer and the correlation between radiation dose and leukemia among atomic bomb survivors. The correlation between exposure to radiation and cancer induction has been qualitatively established for animal exposure and is generally accepted for human exposures, although the physiological mechanisms involved are not understood. 6 g-1l Statistical analysis of large numbers of exposed personnel, such as Japanese atomic bomb survivors, uranium miners, fluorspar miners, radium dial painters, etc., permits rough predictions of numbers of latent cancer fatalities per million person rem of population exposure. In general, this information is based on investigations of situations involving relatively high doses and dose rates delivered by either specific radionuclides (such as Ra-226) or specific sources (such as nuclear weapon explosions). In this assessment and in other studies, however, the use of these values is expanded with the understanding that the effects of varying the nature of the radiation may alter the results.

In the quantification of carcinogenesis in this assessment, a linear dose-response model is assumed. However, for doses from low-LET radiation sources which are accrued at dose rates less than 1 rem per day ( 400 rem per year), a "dose-rate effectiveness factor" of 0.2 is assumed, based on conclusions extracted from Reference 5. For doses from low-LET radiation sources at dose rates greater than 1 $\mathrm{rem} / \mathrm{d}$, a dose-rate effectiveness factor of 1.0 is assumed. This means that if a population segment receives a given total dose at a rate of less than $1 \mathrm{rem} / \mathrm{d}$, the cancer induction rates in that population segment would be $20 \%$ of those predicted by the dose response model for a similar population segment receiving the same total dose at a rate greater than $1 \mathrm{rem} / \mathrm{d}$. For populations exposed to mixed dose rates, a population weighted dose rate effectiveness factor is used. In all cases, high-LET radiation is assigned a factor of 1.0 .

Expected latent cancer fatality rates for a specified integrated population exposure to various organs are shown in Table G-2 for a dose-rate effectiveness factor of 1.0. A brief discussion of the origin of each of these values follows.

Leukemia

The source of data for expected leukemia fatalities from radiation is the BEIR Report 6 as modified by the age' distribution of the U.S. population. 5 Thus, although the in utero leukemia death rate due to irradiation is much higher than the corresponding death rate for other age groups, the fraction of pregnant women in the general population is small, and the overall leukemia death rate is somewhat lower than the fetal rate alone. The computed value of 28.4 leukemia fatalities per million person rem is consistent with values suggested for high dose rates in Reference 12 . 
Table G-2

Expected Latent Cancer Fatalities per $10^{6}$
Person Rem Exposure to the Population
(see References 1,8 , and 12)

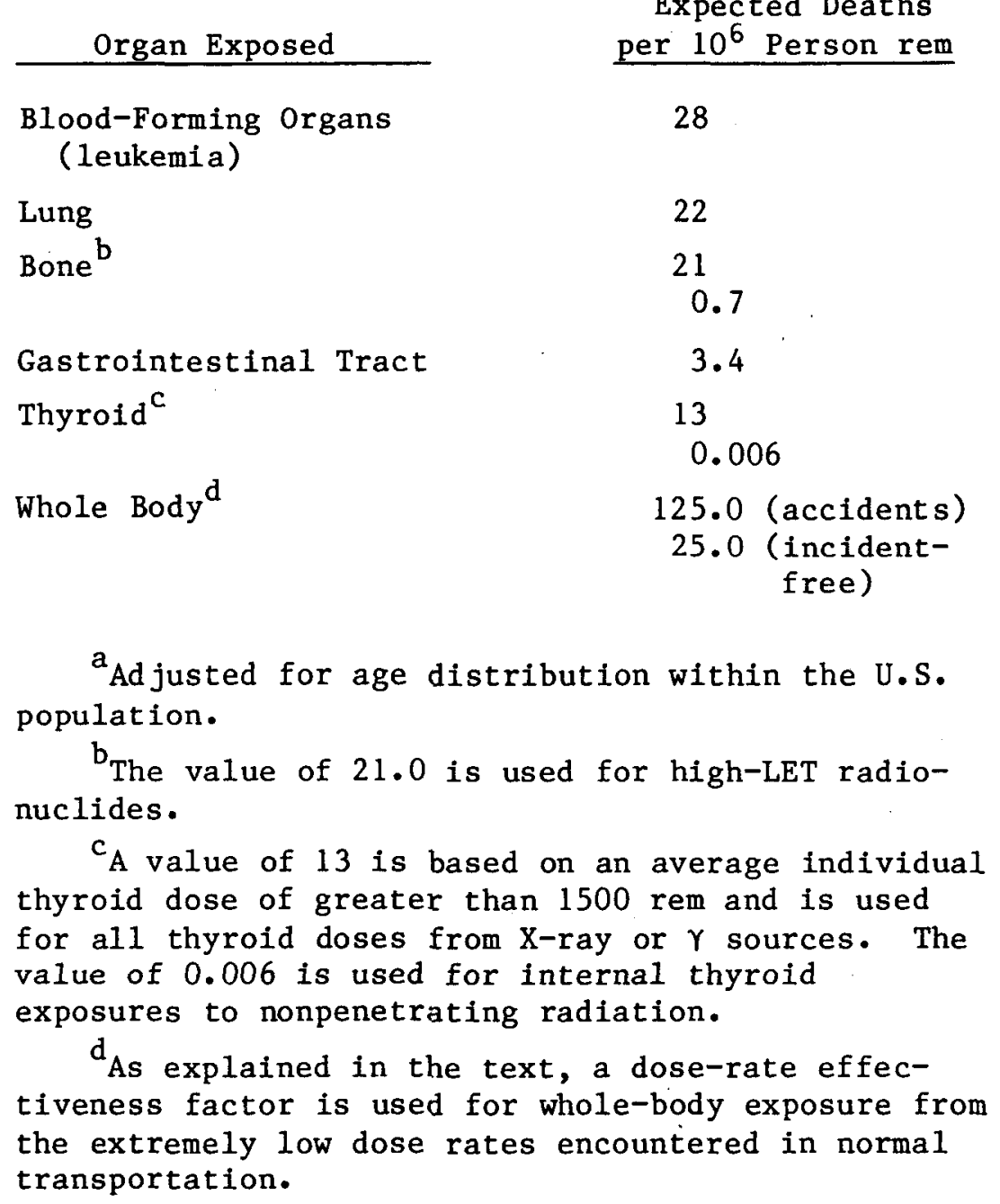

Lung

The primary sources of data on lung cancer rates are also References 5, 12, and 18. These data do not distinguish between smokers and non-smokers and do not consider the "hot particle hypothesis"13 which has not received widespread acceptance in the scientific community. 1417 Reference 13 gives a value of 200 lung cancers per million person rad for high-LET radiation which is consistent with the value of 22 per million person rem chosen for this assessment. 18 
Bone

The principle data source used for bone cancer fatality risk values is Reference 5, which uses age group adjustment factors to derive a value of 6.9 bone cancer fatalities per million person rem based on information in Reference 8 . More recent information suggests that this value may be low by as much as a factor of 3 for longlived, bone-seeking alpha emitters such as $\mathrm{Pu}-239$ and Am-241, and high by as much as a factor of 10 for shorter half-life nuclides emitting low-LET radiation. 18 19 Two values are therefore included, and bone seekers are segregated into high-LET/ long half life or low-LET/short half life groups.

\section{Gastrointestinal Tract}

The data base for radiation-induced cancers of the GI tract derives principally from high-dose-rate X-ray exposures. Current animal experimentation at varying doses and dose rates has failed to show pathological changes in these tissues.20 There does, however, appear to be significant variation of radiation damage in the various portions of the GI tract. 52021 The value of 3.4 cancer fatalities per million person rem was chosen based on Reference 5. This does not account for the variable sensitivity of the different segments of the GI tract.

\section{Thyroid}

The degree of susceptibility of the thyroid to carcinogenesis is not universally agreed upon. In terms of cancer incidence (not fatalities), values ranging from 0.064 per million person rem (see Reference 5) to 230 per million person rem can be obtained (see References 5, 22-25). The two most significant factors in this wide variation are (1) age at irradiation and (2) whether the radiation source was external (as in patients treated for head and neck disorders with X-rays) or internal (as in persons receiving doses from I-131 in fallout). For the quantification of fatalities from thyroid cancers, this assessment uses a $10 \%$ fatality rate as suggested in Reference 5 ( $p$ 9.26), and uses the value of 134 thyroid cancers per million person rem for external irradiation (consistent with References 5 and 22), and 0.06 thyroid cancers per million person rem for internal irradiation (see again Reference 5). This choice of values is consistent with References 5 and 22 and is an intermediate value among those found in the literature.

\section{External Whole-Body Irradiation}

Using a linear dose response model, external whole-body exposure has been estimated to result in 125 fatal malignancies (including 25 leukemias) at high dose rates (above about $10 \mathrm{rad} / \mathrm{min}$ ), and 25 fatal malignancies (including 5 leukemias) at lower dose rates (less than $0.01 \mathrm{rad} / \mathrm{min}$ ) per million person rem. 18 If a sigmoid dose response relationship is assumed for low-LET radiation, the values for fatal malignancies become 0.5 and 0.02 respectively.18 For the purposes of this study, the linear model is assumed, so that 125 fatal malignancies per million person rem whole-body exposure is used in the case of accidents where dose and dose rates can be large, and 25 fatal malignancies per million person rem whole-body exposure is used in the case of radiation exposure from accidents where individual doses and dose rates are small, and also for incident-free transport of radioactive material where the individual doses and dose rates are extremely small. 


\section{G1.4 Genetic Effects}

Genetics is the study of heredity. Specific linear base sequences of the nucleic acids in a cell determine the activities of the cell and the characteristics of the individual. The base sequences are carried in the chromosomes and are transmitted to the next generation when the cell divides. A change in any specific linear sequence, commonly called a mutation, changes the information which is passed on.

Mutations are usually detrimental, and every individual appears to carry a "load" of defective genes which collectively tends to reduce his overall fitness to some degree. Favorable and unfavorable mutations tend to equalize through the evolutionary process. 21 Concern has arisen in the radiobiology community because of laboratory work which has shown radiation to be mutagenic in lower life forms such as drosophila (fruit flies) and various species of mice. These data have been extrapolated to dose-effect relationships in man, 52126 although this extrapolation is tenuous and possibly inaccurate.

When evaluating genetic effects, the significant dose is that received by the gonads. If integrated gonadal exposure is known, statistical data similar to that used to quantify carcinogenesis can be used to estimate the number of various types of genetic effects which might be expected to occur in all subsequent generations as a result of that exposure. Values for the four types of genetic effects considered are shown in Table G-3, assuming a doubling dose (the dose of radiation which induces the same number of mutations as arise spontaneously in one generation) of $100 \mathrm{rem}$. These values account for the variation in child-bearing probability as a function of parental age by using statistics on live births as a function of paternal age. 5

Table G-3

Genetic Effects Risk Coefficients ${ }^{a}$ (see Reference 5)

\section{Genetic Effect}

Single-gene disorders

Multifactorial disorders

Congenital disorders

Spontaneous abortions

Total Genetic Effects
Cases (in all subsequent generations) per $10^{6}$ Person-Rem to Gonads

\footnotetext{
${ }^{\mathrm{a}}$ Assuming a doubling dose of $100 \mathrm{rem}$

$b_{\text {Upper limit of range } 8.4-84}$
} 


\section{G2. Radiation Exposure Pathways}

To relate the health effects resulting from individual and integrated radiation exposure to an organ dose, specific radiation exposure pathways must be considered. These radiation exposure pathways include ingestion, external irradiation by radionuclides in the environs of persons, and inhalation of radionuclides.

\section{G2.1 Ingestion of Radionuclides}

Of all transported radionuclides, only isotopes of iodine, strontium, and cesium are important from an ingestion viewpoint. 527 The only credible means by which these radionuclides might be accidentally ingested is by consumption of either foodstuffs or drinking water.

The scope of this assessment is limited to events occurring in urban areas. Under this constraint the following assumptions are made:

- Very little food will be both produced and consumed within the urban area (i.e., from home gardens, etc.);

- Contamination of foodstuffs in markets will be minimal, and items known to be contaminated will be confiscated by health authorities; and

- For transportation accidents, outlying areas where significant food production occurs will be far enough from the high-density urban areas that contamination may not be significant.

Under these assumptions, consumption of contaminated drinking water becomes the only potential pathway for accidental ingestion of radionuclides released to the environment from a transportation accident in an urban area.

The capacity of the various reservoirs in the New York City water system is between $0.9 \times 10^{9}$ and $144 \times 10^{9}$ gallons.28 The maximum permissible concentrations (MPC) in water for the radionuclides of primary interest are $2 \times 10^{-} 5 \mu \mathcal{C}_{\mathrm{m} / \mathrm{m} \ell}(\mathrm{I}-131), 10^{-} 6$ $\mu \mathrm{Ci} / \mathrm{m} \ell(\mathrm{Sr}-90)$, and $10^{-8} \mu \mathrm{Ci} / \mathrm{ml}(\mathrm{Cs}-137) .29$ Therefore, a release of between 68 and $1.09 \times 10^{4}$ curies of $\mathrm{I}-131,3.4$ and 544 curies of $\mathrm{Sr}-90$, or 0.034 and 5.45 curies of Cs-137 directly into reservoirs within this water system would be required to exceed their respective MPCs. A typical I-131 package shipped in 1974 contained less than 10 curies (see Reference 27 ), so I-131 can be eliminated as an ingestion hazard by assuming that the contaminant is completely soluble and uniformly mixed in the reservoir and by assuming concentrations of less than the MPC will not make a significant radiological dose contribution to the overall environmental impact. Although $\mathrm{Cs}-137$ and $\mathrm{Sr}-90$ are occasionally shipped in quantities large enough to exceed the MPC, it is anticipated that if either of these radionuclides were spilled into a reservoir, action would immediately insure that a minimal amount of contaminated water would be consumed. This would minimize the radiological consequence at the expense of a potentially high socioeconomic consequence.

\section{G2.2 Externa1 Radiation Exposure}

External irradiation can result from incident-free transport, from an accident involving loss of shielding from a nondispersible radioactive material, from cloudshine (external dose from radionuclides in a passing cloud), or from groundshine (external dose from deposited radionuclides) following an accident finvolving dispersal of radioactive material. This type of radiation exposure is assumed to be 
whole-body, low-LET, penetrating radiation. The critical organs are total bone marrow and gonads. - Doses to the skin, lens of the eye, or other external doses are not specifically evaluated.

\section{G2.3 Inhalation of Radionuclides}

The basic model used to describe the inhalation and physiological transport of radionuclides is the ICRP Task Group II Lung Mode1, 30 shown schematically in Figure G-3. The model has been used extensively and is only briefly described here.

Large particles ( $>10$ micrometres $(\mu \mathrm{m})$ in aerodynamic diameter [AMAD]) are selectively deposited from inspired air in the nasopharyngeal passages. They are captured in the mucoid lining of the passages, transported by the cilia with the mucus drainage, and eventually swallowed (pathway (b) on Figure G-3). Intermediate sized particles ( 1 to $10 \mu \mathrm{m}$ in AMAD) are deposited principally in the pulmonary or nasopharyngeal region, with a small fraction depositing in the tracheobronchial region. Some of the particles also become entrained in the mucoid lining and are moved upward towards the pharynx by muco-ciliary action for eventual deposition into the upper GI tract (pathway (d) in Figure G-3). In addition, a small number of these particles are dissolved in blood (pathway (c) on Figure G-3). Small particles $(<1 \mu \mathrm{m}$ in $A M A D)$ are preferentially deposited in the pulmonary region. They come in direct contact with the alveoli and are rapidly phagocytized and localized in the reticuloendothelial cells of the alveoli.

The effects of particle size on the pulmonary solubilization rate (pathway (e) on Figure G-3) have been investigated. $31-35$ The consensus is that, for spherical particles, the dissolution rate for a chemical form of given physiological solubility varies according to particle size. Thus, the lung dose for a given lung burden would be smaller for smaller particles since they would dissolve more rapidly. However, other organ doses would be correspondingly larger. This effect is not specifically included in the model. Some work has also been done on phagocytosis rates (pathway ( $g$ ) on Figure G-3) with the general conclusion that the effect of particle size is a small one, particularly for particles larger than $1 \mu \mathrm{m} .36$ No information is currently available on particle size effects on ciliary transport (pathways ( $f$ ) and $(k)$ on Figure G-3) although those effects are also considered to be small.

Depending on its chemical nature, the radionuclide may translocate after being deposited in the lung and will cause the most significant biological damage to the critical organ (or organs). The dose received by the organ or organs determines the most significant biological effects of the exposure.

\section{G3. Dosimetric Data}

This section is divided into two subsections. The first discusses the values used for rem per curie inhaled for each isotope. The second discusses miscellaneous additional dosimetric factors.

\section{G3.1 Rem-per-Curie Values}

The values for rem per curies have been obtained from two main sources. The principal sources are the INREM codes which provide dose equivalents in units of rem per microcurie for inhalation of $1-\mu$ m particles. 3839 


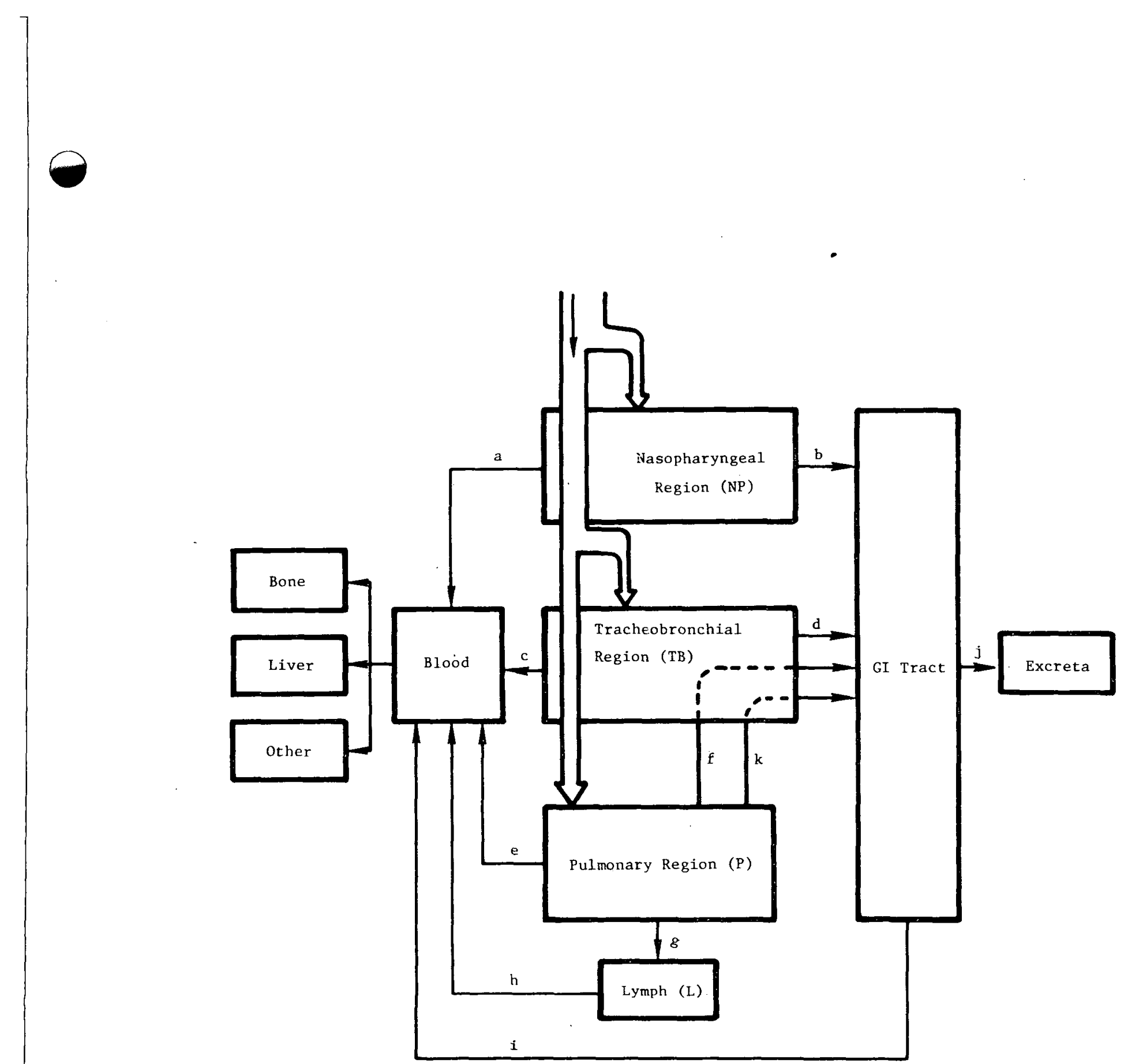

i

a. Nasopharyngeal absorption in blood

b. and d. Muco-ciliary translocation to upper GI tract

c. Tracheobronchial absorption in blood

e. Alveolar diffusion (solubilization)

f. Short-term and $k$. Long-term muco-ciliary translocation of phagacytized material to tracheobronchial region

g. Absorption into lymphatic system

h. Transfer to venous system

i. Gastrointestinal absorption in.blood

j. Excretion from GI tract as feces or absorption from GI tract and excretion as urine

Figure G-3, Biological Pathways for Inhaled Material 
Dispersal of spent fuel presents a unique problem in that the exposed population may be exposed to a mixture of radionuclides. This may also be true of mixtures of plutonium isotopes. In order to address this aspect of the problem, the rem-percurie values for the various released isotopes were weighted by the curies released from a postulated incident. These values were then combined to give a single set of rem-per-curie inhaled values for the release of the postulated mixture of isotopes.

Because the analysis used for this assessment adjusts the pulmonary deposition fraction based on actual particle size, the rem-per-curie inhaled values have been converted to rem-per-curie deposited values. Values for all isotopes are given in Table G-4.

\section{G3.2 Additional Dosimetric Parameters}

Several additional dosimetric parameters are required to perform the calculations. These include total photon energy per disintegration, average photon energy, airborne fraction, particle size, resuspension dose factor, lung mortality type, bone cancer type, thyroid cancer type, and cloudshine dose factor. These parameters are discussed in the following subsection, and the results are summarized in Table G-5. Also included in this table are values for radiological half life of the materials which are needed for the economic impact calculations to be discussed in Appendix $\mathrm{K}$.

\section{Total Photon Energy per Disintegration}

The values for total photon energy per disintegration were computed using energy level diagrams in Reference 40. Each decay scheme was examined, and each fractional occurrence and its associated photon energy were multiplied and summed. The effects of daughter products were included where appropriate. An average value for spent fuel was computed using the previously discussed isotopic mixtures together with energy level diagrams in Reference 40.

Average Energy of Emitted Photons

The average energy of a photon emitted by a particular radionuclide was calculated using decay schemes in Reference 40. The photon energies were weighted by their respective fractional occurrences.

\section{Airborne Fraction 27}

The fraction of material released in an accident which becomes airborne depends upon the accident environment. A container may be crushed beneath a truck, in which case very little material becomes airborne; or it may bounce into the air following the impact and disperse its entire contents. For most small packages, the fraction which becomes airborne is assumed to be 1.0 . However, certain shipments, notably fuel cycle and waste material, may involve large quantities of material ( $10^{5}$ to $10^{6}$ grams per package). An assumption of unity airborne fraction for such shipments would be excessively conservative, since it would be difficult, if not impossible, to make such large amounts of material airborne.

The methods by which material becomes airborne can be divided into four principal categories: (1) wind resuspension of spilled contents (also covered under resuspension), (2) impact or fire-driven pressure rupture, (3) fire entrainment of 
Table G-4

Values Used for Rem per Curfe Deposited

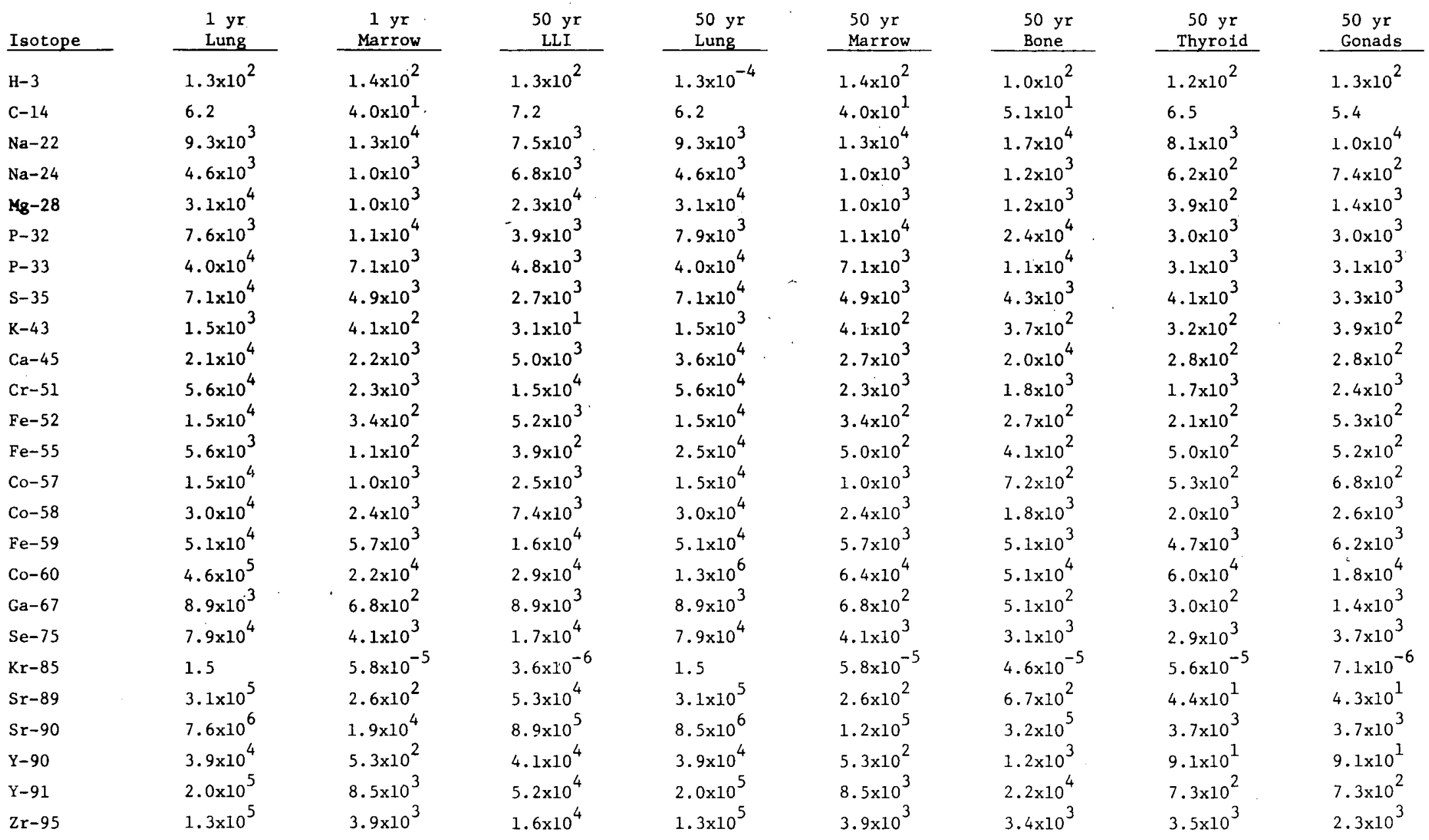


Table G-4 (Contjnued)

\begin{tabular}{|c|c|c|c|c|c|c|c|c|}
\hline Isotope & $\begin{array}{l}1 \mathrm{yr} \\
\text { Lung }\end{array}$ & $\begin{array}{c}1 \mathrm{yr} \\
\text { Marrow }\end{array}$ & $\begin{array}{c}50 \mathrm{yr} \\
\mathrm{LLI}\end{array}$ & $\begin{array}{l}50 \mathrm{yr} \\
\text { Lung }\end{array}$ & $\begin{array}{l}50 \text { yr } \\
\text { Marrow }\end{array}$ & $\begin{array}{l}50 \text { yr } \\
\text { Bone } \\
\end{array}$ & $\begin{array}{c}50 \text { yr } \\
\text { Thyroid }\end{array}$ & $\begin{array}{l}50 \mathrm{yr} \\
\text { Gonads }\end{array}$ \\
\hline $\mathrm{Nb}-95$ & $3.1 \times 10^{4}$ & $1.6 \times 10^{3}$ & $7.2 \times 10^{3}$ & $3.1 \times 10^{4}$ & $1.6 \times 10^{3}$ & $1.2 \times 10^{3}$ & $1.4 \times 10^{3}$ & $1.6 \times 10^{3}$ \\
\hline Mo-99 & $4.0 \times 10^{3}$ & $7.1 \times 10^{2}$ & $5.0 \times 10^{2}$ & $4.0 \times 10^{3}$ & $7.1 \times 10^{2}$ & $1.3 \times 10^{3}$ & $3.7 \times 10^{2}$ & $3.5 \times 10^{2}$ \\
\hline Tc-99m & $8.5 \times 10^{1}$ & $1.5 \times 10^{1}$ & $1.7 \times 10^{1}$ & $8.5 \times 10^{1}$ & $1.5 \times 10^{1}$ & $1.1 \times 10^{1}$ & $1.3 \times 10^{2}$ & $1.2 \times 10^{1}$ \\
\hline Ru-103 & $5.8 \times 10^{4}$ & $1.2 \times 10^{3}$ & $1.2 \times 10^{4}$ & $5.8 \times 10^{4}$ & $1.2 \times 10^{3}$ & $9.2 \times 10^{2}$ & $1.0 \times 10^{3}$ & $1.2 \times 10^{3}$ \\
\hline Ru-106 & $2.5 \times 10^{6}$ & $5.6 \times 10^{3}$ & $1.4 \times 10^{5}$ & $3.9 \times 10^{6}$ & $9.3 \times 10^{3}$ & $1.0 \times 10^{4}$ & $9.2 \times 10^{3}$ & $7.7 \times 10^{3}$ \\
\hline In-111 & $2.7 \times 10^{3}$ & $4.3 \times 10^{2}$ & $2.7 \times 10^{3}$ & $2.7 \times 10^{3}$ & $4.3 \times 10^{2}$ & $2.1 \times 10^{2}$ & $8.3 \times 10^{1}$ & $6.0 \times 10^{2}$ \\
\hline$S n-113$ & $6.7 \times 10^{4}$ & $3.5 \times 10^{3}$ & $1.4 \times 10^{4}$ & $6.7 \times 10^{4}$ & $3.5 \times 10^{3}$ & $5.7 \times 10^{3}$ & $1.5 \times 10^{3}$ & $1.9 \times 10^{3}$ \\
\hline In-114m & $2.7 \times 10^{5}$ & $4.7 \times 10^{4}$ & $7.6 \times 10^{4}$ & $2.7 \times 10^{5}$ & $4.7 \times 10^{4}$ & $2.4 \times 10^{4}$ & $4.0 \times 10^{3}$ & $4.3 \times 10^{3}$ \\
\hline I-123 & $2.4 \times 10^{2}$ & $2.1 \times 10^{1}$ & 4.3 & $2.4 \times 10^{2}$ & $2.1 \times 10^{1}$ & $1.8 \times 10^{1}$ & 1. $2 \times 10^{4}$ & 8.7 \\
\hline I-125 & $3.7 \times 10^{2}$ & $1.5 \times 10^{2}$ & $1.4 \times 10^{1}$ & $3.7 \times 10^{2}$ & $1.5 \times 10^{2}$ & $1.3 \times 10^{2}$ & $7.5 \times 10^{5}$ & $5.6 \times 10^{1}$ \\
\hline $\mathrm{Xe}-127$ & $3.0 \times 10^{-1}$ & $1.0 \times 10^{-2}$ & $5.6 \times 10^{-4}$ & $3.0 \times 10^{-1}$ & $1.0 \times 10^{-2}$ & $7.9 \times 10^{-3}$ & $5.0 \times 10^{-3}$ & $6.8 \times 10^{-4}$ \\
\hline $\mathrm{Te}-127$ & $2.5 \times 10^{3}$ & $5.2 \times 10^{1}$ & $8.1 \times 10^{2}$ & $2.5 \times 10^{3}$ & $5.2 \times 10^{1}$ & $3.1 \times 10^{1}$ & $5.2 \times 10^{1}$ & $5.3 \times 10^{1}$ \\
\hline $\mathrm{Te}-127 \mathrm{~m}$ & 1. $2 \times 10^{5}$ & $2.6 \times 10^{3}$ & $1.0 \times 10^{4}$ & $1.2 \times 10^{5}$ & $2.6 \times 10^{3}$ & $2.9 \times 10^{3}$ & $2.5 \times 10^{3}$ & $2.5 \times 10^{3}$ \\
\hline $\mathrm{Te}-129$ & $1.1 \times 10^{3}$ & 8.1 & 6.0 & $1.1 \times 10^{3}$ & 8.1 & 5.3 & $1.6 \times 10^{1}$ & 7.8 \\
\hline $\mathrm{Te}-129 \mathrm{~m}$ & $1.5 \times 10^{5}$ & $4.0 \times 10^{3}$ & $3.9 \times 10^{4}$ & $1.5 \times 10^{5}$ & $4.0 \times 10^{3}$ & $3.2 \times 10^{3}$ & $4.4 \times 10^{3}$ & $4.2 \times 10^{3}$ \\
\hline$I-131$ & $2.4 \times 10^{3}$ & $2.0 \times 10^{2}$ & $3.4 \times 10^{1}$ & $2.4 \times 10^{3}$ & $2.0 \times 10^{2}$ & $2.4 \times 10^{2}$ & $1.1 \times 10^{6}$ & $4.0 \times 10^{1}$ \\
\hline $\mathrm{Xe}-133$ & $8.5 \times 10^{-1}$ & $3.1 \times 10^{-3}$ & $2.8 \times 10^{-5}$ & $8.5 \times 10^{-1}$ & $3.1 \times 10^{-3}$ & $2.6 \times 10^{-3}$ & $6.8 \times 10^{-4}$ & $5.3 \times 10^{-5}$ \\
\hline Cs-134 & $3.0 \times 10^{4}$ & $5.6 \times 10^{4}$ & $3.7 \times 10^{4}$ & $3.4 \times 10^{4}$ & $6.2 \times 10^{4}$ & $5.9 \times 10^{4}$ & $5.2 \times 10^{4}$ & $6.5 \times 10^{4}$ \\
\hline $\mathrm{Cs}-137$ & $1.4 \times 10^{4}$ & $4.1 \times 10^{4}$ & $1.6 \times 10^{4}$ & $1.6 \times 10^{4}$ & $4.9 \times 10^{4}$ & $5.3 \times 10^{4}$ & $4.5 \times 10^{4}$ & $5.0 \times 10^{4}$ \\
\hline $\mathrm{Ce}-141$ & $4.2 \times 10^{4}$ & $9.3 \times 10^{2}$ & $1.4 \times 10^{4}$ & $4.2 \times 10^{4}$ & $9.3 \times 10^{3}$ & $3.0 \times 10^{3}$ & $1.4 \times 10^{2}$ & $2.8 \times 10^{2}$ \\
\hline $\operatorname{Pr}-143$ & $4.9 \times 10^{4}$ & $3.4 \times 10^{1}$ & $2.5 \times 10^{4}$ & $4.9 \times 10^{4}$ & $3.4 \times 10^{1}$ & $8.6 \times 10^{1}$ & $1.0 \times 10^{2}$ & $3.4 \times 10^{1}$ \\
\hline $\mathrm{Ce}-144$ & $2.1 \times 10^{6}$ & $3.7 \times 10^{3}$ & $1.3 \times 10^{5}$ & $2.9 \times 10^{6}$ & $9.0 \times 10^{3}$ & $2.2 \times 10^{4}$ & $8.2 \times 10^{2}$ & $6.3 \times 10^{2}$ \\
\hline Eu-152 & $8.0 \times 10^{4}$ & $6.0 \times 10^{3}$ & $4.1 \times 10^{4}$ & $1.5 \times 10^{6}$ & $1.2 \times 10^{5}$ & $1.6 \times 10^{5}$ & $5.2 \times 10^{4}$ & $3.4 \times 10^{4}$ \\
\hline I $r-192$ & $1.9 \times 10^{5}$ & $3.6 \times 10^{3}$ & $2.4 \times 10^{4}$ & $1.9 \times 10^{5}$ & $3.6 \times 10^{3}$ & $2.7 \times 10^{3}$ & $2.5 \times 10^{3}$ & $2.3 \times 10^{3}$ \\
\hline $\mathrm{Hg}-197$ & $3.0 \times 10^{3}$ & $1.1 \times 10^{2}$ & $2.9 \times 10^{3}$ & $3.0 \times 10^{3}$ & $1.1 \times 10^{2}$ & $7.1 \times 10^{1}$ & $3.5 \times 10^{1}$ & $1.4 \times 10^{2}$ \\
\hline$A u-198$ & $1.6 \times 10^{4}$ & $2.3 \times 10^{2}$ & $1.6 \times 10^{4}$ & $1.6 \times 10^{4}$ & $2.3 \times 10^{2}$ & $1.6 \times 10^{2}$ & $1.1 \times 10^{2}$ & $5.5 \times 10^{2}$ \\
\hline
\end{tabular}


Table G-4 (Continued)

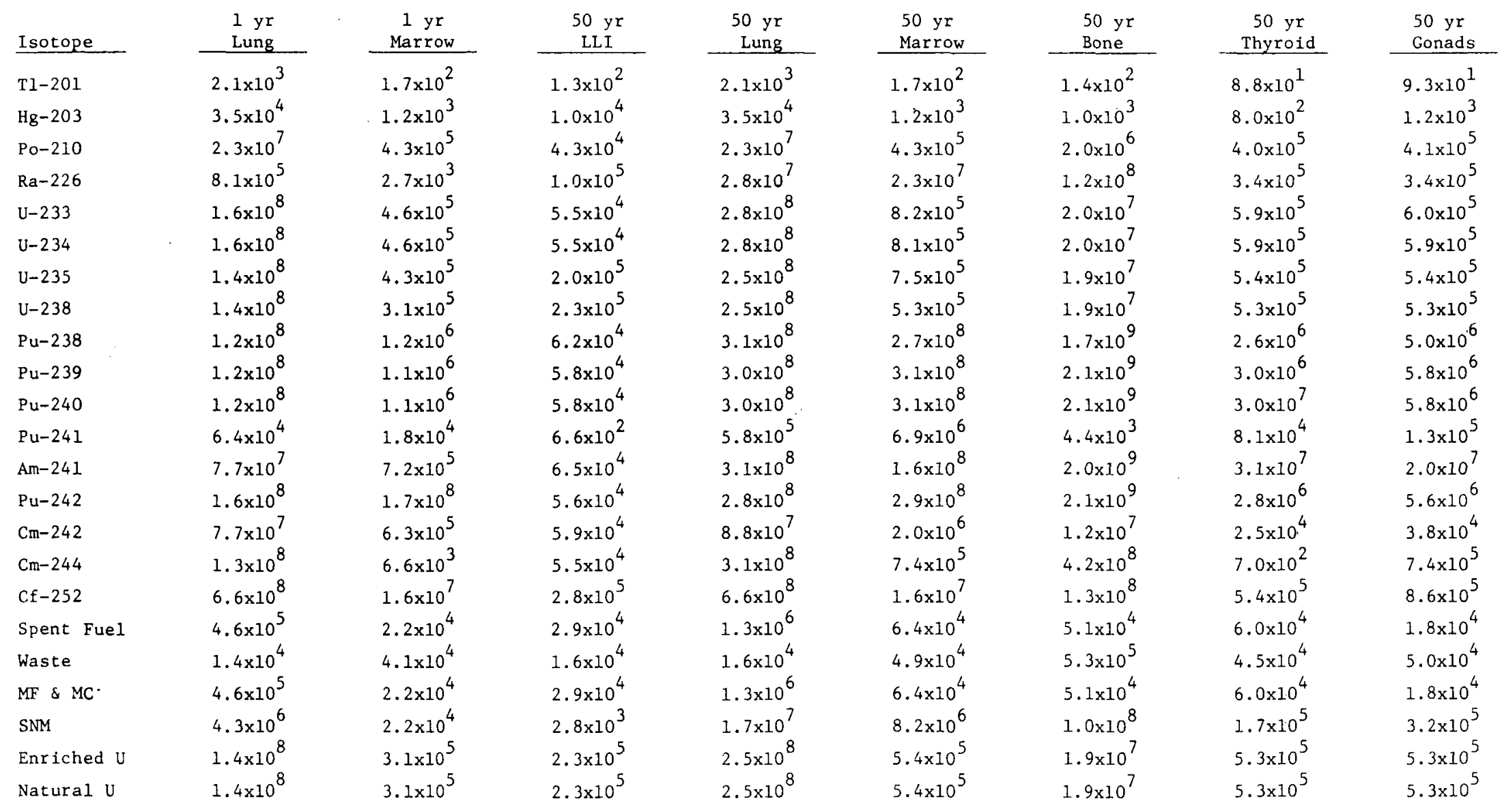


Other Dosimetric Parameters

\begin{tabular}{|c|c|c|c|c|c|c|c|c|c|c|}
\hline Isotope & $\begin{array}{l}t 1 / 2 \text { (days) } \\
\text { (see Ref. 40) } \\
\end{array}$ & $\begin{array}{l}\text { Average } \\
\text { Photon } \\
\text { Energy } \\
\text { (MeV) } \\
\end{array}$ & $\begin{array}{l}\text { Average Total } \\
\text { Photon Energy/ } \\
\text { dis. (MeV) } \\
\end{array}$ & $\begin{array}{l}\text { Aerosolized } \\
\text { Fraction }\end{array}$ & $\begin{array}{l}\text { Particle } \\
\text { Size (4m) }\end{array}$ & $\begin{array}{l}\text { Resuspension } \\
\text { Dose Factor }\end{array}$ & $\begin{array}{l}\text { Lung Early } \\
\text { Mort. Type } \\
\end{array}$ & $\begin{array}{l}\text { Bone } \\
\text { Type } \\
\end{array}$ & $\begin{array}{l}\text { Thyroid } \\
\text { Type } \\
\end{array}$ & $\begin{array}{c}\text { Cloudshine } \\
\text { Dose Factor } \\
\text { (mrem/yr/ } \\
\mu_{\mathrm{Ci}} / \mathrm{m} \ell \text { ) } \\
\end{array}$ \\
\hline $\mathrm{H}-3$ & 4,480 & 0 & 0 & 1.0 & 1.0 & $1.0 *$ & 1 & 2 & 2 & 0 \\
\hline$C-14$ & 20,900 & 0 & 0 & 1.0 & 1.0 & 1.6 & 1 & 2 & 2 & 0 \\
\hline $\mathrm{Na}-22$ & 949. & 0.78 & 1.27 & 1.0 & 1.0 & 1.59 & 1 & 2 & 1 & $1.88 \times 10^{10}$ \\
\hline $\mathrm{Na}-24$ & 0.623 & 2.0 & 4.12 & 1.0 & 1.0 & 1.0 & 1 & 2 & 1 & $3.14 \times 10^{11}$ \\
\hline $\mathrm{Mg}-28$ & 0.896 & 1.1 & 3.15 & 1.0 & 1.0 & 1.09 & 1 & 2 & 1 & $1.1 \times 10^{10}$ \\
\hline$P-32$ & 14.3 & 0 & 0 & 1.0 & 1.0 & 1.09 & 1 & 2 & 2 & 0 \\
\hline$P-33$ & 24.8 & 0 & 0 & 1.0 & 1.0 & 1.1 & 1 & 2 & 2 & 0 \\
\hline$s-35$ & 88. & 0 & 0 & 1.0 & 1.0 & 1.4 & 1 & 2 & 2 & 0 \\
\hline$K-43$ & 0.925 & 0.49 & 0.99 & 1.0 & 1.0 & 1.0 & 1 & 2 & 1 & $8.72 \times 10^{9}$ \\
\hline $\mathrm{Ca}-45$ & 160. & 0.0 & $2.5 \times 10^{-7}$ & 1.0 & 1.0 & 1.47 & 1 & 2 & 2 & 0 \\
\hline $\mathrm{Cr}-5 \mathrm{l}$ & 27.8 & 0.3 & 0.029 & 1.0 & 1.0 & 1.1 & 1 & 2 & 1 & $1.52 \times 10^{8}$ \\
\hline $\mathrm{Fe}-52$ & 0.333 & 0.8 & 1.6 & 1.0 & 1.0 & 1.1 & 1 & 2 & 2 & $6.52 \times 10^{9}$ \\
\hline $\mathrm{Fe}-55$ & $5,280$. & 0.0 & 0 & 1.0 & 1.0 & 1.59 & 3 & 2 & 2 & $1.16 \times 10^{5}$ \\
\hline Co-57 & 268. & 0.12 & 0.137 & 1.0 & 1.0 & 1.52 & 1 & 2 & 1 & $1.65 \times 10^{9}$ \\
\hline Co-58 & 71.5 & 0.81 & 0.19 & 1.0 & 1.0 & 1.35 & 1 & 2 & 1 & $8.66 \times 10^{9}$ \\
\hline $\mathrm{Fe}-59$ & 45. & 1.18 & 1.18 & 1.0 & 1.0 & 1.31 & 1 & 2 & 1 & $9.47 \times 10^{9}$ \\
\hline Co- 60 & $1,910$. & 1.25 & 2.5 & 1.0 & 1.0 & 1.61 & 3 & 2 & 1 & $1.59 \times 10^{10}$ \\
\hline $\mathrm{Ga}-67$ & 3.28 & 0.17 & 0.208 & 1.0 & 1.0 & 1.1 & 1 & 2 & 1 & $2.02 \times 10^{9}$ \\
\hline Se-75 & 120. & 0.22 & 0.391 & 1.0 & 1.0 & 1.44 & 1 & 2 & 1 & $5.7 \times 10^{9}$ \\
\hline $\mathrm{Kr}-85$ & 3,930 . & 0.5 & 0.002 & 1.0 & 1.0 & 1.0 & 1 & 2 & 1 & $1.53 \times 10^{3}$ \\
\hline Sr-89 & 52.7 & 0 & 0 & 1.0 & 1.0 & $1.31 *$ & 1 & 2 & 2 & 0 \\
\hline $\mathrm{Sr}-90$ & 10,200 & 0 & 0 & 1.0 & 1.0 & $1.52 *$ & 3 & 2 & 2 & 0 \\
\hline$Y-90$ & 2.67 & 0 & 0 & 1.0 & 1.0 & 1.0 & 1 & 2 & 2 & 0 \\
\hline$Y-91$ & 58.8 & 0 & 0 & 1.0 & 1.0 & $1.34 *$ & 1 & 2 & 2 & $2.15 \times 10^{7}$ \\
\hline $2 x-95$ & 65.5 & 0.74 & 0.73 & 1.0 & 1.0 & $1.35 *$ & 1 & 2 & 1 & $6.45 \times 10^{9}$ \\
\hline $\mathrm{Nb}-95$ & 35.0 & 0.74 & 0.74 & 1.0 & 1.0 & $1.36 *$ & 1 & 2 & 1 & $6.79 \times 10^{9}$ \\
\hline Mo-99 & 2.78 & 0.5 & 0.279 & 1.0 & 1.0 & 1.1 & 1 & 2 & 1 & $1.4 \times 10^{9}$ \\
\hline TC $-99 m$ & .252 & 0.14 & 0.143 & 1.0 & 1.0 & 1.0 & 1 & 2 & 1 & $1.7 \times 10^{10}$ \\
\hline
\end{tabular}


Table G-5 (Continued)

Other Dosimetric Parameters

\begin{tabular}{|c|c|c|c|c|c|c|c|c|c|c|}
\hline Isotope & $\begin{array}{c}t / 2 \text { (days) } \\
\text { (See Ref. 40) } \\
\end{array}$ & $\begin{array}{c}\text { Average } \\
\text { Photon } \\
\text { Energy } \\
(\mathrm{MeV}) \\
\end{array}$ & $\begin{array}{l}\text { Average Total } \\
\text { Photon Energy/ } \\
\text { d1s. (MeV) } \\
\end{array}$ & $\begin{array}{l}\text { Aerosolized } \\
\text { Fraction } \\
\end{array}$ & $\begin{array}{l}\text { Particle } \\
\text { Size }\left(\psi_{\mathrm{m}}\right) \\
\end{array}$ & $\begin{array}{l}\text { Resuspension } \\
\text { Dose Factor } \\
\end{array}$ & $\begin{array}{l}\text { Lung Early } \\
\text { Mort. Type } \\
\end{array}$ & $\begin{array}{l}\text { Bone } \\
\text { Type } \\
\end{array}$ & $\begin{array}{l}\text { Thyroidd } \\
\text { Type } \\
\end{array}$ & $\begin{array}{c}\text { Cloudshine } \\
\text { Dose Factor } \\
\text { (mrem/yr/ } \\
\mathrm{MCi}_{\mathrm{C} \ell} \text { ) }\end{array}$ \\
\hline $\mathrm{Pu}-103$ & 39.5 & 0.55 & 0.88 & 1.0 & 1.0 & $1.27 *$ & 1 & 2 & 1 & 0 \\
\hline Ru-106 & 368 . & 0 & 0 & 1.0 & 1.0 & $1.55 *$ & 1 & 2 & 2 & 0 \\
\hline In-111 & 2.81 & 0.20 & 0.419 & 1.0 & 1.0 & 1.1 & 1 & 2 & 1 & $6 \times 10^{9 *}$ \\
\hline $5 n-113$ & 115 . & 0.3 & 0.398 & 1.0 & 1.0 & 1.44 & 1 & 2 & 1 & $9.94 \times 10^{7}$ \\
\hline In-114m & 50 . & 1.3 & 0.23 & 1.0 & 1.0 & 1.32 & 1 & 2 & 1 & $7.54 \times 10^{8}$ \\
\hline I-123 & .554 & 0.16 & 0.168 & 1.0 & 1.0 & 1.0 & 1 & 2 & 2 & $1.72 \times 10^{9}$ \\
\hline I- 125 & 60.2 & 0.04 & 0.035 & 1.0 & 1.0 & 1.35 & 1 & 2 & 2 & $2.61 \times 10^{8}$ \\
\hline $\begin{array}{l}x-127 \\
T e-127\end{array}$ & $\begin{array}{r}36.4 \\
.392\end{array}$ & $\begin{array}{l}0.15 \\
0 .\end{array}$ & $\begin{array}{l}0.284 \\
0\end{array}$ & $\begin{array}{l}1.0 \\
1.0\end{array}$ & $\begin{array}{l}1.0 \\
1.0\end{array}$ & $\begin{array}{l}1.0 \\
1.0\end{array}$ & $\begin{array}{l}1 \\
1\end{array}$ & $\begin{array}{l}2 \\
2\end{array}$ & $\begin{array}{l}1 \\
2\end{array}$ & $\begin{array}{r}7 \times 10^{8 *} \\
3.61 \times 10^{7}\end{array}$ \\
\hline $\mathrm{Te}-127 \mathrm{~m}$ & 109. & 0.08 & 0.08 & 1.0 & 1.0 & 1.4 & 1 & 2 & 1 & $8.07 \times 10^{7}$ \\
\hline Te-129 & .0471 & 0 & 0 & 1.0 & 1.0 & 1.0 & 1 & 2 & 2 & $5.92 \times 10^{8}$ \\
\hline Te-129m & 34.1 & 0.03 & 0.02 & 1.0 & 1.0 & 1.2 & 1 & 2 & 1 & $3.35 \times 10^{8}$ \\
\hline$I-131$ & 8.05 & 0.64 & 0.385 & 1.0 & 1.0 & $1.09 *$ & 1 & 2 & 2 & $3.41 \times 10^{9}$ \\
\hline$x e-133$ & 5.27 & 0.08 & 0.081 & 1.0 & 1.0 & 1.0 & 1 & 2 & 1 & $5.02 \times 10^{8}$ \\
\hline $\mathrm{C}_{8}-134$ & 747. & 0.7 & 1.57 & 1.0 & 1.0 & $1.58^{*}$ & 1 & 2 & 2 & $1.38 \times 10^{10}$ \\
\hline$C 8-137$ & 11,000 . & 0.66 & 0.619 & 1.0 & 1.0 & $1.62 *$ & 3 & 2 & 1 & $0.499 \times 10^{10}$ \\
\hline Ce-141 & 32.5 & 0.15 & 0.1 & 1.0 & 1.0 & 1.3 & 1 & 2 & 1 & $6.89 \times 10^{8}$ \\
\hline $\operatorname{Pr}-143$ & 13.6 & 0 & 0 & 1.0 & 1.0 & 1.28 & 1 & 2 & 2 & 0 \\
\hline $\mathrm{Ce}-144$ & 284. & 0.13 & 0.03 & 1.0 & 1,0 & $1.53^{*}$ & 1 & 2 & 2 & $1.72 \times 10^{8}$ \\
\hline Eu-152 & 4,640 & 0.68 & 0.903 & 1.0 & 1.0 & 1.62 & 3 & 2 & 1 & $1 \times 10^{10 *}$ \\
\hline Ir -192 & 74.2 & 0.37 & 0.827 & 1.0 & 1.0 & 1.3 & 1 & 2 & 1 & $1 \times 10^{10 *}$ \\
\hline $\mathrm{Hg}-197$ & 2.71 & 0.08 & 0.081 & 1.0 & 1.0 & 1.1 & 1 & 2 & 1 & $9 \times 10^{8}$ \\
\hline $\mathrm{Au}-198$ & 2.70 & 0.4 & 0.4 & 1.0 & 1.0 & 1.1 & 1 & 2 & 1 & $3.6 \times 10^{9}$ \\
\hline $\mathrm{T} 1-201$ & 3.08 & 0.16 & 0.06 & 1.0 & 1.0 & 1.1 & 1 & 2 & 1 & 0 \\
\hline $\mathrm{Hg}-203$ & 46.9 & 0.3 & 0.279 & 1.0 & 1.0 & 1.32 & 1 & 2 & 1 & $2.66 \times 10^{9}$ \\
\hline Po-210 & 138 & 0.8 & 0 & 1.0 & 1.0 & 1.62 & 2 & 1 & 2 & 0 \\
\hline$R a-226$ & $5.85 \times 10^{5}$ & 0.2 & 1.64 & 1.0 & 1.0 & 1.62 & 4 & 1 & 2 & $7.52 \times 10^{7}$ \\
\hline
\end{tabular}




\begin{tabular}{|c|c|c|c|c|c|c|c|c|c|c|}
\hline Isotope & $\begin{array}{c}t \\
{ }^{1} 1 / 2 \text { (days) } \\
\left.\text { See }^{2} \text { Ref. } 40\right) \\
\end{array}$ & $\begin{array}{l}\text { Average } \\
\text { Photon } \\
\text { Energy } \\
\text { (MeV) } \\
\end{array}$ & $\begin{array}{l}\text { Average Total } \\
\text { Photon Energy/ } \\
\text { dis. (MeV) } \\
\end{array}$ & $\begin{array}{l}\text { Aerosolized } \\
\text { Fraction } \\
\end{array}$ & $\begin{array}{l}\text { Particle } \\
\text { Size ( m) }\end{array}$ & $\begin{array}{l}\text { Resuspension } \\
\text { Dose Factor }\end{array}$ & $\begin{array}{l}\text { Lung Early } \\
\text { Mort. Type }\end{array}$ & $\begin{array}{l}\text { Bone } \\
\text { Type } \\
\end{array}$ & $\begin{array}{l}\text { Thyroid } \\
\text { Type }\end{array}$ & $\begin{array}{c}\text { Cloudshine } \\
\text { Dose Factor } \\
\text { (mrem/yr/ } \\
\mu C i / m \ell)\end{array}$ \\
\hline $\mathrm{v}-233$ & $5.91 \times 10^{7}$ & 0.13 & 0.29 & 1.0 & 1.0 & $1.63 *$ & 4 & 1 & 2 & $1.45 \times 10^{7}$ \\
\hline $\mathrm{U}-234$ & $9.01 \times 10^{7}$ & 1.01 & 1.35 & 0.05 & 1.0 & $1.63 *$ & 4 & 1 & 2 & $1.36 \times 10^{7}$ \\
\hline $\mathrm{U}-235$ & $2.59 \times 10^{11}$ & 0.126 & 0.126 & $0.05^{\mathrm{b}}$ & 7.0 & $1.63 *$ & 4 & 1 & 2 & $1.7 \times 10^{9}$ \\
\hline $\mathrm{U}-238$ & $1.65 \times 10^{12}$ & 0.02 & 2.88 & $0.05^{\mathrm{b}}$ & 7.0 & $1.63 *$ & 4 & 1 & 2 & $6.6 \times 10^{6}$ \\
\hline $\mathrm{Pu}-238$ & 31,500 & 1.02 & 1.36 & 0.05 & 1.0 & $1.61^{*}$ & 4 & 1 & 2 & $1.33 \times 10^{7}$ \\
\hline$P u-239$ & $8.90 \times 10^{6}$ & 0.133 & 0.137 & 0.05 & 1.0 & $1.6 *$ & 4 & 1 & 2 & $5.2 \times 10^{6}$ \\
\hline $\mathrm{Pu}-240$ & $2.40 \times 10^{6}$ & 0.22 & 1.21 & 0.05 & 1.0 & $1.6 *$ & 4 & 1 & 2 & $1.15 \times 10^{7}$ \\
\hline $\mathrm{Pu}-241$ & 4,820 & 0.06 & 0.066 & 0.05 & 1.0 & $1.6 *$ & 3 & 2 & 2 & 0 \\
\hline $\mathrm{Am}-241$ & $1.67 \times 10^{5}$ & 0.06 & 0.066 & 1.0 & 1.0 & $1.6 *$ & 4 & 1 & 2 & $2.94 \times 10^{8}$ \\
\hline $\mathrm{Pu}-242$ & $1.38 \times 10^{8}$ & 0.02 & 2.88 & 0.05 & 1.0 & $1.6 *$ & 4 & 1 & 2 & $1.13 \times 10^{7}$ \\
\hline $\mathrm{Cm}-242$ & 163. & 1.02 & 1.37 & 1.0 & 1.0 & $1.6 *$ & 4 & 1 & 2 & $1.3 \times 10^{7}$ \\
\hline $\mathrm{Cm}-244$ & $6,420$. & .22 & 1.22 & 1.0 & 1.0 & 1.6 & 4 & 1. & 2 & $6.37 \times 10^{7}$ \\
\hline Cf -252 & 966. & 1.0 & 0.42 & 1.0 & 1.0 & 1.59 & 4 & 1 & 2 & $4.74 \times 10^{9}$ \\
\hline $\begin{array}{l}\text { Spent } \\
\text { Fue1 }\end{array}$ & 1,910 . & 1.25 & 2.5 & 1.0 & 1.0 & 1.61 & 3 & 2 & 1 & $1.59 \times 10^{10}$ \\
\hline Waste & 11,000 & 0.66 & 0.619 & 0.05 & 1.0 & 1.62 & 1 & 2 & 1 & $0.499 \times 10^{10}$ \\
\hline $\mathrm{MF}+\mathrm{MC}$ & 1,910 . & 1.25 & 2.5 & 1.0 & 1.0 & 1.61 & 1 & 2 & 1 & $1.59 \times 10^{10}$ \\
\hline
\end{tabular}

${ }^{a}$ Asterisked values are taken from Reference 42. All others are taken from Figure 5 by half-1ife

bormally shipped as large shipments of LSA material:

1 -- Short $t_{1 / 2}$, low LET

2 -- Short $\mathrm{t}_{1 / 2}$, high LET

3 -- Long $t_{1 / 2}$, low LET

4 -- Long $t_{1 / 2}$, high LET

$\mathrm{c}_{1}$-. high LET

2 -- low LET

$\mathrm{d}_{1}$-- penetrating radiation

2 -- nonpenetrating radiation

eNon-asterisked values are taken from Reference 42; asterisked values were estimated using photon energy and comparison with known materials. 
spilled contents, and (4) explosion. By examination of potential accident environments, it has been determined that the pressure-rupture accident is the only mechanism which occurs in a significant proportion of accidents and with a significant potential release. Even when it does occur, not all of the material ejected from the container would become airborne. It was estimated that on the average, no more than $5 \%$ of the released material from a large shipment will become airborne.

\section{Resuspension Dose Factor (RDF)}

In order to make a full analysis of actual inhalation hazard, the phenomena of deposition and resuspension must be considered. As the cloud of aerosolized material is transported by the wind, material is removed from the cloud by various deposition processes and deposited on the ground. Dry deposition continually removes material from the cloud and reduces the downwind concentration. Its effect is estimated by depleting the total quantity of material which would contribute to inhalation dose by the amount of material deposited between the source release point and a point of interest. The amount of material deposited at any point is calculated using a deposition velocity, $v_{d}(\mathrm{~m} / \mathrm{s})$, which, when multiplied by the time integrated concentration $\left(\mathrm{Ci} \cdot \mathrm{s} / \mathrm{m}^{3}\right)$, yields the amount deposited $\mathrm{D}\left(\mathrm{Ci} / \mathrm{m}^{2}\right)$. A value of $0.01 \mathrm{~m} / \mathrm{s}$ is used for $v_{d}$ based on a previous analysis ${ }^{5}$ and for consistency with the resuspension model used in this document.41 (Wet deposition, i.e., deposition caused by scavenging due to rain or snow, is not considered. This neglect of wet deposition will mean that the inhalation dose calculation overestimates the population dose in areas where precipitation can interact with the aerosol cloud.)

Resuspension occurs when particulate material deposited on a surface is made airborne as a result of mechanical forces (walking, vehicular traffic, plowing, etc.), and/or surface wind stress (as in sandstorms or blowing snow). The resuspended material becomes available for inhalation by people in the contaminated area and can cause an additional component of radiation dose which accumulates with time. Methods used to calculate resuspension effects involve an empirical "resupsension factor," $\mathrm{K}\left(\mathrm{m}^{-1}\right)$, which is the ratio of the air concentration at a point to the surface concentration just below that point in the contaminated area. An initial value of $10^{-5} / \mathrm{m}^{-1}$ decreasing exponentially with a 50-day half life to a constant value of $10^{-9} \mathrm{~m}^{-1}$ is used in this model to evaluate the dose contributed by resuspension. 541 Because of radioactive decay, materials with short radioactive half lives provide little resuspension dose, whereas nuclides with long radioactive half lives may increase the initial dose by as much as a factor of 1.6 over the dose received during actual cloud passage.

Since Reference 41 does not include all the isotopes of interest in this report, Figure G-4, which is a plot of radioactive half life versus resuspension dose factor (RDF), was compiled from data in Reference 41, and this curve was used to determine RDF for untabulated isotopes.

Although one might expect that the deposition and resuspension phenomena might be somewhat different inside buildings, the experimental evidence summarized in Table VI-E-3 of Reference 5 indicates that this is probably not true. Values for interior resuspension factors are essentially the same (within the large range of experimental uncertainty) as exterior resuspension factors. Hence, the same value for $\mathrm{RDF}$ is used in both cases in the model. 


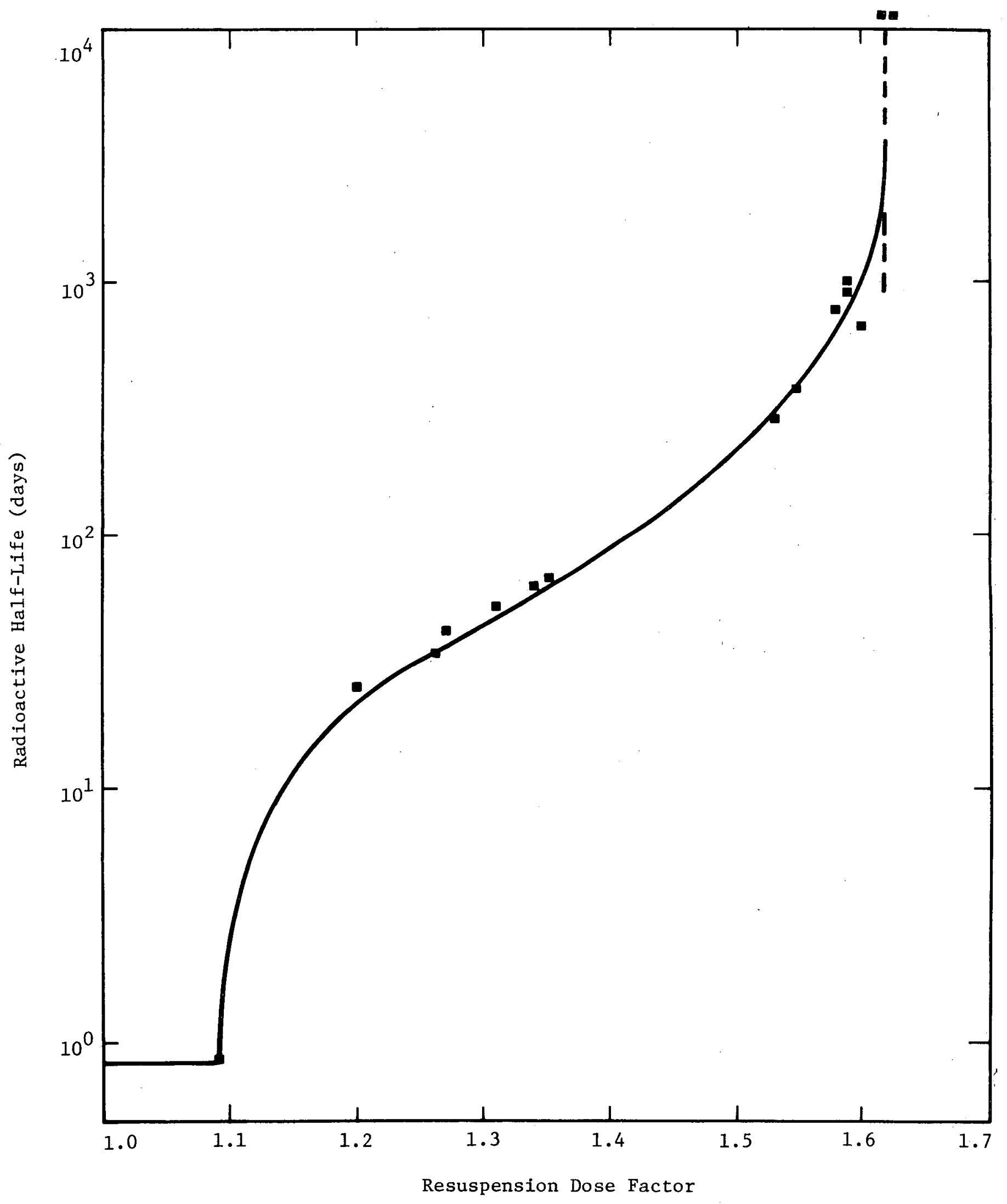

Figure G-4. Plot of Radioactive Half Life versus Resuspension Dose Factor 
Bone Cancer Type, Thyroid Cancer Type

As discussed earlier, the LET of a radiation can greatly affect its potential as a carcinogen. High-LET materials (referred to as bone Type 1) are more effective at inducing bone cancer than low-LET materials (referred to as bone Type 2). Similarly, thyroid cancer seems to be more effectively induced by irradiation of the tissue surrounding the thyroid gland than by direct thyroid irradiation by shortrange particulate emitters. The external irradiators are referred to as thyroid Type 1 , and the internal emitters are referred to as thyroid Type 2.

Lung Fatality Type

The four dose-effect relationships for early fatalities from acute pulmonary dysfunction have been discussed earlier and are shown in Figure G-2. Categories 1 and 2 both use curve A. Category 3 uses curve B, and Category 4 uses curve $C$. Values are selected for each material based on half life and LET.

Cloudshine Dose Factors (CDF)

Cloudshine dose factors representing values for dose received due to immersion for 1 year in a cloud of a fixed concentration have been extracted from Reference 42 . The units are $\mathrm{mrem} / \mathrm{yr} / \mu \mathrm{Ci} / \mathrm{ml}$. The effects of daughter products are, in general, not included. In cases where the isotope does not appear in Reference 42, values have been estimated using isotopes with similar photon energy per disintegration.

\section{NOTES}

lJ. F. Fabrikant, Radiobiology (Chicago: Year Book Medical Publishers, Inc., 1972), pp 4-5.

2J. a. Auxier, Ichlban: Radiation Dosimetry for the Survivors of the Bombings of Hiroshima and Nagasaki, TID27080 (Oak Ridge, TN: Oak Ridge National Laboratory, 1977).

${ }^{3}$ J. M. Yuhas, T. R. Stokes, and C. C. Lushbaugh, "Multifactorial Analysis of Human Blood Cell Response to Clinical Total Body Irradiation," NSA-TM-X-2440, National Symposium on Natural and Man-Made Radiation in Space, January 1972.

${ }^{4}$ U.S. Atomic Energy Commission, Division of Operational Safety, Operational Accidents and Radiation Exposure Experience Within the United States Atomic Energy Commission, 1943-1975, WASH-1192 (USAEC, 1975).

${ }^{5}$ U.S. Nuclear Regulatory Commission, Reactor Safety Study, Appendix VI, WASH-1400 (NUREG-75/014) (Washington: USNRC, October 1975).

${ }^{6}$ National Academy of Sciences, Division of Medical Sciences, Advisory Committee on the Blological Effects of. Ionizing Radiation, "The Effects on Population of Exposure to Low Levels of Ionizing Radiation," November 1972.

${ }^{7}$ American Cancer Society, 1977 Cancer Facts and Figures, 1976. 
8J. Cairns, "The Cancer Problem," Scientific American, 233(5):64 (Novembei 1975).

9W. V. Mayneord and R. H. Clarke, "Carcinogenesis and Radiation Risk," Supplement No. 12 to British Journal of Radiology, 1975.

10 J. W. Baum, "Multiple Simultaneous Event Model for Radiation Carcinogenesis," Hea1th Physics, 30:85-90, January 1976.

$11 \mathrm{~J}$. W. Baum, "Population Heterogeneity Hypothesis on Radiation Induced Cancer," Health Physics, 25:97-104, August 1973.

12C. W. Mays, R. D. Lloyd and J. H. Marshal1, "Malignancy Risk to Humans from Total Body $\gamma$-ray Irradiation," Proceedings of the Third International Congress of the ICRP, USAEC Conference 74-0907, 1974.

13A. R. Tamplin and J. B. Cochran, "Radiation Standards for Hot Particles," Natural Resources Defense Council, February 1974.

${ }^{14} \mathrm{C}$. R. Richmond, "Current States of the Plutonium Hot Particle Problems," Oak Ridge National Laboratory Conference 751105-17, 1975.

15 G. W. Dolphin, Hot Particles, (Harwell, England: National Radiological Protection Board).

${ }^{16}$ National Council on Radiation Protection and Measurements, Alpha Emitting Particles in the Lung, NCRP Report 46 (Washington: NCRP, August 1975).

17"Health Effects of Alpha-Emitting Particles in the Respiratory Tract," Report of the Ad Hoc Committee on "Hot Particles" of the Advisory Committee on the Biological Effects of Ionizing Radiation, National Academy of Sciences, National Research Council, to the U.S. Environmental Protection Agency, October 1976.

$18 \mathrm{C}$. W. Mays, Estimates Risk from Pu-239 to Human Bone, Liver, and Lung, IAEASM-202/806, (Vienna: International Atomic Energy Agency).

$1{ }^{9} \mathrm{C}$. W. Mays et al, Estimated Risk to Human Bone from Pu-239, Biological Effects of Plutonium and Radium, ed. W.S.S. Jee, (Salt Lake City: J. W. Press, 1976).

20M. Goldman, Annual Report, Radiobiology Laboratory, University of CaliforniaDavis, UCD472-121 (Davis, CA: University of California-Davis, 1974), pp 116-162.

${ }^{21}$ National Council on Radiation Protection and Measurements, Basic Radiation Protection Criteria, NCRP Report 39 (Washington: NCRP, 1971).

${ }^{22} \mathrm{~L}$. H. Hamplemann et al, "Neoplasms in Persons Treated with X-rays in Infancy: Fourth Survey in 20 years," Journal of the National Cancer Institute, 55(3):519 (September 1975).

${ }^{23} \mathrm{G}$. W. Dolphin, "The Risk of Thyroid Cancers Following Irradiation," Health Physics Journa1, 15:219-228, 1968. 
${ }^{24}$ B. Modan et al, "Radiation-Induced Head and Neck Tumors," Lancet, 1:277-279, February 1974 .

${ }^{25} \mathrm{C}$. Silverman and D. A. Hoffman, "Thyroid Tumor Risk from Radiation During Childhood," Preventative Medicine, 4:100-105, 1975.

${ }^{26} \mathrm{~J}$. Shapiro, Radiation Protection: A Guide for Scientists and Physicians, (Cambridge: Harvard University Press, 1972).

27U.S. Nuclear Regulatory Commission, Generic Environmental Impact Statement on the Transportation of Radioactive Material by Air and 0ther Modes, NUREG-0170

(Washington: USNRC).

${ }^{28}$ New York City Planning Commission, P1an for New York City (Cambridge: MIT Press, 1969).

${ }^{29}$ U.S. Department of Health, Education and Welfare, U.S. Public Health Service, Radiological Health Handbook, January 1970.

${ }^{30}$ ICRP Task Group on Lung Dynamics, "Deposition and Retention Models for Internal Dosimetry of the Human Respiratory Tract," Health Physics, 12:173-207, 1966.

${ }^{31}$ T. T. Mercer, "On the Role of Particle Size in the Dissolution of Lung Burdens," Health Physics, 13:1211, 1967.

32M. A. Parsont, W. L. Holley, and W. D. Burnett, "The Effect of Particle Size on Organ Distribtuion of Radioactive Material Deposited in the Lungs," Health Physics, $22: 143-8,1972$.

${ }^{33}$ G. M. Kanapilly, O. G. Raabe, and C. H. T. Goh, "Measurement of In Vitro Dissolution of Aerosol Particles for Comparison to In Vivo Dissolution in the Lower Respiratory Tract after Inhalation," Health Physics, 24:297-507, 1973.

${ }^{34}$. L. Fleischer and O. G. Raabe, "Fragmentation of Respirable $\mathrm{PuO}_{2}$ Particles in Water by Alpha Decay--A Mode of 'Dissolution,'" Health Physics, 32:253-7, 1977.

35 . L. Fleischer, "On the Dissolution of Respirable $\mathrm{PuO}_{2}$ Particles," Health Physics, $29: 69-73,1975$.

$36 \mathrm{~F}$. F. Hahn et al, Influence of Particle Size on Phagocytosis by Rabbit Alveolar Macrophages In Vitro, (Albuquerque: Lovelace Foundation Annual Report, 1972).

${ }^{37}$ M. Goldman, An Estimate of Early Mortality and Morbidity Following Inhalation of Plutonium, (Davis, CA: University of California-Davis, October 1976).

${ }^{38}$ G. G. Killough et al, INREM - A Fortran Code which Implements ICRP 2 Models of Internal Radiation Dose to Man, ORNL5003 (Oak Ridge, TN: Oak Ridge Nationa1 Laboratory, February 1975).

${ }^{39}$ G. G. Killough et al, Estimates of Internal Dose Equivalent to 22 Target Organs for Radionuclides Occurring in Routine Releases from Nuclear Fuel Cycle Facilities, Volume I, ORNL/NUREG/TM-190 (Oak Ridge, TN: Oak Ridge National Laboratory, June 1978). 
${ }^{40} \mathrm{C}$. M. Lederer, J. M. Hollander, and I. Perlman, Table of the Isotopes (New York: John Wiley and Sons, Inc.).

41 E. Flack and W. Ray, "RemperSigh Manua1," ORNL unpublished report, May 1974.

${ }^{42}$ G. G. Killough and L. R. McKay, Methodology for Calculatory Radiation Doses from Radioactivity Released to the Environment, ORNL-4992 (Version of Inner) (Oak Ridge, TN: Oak Ridge National Laboratory, March 1976). 


\section{$\mathrm{H}$}

DOT RECORDS FOR RADIOACTIVE MATERIAL TRANSPORTATION INCIDENTS, 1971-1977

In 1971, the U.S. Department of Transportation (DOT) received a mandate from Congress to establish, among other items, a system for collecting information on transportation accidents involving hazardous materials. Radioactive materials were to be included in the hazardous material incident reporting system. Table $\mathrm{H}-1$ represents an accumulation of the DOT records for 1971-1977 incidents involving radioactive materials. Only those are included for which sufficient information exists to allow determination of the cause of the incident, specifically those in which human errors were involved. 
Table $\mathrm{H}-1$

DOT Transportation Incidents in Urban Environs Involving Radioactive Materials, 1971-1977

\begin{tabular}{|c|c|c|c|c|c|c|c|}
\hline $\begin{array}{l}\text { Involved } \\
\text { Material }\end{array}$ & $\begin{array}{l}\text { Class } \\
\text { By Use }\end{array}$ & $\begin{array}{c}\text { Date } \\
\text { of Incidents } \\
\end{array}$ & DOT No. & $\begin{array}{l}\text { Mode of } \\
\text { Transport } \\
\end{array}$ & $\begin{array}{l}\text { Location } \\
\text { In Urban Area }\end{array}$ & $\begin{array}{l}\text { Nature of } \\
\text { Incident }\end{array}$ & $\begin{array}{l}\text { Human } \\
\text { Error }\end{array}$ \\
\hline $\mathrm{Cr}-51$ & Research & $9 \mathrm{Feb} 76$ & 6020670 & Alr passenger & $\begin{array}{l}\text { Alr freight facility, } \\
\text { San Antonfo, TX }\end{array}$ & $\begin{array}{l}\text { Damaged by other } \\
\text { freight }\end{array}$ & Stowage \\
\hline $\mathrm{Cr}-51$ & Research & $2 \operatorname{Dec} 75$ & 5120504 & Atr passenger & $\begin{array}{l}\text { Alr freight factlity, } \\
\text { Chicago, IL }\end{array}$ & Dropped in handling & Handling \\
\hline Cr-51 & Research & 13 May 75 & 5050732 & Air passenger & $\begin{array}{l}\text { Freight facility, } \\
\text { Boston, MA }\end{array}$ & Sp111 handled by NEN & Unknown \\
\hline $\begin{array}{ll}\mathrm{Cr}-51, & \text { АM-198 } \\
\mathrm{I}-125, & \text { Mo-99 }\end{array}$ & Industrial & $9 \operatorname{Mar} 74$ & 4030232 & Alr passenger & $\begin{array}{l}\text { Aircraft aft bulk } \\
\text { cargo bin, } \\
\text { Minneapolis, MN }\end{array}$ & $\begin{array}{l}\text { Damaged by other } \\
\text { freight, prior water } \\
\text { damage }\end{array}$ & Stowage \\
\hline $\mathrm{Cr}-51$ & Research & 20 Apr 73 & None & Air passenger & $\begin{array}{l}\text { Freight area, } \\
\text { Washington, DC }\end{array}$ & Dropped in handling & Handling \\
\hline $\begin{array}{l}\text { Co-60 and } \\
\text { Cs }-137 \text { LSA }\end{array}$ & Fuel. cycle waste & 4 oct 76 & 6110102 & Truck & $\begin{array}{l}\text { Freeway--195 at } \\
\text { NC rte } 46 \text {, Gaston, NC }\end{array}$ & $\begin{array}{l}\text { Top of box opened } \\
\text { during transport }\end{array}$ & Packaging \\
\hline $\begin{array}{l}\text { Co-57 flood } \\
\text { source }\end{array}$ & Research & 11 Feb 76 & 6030626 & Truck & $\begin{array}{l}\text { Clark Ave. Bridge, } \\
\text { Cleveland, OH }\end{array}$ & $\begin{array}{l}\text { Box fell off trailer } \\
\text { run over by } \\
\text { following vehicle }\end{array}$ & Stowage \\
\hline $\mathrm{Co}-57$ & Industrial & 16 Sep 75 & 5100211 & $\begin{array}{l}\text { Alr freight } \\
\text { forwarder }\end{array}$ & $\begin{array}{l}\text { Terminal, } \\
\text { Atlanta, GA }\end{array}$ & Damaged in handling & Handling \\
\hline $\mathrm{Co}-60 \mathrm{SF}$ & Industrial & 2 oct 74 & 4100433 & Truck & In trailer, Tulsa, OK & Fell in transit & Stowage \\
\hline Co-60 & Research & 24 Dec 74 & 4080493 & Truck & $\begin{array}{l}\text { In trailer at } \\
\text { terminal, San } \\
\text { Antonio, TX }\end{array}$ & $\begin{array}{l}\text { Outside container } \\
\text { cracked }\end{array}$ & Stowage \\
\hline Co-60 & Industrial & $10 \mathrm{Feb} 74$ & 4020263 & Truck & $\begin{array}{l}\text { Freight terminal, } \\
\text { Memphis, TN }\end{array}$ & $\begin{array}{l}\text { Damaged by other } \\
\text { freight - crate } \\
\text { bashed in }\end{array}$ & Stowage \\
\hline Co-60 & Industrial & 10 Sep 71 & 1160076 & Truck & $\begin{array}{l}\text { Industrial Company, } \\
\text { Leechburg, PA }\end{array}$ & $\begin{array}{l}\text { Loose fittings } \\
\text { valves or closures }\end{array}$ & Packaging \\
\hline Co-60 & Industrial & $10 \mathrm{JuI} 71$ & 1080013 & Truck & $\begin{array}{l}\text { U.S. } 63 \text { at city } \\
\text { limits, Cabol, } \\
\text { MO }\end{array}$ & Vehicle accident & Accident \\
\hline Ga-67 citrate & Industrial & $29 \mathrm{Jan} 77$ & 7020741 & Truck & $\begin{array}{l}\text { Freeway--NJ turnpike } \\
\text { between } 82 \text { and } 92 \\
\text { northbound }\end{array}$ & Fell out of truck & Stowage \\
\hline$I-131$ & Industrial & 7 Jan 75 & 5010253 & Air freight & $\begin{array}{l}\text { Air freight terminal, } \\
\text { Washington, DC }\end{array}$ & $\begin{array}{l}\text { Water damage, body } \\
\text { or side failure }\end{array}$ & Handling \\
\hline$I-125$ & Research & 31 Aug 74 & 4090307 & Air passenger & $\begin{array}{l}\text { Freight warehouse, } \\
\text { Minneapolis, } \mathbb{N}\end{array}$ & Run over by forklift & Stowage \\
\hline
\end{tabular}




\section{Table H-1 (Continued)}

\begin{tabular}{|c|c|c|c|c|c|c|c|}
\hline $\begin{array}{l}\text { Involved } \\
\text { Materlal }\end{array}$ & $\begin{array}{l}\text { Class } \\
\text { By Use }\end{array}$ & $\begin{array}{c}\text { Date } \\
\text { of Incidents } \\
\end{array}$ & DOT No. & $\begin{array}{c}\text { Mode of } \\
\text { Transport }\end{array}$ & $\begin{array}{c}\text { Location } \\
\text { in Urban Area } \\
\end{array}$ & $\begin{array}{l}\text { Nature of } \\
\text { Incident }\end{array}$ & $\begin{array}{l}\text { Human } \\
\text { Error }\end{array}$ \\
\hline$I-125$ & Industrial & 20 Aug 74 & 4090003 & Air passenger & $\begin{array}{l}\text { Air frelght ware- } \\
\text { house, Chicago, IL }\end{array}$ & $\begin{array}{l}\text { Damaged container } \\
\text { put in trash }\end{array}$ & Disposal \\
\hline$I-131$ & Industrial & 9 Aug 74 & 4080630 & Truck & $\begin{array}{l}\text { Freight facility, } \\
\text { Great Falls, MT }\end{array}$ & $\begin{array}{l}\text { Damaged by other } \\
\text { freight }\end{array}$ & Stowage \\
\hline$I-131$ & Industrial & $17 \mathrm{Jul} 74$ & 4070805 & Ait passenger & $\begin{array}{l}\text { Cargo B1dg. } 81 \text {, } \\
\text { (TWA) JFK, NY }\end{array}$ & Dropped in handling & Handling \\
\hline $\mathrm{I}-125$ & Industrial & $4 \mathrm{Jul} 74$ & 4070349 & Alr passenger & $\begin{array}{l}\text { Tractor tug pulling } \\
\text { full baggage cart, } \\
\text { San Francisco, CA }\end{array}$ & $\begin{array}{l}\text { Jet blast knocked } \\
\text { box off, then } \\
\text { tractor crushed }\end{array}$ & Stowage \\
\hline$I-123$ & Research & 16 Jun 74 & 4080497 & Alr passenger & $\begin{array}{l}\text { Air freight terminal, } \\
\text { Boston, MA }\end{array}$ & Body or side faflure & Packaging \\
\hline$I-125$ & Industrial & 18 Apr 74 & 4050132 & Alr passenger & $\begin{array}{l}\text { Air freight terminal, } \\
\text { forklift, JFK, NY }\end{array}$ & Outer carton damage & Handling \\
\hline I-123, Tc99m & Radiopharm. & 11 Apr 74 & 4040404 & A1r passenger & $\begin{array}{l}\text { DC-8-61 aircraft, } \\
\text { Dallas-Ft. Worth, TX }\end{array}$ & $\begin{array}{l}\text { Dropped in handling, } \\
\text { external puncture }\end{array}$ & Handling \\
\hline$I-125$ & Research & 6 Aug 73 & 3080191 & Air passenger & At aircraft JFK, NY & $\begin{array}{l}\text { Fell from cart, } \\
\text { subsequently crushed }\end{array}$ & Stowage \\
\hline$I-131$ & Research & $26 \mathrm{Jul} 73$ & 3100274 & A1r passenger & $\begin{array}{l}\text { Cargo area, Dallas } \\
\text { Love Fleld, TX }\end{array}$ & Bottom failure & Packaging \\
\hline$I-131$ & Research & 24 May 73 & 5020002 & Air passenger & $\begin{array}{l}\text { Air freight terminal, } \\
\text { Los Angeles, CA }\end{array}$ & $\begin{array}{l}\text { Dropped in handling } \\
\text { external puncture }\end{array}$ & Handling \\
\hline $1-131$ & Research & 22 Jun 72 & None & Afr passenger & $\begin{array}{l}\text { Afrcraft cargo bin, } \\
\text { Houston, TX }\end{array}$ & External puncture & Handling \\
\hline $\operatorname{Ir}-192$ & Industrial & 18 Dec 74 & 4120638 & Air passenger & $\begin{array}{l}\text { A1r freight terminal, } \\
\text { Baton Rouge, LA }\end{array}$ & $\begin{array}{l}\text { Defective valves } \\
\text { fittings or closures }\end{array}$ & Packaging \\
\hline $\mathrm{Ir}-192 \mathrm{SI}$ & Industrial & 5 Sep 74 & 4100206 & Alr passenger & $\begin{array}{l}\text { In aircraft, } \\
\text { Syracuse, NY }\end{array}$ & $\begin{array}{l}\text { Bottom fallure, } \\
\text { corrosion or rust }\end{array}$ & Packaging \\
\hline Ir -192 & Industrial & 27 Aug 74 & 4090359 & Alr passenger & $\begin{array}{l}\text { Alrport freight, } \\
\text { Newark, NJ }\end{array}$ & $\begin{array}{l}\text { Probable container } \\
\text { defect }\end{array}$ & Packaging \\
\hline $\operatorname{Ir}-192$ & Industrial & 8 Apr 74 & 4040403 & Air passenger & $\begin{array}{l}\text { In transit, } \\
\text { Baton Rouge, LA }\end{array}$ & Improper packaging & Packaging \\
\hline Ir -192 & Industrial & $10 \operatorname{Mar} 74$ & 4030399 & Truck & $\begin{array}{l}\text { Route } 422 \text { at } 645, \\
\text { Myerstown, PA }\end{array}$ & $\begin{array}{l}\text { Vehicle accident, no } \\
\text { damage to shipment }\end{array}$ & Accident \\
\hline Ic-99 & Industrial & 1 Aug 73 & N3080034 & Atr passenger & Freight area, JFK, NY & $\begin{array}{l}\text { Outer container } \\
\text { open }\end{array}$ & Packaging \\
\hline $\mathrm{P}-32$ & $\begin{array}{l}\text { Industrial- } \\
\text { research }\end{array}$ & 15 Aug 73 & N3090017 & Air passenger & $\begin{array}{l}\text { Freight area, } \\
\text { Denver, CO }\end{array}$ & $\begin{array}{l}\text { Dropped in handling } \\
\text { cart ran over end } \\
\text { of carton }\end{array}$ & Stowage \\
\hline
\end{tabular}


Table H-1 (Continued)

\begin{tabular}{|c|c|c|c|c|c|c|c|}
\hline $\begin{array}{l}\text { Involved } \\
\text { Material } \\
\end{array}$ & $\begin{array}{l}\text { Class } \\
\text { By Use } \\
\end{array}$ & $\begin{array}{c}\text { Date } \\
\text { of Incidents } \\
\end{array}$ & DOT No. & $\begin{array}{c}\text { Mode of } \\
\text { Transport }\end{array}$ & $\begin{array}{c}\text { Location } \\
\text { In Urban Area }\end{array}$ & $\begin{array}{l}\text { Nature of } \\
\text { Incident }\end{array}$ & $\begin{array}{l}\text { Human } \\
\text { Error }\end{array}$ \\
\hline $\begin{array}{l}\text { Plutonfum } \\
\text { samples }\end{array}$ & Industrial & 6 Dec 75 & 5120517 & Truck & $\begin{array}{l}\text { I70 at Ind. 227, } \\
\text { Richmond, IN }\end{array}$ & $\begin{array}{l}\text { Fell out of moving } \\
\text { trailer }\end{array}$ & Stowage \\
\hline $\begin{array}{ll}\text { Po-210: lonizing } \\
\text { annozzle (NOS) }\end{array}$ & Industrial & 13 Jan. 77 & 7020163 & $\begin{array}{l}\text { Air freight } \\
\text { forwarder }\end{array}$ & $\begin{array}{l}\text { Air cargo terminal, } \\
\text { DFW, TX }\end{array}$ & Dropped in handling & Handling \\
\hline $\mathrm{Ra}-226$ & $\begin{array}{l}\text { Molsture Density } \\
\text { Nuclear Testing } \\
\text { Equip-Industrial }\end{array}$ & 8 Mar 76 & 6030462 & Truck & $\begin{array}{l}\text { On truck, Beckely, } \\
\text { W. Va., or Research } \\
\text { Pk., NC }\end{array}$ & $\begin{array}{l}\text { Damage by other } \\
\text { freight }\end{array}$ & Stowage \\
\hline $\mathrm{Ra}-226$ & Research & 24 Mar 75 & 5040001 & Air passenger & $\begin{array}{l}\text { NW gate D8 ramp-on } \\
\text { craft Chicago, IL }\end{array}$ & $\begin{array}{l}\text { Outside container } \\
\text { crushed }\end{array}$ & Stowage \\
\hline Radium eqpt. & Industrial & 4 Sep 74 & 4090528 & Air passenger & $\begin{array}{l}\text { In aircraft at ramp } \\
\text { gate, Minneapolis, } M N\end{array}$ & $\begin{array}{l}\text { Loose fittings, top } \\
\text { closeness }\end{array}$ & Packaging \\
\hline $\mathrm{Ra}-226$ & Industrial & 30 May 73 & None & Truck & $\begin{array}{l}\text { Dock, freight termi- } \\
\text { nal, Memphis TN }\end{array}$ & Damaged crate & Handling \\
\hline Ru-106 & Industrial & 27 Feb 76 & 6030304 & Air freight & $\begin{array}{l}\text { SAS cargo bldg., } \\
263-J F K, N Y\end{array}$ & $\begin{array}{l}\text { Carton crushed- } \\
\text { cause unknown }\end{array}$ & Stowage \\
\hline Se-75, NOS & Research & $4 \operatorname{Mar} 77$ & 7030383 & Air passenger & $\begin{array}{l}\text { Passenger aircraft } \\
\text { baggage cart, } \\
\text { Montreal, } \mathrm{CN}\end{array}$ & $\begin{array}{l}\text { No actual } \\
\text { contamination }\end{array}$ & Unknown \\
\hline $\mathrm{Se}-75$ & Research & $3 \mathrm{Ju} 173$ & 3070241 & Air passenger & $\begin{array}{l}\text { On DC9 alrcraft } \\
\text { New Orleans, LA }\end{array}$ & Dropped in handling & Handling \\
\hline $\mathrm{Sr}-85, \mathrm{Y} 6169$ & Research & 29 Jul 72 & None & Alr passenger & $\begin{array}{l}\text { Aircraft and air } \\
\text { freight, Denver, co }\end{array}$ & $\begin{array}{l}\text { Damaged by other } \\
\text { freight }\end{array}$ & Stowage \\
\hline Thoric oxide & Industrial & 5-6 Ju1 77 & 7070342 & Truck & $\begin{array}{l}\text { Dock facility Port } \\
\text { Seatrain LTL Facil- } \\
\text { ity, Weehawken, NJ }\end{array}$ & $\begin{array}{l}\text { Damaged by other } \\
\text { freight }\end{array}$ & Stowage \\
\hline $\begin{array}{l}\text { Natural thorium } \\
23 \% \text { as } \mathrm{ThO}_{2}\end{array}$ & Industrial & 21 Feb 77 & 7030876 & Truck & $\begin{array}{l}\text { Freight terminal, } \\
\text { Portland, OR }\end{array}$ & $\begin{array}{l}\text { No actual contami- } \\
\text { nation side of con- } \\
\text { tainers crushed by } \\
\text { other freight }\end{array}$ & Stowage \\
\hline Thorium & Government & 11 oct 75 & 5100990 & Truck & $\begin{array}{l}\text { Freight terminal on } \\
\text { truck, Atlanta, GA }\end{array}$ & Improper stowage & Stowage \\
\hline $\begin{array}{l}\text { Depleted } \\
\text { urantum } \mathrm{UF}_{4}\end{array}$ & Industrial & 28 Sep 75 & 5090817 & Truck & $\begin{array}{l}\text { Freight terminal, } \\
\text { Johnson City, TN }\end{array}$ & External puncture & Handling \\
\hline U-235 fissile & $\begin{array}{l}\text { Industrial- } \\
\text { research }\end{array}$ & 13 Feb 75 & 5020420 & Truck & $\begin{array}{l}\text { 6th St. at Almond } \\
\text { St., Moscow, ID }\end{array}$ & Dropped off truck & Stowage \\
\hline Uranium ore & Industrial & 5 Feb 75 & None & Truck & $\begin{array}{l}\text { Freight terminal, } \\
\text { St. Louis, MO }\end{array}$ & Drum ruptured & Packaging \\
\hline Ore samples & Industrial & 15 Aug 74 & 4090721 & Truck & $\begin{array}{l}\text { Freight dock, } \\
\text { Phoenix, AZ }\end{array}$ & $\begin{array}{l}\text { Loose fittings } \\
\text { valves or closures }\end{array}$ & Packaging \\
\hline
\end{tabular}


Table H-1 (Continued)

\begin{tabular}{|c|c|c|c|c|c|c|c|c|}
\hline $\begin{array}{l}\text { Involved } \\
\text { Materlal } \\
\end{array}$ & $\begin{array}{l}\text { Class } \\
\text { By Use } \\
\end{array}$ & of & $\begin{array}{l}\text { Date } \\
\text { Incidents } \\
\end{array}$ & DOT No. & $\begin{array}{l}\text { Mode of } \\
\text { Transport }\end{array}$ & $\begin{array}{c}\text { Location } \\
\text { In Urban Area }\end{array}$ & $\begin{array}{l}\text { Nature of } \\
\text { Inc1dent } \\
\end{array}$ & $\begin{array}{l}\text { Human } \\
\text { Error }\end{array}$ \\
\hline $\begin{array}{l}\text { Urantum oxide } \\
\text { yellow cake }\end{array}$ & Industrial & 22 & Dec 73 & 4020081 & Truck & $\begin{array}{l}\text { On trailer at } \\
\text { freight terminal, } \\
\text { Oklahoma C1ty, OK }\end{array}$ & $\begin{array}{l}\text { Body or side fallure } \\
\text { corrosion or rust }\end{array}$ & Packaging \\
\hline $\begin{array}{l}\text { Nat'l uranium } \\
\text { conc. }\end{array}$ & Fuel Cycle & 17 & Nov 73 & 3120045 & Truck & $\begin{array}{l}\text { Dock and traller, } \\
\text { Denver, } c 0\end{array}$ & External puncture & Handling \\
\hline $\begin{array}{l}\text { Urantum con- } \\
\text { centrate, LSA }\end{array}$ & Industrial & 5 & Dec 72 & 2120186 & Truck & $\begin{array}{l}\text { Truck terminal, } \\
\text { Denver, co }\end{array}$ & Weld failure & Packaging \\
\hline $\begin{array}{l}\text { Enriched uranium, } \\
\text { fissile }\end{array}$ & Industrial & 27 & $\operatorname{Mar} 72$ & 2040118 & Truck & $\begin{array}{l}\text { Freight facility, } \\
\text { Bristol, VA }\end{array}$ & External puncture & Handling \\
\hline$X e-133$ & Research & 3 & Feb 76 & 6030303 & A1r passenger & $\begin{array}{l}\text { Alr cargo terminal, } \\
\text { Knoxville, TN }\end{array}$ & $\begin{array}{l}\text { Fallure of Inner } \\
\text { receptacle }\end{array}$ & Packag1ng \\
\hline$X e-133$ & Research & 2 & May 75 & 5050185 & Alr freight & $\begin{array}{l}\text { Fed. Exp. hub } \\
\text { sorting facility, } \\
\text { Memph1s, TN }\end{array}$ & $\begin{array}{l}\text { Damaged by other } \\
\text { freight }\end{array}$ & Stowage \\
\hline $\mathrm{Xe}-133$ & Research & 22 & Dec 72 & 2120341 & Air passenger & $\begin{array}{l}\text { On plane at airport, } \\
\text { Newark, NJ }\end{array}$ & $\begin{array}{l}\text { Damaged by other } \\
\text { freight }\end{array}$ & Stowage \\
\hline$Y t-169$ & Industrial & 28 & Aug 74 & 4090112 & Alr passenger & $\begin{array}{l}\text { LAX afrcraft ramp, } \\
\text { gate conveyor belt } \\
\text { Los Angeles, CA }\end{array}$ & $\begin{array}{l}\text { Fell off belt, } \\
\text { dented }\end{array}$ & Handling \\
\hline Radionuclide & Industrial & 6 & Ju1 77 & 7070949 & Truck & $\begin{array}{l}\text { Freight terminal, } \\
\text { Brisbane, CA }\end{array}$ & $\begin{array}{l}\text { Container shield } \\
\text { failure }\end{array}$ & Packaging \\
\hline $\begin{array}{l}\text { Radioactive } \\
\text { mat'l (NOS) }\end{array}$ & Unknown & 23 & Jun 77 & 7061277 & Alr frelght & $\begin{array}{l}\text { Freight terminal, } \\
\text { Denver, CO }\end{array}$ & $\begin{array}{l}\text { Box slightly wet } \\
\text { on bottom }\end{array}$ & Stowage \\
\hline $\begin{array}{l}\text { Radloactive } \\
\text { mat'1 }\end{array}$ & Industrial & 10 & Feb 77 & 7030123 & Truck & $\begin{array}{l}\text { Truck terminal, } \\
\text { Ruby, SC }\end{array}$ & $\begin{array}{l}\text { Trailer contami- } \\
\text { nation }\end{array}$ & Unknown \\
\hline $\begin{array}{l}\text { Radioactive } \\
\text { pharmaceutical }\end{array}$ & Research & 4 & Oct 76 & 6100469 & Truck & $\begin{array}{l}\text { US } 41 \text { between } \mathrm{N} \text {. } \\
\text { Chicago \& Highland } \\
\text { Park, IL }\end{array}$ & $\begin{array}{l}\text { Dropped on roadway } \\
\text { from truck }\end{array}$ & Stowage \\
\hline $\begin{array}{l}\text { Waste mat' } 1 \\
\text { (most recent ship- } \\
\text { ment in vehicle) }\end{array}$ & Fuel cycle waste & 9 & Jan 77 & 7061103 & Truck & $\begin{array}{l}\text { Freight terminal, } \\
\text { Seneca, IL }\end{array}$ & $\begin{array}{l}\text { Residual contamina- } \\
\text { tion on traller }\end{array}$ & Packaging \\
\hline Waste mat' 1 & Fuel cycle waste & 9 & Jun 77 & 7061102 & Truck & $\begin{array}{l}\text { Freight terminal, } \\
\text { Seneca, IL }\end{array}$ & Residual on trailer & Packaging \\
\hline Waste mat' 1 & Fuel cycle waste & 9 & Jun 77 & 7061101 & Truck & $\begin{array}{l}\text { Freight terminal, } \\
\text { Seneca, IL }\end{array}$ & Residual on traile: & \\
\hline $\begin{array}{l}\text { Radfoactive } \\
\text { waste, LSA }\end{array}$ & Fuel cycle waste & 9 & Feb 77 & 7030122 & Truck & $\begin{array}{l}\text { Truck terminal, } \\
\text { Barnwell, SC }\end{array}$ & $\begin{array}{l}\text { Trailer contamina- } \\
\text { tion }\end{array}$ & Packaging \\
\hline $\begin{array}{l}\text { Radioactive } \\
\text { waste, LSA }\end{array}$ & Fuel cycle & 10 & Aug 76 & 6090620 & Truck & $\begin{array}{l}\text { Truck terminal, } \\
\text { Joplin, Mo }\end{array}$ & Spillage on trailer & Packaging \\
\hline
\end{tabular}


Table H-1 (Continued)

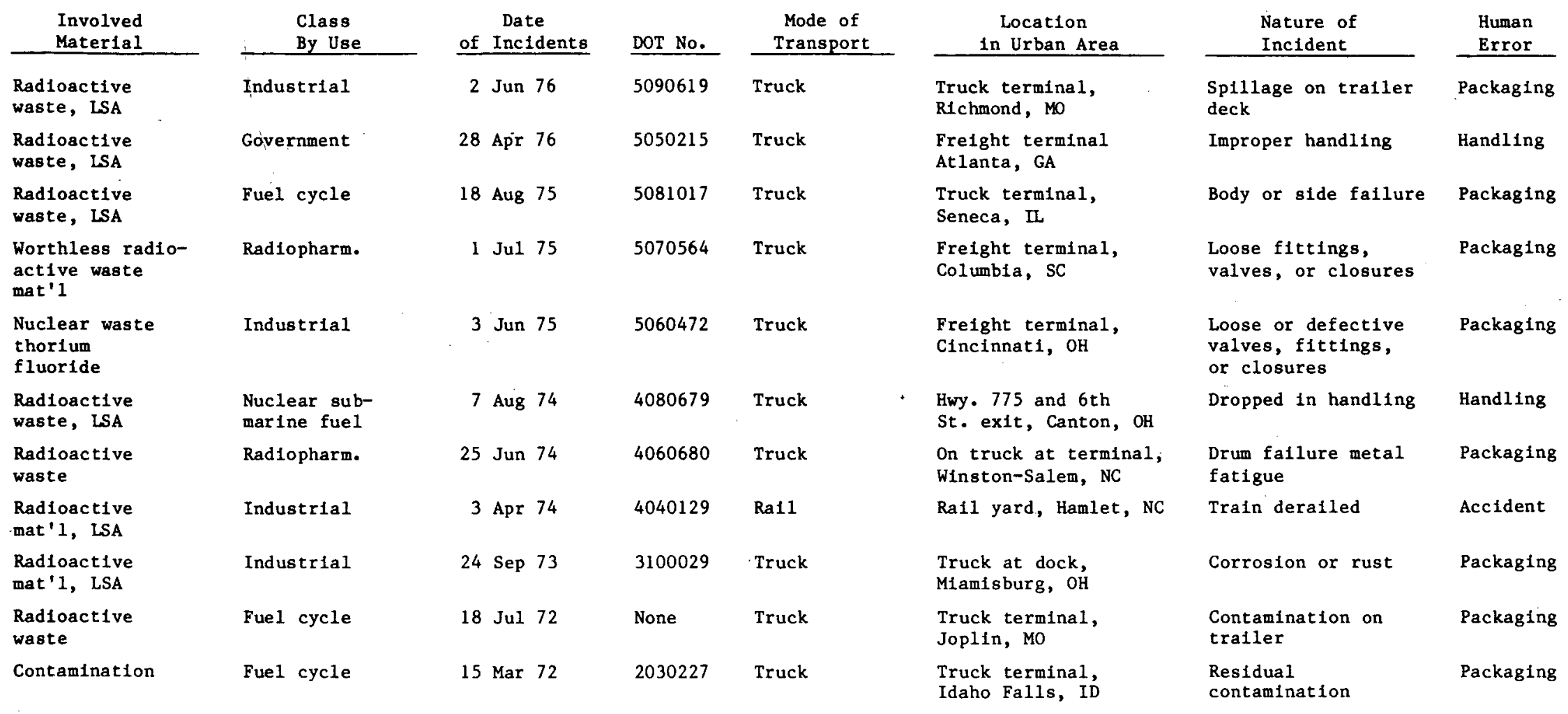


RADIOACTIVE MATERIAL TRANSPORTATION INCIDENTS IN 1975

IN NRC AGREEMENT STATES

Table I-1 summarizes the transportation incidents involving radioactive materials which were reported to the Nuclear Regulatory Commission by its agreement states for 1975.

Table I-1

NRC Transportation Incidents in Urban Environs, 1975

\begin{tabular}{|c|c|c|c|c|c|c|c|}
\hline Involved Material & $\begin{array}{r}\text { Class } \\
\text { by Use } \\
\end{array}$ & & $\begin{array}{l}\text { ate of } \\
\text { cident }\end{array}$ & $\begin{array}{l}\text { Mode of } \\
\text { Transport }\end{array}$ & $\begin{array}{r}\text { Location in } \\
\text { Urban Area }\end{array}$ & $\begin{array}{l}\text { Nature of } \\
\text { Incident }\end{array}$ & Human Error \\
\hline$c-14$ & Radiopharm. & 2 & Dec 75 & Air passenger & $\begin{array}{l}\text { Airport, } \\
\text { Los Angeles, CA }\end{array}$ & Lost source & None \\
\hline $\begin{array}{l}\text { Cs-137 } \\
\text { (sealed source) }\end{array}$ & Industrial & & - & Auto & $\begin{array}{l}\text { City street, } \\
\text { Linden, NJ }\end{array}$ & Thef $t$ & None \\
\hline $\mathrm{H}-3$ & Radiopharm. & & - & Auto & $\begin{array}{l}\text { City street, } \\
\text { Bridgeport, co }\end{array}$ & Thef $t$ & None \\
\hline $\mathrm{H}-3$ & Rad iopharm. & & - & Alr freight & JFK Airport, NY & Mishandling & Handling \\
\hline $\begin{array}{l}\mathrm{H}-3, \mathrm{~S}-35, \mathrm{C}-14, \\
\mathrm{I}-125, \mathrm{Cr}-51\end{array}$ & Radiopharm. & - & - & Truck & $\begin{array}{l}\text { New York Thruway, } \\
\text { Bronx, NY }\end{array}$ & Accident & None \\
\hline $\mathrm{H}-3$ & Radiopharm. & 17 & Dec 75 & Air passenger & $\begin{array}{l}\text { Airport, } \\
\text { Anchorage, AL }\end{array}$ & Accident & None \\
\hline $\mathrm{H}-3$ & Research & 9 & Sep 75 & Air freight & Fremont, MI & Lost source & None \\
\hline I compounds & Radiopharm. & 11 & Mar 75 & Air freight & $\begin{array}{l}\text { O'Hare Airport, } \\
\text { Chicago, IL }\end{array}$ & $\begin{array}{l}\text { Crushed and } \\
\text { leaking } \\
\text { package }\end{array}$ & Stowage \\
\hline$I-125$ & Radiopharm. & & - & Truck & $\begin{array}{l}\text { City street, } \\
\text { Paramus, NJ }\end{array}$ & The f $t$ & None \\
\hline I-125 & Radlopharm. & 23 & Jul 75 & Unknown & Edmonton, Canada & Leakage & Packaging \\
\hline$I-131$ & Rad1opharm. & & - & Truck & Ladysmith, VA & Lost source & None \\
\hline$I-131$ & Radiopharm. & 10 & Feb 75 & Air freight & $\begin{array}{l}\text { O'Hare Airport } \\
\text { Chicago, Il }\end{array}$ & Lost source & None \\
\hline $\begin{array}{l}\text { Ir-192 } \\
\text { (sealed source) }\end{array}$ & Industrial & & - & Alr freight & $\begin{array}{l}\text { Philadelphia } \\
\text { Internat' } 1 \text { Alrport } \\
\text { Philadelphia, PA }\end{array}$ & Mishandling & Handling \\
\hline
\end{tabular}


IMPACTS OF CHEMICAL TOXICITY

Any exposure to toxic chemicals as a result of transportation incidents will be short term. Only in certain cases will there be sufficiently high concentrations present to produce chronic and/or acute effects. Toxicological information on short-term exposures to these materials is somewhat limited. Reference 1 establishes four basic levels of toxic hazard, with class 0 compounds producing effects only under the most unusual conditions and class 3 materials producing toxic effects of sufficient severity to threaten life or cause permanent physical damage. Compounds within these classes are not further differentiated. Using qualitative information on the toxicity of the material from short-term exposures, a listing is made of the relative chemical hazard of the radioactive materials transported. This list of chemicals is reduced by removing compounds which produce little, if any effects. A second reduction is made by eliminating those compounds shipped in such small quantities that toxic effects would be produced in only an extremely limited number of exposed individuals, if at all. The nature of each of the remaining materials: is then investigated.

Using data developed from information in Reference 2, the actual chemical formula for a compound is established by considering the common chemical valence of the radioactive isotope and combining this information with the normal configurations of the remainder of the compound (e.g., sulfate $=\mathrm{SO}_{4}{ }^{2}$, sodium ion $=\mathrm{Na}$, etc.).* The molecular weight of each compound and the percentage of the radioactive isotope are then determined. These are combined with the specific activity of the isotope involved and related to shipment size. ${ }^{2}$ This produces an upper limit to number of grams of a given chemical form that could be involved in an accidental release. This information is summarized in Table $\mathrm{J}-1$.

Following the guidelines in Reference 1, four broad toxicity classes are used to specify the relative hazards of the materials from short-term exposures. A brief description of the biological responses to a compound in each class is given below:

Class 0: Materials cause either no harm under any conditions or produce toxic effects under only the most unusual conditions or in overwhelming doses.

Class 1: Materials produce only slight effects following single exposures -regardless of the quantity absorbed or the extent of the exposure. In general, effects are readily reversible and disappear following termination of exposure, with or without medical treatment.

\footnotetext{
* This will produce, in some instances, more than one useful form. In most cases, only one form has been chosen. Both common forms of uranium oxide, UO 2 and $\mathrm{U}_{3} \mathrm{O}_{8}$, are used.
} 
Class 2: Materials produce moderate effects after intense exposure for a matter of seconds, after moderate exposure for prolonged periods, or after ingestion of a single dose. Although these materials may produce irreversible as well as reversible changes, they are not life threatening nor do they produce serious or permanent physical impairment.

Class 3: Materials produce effects of sufficient severity to threaten life or cause permanent physical impairment or disfigurement following a single exposure or ingestion of a single dose.

Reference 1 classifies toxicity from short-term local exposure and systemic exposure. Occasionally, different types of toxicity (irritant, ingestion, inhalation, etc.) are classified separately. In these instances, the highest listed value for a given compound is used. Table $\mathrm{J}-2$ summarizes the information on toxicity from both local and systemic short-term exposures.

Most of the listed compounds are classified 0 to 1 , i.e., the effects of exposure to them will be slight enough to disappear readily following termination of exposure, with or without medical treatment. These materials are not considered further. For many of the remaining compounds to produce effects of any consequence, it would be necessary for the entire maximum shipment of material to be aerosolized and inhaled by a single individual. Since this is unlikely, the number of materials can be further reduced, leaving for consideration only the compounds in Table J-3.

The first three compounds in Table J-3, shipped in tens of grams, do not present as significant a problem as the uranium compounds, shipped by the millions of grams. Ethylene trichloride and strontium nitrate, though moderately toxic, are shipped in smal1 quantities (less than $20 \mathrm{~g} / \mathrm{shipment}$ maximum) requiring a minimum dilution for maximum effect. Although Reference 3 indicates no record of sensitivity to iridium, the possibility of such should not be discounted.

Reference 4 ranks uranium compounds on a relative toxicity scale. Uranium hexafluoride is the most toxic, followed by $\mathrm{UO}_{2} \mathrm{~F}_{2}, \mathrm{UCl}_{4}$, and $\mathrm{UO}_{2}\left(\mathrm{NO}_{3}\right) \cdot 6 \mathrm{H}_{2} \mathrm{O}$. Uranium oxides are among the least toxic. Large shipments of uranium compounds pose the greatest toxicity hazard. The toxicity of $\mathrm{UF}_{6}$ is due largely to the toxicity of $\mathrm{HF}$, a by product of the decomposition of the material. In a transportation accident involving $\mathrm{UF}_{6}$, it is likely that large quantities of $\mathrm{HF}$ would be produced and a much smaller amount of uranium would be aerosolized. 
Table J-1

Chemical Forms and Shipment Sizes for Materials in the New York City Shipment Mode1

Isotope

Am-241

$\mathrm{Au}-918$

C-14

$\mathrm{Ca}-45$

Cf -252

Co-57

Co- 60

$\mathrm{Cr}-51$

Cs-137

$\mathrm{Eu}-152$

$\mathrm{Fe}-52$
Chemical Form(s) ${ }^{a}$

$\mathrm{Am}_{2} \mathrm{O}_{3}$ (americium oxide)

$\mathrm{Au}$ (colloidal gold)

$\mathrm{AuCl}_{3}$ (gold (III) chloride)

Ethylene trichloride $\left(\mathrm{C}_{2}^{\mathrm{x}} \mathrm{H}_{3} \mathrm{Cl}_{3}\right)$ and other organic chlorides

$\mathrm{C}_{6} \mathrm{H}_{22} \mathrm{O}_{6}$ (glucose)

$\mathrm{CaCl}_{2}$ (calcium chloride)

$\mathrm{Cf}_{2} \mathrm{O}_{3}$ (californium oxide) $^{b}$

$\mathrm{CoCl}_{2}$ (cobalt (II) chloride)

Co (elemental cobalt)

$\mathrm{CoCl}_{2}$ (cobalt (II) chloride)

$\mathrm{Na}_{2} \mathrm{CrO}_{4}$ (sodium chromate)

$\mathrm{CrCl}_{3}$ (chromium (II) chḷoride)

CsC1 (cesium chloride)

$\mathrm{CsNO}_{3}$ (cesium nitrate)

Cs deposited on Zeolite ion;

- exchange resin

$\mathrm{EuCl}_{2}$ (europium chloride)

$\mathrm{FeC}_{6} \mathrm{H}_{5} \mathrm{O}_{7}$ - $5 \mathrm{H}_{2} \mathrm{O}$ (iron (III)
Maximum Shipment

Size for

Compounds (grams)

$3.4 \times 10^{2}$

$4.1 \times 10^{-4}$

$6.3 \times 10^{-4}$

19

4.8

$1.4 \times 10^{-5}$

$2.1 \times 10^{-1}$

$4.3 \times 10^{-3}$

$8.9 \times 10^{2}$

$4.3 \times 10^{-3}$

$3.5 \times 10^{-5}$

$3.4 \times 10^{-5}$

$1.4 \times 10^{3}$

$1.6 \times 10^{3}$

$1.2 \times 10^{3}$

(as cesium)

$7.5 \times 10^{-5}$

$9.1 \times 10^{-6}$

${ }^{a}$ Compounds listed here are only a sample of the materials that could have been extracted from the chemical forms in Reference 2 .

${ }^{b} \mathrm{Also}$, it should be noted that the shipment-size values for americium and californium are excessively high in that a single shipment of the specified size would exceed the total separated stockpile of these materials. 
Table J-1 (Continued)

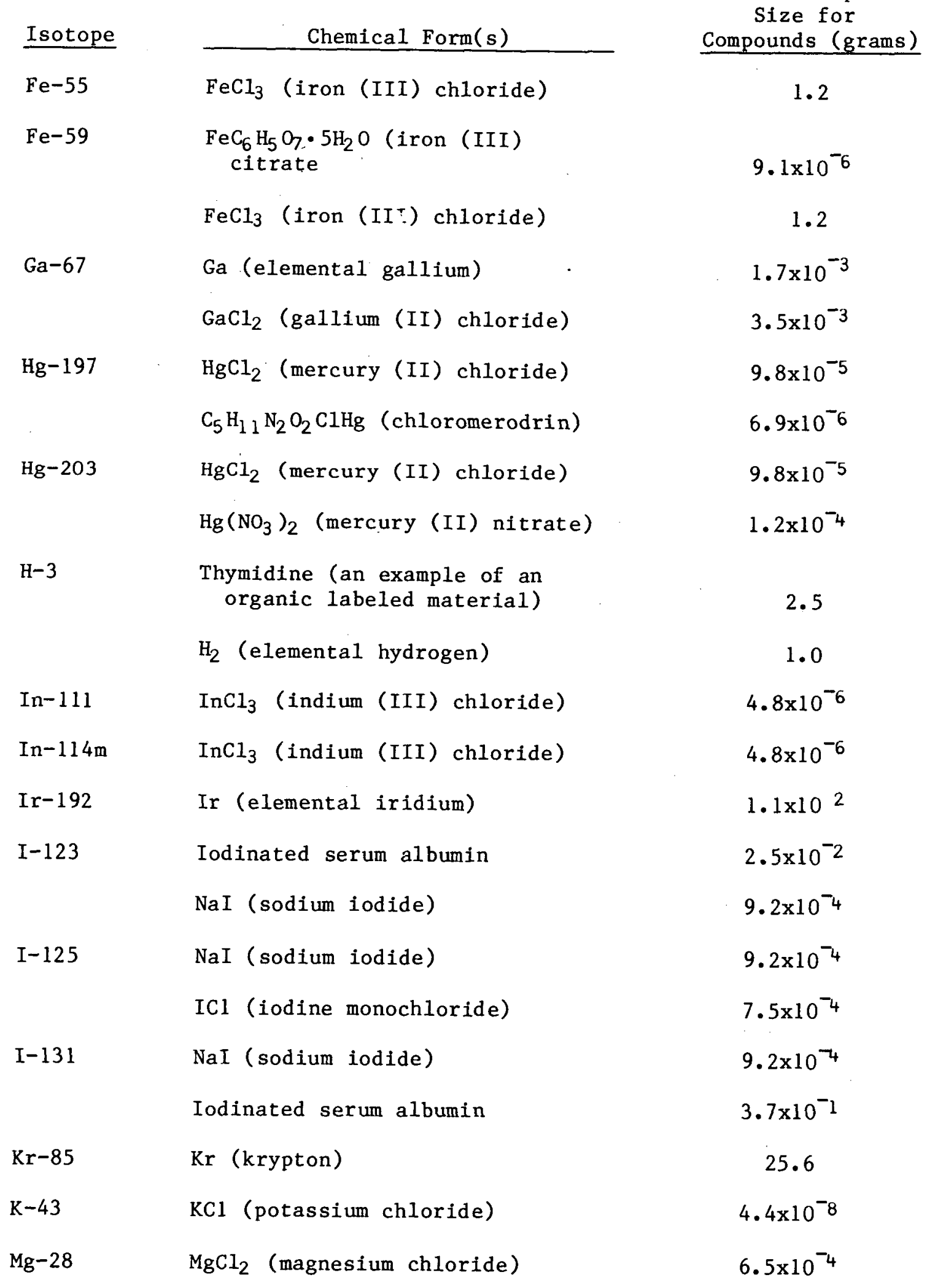

\section{Maximum Shipment \\ ze for}

1.2

$9.1 \times 10^{-6}$

1.2

$\mathrm{HgCl}_{2}$ (mercury (II) chloride)

$1.2 \times 10^{-4}$

2.5

$4.8 \times 10^{-6}$

$4.8 \times 10^{-6}$

$1.1 \times 10^{2}$

$2.5 \times 10^{-2}$

$9.2 \times 10^{-4}$

$9.2 \times 10^{-4}$

$7.5 \times 10^{-4}$

$9.2 \times 10^{-4}$

$3.7 \times 10^{-1}$

25.6

$4.4 \times 10^{-8}$

$6.5 \times 10^{-4}$ 
Table J-1 (Continued)

\begin{tabular}{|c|c|c|}
\hline Isotope & Chemical Form(s) & Compound (grams) \\
\hline Mo-99 & $\mathrm{K}_{2} \mathrm{MoO}_{4}$ (potassium molybdate) & $5.1 \times 10^{-3}$ \\
\hline & $\mathrm{MoO}_{2}$ (molybdenum (IV) oxide) & $2.5 \times 10^{-3}$ \\
\hline & $\mathrm{MoCl}_{4}$ (molybdenum (IV) chloride) & $5.1 \times 10^{-3}$ \\
\hline $\mathrm{Na}-22$ & $\mathrm{NaCl}$ (sodium chloride) & $5.1 \times 10^{-3}$ \\
\hline $\mathrm{Na}-24$ & NaC1 (sodium chloride) & $5.1 \times 10^{-3}$ \\
\hline Po- 210 & Po (elemental polonium) & $2.3 \times 10^{-1}$ \\
\hline $\mathrm{P}-32$ & $\begin{array}{l}\mathrm{Na}_{3} \mathrm{PO}_{4} \cdot 1 \mathrm{OH}_{2} \mathrm{O} \text { (sodium } \\
\text { phosphate) }\end{array}$ & $3.8 \times 10^{-4}$ \\
\hline & $\mathrm{H}_{3} \mathrm{PO}_{4}$ (orthophosphoric acid) & $1.1 \times 10^{7}$ \\
\hline $\mathrm{P}-33$ & $\begin{array}{l}\mathrm{Na}_{3} \mathrm{PO}_{4} \cdot 1 \mathrm{IH}_{2} \mathrm{O} \text { (sodium } \\
\text { phosphate) }\end{array}$ & $3.8 \times 10^{-4}$ \\
\hline $\mathrm{Ra}-226$ & RaO (radium (II) oxide) & 1.1 \\
\hline $\mathrm{Se}-75$ & $\begin{array}{l}\mathrm{Se}\left(\mathrm{MoO}_{4}\right)_{2} \text { (selenium (IV) } \\
\text { molybdate) }\end{array}$ & $3.6 \times 10^{-4}$ \\
\hline & $\mathrm{SeCl}_{4}$ (selenium (IV) chloride) & $2.0 \times 10^{-4}$ \\
\hline$S n-113$ & $\mathrm{SnCl}_{2}$ (tin (II) chloride) & $1.7 \times 10^{-4}$ \\
\hline $\mathrm{Sr}-89$ & $\mathrm{SrCl}_{2}$ (strontium chloride) & $6.3 \times 10^{-6}$ \\
\hline $\mathrm{Sr}-90$ & $\mathrm{Sr}\left(\mathrm{NO}_{3}\right)_{2}$ (strontium nitrate) & 1.7 \\
\hline & Sro (strontium oxide) & $8.2 \times 10^{-1}$ \\
\hline$S-35$ & $\begin{array}{l}\mathrm{Na}_{2} \mathrm{SO}_{4} \text { (sodium sulfate) } \\
\mathrm{C}_{3} \mathrm{H}_{4} \mathrm{~N}_{2} \mathrm{~S} \text { (2-aminothiazole -- } \\
\text { assumed as form for organic } \\
\text { shipments of } \mathrm{S}-35 \text { labeled } \\
\text { compounds) }\end{array}$ & $9.6 \times 10^{-5}$ \\
\hline $\mathrm{Tc}-99 \mathrm{~m}$ & $\mathrm{TcCl}_{6}$ (technetium chloride) & $6.0 \times 10^{-3}$ \\
\hline
\end{tabular}


Table J-1 (Continued)

Isotope

T1-201

$\mathrm{Xe}-1.27$

$\mathrm{Xe}-133$

$\mathrm{U}-233$

$\mathrm{U}-235$

$\mathrm{U}-238$
Chemical Form(s)

\section{$\mathrm{KtcO}_{4}$ (potassium} pertechnetate)*

T1C1 (thallium chloride)

Xe (xenon)

Xe (xenon)

$\mathrm{UO}_{2}$ (uranium (IV) oxide)

UF6 (uranium (IIV) hexafluoride)

U (elemental uranium)

$\mathrm{UO}_{2}$ (uranium (IV) oxide)

U (elemental uranium)

$\mathrm{UO}_{2}\left(\mathrm{NO}_{3}\right)_{2} \cdot 6 \mathrm{H}_{2} \mathrm{O}$
(uranyl nitrate)

$\mathrm{U}\left(\mathrm{SO}_{4}\right)_{2} \cdot 9 \mathrm{H}_{2} \mathrm{O}$ (urany1 sulfate)
Maximum Shipment

Size for

Compound (grams)

$3.9 \times 10^{-3}$

$5.6 \times 10^{-5}$

$5.4 \times 10^{-3}$

$5.4 \times 10^{-3}$

$1.1 \times 10^{7}$

$1.5 \times 10^{7}$

$1.0 \times 10^{7}$

$1.1 \times 10^{7}$

$1.0 \times 10^{7}$

$2.1 \times 10^{7}$

$2.5 \times 10^{7}$

*Although the pertechnetate has not been listed as a chemical form for technetium, its wide use in the medical field dictates its inclusion in this section. 
Table J-2

Toxicity Classes for Chemicals ${ }^{a}$

\section{Toxicity Classifications}

Chemical Form

Americium oxide $\left(\mathrm{Am}_{2} \mathrm{O}_{3}\right)$

Gold ( $\mathrm{Au})$

Gold (III) chloride $\left(\mathrm{AuCl}_{3}\right)$

Ethylene trichloride

Glucose $\left(\mathrm{C}_{6} \mathrm{H}_{12} \mathrm{O}_{6}\right)$

Calcium chloride $\left(\mathrm{CaCl}_{2}\right)$

Californium oxide $\left(\mathrm{Cf}_{2} \mathrm{O}_{3}\right)$

Cobalt (II) chloride $\left(\mathrm{CoCl}_{2}\right)$

Cobalt (Co) $[\mathrm{F}]^{\mathrm{b}}$

Sodium chromate $\left(\mathrm{Na}_{2} \mathrm{CrO}_{4}\right)$

Chromium (III) chloride $\left(\mathrm{CrCl}_{3}\right)$

Cesium chloride ( $\mathrm{CsCl}$ ) [F,E]

Cesium nitrate $\left(\mathrm{CsNO}_{3}\right)$ [F,E]

Cesium deposited in zeolite ion exchange resin $[F, E]$

Europium chloride $\left(\mathrm{EuCl}_{2}\right)$

Iron (III) citrate $\left(\mathrm{FeC}_{6} \mathrm{H}_{5} \mathrm{O}_{7}\right.$. $5 \mathrm{H}_{2} \mathrm{O}$ )

Iron (III) chloride $\left(\mathrm{FeCl}_{3}\right)$

\begin{tabular}{c} 
Short-term \\
Local Exposure \\
\hline
\end{tabular}

R

0

2

2

0

1

$\mathrm{R}$

1

$\mathrm{R}+1$

3

3

$\mathrm{R}+$ ?

$\mathrm{R}+$ ?

$\mathrm{R}+$ ?

$R+$ ?

1

1
Short-term Systemic Exposure

R

0

2

2

0

?

R

1

$\mathrm{R}+$ ?

$?$

?

$\mathrm{R}+1$

$\mathrm{R}+1$

$\mathrm{R}+1$

$\mathrm{R}+$ ?

0

$?$

"Each material not classified in Reference 1 is labeled "?" and should be interpreted as "one whose toxicity is unknown and warrants further study." Where Reference 1 indicates that radiotoxicity predominates, the letter $R$ is used. If the material presents hazards other than toxicity, they will be labeled F--fire, or E-explosive hazard in brackets following the compound name.

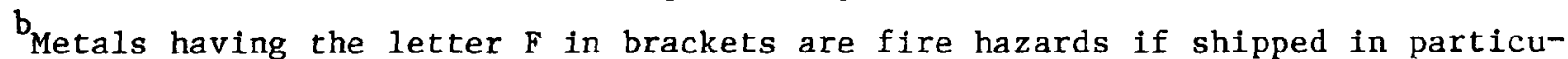
late form, e.g., powder. 
Table J-2 (Continued)

Toxicity Classifications

Chemical Form

Gallium (Ga)

Gallium (II) $\left(\mathrm{GaCl}_{2}\right)$

Mercury (II) chloride $\left(\mathrm{HgCl}_{2}\right)$

Chloromerodrin $\left(\mathrm{C}_{5} \mathrm{H}_{11} \mathrm{~N}_{2} \mathrm{O}_{2} \mathrm{ClHg}\right)$

Mercury (II) nitrate $\left(\mathrm{Hg}\left(\mathrm{NO}_{3}\right)_{2}\right)$

Thymidine

Hydrogen $\left(\mathrm{H}_{2}\right.$-tritium) [F,E].

Indium (III) chloride $\left(\operatorname{INc}_{3}\right)$ [F]

Iridium ( Ir) $[\mathrm{F}, \mathrm{E}]$

Iodinated serum albumin

Sodium iodide ( $\mathrm{NaI}$ )

Iodine monochloride (IC1) [E]

Krypton ( $\mathrm{Kr}$ )

Potassium chloride (KC1)

Magnesium chloride $\left(\mathrm{MgCl}_{2}\right)$

Potassium molybdate $\left(\mathrm{K}_{2} \mathrm{MoO}_{4}\right)$

Molybdenum oxide $\left(\mathrm{MoO}_{2}\right.$ or $\left.\mathrm{MoCl}_{2}\right)$

Molybdenum chloride $\mathrm{MoCl}_{4}$ or $\left.\mathrm{MoCl}_{2}\right)$

Sodium chloride ( $\mathrm{NaCl}$ )

Polonium (Po)

Sodium phosphate $\left(\mathrm{Na}_{3} \mathrm{PO}_{4} \cdot 10 \mathrm{H}_{2} \mathrm{O}\right)$

\section{Short-term}

Local Exposure

$\mathrm{R}+$ ?

$\mathrm{R}+$ ?

3

3

3

?

$\mathrm{R}+0$

$\mathrm{R}+2$

$\mathrm{R}+$ ?

?

variable*

3
Short-term

Systemic Exposure

$\mathrm{R}+1$

$\mathrm{R}+1$

3

3

3

$?$

$\mathrm{R}+1$

$\mathrm{R}+3$

$\mathrm{R}+2$

$?$

2

0

$\mathrm{R}+$ simple asphyxiant in high concentrations

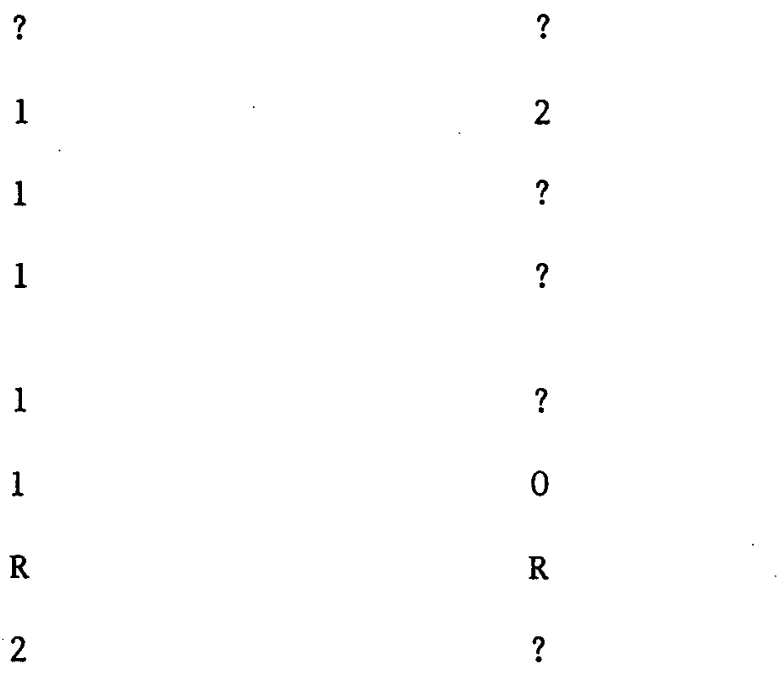


Table J-2 (Continued)

Chemical Form

Phosphoric acid $\left(\mathrm{H}_{3} \mathrm{PO}_{4}\right)$

Radium oxide ( $\mathrm{RaO})$

Selenium (IV) molybdate

$\left(\mathrm{Se}\left(\mathrm{MoO}_{4}\right)_{2}\right)$

Selenium chloride $\left(\mathrm{SeCl}_{4}\right)$

Tin (II) chloride $\left(\mathrm{SnCl}_{2}\right)$ [F]

Strontium chloride $\left(\mathrm{SrCl}_{2}\right)$

Strontium nitrate $\left(\mathrm{Sr}\left(\mathrm{NO}_{3}\right)_{2}\right)[\mathrm{F}, \mathrm{E}]$

Strontium oxide (SrO)

Sodium sulfate $\left(\mathrm{Na}_{2} \mathrm{SO}_{4}\right)$

2-aminothiazole $\left(\mathrm{C}_{3} \mathrm{H}_{4} \mathrm{~N}_{2} \mathrm{~S}\right)$

Technetium chloride $\left(\mathrm{TcCl}_{6}\right)$

Potassium pertechnetate $\left(\mathrm{KTcO}_{4}\right)$

Thallium chloride (TICI)

Xenon (Xe)

Uranium (IV) oxide $\left(\mathrm{UO}_{2}\right)$ [F,E]

Uranium hexafluoride $\left(\mathrm{UF}_{6}\right)[\mathrm{F}, \mathrm{E}]$

Urany1 nitrate $\left(\mathrm{UO}_{2}\left(\mathrm{NO}_{3}\right)_{2} \cdot\right.$ $\left.6 \mathrm{H}_{2} \mathrm{O}\right)[\mathrm{F}, \mathrm{E}]$

Uranyl sulfate $\left(\mathrm{U}\left(\mathrm{SO}_{4}\right)_{2} \cdot 9 \mathrm{H}_{2} \mathrm{O}\right)$

Uranium (U) $[F, E]$

Toxicity Classifications

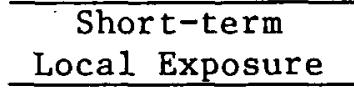

2

R

3

3

2

$\mathrm{R}+0$

$\mathrm{R}+$ ?

$\mathrm{R}+0$

0

?

$\mathrm{R}+$ ?

$\mathrm{R}+$ ?

2

Simple asphyxiant

$\mathrm{R}+3$ *

$\mathrm{R}+3$

$\mathrm{R}+$ ?

$\mathrm{R}+3$ *

$\mathrm{R}+3$ *
Short-term

Systemic Exposure
?

$\mathbf{R}$

3

3

?

$\mathrm{R}+0$

$\mathrm{R}+2$

$\mathrm{R}+0$

0

1

$\mathrm{R}+$ ?

$\mathrm{R}+$ ?

3

$1+$ simple asphyxiant

$\mathrm{R}+3$ *

$\mathrm{R}+$ ?

$\mathrm{R}+2$

$\mathrm{R}+3 *$

$\mathrm{R}+3$ *

\footnotetext{
* Permissible levels for soluble uranium compounds and for insoluble compounds in atr are based on chemical toxicity, while the body levels for insoluble compounds are based on radiotoxicity. 1
} 
Table J-3

Compounds Remaining after Consideration of Minimum Exposable Population*

\begin{tabular}{|c|c|c|}
\hline \multirow[b]{2}{*}{ Chemical Form } & \multicolumn{2}{|c|}{ Toxicity Classification } \\
\hline & Acute Loca1 & Acute Systemic \\
\hline Ethylene trichloride & 2 & 2 \\
\hline Iridium & $\mathrm{R}+$ ? & $\mathrm{R}+2$ \\
\hline Strontium nitrate & $\mathrm{R}+$ ? & $\mathrm{R}+2$ \\
\hline Uranium oxide & $\mathrm{R}+3$ & $\mathrm{R}+3$ \\
\hline Uranium hexafluoride & $R+3$ & $\mathrm{R}+?$ \\
\hline Uranyl nitrate & $\mathrm{R}+$ ? & $\mathrm{R}+2$ \\
\hline Uranyl sulfate & $\mathrm{R}+3$ & $R+3$ \\
\hline Uranium (elementa1) & $R+3$ & $R+3$ \\
\hline
\end{tabular}

NOTES

${ }^{1}$. Irving Sax, Dangerous Properties of Industrial Materials (4th ed; New York: Van Nostrand Reinhold Company, Division of Litton Educ. Publications, Inc., 1975 ).

2 Battelle Pacific Northwest Laboratories, Survey of Radioactive Material in the United States, BNWL-1972 (Hanford, WA, Apri1 1976).

3F. P. Patty, ed, Industrial Hygiene and Toxicology, II (2d ed rev; New York: John Wiley \& Sons/Interscience Publishers, 1963).

${ }^{4}$ C. Voegtlin and H. C. Hodge, eds, Pharmacology and Toxicology of Uranium Compounds (New York: McGraw Hili, 1953). 
In addition to radiological health effects, accidents involving radioactive materials can cause economic impacts including costs for surveillance, cleanup, evacuation, long-term or temporary relocation or permanent land-use denial, and litigation. In this section the model which has been developed to quantify these effects is described. Scenarios are modeled in which specific approaches are taken by civil authorities, and the costs of these are estimated. In many cases cost data are several years old and have been converted to effective 1979 dollars by compounding inflation rates.

The type and extent of economic consequence are both functions of the dispersibility of the material and its half life. In the case of nondispersible materials, the on-scene radiation will be surveyed; recovery procedures and perimeter security will follow. In the case of dispersible materials, the procedures may consist of up to eight steps:

1. On-scene survey and bulk material recovery

2. Pre-cleanup radiation survey

3. Cleanup

4. Intermediate radiation surveys

5. Evacuation

6. Land-use denia1

7. Perimeter security

8. Final radiation surveys

This section will outline the various scenarios used and detail the cost determination formulation.

\section{Kl. Scenarios for Economic Impact Calculations}

\section{K1.1 Nondispersible Shipments and Nonrelease Accidents}

If the accident in question involves a nondispersible radionuclide or if the accident is not severe enough to cause a release of a potentially dispersible material, it will be necessary for response forces to determine the extent of unacceptably high radiation fields. These areas should then be cordoned off and perimeter security established to insure that members of the general public are not overexposed. After initial safety and perimeter security precautions have been taken, the unshielded radioactive material will be recovered and moved from the accident scene. 


\section{K1.2 Dispersible Shipments}

Long Half-Life Radionuclides

In the context of economic impact, a long half-life radionuclide is considered to be one with an environmental residence* of more than seven half lives. If a material of this type is released, the scenario proceeds as follows:

- Response forces conduct on-scene survey and recovery operations.

- A general survey is made downwind to determine the locations and levels of contamination.

- Streets and buildings are cleaned to permissible residual levels if the level of contamination is not greater than a value above which cleanup is not considered reasonable.

- In certain heavily contaminated areas, it is assumed that residents are evacuated and temporarily relocated and that businesses are closed for a period of time during the cleanup operation. In addition, personal and corporate income losses and perimeter security costs are incurred.

- If areas are contaminated above levels for, which cleanup is considered to be reasonable, long-term or permanent land-use denial would result. That is to say, the areas would be placed off-limits to all residents, workers, and businesses. Presumably residents would be forced to relocate. These restrictions would be enforced by permanent barriers and a full-time security force.

- In areas which are considered cleanable, periodic radiation surveys could be required during cleanup and a final survey would be required before the area is released for general occupancy.

Short Half-Life Radionuclides

Short half-life materials may be left to decay (in which case evacuation of the area is necessary) or they can be cleaned up. The choice of techniques will probably be based on how short-lived the material actually is. This model uses a threshold value of 10 days. Thus, if seven environmental half lives amount to less than 10 days and if the "cleanup factor" achieved by seven half lives of radioactive decay is less than the allowable residual level, it is assumed that the material is left to decay and that the area is evacuated and the affected population temporarily relocated.

K2. Decontamination Factor Determination

Economic impacts from transportation accidents are, in general, dependent on the actual level of contamination as well as the level to which the area

*The environmental residence time is conservatively assumed to be seven radioactive half lives. In an actual deposition incident, environmental forces such as weathering, fixation in ecological systems, etc. will generally serve to make environmental residence less than indicated by simply using radioactive half life. 
must be decontaminated. These two factors are related by a term called decontamir nation factor, or DF. DF is defined as the ratio of the actual contamination level to the desired or allowable level. The model used in this analysis considers five DF levels:

\begin{tabular}{|c|c|}
\hline Level & DF Range \\
\hline 1 & $\overline{\mathrm{DF}} \leq 1$ \\
\hline 2 & $1<\mathrm{DF} \leq \mathrm{DF} 1$ \\
\hline 3 & $\mathrm{DF} 1 \leq \mathrm{DF} 2$ \\
\hline 4 & $\mathrm{DF} 2<\mathrm{DF} \leq \mathrm{DF} 3$ \\
\hline 5 & $\mathrm{DF}>\mathrm{DF} 3$ \\
\hline
\end{tabular}

where values selected for DF1, DF2, and DF3 are assigned by the analyst.

The contamination level and a DF value for each cell are computed based on the total amount of material deposited in the cell during cloud passage, the total area of the cell covered by the cloud at its maximum extent in the cell, and the allowable residual contamination level. Streets and open areas are considered separately from buildings because a considerable amount of material may be captured in air conditioning system filters, precipitators, and cooling coils. This scavenging effect is accounted for using the building dose factor (BDF), introduced in the dispersion analysis in the computation of building decontamination factor (Appendix C):

Some typical values for residual contamination level together with the values for DFl through DF3 selected for the present analysis are given in Table $\mathrm{K}-1$.

K3. Cost Models

K3.1 Immediate On-Scene Cost Evaluation

The costs of immediate emergency response can be broken down into three parts:

1. Salaries of emergency response personne1

2. Costs of emergency response vehicles

3. Costs of continued security once immediate on-scene actions have been taken

Reference 1 indicates that a typical salary is $\$ 7.25$ per hour for a police officer and $\$ 7.55$ per hour for a firefighter. Average loaded salaries are assumed to be double these.

It is assumed that a single police vehicle (2 officers) responds to a severity I accident and remains at the scene for the entire accident delay time of $1 / 2$ hour. For severities II through IV, the following response force is assumed:

- A single police vehicle (2 officers)

- One engine company consisting of 2 fire trucks (10 firefighters)

- One rescue vehicle (3 paramedics)

- One ambulance (2 attendants) 
Table $\mathrm{K}-1$

Values for Residual Decontamination

Leve1 and Assumed Decontamination Factors for Current Analysis

A. Residual Level Tabulation

Proposed Leve1

$\left(\mu \mathrm{Ci} / \mathrm{m}^{2}\right)$

12

Comments

Source (Reference No.)

0.0016

EPA leve1

7

11

0.02

Most $\alpha$ emitters

11

0.08

Radioiodines; Sr-90

Al1 radionuclides

except $\alpha$ emitters,

radioiodines, and

Sr-90

11

$0.3-30$

Palomares, Spain, actual cleanup levels

(plutonium)

0.02

DOT vehicle contami-

nation limit

(plutonium)

$0.003-0.01$

Urban area proposed

guidelines (plutonium)

B. DF Levels Selected for Current Analysis

\section{DF Parameters}

DF 1

DF2

DF3
Value Assigned

2.0

20.0

40.0

Firefighting personnel are assumed to remain at the accident scene for 1 hour. Police officers are assumed to remain on the scene for the entire accident delay time (time over 1 hour is considered as cost of continued security).

In extremely severe accidents (severities $V$ through VIII), where the uncontrolled fire duration may be greater than 2 hours, it is assumed that two engine companies 
and two police vehicles would respond. Firefighting personnel are assumed to remain at the accident scene for a maximum of 2 hours; rescue and ambulance personnel are assumed for 1.hour. Police officers are assumed to be involved in immediate emergency response for 2 hours and to remain for continued security through the entire accident delay time with a shift change assumed after the first 8 hours. Salaries for immediate emergency response personnel as well as estimated costs for continued security are listed in Table $\mathrm{K}-2$, columns 3 and 4 .

Costs for typical emergency vehicles for metropolitan police and firefighting personnel are estimated as below. ${ }^{2}$

A new police vehicle costs approximately $\$ 6000$, and the lifetime cost of operating the vehicle including depreciation, repairs, and maintenance is about $\$ 0.25$ per mile. For firefighting equipment analogous values are

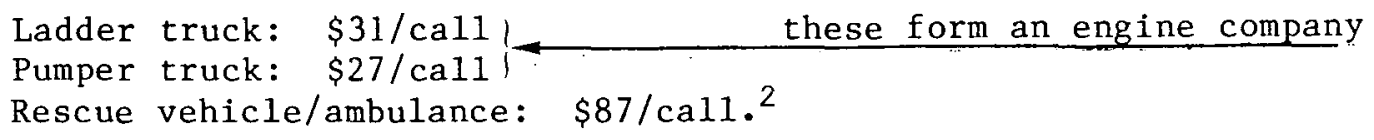

The costs of emergency vehicles for the response to an accident and overall totals for each accident severity are given in Table $\mathrm{K}-2$, column 5 .

\section{K3.2 Cleanup and Recovery Costs}

Once the accident scene is secure, immediate hazards have been identified, and injured personnel attended to; monitoring and recovery must begin, the area must be cleaned up, the radioactive material recovered, and the area returned to normal use. As shown in Table $\mathrm{K}-2$, column 6 , health physics personnel and other workers are required. As the accident severity increases, the requirements for cleanup and monitoring also increase as shown.

Column 7 of Table K-2 summarizes the total on-scene costs for accidents of various severities. These costs are computed for all affected cells, regardless of the DF value.

\section{K3.3 Survey Cost Evaluation}

A survey to determine the extent and hazard of contamination beyond the immediate vicinity of the accident will be conducted by health physics technicians. The expense incurred during this survey depends on the area surveyed, how quickly the technician works, and the technician's loaded salary. These latter two are model parameters and can be modified as desired by the analyst. It is assumed that the area to be surveyed includes all downwind cells affected by the cloud. Since personnel will probably be conservative in choosing their starting point for their pre-cleanup and prerelease surveys, it is further assumed that all horizontal areas in each cell, including streets, open areas, and all floors of all buildings will also be surveyed. This "total cell horizontal area" can be expressed as shown in Equation 1 .

$$
\left[\begin{array}{c}
\text { Total cell } \\
\text { horizontal } \\
\text { area }
\end{array}\right]=\left[\begin{array}{c}
\text { street } \\
\text { area }
\end{array}\right]+\left[\begin{array}{c}
\text { open } \\
\text { area }
\end{array}\right]+\left[\begin{array}{c}
\text { total building } \\
\text { horizontal } \\
\text { area }
\end{array}\right]
$$


TABLE $\mathrm{K}-2$

On-Scene Cost Summary

\begin{tabular}{|c|c|c|c|c|c|c|}
\hline $\begin{array}{l}\text { Accident } \\
\text { Severity } \\
\text { Category } \\
\end{array}$ & $\begin{array}{l}\text { Delay } \\
\text { Time } \\
\text { (hours) }\end{array}$ & $\begin{array}{c}\text { Immediate } \\
\text { Emergency } \\
\text { Response Costs } \\
\end{array}$ & $\begin{array}{l}\text { Continued } \\
\text { Security Past } \\
\text { (Occurrence) } \\
\end{array}$ & $\begin{array}{c}\text { Cost of } \\
\text { Emergency } \\
\text { Vehicles for } \\
\text { Indicated Times } \\
\end{array}$ & $\begin{array}{c}\text { Cost of } \\
\text { Recovery } \\
\& \text { Monitoring } \\
\text { Personnel } \\
\end{array}$ & Totals \\
\hline I & 0.5 & $\begin{array}{l}\$ 14.50 \text { ( } 2 \text { officers } \\
\text { veh. } 1 / 2 \text { hour each) }\end{array}$ & 0 & $\begin{array}{l}\$ 5 \text { (1 police } \\
\text { vehicle) }\end{array}$ & $\begin{array}{l}\$ 10(1 \mathrm{moni}- \\
\text { tor } / 1 \mathrm{~h})\end{array}$ & $\$ 29.50$ \\
\hline II & 1 & 255.50 & 0 & $\begin{array}{l}\$ 203 \text { (1 police } \\
\text { vehicle, } 1 \text { fire } \\
\text { co., 1 rescue } \\
\text { vehicle, } 1 \\
\text { ambulance) }\end{array}$ & $\begin{array}{l}\$ 10(1 \text { moni- } \\
\text { tor } / 1 \mathrm{~h})\end{array}$ & 468.50 \\
\hline II I & 2 & 255.50 & $\begin{array}{l}\$ 29(2 \text { off } i- \\
\text { cers } / 1 \text { h) }\end{array}$ & $\begin{array}{l}\$ 203 \text { ( } 1 \text { police } \\
\text { vehicle, } 1 \text { fire } \\
\text { co. I rescue } \\
\text { vehicle, } 1 \\
\text { ambulance) }\end{array}$ & $\begin{array}{l}\$ 45(2 \text { moni- } \\
\text { tors, } 5 \\
\text { laborers/1 h) }\end{array}$ & 532.50 \\
\hline IV & 4 & 255.50 & $\begin{array}{l}\$ 87(2 \text { offi- } \\
\text { cers } / 3 \mathrm{~h})\end{array}$ & $\begin{array}{l}\$ 203 \text { (1 police } \\
\text { vehicle, l fire } \\
\text { co., l rescue } \\
\text { vehicle, } 1 \\
\text { ambulance) }\end{array}$ & $\begin{array}{l}\$ 135(2 \text { moni- } \\
\text { tors, } 5 \\
\text { laborers } / 3 \mathrm{~h})\end{array}$ & 680.50 \\
\hline v & 8 & 755.00 & $\begin{array}{l}\$ 348(4 \text { offi- } \\
\text { cers } / 6 \mathrm{~h})\end{array}$ & $\begin{array}{l}\$ 406 \text { ( } 2 \text { police } \\
\text { vehicles, } 2 \\
\text { fire co., } \\
2 \text { rescue vehi- } \\
\text { cles, } 2 \\
\text { ambulances) }\end{array}$ & $\begin{array}{l}\$ 390 \text { ( } 3 \text { moni- } \\
\text { tors, } 7 \\
\text { laborers } / 6 \mathrm{~h} \text { ) }\end{array}$ & 1899.00 \\
\hline VI & 12 & 755.00 & $\begin{array}{l}\$ 580(4 \text { offi- } \\
\text { cers } / 10 \mathrm{~h})\end{array}$ & $\begin{array}{l}\$ 416 \text { ( } 4 \text { police } \\
\text { vehicles, } 2 \\
\text { fire co., } 2 \\
\text { rescue vehicles, } \\
2 \text { ambulances) }\end{array}$ & $\begin{array}{l}\$ 900(4 \text { moni- } \\
\text { tors, } 10 \\
\text { laborers } / 16 \mathrm{~h})\end{array}$ & 2651.00 \\
\hline VII & 18 & 755.00 & $\begin{array}{l}\$ 928(4 \text { offi- } \\
\text { cers } / 16 \mathrm{~h})\end{array}$ & $\begin{array}{l}\$ 426 \text { ( } 6 \text { police } \\
\text { vehicles, } 2 \\
\text { fire co., } 2 \\
\text { rescue vehicles, } \\
2 \text { ambulances) }\end{array}$ & $\begin{array}{l}\$ 1404 \text { (4 moni- } \\
\text { tors, } 10 \\
\text { laborers } / 10 \mathrm{~h} \text { ) }\end{array}$ & 3549.00 \\
\hline VIII & 24 & 755.00 & $\begin{array}{l}\$ 1276(4 \\
\text { officers/ } \\
22 \mathrm{~h})\end{array}$ & $\begin{array}{l}\$ 436 \text { ( } 8 \text { police } \\
\text { vehicles, } 2 \\
\text { fire co., } 2 \text { res- } \\
\text { cue vehicles, } \\
2 \text { ambulances) }\end{array}$ & $\begin{array}{l}\$ 1980 \text { ( } 4 \text { moni- } \\
\text { tors, } 10 \\
\text { laborers } / 22 \mathrm{~h} \text { ) }\end{array}$ & 4447.00 \\
\hline
\end{tabular}


If $\mathrm{A}=$ cell area in $\mathrm{m}^{2}$, then

$$
\underset{\left.\begin{array}{c}
\text { total cel1 } \\
\text { horizontal } \\
\text { area }
\end{array}\right]=}{\left.\left(f_{s t} \cdot A\right)+f_{0} \cdot A\right)+\left[\left(1-\left(f_{s t}+f_{o}\right)\right)\right.}
$$

where

$$
\begin{aligned}
\mathrm{f}_{\mathrm{st}} & =\text { fraction of cell area occupied by streets } \\
\mathrm{f}_{\mathrm{o}} & =\text { fraction of cell area occupied by open areas } \\
\mathrm{n} & =\text { number of floors of buildings affected }
\end{aligned}
$$

The expression can be simplified to yield

$$
\left[\begin{array}{c}
\text { total cell } \\
\text { horizontal } \\
\text { area }
\end{array}\right]=A \cdot\left[\left(f_{s t}+f_{o}\right)(1-n)+n\right]
$$

Thus, the total survey cost is approximated by

$$
(\text { survey } \cos t)=A \cdot \sum_{z=1}^{P}\left[\left(f_{s t}+f_{0}\right)\left(1-n_{z}\right)+n_{z}\right] \cdot \frac{H P S}{S R} \cdot 2
$$

where

$$
\begin{aligned}
z= & \text { summation variable over cells in grid } \\
P= & \text { number of cells in grid with nonzero atmospheric } \\
& \text { dilution factors } \\
H P S= & \text { loaded salary for health physics technicians }(\$ / h) \\
S R= & \text { survey rate }\left(\mathrm{m}^{2} / \mathrm{h}\right)
\end{aligned}
$$

If the value for street $D F$ is $\leq 1$ in any affected cell, no survey costs are included for that cell.

\section{K3.4 Street Cleanup Costs - Long Half-Life Material}

The model assumes one of two basic cleanup procedures for areas covered by pavement and/or sidewalk: for relatively low contamination levels, hosing and/or sandblasting may be sufficient; for higher levels, pavement would probably be removed and replaced.

If the street cleanup cost values from the SL-1 research reactor incident in Idaho are typical, a cleanup cost of about $\$ 4 / \mathrm{m}^{2}$ can be inferred. Contact with paving contractors indicates that repaving costs would be approximately $\$ 2.50 / \mathrm{m}^{2}$, including $\$ 1.20 / \mathrm{m}^{2}$ for removal of old asphalt. This average value assumed by the model is a variable and can be adjusted by multiplying the contaminated area by the cost per unit area: 


$$
(\text { street cleanup } \cos t)=A \cdot \gamma \cdot f_{s t} \cdot V_{s t}
$$

where

$$
\begin{aligned}
\gamma & =\text { fraction of cell area covered by cloud } \\
\mathrm{V}_{\mathrm{st}} & =\text { cost for street cleanup }\left(\$ / \mathrm{m}^{2}\right)
\end{aligned}
$$

It should be noted that this estimate does not include costs associated with attendant business and traffic disruption.

\section{K3.5 Building Cleanup Costs -- Long Half-Life Material}

Building cleanup costs depend on four factors:

1. Height of the buildings

2. Building usage (commercial/industrial or residential)

3. Population density

4. Contamination leve1

Costs to clean up parks and other public areas are also included in this portion of the analysis.

Affected cells are initially classified as either principally commercial/ industrial or principally residential. If they are commercial/industrial, the cost estimate equation takes this form:

$$
\left[\begin{array}{l}
\text { building } \\
\text { cleanup } \\
\text { cost }
\end{array}\right]=A \cdot \gamma \cdot\left[\left(\mathrm{PD}_{3}-\mathrm{PD}_{1}\right) \cdot \mathrm{V}_{\mathrm{B}}\right]+\left[\mathrm{PD}_{1} \cdot \mathrm{V}_{\mathrm{P}}\right]
$$

where

$$
\begin{aligned}
\mathrm{PD}_{3} & =\text { population density in time span } 3 \text { (morning work period) } \\
\mathrm{PD}_{1} & =\text { population density in time span } 1 \text { (nighttime) } \\
\mathrm{V}_{\mathrm{B}} & =\text { building cleanup cost ( } \$ \text { per capita) } \\
\mathrm{V}_{\mathrm{P}} & =\text { park and public area cleanup cost ( } \$ \text { per capita) }
\end{aligned}
$$

Since the term PD refers to people in buildings, the fraction of area occupied by buildings is not required. The term $\left(P D_{3}-P D_{1}\right)$ describes the number of workers in the cell since $V_{B}$ depends on worker population. $V_{P}$ is dependent only on the resident population, so the applicable population density term is $\mathrm{PD}_{1}$. The calculations for building cleanup for commerical/industrial cells is assumed to be independent of building height.

If the cell is principally residential, three building heights are evaluated: 1 and 2 floors; 3, 4, or 5 floors; and 6 or more floors. The general form of the cost expression is the same in all three cases:

$$
\left[\begin{array}{c}
\text { building } \\
\text { cleanup } \\
\text { cost }
\end{array}\right]=A \cdot \gamma \cdot \mathrm{PD}_{1} \cdot\left(\mathrm{V}_{\mathrm{B}}+\mathrm{V}_{\mathrm{P}}\right)
$$


As discussed in Section K2, there are four DF levels of interest. For DF $>\mathrm{DF} 3$, other assumptions are made. At the fourth level, the cleanup technique is assumed to be the same as in the third except that it is performed twice and a radiation survey is included between the two cleanup cycles to assess the success of the operation. Thus, the form of the equation becomes

$$
\left[\begin{array}{c}
\text { building } \\
\text { cleanup } \\
\text { cost }
\end{array}\right]=A \cdot \gamma \cdot \mathrm{PD}_{1} \cdot\left[\left(\mathrm{V}_{\mathrm{B}} \cdot 2\right)+\mathrm{V}_{\mathrm{P}}\right]+\frac{\mathrm{HPS}}{\mathrm{SR}} \cdot \mathrm{f}_{\mathrm{b}} \cdot \mathrm{n}
$$

Note that, because the park cleanup technique is turf removal and replacement, its cost is not doubled. The survey cost format is similar to that given in Equation 4 with the modification that only building areas are assumed to be surveyed.

If buildings use central air-conditioning. systems, a substantial amount of the radioactive material may be trapped in filters. These filters would have to be removed and disposed of as contaminated waste and new filters would have to be installed. It is assumed that approximately $l$ hour per filter is required for removal, bagging, monitoring, and reinstallation. If heavy contamination is present requiring extensive ductwork cleanup (i.e., DF2 < DF $\leq \mathrm{DF} 3$ ), two change-out cycles are assumed, whereas one change-out is considered sufficient in less contaminated areas.

\section{K3.6 Evacuation Costs -- Long Half-Life Material}

Expense incurred as a result of evacuation has three components: 1odging and travel expense, personal income loss for affected workers for the period of the evacuation, and corporate income loss. The baseline cost expression for evacuation is given by

$$
\begin{aligned}
\left.\begin{array}{c}
\text { evacuation } \\
\text { cost })
\end{array}\right]= & \gamma \cdot A \cdot\left\{\left(P D_{1} \cdot V_{e}\right)+\left[\left(P D_{3}-P D_{1}\right) \cdot V_{P I}\right]+\right. \\
& {\left.\left[\left(\mathrm{PD}_{3}-\mathrm{PD}_{1}\right) \cdot \mathrm{V}_{\mathrm{CI}}\right]\right\} }
\end{aligned}
$$

where

$$
\begin{aligned}
\mathrm{V}_{\mathrm{e}} & =\text { evacuation cost }(\$ \text { per capita) } \\
\mathrm{V}_{\mathrm{PI}} & =\text { personal income loss ( } \$ \text { per person) } \\
\mathrm{V}_{\mathrm{CI}} & =\text { corporate income loss ( } \$ \text { per worker) }
\end{aligned}
$$

Because the evacuation is assumed to last a minimum of 10 days, personal and corporate income losses are figured on that basis.

As the required level of cleanup increases, so does the period of assumed evacuation. For example, when the $D F$ goes from $1<D F \leq D F 1$ to $D F 1<D F \leq D F 2$, the cost doubles; and when the DF goes from DF1 $<\mathrm{DF} \leq \mathrm{DF} 2$ to $\mathrm{DF} 2<\mathrm{DF} \leq \mathrm{DF} 3$, the cost doubles again.

\section{K3.7 Long-Term or Permanent Land-Use Denial -- Long Half-Life Materials}

Under certain conditions, civic authorities may decide that the use of an area must be denied entirely. If this should happen, the economic impact has four components: land use costs, moving costs (personal and business), personal income loss, and corporate income loss. 
Land-use costs are assumed to include the cost of property taxes (at $3 \%$ ) and the interest (at $9 \%$ ) which could be earned if the money tied up in land were available for other investments. Personal income loss is predicated on relocation and reemployment after 90 days; corporate losses assume a "dead" period of 180 days. The period of land-use denial is assumed to be seven half lives or 50 years, whichever is less. The general form of the cost expression, based on information from Reference 4 , is given by

$$
\begin{aligned}
{\left[\begin{array}{c}
\text { land use } \\
\text { denial cost }
\end{array}\right]=} & \gamma \cdot \mathrm{A} \cdot \mathrm{PD}_{1} \cdot \mathrm{V}_{\mathrm{L}} \cdot\left\{1-\left[\exp \left(-0.12 \cdot \frac{7 \cdot \mathrm{t}_{1 / 2}}{365}\right)\right] .\right. \\
& {\left.\left[0.3+0.3 \exp \left(-0.12 \cdot \frac{7 \cdot \mathrm{t}_{1 / 2}}{365}\right)\right]\right\}+ } \\
& {\left[\mathrm{PD}_{1} \cdot \mathrm{V}_{\mathrm{PI}}\right]+\left[\left(\mathrm{PD}_{3}-\mathrm{PD}_{1}\right) \cdot \mathrm{v}_{\mathrm{CI}}\right]+\left[\mathrm{PD}_{1} \cdot\left(\mathrm{V}_{\mathrm{MI}}+\mathrm{Vc}\right)\right] }
\end{aligned}
$$

where

$$
\begin{aligned}
\mathrm{V}_{\mathrm{L}} & =\text { assumed purchase value of land ( } \$ \text { per capita) } \\
\mathrm{t}_{1 / 2} & =\text { radionuclide half life (days), } \\
\mathrm{V}_{\mathrm{MI}} & =\text { individual moving cost ( } \$ \text { per capita) } \\
\mathrm{V}_{\mathrm{MB}} & =\text { business and government agencies moving cost ( } \$ \text { per capita) }
\end{aligned}
$$

The factor of 0.3 is the assumed ratio of the value of improvements to the total value of the property.

\section{K3.8 Security Costs}

If an area is evacuated for any period of time, security will be required to prevent looting, vandalism, and spread of contamination. The total perimeter of the contaminated area to be secured is given by

$$
\left[\begin{array}{l}
\text { Perimeter } \\
\text { Distance }
\end{array}\right]=4 \cdot \sqrt{\mathrm{CAREA} \cdot \gamma_{\mathrm{DF}}}
$$

where CAREA $=$ cell area .

It is further assumed that one guard can effectively patrol a 1/4-mile (402-metre) section, that three full shifts will be required per day, and that each shift will include at least one supervisor. Thus, the number of security personnel required per shift is given by

$$
\left[\begin{array}{c}
\text { No. } \text { of security } \\
\text { personnel } \\
\text { per shift }
\end{array}\right]=\frac{4 \cdot \sqrt{\text { CAREA } \cdot \gamma_{D F}}}{402}+1
$$

By assigning a number of days appropriate to the evacuation and loaded salaries for security force personnel, the total security cost can be computed. 
If permanent land-use denial is assumed, the security must be long-term. In this case, it is assumed that a 6-foot chainlink fence is erected around the area and that a 24-hour security force of four guards is assigned for either seven radioactive half lives or 20 years, whichever is less.

\section{K3.9 Modifications for Short Half-Life Materials}

Short half-1ife materials may have hazard times of more than 10 days or less than 10 days. In this context, "hazard time" is defined as seven half lives, i.e., the time in which more than $99 \%$ of the original material will have decayed.

If the material has a hazard time of less than 10 days and if the residual contamination after 10 days is less than the selected cleanup level, it is assumed that the material will be left to decay (see K1.2). Under these circumstances, building cleanup costs, street cleanup costs, and land-use denial costs are set to zero. In addition, the low-level contaminated area $(1<\mathrm{DF}<\mathrm{DF} 1)$ is assumed to be nonresidential. Because evacuation time may vary in this approach, evacuation costs are multiplied by a factor of $7 \times t_{1 / 2} / 10$.

If the material has a hazard time greater than 10 days or if 10 days of radioactive decay does not reduce the level sufficiently, the procedure outlined for long half-life materials are followed with the following exceptions :

- No land-use denial is assumed.

- All evacuation costs are multiplied by $7 \times t_{1 / 2} / 10$.

K4. Economic Risk Calculation

Once total costs are computed for each accident, they are combined with their probability of occurrence. The results are summed over all cells on each route, all accident severity categories, and all transported radionuclides to give economic risk.

$$
\begin{aligned}
& {\left[\begin{array}{c}
\text { economic } \\
\text { isk }
\end{array}\right]=\sum_{i=1}^{\text {isotopes }} \sum_{j=1}^{\text {\#severities }} \sum_{k=1}^{\text {cells }}\left(\begin{array}{l}
\text { cost } \\
\text { acc. })_{i, j, k}
\end{array} .\right.} \\
& \mathrm{SPY}_{i} \cdot \mathrm{L}_{i, k}
\end{aligned}
$$

\section{K5. Summary}

Figure $\mathrm{K}-\mathrm{l}$ shows the basic calculated flow path with the economic impact assessment model. Table $\mathrm{K}-3$ shows the required parameters and the values assumed for the current evaluation. Those items with an asterisk in column 5 are model variables and can be modified to suit other analyses as desired. Principal modeling assumptions are outlined in the table. 


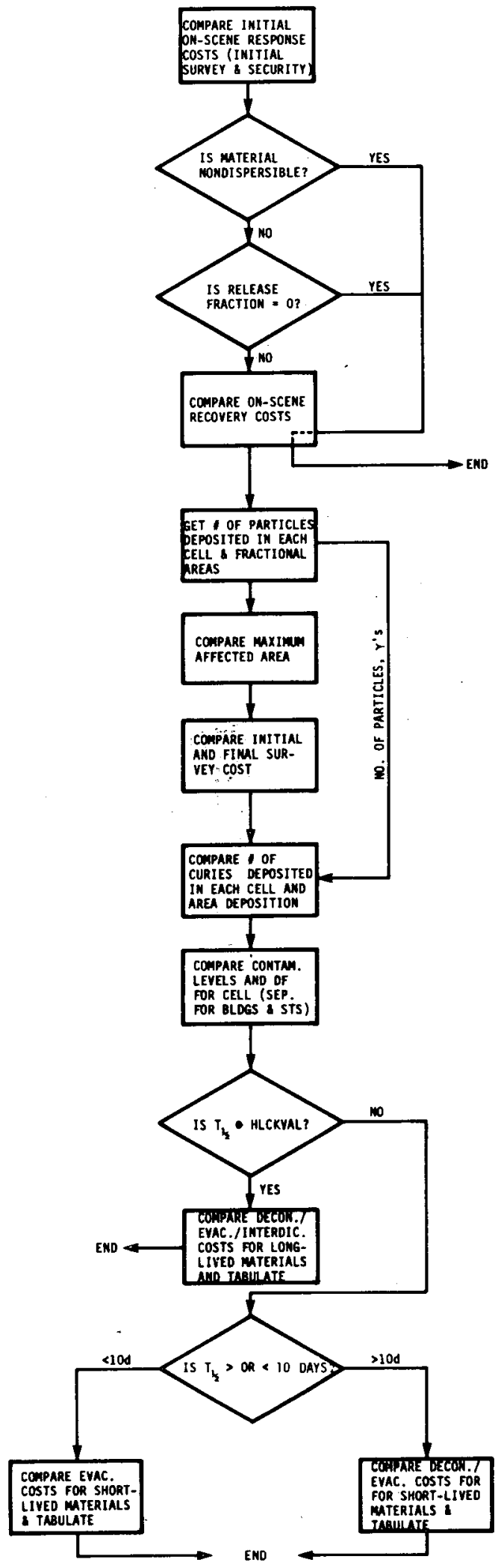

Figure K-1. Calculational Flow Diagram for Economic Impact Assessment 
Table $\mathrm{K}-3$

Economic Cost Parameter Summary

\begin{tabular}{|c|c|c|c|c|c|c|}
\hline Parameter & $\underline{\text { DF Range }}$ & General Technique & Symbol & $\begin{array}{l}\text { Value } \\
\text { Chosen* }\end{array}$ & Comments & Reference \\
\hline $\begin{array}{l}\text { Acceptable cleanup } \\
\text { level }\end{array}$ & NA & NA & CULVL & $0.2 \star$ & EPA proposed & 7 \\
\hline $\begin{array}{l}\text { Upper limit of } \\
\text { lightly contaminated } \\
\text { regime }\end{array}$ & NA & $\mathrm{NA}$ & DF 1 & 2 & $\begin{array}{l}\text { Some data } \\
\text { available }\end{array}$ & 5 \\
\hline $\begin{array}{l}\text { Lower limlt of } \\
\text { moderately contami- } \\
\text { nated regime }\end{array}$ & NA & $\mathrm{NA}$ & DF 2 & 20 & $\begin{array}{l}\text { Some data } \\
\text { available }\end{array}$ & 5 \\
\hline $\begin{array}{l}\text { Upper limit of } \\
\text { moderately contami- } \\
\text { nated regime }\end{array}$ & $\mathrm{NA}$ & $\mathrm{NA}$ & $\mathrm{DF} 3$ & 40 & $\begin{array}{l}\text { Models assumes } \\
\text { techniques for } \\
D F=20 \text { done twice }\end{array}$ & - \\
\hline $\begin{array}{l}\text { Loaded salary for } \\
\text { health physics } \\
\text { technician }\end{array}$ & NA & $\mathrm{NA}$ & HPS & $9 \$ / h^{*}$ & $\begin{array}{l}\$ 20,000 / \mathrm{yr}(270 \\
8-\mathrm{h} \text { days })(1979 \\
\text { salary information })\end{array}$ & 8 \\
\hline Survey rate & NA & $\begin{array}{l}\text { Hand-carried } \\
\text { radiac equip. }\end{array}$ & $\mathrm{SR}$ & $\begin{array}{l}60-120 \star \\
\mathrm{m}^{2} / \mathrm{h}\end{array}$ & $\begin{array}{l}\text { Higher values } \\
\text { for -, lower } \\
\text { for }\end{array}$ & 8 \\
\hline Half-life check value & NA & - NA & HLCKVAL & $\begin{array}{l}180 \\
\text { days* }\end{array}$ & $\begin{array}{l}\text { Threshold for } \\
\text { long-lived } \\
\text { radionuclides }\end{array}$ & - \\
\hline $\begin{array}{l}\text { Cost for street } \\
\text { cleanup }\end{array}$ & $<D F<D F 1$ & $\begin{array}{l}\text { Sandblast/Vl } \\
\text { firehose }\end{array}$ & & $\begin{array}{l}4.38 \\
\$ / \mathrm{m}^{2} \star\end{array}$ & $\begin{array}{l}\text { SL-1 accidents } \\
\text { data (from } 1961 \\
\text { data) }\end{array}$ & 4 \\
\hline $\begin{array}{l}\text { Cost for building } \\
\text { cleanup }\end{array}$ & $1<\mathrm{DF} \leq \mathrm{DF} 1$ & $\begin{array}{l}\text { Replace lawn/ } \\
\text { firehose roofs } \\
\text { and pavement }\end{array}$ & v2 & $\begin{array}{l}506 \$ / \\
\text { capita* }\end{array}$ & $\begin{array}{l}1,2 \text { story } \\
\text { residential } \\
\text { buildings } \\
\text { (from } 1974 \\
\text { data) }\end{array}$ & $\begin{array}{l}5, \text { Table } \\
\text { VI, }, \cdot 2,3\end{array}$ \\
\hline
\end{tabular}

*A11 cost values in 1979 dollars assuming the following compound inflation rates: 1960-1965: $1.25 \%$; 1965-1970: 4.24\%; 1970-1975: 6.75\%; 1975-current: $7.51 \%$. 


\begin{tabular}{|c|c|c|c|c|c|c|}
\hline Parameters & DF Range & General Technique & Symbol & $\begin{array}{l}\text { Value } \\
\text { Chosen* } \\
\end{array}$ & Comments & Reference \\
\hline $\begin{array}{l}\text { Cost for building } \\
\text { cleanup }\end{array}$ & $1<D F \leq D F 1$ & $\begin{array}{l}\text { Replace lawn/ } \\
\text { firehose roofs } \\
\text { and pavement }\end{array}$ & v3 & $\begin{array}{l}44 \$ / \\
\text { capita* }\end{array}$ & $\begin{array}{l}3,4,4-\text { story } \\
\text { residential } \\
\text { buildings (from } \\
1974 \text { data) }\end{array}$ & $\begin{array}{l}\text { 5, Table } \\
\text { vI, } \ell .2,3\end{array}$ \\
\hline $\begin{array}{l}\text { Cost for building } \\
\text { cleanup }\end{array}$ & $1<\mathrm{DF} \leq \mathrm{DF} 1$ & $\begin{array}{l}\text { Replace lawn/ } \\
\text { firehose roofs } \\
\text { and pavement }\end{array}$ & v4 & $\begin{array}{l}22 \$ 1 \\
\text { capita* }\end{array}$ & $\begin{array}{l}6 \text { floors or } \\
\text { more (from } \\
1974 \text { data) }\end{array}$ & $\begin{array}{l}\text { 5, Table } \\
\mathrm{VI}, \ell .2,3\end{array}$ \\
\hline $\begin{array}{l}\text { Cost for bullding } \\
\text { cleanup }\end{array}$ & $1<\mathrm{DF} \leq \mathrm{DF} 1$ & Not specified & v5 & $\begin{array}{l}31 \$ 1 \\
\text { capita* }\end{array}$ & $\begin{array}{l}\text { Commercial } \\
\text { (from } 1974 \\
\text { data) }\end{array}$ & $\begin{array}{l}\text { 5, Table } \\
\text { VI, } l .2-7\end{array}$ \\
\hline $\begin{array}{l}\text { Cost for park/open } \\
\text { area cleanup }\end{array}$ & $1 \leq \mathrm{DF} \leq \mathrm{DF} 1$ & Not spectfied & v6 & $\begin{array}{l}101 \$ / \\
\text { capita* }\end{array}$ & $\begin{array}{l}\text { (from } 1974 \\
\text { data) }\end{array}$ & $\begin{array}{l}\text { 5, Table } \\
\text { VI, } \ell .2-7\end{array}$ \\
\hline Evacuation cost & NA & NA & v7 & $\begin{array}{l}200 \$ 1 \\
\text { capita* }\end{array}$ & $\begin{array}{l}\text { 10-day evacua- } \\
\text { t1on (from } 1974 \\
\text { data) }\end{array}$ & $\begin{array}{r}5 \text { Table } \\
v I, \ell .2-9\end{array}$ \\
\hline $\begin{array}{l}\text { Individual income } \\
\text { loss }\end{array}$ & NA & NA & v8 & $\begin{array}{l}718 \$ 1 \\
\text { capita }\end{array}$ & $\begin{array}{l}\text { 10-day evacua- } \\
\text { t1on (from } \\
1974 \text { data) }\end{array}$ & $\begin{array}{l}\text { 5, Table } \\
12.4 .2 .2\end{array}$ \\
\hline $\begin{array}{l}\text { Corporate income } \\
\text { loss }\end{array}$ & NA & NA & v9 & $\begin{array}{l}76 \$ / \\
\text { capita }\end{array}$ & $\begin{array}{l}\text { 10-day evacua- } \\
\text { tion (from } \\
1974 \text { data) }\end{array}$ & $\begin{array}{c}5 \\
12.4 .2 \cdot 2\end{array}$ \\
\hline $\begin{array}{l}\text { Cost for street } \\
\text { cleanup }\end{array}$ & $\mathrm{DF} 1 \leq \mathrm{DF} \leq \mathrm{DF} 3$ & $\begin{array}{l}\text { Replace pave- } \\
\text { ment }\end{array}$ & v10 & $\begin{array}{l}247 \\
\$ / m^{2}\end{array}$ & (1979 data) & 4 \\
\hline $\begin{array}{l}\text { Cost for building } \\
\text { cleanup }\end{array}$ & $\mathrm{DF} 1 \leq \mathrm{DF} \leq \mathrm{DF} 2$ & Not specifled & V11 & $\begin{array}{l}1607 \$ / \\
\text { capita }\end{array}$ & $\begin{array}{l}1,2-\text {-story } \\
\text { residential } \\
\text { bulldings } \\
\text { (from } 1974 \\
\text { data) }\end{array}$ & $\begin{array}{c}5 \\
12.4 \cdot 1.2\end{array}$ \\
\hline $\begin{array}{l}\text { Cost for buflding } \\
\text { cleanup }\end{array}$ & $\mathrm{DF} 1 \leq \mathrm{DF} \leq \mathrm{DF} 2$ & Not specified & v12 & $\begin{array}{l}441 \$ 1 \\
\text { capita* }\end{array}$ & $\begin{array}{l}3,4,5 \text {-story } \\
\text { residential } \\
\text { buildings (from } \\
1974 \text { data) }\end{array}$ & $12.5^{5} \cdot 1.2$ \\
\hline
\end{tabular}

*A1l cost values in 1979 dollars assuming the following compound Inflation rates: 1960-1965: $1.25 \%$; 1965-1970: 4.24\%; 1970-1975: $6.75 \%$; 1975-current： $7.51 \%$. 
Table K-3 (Continued)

\begin{tabular}{|c|c|c|c|c|c|c|}
\hline Parameters & DF Range & General Technique & Symbol & $\begin{array}{l}\text { Value } \\
\text { Chosen* }\end{array}$ & Comments & Reference \\
\hline $\begin{array}{l}\text { Cost for building } \\
\text { cleanup }\end{array}$ & $\mathrm{DF} 1 \leq \mathrm{DF} \leq \mathrm{DF} 2$ & Not specified & V13 & $\begin{array}{l}205 \$ / \\
\text { capita* }\end{array}$ & $\begin{array}{l}\text { 6-story } \\
\text { residential } \\
\text { buildings } \\
\text { (from } 1974 \text { data) }\end{array}$ & $\begin{array}{l}\text { 5, Table } \\
12.4 .1 .2\end{array}$ \\
\hline $\begin{array}{l}\text { Cost for building } \\
\text { cleanup }\end{array}$ & $\mathrm{DF} 1<\mathrm{DF} \leq \mathrm{DF} 2$ & Not specified & V14 & $\begin{array}{l}462 \$ / \\
\text { capita* }\end{array}$ & $\begin{array}{l}\text { Commercial } \\
\text { (from } 1974 \text { data) }\end{array}$ & $\begin{array}{l}\text { 5, Tabie } \\
\text { Ve.2.7 }\end{array}$ \\
\hline $\begin{array}{l}\text { Cost for parks/ } \\
\text { open area cleanup }\end{array}$ & $\mathrm{DF} 1<\mathrm{DF} \leq \mathrm{DF} 3$ & Replace turf & V15 & $\begin{array}{l}673 \$ / \\
\text { capita* }\end{array}$ & (Erom 1974 data) & $\begin{array}{l}\text { 5, Table } \\
\text { Ve. } 2-7\end{array}$ \\
\hline Land value & NA & $\mathrm{NA}$ & V16 & $\begin{array}{l}24,965 \\
\text { \$/capita* }\end{array}$ & (from 1974 data) & $\begin{array}{l}\text { 5, Table } \\
\text { VI, I } 2-9\end{array}$ \\
\hline $\begin{array}{l}\text { Moving expense- } \\
\text { people }\end{array}$ & NA & $\mathrm{NA}$ & V17 & $\begin{array}{l}587 \$ / \\
\text { capita* }\end{array}$ & (from 1974 data) & $\begin{array}{l}5, \text { Sec. } \\
12.4\end{array}$ \\
\hline $\begin{array}{l}\text { Moving expense- } \\
\text { agencies }\end{array}$ & NA & NA & V18 & $\begin{array}{l}690 \$ / \\
\text { capita* }\end{array}$ & (from 1974 data) & $\begin{array}{l}5, \mathrm{Sec} . \\
12.4\end{array}$ \\
\hline $\begin{array}{l}\text { Security guard } \\
\text { loaded salary }\end{array}$ & NA & NA & V19 & $\begin{array}{l}5.80 \\
\$ / h^{*}\end{array}$ & (from 1977 data) & 6 \\
\hline Fencing costs & NA & $\mathrm{NA}$ & V20 & $\begin{array}{l}9.80 \\
\$ / \mathrm{m} *\end{array}$ & $\begin{array}{l}6 \mathrm{ft} \text { chainlink } \\
\text { fence installed } \\
\text { (1979 data) }\end{array}$ & 9 \\
\hline Filter "density" & NA & NA & FA & $0.02 *$ & $\begin{array}{l}\text { filters } / \mathrm{m}^{2} \\
(1979 \text { data })\end{array}$ & 10 \\
\hline Filter cost & NA & NA & v21 & $\begin{array}{l}20 \$ 1 \\
\text { filter* }\end{array}$ & $\begin{array}{l}\$ 10 \text { per oiled } 20 x \\
20 \times 2 \text { in. metal fil- } \\
\text { ter, (SLA stock } \\
\$ 717-323,717-327) \\
\text { \$10 labor charge } \\
\text { for installation } \\
\text { (1979 data) }\end{array}$ & 10 \\
\hline
\end{tabular}

*All cost values in 1979 dollars assuming the following compound inflation rates: 1960-1965: 
NOTES

lU.S. Department of Commerce, U.S. Statistical Abstract, 1978.

${ }^{2}$ E. R. Voth, "Commercial Ambulance Rates," Emergency Magazine, 11(7): 15-16 (July 1979).

3 "Handling of Radiation Accidents," IAEA Symposium, IAEA-SM-119/53, Vienna, Austria, 19-23 Mà 1969.

${ }^{4}$ J. M. Taylor, Sandia Laboratories Division 1233, private .communication with Hale Construction, Albuquerque, NM, March 1979.

${ }^{5}$ U.S. Nuclear Regulatory Commission, Reactor Safety Study, Appendix VI, WASH-1400 (Washington: USNRC, October 1975).

${ }^{6}$ J. M. Taylor, Sandia Laboratories Division 1233, private communication with Sandia Laboratories Security, March 1979.

${ }^{7}$ U.S. Environmental Protection Agency, Proposed Guidance on Dose Limits for Persons Exposed to Transuranic Elements in the General Environment, EPA 520/4-77-016 (Washington: USEPA, September 1977).

8 J. M. Taylor, Sandia Laboratories Division 1233, private communication with J. Metcalf, Sandia Laboratories Division 3312 (Health Physics), March 1979.

${ }^{9}$ J. M. Taylor, Sandia Laboratories Division 1233, private communication with Albuquerque, NM, fencing distributors, March 1979.

10 J. M. Taylor, Sandia Laboratories Division 1233, private communication with R. Hole, Sandia Laboratories Division 3742 (Stores Management), March 1979.

11 American National Standards Institute, Proposed America National Standard N328: "Control of Radioactive Surface Contamination on Materials, Equipment, and Facilities to be Released for Uncontrolled Use," 1979.

${ }^{12}$ L. G. Faust et a1, A Guide to Good Practices at Plutonium Facilities, BNWL-2086 (Richland, WA: Battelle Pacific Northwest Laboratories,

September 1977). 
SOCIAL IMPACTS

\section{L1. Background}

This appendix summarizes a report on social impacts resulting from the transportation of radioactive materials through urban environments.* Social impacts are defined as significant changes in the conditions of daily life that can be attributed to specific events. These social impacts are identified by retrospectively examining representative cases and reviewing selections from the theoretical and the technical literatures on social impacts. The likelihood of social impacts occurring in the future are assessed on the basis of observed impacts to date.

\section{L2. Framework for Analysis}

This study analyzes five categories of social impacts resulting from four transportation events (causative events). The causative events are (1) incident-free transportation, in which nothing unusual happens to the radioactive package or transportation vehicle; (2) vehicular accidents;

(3) human errors and deviation from accepted quality assurance practices; and (4) malevolent acts, such as sabotage or theft.

The social impact categories include: (1) psychological impacts, defined as psychological distress and changes in an individual's beliefs, attitudes, and behavior; (2) sociological impacts, defined as changes or attempted changes in social structure, the organization of social life, and collective behavior; (3) political impacts, defined as changes or attempted changes in those activities by which opposing viewpoints are reconciled, including both governmental politics and interest group activity; (4) legal impacts, defined as changes or attempted changes in statutes, regulations, and adjudicatory decisions; and (5) organizational impacts, defined as changes in organizations with respect to their existence, structure, procedures, and capabilities. These impact categories are not mutually exclusive. Economic impacts were not included in this definition of social impacts, except as they indirectly affect the five impact categories.

Causative events and social impacts are analyzed within the urban environment, which is composed of local social and physical attributes as well as links to people and institutions outside the impacted community. The impact process is continuous as people and institutions adapt to the changes induced by the causative event. Social impacts are defined in

*This appendix is the executive summary to a report entitled "Identification and Assessment of the Social Impacts of Transportation of Radioactive Materials in Urban Environments," prepared by Battelle Human Affairs Research Centers, December, 1979. Detailed references are available in this larger document. 
each of the five impact categories as the difference between pre-impact baseline conditions and post-impact conditions less changes due to factors not associated with the causative event.

\section{L3. Data and Methods}

Social impact analysis of radioactive materials transportation is a new undertaking. Standard methods used in socioeconomic impact assessments have been modified to fit the needs of this study. Data and methods include

1. An evaluation of public opinion surveys regarding nuclear power.

2. Reviews of selected 1 iterature on social impact assessment methodologies, community decision making processes, including public participation in urban political processes, and individual community responses to natural disasters.

3. An analysis of selected case studies.

4. Telephone interviews with officials and citizens who were involved in or were knowledgeable about selected transportation events.

5. Legal analysis involving a review of statutes, regulations and adjudicatory decisions pertaining to radioactive materials transportation.

6. Compilation and review of organizational procedures and emergency response plans for dealing with these transport events.

7. Reference to other types of events as analogies to help fill in gaps in the data.

8. Informed judgment in the assessment of the significance of social impacts, their likelihood of occurrence given similar events in the future, and the consequences for urban social life and nuclear transportation policy if the impact does occur.

\section{L4. Findings}

The findings of this study are presented below, by category of social impact for each of the four causative events.

\section{L4.1 Incident-Free Transportation}

The purpose of this section is to discuss the present baseline conditions with respect to the five impact categories. If a present condition is clearly due to anticipation of or response to a vehicular accident, a human error, or a malevolent act, then it will be discussed in the section dealing with that event category. Where causality is unclear, the baseline condition will be discussed in this section. 
The urban public generally is not knowledgeable about the transportation of radioactive materials, in part because there have not been any serious incidents to date. Routine transportation results in a low level of media coverage and public awareness. A majority of the general public believes that the transportation of nuclear wastes is very dangerous, but probably does not hold the attitude that nuclear waste transportation or other radioactive materials transportation should be banned in urban areas. Transportation of radioactive materials for medical purposes appears to be acceptable to almost everyone. In summary, incident-free transportation has not caused significant psychological impacts.

'Sociological Impacts

Most collective response in opposition to incident-free transportation reflects a concern that is grounded in the history of the environmental movement and is closely allied with antinuclear sentiment. Responses to date have focused on the role of transportation in waste disposal and further power generation, rather than on the safety of transportation per se. Most collective response from groups supporting continued transportation reflects a belief in the fundamental safety and usefulness of such transportation for the nuclear industry. The evidence suggests that transportation has become an issue of concern primarily to existing interest groups, although a few new groups have formed in response to incident-free transportation. This small number of new groups includes both those in support of and in opposition to continued transportation. There is little evidence to suggest that the interest or membership of existing groups is changing as a result of adopting transportation as an issue.

\section{Political Impacts}

Few political impacts have been generated solely by incident-free transportation of radioactive materials. Electoral politics, including elections, initiatives, referenda, or recalls, have not been impacted by incident-free transportation. There has been some impact on the nonelectoral process, including increased city council and state government inquiry into regulating radioactive materials transportation.

Legal Impacts

Perhaps the most important legal impact from incident-free transportation has been the passage of 1 aws by states and local governments for the purpose of regulating nuclear transportation. Some of these regulations pertain to issues that are relevant to incident-free transportation, such as advance notification, records and manifests, and registration. Other regulations, of course, pertain to other causative events, such as regulations regarding safeguarding of certain shipments, accident notifaction, accident and emergency procedures and financial liability. Seven states now have statutes that restrict routing. 
An additional legal impact is uncertainty regarding the question of whether federal authority preempts some or all state and local restrictions on transportation. Recent court decisions holding that the Atomic Energy Act of 1954 preempts certain California and New York restrictions on fixed nuclear facilities, coupled with Nuclear Regulatory Commission (NRC) and Department of Transportation (DOT) routing requirements, point strongly to federal preemption of at least some state and local transportation restrictions. Because the exertion of control by states and localities has been a major outlet for their concerns about the adequacy of federal standards and procedures, preemption could force this concern into other political channels unless accompanied by measures that enhance confidence in federal standards and procedures. On the other hand, without definitive legislative or judical resolution of the preemption issue, states and localities are likely to continue to enact transport restrictions.

Organizational Impacts

Urban transportation has resulted in few impacts on the federal regulatory structure except for those required to implement routing restrictions. At the state and local level, the passage of statutes and ordinances mentioned above has in turn resulted in organizational impacts. First, these statutes and ordinances have created new organizations or given new responsibilities to existing organizations for the regulation of incident-free transportation. Second, these laws have resulted in the development of procedures such as permitting systems. Third, legislative developments have resulted in the acquisition of capabilities in the form of trained personnel and budgetary resources for the promulgation and enforcement of regulatory requirements.

\section{L4.2 Vehicular Accidents}

Psychological Impacts

An analysis of impacts due to accidents must account for the fact that, of the millions of individual shipments that have taken place over the last decade, only a handful of accidents have occurred. In terms of radioactive release and contamination, the effects have been smal1, and no one has been killed or seriously injured. Yet, in reacting to a vehicular accident people are also reacting in part to the possibility of a more serious accident in the future. Accidents are more likely than incident-free transportation or human error to cause psychological impacts because of the potential for future consequences from an accident. These impacts are influenced both by media treatment and by the actual seriousness of the accident. The greatest potential effects from a serious accident involve changed beliefs and attitudes on the part of the uncommitted general public. The relatively minor accidents that have occurred have likely tended to reinforce the existing beliefs and attitudes of both the antinuclear and pronuclear members of the general public concerning nuclear power in general and transportation in particular. 
A serious accident resulting in deaths or contamination of people or property in densely populated parts of a city could produce substantial psychological impacts. These effects would probably be greater than those that would result from a nonnuclear hazardous materials transportation accident of equivalent magnitude, given a generally greater public apprehension regarding nuclear materials compared to other hazardous materials. Beliefs about the safety of radioactive materials transport and attitudes about banning such transport would likely change significantly in the local community. Belief and attitude change would also be expected, although to a lesser degree, for those not residing in the local community. Psychological distress would also likely occur to anyone who might have been contaminated. A serious accident would provide the behavioral motivation for greater sociological, political, legal and organizational impacts than have occurred to date. This conclusion is speculative, given the lack of such nuclear accidents and a large range of variation in public response to other hazardous materials accidents.

\section{Sociological Impacts}

Sociological impacts depend on population density, severity of consequences, and media treatment of the facts and associated issues. Some data are available regarding a fairly broad range of urban groups that have been observed to actively respond to accidents. Even though an accident may take place outside the city, organized groups tend to be based in cities. Thus, impacts in the form of altered group behavior are largely an urban phenomenon. No impacts have been observed to date on the organization of social life in urban communities; population composition of residential neighborhoods in which accidents have occurred has remained stable.

If a serious accident resulted in a release of radioactivity, social organization might be disrupted. For example, rapid evacuation might occur, and there would likely be a temporary disruption of some public services, such as education and some medical services. Businesses in the immediate vicinity would likely close at least temporarily, with possible long-term effects for the local economy. However, evidence from community response to natural disasters suggests that the accident initially might help unite the community to deal with the problem. Social order would depend in large part on the capability of government and private groups to effectively deal with the accident and its human consequences. A special difficulty in dealing with radioactive materials is that of convincing a public, who must rely on statements made by others, that any possible longer term dangers are under control, because the public generally is not aware of the capability or efficacy of decontamination technologies.

\section{Political Impacts}

Political response has been fairly rapid following some accidents and has been associated most directly with concern for public safety, clarification of lines of responsibility with respect to organizational 
response, and the acquisition of public knowledge. Participants in the political process use various strategies in anticipation or in response to accidents, including petitions, testimony at hearings and other public meetings, demonstrations, the initiation and support of new legislation, interest group lobbying, and court suits. These activities occur both in opposition to and in support of continued transport. Experience suggests that transportation accidents can affect campaign issues and strategies, as well as choice of candidates. It is reasonable to expect an increased number and intensity of these kinds of political impacts if more accidents (or a serious accident) were to occur in the future. To date, however, political impacts have been small and have occurred mainly in the communities near to or in which the accident took place. Again, the media plays a formative role in the political process by their choice of how to handle and present information about incidents of this type.

\section{Legal Impacts}

The primary legal impacts of transportation accidents have been the passage of state statutes and local ordinances. However, such laws are rarely attributable solely to the occurrence of a particular incident or to the anticipation of one. Rather, legal activity originates in fairly long-standing concern for broader nuclear energy issues. The actual occurrence of an incident creates a perceived need to act soon and gives proponents of legal change an issue to exploit.

The occurrence of vehicular accidents in urban areas, compared to accidents in rural areas, is especially likely to give rise to the passage of statutes and ordinances because media coverage is more extensive, speculation about the consequences had the accident been more serious are especially compelling, the area immediately affected closely corresponds to a political jurisdiction with authority to act, and the constituency of activists in this area is primarily urban. For these reasons an accident might act as a precipitating event that sets in motion legal responses that may already have been well formed before the actual event.

\section{Organizational Impacts}

In the area of emergency response, evidence suggests that many cities do not have their own emergency response plans in place and must rely on state procedures. Most formal procedures do not differ with respect to the density of an area. Accordingly, problems unique to the urbanness of an area must be handled on an ad hoc basis. Thus, the adequacy of emergency response in urban areas is very difficult to judge prospectively.

Impacts that have occurred as a result of minor vehicular accidents include small adjustments to state and local emergency response capabilities, including training programs, small procedural changes, and the purchase of new equipment. These kinds of impacts typically occurred when an accident revealed inadequacies in emergency response procedures and capabilities. An accident with more serious consequences for the 
urban public could cause more substantial organizational impacts at the local, state, and federal levels. Such major accidents probably would indicate inadequacies in emergency preparedness at the city and state levels.

\section{L4.3 Human Errors and Deviations from Accepted Quality Assurance Practices}

Psychological Impacts

Although the majority of incidents reported to the DOT and the NRC fall into this category, these events rarely result in radioactive release or contamination. As a result of their relatively benign nature, they also tend not to be brought to public attention. Thus, psychological impacts associated with human error events associated with transportation have been quite small. If such events became more numerous and received widespread media coverage, they could lead to the belief that the human error component of transportation is a serious area of concern that has not been adequately addressed. Evidence of human errors at fixed facilities, such as those associated with Three Mile Island, may lead to increased attention to human errors in radioactive materials transportation.

\section{Sociological Impacts}

There is no evidence that new groups have formed in response to human error incidents. Existing groups have expressed concern for packaging safety and proper labeling and handling procedures.

\section{Political Impacts}

Political impacts have included the emergence of quality assurance as a political issue and increased pressure for strict adherence to quality assurance practices. State officials in states with low-level waste disposal facilities have temporarily or permanently closed the facilities at least partially in response to deviations from accepted quality assurance practices in transportation. Human error problems could become an election issue if an acceptable approach to ensuring the quality of transport procedures has not been placed convincingly before the public and if the candidates and the media felt such an issue warranted their attention.

Legal Impacts

Legal impacts have included lawsuits, modification of federal quality assurance regulation, litigation involving federal standards, congressional legislation, and local ordinances. The principal legal impacts of deviation from accepted quality assurance practices are lawsuits initiated by those directly affected. Human errors may lead to lawsuits by carriers, carriers' employees, the public, or state and local governments. Because who is affected does not depend greatly on whether the incident occurs in urban or nonurban areas, these impacts are not 
likely to vary with the location of the incident. Deviations from accepted quality assurance practices are less likely than vehicular accidents to give rise to the passage of state statutes and local ordinances, although three states have temporarily banned or restricted shipments to low-level waste facilities due to failure to meet accepted quality assurance standards. This is because it is more difficult to give such incidents graphic media coverage and because the consequences of such incidents, except in the case of air transport, have not been significant to members of the general public.

Organizational Impacts

The establishment and enforcement of quality assurance standards are primarily the responsibility of the NRC and the DOT. Conformity with these standards is the responsibility of carriers, licensees, and handlers. The organizational impacts from deviations from accepted quality assurance practices, whether occurring in urban or nonurban areas, appear to be slight. In general, past incidents have not prompted changes in organizational arrangements nor would future incidents be expected to do so. Major organizational change usually depends on a clear demonstration of failure. Even significant deviations from quality assurance, such as a leaking container, do not lend themselves to such demonstration. In contrast to vehicular accidents or malevolent acts, the consequences are less visible, fewer agencies are involved in responding, and the potential for extensive media coverage is smaller. Also, since persons other than the shippers' or carriers' employees are less likely to be at risk, the outside constituency for change is likely to be smaller. However, the impacts of a deviation from quality assurance practices with large consequences are likely to be greater if the incident occurs in an urban rather than nonurban area. Accordingly, urban incidents are probably more likely than nonurban incidents to give rise to organizational impacts in the form of tighter standards and expanded capabilities for inspection and enforcement, including the possibility of expanded state roles.

\section{L4.4 Malevolent Acts}

Psychological Impacts

Because incidents of this type have been infrequent, data on which to judge social impacts are limited. Compared to other public concerns regarding nuclear power--such as reactor safety, waste management, and thermal pollution--proliferation, sabotage, and terrorism are not major nuclear power concerns of the public. Successful malevolent acts would likely change beliefs and attitudes regarding the adequacy safeguards and the vulnerability of transportation to such acts. Psychological impacts would strongly depend on the severity of the event consequences. Impacts would tend to be largest in urban, densely populated areas where the potential for harm is greatest. A terrorist threat to disperse radioactive material being shipped through a city would cause fear and possible panic. Radioactive materials in transit are probably believed by the public to be more vulnerable to malevolent acts than the same materials confined within a fixed facility. 
If a serious sabotage attempt were made, the impacts might include extensive group concern for public well-being, voluntary or supervised evacuation from a threatened area, the prospect of social disruption implied by that experience, and increased activist group demand for transportation security to prevent future occurrences. If dispersal of high-level materials in a highly urban area were successful, the social disruption might continue from several weeks to several months while decontamination was occurring. Large economic costs might be incurred if the contaminated area had to be decontaminated to previous background radiation levels.

\section{Political Impacts}

The anticipation of sabotage events has caused political impacts in at least one election campaign. Further political issues and impacts from potential or actual events are more likely to arise in the city where the event may occur or has occurred, though there would probably be political repercussions in other urban areas as well. Citizen group reaction and official reaction to the potentially disastrous consequences of a malevolent act involving transportation would likely be intense. Increased security and careful regulation would be a likely impact.

Legal Impacts

Legal impacts, particularly with regard to NRC safeguards requirements involving spent fuel, have been in anticipation of malevolent acts. If such an act were successfully carried out, possible legal impacts might include more stringent federal safeguards requirements, express congressional authorization for the use of firearms by private guards, the provision of better escorts and protection for drivers and cargoes, and resolution of uncertainties concerning jurisdiction over acts of terrorism and sabotage and the scope of Price-Anderson coverage. It is also possible that the community or the state in which such an event occurred would declare an outright ban on all shipments within its jurisdiction.

Organizationa1 Impacts

Successful malevolent acts can be expected to expose the fragmentary nature of emergency preparedness that exists in many cities and states. Thus, the organizational impacts of a serious incident could include the clarification of current responsibilities for responding to malevolent acts and the possible creation of a federal guard force. As long as a major malevolent act has not occurred, activity to resolve ambiguities about responsibilities at the federal, state, and local levels are likely to proceed slowly. If such an incident occurs, pressures for rationalizing organizational responsibilities are likely to arise. 


\section{L5. Conclusions}

Findings from this study are summarized in Table L-1, and several broad conclusions have been drawn from then, as follows:

1. The general public is more concerned with the potential health and safety consequences of a transportation incident than with the probability that such an incident will occur. Such concern helps explain public opposition to nuclear power and the transportation of radioactive materials. If transportation policy fails to account for public concern for the potential consequences of future incidents, social impacts will likely increase, and radioactive materials transportation will encounter increased resistance.

2. The social impacts of nuclear transportation are different in some respects from the social impacts of fixed nuclear facilities. Although psychological and sociological impacts associated with fixed facilities are typically large for facilities sited in rural areas, such impacts have not been large for urban transportation. This is because transportation incidents have not caused serious physical consequences. Political and legal impacts associated with transportation have been more substantial than psychological and sociological impacts, though not very different compared with fixed facilities. State and local restrictions on radioactive materials transportation are particularly attractive to nuclear power opponents as a means of restricting the growth of nuclear power. Thus, local transportation restrictions can have important effects upon nuclear power development in many locations, including fixed facilities.

3. For a given magnitude of physical consequences, nuclear materials transportation has greater social impacts than hazardous materials transportation generally. The general public is probably more concerned about radioactive materials transportation than other hazardous materials transportation, even though the physical consequences of nuclear materials transportation has been much less. In addition, radioactive materials have generated more organized political and legal attention than hazardous materials.

4. Radioactive materials transportation through urban areas results in greater social impacts compared with transportation through other areas. Urban transportation, as opposed to transportation in rural areas, involves additional jurisdictions which may assert legal authority, additional agencies which may have roles to play in emergency response, and more people and groups whose attitudes, beliefs, and activities are affected. Urban transportation is heavily politicized because of intensive media coverage and the number of active interest groups on both sides of the issue. However, although nonurban transportation might reduce the severity of the consequences of an incident to local populations, it may increase the probability that such an event would occur, due to poorer road conditions, higher speeds, and increased communication problems. 
5. Malevolent acts may hold the greatest potential for social impacts. Such acts, involving the dispersal of high-level radioactive materials to cause or threaten harm may be unlikely; however, a malevolent act may be the only way in which high-level materials may get dispersed in an urban area from a transportation event. Such an incident would cause significant social impacts.

In sum, a relatively small minority of the public is involved in active, organized support of or opposition to radioactive materials transportation, although a large majority of the public is aware of the controversy over nuclear power. The activist proponents and opponents of continued transportation are generally also activists in this larger controversy. While transportation incidents have so far been few and of relatively small consequence, transportation issues seem to be a particularly visible component of nuclear energy development. Activists who oppose nuclear energy have started to focus on transportation as one way of hindering the industry. Thus, while it is concluded that in the absence of a major incident social impacts of radioactive materials transportation are not likely to increase, the political and legal attention given such transportation may escalate with the general controversy surrounding nuclear power. This attention may ultimately prove to be of more significance in decisions regarding the transportation of radioactive materials than strictly technical concerns. 
Table L-1

Social Impacts by Impact Category and Causative Event

Social

Impact Category

Psychological

Impacts

Sociological

Impacts

Political
Impacts

Legal

Impacts

Organizational Impacts

Incident-free Transport

No widespread impacts to date. Public is generally not

knowledgeable about transportation but is concerned portation but is concerned about radioactive materials troversy over the use of nuclear power.

\section{Scattered evidence of new} interest. group or coalition formation--impacts small to

Impacts closely associated with basic nuclear power issues.

Transportation has been political issue in a limited number of cases (with respect to public debate, pressure group activity, campaign strategy).

Uncertainty about federal pre-emption is the principal impact to date. Other impacts include passage of state and local statutes and ordinances.

Only minor impacts to date at the federal or state level. primarily implementation

of new regulatory requirements.

Causative Event

\section{Vehicular Accident}

Relatively little psychological impact to date.

A serious accident would likely cause belief and attitude change regarding transport and psychological distress to those directly affected by the accident.

No observed impacts on community organization or composition.

Existing interest groups may take on transportation as new issue.

Accidents involving large release could cause serious social disruption.

Impacts to date small and localized.

Major concern with public safety, emergency response management, public information.

More serious or more frequent accidents will likely intensify these concerns.

Passage of state and local statutes and ordinances the principal impact to date.

Major accident would result in lawsuits, possible new safety requirements.

No federal level impacts observed to date.

Minor changes in state and local emergency response capabilities.

Larger accidents would likely result in major shifts in emergency response measures.
Human Errors

Very sma11 impacts to date. If events increase in frequency of consequences, beliefs about the human component of transport may change.

No evidence to date that human error has caused important impacts for group or community organization.

Quality assurance has emerged as a political issue, in a few isolated instances.

A serious transport error could lead to pressure on government for tighter quality control.

Lawsuits; federal and state statutes and regulations are principal impacts to date. Major incidents may result in state attempts to exert control over quality assurance.

Minor impacts to date.

More significant errors may result in upgrading of enforcement, inspection, and monitoring capabilities.
Malevolent Acts

Malevolent acts are not a major public concern compared to other nuclear power concerns. A successful sabotage incident could cause public fear fol lowed by belief and attitude

Analogy with other disasters suggests prospect of evacuation and associated social disruption.

Political impacts could be widespread, beyond urban site.

Likely emphasis on tight security and regulation.

Possible impacts: Upgraded safeguards, clarification of permissible force, clarificaprospect of outright ban on urban routing.

Possible impacts: clarification of responsibility for response to incident, possible creation of federal guard force. 
TASK GROUP

Opening the process of impact assessment to public participation at an early stage is one way to assure that the information obtained is complete and comprehensive. For this purpose, Sandia Laboratories formed a task group to provide a vehicle for public involvement in the impact assessment during the early stages of its development. This task group is composed of individuals affiliated with federal, state, and local government agencies as well as persons involved in industry, public interest groups, and universities. Members of the task group were asked to express their opinions rather than act as official spokespersons. No consensus was expected on issues to be discussed during meetings of this group nor during other operations of the organization. Meetings of the task group were advertised in order to obtain local public input during the sessions. In all, five public task group meetings were held, with the last involving a review and comments on the Working Draft of the study. Task group members have been kept informed of project developments by NRC staff since the last meeting in July 1978. Members of the Task Group with their affiliations at the time of their inclusion in the organization are listed in Table $\mathrm{M}-1$. 
Table $M-1$

Task Group on the Transportation of Radioactive Materials Through Urban Environs

Member

Ludwig Benner

National Transportation Safety Board Washington, $D C$

202/472-6124

Donald J. Binder

Manager, Nuclear Engineering Division Long Island Lighting Co.

175 East 01d Country Road

Hicksville, L. I., NY 11801

516/733-4373

Calvin Brantley

Vice President

New England Nuclear Corporation

549 Albany Street

Boston, MA 02118

617/482-9595

Sherwood Davies, Director

Bureau of Radiological Health

New York State Department of Health

Empire State Plaza, Room 359

Albany, NY 12226

$518 / 564-2886$

Jack Edlow

Edlow International

Nuclear Transport Division

110017 th Street, N.W.

Washington, DC 20036

202/833-8237

Paul Giardina

U.S. Environmental Protection Agency

26 Federal Plaza

New York, NY 10007

$212 / 264-4418$

David Harris

Health Services Department

Veterans Memorial Highway

Hauppauge, NY 11787

516/979-2133

\section{Alternate}

Tony Lasseigne

National Transportation Safety Board

Washington, DC

William J. Tunney

Section Head for Nuclear Fuels

Management

Long Island Lighting Co.

175 East 0ld Country Road

Hicksville, L.I., NY 11801
Diane Harmon

Edlow Internationa1

110017 th Street, N.W.

Washington, DC 20036

Jeannette Eng

U.S. Environmental Protection Agency

26 Federal Plaza

New york, NY 10007 
Arthur T. Heubner

Assistant Director of Compliance Radiation

State Dept. of Environmental Protection

State Office Building

Hartford, CT 06115

203/641-5668

\section{Ida Hoos}

Research Sociologist

Space Science Laboratory

University of California at Berkeley

Berkeley, CA 94720

415/642-1347

Ralph Jacobs

RAD Services, Inc.

9381 C. Davis Avenue

Laure 1, MD 20810

202/953-9583

Joseph Karbus, Director

Radlological Heàlth

County of Los Angeles

Department of Health Services

313 North Figueroa Street

Los Angeles, CA 90012

213/974-7891

William Luch

Chairman, Western Interstate Nuclear Board Committee on Transportation

9212 North Reno

Portland, OR 97205

503/286-1963

Richard Pollock

Critical Mass

133 C Street, SE

Washington, DC 20003

202/546-4790

Marvin Resnikoff

Rachel Carson College

State University of New York

Amherst, NY 14261

$636 / 2319$
Don L. Collins

Don L. Collins \& Associates

418 N. Glendale Avenue

Glendale, CA 91206
Walter E. Pollock

Oregon Department of Energy

Western Interstate Nuclear Board

528 Cottage, NE

Salem, OR 97310

John Abbotts

c/o PIRG

1346 Connecticut Avenue NW

Suite 401

Washington, DC 20016

Marc Alhonte

Rachel Carson College

State University of New York

Amherst, NY 14261 
Marc Ross

Physics Department

University of Michigan

Ann Arbor, MI 48109

964/4459 (Physics Dept.--4437)

David Schweller

Acting Area Manager

Department of Energy

Brookhaven Area office

Upton, NY 11973

$664 / 3430$

Leonard R. Solon, Director

Bureau for Radiologic Control

325 Broadway

New York, NY 10007

212/566-7750

John P. Spath

NY State Energy Office

Agency Building \#2

Empire State Plaza

Albany, NY 12223

$518 / 474-4160$

Bil1 R. Teer

Vice President

Trans Nuclear, Inc.

North Broadway

White Plains, NY 10601

914/761-4060
Mary P. Sinclair

University of Michigan

5711 Summerset Drive

Midland, MI 84640

Robert Friess

Department of Energy

Brookhaven Area Office

Upton, NY 11973
John Mangusi

Trans Nuclear, Inc.

North Broadway

White Plains, NY 10601 
BIBLIOGRAPHY

NOTE: A11 NUREG and NUREG/CR reports are available for purchase from the NRC/GPO Sales Program, U.S. Nuclear Regulatory Commission, Washington, DC 20555, and the Nationa1 Technical Information Service, Springfield, VA 22161. 
Abler, R., and Adams, J. S. A Comparative Atlas of America's Great Cities--20 Metropolitan Regions. Minneapolis: University of Minnesota Press, 1976.

Adam, J. A., and V. L. Rogers. A Classification System for Radioactive Waste Disposa1--What Waste Goes Where?, NUREG-0456, FBDU-224-10. Washington: USNRC Office of Nuclear Material Safety and Safeguards, June 1978.

Adams, J. S. Contemporary Metropolitan America, vols. 1-4. Cambridge, MA: Ballinger Publishing Company, 1976.

Advisory Committee on Reactor Safeguards Working Group. "Transportation of Radioactive Materials," transcript of meeting. New York, 23 August 1977.

Agres, T. "Low Level Radiation: How Much Is Too Much?" Industrial Research/ Development, May 1979.

Airline Pilots Association International v Civil Aeronautics Board. 516 F.2d.

Ajzen, I., and M. Fishbein. "Attitudinal and Normative Variables as Predictors of Specific Behaviors." Journal of Personality and Social Physcology 27: 41-57, 1973.

Albrecht, S. L. "Environmental Social Movements and Counter-Movements: An Overview and Illustration." Journal of Voluntary Action Research 1:2-11, October 1972.

Alcaly, R. E. "Transportation and Urban Land Values: A Review of the Theoretical Literature." Land Economics 52:1, February 1976.

Aldrich, D. C., D. M. Ericson, and J. D. Johnson. Public Protection for Potential Nuclear Reactor Accidents: Sheltering Concepts with Existing Public and Private Structures, SAND77-1725. Albuquerque: Sandia Laboratories, February 1978.

Aldrich, D. C., N.C. McGrath, and Rasmussen. Examination of Offsite Radiological Emergency Protective Measures for Nuclear Reactor Accidents Involving Core Me1t, NUREG/CR-1131, SAND78-0454. Albuquerque: Sandia Laboratories, June 1978.

Alexander, Y. International Terrorism--National, Regional and Global Perspectives. New York: Praeger, 1976.

Allen, J. H. U. S. LWR Spent Fuel Inventories, ORNL/SUB-78/4258/1. Atlanta: Nuclear Assurance Corp., September 1978.

Allen, T. M. "A Factor Analysis of Accident Records." Highway Research Record 79:17-25, 1965.

Allied-General Nuclear Services. Current Status and Future Considerations for a Transportation System for Spent Fuel and Radioactive Waste, OWI/SUB-77/42513. Barnwell SC: AGNS, February 1978.

Allison, G. T. Essence of Deciston: Explaining the Cuban Missile Crisis. Boston: Little, Brown, 1971 . 
Allport, G., and L. Postman. The Psychology of Rumor. New York: Holt, 1947.

American Association of State Highway Officials. Policy on Design of Urban Highways and Arterial Streets, Publication GU-2, 1973.

American Cancer Society. Cancer Facts and Figures 1976. New York: ACS, 1977.

American Conference of Governmental Industrial Hygienists, Committee on Threshold Limit Values. Documentation of Threshold Limit Values.

Cincinnati: ACGIH, 1966.

American National Standards Institute, Inc. Administrative Guide for Obtaining Department of Transportation Special Permits for Radioactive Materials Shipments, ANSI N14.10.2. New York: ANSI, 1973.

American National Standards Institute, Inc. Packaging and Transportation of Radioactivity Contaminated Biological Materials, ANSI N14.3. New York: ANSI, 1973.

American National Standards Institute, Inc. Proposed American National Standard Agency Responsibilities and Emergency Procedures for Highway Transportation Accidents Involving Radioactive Materials. New York: ANSI, 23 November 1976.

American National Standards Institute, Inc. Proposed American National Standard: Control of Radioactive Surface Contamination on Materials, Equipment, and Facilities To Be Released for Uncontrolled Use, N328. New York: ANSI, 1978.

American Public Transit Association. Transit Fact Book, 1976-77, ISSN 0082-5913. Washington: APTA.

Amos, D. E., and S. L. Daniel. CDC 6600 Subroutines for Besse1 Functions $1 \nu(x), v=0,1 ;-\infty<x<\infty$; and $K(x), \nu=0,1 ; x>0$, SAND75-0149. Albuquerque: Sandia Laboratories, February 1976.

Amos, D. E., and S. L. Danie1. CDC 6600 Subroutines for Integrals of $(\sin t / t),(\cos t) / t, K_{0}(t)$, SAND76-0062. Albuquerque: Sandia Laboratories, February 1976 .

Anderson, J., and L. Whitten. "Nuclear Container Research Hit." Washington Post, 11 November 1977, P D17, col. 2.

Anderson, M. Fallout on the Freeway: The Hazards of Transporting Radioactive Wastes in Michigan. Lansing: Public Interest Research Group in Michigan (PIRGIM), 1974.

Anderson, R. T. Cooldown Testing of Nuclear Fuel Casks. Barnwell SC: Allied General Nuclear Services, August 1979.

Anderson, R. T. Thermal Characteristics of Aged Solvent Fuel in a Dry Environment, AGNS-35900-CONF-6. Barnwell SC: Allied General Nuclear Services, August 1979. 
Andrews, W. B. An Assessment of the Risk of Transporting Liquid Chlorine by Rail, draft. Richland, WA: Battelle Pacific Northwest Laboratories, September 1979.

Anno, G. H., and M. A. Dore. Evacuation and Sheltering Protective Measures Aga inst Nuclear Accidents Involving Gaseous Releases, PSR Report 517. Santa Monica, CA: Pacific-Sierra Research Corporation, December 1975.

Anno, G. H., and M. A. Dore. The Effectiveness of Sheltering as a Protective Measure Against Nuclear Accidents Involving Gaseous Releases, PSR Report

515. Santa Monica,CA: Pacific Sierra Research Corporation, December 1975.

"Anti-Nuclear Cult Hero Backs Atomic Power Safety." Washington Post, 17 February 1979, p A2.

"Antinuke Group Seeks N-Waste Transport Ban." Nashville Tennessean, 18 February 1979.

"Applying the Brakes to Hazardous Cargoes," editorial. Seattle Times, 26 January 1979.

Archer, V. E. "Effects of Low-Level Radiation: A Critical Review." Nuclear Safety 21(1):68-82, January-February 1980.

Arkansas Department of Health, Bureau of Environmental Health Sciences, Division of Radiological Health. "Summary of RAM Incidents for the State of Arkansas." Little Rock, 1977.

Arkansas Department of Health. State of Arkansas Emergency Operations Plan: Annex Q, Radiological Incident Response. Little Rock, nd.

Arizona Revised Statutes. Phoenix.

Arkansas Statutes Annotated. Little Rock.

Arthur D. Little, Inc., for the Automobile Manufacturers Association, Inc. The State of the Art of Traffic Safety, June 1966.

American Society of Heating, Refrigeration, and Air Conditioning Engineers. Handbook of Fundamenta1s. New York: ASHRAE, 1972.

Association of American Railroads. Railroad Tank Car Safety Research and Test Project. Final Phase 02 Report on Accident Review, RA-02-2-18. Washington: AAR, August 1972 .

Association of American Railroads, Bureau of Explosives. Emergency Handling of Hazardous Materials in Surface Transportation, BE Pamphlet No. 1, September 1977.

"Atomic Energy Act of 1954." United States Code, Title 42, 2011-2296.

Atomic Industrial Forum, Inc., Committee on Nuclear Fuel Cycle Services. Spent Fuel Storage Study 1976-1986. Washington: AIF, April 1977. 
Atomic Industrial Forum Study Group on Fuel Cycle Safeguards. Technical Options for Plutonium Safeguards. Washington: AIF, nd.

Atomics International Division, Rockwell Hanford Operations. Spent Fuel Demonstration Tests: Best Effort Schedule, RHO-BWI-CD-13. Richland, WA: RHO, March 1978.

"Atomic Waste Truck Crashes." The Newburgh [New York] News, 16 March 1976, p A1, col. 1 .

Auerbach, C., et al. "Issues Related to Choosing a Guard Force Structure," BNL-26129. Paper presented at the Sixteenth Annual Meeting of INMM, New Orleans, 18-20 June 1975.

Auxier, J. A. Ichiban: Radiation Dosimetry for the Survivors of the Bombings of Hiroshima and Nagasaki, TID 27080. Oak Ridge, TN: Oak Ridge National Laboratory, 1977.

Ayres, R. W. "Policing Plutonium: The Civil Liberties Fallout." Harvard Civil Rights--Civil Liberties Law Review 10:343-369, Spring 1975.

Back, K. W. Social Psychology. New York: John Wiley and Sons, 1977.

Baerwald, J. E., ed. Transportation and Traffic Engineering Handbook. Englewood C1iffs, NJ: Prentice-Hall, 1976.

Bagdikian, B. H. "The Policies of American Newspapers." Columbia Journalism Review $10: 8-13,1972$.

Bahme, C. W. Fire Officer's Guide to Dangerous Chemicals. Boston: National Fire Protection Association, 1972.

Bair, W. J., and D. H. Willard. "Plutonium Inhalation Studies -- III: Effect of Particle Size and Total Dose on Deposition, Retention and Translocation." Health Physics 9:253-266, 1963.

Bair, W. J. and J.M. Thomas. Prediction of the Health Effects of Inhaled Transuranium Elements from Experimental Animal Data, BNWL-SA-5388, IAEA-SM-199/58. Richland, WA: Battelle Pacific Northwest Laboratories, 1958.

Baker, G. W., and D. W. Chapman. Man and Society in Disaster. New York: Basic Books, 1962.

Baker, J. S., and H. L. Ross. "Concepts and Classification of Traffic Accident Causes." International Road Safety and Traffic Review. Part 1 -9(3):11ff, 1961; part 2-9(4):17ff, 1961.

Baker, S. "Group Asks Local Regulation of Nuclear Waste Transport." [Chattanooga, TN] News-Free Press, 2 September 1978, p A8.

Bakke, S. W. "Concept of the Social Organization." In Modern Organizational Theory, M. Haire, ed. New York: John Wiley and Sons, 1959. 
Balakrishnam, P. V., and G. M. Allison. "Sonic In-Reactor Loop Experiments on Corrosion Product Transport and Water Chemistry." Nuclear Technology 39: 105ff, July 1978 .

Baldonado, 0. C., and C. V. Hodge. Evaluation of Routine Exposure from the Shipment of Radioactive Material for the Nuclear Power Industry, NSS-8172.2. Anaheim, CA: Nuclear and Systems Sciences Group, September 1974.

Baldonado, 0. C. et al. Executive Summary of Safeguards Systems Concepts for Nuclear Material Transportation, NUREG-0334. McLean, VA: Systems Development Corporation, September 1977.

Baldonado, 0. C. et al. Safeguards Systems Concepts for Nuclear Material Transportation, NUREG-0335. Washington: Office of Nuclear Regulatory Research, Washington: September 1977.

Banfield, E. C., and J. Q. Wilson. City Politics. Cambridge: Harvard University Press, 1963.

Barker, R. F. Regulatory and Other Responsibilities as Related to Transportation Accidents, NUREG-0179. Washington: U.S. Nuclear Regulatory Commission Office of Standards Development, June 1977.

Bartholomew, R. J. "Cargo Response to Railcar Impact and Tiedown Analysis," Informal report LA-7469-MS. Los Alamos, NM: Los Alamos Scientific Laboratory, September 1978.

Barton, A. Social Organization Under Stress: A Sociological Review of Disaster Studies, Study Number 17, Publication No. 1032. Washington: National Academy of Sciences, National Research Council, Disaster Research Group, 1963.

Barton, J. H. Intensified Nuclear Safeguards and Civil Liberties. Washington: U.S. Nuclear Regulatory Commission, 1975.

Basowitz, H. Anxiety and Stress. New York: McGraw-Hill, 1955.

Baston, V. F., J. H. McFadden, and W. A. Yuill. An Analytical Method for Calculating Steady State Fission Gas Release-Fission Product Fuel Model (FPFM) Code, ANCR-1010, September 1971 .

Bates, D. V., et al. "Deposition and Retention Models for Internal Dosimetry of Human Respiratory Tract." Health Physics 12:173-207, 1966.

Bates, F. L., and L. Bacon. "The Community as a Social System." Social Forces 50:371-379, March 1972.

Bates, F. L., et al. The Social and Psychological Consequences of a National Disaster: A Longitudinal Study of HurricaneAudrey. Washington: National Academy of Sciences, National Research Counc11, 1963.

Battelle Columbus Laboratories. Methodology and Analysis. Volume 1 of Socioeconomic Effects of the DOE Gas Centrifuge Enrichment Plant, ORD-EP-111-P1. Columbus, OH: Battelle, May 1979. 
Battelle Pacific Northwest Laboratories. Assessment of the Environmental Impact of the FAA Proposed Rulemaking Affecting the Conditions of Transport of Radioactive Material in Aircraft, BNWL-B-241. Richland, WA: BNWL, September 1975.

Battelle Pacific Northwest Laboratories. Alternative for Interim Storage and Transportation. Volume 3 of Alternatives for Managing Wastes from Reactors and Post-Fission Operations in the LWR Fuel Cycle, ERDA-76-43. Richland, WA: BNWL, May 1976.

Battelle Pacific Northwest Laboratories. An Assessment of the Risk Transporting Plutonium Oxide and Liquid Plutonium Nitrate by Truck, BNWL-1846. Richland, WA: BNWL, August 1975.

Battelle Pacific Northwest Laboratories, USAEC Division of Biomedical Research. Radiation and the Lymphatic System. Proceedings of the Fourteenth Annual Hanford Biology Symposium, 30 September to October 1974, CONF-740930. Richland, WA: BNWL, 1976.

Battelle Pacific Northwest Laboratories. "Task 19, Transportation System Description," draft. Richland, WA: BNWL, December 1976.

Bauer, R. A., and A. H. Bauer. "America, Mass Society and Mass Media." Journal of Social Issues $16: 3-66,1960$.

Baum, J. W. "Multiple Simultaneous Event Model for Radiation Carcinogenesis." Hea1th Physics 30:85-90, 1976.

Baum, J. W. "Population Heterogeneity Hypothesis on Radiation Induced Cancer." Health Physics 25:97, 1973.

"Beam That Supported Trailer Floor?" St. Louis Post-Dispatch, 28 January 1979.

Behnam, J., and B. G. Patel. "Pedestrian Volume Estimation by Land-Use Variables." Transportation Engineering Journa1-Proceedings 103(TE4):507, 1977.

Be11, J. B. Transnational Terror. Washington: American Enterprise Institute for Public Policy Research, and Palto Alto, CA: Hoover Institution on War, Revolution and Peace; 1975.

Bell, M. J. ORIGEN, the ORNL Isotope Generation and Depletion Code, ORNL-4628. Oak Ridge, TN: Oak Ridge National Laboratory, May 1973.

Benington; H., et al. Public Participation in Energy Related Decision Making: Workshop Proceedings, NSF/PRA-7521960/3/7. McLean, VA: Mitre Corporation, January 1977.

Benjamin, A. S., et al. Spent Fuel Heatup Following Loss of Water During Storage, NUREG/CR-0649. Albuquerque: Sandia Laboratories, March 1979.

Benjamin, J. R., M. Shinozuka and H. C. Shah. "Acceptable Risk on Reactor Safety Studies. "In Proceedings of the 3rd International Conference on Structura1 Mechanics in Reactor Technology, September 1975. 
Berelson, B. "The Effects of Print Upon Public Opinion." In Print, Radio and Film in a Democracy, 0. Waples, ed. Chicago: University of Chicago Press, $1942, \mathrm{p} 41-65$.

Berk, R. A. "The Controversy Surrounding Collection Behavior: Some Methodological Notes." In Collective Violence, J. E. Short and M. Wolfgang, eds. Chicago: Aldine Publishing Co., 1972.

Berk, R. A. "The Emergence of Muted Violence in Crowd Behavior." In Collective Violence, J. S. Short and M. E. Wolfgang, eds. Chicago: Aldine Publishing, 1972.

Berkbigler, K. P. Estimating the Availability of LLEA Officers, SAND 77-8626. Livermore, CA: Sandia Laboratories, July 1977.

Berkey, E. Social Impact Assessment, Monitoring and Management by the Electric Energy Industry: State-of-The-Practice. Pittsburgh: Energy Impact Associates, Inc., December 1977.

Berkowitz, D. A., ed. Power Generation and Environmental Change. Cambridge MA: MIT Press, 1971.

Berkun, M. M., et al. Experimental Studies of Psychological Stress in Man. Psychological Monographs 76, Whole No. 534, 1962.

Berry, B. J., and J. D. Kasarda. Contemporary Urban Ecology. New York: Macmillan, 1977.

Berry, W. E., and R. B. Diegle. Survey of Corrosion Product Generation, Transport and Deposition in Light Water Nuclear Reactors, final report, EPRI NP-522, TPS 76-663. Palo Alto, CA: Electric Power Research Institute, March 1979.

Best, R. E., Manager, Engineering Services, Nuclear Assurance Corporation, Atlanta. Letter and enclosure to Alfred Grella, U.S. Department of Transportation, Washington DC, 22 February 1978.

Best, R. E., et al. Capabilities of U.S. Domestic Transportation Systems for the Shipment of Radioactive Wastes, Y/OWI/SUB-77/22330. Atlanta, GA: Nuclear Assurance Corp., September 1977.

Bethe, H. "The Necessity of Fusion Power." Scientific American, January 1976, pp 122-134.

Bishop, W. P. and F. J. Miraglia, Jr. Environmental Survey of the Reprocessing and Waste Management Portions of the LWR Fuel Cycle: A Task Force Report, NUREG-0116. Washington: U.S. Nuclear Regulatory Commission, October 1976.

Blomeke, J. 0., C. W. Kee, and J. P. Nickols. Projections of Radioactive Wastes To Be Generated by the U. S. Nuclear Power Industry. ORNL-TM-3965. Oak Ridge, TN: Oak Ridge National Laboratory, Febuary 1974. 
Blond, R. "Reactor Safety Study Consequence Model-CRAC." U.S. Nuclear Regulatory Commission, Probabilistic Analysis Branch, unpublished.

Blumer, H. "Collective Behavior." In Part IV of Principles of Sociology, 2nd ed, A. M. Lee, ed. New York: Barnes and Noble, 1946.

Blumer, H. Symbolic Interactionism: Perspective and Method. Englewood Cliffs, NJ: Prentice-Ha11, 1969.

Bone, Martin, and Harvey. Selection of Cycle Length for Fixed Time Signals, Research Report R62-37. Cambridge, MA: Massachusetts Institute of Technology, Department of Civil Engineering, 1962.

Bonzon, C, and J. T. Schauman. "Container Damage Correlation with Impact Velocity and Tanget Hardness." Presented at the IAEA Seminar on Radioactive Materials Packaging and Transportation, IAEA-SR-10/21. Vienna, Austria, August 1976.

Bonzon, L. L. Final Report on Special Impact Tests of Plutonium Shipping Containers, SAND76-0437. Albuquerque: Sandia Laboratories, February 1977.

Bonzon, L. L. and R. McWhirter. "Special Tests on Plutonium Shipping Containers," IAEA-SR-10/22. Pressented at the IAEA Seminar in Radioactive Materials Packaging and Transportation, Vienna, Austria, August 1976.

Book, S. A. and M. Goldman. "Thyroidal Radiolodine Exposure of the Fetus." Health Physics 29:874-77, 1975 .

Booth, A. H. A Method of Calculating Fission Gas Diffusion from $\mathrm{UO}_{2}$ Fuel and Its Application to the X-2-f Loop Test, CRDC-721. Challe River, Ontario: . Atomic Energy of Canada Ltd., September 1957.

Bornstein, R. C., and D. S. Johnson. "Urban-Rural Wind Velocity Differences." Atmospheric Environment 2:597-604, 1977.

Bornstein, R. D. The New York Urban Air Pollution Project of 1964-69: The Data, Report 76-02. San Jose, CA: Dept. of Meterology, San Jose State University, 1976.

Boulton, J, ed. Management of Radioactive Fuel Wastes: The Canadian Disposal Program, AECL-6314. Pinowa, Ontario: Atomic Energy of Canda Ltd., October 1978.

Box, G. E. P. "The Exploration and Exploitation of Response Surfaces: Some General Considerations and Examples." Biometrics, March 1954, pp 16-60.

Box, G. E. P. and D. W. Behnken. "Simplex-Sum Designs: A Class of Second Order Rotatable Designs Derivable from Those of First Order." Annals of Mathematical Statistics 33:838, 1962.

Box, G. E. P. and J. S. Hunter. "Multifactor Experimental Designs for Exploring Response Surfaces." Annals of Mathematical Statistics 28:1, 1957. 
Box, G. E. P., and N. R. Draper. "The Choice of a Second Order Rotatable Design." Biometrika 50(3-4):335, 1963.

Boyd, E. M. Predictive Toxicometrics. Baltimore: The Williams and Wilkins Co., 1972.

"Bradley Citizens in Nuclear Protest." [Chattanooga,TN] News-Free Press, 30 January 1979, p F3.

Bradley, R. A. "Determination of Optinum Operating Conditions by Experimental Methods; Part I: Mathematics and Statistics Fundamental to the Fitting of Response Surfaces." Industrial Quality Control, vol. 15, No. 6, July 1958.

Branchley, D. L., et al. Environmental Assessment Methodology for the Nuclear Fuel Cycle, BNWL-2219. Richland, WA: Battelle Pacific Northwest Laboratories, July 1977.

Braniff Airways, Inc. v Minneapolis-St. Paul Metropolitan Airport Commission. 377 F. Supp 1190 (D. Minn. 1974).

Brannigan, F. L. Living with Radiation. The Problems of the Nuclear Age for the Layman, ERDA-76/89. Washington: U.S. Energy Research and Development Administration Division of Safety, Standards and Compliance, 1976.

Braungart, R. E., ed. Society and Politics: Readings in Political Sociology. Englewood Cliffs, NJ: Prentice Hall, 1976.

Breckenridge v Rumsfeld. 537 F.2d 864, 6 Envt1. L. Rep. 20597; 6th Cir. 1976.

Brenner, R., et al. Statistical Analysis of Accident Data as a Basis for Planning Selective Enforcement, PB177354. Distributed by the Clearinghouse for Federal Scientific and Technical Information, June 1966.

Brobst, W. A. "The Probability of Transportation Accidents." Presented before the 14th Annual Explosives Safety Seminar, New Orleans, 10 November 1972.

Brobst, W. A. "Transportation Accidents: How Probable?" Nuclear News $16(7): 48-54,1973$.

Brobst, W. A. "Transportation of Nuclear Fuel and Waste." Nuclear Technology 24:343-355, December 1974 .

Broder, J. "Atom Waste Hauls Defy Safety Laws." [Detroit] News, 25 February 1979.

Bromley, D. G. "Power, Politics, and Decision Making." In Contemporary Topics in Urban Sociology, K. P. Schwirian, ed. Morristown, NJ: General Learning Press, 1977.

Brown, 0. F., II, of Le Boeuf, Lamb, Lleby \& MacRae, Washington. Letter, 31 May 1979.

Bruce, J. A. One Way Major Arterial Streets, Highway Research Board Special Report 93, 1967 . 
Brunner, P. N. "Transportation of Radioactive Material in Illinois." Radiation Benefits and Risks: Facts, Issues and Opinion. HEW Publication (FDA) 77-8021, 1976.

Bryson, J. D., and D. Gross. Techniques for the Survey and Evaluation of Live Floor Loads and Fire Loads in Modern Office Buildings, NBS-BSS 16. Fredericksburg, MD: National Bureau of Standards, December 1967.

Bucher, R. "Blame and Hostility in Disaster." American Journal of Sociology $62: 467-475,1957$.

Buckner, H. T. "A Theory of Rumor Transmission." Public Opinion Quarterly $29: 54-70,1965$.

Bugen, L. "Human Grief." American Journal of Orthopsychiatry 47:196-206, 1977.

Bukro, C. "Ban O'Hare A-Shipments, Scott Urges." Chicago Tribune, 1 December 1977, sec. 3, p 4, col. 2.

Burchett, 0., and C. M. Henning. Shipping Containers for Nuclear Material: A Descriptive Bibliography, NUREG/CR-0614, SAND77-1977. Albuquerque: Sandia Laboratories, May 1979.

Burgess, R. A. Helplessly Hoping: A Review of the State of Maine Radiological Response Plan for Accidents at Maine Yankee. Augusta, ME: Maine Public Interest Research Group, 31 October 1975.

Burleson, C. The Day the Bomb Fell on America: True Stories of the Nuclear Age. Englewood Cliffs NJ: Prentice-Ha11, 1978.

Burns, W. J. "Transportation of Radioactive Materials." In States' Role in Radioactive Material Management. Proceedings of a workshop held in Las Vegas, 9-11 December 1974, pp 7-13.

Burson, G., and A. E. Profia. Structure Shielding from Cloud and Fallout Gamma Ray Sources for Assessing the Consequences of Reactor Accidents, EGG-1183-1670, December 1975.

Burt, R. "Tampering With Nuclear Fuel Shipment Reported." New York Times, 5 January 1979, p A2l.

Burt, R. S., et al. Resolving Community Conflict in the Nuclear Power Issue: A Report and Annotation Bibliography, Y/OWI/SUB-78/2237, February 1978 .

Busch, R. H., J. E. Ballow and D. D. Mahlum, eds. Radionuclide Carcinogenesis, CONF-720505. Proceedings of Twelfth Annual Hanford Biology Symposium, 10-12 May 1972. Richland, WA: Battelle Pacific Northwest Laboratories, 1973.

Cain, H., E. Egner, and J. Ruska. "Deposits of Rare Earth Metals in the Lungs of Man and in Experimental Animals." Virchow's Arch. A. Path. Anat. and Histo1. $374: 249-261,1977$. 
Cairns, Jr. "The Cancer Problem." Scientific American 233(5):64, November 1975.

"Calavan Cites Nuclear Safety Hazards." N.C. Anvil, 17 November 1978.

California Energy Resources Conservation and Development Commission et al. "Comments on the Draft Report to the President by the Interagency Review Group on Waste Management." Sacramento, 1 December 1978.

California Health and Safety Code. Sacramento.

California Office of Emergency Services et al. Evacuation Plan for the Area Surrounding San Onofre Generating Station. Sacramento, July 1975.

California Office of Emergency Services. Nuclear Blackmail or Nuclear Threat Emergency Response Plan for the State of California. Sacramento, January 1978.

California Office of Emergency Services. Standard Operating Procedures --Nuclear Blackmail or Threat, SOP-12. Sacramento, April 1979.

California Office of Emergency Services. Standard Operating Procedures --Peacetime Radiological Incident, SOP-9. Sacramento, April 1979.

California Office of Emergency Services and Department of Health. Nuclear Power Plan Emergency Response Plan. Sacramento, July 1975; rev. August 1978.

California Population Research Unit. Provisional Projections of California Counties to 2000. 15 September 1971 .

California State Assembly Committee on Resources, Land Use, and Energy. Hearings on Proposition 15. Vol. 7, Nuclear Waste Disposal and Transportation, Part 1, 4 November 1975.

California State Assembly Committee on Resources, Land Use and Energy. Hearings on Proposition 15. Vol. 8, Nuclear Waste Disposal and Transportation, Part 2, 5 November 1975.

Calvin, M. Chemical Carcinogenesis, LBL-5398. Berkeley, CA: Lawrence Radiation Laboratory, 20 September 1976.

Cantril, H., H. Gaudet, and H. Herzog. Invasion from Mars. Princeton, NJ: Princeton University Press, 1940.

Cantril, H. The Psychology of Social Movements. New York: John Wiley and Sons, 1941 .

Carolina Environmental Study Group, Inc., et al v USAEC et al, 98 S.C.T. 2620; 1978.

Carter, R. E., Jr. "Newspaper Gatekeepers and the Sources of News." Public Opinion Quarterly 22:133-144, Summer 1958. 
Ceder, A. "Drivers' Behavior, Traffic Flow, and Road Safety Studies." Hazard Prevention 15:1, September/October 1978.

Centre-Mark Company. CENTRE-US: The Data Base of Geography. Elmhurst, IL.

Chandler, J. M. Peach Bottom Spent Fuel Element Shipping Cask Accident, December 8, 1971, ORNL-TM-3844. Oak Ridge, TN: Oak Ridge National Laboratories, July 1972.

Chang, K. H., M. P. Labar, and R. J. Campana. Modeling of Isotopic Fission Gas Release in the GCFR Vented Fuel Rod, GAA 15175. October 1978.

Chang, P. C., P. N. Wang, and A. Lin. "Turbulent Diffusion in a City Street." In Proceedings of the Symposium on Air Pollution, Turbulence and Diffusion, SC-M-71-0913. Albuquerque: Sandia Laboratories, March 1972, pp 137-144.

Chang, Y. B., and D. S. Berry. "Examination of Consistency in Signalized Intersection Capacity Charts of the Highway Capacity Manual." Highway Research Record 289, 1969.

"Chicago is Depicted as Depot for Uranium Banned by N.Y." Washington Post, 27 November 1977,P A10, col. 2.

"Chicago New Hub for Nuke Traffic." San Francisco Sunday Examiner and Chronicle, 27 November 1977, p A24.

Chiles, W. D. Psychological Stress as a Theoretical Concept. Dayton, OH: Wright Patterson AFB, Wright Air Development Center, July 1957.

Choldin, H. M. "Social Life and the Physical Environment." In Handbook of Con temporary Urban Life. San Francisco: Jossey-Bass, 1978.

Church, H. W., R. E. Luna, and S. M. Milly. Operation Roller Coaster: Near Ground Level Air Sample Measurements, SC-RR-69-788. Albuquerque, NM: Sandia Laboratories, 6 February 1970.

"Civilian Airport Ban: Mikva Bill Restricts A-Cargo." Chicago Tribune, 8 December 1977, sec. 7, p 13, col. 3.

Clarke, R. K., et al. The Analysis of Transportation Accident Environments-The Purpose, Method and Indroduction. Proceedings of the 4th International Symposium on Packaging and Transportation of Radioactive Material, Miami Beach, Florida, 22-27 September 1974.

Clarke, R. K., et al. "Quantitative Characterization of the Environment Experienced by Cargo in Aircraft Accidents." In Proceedings of the 4th International Symposium on Packaging and Transportation of Radioactive Material, Miami Beach, Florida, 22-27 September 1974.

Clarke, R. K., et al. "Quantitative Characterization of the Environment Experienced by Cargo in Motor Carrier Accidents." In Proceedings of the 4th International Symposium on Packaging and Transportation of Radioactive Material, Miami Beach, Florida, 22-27 September 1974 . 
Clarke, R. K., et al. "Quantitative Characterization of the Environment Experienced by Cargo in Railroad Accidents." In Proceedings of the 4th International Symposium on Packaging and Transportation of Radioactive Materia1, Miami Beach, Florida, 22-27 September 1974 .

Clarke, R. K., et al. Severities of Transportation Accidents, SLA-74-0001. Albuquerque: Sandia Laboratories, July 1976.

Clarke, R. K., et al. "Severities of Transportation Accidents, Vol. 1, Summary." In Proceedings of the 4th International Symposium on Packaging and Transportation of Radioactive Materials, Miami Beach, Florida, 22-27 September 1974.

Clark, T. N. Community Power and Policy Outputs: A Review of Urban Research. Beverly Hilis, CA: Sage, 1973.

Cleary, P. G., and D. D. Comey. Nuclear Power Plant Evacuation Plans, CBE-7865. Chicago: Citizens for a Better Environment, 25 May 1978.

Clifford, R. A. The Rio Grande Flood: A Comparative Study of Border Communities in Disaster. Washington: National Academy of Sciences, National Research Council, 1956.

Cloyd, D. R., and W. J. Murphy. Handling Hazardous Materials. Washington: NASA Technology Utilization Division, September 1965.

Cockerham, W. "State Energy Chief Opposes Bill Controlling N-Shipments." The Hartford Courant, 3 April 1976.

Code of Federal Regulations. Title 10.

Cohan, S. C., and K. D. Dance. Scope Assessment of the Environmental Health Risk Associated with Accidents in the LWR Supporting Fuel Cycle.

Washington: Teknekron, Inc., November 1975.

Cohen, A. F., and B. L. Cohen. Infiltration of Particulate Matter into Buildings, NUREG/CR-1151, SAND79-2079. Albuquerque: Sandia Laboratories, November 1979.

Cohen, B. "The Potentialfties of Terrorism: Plutonium is an Overrated Weapon for Terrorist Use." Bulletin of the Atomic Scientists 6:34-35, 1976.

Cohen, B. L. "Conclusions of the BEIR and UNSCEAR Reports on Radiation Effects per Man-rem." Health Physics 30:351-352, 1976.

Cohen, B. L. "Hazards from P1utonium Toxicity." Health Physics 32:359-379, 1977.

Cohen, B. L. The Hazards of Plutonium Dispersal, TID 26794. Institute for Energy Analysis, July 1975.

Cohen, B. L. Hazards from Radioactive Waste In Perspective, CONF-790204-9. Argonne, IL: Argonne National Laboratory, 27 February 1979. 
Cohen, J. J. "On Determining the Cost of Radiation Exposure to Populations for Purposes of Cost-Benefit Analysis." Health Physics 25:527, 1973.

Cohen, S. C., and K. D. Dance. Scoping Assessment of the Environmental Health Risk Associated with Accidents in the LWR Supporting Fuel Cycle, PB-258801. Washington: Teknekron, Inc., November 1975.

Cole, R. Inhalation of Radioiodine from Fallout: Hazards and Countermeasures-Summary, final report, ESA-TR-72-01. For the Defense Civil Preparedness Agency, Office of the Secretary of Defense. Burlingame, CA: Environmental Science Associates, August 1972.

Coleman, A. L., et al. "Threshold Limit Values for 1959." AMA Archives of Industrial Health 20:266-270 1959.

Coleman, J. S., and L. Katz. Medial Innovation: A Diffusion Study. Indianapolis : Bobbs-Merrill, 1966.

Coleman, J. S., and L. Katz. The Problem of Panic, TR-08 (formerly TB-19-2). Washington: National Academy of Sciences, National Research Council, Committee on Disaster Studies; for the Office of Civil and Defense Mobilization, June 1955.

Collins, H. E., B. K. Grimes, and F. Galpin. Planning Bases for the Development of State and Local Government Radiological Emergency Response Plans in Support of Light Water Nuclear Power Plants, NUREG-0396, EPA 520/1-78-016. December 1978.

Colorado Department of Health, Radiation and Hazardous Waste Control. "Summary of RAM Incidents for the State of Colorado, 1968-1977. Denver.

Colorado Department of Health. Radiation Incident Report--Uranium 'Yellowcake' Spill, September 27, 1977--Southeastern Colorado, 1978.

Colorado Department of Health, Division of Occupational and Radiological Health. Emergency Handling of Radioactive and Metallic Fires: A Handbook for Fire Departments, nd.

Colorado Department of Health, Division of Occupational and Radiological Health. Emergency Handling of Radiation Incidents: A Handbook for Police Officers, nd.

Colorado Department of Health, Division of Occupational and Radiological Health. Handling the Radiation Accident Victim: A Guide for Hospital Personnel, nd.

Colorado Department of Health, Radiation and Hazardous Waste Division. Radiological Emergency Response Plan for Transportation Incidents, 1978.

Colorado Revised Statutes. Denver.

Colton, J. D., and R. E. Emerson. Study of the Mechanics of a Transportation Accident Involving Natural Uranium Concentrate, NUREG/CR-0558. Menlo Park, CA: Stanford Research Institute International, Poulter Laboratory, 8 September 1978. 
Comar, C. L., and L. A. Sagan. "Health Effects of Energy Production and Conversion." Annual Review of Energy 1:581, 1976.

Comey, D. D. "The Perfect Trojan Horse." Bulletin of the Atomic Scientists $6: 34-35,1976$.

Commoner, B. "The Dual Crisis in Science and Society." In Stress and Campus Response, G. K. Smith, ed. San Francisco: Jossey-Bass, Inc., 1968, pp 15-26.

Comptroller General of the United States. Industrial Capacity to Produce Rail and Cross-Ties for Nationwide Railroad Track Rehabilitation, CED-76-150. Washington, 23 September 1976.

Comptroller General of the United States. Information Available on Estimated Costs to Rehabilitate the Nation's Railroad Track and a Summary of Federal Assistance to the Industry, RED-76-44. Washington, 21 November 1975.

Comptroller General of the United States. Problems Resulting from Deterioration of Pavement on the Interstate Highway System. For the Deptartment of Transportation, Federal Highway Administration. 30 June 1970.

Conant, R. "Rioting, Insurrection, and Civil Disorder." American Scholar $37: 420-433,1968$.

"Concerned Citizens of Southern Ohio Sponsored Advertisement on Uranium Enrichment." Washington Post, 11 May 1977, p A8, col. 2.

Conley, B. C. "The Value of Human Life in the Demand for Safety." American Economic Review 66(1):45-55, 1976.

"Connecticut Acts on Atom Carriers." New York Times, 6 April 1976.

Connecticut General Statutes Annotated. Hartford.

"? Contained by Rain." Wichita Eagle-Beacon, 22 March 1979.

Cook, M. A. The Science of High Explosives, ACS Monograph 139. New York: Reinhold Publishers, 1958.

Cooley, P. Fire in Motor Vehicle Accidents, UM-HSRI-SA-74-3. Ann Arbor: Highway Safety Research Institute, University of Michigan, April 1974.

Cortese, C. F. "The Social Impacts of Energy Development in the West: An Introduction." The Social Change Journal 16(2), 1979.

Cortese, C. F., and B. Jones. "The Sociological Analysis of Boomtowns." Western Sociological Review 8(1):76-90, 1977.

Corum, J. M. Overview of U.S. Efforts to Validate Analysis Methods and Design Criteria, CoNF-790363-1. Oak Ridge TN: Oak Ridge National Laboratory, 1979. 
Coser, L. "Conflict: Social Aspects." International Encyclopedia of the Social Sciences 3, 1968.

Couch, C. J. "Collective Behavior: An Examination of Source Stereotypes." Social Problems 15:310-322, 1968.

Council for Science and Society (Britain). The Acceptability of Risks. London: Barry Rose Publishers, Ltd., 1976.

Council of National Library Associations. American National Standard Guidelines for Format and Production of Science and Technical Reports, ANSI 239.18. New York: American National Standards Institute, 1974.

Council of State Governments. The Book of the States 1978-1979. Lexington, KY: The Council, 1978.

Craig, A. G. "Alternatives to the Linear Risk Hypothesis." Health Physics $31: 81-82$, 1976. Letters to the editor.

"'Crash Testing' of Nuclear Fuel Shipping Casks Helps Scientists Assess Transportation Safety Risks." Information From ERDA, News Feature No. NF-77-5, 25 February 1977.

Creighton, R. L., et al. "Estimating Efficient Spacing for Arterials and Expressways." Highway Research Board Bulletin 253:1-43, 1960.

Critical Mass Energy Project. The Uranium Accident of September 27, 1977: The Case For Emergency Preparedness Plans and the Adequacy of Transportation Standards. Washington: Critical Mass, 31 October 1977.

Critical Mass Energy Project. Radioactive Risks on the Roadway, the Nuclear Cargo Accident Record 1974-1978. Washington, 1979.

Critical Mass Journal, vol. 3, No. 2 to vol. 3, No. 12 (May 1977 to March 1978); and vol. 4, No. 10 (January 1979 to present).

Croff, A. G., et al. Reused Uranium-Plutonium Cycle PWR and BWR Models for the ORIGEN Computer Code, ORNL/TM-6051. Oak Ridge, TN: Oak Ridge National Laboratory, September 1978. 
Cronan, R. T. "Nuclear Safeguards: Uranium Diversion Hazard." Critical Mass Journal 4(6):6, September 1977 .

Croucher, D. W. Behavior of Defective PWR Fuel Rods During Power Ramp and Film Boiling Operation, NUREG/CR-0283. Idaho Falls: Idaho National Engineering Laboratories, February 1979.

Cummings, L. G. Third Party Liability Insurance and Government Indemnity Associated with the Transportation of Radioactive Material, CONF-710801. August 1971.

"Curb Asked on Nuclear Waste Hauls." New London [CT] Day, 17 March 1976.

Curran, F. B., and J. T. Stegmaier. "Travel Patterns in 50 Cities." Highway Research Board Bulletin 203:99-130, 1958.

Curtis, H. W. "European Experience in the Transport of Irradiated Light-Water Reactor Fue1." International Atomic Energy Agency Bulletin 21(6):57-66, December 1979.

Cuyahoga County, Ohio, Disaster Services Agency. Cuyohoga County Disaster Operations Plan, Part E: Major Explosions/Accidents Operations, nd.

Cyert, R. M., and J. G. March. A Behavioral Theory of the Firm. Englewood Cliffs, NJ: Prentice-Ha11, 1963.

Dabbert, W. F., F. L. Ludwig, and W. B. Johnson. "Validation and Applications of an Urban Diffusion Model for Vehicle Pollutants." Atmospheric Environment 7:603-618, 1973 .

Dalrymple, L. "Nuclear Waste Ship Granted OK to Dock in Pear1 Harbor." The Honolulu Advertiser, 8 June 1979, p Al.

Daly, C. The Media and the Cities. Chicago: University of Chicago Center for Policy Studies, 1968.

"Danger Disputed in I-70 Uranium Spill." St. Louis Post-Dispatch, 18 January 1979.

Danzger, M. H. "Validating Conflict Data." American Sociological Review $40: 570-584,1975$.

Danzig, E. R., P. W. Thayer, and L. Galanter. The Effects of a Threatening Rumor on a Disaster-Stricken Community, Disaster Study No. 10. Washington: National Academy of Sciences, National Research Council, 1958.

"Data on Mystery Uranium Carrier Added by EEC." Washington Post, 6 May 1977, p A12, col. 6 .

Davidson, B. "A Summary of the New York University Urban Air Pollution Dynamics Research Program." J. of the Air Pollution Control Assoc 17:154, 1967. 
Davidson, C. A. and J. T. Foley. Transportation Environment Data Bank Index. SAND-75-0248C. Albuquerque: Sandia Laboratories, April 1977.

Davies, J. C. When Men Revolt and Why. New York: Free Press, 1971.

Davis, R. "Uranium Cargoes Halted at O'Hare." Chicago Tribune, 3 December 1977, sec. 1, p 1, col. 4 .

Davis, W. Jr, G. A. West, and R. G. Stacy. Oxide Particle Size Distribution from Shearing Irradiated and Unirradiated LWR Fuels in Zircaloy and Stain1ess Steel Cladding, NUREG/CR-0866, March 1979.

Davison, W. P. "On the Effects of Communication." Public Opinion Quarterly, $23: 343-360,1959$.

"Deadly-Cargo Unit Waits in Tukwila." Seattle Post-Intelligencer, 21 February 1979, P A6.

"Dean Wants Warnings of Radioactive Moves." [Chattanooga, TN] News-Free Press, 31 January 1979, p A12.

De Boer, T. K. "New York State's Program for Transportation of Radioactive Materials." In States' Role in Radioactive Material Management, proceedings of a workshop held in Las Vegas, Nevada, 9-11 December 1974, pp 30-34.

"Lıcision Postponed on N-Waste Transport." [Eugene, OR] Register Guard, $\therefore$ December 1978.

Deeter, C. R, and A. A. Hoffman. "Energy Related Mathematical Models: Annotated Bibliography." Energy Conversion 1r:189-227, 1978.

De Fleur, M. L. "Mass Communication and the Study of Rumor." Sociological Inquiry 32:51-70, 1962 .

De Fleur, M. L., and O. N. Larsen. The Flow of Information: Experiment in Mass Communication. New York: N. T. Harper, 1958.

Delcoigne, G. "Education and Public Acceptance of Nuclear Power Plants." Nuclear Safety 20(6):655ff, November-December 1979 .

De Mangin, C. "Suffolk Weighs Nuclear Waste Transport Law." Long Island Press, 30 March 1976.

Deming, A., et a1. "War on Terrorism." Newsweek, 31 October 1977.

Dennis, A. W., et al. Severities of Transportation Accidents Involving Large Packages, SAND77-0001. Albuquerque: Sandia Laboratories, 1976.

Department of Energy, Office of Health \& Environmental Research. Nuclear Medicine Instrumentation Development: DOE Program, Study Group Report, DOE/EV-0034. Washington: USDOE, February 1979. 
Department of the Environment (London). Code of Practice for the Carriage of Radioactive Materials By Road. London: Her Majesty's Stationery Office, 1975.

De Steese, J. G., and R. E. Rhoads. National Waste Terminal Storage Program: Potential Problems in the Waste Transportation System, PNL-2444. Richland, WA: Battelle Pacific Northwest Laboratories; December 1977.

De Vries, G. W., and V. L. Tarrance. The Ticket Splitter: A New Force in American Politics. Grand Rapids, MI: William B. Eerldmans Publishing, 1972.

Diggory, J. C. "Some Consequences of Proximity to a Disease Threat." Sociometry 19:47-53 1956 .

"Dillon Studies a Request for A-Waste Problem." Long Island Press, 31 March 1976.

Di Pietro, C. M., and L. E. King. "Pedestrian Gap-Acceptance." Highway Research Record 308, 1970.

Distenfeld, C., and J. Klemish. Air Sampling for Evaluating the Thyroid Dose Commitment Due Fission Products Released from Reactor Containment. Final Report, NUREG/CR-0314. Upton, NY: Brookhaven National Laboratory, 1978.

Dolphin, G. W. Hot Particles, Radiological Protection Bulletin No. 8. Harwell, England: National Radiological Protection Board, July 1974.

Dolphin, G. W. "The Risk of Thyroid Cancers Following Inradiation." Health Physics Journal 15:219-228, 1968 .

Doob, L. W. Public Opinion and Propaganda. New York: Holt, Rinehart and Winston, $19 \overline{48 .}$

Draper, N. R. "Second Order Rotatable Designs in Four or More Dimensions." Annals of Mathematical Statistics 31:23, 1960.

Drabeck, T. E. "Social Processes in Disaster: Family Evacuation." Social Problems 16:337-349, Winter 1969.

Drabeck, T. E., E. Haas, and S. Krane. "System Shock: Response and Recovery." Paper presented at ASA Meeting in New York City, August 1973.

Drabeck, T. E., and E. L. Quarantelli. "Scapegoats, Villians, and Disasters." Transaction 4(4):12-17, 1967.

Drabeck, T. E., and K. S. Boggs. "Families in Disaster: Reactions and Relatives." Journal of Marriage and The Family 30:443-451, August 1968.

Drabeck, T. E., et al. "The Impact of Disaster on Kin Relationships." Journal of Marriage and the Family 37:481-494, August 1975.

Drabeck, T. E., and W. C. Key. "The Impact of Disaster on Primary Group Linkages." In Mass Engineering. Amsterdam, Holland: El Sevier Scientific Publishing Co., 1976. 
Dresch, F. W., and H. Ellis. Methodology for Assessing Total Vulnerability, OCD-PS-64-201. Palo Alto, CA: Stanford Research Institute, August 1966.

"Drive to Halt Terror Bombings." U.S. News and World Report, 15 March 1971, p 17.

Drozdiak, W. "Odyssey of Nuclear Shipment Baffles Experts in Europe." Washington Post, 17 May 1977, p A12, col. 1.

Druyan, L. M. "A Comparison of Low-Level Trajectories in an Urban Atmosphere." J of Applied Meteorlogy 7:583-590, 1976.

DuCharme, A. R., et al. Transport of Radionuclides in Urban Environs: Working Draft Assessment, SAND77-1927. Albuquerque: Sandia Laboratories, May 1978.

Duhame1, F, J. M. Lavie, and L. Fitoussi. "Data Provided by Monitoring and Resulting Rules for Protection against Radiation." In Proceedings of 2 nd U.N. International Conference on Peaceful Uses of Atomic Energy, Geneva, September 1958, vol. 23; pp 221ff.

Dukert, J. M. Atoms on the Move, Transporting Nuclear Material. Washington: U.S. Energy Research and Development Administration, 1975.

"Dumped Uranium Removed From I-70." Kansas City Star, 18 January 1979.

Dupont de Nemours, E. I. Blasters Handbook, 15th ed. Wilmington, DE: Dupont.

Dupree, S. A. Neutron and Gamma-Ray Sources in LWR High-Level Nuclear Waste, SAND 77-0369. Albuquerque: Sandia Laboratories, 1977.

Dynes, R. R., and E. L. Quarantelli. "Organization as Victim in Mass Civil Disturbances." Issues in Criminology 5:181-193, Summer 1970.

Dynes, R. R. Organized Behavior in Disasters: Analysis and Conceptualization. Washington, 1978.

Dynes, R. R., E. L. Quarante1li, and G. A. Kreps. A Perspective in Disaster Planning. Columbus, $\mathrm{OH}$ : Disaster Research Center, Ohio State University, 1972.

Dynes, R. R., and E. L. Quarantelli. "Redefinitions of Property Norms in Community Emergencies." The International Journal of Legal Research 3:100-112, December 1968 .

Dynes, R., and E. L. Quarante11i. "What Looting in Civil Disturbances Really Means." Trans-Action 5:9-14, May 1968.

Eagly, A.-H., and S. Himmelfarb. "Attitudes and Opinions." Annual Review of Psychology 29:517-554, 1978 .

Ebbin, S., and R. Kasper. Citizen Groups and the Nuclear Power Controversy: Use of Scientific and Technical Information. Cambridge, MA: M.I.T. Press, 1974. 
Eckhoff, N. D., et al. "Correlation of Leukemia Mortality Rates with Altitude in the United States." Health Physics 27:377, 1974.

Edel, M., and E. Sclar. "Taxes, Spending, and Property Values: Supply Adjustment in a Tiebout-Oates Mode1." Journal of Political Economy 82:941, 1974.

Edelhertz,H. et al. The White Collar Threat in Nuclear Safeguards NU0156. Batelle Human Affairs Research Center, January 1977.

Edie, L. C. "Car Following and Steady State Theory for Non-Congested Traffic." Operations Research 9(1):66-75, 1961 .

Egan, T. "McDermott Chides Ray on N-Dump" Seattle Post-Intelligencer, 29 August 1979. p. A4.

Eger, K. J. and G. E. Zima. Commentary on Spent Fuel Storage of Morris Operation, NUREG/CR-0956, PNL-3065. Richland, WA: (Battelle Pacific Northwest Laboratories, July 1979.

Eicholz, G. G. Environmental Aspects of Nuclear Power. Ann Arbor, MI: Ann Arbor Science, 1976 .

Eisenbers, N. A, C.J. Lynch and R. J. Breeding. Vulnerability Mode1--A Sumulation System for Assessing Damage Resulting from Marine Spills, Report No. CG-D-136-75. Final Report. Prepared for Department of Transportation U.S. Coast Guard Office of Research \& Development. RockVille, MD: June 1975.

Eisenbud, Mr. Environmental Radioactivity,2nd ed. Academic Press, New York: 1973.

Eisinger, P. K. "The Conditions of Protest Behavior in American Cities." American Political Science Review 67:11-28, 1973.

Elder, H. K., et al. An Assessment of the Risk of Transporting Spent Nuclear Fue1 By Truck, PNL-2588. Richland, WA: Battelle Pacific Northwest Laboratories, November 1978.

Elder, J. C., M. Gonzales, and H. J. Ettinger. "Plutonium Aerosol Size Characteristics." Health Physics 27:45, 1974.

Electric Power Research Institue. Plutonium: Facts and Inferences, EPRI EA-43-SR, Palo Alto, CA: EPRI, August 1976.

E1 Gente, M. S. Potental for Cladding Thermal Failure in LWRs During High Temperature Transients, Conf-790602-21. 1979.

Energy Planning Report, 12 February 1979.

Energy Policy Information CenterRadioactive Waste in New England: A Handbook for Citizen Activists. Boston: EPIC, June 1979.

England, W. T. "Recent Regulatory Developments Concerning the Transportation of Nuclear Fuel and Other Radioactive Materials" Environmental Law

$7: 203-222,1977$. 
Enviro Control, Inc. "Continuing Development of the Vulnerability Model," draft final report. Rockville, Md: Enviro Control, February 1977, PP 137-162.

Epstein, S. S., and R. 0. Grundy, eds. Consumer Health and Product Hazards--Chemicals, Electronic Products, Radiation. Vol. 1 of The Legislation of Product Safety. Cambridge, Mass: M. I. T. Press 1974.

Erdmann, R. C., et al. Status Report on the EPRI Fuel Cycle Accident Risk Assessment, EPRI NP-1128. Palo Alto, CA: Science Applications, Inc., July 1979.

Erickson, P. E. et al. "Families in Disaster: Patterns of Recovery." In Mass Emergencies. Amsterdam, Holland: Elsevier Scientific Publishing Co., 1976.

Ericson, D. M., Jr. "Accident Descriptions for Emergency Response Exercise Scenarios," draft, SAND78-0269. Albuquerque: Sandia Laboratories, May 1978.

Erikson, K. T. Everything in Its Path. New York: Simon and Schuster, 1976.

Estey and Nechodon. Fuel Rod Drop Test, JN-55. Jersey Nuclear Company, October 1971.

"Evacuation Plans Found Inadequate." Not Man Apart--Nuclear Blowdown, August 1978 , p 10.

Evans, R. D. Physical, Biological, and Administrative Problems Associated With the Transportation of Radioactive Substances, Nuclear Science Series Preliminary Report No. 11. Washington: National Academy of Sciences, National Research Council, 1951.

Ewing, R. A., et al. Industrial Environmental Conditions of Sealed Radiation Sources, AEC Report BMI-1648. Columbus, OH: Battelle Memorial Institute, 10 September 1963.

Executive Office of the President, Office of Management and Budget, Statistical Policy Division. Standard Metropoliton Statistical Areas, rev ed. Washington: OMB, 1975.

Fabrikant, J. I. Health Effects of Low-Level Ionizing Radition, LBL-9018. Berkeley, CA: Lawrence Berkeley Laboratory, April 1979.

Fabrikant, J. F. Radiobiology. Chicago: Yearbook Medical Publishers, Inc., 1972.

Faich, R. G., and R. P. Gale. "The Environmental Movement, From Creation to Politics." Pacific Sociological Review 14:270-287, July 1971.

Fairbairn, A. "The Development of the IAEA Regulations for the Safe Transport of Radioactive Materials." IAEA Atomic Energy Review 11(4):843-899, 1973.

Farmer, F. R. "Some Considerations of Major Non-Nuclear Hazards." Presented at ENS/ANS Topical Meeting on Nuclear Power Reactor Safety, Brussels, Belgium, 16-19 October 1978.

Faust, L. G., et al. A Guide to Good Practtices at Plutonium Facilities, BNWL-2086. Richland, WA: Battelle Pacific Northwest Laboratories, September 1977.

By-22 
Fearing, F. "Social Impact of the Mass Media of Communication." In Mass Media and Education, N. B. Henry, ed. Chicago: University of Chicago Press, 1954, pp 165-191.

Federal Aviation Administration, Flight Standards Service. "Survey to Determine the Percent of Passenger Aircraft Departures Carrying Hazardous Materials." Washington: FAA, 20 June 1974.

Federal Railroad Administration. "Summary and Analysis of Accidents on Rai1roads in the U.S. 1969," FRA Accident Bulletin No. 138. Washington: FRA.

"Federal-State Roles: Transportation of Nuclear Material." Energy Report to the States $4(6): 85-95,1978$.

Feige, Y., et al. The Internal Radiation Dose to the Population Resulting from Radionuclides in the Environment, IAEA-R-1015-F. International Atomic Energy Agency, November 1974.

Feiveson, H. A., et al. "The Plutonium Economy: Why We Should Wait and Why We Can Wait?" Bulletin of the Atomic Scientists, December 1976, pp 11-14.

Fellows, L. "Connecticut Will Escort Atom Waste Shipments." Torrington [CT] Register, 10 March 1976, p 1.

Festinger, L, et al. "A Study of Rumor: Its Origin and Spread. Human Relations $1: 464-486,1948$.

Festinger, L. A Theory of Cognitive Dissonance. Palo Alto, CA: Stanford University Press, 1957.

Fields S. R., and S. J. Mech. Dynamic Analysis to Establish Normal Shock and Vibration of Radioactive Material Shipping Packages, NUREG/CR-0589. Richland, WA: Hanford Engineering Development Laboratory, November 1978.

Fink, D. "Radioactive Shipment Bill Before Board of Aldermen." St. Louis Post Dispatch, 20 April 1979.

Finsterbusch, K. A Methodology for Analyzing Social Impacts of Public Policies, BDM/W-75-709-TR. Vienna, VA: The BDM Corporation, May 1975.

Finsterbusch, K., and C. P. Wolf, eds. Methodology Social Impact Assessment, Community Development Service, vol. 32. Stroudsburg, PA: Dowden, Hutchinson, and Ross, 1977.

Fish, B. R. Surface Contamination. Proceedings of a Symposium, Gatlinburg, Tennessee, June 1974. New York: Pergamon Press, 1974.

Fischoff, B., et al. "How Safe is Safe Enough?: A Psychometric Study of Attitudes Towards Technological Risks and Benefits." Policy Sciences 9:127-152, 1978 .

Fishbein, M. Readings in Attitude Theory and Measurement. New York: John Wiley and Sons, 1967. 
Fisher, C. S. "Observing a Crowd: The Structure and Description of Protest Demonstrations." In Research on Deviance, J.D. Douglas, ed. New York:

Random House, 1972, pp 187-211.

Flack, E., and W. Ray. "Rempersigh Manual." Oak Ridge TN: Oak Ridge National Laboratory, May 1974, unpublished.

Fleischer, R. L., and 0. G. Raabe. "Fragmentation of Respirable $\mathrm{PuO}_{2}$ Particles in Water by Alpha Decay--A Mode of 'Dissolution.' " Health Physics $32: 253-257,1977$.

Fleischer, R. L. "On the Dissolution of Respirable $\mathrm{PuO}_{2}$ Particles." Health Physics 29:69-73, 1975.

Florida Department of Health and Rehabilitative Services. "Summary of RAM Incidents for the State of Florida, 1975-1977." Tallahassee.

Florida Lime and Avocado Growers Inc. v Paul, 37 U. S. 132; 1963.

Florida Statutes Annotated. Tallahassee.

Fogelson, R. Violence as Protest. New York: Doubleday, 1971.

Foley, J. T. "Accident Rates for Various Modes of Transport." A Listing of applicable accident rates available from ERDA Trans. Environmental Data-Bank files, August 1975.

Foley, J. T., and M. B. Gens. "Shock and Vibration Measurements During Normal Rail and Truck Transport." International Journal of Radiation Engineering $1(5): 453-4741971$.

Foley, J. T., D. W. Larson, and R. K. Clarke. Predictive Models of the Environments Produced in Helicopter Accidents, SLA-74-0241. Albuquerque: Sandia Laboratories, May 1974.

Foote, N. N., and C. W. Hart. "Public Opinion and Collective Behavior." Group Relations at the Crossroads, M. Sherity and M. O. Wilson, eds. New York: Harper \& Row, 1953, pp 308-331.

Forbes T. W., and F. A. Wagner, Jr. "Effect of Small and Compact Cars on Traffic Flow and Safety." Highway Research Board Bulletin 351, 1965.

Form, W. H., and S. Nosow. Community in Disaster. New York: Harper and Row, 1958.

Form, W. H., and C. P. Loomis. "The Persistence and Emergencies of Social and Cultural Systems in Disasters." American Sociological Review 21:180-185, 1956.

Forman, R. E. "Resignation as a Collective Behavior Response." American Journal of Sociology 69:285-290, 1963.

Foster, A. R., and R. L. Wright. Basic Nuclear Engineering. Boston: Allyn and Bacon, 1969. 
French, J. R. P., Jr. "Organized and Unorganized Groups Under Fear and Frustration." In Authority and Frustration, K. Levin et al, eds. Iowa City: University of Iowa Press, 1944, pp 229-308.

Freud, S. Group Psychology and the Analysis of the Ego, J. Strachey, trans. London: The Logarth Press, 1922.

Fritz, C. E., J. F. Raynet, and S. L. Guskin. Behavior in an Emergency Shelter: A Field Study. Washington: National Academy of Sciences, National Research Council, Disaster Research Group, 1958.

Fritz, C. E., and J. H. Mathewson. Convergence Behavior in Disaster: A Problem in Social Control, NAS-NRC Publication 476. Washington: National Academy of Sciences, National Research Council, 1957.

Fritz, C. E. "Disaster." In Contemporary Social Problems, 1st ed, R. K. Merton and R. A. Nisbet, eds. New York: Harcourt, Brace and World, 1961, pp 651-694.

Fritz, C. E., and H. B. Williams. "The Human Being in Disasters: A Research Perspective." Ann. Amer. Acad. of Political Social Science 309:42-51, 1967.

Fritz, C., and E. Marks. "The NORC Studies of Human Behavior in Disaster," Journal of Social Sciences 10(3):26-41, 1954.

Froman, A., Jr. "A Realistic Approach to Campaign Strategies and Tactics." In The Electoral Process, M. K. Jennings and L. H. Zugler, eds. Englewood C1iffs, NJ: Prentice-Ha11, 1966.

Fry, R. J. M. Radiation Carcinogenesis, CONF-760946-6. Argonne, IL: Argonne National Laboratory, 1976.

Fullwood, R. R., and R. C. Erdmann. Review of Current Risk Analysis Methods Applied to Nuclear Waste Transportation, SAI-016-76-PA. Palo Alto, CA: Science Applications, Inc., 12 September 1976.

Funk, C. W., and L. D. Jacobsen. Spent Fuel Integrity During Transportation, HEDL-TME-78-58. Richland, WA: Hanford Engineering Development Laboratory, January 1980.

Futter, J. L. "Transpontion of Radioactive Materials--A Utility View." Presented at the Atomic Industrial Forum Conference, Kansas City, Missouri, 26 February 1979.

"FY-80 NRC Authorization Bill." Nucleonics Week, 19 July 1979, pp 3-4.

Gallagher, R. J., K. G. Stemwell, and N. R. Wagner. The Configuration of Road Convoys: A Simulation Study, SAND77-8625. Livermore, CA: Sandia Laboratories, July 1977.

Gamble, H. B., R. H. Downing, and 0. H. Sauerlender. Effects of Nuclear Power Plants on Community Growth and Residential Proverty Values, Final report, Sept. 30, 1977- Nov. 15, 1978, NUREG/CR-0454. University Park, PA: Pennsylvania State University, 1978. 
Gamson, W. A. The Strategy of Social Protest. Homewood, IL: Dorsey Press, 1975.

Garfinkel, S. A., and F. A. Bryan, Jr. "Radioactive Emergency Response System Test," Research Memorandum RM-24U-901-4. Research Triangle Park, NC:

Center for Health Studies, 1974.

Garofalo, F. "Groups Ask City for Radioactivity Controls." Wichita EagleBeacon, 3 April 1979.

Garofalo, F. "Hazardous Shipment Study Sought." Wichita Eagle-Beacon, 18 April 1979, p Cl.

Garvey, P. "Rushing Roulette." Ohio Magazine 17:31, August 1979.

Gay, W. F. National Transportation Statistics, DOT-TSC-RSPA-79-19. Cambridge, MA: U. S. Department of Transportation, Research and Special Programs Administration, August 1979.

Geffen, C. A., et al. An Assessment of the Risk of Transporting Uranium Hexafluoride by Truck and Train, PNL 2211. Richland WA: Battelle Pacific Northwest Laboratories, August 1978.

General Electric Company, Knolls Atomic Power Laboratory. "Chart of the Nuclides," 1968.

Georgia Code Annotated. Atlanta.

Georgia Department of Human Resources, Radiological Health Unit. "Summary of RAM Incidents for State of Georgia, 1972-1977." Atlanta.

George, H. W., II, E. Busch, and E. Weber. Investigation of the Temporal and Spatial Distribution of the Emmission Concentration of Carbon Monoxide in Frankfurt am Main. Frankfurt am Main, Germany: Institute for Meterology and Geophysics of the University of Frankfurt am Main, May 1967.

Gerlach, L., and N. Hines. People, Power and Change: Movements of Socia1 Transformation. Indianapolis, IN: Bobbs-Merrill, 1970.

Gibbons v Ogden. 22 U. S. 1, 209; 1824.

Gifford, F. A., Jr. "Atmospheric Transport and Disperson Over Cities." Nuclear Safety 13(5):391, 1972 .

Gifford, F. A. "Turbelent Diffusion-Typing Schemes: A Review." Nuclear Safety $17(1), 1976$.

Gilette, R. "200 Tons of Uranium Lost." Los Angeles Times, 29 April 1977 $\mathrm{p} \mathrm{Al}, \mathrm{col} .4$.

Gailmore, J. S., and M. K. Duff. Boom-town Growth Management: A Case Study of Rock Springs-Green River, Wyoming. Boulder, CO: Westview Press, 1975.

Gilmore, J. S. et al. Socioeconomic Impact Mitigation Mechanisms in Six States: Categories, Generalizations, and Unresolved Issues, SAN/1379-1. Denver: Denver Research Institute, September 1977.

By -26 
Glasstone, S., ed. The Effects of Nuclear Weapons. Washington: U.S. Atomic Energy Commission, April 1962. Also published as Air Force Pamplet 136-1-3.

Glasstone, S., and A. Sesonske. Nuclear Reactor Engineering. New York: Van Nostrand-Reinhold Compnay, 1967.

Gofman, J. W. The Cancer Hazard from Inhaled Plutonium, CNR Report 1975-1R. Dublin, CA: Committee for Nuclear Responsibility, May 1975.

Gofman, J. W. Estimated Production of Human Lung Cancers by Plutonium from Worldwide Fallout, CNR Report 1975-2. Dublin, CA: Committee for Nuclear Responsibility, July 1975.

Goland, S. and I. Burton. Avoidance Response to the Rich Environment. Natural Hazard Research Working Paper No. 6. Toronto: University of Toronto Department of Geography, 1969.

Goldberg, J. "Official Claims No Danger in Nuclear Waste Transport." Waterbury [CT] Republican, 13 March 1976, p Al.

Goldman, M. An Estimate of Early Mortality and Morbidity Following Inhalation of Plutonium. Davis, CA: University of California, October 1976.

Goldman, M. Annual Report of the Radiobiology Laboratory, UCD472-121. Davis, CA: University of California, 1974, pp 116-162.

Goldstein, H. Fundamental Aspects of Reactor Shielding. Reading, MA: Addison Wesley, 1959.

Gomes, L. "Court Fight Over Ship Continues." Honolulu Star-Bulletin, 7 June 1979, p A4.

Gomes, L. "Pacific Fisher Sails, Leaves Issue in Wake." Honolulu Star-Bulletin, 9 June 1979, p Al.

Gomes, L. "Pacific Swan Ready to Sail." Honolulu Star-Bulletin, 16 June 1979, P A2.

Gordon, C., et al. Review and Integration of Existing Literature Concerning the Social Impacts of Transportation of Radioactive Materials in Urban Environs, SAND 78-7017. Albuquerque: Sandia Laboratories, May 1978.

Gossick, L. V. "Regulation of Nuclear Material Transportation: the Role of NRC." Presented before the AIF Conference on Transportation for the Nuclear Ind ustry, 26 May 1976.

Gouldrer, A. W. "Organizational Analyisis." In Sociology Today, R. K. Morton et al, eds. New York: Basic Books, 1959, pp 400-428.

Graham, H. D., and T. R. Gurr, eds. The History of Violence in America: Report of National Commission on the Causes and Prevention of Violence. New York: Bantam Books, 1970. 
Greenberg, B. S. "Dimensions of Informal Communciation." One of the Paul J. Deutschmann Memorial Papers in Mass Communication Research. Cincinnati:

Scripps Howard Research, 1963, pp 35-43.

Greenborg, J., and W. B. Andrews. Nuclear Industry Alternatives--Their Effect on Transportation. Richand, WA: Battelle Pacific Northwest Laboratories, January 1977.

Greenshields, B. D., et al. Quality and Theory of Traffic Flow-A Symposium, New Haven: Yale University, 1961.

Greenshields, B. D. "Traffic Accidents and the Quality of Traffic Flow." Highway Research Board Bulletin 208:1-15, 1959.

Grella, A. G. Today's Role of DOT in Regulating the Transport of Radioactive Material, CONF-710801, August 1971 .

Grella, A. W. "A Review of Five Years' Accident Experience in the USA Involving Nuclear Transportation, 1971-1975," IAEA-SR-10/5. Washington: U.S. Department of Transportation, 1976.

Grella, A.W. "State of Current and Proposed Regulations and Legislation for Transport of Radioactive Materials." In Radiation Benefits and Opinion, proceedings of the 8 th National Conference on Radiation Control, HEW Publication (FDA) 77-8021. Springfield, IL: U. S. Department of Health, Education and Welfare, 7 May 1976.

Grendon, A. "Some Plutonium Fallacies." Presented at the 21 st annual meeting of the Health Physics Society, San Francisco, 27 June - 2 July 1976.

Grieson, R. E. "The Economics of Property Taxes and Land Values: The Elasticity of Supply--of Structures." Journal of Urban Economics 1:367-382 1974.

Grimm, E. A. "Design and Operational Characteristes of High Density Fuel Storage Facilites." Nuclear Technology 43:146-154, Mid-April 1979.

Gros, J. E. et al. "A System Analysis Approach to Nuclear Facility Siting." Behavioral Science 21, 1976.

Grosser, G. H., ed. The Threat of Impending Disaster. Cambridge, MA: M. I.T. Press, 1971 .

Grossman, L. N., and J. O. Hegland. Tritium Distribution in High Power Zircaloy Fuel Elements, GEAP-12205, June 1971.

Gwynn, D. W. "Accident Rates and Control of Access." Traffic Engineering $37(11): 18-21,1966$.

Gwynn, D. W. "Relationship of Accident Rates and Accident Involvements with Hourly Volumes." Traffic Quarterly 21(3):407, 1967.

Haas, J. E., and T. E. Drabeck. "Community Disaster and Systen Stress: A Sociological Perspective." In Social and Psychological Factors in Stress, J. E. McGrath, ed. New York: Holt, Rinehart and Winston, 1970, pp 264-286. 
Haas, J. E. Role Conception and Group Consensus. Columbus, OH: Ohio State University Bureau of Business Research, 1964.

Haas, J. E. "Role, Position and Social Organization: A Conceptual Formulation." Midwest Sociologist 19:33-37, December 1956.

Hachiya, M. Hiroshima Diary. Chapel Hill, NC: University of North Carolina Press, 1955 .

Haff, K. W., R. G. Niemeyer, and R. A. Robinson. Radioisotope Source Safety Testing, ORNL-4092. Oak Ridge, TN: Oak Ridge National Laboratory, May 1967.

Hagrman, D. L., and G. A. Reymann. MATPRO-Version II: A Handbook of Materials Properties for Use in the Analysis of Light Water Reactor Fuel Rod Behavior, NUREG/CR-0497. Idaho Falls: Idaho National Engineering Laboratory, Febuary 1979.

Hahn, F. F., et al. Influence of Particle Size on Phagocytosis by Rabbit Alveolar Macrophages, Annual Report 257-260. Albuqueruque: Lovelace Foundation, 1972 .

Hall, P. M., and J. P. Hewitt. "The Grasi-theory of Communication and the Management of Dissent." Social Problems 18:17-27, Summer 1970.

Ha11, R. J., et al. An Assessment of the Risk of Transporting Plutunium Dioxide and Liquid Plutonium Nitrate by Train, BNWL-1996. Richland, WA: Battelle Pacific Northwest Laboratories, February 1977.

Halperin, M. H. Bureaucratic Politics and Foreign Policy. Washington: The Brookings Institution, 1974.

Hamard, J. Thoughts on Radiological Hazards During Transport of Radioactive Materials, BNWL-tr-212. Fonlenay-aux-Roses, France: CEA Cenre d'Etudes Nucleaires de Fonlenay-aux-Roses, 92, Dept. de Protection, September 1976. Translated at Battelle Pacific Northwest Laboratories, Richland, WA.

Hamilton, R. Y., R. M. Taylor, and G. E. Rice. A Social Psychological Interpretation of the Udall, Kansas, Tornado. Washington: National Academy of Sciences, National Research Council, 1955.

Hammond, R. D. Quality Assurance in Alternative Energy Sources, RHO-SA-107. Richland, WA: Atomics International Divison, Rockwell Hanford Operations, February 1979.

Hammond, R. P. "Nuclear Power Risks." American Scientist 62:155-160, March-April 1974.

Handley, T. H., and C. J. Barton. Home Ventilation Rates: A Literature Survey, ORNL-TM-4318. Oak Ridge, TN: Oak Ridge National Laboratory, 1973.

Hanford Engineering Development Laboratory. FFTF Final Safety Analysis Report, HEDL-TI-75001. Richland, WA: HEDL, December 1975. 
"Hanford Safety Good, Says Official." Seattle Times, 23 April 1978.

Hardin, G. "Living with the Faustian Bargain." Bulletin of the Atomic Scientists 10:25-29, 1976.

Hardman, J. B. S. The Encyclopedia of the Social Sciences, vol. 4. New York: Crowe11-Collier, 1933.

Harmon, K. M. International Source Book: A Compendium of Worldwide Programs in Nuclear Energy Supply and Radioative Waste Management, vols. 1-4, PNL-2478. Richland, WA: Battelle Pacific Northwest Laboratories, January 1978.

Harris, R. W. "Proposed Transportation and Visitor Center." Trans Eng Journal, Proceedings of the ACRE TE3:301, 1970.

Hart, H., and A. Sacks. "The Legal Process: Basic Problems in the Making and Application of Law," draft. Cambridge, MA, 1958.

Hart, Ritchie, and Vornadore, eds. Population Exposures, Proceedings of the Eighth Topical Symposium of the Health Physics Society, Knoxville, Tennessee, 21-24 October 1974; CONF-741018. Oak Ridge, TN: Oak Ridge National Laboratory, October 1974.

Hartley, H. O. "Smallest Composite Designs for Quadratic Response Surfaces. Biometrics 15:611, 1959.

Hartman, W. F., C. A. Davidson, and J. T. Foley. Statistical Description of Heavy Truck Accidents on Representative Segments of Interstate Highway, SAND 76-0409. Albuquerque: Sandia Laboratories, January 1977.

Hastings, B. "Ship Full of Radioactive Waste Due to Stop Here for Provisions." The Honolulu Advertiser, 1 June 1979, p A3.

Hawley, A. H. Human Ecology: A Theory of Community Structure. New York: Ronald Press, 1950.

Hawley, A. H., and V. P. Rock, eds. Metropolitan America in Contemporary Perspective. New York: John Wiley and Sons, Halsted Press Division, 1975.

"Hazard Questioned: Nuclear Freight Shipped at 0'Hare." Chicago Tribune, 27 November 1977, sec. 1, p 2, col. 1.

"Hazardous-Cargo Trucks Detained." Seattle Times, 30 January 1979, p C4.

"Hazardous Shipments Study Sought." Wichita [KS] Eagle-Beacon, 8 April 1979.

Heaberlin, S. W., et al. Evaluation of the Consequences of LWR Spent Fuel and Plutonium Shipping Packages Lost at Sea, BNWL-2093. Richland WA: Battelle Pacific Northwest Laboratories, December 1976. Also published as paper No. IAEA-SR-10/14 of the IAEA Seminar on the Design, Construction and Testing of Packaging for the Safe Transport of Radioactive Materials, Vienna, 23-27 August 1976. 
Heaberlin, S. W., and D. A. Baker. Evaluating the Loss of a LWR Spent Fuel or Plutonium Shipping Package into the Sea, BNWL-SA-5744. Richland, WA: Battelle Pacific Northwest Laboratories, June 1976.

Healey, J. W., et al. A Brief Review of the Plutonium Lung Estimates by John W. Gofman, LA-UR-75-1779. Los Alamos, NM: Los Alamos Scientific Laboratory, October 1975.

Hebert, J. A., et al. Nontechnical Issues in Waste Management: Ethical, Institutional, and Political Concerns, PNL-2400. Seattle, WA: Battelle Human Affairs Research Centers, May 1978.

Heirich, M. A. The Spiral of Conflict: Berkeley 1964. Berkeley, CA: University of California Press, 1971.

Hempelmann, L. H., et al. "Neoplasms in Persons Treated with X-rays in Infancy: Fourth Survey in Twenty Years." $J$ of the National Cancer Institute $55(3): 519,1975$.

Henderson, C., and W. E. Strepe. Crisis Relocation of the Population at Risk in the New York Metropolitan Area, SRI Report No. 5591. Menlo Park, CA: Stanford Research Institute International, September 1978.

Hendrickson, P. L., et al. Review of Decision Methodologies for Evaluating Regulator Actions Affecting Public Health and Safety, BNWL-2158. Richland, WA: Battelle Pacific Northwest Laboratories, December 1976.

Herring, F. W. "Metropolitan Growth and Metropolitan Travel Patterns." Highway Research Board Bulletin 293, 1961.

Heyns, R. W. "Stress and Administrative Authority." In Stress and Campus Response, G. K. Smith, ed. San Francisco: Jossey-Bass, Inc., 1968, pp 163-172.

Highway Users Federation. Traffic Control and Roadway Elements-Their Relationship to Highway Safety, rev. ed, 1969-1971. Washington: The Federation.

Hill, Sir J. "Quest for Public Acceptance of Nuclear Power." Nuclear Energy $18(5): 301-309$, October 1979 .

Hill, W. J., and W. G. Hunter. "A Review of Response Surface Methodology: A Literature Survey." Technometrics vol. 8, No. 4, November 1966.

Hilst, G. R., and N. E. Bourne. "Diffusion of Aerosols Released Upward of an Urban Complex." Environmental Science and Technology, vol. 5, No. 4, 1971.

Hines v Davidowitz, 312 U.S. 67; 1941.

Hirt, C. W., and J. L. Gook. "Calculating Three-Dimensional Flows around Structures and over Rough Terrain." Journal of Computational Physics 10:324, 1972 .

Hobson, D. 0., M. F. Osborne, and G. W. Parker. "Comparison of Rupture Data From Irradiated Fuel Rods and Unirradiated Cladding." Nuclear Technology $11: 479,1971$. 
Hobson, D. O., and P. L. Rittenhouse. Deformation and Rupture Behavior of Light-Water Reactor Fuel Cladding, ORNL-4727. Oak Ridge, TN: Oak Ridge National Laboratory, October 1971.

Hodge, C. V., et al. Transportation Accident Risks in the Nuclear Power Industry 1975-2020, NSS-8191.1. Holmes and Marver, Inc., Nuclear and Systems Sciences Group, November 1974.

Hodge, H. W., and R. G. Thomas. "Health Hazards from the Inhalation of Insoluble Uranium and Thorum Oxides." In Proceedings of 2nd UN International Conference on Peaceful Uses of Atomic Energy, Geneva, September 1958, vol. 23, pp 302ff.

Hodge, H. C., J. N. Stannard, and J. B. Hursh. Uranium, Plutonium, Transplutonic Elements. Vol. 36 of the Handook of Experimental Pharmacology. New York: Springer-Verlag, 1973.

Hoenes, G. R., and J. K. Sordat. Age Specific Radiation Dose Commitment Factors for a One-Year Chronic Intake, NUREG-0172. Richland, WA: Battelle Pacific Northwest Laboratories for the Nuclear Regulatory Commission Office of Standards Development, November 1977.

Hoffman, F. O., et al. "Computer Codes for the Assessment of Radionuclides Released to the Environment." Nuclear Safety 18(3):343, 1977.

Hogerton, J. F., et al. Nuclear Power Waste Management. New York: Atomic Industrial Forum, 1971.

Holley, W. L. Methods of Assessing the Lung Dose from Inhaled 238-PuO 2 , SC-DC-67-1854. Albuquerque: Sandia Laboratories, 1967.

Honolulu Advertister, June 1979.

Honolulu Star Bulletin, June 1979.

Hornblower, M. "Uranium Oxide in Colorado: Crash Spills Toxic Matter." Washington Post, 8 October 1977, p Al, col. 1 .

Hotchkiss, R. S. "The Numerical Calculation of Three-Dimensional Flows of Air and Particulates About Structures." In Proceedings of the Symposium on Air Pollution, Turbulence, and Diffusion, SC-M-71-0913. Albuquerque: Sandia Laboratories, March 1972, pp 35-42.

Hovland, C. I. "Effects of the Mass Media on Communication." In Handbook of Social Psychology, vol. 2, G. Lindsey, ed. Reading, MA: Addison Wesley, 1954, pp 1062-1103.

Hovland, C., et al. Communication and Persuasion. New Haven: Yale University Press, 1953.

Hovland, C. I. "Psychology of the Communication process." In Communications in Modern Society, W. Schramm, ed. Urbana, IL: University of Illinois Press, 1948, pp 59-65. 
Hoydysh, W. G., et al. "A Combined Experimental and Numerical Study of Flow in Street Canyons." In Proceedings of the Second National Conference on Wind Engineering Research. Ft. Collins, C0: June 1975, pp I-17-1 to I-17-3.

Huerta, M., A. W. Dennis, and R. H. Yoshimura. Impact Analysis of Spent Nuclear Fuel Shipping Casks, SAND77-0466. Albuquerque: Sandia Laboratory, July 1978.

Hull, A. P. Current Events: Transportation of Radioactive Materials. Washington: U. S. Department of Health, Education, and Welfare, 1976.

Hundley, J. R., Jr. "The Dynamics of Recent Ghetto Riots." Detroit Journal of Urban Law 45:627-639, 1968.

Hundley, J.R., Jr. "Interaction Between the Crowd and Social Control Agencies." In Rift in the Cities, R. A. Chikita and M. C. Moran, eds. Rutherford, NJ: Fairleigh Dickinson University Press, 1968, pp 147-148.

Hurst, D G. Diffusion of Fission Gas: Calculated Diffusion from a Sphere Taking into Account Trapping and Return From the Traps, CRRP-1124 (AECL-1550). Atomic Energy of Canada, Ltd., 15 November 1962.

Hurst, P. M. Errors in Driver Risk-Taking, Report No. 2. State College, PA: Division of Highway Studies, Institute for Research, 1964.

Hurst, P. M., T. C. Perchonok, and E. L. Seguin. Measurement of Subjective Gap Size, Report No. 7. State College, PA: Division of Highway Studies, Institute for Research, 1965.

"I-5 Inspectors Corral Herd of Trucks." Seattle Post-Intelligencer, 30 January 1979, p C20.

Idaho Code. Boise.

Idaho Department of Health and Welfare, Environmental Division, Radiation Control. "Summary of RAM Indidents for the State of Idaho, 1968-1977." Boise.

Idaho Department of Health and Welfare. State of Idaho Radiation Emergency Response Plan, nd.

Illinois Annotated Health Statutes. Springfield.

Illinois Dept. of Public Health, Division of Radiological Health. Transportation of Radioactive Material in Illinois: June 6, 1977-June 6, 1978, NUREG/CR-0756. Springfield, IL, March 1979.

Iman, R. L. STEPWISE Regression, SAND76-0364. Albuquerque: Sandia Laboratories, 1976.

"Inadequate Safety." Critical Mass Journal 3(2):2, 1977.

Indiana, State of. Nuclear Blackmail: Emergency Response Plan for the State

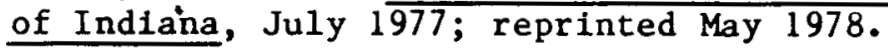


Indiana Board of Health. Indiana State Board of Health Radiological Assistance Program, nd.

Ingersol1, B. "Interstate 80: America's Nuclear Highway." San Francisco Sunday Examiner and Chronicle, 27 November 1977, p A22.

Ingersoll, B. "O'Hare Uranium Storage Despite Lax Security Bared." Chicago Sun-Times, 16 March 1977.

Inglis, D. A. Nuclear Energy: Its Physics and Its Social Challenge. Reading, MA: Addison-Wesley, 1973.

"Injunction Eyed on N-Shipment." The Bridgeport [CT] Post, 23 March 1976.

Insurance Information Institute. NEL-PIA: Insurance for the Nuclear Industry, March 1976.

Interagency Review Group on Nuclear Waste Management. "Report to the President," draft, TID-28817. Springfield, VA: National Technical Information Service, October 1978.

Interagency Review Group on Nuclear Waste Management. "Subgroup Report on Alternative Technology Strategies for the Isolation of Nuclear Waste," draft, TID-28818. Springfield, VA: National Technical Information Service, October 1978.

International Association of Fire Chiefs. Nuclear Hazard Management for the Fire Service. Washington: IAFC, 1975.

International Atomic Energy Agency. Handling of Radiation Accidents 1977: Proceedings of A Symposium, 28 February to 4 March 1977. Vienna: IAEA, 1977.

International Atomic Energy Agency. Handling of Radiation Accidents: Proceedings of a Symposium, 19-23 May 1969. Vienna: IAEA, 1969.

International Atomic Energy Agency. Nuclear Power and Its Fuel Cycle: Proceedings of a 1977, Conference Held at Salzburg. Vienna: IAEA, 1978.

International Atomic Energy Agency. Risk Analysis for the Protection of the Public in Radiation Accidents, IAEA Safety Series 21, STI/PUB/124. Vienna: IAEA, April 1967.

International Atomic Energy Agency. Technical Packaging Requirements: An Analysis of the Criteria and Tests. Vol. 1 of IAEA Regulations for the Safe Transport of Radioatice Materials, EUR-385 e,f.; together with a study of practical means of compliance. Vienna: IAEA, 1968.

International Atomic Energy Agency. Transport Packaging for Radioactive Materials: Proceedings of a Seminar, 23-27 August 1976. Vienna: IAEA, 1976.

International Commission on Radiological Protection. The Assessment of Internal Contamination Resulting From Recurrent or Prolonged Uptakes, ICRP Publication 10A. Elmsford, NY: Pergamon Press, 1969. 
International Commission on Radiological Protection. The Metabolism of Plutonium and Other Actinides, ICRP Publication 19. Elmsford, NY: Pergamon Press, 1972.

International Commission on Radiological Protection. Protection of the Patient in Radionuclicle Investigations, ICRP Publication 17. Elmsford, NY: Pergamon Press, 1971.

International Commission on Radiological Protection. Radiosensitivity and Spatial Distribution of Dose, ICRP Publication 14. Elmsford, NY: Pergamon Press, 1969.

International Commission on Radiological Protection. Report of Committee IV on Evaluation of Radiation Doses to Body Tissue from Internal Contamination Due to Occupational Exposures, ICRP Publication 10. E1msford, NY: Pergamon Press, 1968.

International Commission on Radiological Protection. Report of Committee II on Permissible Dose for Internal Radiation, ICRP Publication 2. Elmsford, NY: Pergamon Press, 1960.

International Commission on Radiological Protection. Report of the Task Group on Reference Man, ICRP Publication 23. E1msford, NY: Pergamon Press, 1975.

International Commission on Radiological Protection. A Review of the Radiosensitivity of the Tissues in Bone, ICRP Publication 11. Elmsford, NY: Pergamon Press, 1967.

International Matematical and Statistical Libraries, Inc. The IMSL Library IMSL LIB03-0006, IMSL Library 3, (FORTRAN) CDC 6000/7000; Series CYBER $70 / 170.6$ th ed., July 1977.

Interstate Commerce Commission. Final Environmental Impact Statement in Transportation of Radioactive Material by Rail. Washington: ICC, August 1977.

Iowa Code Annotated. Des Moines.

"Israel Denies Involvement in Uranium Disappearance." Washington Post, 30 April 1977, p Al6, col. 3.

Jackman, N. R. "Collective Protest in Relocation Centers." American Journal of Sociology 63:264-272, 1958.

Jaeger, R. G., et al. Engineering Compendium on Radiation Shielding. New York: Springer-Verlag, 1975.

Jammeth, H., and G. Vacca. "Various Possible Expressions of the Toxicity of Radioactive Substances as Defined by the Practical Problems It Raises." In Proceedings of the 2nd UN International Conference in the Peaceful Uses of Atomic Energy, Geneva, September 1958, Vo1. 23, pp 346ff.

Janis, I. L. "Group Identification under Conditions of External Danger." British Journal of Medical Psychology 36:227-238, 1963. 
Janis, I. L. "Problems of Theory in the Analysis of Stress Behavior." Journal of Social Issues 10:12-24, 1954.

Janis, I. L. "Psychological Effects of Warnings." In Man and Society in Disaster, G. W. Baker and D. W. Chasman, eds. New York: Basic Books, 1962, pp 55-92.

Joy D. S., and L. B. Shappert. Effects of AFR Storage Location on Spent Fuel, CONF-790602-58. Oak Ridge, TN: Oak Ridge National Laboratory, 1979.

Jee, W. S. E. "Distribution and Toxicity of Pu-239 in Bone." Health Physics $22: 583-595,1972$.

Jefferson, R. M. "Cask Demolishes Train Superstructure, Sustains Minor Damage." Nuclear Engineering International, vol. 22, No. 260, July 1977.

Jefferson, R. M. Transportation: The Vital Tendons of the Nuclear Industry, SAND-79-0466C. Albuquerque: Sandia Laboratories, 1979.

Jefferson, R. M., and H.K. Yoshimura. Crash Testing of Nuclear Fuel Shipping Containers, SAND77-1962. Albuquerque: Sandia Laboratories, February 1978.

Jenkins, B. International Terrorism, A New Mode of Conflict, Research Paper No. 48 of California Seminar on Arms Control and Foreign Policy. Los Angeles: Crescent Publications, 1974.

Jenkins, B. M. The Potential for Nuclear Terrorism, P-5876 of The Rand Paper Series. Santa Monica, CA: The Rand Corporation, May 1977.

Jenkins, B. M. Will Terrorists Go Nuclear?, P-5541 of The Rand Paper Series. Santa Monica, CA: The Rand Corporation, November 1975.

Jenkins, C. E., E. S. Murphy, and K. J. Schneider. Technology, Safety and Costs of Decommissioning a Reference Small Mixed oxide Fuel Fabrication Plant, 2 vols., NUREG/CR-0129. February 1979.

Jet Research Center. Commercial Catalog. Arlington, TX.

John, P. W. M. Statistical Design and Analysis of Experiments. New York: MacMillan and Co., 1971.

Johnson, A. B., Jr. "Spent Fuel Storage Experience." Nuclear Technology 43:165ff, Mid-April 1979.

Johnson, C. J., and R. R. Tidball. "Plutonium Hazard in Respirable Dust on the Surface of Soil." Science 193:488-90, 1976.

Johnson, D. M. "The Phantom Anesthetist of Matoon. A Field Study of Mass Hysteria." Journal of Abnormal and Social Psychology 40:175-186, 1945.

Johnson, J. F., and R. J. Ha1l. Usefulness of the Risk Assessment Technique in Solving Transportation Problems, BNWL-SA-5947. Richland, WA: Battelle Pacific Northwest Laboratories, 1976. 
Johnson, N. R., and W. E. Feinberg. "A Computer Circulation of the Emergence of Consensus in Crowds." American Sociological Review 42:505-521, June 1977.

Johnson, N. R., et al. "Crowd Behavior as 'Risky Shift': A Laboratory Experiment." Sociometry 40(2):183-187, 1977.

Johnson, W. B., et a1. "An Urban Diffusion Simulation Model for Carbon Monoxide." Journal of the Air Pollution Control Association 23(6):490, 1973.

Jonasson, et al. The Release of Radioactive Material in the Event of the Destruction of a Full Type A Package, CONF-681001, October 1968.

Jones, E. E., et al. Attribution: Perceiving the Causes of Behavior. Morristown, NJ: General Learning Press, 1972.

Jones, R. E., and C. B. Bailey. Brief Instructions for Using MATHLIB (Version 6.0), SAND75-0545. Albuquerque: Sandia Laboratories, February 1976.

Jones, J. M., and T. W. Hoover. Department of Energy (DOE) Transportation System for Nuclear Materials and the Role of State Law Enforcement Agencies, SAND78-0150C. Albuquerque: U.S. DOE Transporation Safety Division/Sandia Laboratories, 1978.

Kahn, R. L., et al. Organizational Stress: Studies in Role Conflict and Ambiguity. New York: John Wiley and Sons, 1964.

Kanapilly, G.M., et al. "Measurement of In Vitro Dissolution of Aerosol Particles for Comparison to In Vivo Dissolution in the Lower Respiratory Tract After Inhalation." Health Physics $24: 497,1973$.

Kansas Department of Health and Environment. "Summary of RAM Incidents for the State of Kansas, 1966-1977." Topeka.

Kansas Department of Health and Environment, Bureau of Radiation Control. Radiation Emergency Handbook. Topeka, May 1978.

Kansas Statutes Annotated. Topeka.

Kaplan and Associates, Inc. Notes for a Workshop on Risk Analysis and Decision Under Uncertainty, UCRL-15007. Irvine, CA, 1979.

Kappe1man v De1ta Air1ines, Inc. 539 F.2d 165; D.C. Cir. 1976.

Karber, P. A., et al. Analysis of the Terrorist Threat to the Commercial Nuclear Industry, BDM/75-176-TR. Vienna, VA: BDM Corp•, 30 September 1975.

Karalekas, A., ed. "Conducting Military Operations." In Appendix K: Adequacy of Current Organization: Defense and Arms Control, vol. 4 of Appendices [to the] Commission on the Organization of the Government for the Conduct of Foreign Policy June 1975, 7 vols. Washing ton: U.S. Government Printing office, 1975.

Karber, P. A. "Urban Terrorism: Baseline Data and a Conceptual Framework." Social Science Quarterly 2(3):521-533, 1971. 
Kasarda, J. D., and M. Janowitz. "Community Attachment in Mass Society." American Sociological Review 39:328-339, June 1974.

Kastenberg, et a1. On Risk Assessment in the Absence of Complete Data, PB-261 573. Los Angeles: Univerrsity of California, Los Angeles, School of Engineering and Applied Sciences, 1976.

Kasun, D. J. Physical Protection of Shipments of Irradiated Reactor Fuel, Interim Guidance, NUREG-0561, June 1979.

Katayama, Y. B. Leaching of Irradiated LWR Fuel Pellets in Deionized and Typical Ground Water, BNWL-2057. Richand, WA: Battelle Pacific Northwest Laboratories, July 1976.

Kates, R. W., et al. "Human Impact of the Managua Earthquake." Science 182:981-990, 7 December 1973.

Katz, E. "The Two-Step Flow of Communication: an Up-to-Date Report on an Hypothesis." Public Opinion Quarterly 21:61ff, Spring 1957.

Kaye, R. A. Analysis and Summary of Accident Investigations 1973-76. Washington: U. S. Department of Transportation, Federal Highway Administration, Bureau of Motor Carrier Safety.

Keaton, S. C., and P. DeLaquell IV. Conflict Simulation for Surface Transport Systems, SAND77-8624. Livermore, CA: Sandia Laboratories, July 1977.

Kee, C. W., A. R. Croft, and J. O. Blomeke. Updated Projections of Radioactive Wastes To be Generated by the U.S. Nuclear Power Industry, ORNL/TM-5427. Oak Ridge, TN: Oak Ridge National Laboratory, 1976.

Keeny, S. M., et al. Nuclear Power: Issues and Choices. Cambridge, MA: Ballinger Publishing Co., 1977.

Kelley, H. H., et al. "Collective Behavior in a Simulated Panic Situation." Journal of Experimental Social Psychology $1: 20-54,1965$.

Kennedy, G. S. Explosive Shocks in Air. New York: MacMillan and Co., 1962.

Kennedy, J. M., and J. H. Kent. "Wind Tunnel Modelling of Carbon Monoxide Dispersal in City Streets." Atmospheric Environment 2:541-547, 1977.

Kentucky Department of Human Resources, Bureau of Health, Radiation Control Branch. "Summary of RAM Incidents for the State of Kentucky, 1963-1977." Frankfort.

Kerckhoff, A. C. "A Theory of Hysterical Contagion." In Human Nature and Collective Behavior, T. Shibutani, ed. Brunswick, NJ: Transaction Books, 1970, pp 81-93.

Kerckhoff, A. C., and K. W. Back. The June Bug: A Study of Hysterical Contagion. New York: Appleton-Century-Crofts, 1968. 
Kerckhoff, A. C., K. W. Back, and N. Miller. "Sociometric Patterns in Hysterical Contagions." Sociometry 28:2-15, 1965.

Key, V. 0., Jr. Politics, Parties and Prssure Groups. New York: Thomas Y. Crowe11, 1942.

Keyrim-Markus, I. B., and V. I. Popov. "About the Dependence of Radiation Quality Factor on L.E.T." Health Physics 30:299-302, 1976.

Kibbey, A. H., and H. W. Godbee. Sources, Amounts and Characteristics of Low Level Radioactive Solid Wastes, CONF-790209-6. Oak Ridge, TN: Oak Ridge National Laboratory, 1979.

Killian, L. M. "The Significance of Multiple Group Membership in Disaster." American Journal of Sociology 57:309-314, 1952 .

Killian, L. M. "Social Movements." In Handbook of Modern Sociology, R.E.L. Faris, ed. Chicago: Rand McNally and Co., 1964, pp 426-455.

Killough, G. G., and L. R. McKay. Methodology for Calculating Radiation Dose from Radioactivity Released to the Environment, ORNL-4992. Oak Ridge, TN: Oak Ridge National Laboratory, March 1976.

Killough, G. G., et al. Estimates of Internal Dose Equivalent to 22 Target Organs for Radionuclides Occurring in Routine Releases from Nuclear Fue1-Cycle Facilities, vol. 1, NUREG/CR-0150, ORNL/NUREG/TM-190. 0ak Ridge, TN: Oak Ridge National Laboratory, 1979.

Killough, G. G., D. E. Dunning, Jr., and J. C. Pleasant. INREM II: A Computer Implementation of Recent Models for Estimating the Dose Equivalent to Organs of Man from an Inhaled or Ingested Radionuclide, NUREG/CR-0114, ORNL/NUREG/TM-84. Oak Ridge, TN: Oak Ridge National Laboratory, 1979.

Killough, G.G., P. S. Rohwer, and W. D. Turner. INREM-A Fortran Code which Implements ICRP2 Models of Internal Radiation Dose to Man, ORNL-5003. Oak Ridge, TN: Oak Ridge National Laboratory, February 1975.

Kilpatrick, F. P. "Problems of Perception in Extreme Situations." Human Organization 16:20-22, 1957.

Klapp, 0. E. "Social Types: Process and Structure." American Sociological Review 23:674-678, December 1958.

Klapper, J. The Effects of Mass Communication. Glencoe, IL: Free Press, 1960.

Klein, G. F., et al. Methods of Evaluation of the Effects of Transportation Systems on Community Values--Final Report, SRI Project MU-8493. Menlo Park, CA: Stanford Research Institute, April 1971.

Koch, R. C., and S. D. Thayer. Validation and Sensitivity Analysis of the Gaussian Plume Multiple-Source Urban Diffusion Model, EPA Report APTD-0935, NTIS No. PB206-951. Springfield, VA: National Technical Information Service, 1971 . 
Kohn, H. "Karen Silkwood Was Right in Plutonium Scandal." Rolling Stone, 20 October 1977.

Lackey, L. L, T. O. Jacobs, and S. R. Stewart. Public Attitudes Toward Hazardous Waste Disposal Facilities, Hum RRO-TR-7407. Alexandria, VA: Human Resources Research Organization, April 1974.

Laitner, S. Citizens Guide to Nuclear Power. Washington: The Center for Study of Responsive Law, 1975.

"Lane County, Ore. Vote on Ordinance Restricting Nuclear Shipments Set for March, 1979." Nucleonics Week, 4 January 1979, p 7.

Lang, K., and G. Lang. "Collective Behavior." In International Encyclopedia of the Social Sciences, 2nd ed., vol II., 1968, pp 556-565.

Lang, K., and Lang, G. E. "Collective Behavior Theory and the Escalated Riots of the Sixties." In Human Nature and Collective Behavior, T. Shibutani, ed. New Brunswick, NJ: Transaction Books, 1970, pp 4-110.

Lang, K., and Lang, G. E. Collective Dynamics. New York: Crowell Publishers, 1961.

Lange, R., et al. Particle-in-Cell vs. Straight-Line Air flow Gaussian Calculations of Concentration and Deposition of Airborne Emissions out to 70 km for Two Sites of Differing Meterological and Topographical Character, UCRL-52133. Livermore, CA: Lawrence Livermore Laboratory, 1976.

Lange, T. "Yellowcake Spilled; Clean Up Slow, Disorganized." High Country News 9:1, 16 December 1977.

Lanoue, R. Evacuation Plan--The Achilles Heel of the Nuclear Industry, 2nd ed. Washington: Critical Mass Energy Project, 1978.

Lanze1, L. H., J. H. Pingel, and J. H. Rust. Radiation Accidents and Emergencies in Medicine, Research and Industry. Springfield, IL: C. C. Thomas.

Lapp, R. E., Dr. The Radiation Controversy. Greenwich, CT: Reddy Communications, 1979 .

Larsen, D. K., and R. O. Chester. Radiation Emergency Response in Illinois, Alabama, and Texas, ORNL-5242. Oak Ridge, TN: Oak Ridge National Laboratory, March 1978.

Larsen, 0. N., and R. J. Hill. "Mass Media and Interpersonal Communications in the Diffusion of a News Event." American Sociological Review 19:426-433, 1954.

Lasswe1l, H. D. Politics: Who Gets What, When, How. Cleveland: World Publishing, Meridian Books, 1958.

Latane, B., and L. Wheeler. "Emotionality and Reactions to Disaster." Journal of Experimental Social Psychology, Suppl. 1, 1966, pp 95-102. 
Lautkaski, R., and T. Mankamo. Chlorine Transportation Risk Assessment, Report 27. Helsinki: Technical Research Centre of Finland Nuclear Engineering Laboratory, February 1977.

Lawrence, L. A., J. W. Weber, and J. L. Devary. Fuel Cladding Chemical Interaction in Mixed Oxide Fuels, HEDL-SA-1527-FP. Richland, WA: Hanford Engineering Development Laboratory, October 1978.

Lawrence, S. "Probe Goof on Uranium at Kennedy." New York News, 9 January 1979.

Lawrence W. Bienlein Law Offices. Transportation of Hazardous Materials by Highway in Illinois, An Appraisal of the Need for and Lawfulness of Regulatory Control by the State of Illinois. March 1977.

Lazarus, R. S. Psychological Stress and the Coping Process. New York: McGraw-Hil1, 1966.

Leachmen, R. B., and P. Althoff. Preventing Nuclear Theft: Guidelines for Industry and Government. New York: Praeger Publishers, 1972.

Leahey, D. M. "An Advective Model for Predicting Air Pollution within an Urban Heat Island with Applications to New York City." Journal of the Air Pollution Control Association 22:548, 1972.

Lebon, G. The Crowd: A Study of the Populat Mind. London: Ernest Benn, Ltd. 1896.

Lederer, C. M., J. M. Hollander, and I. Perlman. Table of the Isotopes. New York: John Wiley and Sons, Inc., 1967.

Leighton, A. Psychological Factors in Major Disasters, University of Rochester Medical Projects Report. Rochester, NY: Univ, of Rochester, 1951

Leimkuhler, F. K. Trucking of Radioactive Materials: Trucking vs. Economy in Highway Transport, NYO-9773. New York: Johns Hopkins Press, 1963.

Leonard, J. "Area Officials Wait for State to Act on Radioactive Shipment." [Danbury, CT] News-Times, 14 March 1976.

Lerch, I. "Risk and Fear." New Scientist, 3 January 1980, pp 8-11.

Letavet, A. A., and E.B. Kurlyandskoya, eds. Strontium, Cesium--Ruthenium, Radon, vol. 1 of The Toxicology of Radioactive Substances; E. Lloyd, trans. Elmsford, NY: Pergamon Press, 1962.

Letavet, A. A., and Kurlyandskoya, eds. Iron-59, vol. 3 of The Toxicology of Radioactive Substances; R. E. Travers, trans. Elmsford, NY: Pergamon Press, 1967.

Lettau, H. H. "Physical and Meteorological Basis for Mathematical Models of Urban Diffusion Processes." In Proceedings of a Symposium on Multiple Source Urban Diffusion Models, $\mathrm{AP}-86$. Washington: U.S. Air Pollution Office, 1970. 
Levine, G. N., and J. Mode11. "American Public Opinion and the

Fallout-Shelter Issue." Public Opinion Quarter1y 290:270-279, 1965.

Levinson, H. S., and F. H. Wynn. "Effects of Density on Urban Transportation Requirements." Highway Research Record 2:38-64, 1963.

Levinson, H. S., and F. H. Wynn. "Some Aspects of Future Transportation in Urban Areas." Highway Research Board Bulletin 326:1-31, 1962.

Lewis, F. One of Our H-Bombs is Missing. New York: McGraw-Hill, 1967.

Lewis, H. W., et al. Risk Assessment Review Group Report to the Nuclear Regulatory Commisson [WASH-1400], NUREG/CR-0400, September 1978.

Libby-Owens-Ford Company. Glass for Construction, LOF 8.26/Li, January 1976.

Library of Congress, Congressional Research Service. Hazardous Materials

Transportation: A Review and Analysis of the Department of Transportation's Regulatory Program. Washington: U.S. Government Printing Office, April 1979.

Lichtenstein, S., et al. "Judged Frequency of Lethal Events." Journal of Experimental Psychology: Human Learning and Memory $4(6): 551-5 \overline{58,1978 .}$

Lifton, R. J. Death in Life--Surivors of Hiroshima. New York: Random House, 1968.

Lifton, R. J. "On Death and Death Symbolism: The Hiroshima Disaster." Psychiatry 27:191-210, August 1964 .

Lifton, R. J. "Psychological Effects of the Atomic Bomb in Hiroshima: The Theme of Death." Daedalus 92:462-487, 1963.

Lin, M. K., and Seinfeld, J. H. "On the Validity of Grid and Trajectory Models of Urban Air Pollution." Atmospheric Environment 9:555, 1975.

Lincoln, J. R. "Organizational Differentiation in Urban Communities: A Study in Organizational Ecology." Social Forces 57(3):915-931, 1979.

Lindell, M. K., et al. Radioactive Waste: Public Attitudes Toward Disposal Facilities, B-HARC-411-004. Seattle: Battelle Human Affairs Research Centers, October 1978.

Linnerooth, J. "Methods for Evaluating Mortality Risk." Futures, August 1976.

Lippek, H. E., and C. R. Schuller. Legal, Institutional and Political Issues in Transportation of Nuclear Materials at the Back End of the LWR Nuclear Fuel Cycle, PNL-2457. Seattle: Battelle Human Affairs Research Centers, 1979.

Litchfield, J. W., and J. C. King. Decommissioning and Decontamination Planning for Hanford Nuclear Facilities Using Multiattributed Decision Analysis, BNWL-SA-6007. Richland, WA: Battelle Pacific Northwest Laboratories, May 1977. 
"Living with Radioactivity," editorial. [Chattanooga, TN] News-Free Press, 1 September 1978 .

Lombardi, D. J., R. S. Thompson, and J. E. Cernak. Physical Modeling of the Transport of Automotive Emissions in a City Street Canyon, Technical Report CEP 74-75, DJL-RST-JEC-22. Fort Collines, CO: Colorado State University School of Engineering, 1974.

Los Alamos Scientific Laboratory. Summary Report of Jointly Sponsored NRC-DOT Transportation Surveillance Program Involving Low-Level Radioactive Materials: Findings and Recommendation, NUREG-0393. Los Alamos, NM: LASL, 1978.

Loscutoff, W. V. A Safety and Economic Study of Special Trains for Shipment of Spent Fuel, draft, BNWL-2263. Richland, WA: Battelle Pacific Northwest Laboratories, May 1977.

"Lose Any Uranium?" Critical Mass Journal 3(10):2, January 1978.

"Lose Your Uranium?" Critical Mass Journal 3(8):4, 1977.

Losswell, H. D. "The Structure and Function of Communication in Society." In Mass Communication, W. Schramin, ed. Urbana, IL: University of Illinois Press, 1949, pp 102-115.

Lovins, A. Soft Energy Paths: Toward a Durable Peace. Cambridge, MA: Ballinger Publishing Co., 1977.

Louisiana Board of Nuclear Energy, Division of Radiation Control. "Summary of RAM Incidents for the State of Louisiana, 1966-1977." Baton Rouge.

Louisiana Revised Statute Annotated. Baton Rouge.

Lowrance, W. W. Of Acceptable Risk. Los Altos, CA: William Kaufman, Inc., 1976.

Loy and Sazena. Processing and Packaging of Solid Wastes from BWR's, CONF-710801, August 1971 .

Ludwig, F. L., and W. F. Dabberdt. "Comparison of Two Practical Atmospheric Stability Classification Schemes in an Urban Application." Journal of Applied Meteorology 15:1172, 1976.

Lushbaugh, C. C., et al. "Medical Aspects of Nuclear Radiation Emergencies." Emergency, September 1978, pp 32ff.

Mabry, R. C., Jr. "Nuclear Theft: Real and Imagined Dangers." Master's thesis, Naval Postgraduate School, Monterey, CA, March 1976.

Macdonald, H. F., and J. H. Mairs. Individual and Collective Doses Associated With the Transport of Irradiated Magnox Fuel within the U. K., RD/B/N 4440 . United Kingdom: Central Electricity Generating Board Research Division, Berkeley Nuclear Laboratories, December 1978. 
Mack, R. W., and R. C. Snyder. "The Analysis of Social Conflict-Toward an Overview and Synthesis." Journal of Conflict Resolution 1:212-248, 1957.

Mack, R. W., and G. W. Baker. The Occasion Instant, Publication 945. Washington: National Academy of Sciences, National Research Council, 1961.

Malonowski, D. D., et al. Radiological Consequences of a Fuel Handling Accident, WCAP-7828, December 1971 .

Manion, W. J., T. S. LaGuardia, and P. Garrett. An Engineering Evaluation of Nuclear Power Reactor Decommissioning Alternatives: Summary Report. New York: Atomic Industrial Forum, Inc., National Environmental Studies Project, November 1976.

Manis, M. "Cognitive Social Psychology and Attitude Change." American Behavioral Scientist 21(5):675-690, 1978.

Manning, D. H. Disaster Technology: An Annotated Bibliography, London Technical Group, Institute of Biology. New York: Pergamon Press, 1976.

Manocha, K. K., and R. K. Mohindra. "Estimate of Natural Internal Radiation Dose to Man." Health Physics 30:487, 1976.

Margolis, J. "Charge Lax Guarding of Uranium at O'Hare." Chicago Tribune, 15 March 1977, p 1.

Marighella, C. "Excerpts from Minimanual of the Urban Guerrilla." Survival 13:95-100, March 1971.

Maring, G. E. "Pedestrian Travel Characteristics." Highway Research Record, vol. 406, 1972.

Marx, G. "Issueless Riots." In Collective Violence, J. F. Short, Jr., and M. E. Wolfgang, eds. Chicago: Aldine Publishers, 1972, pp 47-59.

Marx, G. and J. Wood. "Strands of Theory and Research in Collective Behavior." In Annual Review of Sociology, A. Inkeles, ed. Palo Alto, CA: Annual Reviews, Inc., 1975.

Marx, J. L. "Low-Level Radiation: Just How Bad Is It?" Science 204:160, 13 April 1979.

Marx, J. L. "Low-Level Radiation: A High-Level Concern." Science 204 (4389): 165-204, 1979.

Maryland Department of Health and Mental Hygiene, Environmental Health Administration, Division of Radiation Control. "Summary of RAM Incidents for the State of Maryland, 1970-1977." Baltimore.

Mason, R. E. Fission Gas Release (FGASRL), rev. ed., CDAP-TR-010. Idaho Falls, ID: Idaho National Engineering Laboratory, March 1978.

Massachusetts Annotated Laws. Boston. 
Massachusetts Public Interest Research Group. Nuclear Evacuation Planning: Blueprint for Chaos. Amherst, MA: MassPIRG, 6 July 1977.

Mathewson, J. H., and R. Brenner. "Indexes of Motor Vehicle Accident Likelihood." Highway Research Board Bulletin 161:1-8, 1957.

Maynard, W. S., et a1. Public Values Associated with Nuclear Waste Disposal, BNWL-1997/VC-70. Seattle: Battelle Human Affairs Research Centers, June 1976.

Mayneord, W. V., and R. H. Clarke. "Carcinogenesis and Radiation Risk: A Biomathematical Reconnaissance." British Journal of Radiology, Supplement No. 12, 1975.

Mays, C. W. "Estimated Risk from 239-Pu to Human Bone, Liver and Lung." In Biological and Environmental Effects of Low Level Radiation, vol. 2, IAEA-SM-202/806. International Atomic Energy Agency, 1976, pp 373-384.

Mays, C. W., et al. "Estimated Risk to Human Bone from Pu-239." In Biological Effects of Plutonium and Radium, W. S. S. Jee, ed. Salt Lake City: J. W. Press, 1976.

Mays, C. W., R. D. Lloyd, and J. H. Marshall. "Malignancy Risk to Humans from Total Body X-ray Irradiation." In Proceedings of the Third International Congress of the ICROP, CONF-74-0907. U.S. Atomic Energy Commission, 1974.

McCarthy, J. D., and M. N. Zald. The Trend of Social Movements in America: Professionalization and Resource Mobilization. Morristown, NJ: General Learning Press, 1973.

McCluggage, W. C. "The AEC Accident Record and Recent Changes in the AEC Manual." In Proceedings of the 3rd Internatural Symposium on Packaging Transport Radioative Materials, USDEL Rept. BNWL-SA-3906. Hanford, WA: Battelle Pacific Northwest Laboratories, 1971.

McConnaughey, W. E., et al. "Radioactive Wastes ... Hazardous Materials Transportation." Chemical Engineering Progress 66:57ff, 1970.

McCusker, K., G. Baecher, and J. Gvos. "Balancing Apples and Oranges: Methodologies for Siting," draft. Laxenburg, Austria: International Institute for Applied Systems Analysis, 1974.

McDonald, J. W. Relation between Number of Accidents and Traffic Volume at Divided Highway Intersections." Highway Research Board Bulletin 74:7-17, 1953.

McDowe11 v Schlesinger. 404 F. Supp. 221, 6 Envt1. L. Rep. 20 224; W.D. MO. 1975.

McEwan, A. C. "The Critical Organ for Tritium Gas." Health Physics Journal 23:742-744, 1972 .

McGrath, E. ed. Social and Psychological Factors in Stress. New York: Holt, Rinehart and Winston, 1970.

McHugh, P. Defining the Situation: the Organization of Meaning in Social Interaction. Indianapolis, IN: Bobbs-Merrill, Co., 1968 . 
McKeown, B. "Containers Seen Making N-Shipments Safe." [Chattanooga, TN] News-Free Press, 30 August 1978.

McPhail, C. "Civil Disorder Participation: a Critical Examination of Recent Resarch.: American Sociological Review 36:1058-1073, December 1971.

McPhail, C., and D. L. Miller. "The Assembling Process: a Theoretical and Empirical Examination." American Sociological Review 38:721-735, December 1971.

McPhee, J. The Curve of Binding Energy. New York: Farrar, Strauss, and Giroux, 1974 .

McPherson, W., ed. Ideology and Change. Palo Alto, CA: National Press Books, 1973.

McSweeney, T. I., and J. F. Johnson. Assessment of the Risk of Transporting Plutonium Dioxide by Cargo Aircraft, BNWL-2030. Richland, WA: Battelle Pacific Northwest Laboratories, June 1977.

McWhirter, M., et al. Preliminary Report on Special Tests of Plutonium Oxide Shipping Containers to FAA Flight Recorder Survivability Standards, R-422207. Albuquerque: Sandia Laboratories, 1975.

Medalia, N., and O. N. Larsen. "Diffusion and Belief in a Collective Delusion: the Seattle Windshield Pitting Epidemic." American Sociological Review 23:221-232, 1958.

Meerloo, A. M. Delusion and Mass Delusion, Nervous and Mental Disease Monographs. New York, 1949.

Meerloo, J. A. H. Patterns of Panic. New York: International University Press, 1950.

Mehner, A. S., and K. Vinjamuri. Fuel Rod Behavior during Test PCM-2, NUREG/CR-0647. Idaho Falls, ID: Idaho National Engineering Laboratory, April 1979.

Meier, A., ed. The Transformation of Activism. Chicago: Transaction Books, 1970.

Melber, B. D., et al. Nuclear Power and the Public: Analysis of Collected Survey Research, PNL-2430. Seattle: Battelle Human Affairs Research Centers, November 1977.

Mercer, T. T. "On the Role of Particle Size in the Dissolution of Lung Burdens." Health Physics 13:1211-1221, 1967.

Merton, R. K. "The Role Set: Problems in Sociological Theory." British Journal of Sociology 8:106-120, June 1957 .

Merton, R. K. Mass Persuasion. New York: Harper, 1946.

Meslin, T., and P. Pages. Evaluation of Transportation Safety of Radioactive Materials: Methodological Approach and Applications. Part 2: Risk

Evaluation, trans. Paris: Centre D'Etude Sur L'Evaluation de la Protection dans le Domaine Nucleaire, April 1978.

By -46 
Messenger and Fairbarn. The Transport of Radioactive Materials: Interim Recommendations for the Application of Environmental Tests to the Approval of Packaging, AHSB-S-R-19, 1963.

Meyer, W., et al. "The Homemade Nuclear Bomb Syndrome." Nuclear Safety $18(4): 427 \mathrm{ff}$, July-August 1967.

"Miami's Cruise Passenger Business." Nucleonics Week, 11 January 1979, p 11.

Michelson, W. H. Man and His Urban Environment: A Sociological Approach, 2nd ed. Reading, MA: Addison-Wesley, 1976.

Michigan Department of National Resources. Proceedings of the 1 st International Conference on Hazardous Materials Management, Detroit, 4-5 December 1978. Lansing, MI: Department of Natural Resources, 1979.

Mileti, D. S., T. E. Drabek, and J. E. Haas. Human System in Extreme Environments. Boulder, CO: University of Colorado Institute of Behavioral Science, 1975 .

Milgram, S., and H. Toch. Collective Behavior: Crowds and Social Movements. Vol. 4 of The Handbook of Social Psychology, G. Lindzey and E. Aionson, eds. Reading, MA: Addison-Wesley, 1969.

Milgram, S. "Some Conditions of Obedience and Disobedience to Authority." Human Relations 18:57-76, 1965.

Miller, A. K. "Zircaloy Cladding Deformation and Fracture Analysis, Report EPRI-NP-856 on research projects 700 and 971. Palo Alto, CA: Stanford University, August 1978.

Minnesota Statutes Annotated. St. Paul.

"Missing Uranium." Critical Mass Journal 3(3):2, 1977.

Mississippi Code Annotated. Jackson.

Mississippi State Board of Health. "Summary of RAM Incidents for the State of Mississippi, 1965-1977." Jackson.

Missouri Statutes Annotated. Jefferson City.

Mitche11, R. E. "The Nuclear Debate." Paper presented at the Annual Meeting of the American Association for the Advancement of Science, Washington, DC, February 1978 .

Modan, B., et al. "Radiation-Induced Head and Neck Tumors." Lancet $1: 277-279$, February 1974 .

Moeller, D. W. "The Advisory Committee on Reactor Safeguards." Health Physics 28:177-187, 1975.

Moeller, D. W., and J. M. Selby. "Planning for Nuclear Emergencies." Nuclear Safety 17(1), January-February 1976. 
Mohr, C. L. Transient Deformation Properties of Zircaloy for LOCA Simulation, vo1. 5, EPRI-UP-526. Richland, WA: Battelle Pacific Northwest

Laboratories, December 1978.

Mole, R. H. "The Biological Basis of Plutonium Safety Standards." Journal of the British Nuclear Energy Society 15:203-213, 1976.

Molotch, H., and M. Lester. "News as Purposive Behavior: On the Strategic Use of Routine Events, Accidents, and Scandals." American Sociological Review 39:101-12, February 1974 .

Montgomery, T. L., and D. J. Rose. "Some Institutional Problems of the US Nuclear Ind ustry." Technology Review, March/April 1979, pp 53ff.

Montana Revised Code Annotated. Helena.

deMontmollin, J. M., and T. A. Sellers. "A System for Communication with Commercial Special Nuclear Material Shipments." Nuclear Technology

23:117-123, August 1974.

Moore, H. E. "Toward a theory of Disaster." American Sociological Review $21: 733-737,1958$.

Moore, H. E., and H. J. Friedsam. "Reported Emotional Stress Following a Disaster." Social Forces 38:135-139, 1959.

Morgan, J. M., Jr., J. W. Knapp, and J. T. Thompson. A Study of the Possible Consequences and Costs of Accidents in the Transportation of High-Level Radioactive Materials, NY0-9772。 U.S. Atomic Energy Commission, 1961 .

Morgan, K. Z., and J. E. Turner, eds. Principles of Radiation Protection. Huntington, NY: Robert E. Krieger Publishing Co., 1973.

Morin, Donald A. "Application of Statistical Concepts to Accident Data." 'Highway Research Record, vol. 188, 1967.

Morrison, D. L., et al. An Evaluation of the Applicability of Existing Data to the Analytical Description of a Nuclear Reactor Accident, BMI-1779. Columbus, OH: Battelle Memorial Institute, August 1966.

Morrison, E. E. Men, Machines, and Modern Times. Cambridge, MA: MIT Press, 1966.

Morrison, P. A. "Dimensions of the Population Problem in the United States." In Population Distribution and Policy, vol. 5 of Commission Research Reports, U.S. Commission on Population and the American Future, Sara Miles Mazie, ed. Washington: U. S. Government Printing Office, 1972.

Moses, H. Mathematical Ürban Air Pollution Models, ANL/ES-RPY-1. Argonne, IL: Argonne National Laboratory, 1969.

"Most of Uranium Spill Cleaned Up." The Wichita Eagle-Beacon, 24 March 1979. 
Motor Vehicle Manufacturer's Association of the United States, Inc. Motor Vehicle Facts and Figures 1977.

Mulinazzi, T. E., and H. L. Michael. "Correlation of Design Characteristics and Operational Controls with Accident Rates on Urban Arterials." In Proceedings of the 53rd Annual Road School of Purdue University, Lafayette, IN, 1967, pp 150-173.

Murphey, A. W., and D. B. LaPierre. Nuclear "Moratorium" Legislation in the States and the Supremacy Clause: a Case of Express Preemption. New York: Atomic Industrial Forum, November 1975.

Murphy, C. E., Jr., and M. M. Pendergast. Environmental Transport and Cycling of Tritium in the Vicinty of Atmospheric Releases, DP-MS-78-25. Aiken, SC: E. I. DuPont de Nemours and Co. for Savannah River Laboratory, 1978.

Myers, D. G., and H. Lamm. "The Group Polarization Phenomenon." Psychological Bulletin 83:602-627, 1976.

Myers, R. C. "Anti-Communist Mob Action: A Case Study." Public Opinion Quarterly $12: 57-67,1948$.

Myers, R. H. Response Surface Methodology. Boston: Allyn and Bacon, Inc., 1971.

Nakamura, S., ed. An Annotated Bibliography: Social and Economic Factors Associated with Electric Power Generation 1978. Washington: Inforum, May 1978 .

Nardine, T. "Theories of Conflict Management." Peace Research Reviews 4:1-87, April 1971.

Narron, D. "Georgia Officials Coordinate Cleanup at Hazardous Truck Crash Site." [Chattanooga, TN] News-Free Press, 30 August 1978.

Narron, D. "No Contamination From I-24 Wreck." [Chattanooga, TN] News-Free Press, 30 January 1979.

National Academy of Sciences, Division of Medical Sciences, Advisory Committee on the Biological Effects of Ionizing Radiation. The Effects on Population of Exposure to Low Levels of Ionizing Radiation. Washington: NAS, November 1972.

National Academy of Sciences, National Research Council, Advisory Committee On The Biological Effects of Ionizing Radiation (BEIR). The Effects on Populations of Exposure to Low Levels of Ionizing Radiation. Washington: NAS, 1972.

National Academy of Sciences, National Research Council, Disaster Research Group. Field Studies of Disaster Behavior: An Inventory, Disaster Study No. 14. Washington: NAS, 1961 . 
National Academy of Sciences, National Research Council, Highway Research Board. Highway Capacity Manual 1965, Highway Research Board Special Report 87. NAS Publication 1328. Washington: NAS, 1965.

National Academy of Sciences, National Research Council, Highway Research Board. Improved Street Utilization Through Traffic Engineering, Special Report 93; proceedings of a conference, 22-24 May 1967. Washington: NAS, 1967.

National Academy of Sciences, National Research Council, Highway Research Board. "Traffic Accident Research." Highway Research Record 188, 1967.

National Academy of Sciences, National Research Council, Highway Research Board. "Traffic Speed and Volume Measurements." Highway Research Board Bulletin 156, 1957.

National Academy of Sciences, National Research Council. Review of Criteria

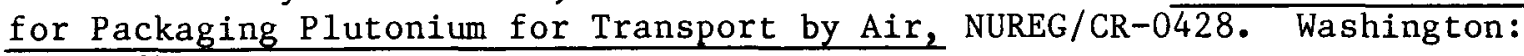
NAS, 1978.

National Academy of Sciences. Principles for Evaluating Chemicals in the Environment. Washington: NAS, 1975.

National Academy of Sciences. Radioactive Wastes at the Hanford Reservation, C00-2708T004-1. Washington: NAS, 1978.

National Academy of Sciences. "Risks Associated with Nuclear Power." Nuclear Safety $20(6): 65 f f$, November-December, 1979 .

National Academy of Sciences. Risks Associated with Nuclear Power: A Critical Review of the Literature, summary and synthesis chapter. Washington: NAS, April 1979.

National Academy of Sciences. Transportation of High-Level Nuclear Wastes, ERDA-8. Washington: NAS, November 1974 .

National Advisory Commission On Civil Disorders. "Patterns of Disorder." Chap. 2 of the Report of NACCD (the Kerner Report). Washington: Government Printing Office, 1968. The Kerner Report was also published in 1968 by Bantam Books, New York.

National Bureau of Standards. Maximum Permissible Body Burdens and Maximum Permissible Concentrations of Radionuclides in Air and in Water for Occupational Exposure, Handbook 69. Frederick, MD: NBS, 1963.

National Committee On Urban Transport. Determining Street Use, Procedure Manua1 1a. Chicago: Chicago Public Administration Service, 1958.

National Council On Radiation Protection and Measurements. Alpha Emitting Particles in the Lung, NCRP Report 46. Washington: NCRP, August 1975.

National Council On Radiation Protection And Measurements. Basic Radiation Protection Criteria, NCRP Report 39, 15 January 1971. 
National Council on Radiation Protection and Measurement. Cesium-137 From the Environment to Man: Metabolism and Dose, NCRP Report 52. Washington: NCRP 1977.

National Council on Radiation Protection and Measurements. Exposure to Radiation in an Emergency, NCRP Report 29. Washington: NCRP, January 1962.

National Council on Radiation Protection and Measurements. Precautions in the Management of Patients Who Have Received Therapeutic Amounts of Radionuclides, NCRP Report 37. Washington: NCRP, 1970.

National Council on Radiation Protection and Measurements. Protection of the Thyroid Gland in the Event of Releases of Radioiodine, NCRP Report 55. Washington: NCRP, 1 August 1977.

National Fire Protection Association. Fire Protection Guide on Hazardous Materials. Boston: NFPA, 1972.

National Fire Protection Association. Fire Protection Handbook, 13th ed. Boston: NFPA, 1969.

National Safety Council. Accident Facts 1974-1978. Chicago: NSC, 1978.

National Safety Council. Accident Facts, 1979 ed. Chicago: NSC.

National Safety Council. Fleet Accident Rates, 1979 ed. Chicago: NSC.

National Transportation Safety Board, Bureau of Surface Transportation Safety. Special Study: Broken Rails--A Major Cause of Train Accidents, NTSB-RSS-741. Washington: NTSB, 2 January 1974.

National Transportation Safety Board. Special Study of the Carriage of Radioactive Materials by Air, NTSB-AAS-72-4. Washington: NTSB, April 1972 .

NATO Committee on the Challenges of Modern Society. Proceedings of the 5th Annual Meeting of the Expert Panel on Air Pollution Modeling, NTIS No. PB-240-582. Springfield, VA, August 1974 .

Nealey, S. M., et al. A Comparative Analysis of Print Media Coverage of Nuclear Power and Coal Issues, B-HARC-411-006. Seattle: Battelle Human Affairs Research Centers, October 1978.

Nealey, S. M., and L. M. Radford. Public Policy Issues in Nuclear Waste Management, B-HARC-411-003. Seattle: Battelle Human Affairs Research Centers, October 1978.

Nealey, S. M., and W. L. Rankin. Nuclear Knowledge and Nuclear Attitudes: Is Ignorance Bliss?, B-HARC-411-002. Seattle: Battelle Human Affairs Research Centers, October 1978.

Nebraska Department of Health, Division of Radiological Health. "Summary of RAM Incidents for the State of Nebraska, 1966-1977." Lincoln. 
Nebraska Department of Health. Radiological Emergency Response Plan, NRH-27. Lincoln, NB, June 1979.

Neff, T. L., and H. D. Jacoby. Nuclear Fuel Assurance: Origins, Trends, and Policy, MIT-EL-79-003. Cambridge, MA: M.I.T. Press, February 1979.

Nelkin, D. Nuclear Power and Its Critics. Ithaca, NY: Cornell University Press, 1971 .

Ness, M. P., J. F. Morral1, and B. G. Hutchinson. "An Analysis of Central Business District Pedestrian Circulation Patterns." Highway Research Record, vol. 283, 1969.

Neuweg, M. "Spent Fuel Transportation Accident--A States Involvement." Springfield, IL: Illinois Department of Public Health, nd.

Nevada Department of Human Resources, Division of Radiological Health, Bureau of Consumer Health Protection Services. "Summary of RAM Incidents for the State of Nevada, 1972-1977." Carson City.

New Hampshire Radiation Control Agency. "Summary of RAM Incidents for the State of New Hampshire, 1966-1977." Concord.

New Jersey Revised Statutes Annotated. Trenton.

New Mexico Radiation Protection Section. Radiological Emergency Response Plan. Santa Fe, 24 January 1979.

New York State Energy Research and Development Authority. Characterization of Selected Low-Level Radioactive Waste Generated by Four Commercial (etc). PB-295360. Albany, December 1977.

Newton, G. N., et al. "The Effect of Particle Size on the In Vitro Phagocytosis of Monodisperse Particles by the Alveolar Macrophage." In The Lovelace Foundation Annual Report. Albuquerque: The Lovelace Foundation, 1972 .

New York City Building Code, Local Law No. 76, effective December 1968. Includes amendments to 10 June 1974. Al so Supplement No. 1, amended to 4 July 1976.

New York City Health Code.

New York City Planning Commission. Community Planning District Profiles, September 1974.

New York City Planning Commission. Plan for New York City, 6 vols. Cambridge, MA: M.I.T. Press, 1969.

New York State Department of Health and Division of Military and Naval Affairs. New York State Emergency Plan for Radiation Accidents. Albany, September 1977 . 
New York Transportation Law. Albany.

"N-Fuel Shipment Raises Question of Route Safety." The Charlotte [NC] Observer, 5 February 1979. p B1.

Nichols, B. D., and C. W. Hirt. Transient Three-Dimensional Fluids Flow in the Vicinity of Large Structures, LA-DC-72-711. Los Alamos, NM: Los Alamos Scientific Laboratory, 1972.

Nicholson, S. E. "A Pollution Model for Street-Level Air." Atmospheric Environment 9:13-31, 1975.

Niebing, H. L. "Agonistics-Ritual of Conflict." In Collective Violence, J. F. Short and M. E. Wolfgang, eds. Chicago: Aldine Publishers, 1972, pp 82-99.

Niemeyer, R. G. Source Application Guide on the ORNL Source Capsule Classification System, ORNL-4427. Oak Ridge, TN: Oak Ridge Nationa1 Laboratory, July 1969 .

Niemeyer, R. G. Leak Testing Encapsulated Radioactive Sources, ORNL-4529. Oak Ridge, TN: Oak Ridge National Laboatory, July 1972.

Nieto, J. M., S.J. Nathan, and B. N. Naft. Determination of Acceptable Site Specific Shipment Frequency (SSSF) of Hazardous Chemical Shipments Past Nuclear Power Plant Sites. Rockville, MD: NUS Corporation, 1978.

Nimmo, D., and R. L. Savage. Candidates and their Images: Concepts, Methods and Findings. Pacific Palisades, CA: Goodyear Publishing, 1976.

Nix, H. L. The Community and Its Involvement in the Study Planning Action Process, HEW Publication No. (CDC) 78-8355. Washington: U. S. Government Printing office, 1977.

Noelle-Neuman, E. "Mass Communciation, Media and Public Opinion." Journalism Quarterly 36:401-409, 1959.

"No Place for Nuclear Waste." Newtown [CT] Bee, 12 March 1976.

North Carolina Department of Human Resources, Radiation Protection Section. Contingency Plan: North Carolina Emergency Response. Raleigh, January 1976; revised March 1978.

North Carolina General Statutes. Raleigh.

Northern States Power. Co. v Minnesota. 447 F.2d 1143, 1 Envt'1 L. Rep. 20451, 20454; 8th Cir. 197I.

Norwood, W. D. Health Protection of Radiation Workers. Springfield, IL: Charles C. Thomas, 1975.

"A Framework for Preemption Analysis," note. Yale Law Journal 88:363-389, December 1978. 
Nowotny, H. "Social Aspects of the Nuclear Power Controversy," Research Memorandum RM-76-33. Laxenburg, Austria: International Institute for Applied Systems Analysis 236, April 1976.

Nuclear Assurance Corporation. "Briefing Paper for Atlanta City Council Members on Radioactive Materials Transportation." Atlanta: NAC, 6 February 1979.

"Nuclear Cargo in NC." Critical Mass Journal 3(4):6, 1977.

Nuclear Energy Policy Study Group. Nuclear Power Issues and Choices. Cambridge, MA: Ballinger, 1977.

"Nuclear Freight Shipped at 0'Hare." Chicago Tribune, 27 December 1977, sec. 2, p 1 .

Nuclear Regulatory Commission. Manual of Respiratory Protection against Airborne Radioactive Materials, PB-258052, NUREG-0041. USNRC, October 1979.

"Nuclear Shipments Banned by Miami's Port." Nucleonics Week, 11 January 1979, pp 11-12.

"Nuclear Shipments: 'Leave the Driving to Us?'" People and Energy, vol. 4, Nos. 8-10, 1978.

Nuclear Transportation Group (ad hoc group of 29 utilities). "State and Local Restrictions on Transportation of Some or All Radioactive Materials."

A list provided by John M. Buchhait, Yankee Atomic Electric Co., Westborough, MA, 6 July 1979.

"Nuclear Trips Rerouted." The New Haven Register, 10 March 1976.

"Nuclear Waste Shipment Bill Hit by Energy Chief." The Bridgeport [CT]

Telegram, 3 April 1976.

Nuclear Waste Transportation Safety Act of 1979, S. 535. 96th Congress, 1st Session, 1979.

Nucleonics Week, 30 August 1979, pp 5-6.

Nunez, M., and T. R. Oke. "The Energy Balance of an Urban Canyon." Journal of Meterology 16:11, 1977 .

Oak Ridge National Laboratory. Comparison of the Socoeconomic Impacts of International Fuel Service Centers versus Dispersed Nuclear Facilities, CONF-790445-4. Oak Ridge, TN: ORNL, 1979.

Oak Ridge National Laboratory. Siting of Fuel Reprocessing Plants and Waste Management Facilities, PNL-4451. Oak Ridge, TN: ORNL July 1970.

Oberschall, A. Social Conflict and Social Movements. Englewood Cliffs, NJ: Prentice-Ha11, 1973. 
0'Brien, J. J. "A Note on the Vertical Structure of the Eddy Exchange Coefficient in the Planetary Boundary Layer." Journal of the Atmospheric Sciences 27:1213, 1970 .

Odaira, T., et al. Using Wind Tunnel Experiments to Forecast Automobile Exhaust Gas Concentrations along Streets, NTIS No. N74-15335. Springfield, VA: National Technical Information Service, 1973.

Office of Civil Defense Mobilization. A Brief Review of Salient Specific Findings on Moral and Human Behavior Under Disaster Conditions, OCDM BC 31196. Battle Creek, MI: OCDM, 19 April 1958.

Oglesby, J. "FPL Nuclear Waste Secretely Shipped to Nevada." Miami Herald, 7 January 1979.

Oklahoma Department of Health. Natural Disaster Plan for the State of Oklahoma, 1975.

Okrent, D., and C. Whipple. An Approach to Societal Risk Acceptance Criteria and Risk Management, UCLA-ENG-7746. Los Angeles: University of California, Los Angeles, School of Engineering and Applied Science.

01son, C. A., M. H. Hall, and H. J. Plagge. Wind Data for the Albuquerque Area, SC-M-70-144. Albuquerque: Sandia Laboratories, May 1970.

01son, M. E. "A Model of Political Participation Stratification." Journal of Political and Military Sociology 1:183-200, Fall 1973.

"One Truck Crash Could Kill a City." Seattle Post-Intelligencer, 11 February 1979.

Oregon State Board of Health, Radiation Control Section. "Summary of RAM Incidents for the State of Oregon not reported to NRC, 1977." Portland.

Oregon Revised Statutes. Salem.

Orol, A. M. Nuclear Industry and the Environment: Some Legal Aspects. ANL-Trans-1143. Buenos Aires: Comision Nacional de Energia Atomica, September 1978. Translated by Argonne National Laboratory, Argonne, IL.

Osgood, C., and P. Tannenbaum. "The Principle of Congruity in the Prediction of Attitude Change." Psychological Review 62:42-55, 1955.

Otway, H. J., and M. Fishbein. The Determinants of Attitude Formation: An Application to Nuclear Power, RM-76-80. Laxenburg, Austria: Internatina1 Institute for Applied Systems Analysis, December 1976.

Otway, H. J., and M. Fishbein. Public Attitudes and Decision Making, RM-77-54. Laxenburg, Austria: International Institute for Applied Systems Analysis, November 1977. 
Otway, H. J. "Review of Research on Identification of Factors Influencing Social Response to Technological Risks." In Nuclear Power and its Fuel Cycle, IAEA-CN-36/4. Proceedings of an International Conference, Salzburg, 2-13 May 1977, vol. 7, pp 95ff.

Otway, H. J., and P. D. Pahner. "Risk Assessment." Futures, April 1976, pp 122-134.

Otway, H. J. Risk Assessment and Societal Choices, RM-75-2. Laxenburg, Austria: International Institute for Applied Systems Analysis, February 1975.

Otway, H. J. et al. "A Risk Estimate for an Urban Sited Reactors." Nuclear Technology 12:173-187, October 1971 .

Otway, H. J., P. D. Pahner, and J. Linnerooth. Social Values in Risk Assessment, RM-75-54. Laxenburg, Austria: International Institute for Applied Systems Analysis, 1975.

Ovcharenko, E. P. "An Experimental Evaluation of the Effects of Transuranic Elements on Reproductive Ability." Health Physics 22:641, 1972.

Owen, W. Transportation for Cities. Washington, DC: The Brookings Institution, 1976.

Pacific Legal Foundation et al v State Energy Resources Conservation and Development Commission, 2 C.C.H. Nuclear Regulation Reporter, para. 20, 109; D.S.Cal 1979 .

Packaging and Transportation of Radioactive Materials: Proceedings of the 5th International Symposium, Las Vegas, 1978.

Paer, P. D. A Psychological Perspective of the Nuclear Energy Controversy, RM-76-67. Laxenburg, Austria: International Institute for Applied Systems Analysis 2361, August 1976.

Paer, P.D. The Psychological Displacement of Anxiety: An Application to Nuclear Energy. Laxenburg, Austria: International Instiutute for Applied Systems Analysis, 1975.

Parker, G. W., et al. Out-of Pile Studies of Fission Product Release from Overheated Reactor Fuels at ORNL, 1955-1965, ORNL-3981. Oak Ridge, TN: Oak Ridge National Laboratory, July 1967.

Parsont, M. A., W. L. Holley, and W. D. Burnett. "The Effect of Particle Size on Organ Distribution of Radioactive Material Deposited in the Lungs." Health Physics 22:143, 1972.

Pasquil1, F. Atmospheric Diffusion. New York: John Wiley and Sons, 1974.

Pasquil1, F. "The Estimation of the Dispersion of Windborne Material." The Meteorological Magazine 90(1063):33-49, 1961. 
Patterson, D. E., and V. P. De Fatta. A Summary of Incidents Involving USAEC Shipments of Radioactive Materia1, 1957-1961, TID 16764. Springfield, VA: National Technical Information Service, 1962.

Patterson, D. E., and A. Mehn. A Summary of Incidents Involving USAEC Shipments of Radioactive Material, 1962, Supplement 1 to TID 16764. Springfield, VA: National Technical Information Service, 1963.

Patty, F. A. Industrial Hygeine and Toxicology, 2 vols. I. General Principles, 1958. II. Toxicology, 1963. New York: Interscience Publishers.

Pearson, J. S. Sociological Analysis of the Reduction of Hazardous Radiation in Uranium Mines, NIOSH-75-171. Denver: University of Denver, April 1975.

Pennsylvania Department of Environmental Resources, Bureau of Radiological Health. Transportation of Radioactive Material in Pennsylvania, NUREG/CR-0286, July 1978.

Pennsylvania Environmental Research Foundation, Inc. The Hazardous Waste Management Directory, B34318-001A. Philadelphia, $19 \overline{79 .}$

Pennsylvania Statutes Annotated. Harrisburg.

Perchonok, K. The Determination of Environmental Factors Controlling Driver Behavior, Report No. 3. State College, PA: Division of Highway Studies, Institute for Research, 1964.

Perchonok, K. The Measurement of Driver Errors, Report No. 1. State College, PA: Division of Highway Studies, Institute for Reasearch, 1964.

Perchonok, K., and P. M. Hurst. The Effect of Lane Closure Signals Upon Driver Decision-Making and Traffic Flow, Report No. 8. State College, PA: Division of Highway Studies, Institute for Research, 1965.

Perchonok, K., and E. L. Seguin. Vehicle Following Behavior: A Field Study, Report No. 5. State College, PA: Division Highway Studies, Institute for Research, 1964.

Perloff, H. S. The Quality of the Urban Environment. Baltimore: Johns Hopkins Press, 1969.

Perry, R. H. Engineering Manual. New York: McGraw Hill, 1967.

Perry, R. W., and M. K. Lindell. "The Psychological Consequences of Natural Disaster: A Review of Research on American Communities." Mass Emergencies 3:105-115, 1978 .

Pervin, L. A. "The Need to Predict and Control under Conditions of Threat." Journal of Personality 31:570-587, 1963.

Peterson, K. R., T. V. Crawford, and L. A. Dawson. CPS: A Continuous-Point Source Computer Code for Plume Dispersion and Deposition Calculations, UCRL-52049. Livermore, CA: Lawrence Livermore Laboratory, 1976. 
Peterson, W. A., and N. P. Gist. "Rumor and Public Opinion." American Journal of Sociology 57:159-167, 1951 .

Petit, C., M. Trinte, and P. Valentin. "Study of Turbulent Diffusion above and within a Forest." Atmospheric Environment 10:1057, 1976.

Phillips, J. W., et al. An Analysis of Low-Level Solid Radioactive Waste from LWRs through 1975, $\mathrm{PB}-2 \overline{95-408 . ~ W a s h i n g t o n: ~ O f ~ f i c e ~ o f ~ R a d i a t i o n ~ P r o t e c t i o n ~}$ Programs, November 1977.

Pike, E. A. Protection Against Bombs and Incendaries for Business, Industrial, and Educational Institutions. Springfield, IL: C. C. Thomas, 1972.

Plackett, R. L., and J. P. Burman. "The Design of Optimum Mulitfactorial Experiments." Biometrika 33:305, 1943-1946.

"Plane With Radioactive Matter Crashes." Washington Post, 23 January 1978, p C3, col. 1 .

Plate, E. J. Aerodynamic Characteristics of Atmospheric Boundary Layers. U.S. Atomic Energy Commission Critical Review Series, May 1971.

Platt, F. N. "Operations Analysis of Traffic Safety": Part 1: "A Classification of Traffic Situations."6(2):8, 1958. Part 2: "A Mathematical Approach to Traffic Situations." 6(4):23, 1958. Part 3: "Methods of Evaluating Traffic Situations." 7(3):11, 1959. International Road Safety and Traffic Review.

Pneumodynamics Corporation. Sea Disposal Container Test and Evaluation, TID-13226. Springfield, VA: National Technical Information Service, 15 June 1961 .

Pochin, E. E. "Problems Involved in Detecting Increased Malignancy Rates in Areas of High Natural Radiation Background." Health Physics 31:148-151, 1976.

Poda, G. A., and R. M. Hall. "Two Californium-252 Inhalation Cases." Health Physics 29:407-409, 1975.

Policy Research Association. Socioeconomic Impacts: Nuclear Power Station Siting, NUREG-0150, June $197 \overline{7}$.

Pollock, R. "Citizens Can Expose Plan Flaws--Citizens Guide to Evacuations." Critical Mass Journal 3(11):1, 1978.

Pollock, R. "Flying the Friendly Skies at O'Hare." Critical Mass Journal $3(10): 1,1978$.

Pollock, R., Director, Critical Mass Energy Project. "Transporation of Radioactive Materials Routing Recommendations." Statement before the U.S. Department of Transportation, 28 November 1978. 
Pollock, R. "Nuclear Emergency Plans Fail in Most States." Critical Mass Journal 3(11):1, 1978 .

Pollock, R. "Ownership of the Atomic Energy Industry." Critical Mass Journal, August 1977, pp 1-6.

Pollock, R. "The Rains of October." Critical Mass Journal, August 1976, p 9.

Pollock, R. "Robin Hood and Radioactive Waste." Critical Mass Journal $3(4): 3,1977$.

Pollock, R. "They 'Muffed' It: Uncle Sam Loses Material." Critical Mass Journa1 3(7):8, 1977 .

Pollock R. "Truck Accident Spills Radioactive Uranium." Critical Mass Journal 3(9):1, 1977 .

Pollock R. "You Can Be Sure if It's Westinghouse." Critical Mass Journal August 1976, pp 1-8.

Pollock, W. E., and W. M. Rogers, Jr., eds. Transportation of Radioactive Materials in the Western States. Western Interstate Nuclear Board, March 1974.

Polzer, W. L., et al. Characteristics of Wastes and Soils which Affect Transport of Radionuclides through the Soil and Their Relationship to Waste Management, NUREG/CR-0842. Los Alamos, NM: Los Alamos Scientific Laboratory, April 1979.

"Preemption Doctrine in the Environmental Context: A Unified Method of Analysis," comment. University of Pennsylvania Law Review 127:197-232, November 1978 .

"Public Hearing Granted on Nuclear Transport Plan." [Anderson, SC] Daily Mail, 14 November 1978.

Program Report, vol 3, No. 8. Conference on Transportation for the Nuclear Industry, Minneapolis, 1976. Washington: Atomic Industrial Forum, February 1977.

Purington, R. G., and H. W. Patterson. Handling Radiation Emergencies. Boston, MA: National Fire Protection Association, 1977.

Pushkarev, B. and M. Zupan. "Pedestrian Travel Demand." Highway Research Record 355, 1971.

Pushkarev, B. and M. Zupan. Urban Space for Pedestrians. Cambridge, MA: M.I.T. Press, 1975.

Quarantelli, E. L. "Emergent Accommodation Groups: Beyond Collective Behavior Typologies." In Human Nature and Collective Behavior, T. Shibutani, ed. Edglewood Cliffs, NJ: Prentice-Ha11, Inc., 1970, pp 111-123. 
Quarantelli, E. L. "A Note on the Protective Function of the Family in

Disasters." Marriage and Family Living 22:263-264, 1960.

Quarantelli, E. L. "A Study of Panic: Its Nature, Types and Conditions."

National Opinion Research Center Survey 308. Chicago, 1953.

Quarantelli, E. L., and R. R. Dynes. "Response to Social Crisis and

Disaster." Annual Review of Sociology 3:23-49, 197.7.

Quarantelli, E. L., and R. R. Dynes. "When Disaster Strikes, It Isn't Much

Like What You've Heard and Read About." Psychology Today 5:66-70, February

1972.

Quarantelli, E. L. and J. R. Hundley, Jr. "A Test of Some Propositions about Crowd Formation and Behavior." In Readings in Collective Behavior, R.R. Evans, ed., 1st ed. Chicago: Rand McNa11y, 1969, pp 538-554.

Quarantelli, E. L., et al. "Initial Findings from a Study of Socio-behavioral Preparations and Planning for Acute Chemical Hazard Disasters." Journal of Hazardous Materials 3(1):77-90, 1979.

Quinn, J. L., III M.D. The American College of Radiology Survey on

Regionalization in Nuclear Medicine. ACR Commission on Nuclear Medicine, March 1975.

"Radiation Feared in Nevada Fire." Seattle Post-Intelligencer, 15 May 1979; p Al, col. 5 .

"Radioactive Cargo." Critical Mass Journal 3(7):11, 1977.

"Radioactive Roadway." Critical Mass Journal 3(10):2, 1978.

"Radioactive Shipment Bill Before Board of Aldermen." St. Louis Post Dispatch, 20 April 1979.

"Radioactive Shipments on City Agenda." Wichita Eagle-Beacon, 16 April 1979.

"Radioactive Spills Here Termed Likely." St. Louis Post-Dispatch, 19 January 1979.

"Radioactive Transport." Critical Mass Journal 3(12):2, 1978.

"Radioactive Transport Bill Is Proposed." St. Louis Post-Dispatch, 23 January 1979.

"Radioactive Waste Disposal Transportation." (24 citations from an online search of NTIS data base, March 1977.)

Radiological Emergency Response Plan for Rocky Flats. np, February 1979.

Ragland, K. W. and J. J. Peirce. "Boundary Layer Model for Air Pollutant Concentrations Due to Highway Traffic." Journal of the Air Pollution Control

Association 25:48-51, 1975. 
Raglund, E. Nuclear Cargo in North Carolina: What are the Risks? Durham, NC: North Carolina Public Interest Research Group, May 1977.

"Railroads and Utilities Clash Over Nuclear Waste Transport." Energy Daily. December 1978, p 3.

Randall, N. "Envisioning How We Might Suffer in Two Kinds of Nuclear Attacks." Washington Post, 29 January 1978, p C4, col. 10.

Rankin, W. L. "Recent Survey Findings Regarding Public Attitudes Toward Nuclear Power." Presented at 25th Annual Meeting of the American Nuclear Society, Atlanta, GA, June 1979.

Rankin, W. L., and S. M. Nealey. A Comparative Analysis of Network Television News Coverage of Nuclear Power, Coal and Solar Stories, B-HARC-411-005. Seattle: Battelle Human Affairs Research Centers, February 1979.

Rankin, W. L. and S. M. Nealey. The Relationship of Human Values and Energy Beliefs to Nuclear Power Attitude, B-HARC-411-007. Seattle: Battelle Human Affairs Research Centers, November 1978.

Ray v Atlantic Richfield Co. 435 U.S. 151, 158; 1978.

Reed, J. W. Wind Power Climatology of the United States, SAND74-0348. Albuquerque: Sandia Laboratories, August 1977.

Reed, R. Design Concepts Study of a Special Nuclear Material Cargo Vehicle, SLA-73-0930. Albuquerque: Sandia Laboratories, 1973.

Reed, R. E. "Design Concepts Study of a Special Nuclear Material Cargo Vehicle." Nuclear Technology 23:112-116, August 1974.

Region VIII and Western Interstate Nuclear Board, Regional Training Committee. Guide and Example Plan for Development of State Emergency Response Plans and Systems for Transportation-Related Radiation Incidents. np, April 1975.

Reilly, E. F., and J. Seifert. "Capacity of Signalized Intersections." Highway Research Record 321, 1970.

Reinig, W. C., ed. Environment Surveillance in the Vicinity of Nuclear Facilities: Proceedings of a Symposium. Springfield, IL: C. C. Thomas, 1970 .

Reinking, A. G., and V. E. Schrock. "Spent Fuel Transporation Risks." Nuclear News, November 1972, pp 71-75.

"Rep. Weiss Opposes Plan to Haul A-Wastes in N.Y.C." Washington Post, 25 September 977, p A3, col. 1 .

Rhoads, R. E. An Overview of Transportation in the Nuclear Fuel Cycle, BNWL-2066. Richland, WA: Battelle Pacific Northwest Laboratories, May 1977.

Rhoads, R. E. and J. F. Johnson. Risks in Transporting Materials for Various Energy Industries, BNWL-SA-6547. Richland, WA: Battelle Pacific Northwest Laboratories, 1978. 
Rhoads, R. E., and J. F. Johnson. "Risks in Transporting Materials for Various Energy Industries." Nuclear Safety 19(2):135-149, 1978.

Rhode Island General Laws. Providence.

Rhyne, W. R., et al. Spent Fuel Cask Transportation Accidents, An Assessment of Existing Information--Interim Report, SAI-0R-78-140-01. Oak Ridge, TN: Science Applications, Inc., 13 December 1978.

Rhyne, W. R., et al. A Scoping Study of Spent Fuel Cask Transporations Accidents, NUREG/CR-0811, SAI-0R-79-140-04. Oak Ridge, TN: Science Applications, Inc., 14 June 1979.

Richmond, C. R. "Current States of the Plutonium Hot Particle Problems," CONF-751105-17. Oak Ridge, TN: Oak Ridge National Laboratory, 1975.

Rinne, R. L. The Evaluation of Safeguards Systems for Nuclear Material in Transit--The Development of the Program Plan, SAND77-8249. Livermore, CA: Sandia Laboratories, July 1977.

Ritzman, R. L., and D. L. Morrison. FRCRL2-A Computer Code for Calculating Fission Product Release in Reactor Accident Analysis, BMI-1913. Columbus, OH: Battelle Memorial Institute, August 1971.

Rizzo, M. J. "The Effect of Crime on Residential Rents and Property Values." American Economist 23:16-21, 1979.

Robinson, J. P. and P. E. Converse. Summary of U.S. Time Use Survey. Ann Arbor, MI: University of Michigan Institute for Social Research, 1966.

Robinson, R. A. "AEC-ORNL Source Safety Testing Program." Isotopes and Radiation Technology 4(3):281, 1967.

Robinson, R. A., et al. Experimental Studies of Dynamic Impact Response with Scale Models of Lead-Shielded Radioactive Material Shipping Containers, Topical Report BMI-2001. Columbus, OH: Battelle Columbus Laboratories, September 1978.

Robinson, R. A. "Radioisotope Source Engineering for Radiation and Thermal App1ications." Trans. Am. Nuc. Soc. 14(1):51, June 1971.

Robinson, R. A. "Safety Aspects of 137-Cs Gamma Radiation Sources." Trans. Am. Nuc. Soc. 15(2):62-63, November 1972 .

Robnett, M. and A. Wilson, Coalition for the Environment, St. Louis; letter, 19 January 1979.

Rockwell, T. Reactor Shielding Design Manual. Cincinnati: Van Nostrand Co., Inc., 1956.

Rogers, R. F. "Spent-Fuel Receiver's Point of View and Experience." In CONF-6810-01. Oak Ridge, TN: Oak Ridge National Laboratory, October 1968. 
APPENDIX L

Social Impacts

\section{Christopher Cluett \\ Majorie Greene \\ Frederic Morris \\ William Rankin \\ Carin Weiss}

Battelle Human Affairs Research Centers

Seattle, Washington 
Rohwer, R. S., and W. H. Wilcox. "Radiological Effects of Environmental Tritium." Nuclear Safety 17(2), March-April 1976.

Rokeach, M. Beliefs, Attitudes, and Values. San Francisco: Jossey-Bass, 1968.

Rokeach, M. The Nature of Human Values. New York: The Free Press, 1973.

Rollins, J. "Transportation of Irradiated Fuels." In Nuclear Power Safety, J. H. Rust and L. E. Weaver, eds. Elmsford, NY: Pergamon Press, 1976, pp 359-380.

Romander, C. M., and J. D. Colton. Mechanics of a Highway Accident at Wichita, Kansas, Involving Natural Uranium Concentrate, Final Report, NUREG/CR-0992. 29 April 1979 - 15 August 1979.

Romney, E. M., and J. J. Davis. "Ecological Aspects of Plutonium Dissemination in Terrestrial Environments." Health Physics 22:551-557, 1972.

Rosengren, K. E., P. Arvidson, and D. Sturesson. "The Barseback Panic: A Radio Programme as a Negative Summary Event." Acta Sociologica 18:303-321, 1975.

Rosenthal, B. "Radiation Causes Evacuation at Colorado Nuclear Reactor." Critical Mass Journal 3(12): 14, 1978.

Rosenthal, B. "Radioactive Waste Disposal Problems Mount." Critical Mass Journal, August 1976, pl.

Rosnow, R. L., and G. A. Fine. Rumor and Gossip: The Sociological Psychology of Hearsay. New York: Elsevier Publishing, 1976.

Ross, M. "The Possibility of Release of Cesium in a Spent-Fuel Transporation Accident." In The Nuclear Fuel Cycle, A Survey of Public Health, Environmental and National Security Effects of Nuclear Power, Union of Concerned Scientists. Cambridge, MA: M.I.T. Press, 1975.

Rowe, W. D. An Anatomy of Risk. New York: John Wiley and Sons, 1977.

Rowland, T. C. U0 Irradiation for ORNL, GEAP-5642, June 1968.

Rudy, B. M. "Operational Route Analysis." Highway Research Board Bulletiri $341: 1-17,1962$.

Rust, S. H., and L. E. Wesney, eds. Nuclear Power Safety. New York: Pergamon Press, 1976.

Rutenkroger, E. 0., of Nuclear Services, Tri-State Motor Transit Co. Letter to B. H. Finley concerning Dedicated Carrier of RAM Incident Record, 20 September 1977 .

"Sabotage Response." Critica1 Mass Journal 3(4):11, 1977. 
"Safety Gear at Three Mile Island Plant was Adequate for Task, NRC Staff Finds." The Wall Street Journa1, 3 August 1979, p 2, col. 7.

Sagan, L. A. "Human Costs of Nuclear Power." Science 177:487-493, 1972.

Sagan, L. A. "Human Radiation Exposures from Nuclear Power: Their Magnitude and Significance." In Human and Ecologic Effects of Nuclear Power Plants, L. A. Sagan, ed. Springfield, IL: C. C. Thomas, 1974, pp 480-493.

Sagi, G. S., and Project Committee 4C-M of the Institute of Traffic Engineers. "Signalized Intersection Capacity Parameters." Traffic Engineering $42(9): 50-55,1972$.

Sagi, G. S. and L. R. Campbel1. "Vehicle Delay at Signalized IntersectionsTheory and Practice." Traffic Engineering 39(5):32-40, 1969.

Salisbury, D. E. "Nuclear Power--Is the Dream Fading?" Christian Science Monitor, 15 February 1979, pp 14-15.

Salomon, S. N. "Beyond Defense In-Depth: Cost and Funding of State and Local Government Radiological Emergency Response Plans and Preparedness in Support of Commercial Nuclear Power Stations," draft, NUREG-0553. Washington: U.S. Nuclear Regulatory Comnission, 30 March 1979.

Salzman, L. "New Yorkers Protest Transport of Nuclear Wastes." Not Man Apart, January 1978, p 9.

Sandia Laboratories. Proceedings of the 5th International Symposium on Packaging and Transportation of Radioactive Materia1s, Las Vegas, NV, 7-12 May 1978. Volumes 1 and 2 and Summary Program. Albuquerque: SLA, 1978.

Sax, N. I. Dangerous Properties of Industrial Materials, 4th ed. New York: Van Nostrand Reinhold Co., 1975.

Scaife, C. "Rig Spills Nuclear Waste near Monteagle." [Chattanooga, TN] Times, 29 January 1979 , p Al.

Schacter, S., and H. A. Burdick. "A Full Experiment on Rumor Transmission and Distortion." Journal of Abnormal Social Psychology 50:363-371, 1955.

Schilling, A. H., et al. Decommissioning Commercial Nuclear Facilities: A Review and Analysis of Curent Regulations, NUREG/CR-0671. Richland, WA: Battelle Pacific Northwest Laboratories, August 1979.

Schilling, A. H., et al. Emergency Response in Transportation of Radiactive Material: An Evaluation Methodology, B-HARC-311-044, TTC-0045. Seattle, WA: Battelle Human Affairs Research Centers, November 1979.

Schmidt, G. D. "Radiation Survey of the Transportation of Radioactive Material." In CONF-710801, vol. 2. Richland, WA: Battelle Pacific Northwest Laboratories, August 1971, pp 552-63. 
Schneider, K. J., C. E. Jenkins, and R. E. Rhoads. Technology, Safety and Costs of Decommissioning a Reference Nuclear Fuel Reprocessing Plant, 2 vols., NUREG-0278. Richland, WA: Battelle Pacific Northwest Laboratories, September/October 1977.

Schwab, J. "Transportation Bans Ring Clear: Concern Over Nuclear Shipments Leapfrogs for City to City." Critical Mass Journal 4(10):1, 1979.

Schwartz, F. W. "A Probabilistic Mass Transfer Model." In Proceedings of the 1975 Canadian Hydrology Symposium, 11-14 August 1975, 1976.

Schwenke, R. L. "N-Ship Owner Seeking Pear1 Berth." Honolulu Advertiser, 6 June 1979, p Al.

Schwirian, K. P., ed. Contemporary Topics in Urban Sociology. Morristown, NJ: General Learning Press, 1977.

Science Applications, Inc. "A Methodology and Data Base for Examining the Health Risks of Electricity Generation From Uranium and Coal Fuel," draft, SAI-OR-79-140-20. Oak Ridge, TN: SAI, December 1979.

Scofield, N. E. and L. G. Haggmark. Penetration of Plane Normal and Plane Slant Gamma Rays Through Slabs of Aluminum and Steel, 2 vols. I. Angular and Energy Distributions (Experimental Pulse Height), USNRDL-TR-272, October 1958. II. Angular and Energy Spectra (Photon Number F1ux), USNRDL-TR-475, October 1960.

Scott, L. M., and C. M. West. "Excretion of 210-Po Oxide Following Accidental Inhalation." Health Physics 28:563-565, 1975.

Scott, M. B. and S. M. Lyman. "Accounts." American Sociological Review, February 1968, pp 46-62.

Scott, R. L. "Browns Ferry Nuclear Power Plant Fire on March 22, 1975." Nuclear Safety 17(5):592-611, 1976.

Scovel, J. "A-Waste 'Overload' Led to City's Shipment Ban." [Garden City, NY] Newsday, 8 April 1979.

"The Secret Train That Goes South." Seattle Post-Intelligencer, 19 September 1978.

Sedgwick County Civil Preparedness Committee. C. P. Log: Monthly Activity Report February - March 1979. Wichita, KS, 1979.

Sedgwick County Civil Preparedness Committee. Sedgwick County Civil Preparedness Emergency Plan, Part D-3: Hazardous Material Incident Standing Operating Procedures. Wichita, KS, May 1979.

Seeman, H. L. "Keeping the Gates at the Chicago Defender." Journalism Quarterly 48:275-278, Summer 1971 .

Seguin, E. L., and K. Perchonok. Vehicle Interactions on An Urban Expressway, Report No. 6. State College, PA: Division of Highway Studies, 1965. 
Sehmel, G. A. "Particle Resuspension from an Asphalt Road Caused by Car and Truck Traffic." In Atmospheric-Surface Exchange of Particulate and Gaseous Pollutants, CONF-74092. Washington: U.S. Energy Research Development Administration, 1976, pp 859-882.

Seinfeld, J. H. Air Pollution--Physical and Chemical Fundamentals. New York: McGraw-Hil1, 1975.

Selby, S. M., ed. Standard Mathematical Tables. Chemical Rubber Company, 1970.

Selph, W. E. Neutron and Gamma-Ray Albedos, ORNL-RSIC-21 (DASA-1892-2). Oak Ridge, TN: Oak Ridge National Laboratory, February 1968.

"A Sequel to Atomic Tragedy (The Houston Incident)." Look, 12 April 1960.

Sevick, S. "N-Shipment Curb Draws Opposition." The Hartford Courant, 16 December 1978.

Shappert, L. B. Cask Designer's Guide: A Guide for the Design, Fabrication, and Operation of Shipping Casks for Nuclear Applications, ORNL-NSIC-68. Oak Ridge, TN: Oak Ridge National Laboratory, February 1970.

Shapiro, F. C. "Radiation Route." New Yorker, 13 November 1978, pp 142-160.

Shapiro, J. Exposure of Airport Workers to Radiation from Shipments of Radioactive Materials--A Review of Studies Conducted at Six Major Airports, NUREG-0154, January 1977.

Shapiro, J. Exposure of Airport Workers to Radiation from Shipment of Radioactive Materials, NUREG-0154. U.S. Nuclear Regulatory Commission, January 1977.

Shapiro, J. Radiation Protection: A Guide for Scientists and Physicians. Cambridge, MA: Harvard University Press, 1972.

Shapley, D. "Plutonium: Reactor Proliferation Threatens a Nuclear Black Market." Science 172:143-146, 1971.

Shapley, D. "Radioactive Cargoes: Record Good But the Problems will Multiply." Science, 172:1318-1322, 1971.

Shappert, L. B. "Shipment of Radioactive Materials." In Human and Ecologic Effects of Nuclear Power Plants, L. A. Sagan, ed. Springfield, IL: C. C. Thomas, 1973, pp 151-176.

Shappert, L. B., et al. Radioactive Waste Transportation Systems Analysis and Program Plan, ORNL-5362. Oak Ridge, TN: Oak Ridge National Laboratory, March 1978.

Shappert, L. R., et al. Probalility and Consequences of Transportation Accidents Involving Radioactive Material Shipments in the Nuclear Fuel Cycle." Nuclear Safety 14(6):597, 1973. 
Sheatsley, P. B., and J. J. Feldman. "The Assassination of President Kennedy: a Preliminary Report on Public Reactions and Behavior." Public Opinion Quarterly 28:189-215, 1964.

Shefelbine, H. C. Preliminary Evaluation of the Characteristics of Defense Transuranic Wastes, SAND78-1850. Albuquerque: Sandia Laboratories, November 1978.

Sheih, L. J. et al. "Air Quality Diffusion Model: Application to New York City." IBM Journal of Research and Development, 1972.

Sheih, C. M. "A Puff-Grid Model for Predicting Pollutant Transport seen on Urban Area." Journal of the Air Pollution Control Association 27:784-785 August 1977.

Shellow, R., and D. V. Roemer. "The Riot That Didn't Happen." Social Problems 14:221-233, 1966.

Sheppart, N., Jr. "Uranium Shipments Banned by Chicago." New York Times, 3 December $1977, \mathrm{p} 11, \mathrm{col} .4$.

Shibutani, T., ed. Human Nature and Collective Behavior. New Brunswick, NJ: Transaction Books, 1970 .

Shibutani, T. Improvised News. New York: Bobbs-Merril1, 1966.

Shields, M. A., J. T. Cowan, and D. J. Bjornstad. Socioeconomic Impacts of Nuclear Power Plants: A Paired Comparison of Operating Facilities, NUREG/CR-0916. Oak Ridge, TN: Oak Ridge Nationa1 Laboratory, July 1979.

Short, J. S., and M. E. Wolfgang, eds. Collective Violence. Chicago: Aldine Publishers, 1972.

Sievert, R. M. "Eine Methode zur Messung von Rontgen-, Radium-, und Ultrastrahlung nebst einiqe Untersuchugen uber die Anwendbarkeit Derselben In Der Physik und Der Medizin." [A Method of Measuring X-, Gamma-, Beta-, and Ultraviolet Rays, in Addition to an Investigation into the Applicability of the Method in Physics and Medicine.] Appendix to Acta Radiological Supplements 12-16. Stockholm, 1932. Translation.

Sigelman, L. "Reporting the News: An Organization Analysis." American Journal of Sociology 79:132-151, 1973.

Silverman, C., and D. A. Hoffman, "Thyroịd Tumor Risk from Radiation During Childhood." Preventative Medicine 4:100-105, 1975.

Simmons, G. L. SAI/EPRI Albedo Information Library, EPRI-NP-1017. Palo Alto, CA: Electric Power Research Institute, March 1979.

Simmons, J. L., et al. Survey of Radioactive Materials Shipments in the U.S., BNWL-1972, NUREG-0073. Richland, WA: Battelle Pacific Northwest Laboratories, April 1976. 
Simon, H. Models of Man. New York: John Wiley and Sons, 1957.

Simon, R. J. Public Opinion in America: 1936-1970. Chicago: Rand-McNally College Publishing Co., 1974.

Sittig, M. Toxic Metals -- Pollution Control and Worker Protection. Park Ridge, NJ: Noyes Data Corporation, 1976.

Skillman, T. S. Road Safety: How to Reduce Road Accidents. New York: David McKay Company, Inc. 1966.

Sklarew, R. C., A. J. Fabrick, and J. E. Prager. "Mathematical Modeling of Photochemical Smog using the PICK Method. Journal of the Air Pollution Control Association 22, November 1972.

Sklarew, R. C., A. J. Fabrick, and J. E. Prager. "A Particle-in-Cell Method for Numerical Solution of the Atmospheric Diffusion Equation and Applications to Air Pollution Problems," vol. 1, 3SRP44. LaJolla, CA: Systems, Science and Software, 1971.

Slade H. H., ed. Meterology and Atomic Energy. Washington: U. S. Atomic Energy Commission, Office of Information Services, July 1968.

"Smart Suggests Squad to Tackle Waste Spi11." [Chattanooga, TN] News-Free Press, 2 February 1979, p AlO.

Smelser, N. J. Theory of Collective Behavior. New York: The Free Press, 1963.

Smith, C. W. Calculated Fuel Rod Perforation Temperatures--Commercial Power Reactor Fuels, NEDO-10093, September 1969.

Smith, D. R., and J. M. Taylor. Analysis of the Radiological Risks of Transporting Spent Fuel and Radioactive Wastes by Truck and by Ordinary and Special Trains, SAND77-1257. Albuquerque: Sandia Laboratories, June 1978.

Smith, D. R., et al. Analysis of the Radiological Risks of Transporting Spent Fue1, SAND 77-1257. Albuquerque: Sandia Laboratories, 1976.

Smith, R. I., G. J. Konzek, and W. E. Kennedy, Jr. Technology, Safety and Costs of Decommissioning a Reference Pressurized Water Reactor Power Station, 2 vols., NUREG/CR-0130. Richland, WA: Battelle Pacific Northwest Laboratories, June 1978.

Smith, W. E. "Reasearch and Worldwide Urban Transportation." Highway Research Record 125:30-39, 1966.

Smith, Wilber, and Associates. Characteristics of Urban Transportation Demand: A Handbook for Transportation Planners,_PB-293220, Apri1 1978 .

"Smooth Ride Seen for Constructive Bills on Hazardous Cargo." Seattle Times, 31 January 1979. 
Smyth, F. F., Jr. "A Toxicologists' View of Threshold Limits." American Industrial Hygiene Association Journal 23:37ff, 1962.

Snipes, M. B., et al. Review of John Gofman's Papers on Lung Cancer Hazard from Inhaled Plutonium, LF-51. Albuquerque: Lovelace Foundation for Medical Education and Research, September 1975.

deSola, I., et al. Candidates, Issues and Strategies: a Computer Simulation of the 1960 and 1963 Presidential Elections. Cambridge: MIT Press, 1964.

Solomon, D. Accidents on Main Rural Highways. Washington: US Dept of Commerce, Bureau of Public Roads, 1964.

Solomon, K. A. Estimate of the Probablity that an Aircraft Will Impact the PVNGS, NUS 1416 , lst rev. ed. Prepared for the Arizona Nuclear Power Project, 25 July 1975.

Solon, L. R. "Public Health Aspects of Transportation of Radioactive Materials in Large Urban Areas." In Radiation Benefits and Risks: Facts, Issues, and Opinion, FDA-77-8021. Proceedings of the 8 th Annual National Conference on Radiation Control. Springfield, IL: U.S. Department of Health, Education, and Welfare, 7 May 1976.

Sousselier, Y. Environmental Impact of Waste Management and Transport of Radioactive Materials Originating From the Nuclear Fuel Cycle, PNL-TR-355. Richland, WA: Battelle Pacific Northwest Laboratories, March 1979.

Sosslau, A. B., et al. Quick-Response Urban Travel Estimation Techniques and Transferable Parameters--User's Guide, Report 187. National Cooperative Highway Research Program, 1978.

South Carolina Code. Columbia.

South Carolina Department of Health and Environmental Control. Peacetime Radiological Emergency Response Plan. Columbia, SC, 30 December 1978 .

South Carolina Department of Health and Environmental Control, Bureau of Radiological Health. Transportation of Radioactive Material in South Carolina, NUREG/CR-0266. Columbia, SC, April 1978.

Southern Interstate Nuclear Board. Radioactive Materials Transportation, Administrator's Guide No. 4. Atlanta, July 1973.

Southern Interstate Nuclear Board. Transportation of Nuclear Spent Fue1, Adminstrator's Guide No. 3. Atlanta, December 1972 .

Southern Interstate Nuclear Board. Transportation of Nuclear Spent Fue1, Administrators Guide No. 3. Atlanta, 1972 .

Southwest Research Institute. Strength Tests of Prototype Sea Disposal Containers for Radioactive wastes, TID-13942. San Antonio, TX: SwRI, October 1961. 
Speth, G., A. Tamplin, and T. Cochran. "Plutonium Recycle or Civil Liberties? We Can't Have Both." Environmental Action, 7 December 1974, pp 10-13.

Stack, K. "A-Shipments Bil1 Ready at Capito1." Danbury [CT] News-Times, 18 March 1976.

Stallings, R. A., and W. Freeling. "Correction Policies for Natural Disaster." Proceedings of American Sociological Association. Chicago, 1977.

Stang, L. G., Jr. Radionuclide Generators: Past, Present and Future, BNL-13595. Richland, WA: Battelle Pacific Northwest Laboratories, 22 April 1969.

Stannard, J. N. "An Evaluation of Inhalation Hazards in the Nuclear Energy Industry." In Proceedings of the 2nd U. N. International Conference on the Peaceful Uses of Atomic Energy, Geneva, September 1958, 23:306ff.

Stark, M. J., et al. "Some Empirical Patterns in a Riot Process." American Sociological Review 39:865-876, December 1974 .

Starr, C. "Social Benefit Versus Technological Risk." Science 165:1232-1238, September 1969.

"State Legislation to Halt Spreading of Local Nuclear Transportation Restrictions." Nucleonics Week, 28 December 1978, p 6.

"State Monitors Radioactive Shipments via Permits." New Haven Register, 28 January 1979.

State of New York and State of Illinois v NRC et al. U.D. District Court SDNY, 75 Civil 2121 .

"State Report: Radioactive Materials." Energy Report to the States 4:93-95, 31 March 1978.

"States, Not Waiting for the Feds, Move to Ban Wastes." Not Man Apart, Nuclear Blowdown. February 1979, p 17.

States' Role in Radioactive Materials Management: Report of a Workshop. Proceedings of the Workshop on States' Role in Radioactive Materials Management, Las Vegas, 1974. Lexington, KY: Council of State Governments, 1975.

Steele, W. A., et al. The Incidence of Hazardous Material Accidents During Transportation and Storage, NBISR 73-412. Frederick, MD: National Bureau of Standards November 1973.

Stein, M. "Social Impact Assessment Techniques and Their Application to Transportation Decisions。" Traffic Quarterly 31(2):297, 1977.

Steinbruner, J. D. The Cybernetic Theory of Decision. Princeton, NJ: Princeton University Press, 1974. 
Sterling, J., T. E. Drabek, and W. H. Key. "The Long-Term Impact of Disaster on the Health Self-Perceptions of Victims." Proceedings of American

Sociological Association. Chicago, 1977.

Stern, A. C., ed. Proceedings of a Symposium on Multi-Source Urban Diffusion Models, 1970, PB 190-400. Springfield, VA: National Technical Information Service, 1970 .

Stewart, K. B. Rail Accident Statistics Pertinent to the Shipment of Radioactive Materials, HW-76299. Richland, WA: General Electric Co., 21 January 1963.

Strange, D. L., E. C. Watson, and J. G. Droppo. Review of Calculational Models and Computer Codes for Environmental Dose Assessment of Radioactive Releases, BNWL-B-454. Richland, WA: Battelle Pacific Northwest Laboratories, June 1976.

Stravasnik, L. F. Special Tests for Plutonium Shipping Containers 6M, SP5795, and L-10, SC-DR-72-0597. Albuquerque: Sandia Laboratories, September 1972 .

Street, D. ed. Handbook of Contemporary Urban Life. San Francisco: Jossey-Bass, $1 \overline{978 .}$

Street, D., and J. L. Davidson. "Community and Politics in City and Suburb." In Handbook of Contemporary Urban Life, David Street, ed. San Francisco: Jossey-Bass, 1978, pp 468-493.

Strom, P. O., and E. C. Watson. Calculated Doses from Inhaled Transuranium Radionuclides and Potential Risk Equivalence to Whole-Body Radiation, BNWL-SA-5588. Richland, WA: Battelle Pacific Northwest Labratories, 1975.

"Study Finds Luck Aided Nuclear Energy Industry." The Washington Post, 7 October 1977 , p B8.

Sutton, H. Comprehensive Emergency Preparedness Planning in State Government, Iron Works Pike. Lexington, KY: The Council of State Goverments, 1976.

Swanson, C. E. "Procedures and Effects of the Printed Media." In Mass Media and Education. Chicago: University of Chicago Press, 1954, pp 13 $\overline{9-164 .}$

Swanson, G. E. "A Preliminary Laboratory Study of the Acting Crowd." American Sociological Review 18:522-533, 1953.

Swanson, T. "A State in Emergency." National Civic Review 53:483-488, 1964.

Syrek, D. "Accident Rates at Intersections." Traffic Engineering $25(8): 312-316,1955$.

Tamplin, A. R., and J. W. Gofman. Population Control through Nuclear Pollution. Chicago: Nelson-Hall Co., 1970 .

Tamplin, A. R., and T. B. Cochran. A Report on the Inadequacy of Existing Radition Protection Standards Related to Internal Exposure of Man to Insoluble Particles of Plutonium and Other Alpha-Emitting Hot Particles. Washington: Natural Resources Defense Council, 14 February 1974. 
Taylor, J. M., and S. L. Daniel. RADTRAN-A Computer Code to Analyze Transportation of Radioactive Material, SAND76-0243. Albuquerque: Sandia Laboratories, 1977.

Taylor, J. B., L. Zurcher, and W. H. Key. Tornado: A Community Responds to Disaster. Seattle: University of Washington Press, 1970.

Taylor, J. M., and S. L. Daniel. "A Model to Predict Radiological Consequences of Transportation Accidents Involving Dispersal of Radioactive Material in Urban Areas." In Proceedings of the 1978 Summer Computer Simulation Conference, 24-26 July 1978, pp 601ff.

Taylor, V. "Good News about Disaster." Psychology Today, October 1977, pp $93 \mathrm{ff}$.

Tennessee Department of Public Health. "Administrative Emergency Procedures for Radiological Incidents at Nuclear Facilities and in Transportation." Appendix II to the Tennessee Disaster Assistance Plan. np, 1 August 1976.

Tennessee Department of Public Health, Division of Occupational and Radiological Health. "Summary of RAM Incidents for the State of Tennessee, 1966-1977." Nashville.

Texas Department of Health Resources, Division of Occupational Health and Radiation Control. "Summary of RAM Incidents for the State of Texas, 1963-1977." Austin.

Texas Revised Civil Statutes. Austin.

Thaldos, L. T. "How to Get Nuclear Transportation Curbs Passed in Your Community." Critical Mass Journal 4(10):8-9, 1979.

"The Ordeal at Three Mile Island." Nuclear News Special Report, 6 April 1979.

Thomas, H. P. "An On-Line Meteorological Information System for Response to Accidental Releases of Radioactivity into the Atmosphere." Nuclear Technology 46:241ff, December 1979 .

Thomas, J. M. and W. G. Bennis, eds. The Management of Change and Conflict. Baltimore: Penguin Books, 1972.

Thompson, J. D. Organizations in Action. New York: McGraw-Hill, 1967.

Thompson, R. C., et al. "Evaluation of Internal Exprosure Hazards for Several Radioisotopes Encountered in Reactor Operations." In Proceedings of 2nd U.N. International Conference on Peaceful Uses of Atomic Energy, Geneva, September 1958, 23:283ff.

Thornton, T. P. "Terror as a Weapon of Political Agitation." In Internal War: Problems and Approaches, H. Eckstein, ed. New York, 1964, p 78.

Tidwel1, J. E., Jr., and J. B. Humphrey. "Relation of Signalized Intersection Level of Service to Failure Rate and Average Individual Delay." Highway Research Record 321, 1970. 
Tittemore, L. H., et al. An Analysis of Urban Area Travel by Time of Day, PB-247776. Boston: Peat, Marwick, Mitchell and Company, for the Federal Highway Administration, January 1972.

Toch, H. The Social Psychology of Social Movements. New York: Bobbs-Merrill, Inc. 1965.

Townley, C. W., et al. The Development and Evaluation of Safety Performance Criteria for Sealed Radiation Sources, BMI-1559. Columbus, OH: Battelle Memorial Institute, 5 December 1961.

"Transport -- Laser-beam M-16's Used in Training Exercises." Nuclear News, November 1978 , p 100.

"Transport of Radioactive Materials in the United Sates." Nuclear Safety 18:291-297, May-June 1977.

Treadway, T. B., and J. C. Oppenlander. "Statistical Modeling of Travel Speeds and Delays on a High-Volume Highway." Highway Research Record 199, 1967.

Tribe, L. H. American Constitutional Law. Mineola, NY: The Foundation Press, 1978.

Tribe, L. H. "California Declines the Nuclear Gamble: Is Such a State Choice Preempted?" Ecology Law Quarterly 7(3):679-729, 1979.

Trinquier, R. Modern Warfare: A French View of Counterinsurgency, D. Lee, trans. New York: Praeger, 1964.

Tri-State Regional Planning Commission. 1970 County-to-County Trave1: Census Journey to Work by Mode; Person Travel by Purpose, Interim Technical Report 4549-1302, 1975.

Troutman, J. E. The Hazards of Radioisotopes under Fire Conditions. Factory Insurance Association; 1959.

"Truck Accident Spills Radioactive Uranium." Critical Mass 3(9):1, 1978.

"Truck Carrying A-pellets Almost Loses Load in Mishap." Chicago Tribune, 10 February 1978, sec. 1, p 2, col. 1.

"Truck Wreck Strews Radioactive Load." [Chattanooga, TN] News-Free Press, 29 January 1979.

Tse, A. N. Measurement of Radiation Exposure Received by Flight Attendants from Shipments of Radioactive Material, NR-DES-0001. U.S. Nuclear Regulatory Commission, November 1.976.

Tuchman, G. "Making News by Doing Work: Routinizing the Unexpected." American Journal of Sociology, vol. 79, 1973, pp 110-131. 
Tucker, J. G. "The Transportation of Radioactive Materials--Federal Preemption versus State Regulation." State Government, Spring 1974, pp 122-127.

Tukey, J. W. The Propagation of Errors, Flucuations and Tolerances; Basic Generalized Formulas, Technical Report No. 10. Princeton University Statistical Research Group, 1958.

Turner, B. A. "The Organizational and Inter-organizational Development of Disasters." Administrative Science Quarterly 21, September 1976, pp 378-397.

Turner, R. H. and L. M. Killian. Collective Behavior, 1st ed. Englewood Cliffs, NJ: Prentice Ha11, 1957.

Turner, R. H., and L. M. Killian. Collective Behavior, 2nd ed. Englewood Cliffs NJ: Prentice Hall, 1972.

Turner, R. H. "Collective Behavior." In Handbook of Modern Sociology,

R. E. L. Faris, ed. Chicago: Rand-McNa11y, 1964, pp 382-425.

Turner, R. H. "Collective Behavior and Conflict: New Theoretical Framework." Sociological Quarterly 5:127-128, 1964.

Turner, R. H. "Determinants of Social Movements Strategies." In Human Nature and Collective Behavior, T. Shibutani, ed. New Brunswick, NJ: Transaction Books, 1970 .

Turner, R. H. "The Public Perception of Protest." American Sociological Review 34:813-815, December 1969.

Turner, R. H. "Types of Solidarity in the Reconstruction of Groups." Pacific Sociological Review, Fal1 1967, pp 10,60-68.

Underhill, D. W., and D. W. Moeller. Development of a Computer Model for Estimating Population Dose Equivalent Rates from Naturally Occurring Radionuclides in Building Materials. Boston: Harvard School of Public Health.

Union of Concerned Scientists. The Nuclear Fuel Cycle. Cambridge, MA: M.I.T. Press, 1974.

U.S. Army Corps of Engineers. Proceedings (of) Social Scientists Conference, Memphis, 20-24 September 1976. Fort Belvoir, VA: U.S. Army Corps of Engineers and Institute for Water Analysis, December 1977.

U.S. Atomic Energy Commission. Environmental Survey of Transportation of Radioactive Materials To and From Nuclear Power Plants, WASH-1238. Washington: USAEC, December 1972.

U.S. Atomic Energy Commission. Directory of Shipping Containers for Radioactive Materials, October 1969. 
U.S. Atomic Energy Commission, Directorate of Fuels and Materials Licensing. Environmental Survey of the Uranium Fuel Cycle, WASH 1248. Washington: USAEC, 1974.

U.S. Atomic Energy Commission, Office of Safeguards and Materials Management. Factual Study of Special Nuclear Material Shipping Patterns of U.S. Commercial Organizations and of Unclassified Exports By the AEC and Its Contractors, Vols. 1 and 2, TID 26232. Springfield, VA: National Technical Information Service, 1 May 1971.

U.S. Atomic Energy Commission. High-Level Radioactive Waste Management Alternatives, WASH-1297. Washington: USAEC, May 1974 .

U.S. Atomic Energy Commission. Operational Accidents and Radiation Exposures Experience Within the U. S. 1943-1975, WASH-1192. Washington: USAEC, 1975.

U.S. Atomic Energy Commission. Proposed Final Environmental Statement on the Liquid Metal Fast Breeder Reactor Program, WASH-1535. Washington: USAEC, December 1974.

U.S. Atomic Energy Commission Regulatory Guide 1.3, "Assumptions Used for Evaluating the Potential Radiological Consequences of a Loss of Codant Accident for Boiling Water Reactors." Revision 2, June 1974.

U.S. Atomic Energy Commission Regulatory Guide 1.86, "Termination of Operating Licenses for Nuclear Reactors," June 1974.

U.S. Atomic Energy Commission. The Safety of Nuclear Power Reactors (Light Water-Cooled) and Related Facilities, WASH-1250. Washington: USAEC, July 1973.

U.S. Atomic Energy Commission. Shipments of Nuclear Fuel and Waste...Are They Really Safe?, WASH-1339. Washington: USAEC, August 1974.

U.S. Atomic Energy Commission. A Summary of Incidents Involving USAEC Shipments of Radioactive Material 1963-1964, Supplement 2 to TID 16764. Springfield, VA: National Technical Information Service, 1966.

U.S. Coast Guard. "Statistical Summary of Casualties to Commercial Vessels." Proceedings of the Merchant Marine Counci1, CG-129, 27(12):228-233, December 1970.

United States Code. Title 42, "Clean Air Act Amendments of 1977."

United States Code. Title 42, "Energy Reorganization Act of 1974," 5801-5891.

United States Code Annotated. Title 42, "The Public Health and Welfare."

United States Code. Title 49, "Hazardous Materials Transportation Act of 1974," 1801-1812.

United States Code Annotated. Title 49, "Transportation." 
U. S. Congress, Senate. "A Bill to regulate commerce by providing for the safe transportation of nuclear waste and radioactive nuclear reactor fuel, and for other purposes," S. 535. Washington: U.S. Government Printing Office, 5 March 1979.

U.S. Comptroller General. Nuclear Energy's Dilemna: Disposing of Hazardous Radioactive Waste Safely. Washington: U. S. General Accounting Office, 1977 .

U.S. Comptroller General. Report to the Congress: Areas Around Nuclear Facilities Should be Better Prepared for Radiological Emergencies, EMD-78-110. Washington: U.S. General Accounting Office, 30 March 1979.

U.S. Comptroller General. Report to the Congress: Federal Actions Are Needed to Improve Safety and Security of Nuclear Materials Transportation, EMD-79-18. Washington: U.S. General Accounting Office, 7 May 1979.

U.S. Congress, Senate Committee on Commerce. Transportation of Hazardous Materials, hearings. 93d Cong., 2d Sess., 1974.

U.S. Department of Agriculture. Treatments for Farmland Contaminated with Radioactive Material, Agriculture Handbook No. 395. Washington: USDA.

U.S. Department of Commerce. Number of Inhabitants, U.S. Summary, 1970 Census of Population, PC(1)-A1. Washington, December 1971.

U.S. Department of Commerce. Social Indicators, 1976. Washington, 1977.

U.S. Department of Commerce. Statistical Abstract of the United States, $97 \mathrm{th}$ Annual Edition, 1976; and 98th Annual Edition, 1977.

U.S. Department of Defense, Department of the Army. Explosives and Demolitions, FM5-25. Washington, 1967.

U.S. Department of Defense, Department of the Army. Fundamentals of Protective Design (Non-Nuclear), TM5-855-1. Washington, Ju1y 1965.

U.S. Department of Energy. Analytical Methodology and Facility Description Spent Fuel Policy, DOE-ET-0054. Washington: USDOE, August 1978.

U.S. Department of Energy, Division of Environaental Control Technology. Everything You Always Wanted to Know About Shipping High-Level Wastes. DOE/EV-0003, revision of WASH-1264. Washington: USDOE, 1978.

U.S. Department of Energy, Division of Environmental Control Technology. Shipments of Nuclear Fuel and Waste...Are They Really Safe?, DOE/EV-0004, October 1977.

U.S. Department of Energy, Division of Solar Applications. Psycho-Economic Factors Affecting the Decision Making of Consumers and the Technology Delivery System. Washington: USDOE, January 1978. 
U.S. Department of Energy. Roles and Responsibilities of Energy-Related Environmental Organizations, DOE/EV-0026. Washington: USDOE, September 1978.

U.S. Department of Health, Education, and Welfare. Radiation Benefits and Risks: Facts, Issues and Options. Proceedings of the 8 th Annual National Conference on Radiation Control, 2-7 May 1976, in Springfield, IL. Rockville, MD: U.S. HEW, April 1977.

U.S. Department of Health, Education and Welfare. Radiation Benefits and Risks: Facts, Issues, and Opinion, FDA 77-8021. Washington: HEW Publications, 1976.

U.S. Department of Health, Education and Welfare, U. S. Public Health Service. Radiological Health Handbook. Rockville, MD: U.S. HEW, January 1970.

U.S. Department of Health Education and Welfare, Public Health Service. Registry of Toxic Effects of Chemical Substances. Rockville, MD: USHEW, 1977.

U.S. Department of Labor, Bureau of Labor Statistics. Occupational Outlook Handbook, 1973-79 ed.; Bulletin 1955. Washington: U.S. DOL.

U.S. Department of Transportation. Annual Report of the Secretary of Transportation on Hazardous Materials Control. Washington: USDOT, 1975.

U.S. Department of Transportation, Federal Highway Administration, Bureau of Motor Carrier Safety. Accidents of Motor Carriers of Property, 1973, 1975, and 1976 eds. Washington: U.S. DOT.

U.S. Department of Transportation, Federal Highway Administration, Bureau of Motor Carrier Safety. 1969 Analysis of Accident Reports Involving Fire, also 1972 report. Washington: U.S. DOT.

U.S. Department of Transportation, Materials Transportation Bureau, Research and Special Program Administration. "Federal Register Reprints Pertaining to 49 CFR." Washing ton: U.S. DOT, June 1978 .

U.S. Department of Transportation, Materials Transportation Bureau. Hazardous Materials Definitions. Washington: U.S. DOT, December 1977.

U.S. Department of Transportation, Materials Transportation Bureau. Hazardous Materials Incident Reports for 1978. Washington: U.S. DOT, 1978.

U.S. Department of Transportation, Materials Transportation Bureau. Hazardous Materials Newsletter, vol. 8, No. 70, June 1978.

U.S. Department of Transportation. Heavy Trucks Special Report: Fatal Accident Reporting System, DOT HS 803259 . Washington: USDOT, May 1978.

U.S. Department of Transportation. Incident Reports involving RAM, 1975. Washington: U.S. DOT. 
U.S. Department of Transportation, Materials Transportation Bureau. Inconsistency Ruling IR-1, 4 April 1978, 43 Fed. Reg. 16954, 1978, 2 C. C. H. Nuclear Regulation Reporter, para. 20, 079.

U.S. Department of Transportation, Office of Hazardous Materials Operations. An Index to the Hazardous Materials Regulations of Title 49, Code of Federal Regulations, Parts 170-180, 1 October 1975, revision.

U.S. Department of Transportation. Public Hearing on the Highway Routing of Radioactive Material, 29 November 1978, HM-164.

U.S. Department of Transportation, Materials Transportation Bureau. Radioactive Materials Incidents for 1971 through 1978. Washington: U.S. DOT, 1979.

U.S. Department of Transportation. Report of the Hazardous Materials Transportation Task Force. Washington: U.S. DOT, September 1978.

U.S. Department of Transportation, Office of Hazardous Materials Operations. A Review of the Department of Transportation (DOT) Regulations for Transportation of Radioactive Materials. Washington: U.S. DOT, December 1977.

U.S. Department of Transportation. Risk Analysis in Hazardous Materials Transportation--Bibliography, DOT-OS-20114. Washington: U.S. DOT, March 1973.

U.S. Department of Transportation. "The Severity of Large Truck Accidents," NHTSA Technical Note, DOT HS-802 332. Washington: National Highway Transportation Safety Administration, April 1977.

U.S. Department of Transportation Federal Highway Administration. A Summary, of Highway Facilities Where Hazardous Materials are Restricted. Washington: U.S. DOT, January 1977.

U.S. Department of Transportation, Materials Transportation Bureau. Advance Notice of Proposed Rulemaking. 43 Fed. Reg. 366-368, 17 August $197 \overline{8 .}$

U.S. Department of Transportation and U. S. Nuclear Regulatory Commission. "DOT and NRC Memorandum of Understanding on Transportation of Radioactive Materials," 44 FR 38690, 2 July 1979.

U.S. Energy Research and Development Administration. "The Environmental Impact of Transportation of Nuclear Materials in the LMFBR Programs." Excerpts from WASH-1535, Proposed Final Environmental Impact Statement, Liquid Metal Fast Breeder Reactor Program, ERDA-40. Washington: U.S. ERDA, May 1975.

U.S. Energy Research and Development Administration. Light Water Reactor Fue1 Reprocessing and Recycling, ERDA-77-75. Washington: U.S. ERDA, July 1977.

U.S. Energy Research and Development Administration. Standards for Radiation Protection. Washington: U.S. ERDA, chapter 0524. 
U.S. Environmental Protection Agency, Office of Radiation Programs. Environmental Analysis of the Uranium Fuel Cycle, Part I: Fuel Supply, EPA-52019-73-003B. Washington: U.S. EPA, October 1973.

U.S. Environmental Protection Agency. Health Effects of Alpha-Emitting Particles in the Resiratory Tract. Report of the Ad Hoc Comittee on "Hot Particles" of the Advisory Committee on the Biological Effects of Ionizing Radiation, National Academy of Sciences, National Research Council. Washington: U.S. EPA, October 1976.

U.S. Environmental Protection Agency. Proposed Guidance on Dose Limits for Persons Exposed to Transuranium Elements in the General Environment, EPA520/4-77-016. Washington: U.S. EPA, September 1977.

U.S. Environmental Protection Agency, Office of Radiation Programs. Transportation Accidents Risks in the Nuclear Power Industry, 1975-2020, EPA-520/3-75-023. Washington: U.S. EPA, 1974.

U.S. Environmental Protection Agency and Nuclear Regulatory Commission Task Force on Emergency Planning. Planning Basis for the Development of State and Local Government Radiological Emergency Response Plans in Support of Light Water Nuclear Reactor Plants, NUREG-0396, December 1978.

U.S. General Accounting office. Commercial Nuclear Fuel Facilities Need Better Security, an unclassified digest of classified report. Washington: U.S. GAO, 2 May 1977.

U.S. General Accounting Office. Federal Actions Are Needed to Improve Safety and Security of Nuclear Materials Transportation. Washington: U.S. GAO, 1979 .

U.S. Geological Survey. National Energy Transportation Systems: Nuclear Fuel Materials Movement by Highways (BTU): 1975, Map No. 16. Washington: USGS, 1976.

U.S. Geological Survey. National Energy Transportation Systems: Nuclear Fuel Materials Movement by Highways (Truckloads): 1975, Map No. 15 . Washington: USGS, 1976.

U.S. Geological Survey. National Energy Transportation Systems: Railroad, Highway, and Water Transportation Systems: 1974. Map No. 18. Washington: USGS, 1976.

U.S. Interstate Commerce Commission, office of Proceedings, Section of Energy and Environment. Transportation of Radioactive Materials by Rail, Final EIS. Washington: U.S. ICC, 23 August 1973.

U. S. Nuclear Regulatory Commission and U.S. Department of Transportation Joint Study Group. "Review and Assessment of Package Requirements (Yellowcake) and Emergency Response to Transportation Accidents: Part I, Executive Summary," draft, NUREG-0535. Washington: U.S. NRC, October 1978. 
U.S. Nuclear Regulatory Commission, Division of Safeguards. Calculations of Radiological Consequences from Sabotage of Shipping Casks for Spent-Fuel and High-Level Waste, NUREG-0194. Washington: U.S. NRC, February 1977.

U.S. Nuclear Regulatory Commission, Office of Nuclear Reactor Regulation. Demographic Statistics Pertaining to Nuclear Power Reactor Sites, NUREG-0348. Washington: U.S. NRC, October 1979.

U.S. Nuclear Regulatory Commission, Office of Nuclear Material Safety and Safeguards. Directory of Certificates of Compliance for Radioactive Materials Packages, vol. 1, rev. 1, NUREG-0383. Washington: U.S. NRC, November 1978.

U.S. Nuclear Regulatory Commission, Office of Standards Development. Draft Environmental Statement on the Transportation of Radioactive Material by Air and Other Modes, NUREG-0034. Springfield, VA: National Technical

Information Service, March 1976.

U.S. Nuclear Regulatory Commission, Office of Standards Development. Environmental Survey of Transportation of Radioactive Materials To and From Nuclear Power Plants, Supplement 1, NUREG-75/038. Washington: U.S. NRC, April 1975.

U.S. Nuclear Regulatory Commission. Final Environmental Statement Related to Manufacture of Floating Nuclear Power Plants by off Shore Power Systems, NUREG-0056. Washington: U.S. NRC, September 1976.

U.S. Nuclear Regulatory Commission. Final Generic Environmental Statement on the Use of Mixed Oxide Fuel in Light Water Cooled Reactors - Health, Safety and Environment, NUREG-0002. Washington: U.S. NRC, August 1976.

U.S. Nuclear Regulatory Commission. "Generic Environmental Impact Statement on Handling and Storage of Spent Light Water Power Reactor Fuel," vols. 1 and 2, draft, NUREG-0404, March 1978.

U. S. Nuclear Regulatory Commission, office of International and State Programs. Guide and Checklist for Development and Evaluation of State and Local Government Radiological Emergency Response Plans in Support of Fixed Nuclear Facilities, NUREG-75/111. Springfield, VA: National Technical Information Service, 1974.

U.S. Nuclear Regulatory Commission, Office of State Programs. Handbook for Federal Assistance to State and Local Governments, NUREG-009371. Springfield, VA: National Technical Information Service, June 1976.

U.S. Nuclear Regulatory Commission. Handling and Storage of Spent Light Water Power Reactor Fuel: Final Generic Environmental Impact Statement, NUREG-0575. Springfield, VA: National Technical Information Service, August 1979 .

U.S. Nuclear Regulatory Commission, Office of State Programs. Information Report on State Legislation, vol. 3, No. 1, January 1977. 
U.S. Nuclear Regulatory Commission, Office of State Programs. Means for Improving State Participation in the Siting, Licensing and Development of Federal Nuclear Waste Facilities: A Report to Congress, NUREG-0539, March 1979.

U.S. Nuclear Regulatory Commission and the Agreement States. "Licensing Statistics and Other Data," January 1976 to May 1976; and "Incident Reports," June 1976 to December 1976; B-180225 (GAO), RO 387. Washington: U.S. NRC.

U.S. Regulatory Commission. Potential Releases of Cesium from Irradiated Fuel in a Transportation Accident, Supplement II to WASH-1238, NUREG-0069. Washington: U.S. NRC, July 1976.

U.S. Nuclear Regulatory Commission. Reactor Safety Study, An Assessment of Accident Risks in U.S. Commercial Nuclear Power Plants, NUREG-75/014, WASH-1400. Washington: U.S. NRC, October 1975.

U.S. Nuclear Regulatory Comnission, Office of Nuclear Material Safety and Safeguards. Physical Protection of Shipments of Irradiated Reactor Fuel, NUREG-0561. Interim Guidance, Section 4, 21 May 1979.

U.S. Nuclear Regulatory Commission. Regulation of Federal Radioactive Waste Activities, NUREG-0527, 1979. Report to Congress on extending the Nuclear Regulatory Commission's licensing and regulatory authority to federal radioactive waste storage and disposal activities.

U.S. Nuclear Regulatory Commission. Regulatory Guide 1.11, "Methods for Estimating Atomospheric Transport and Dispersion of Gaussian Effluents in Routine Releases from Light-Water Cooled Reactors." Revision 1, Ju1y 1977.

U.S. Nuclear Regulatory Commission. "Review and Assessment of Package Requirements (Yellocake) and Emergency Response to Transportation Accidents," draft, NUREG-0535, March 1979.

U.S. Nuclear Regulatory Commission. Release No. 76-205, 2 C.C.H. Nuclear Regulation Reporter, para. 20, 039, 7 September 1976.

U.S. Nuclear Regulatory Commission. Safeguards Program, 1 C.C.H. Nuclear Regulation Reporter, para. 1015.

U.S. Nuclear Regulatory Commission. Staff Study Concerning Financial Protection against Potential Harm Caused by Sabotage or Theft of Nuclear Materials, June 1975.

U.S. Nuclear Regulatory Commission. Standards for Protection Against Radiation, 10CFR20, 1977.

U.S. Nuclear Regulatory Commission. Summary Report of Jointly Sponsored NRC-DOT Transportation Surveillance Program Involving Low-Leve1 Radioactive Materials: Findings and Recommendation, NUREG-0393, March 1978.

U.S. Nuclear Regulatory Commission. Transportation Incident Date for 1975, NRC Regional offices, 1977. 
U.S. Nuclear Regulatory Commission. Transport of Radioactive Material in the U.S., NUREG-0073. Washing ton: U.S. NRC, 1976.

U.S. Nuclear Regulatory Commission, Office of Standards Development. Transport of Radioactive Material in the U.S., A Detailed Summary of "Survey of Radioactive Material Shipments in the United States," BNWL-1972, NUREG-0073. May 1976.

U.S. Nuclear Regulatory Commission Study Group. The Urban Transportation Study--Looking Ahead: Status, Goals, and Approaches. Transcription of a panel discussion in New York, 25 July 1978. Washington: AEC-Federal Reporters, 1978.

United States of America and Trustees of Columbia University v City of New York. 2 C.C. H. Nuclear Regulation Reporter, para. 20, 101; D.S.N.Y. 1978.

"U.S. To Study City Uranium Transport." Chicago Tribune, 2 December 1977, sec. $1, \mathrm{p} 3$.

"Uranium Loss is Admitted." Washington Post, 3 May 1977, p Al, col. 6.

"Uranium Ore Spilled in Kansas." Washington Post, 23 March 1979, p A9.

Urbon, S. F. "Second Nuclear Waste Load Meets Little Fanfare." [Norwich, CT] Bulletin, 23 March 1976.

Uselman, J., and J. W. McKlveen. "Radiation Exposures Aboard Commercial Aircraft." Health Physics 29:881-83, 1975.

Van Der Zarden, J. W. "Resistance and Social Movements." Social Forces $37: 312-315,1959$.

Vanslager, F. E., et al. Fission Product Transport in HTGR System--A Summary, GA-10073, 22 April 1970.

Veltfort, H. R., and G. E. Lee. "The Cocoanut Grove Fire: A Study in Scapegoating." Journal of Abnormal and Social Psychology 38:138-154, April 1943.

Verba, S., and N. Nie. Participation in America: Political Democracy and Social Equality. New York: Harper and Row, 1972.

Verdon, R. "Atomic Shipment Checked for Safety." [Garden City, NY] Newsday, 9 March 1976.

Vermont Public Interest Research Group, Inc. Radwaste on the Roadway: The Transportation of Radioactive Materials in Vermont. Montpelier, VT: VPIRIG, February 1977.

Vermont Statutes Annotated. Montpelier.

"Vermont Town Meetings Reject Nuclear Power." Not Man Apart, Mid-March/April 1977 , p 16. 
Vermont Yankee $v$ NRCD, 98 S. Ct. 1197, 1978.

Versace, J. "Factor Analysis of Roadway and Accident Data." Highway Research Board Bulletin 240:24-32, 1960.

Virginia Code. Richmond.

Virginia Sunshine Alliance and Others v Joseph Hendrik and Others, Civil No. 79-1989 (D.D.C. 1979).

Vittek, J. F., Jr. "Is There an Airship in Your Future?" Technology Review $77(8): 23-29,1975$.

Vogt, S., and W. G. Hubschmann. "The Influence of Meterological Parameters on the Consequences of Accidental Activity Releases from Nuclear Power Plants." Nuclear Technology 46:300ff, December 1979 .

Voth, E. R. "Commercial Ambulance Rates." Emergency $11(7): 15-16$, July 1979.

Wagman, P. "Danforth Backs Bill to Oversee Nuclear Shipments." St. Louis Post Dispatch, 19 March 1979, p A3.

Wagman, P. "Luck Averted Radioactive Spill in Truck Accident, Official Says." St.Louis Post-Dispatch, 21 March 1979, p A8.

Wagman, P., and S. Defty. "Radioactive Waste in Truck in Crash." St. Louis Post-Dispatch, 20 March 1979, p Al.

Wagner, N. R. A Survey of Threat Studies Related to the Nuclear Power Industry, SAND 77-8254. Albuquerque: Sandia Laboratories, August 1977.

Wa11, I. B., et al. Reactor Safety Study Consequence Model CRAC, Computer Code Users Manual. Argonne, IL: Argonne National Laboratory, Argonne Code Center.

Wall, I. B., et al. Overview of the Reactor Safety Study Consequence Model, NUREG-0340, October 1977.

Wallace, A. F. C. Human Behavior in Extreme Situations: A Survey of the Literature and Suggestions for Future Research. Washington: National Academy of Sciences, National Research Council, 1956.

Walton, J. "The Structural Basis of Political Change in Urban Communities." Sociological Inquiry $43: 3-4,1973$.

Warren, R. I. The Community in America, 2nd ed. Chicago: Rand McNally, 1972.

Washington Public Interest Research Group. A National Radioactive Waste Repository at Hanford: A Statewide Opinion Survey. Seattle: University of Washington, March 1979.

Washington Public Interest Research Group. Nuclear Waste: A National Repository at Hanford. Seattle: University of Washington, October 1978 . 
Washington Revised Code Annotated. Olympia.

Waste Handling and Transportation. Nucleonics Week Special Report, Series 5, 1978.

Waxman, J. J. "Local Broadcast Gatekeeping during National Disasters." Journalism Quarterly 50(4):751-758, Winter 1973.

Wayland, J. R. Conditional Probability of Intense Rainfall Producing High Ground Concentrations from Radioactive Plumes, SAND76-9746, NUREG-0209. Albuquerque: Sandia Laboratories, March 1977.

Webber, D. L. "Darwin Cyclone: An Exploration of Disaster Behavior." Australian Journal of Social Issues, vol. 11, No. 1, February 1976.

Wedding, J. B., D. J. Lombardi, and J. E. Cermak. "A Wind Tunnel Study of Gaseous Pollutants in City Street Canyons." J. of the Air Pollution Control Association 27:557, 1977.

Weick, K. E. "The 'ess' in Stress: Some Conceptual and Methodological Problems." In Social and Psychological Factors in Stress, J. E. McGrath, ed. New York: Holt, Rinehart and Winston, 1970, p 287-347.

Weinberg, A. H. "Social Institutions and Nuclear Energy." Science 177:27-34, 7 July 1972 .

Weinberg, A. M. "Energy Needs, Nuclear Power, and the Environment." In Radiation Research: Biomedical, Chemical, and Physical Perspectives. Proceedings of the 5th International Congress of Radiation Research, Seattle, July 1974, pp 32-38.

Weinberg, A. M. "Social Institutions and Nuclear Energy." Science 177(7): $27-34,1972$.

Weiner, R. I. "The Potential of Nuclear Pacemakers as a Terrorist Tool." Hazard Prevention, November/December, 1978, p 23.

Weiss, W. "Effects of the Mass Media of Communication." In The Handbook of Social Psychology, vol. 5, G. Lindzey, and E. Aronson, eds. Reading, MA: Addison-Wesley, 1969.

Wertham, F. "The Scientific Study of Mass Media Effects." Am Journal of Psychiatry 119:306-311, 1962 .

Westley, W. A. "The Formation, Nature and Control of Crowds." Report to the Defense Research Board of Canda, April 1956.

"What Urban Guerrillas Read." Scanlan's 1:67-68, January 1971.

White, D. M. "The 'Gatekeeper': A Case Study in the Selection of News." Journalism Quarterly 27:383-390, 1950.

Wildavsky, A. "No Risk is the Highest Risk of All." American Scientist $67: 32 \mathrm{ff}, 1979$. 
Williams, R. M. "Massing at the Grass Roots." Saturday Review, 22 January 1977.

Williamson, H. E., and D. C. Ditmore. Current State of Knowledge, High Performance Zircaloy Clad UO 2 Fue1, NEDO-10173, May 1970 .

Willrich, M., and T. B. Taylor. Nuclear Theft: Risks and Safeguards. A report to the Energy Policy Project of the Ford Foundation. Cambridge, MA: Ballinger, 1974.

Willrich, M. "Terrorists Keep Out!" Bulletin of the Atomic Scientists, May 1975, pp 12-16.

Willrich, M., et al. Radioactive Waste--Management and Regulation. New York: Free Press, 1977.

Wing, J. Toxic Vapor Concentrations in the Control Room Following a Postulated Accidental Release, NUREG-0570. Washington: Nuclear Regulatory Commission, May 1976.

Wirth, L. "Consensus and Mass Communication." American Sociological Review 13:1-15, 1948.

Wisconsin Legislative Council. Recent Legislative Council Publications, 30 April 1979.

Wisconsin Legislative Council Staff. Additional Information on the Transportation of Radioactive Wastes and Related Emergency Planning, Information Memo 77-20. Madison, WI, 1 November 1977.

Wisconsin Legislative Council Staff. An Introduction to Radioactive Waste Management Issues in Wisconsin, SB 77-4. Madison, WI, 12 May 1977.

Wolf, C. P. "Social Impact Assessment: The State of the Art." In Social Impact Assessment, ERDA-5, C. P. Wolf, ed. Milwaukee: Environmental Design Research Association, 1974.

Wolfenstein, M. Disaster: A Psychological Essay. Glencoe, IL: Free Press, 1957.

World Meteorological Organization. "Building Climatology," Technical Note 109. WMO No. 255, TP 142, 1970.

World Meteorogical Organization. "Urban Climates," Technical Note 108. WMO No. 254, TP141, 1970.

Wright, A. L., et al. Effect of Energy Density on Aerosol Yield and Primary Particle Sizes Produced by the Capacitor Discharge Vaporization (CDV) of $\overline{\mathrm{UO}}^{2}$, NUREG/CR-0120, ORNL/NUREG/TM-163. Oak Ridge, TN: Oak Ridge National Laboratory, October 1978.

Yadigaroglu, G. "Spent Fuel Transportation on Highways -- The Radioaciive Dose to the Traffic." Nuclear Technology 27:258, 1975. 
Yadigaroglu, G., A. G. Reinking, and V. E. Schrock. "Spent Fuel Transporation Risks." Nuclear News 15(11):71-75, 1972.

Yavitz, R. S. "Federal, State, and Common Carrier Efforts to Safeguard the Transportation of Radioactive Materials." Stanford Environmental Law Annual, 1978, Energy Production: Selected Issues 1:202-248, 1979.

Yoshimura, H. R. Crash Testing of Spent-Nuclear-Fuel Shipping Systems, SAND 78-0451C. Albuquerque: Sandia Laboratories, April 1978.

Yoshimura, R. "Full Scale Tests of Spent-Fuel Shipping Systems." In Transactions of the American Nuclear Society Winter Meeting, 28 November to 2 December, 1977.

Yoshimura, R., and M. Huerta. A Crash Test of a Nuclear Spent Fuel Cask and Truck Transport System, SAND77-0419. Albuqueruqe: Sandia Laboratories, January 1978.

Zima, G. E. Comments in Fuel Crud as a Safety and Operational Factor of Independent Spent Fuel Storage Installations (ISFSI). NUREG/CR-0163, PNL-2657. Richland, WA: Battelle Pacific Northwest Laboratories, November 1978.

Yuhas, J. M., T. R. Stokes, and C. C. Lushbaugh. "Multifactorial Analysis of Human Blood Cell Response to Clinical Total Body Irradiation,"

NSA-TM-X-2440. Presented at National Symposium on Natural and Man-Made Radiation in Space, January 1972. 